Basendowski, Sven

\title{
Das andere Lesen. Das Lesen multikodaler Textualität an Einfach(st)arbeitsplätzen im Spiegel der Relation aus Bildung und Teilhabe
}

\author{
Bad Heilbrunn : Verlag Julius Klinkhardt 2020, 261 S. - (Literalität und Arbeitswelt)
}

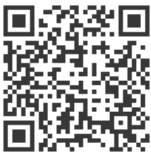

Quellenangabe/ Reference:

Basendowski, Sven: Das andere Lesen. Das Lesen multikodaler Textualität an

Einfach(st)arbeitsplätzen im Spiegel der Relation aus Bildung und Teilhabe. Bad Heilbrunn : Verlag Julius Klinkhardt 2020, 261 S. - (Literalität und Arbeitswelt) - URN: urn:nbn:de:0111-pedocs-209468 DOI: 10.25656/01:20946

https://nbn-resolving.org/urn:nbn:de:0111-pedocs-209468

https://doi.org/10.25656/01:20946

in Kooperation mit / in cooperation with:

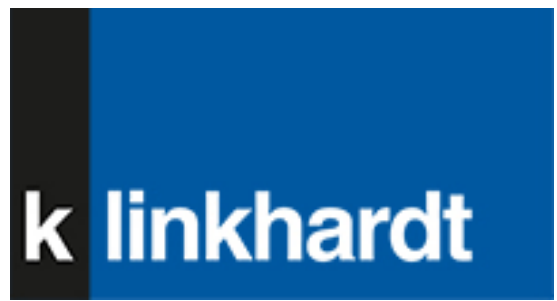

http://www.klinkhardt.de

\section{Nutzungsbedingungen}

Dieses Dokument steht unter folgender Creative Commons-Lizenz: http://creativecommons.org/licenses/by-nc-sa/4.0/deed.de - Sie dürfen das Werk bzw. den Inhalt unter folgenden Bedingungen vervielfältigen, verbreiten und öffentlich zugänglich machen sowie Abwandlungen und Bearbeitungen des Werkes bzw. Inhaltes anfertigen: Sie müssen den Namen des Autors/Rechteinhabers in der von ihm festgelegten Weise nennen. Dieses Werk bzw. der Inhalt darf nicht für kommerzielle Zwecke verwendet werden. Die neu entstandenen Werke bzw. Inhalte dürfen nur unter Verwendung von Lizenzbedingungen weitergegeben werden, die mit denen dieses Lizenzbedingungen weitergegeben werden,

Mit der Verwendung dieses Dokuments erkennen Sie die Nutzungsbedingungen an.

\section{Terms of use}

This document is published under following Creative Commons-License: http://creativecommons.org/licenses/by-nc-sa/4.0/deed.en - You may copy, distribute and transmit, adapt or exhibit the work in the public and alter, transform or change this work as long as you attribute the work in the manner specified by the author or licensor. You are not allowed to make commercial $\mathrm{k}$ in any way, you
able license. may distribute the resulting work only under this or a comparable license.

By using this particular document, you accept the above-stated conditions of

\section{Kontakt / Contact:}

peDOcs

DIPF | Leibniz-Institut für Bildungsforschung und Bildungsinformation Informationszentrum (IZ) Bildung

E-Mail: pedocs@dipf.de

Internet: www.pedocs.de

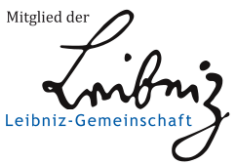




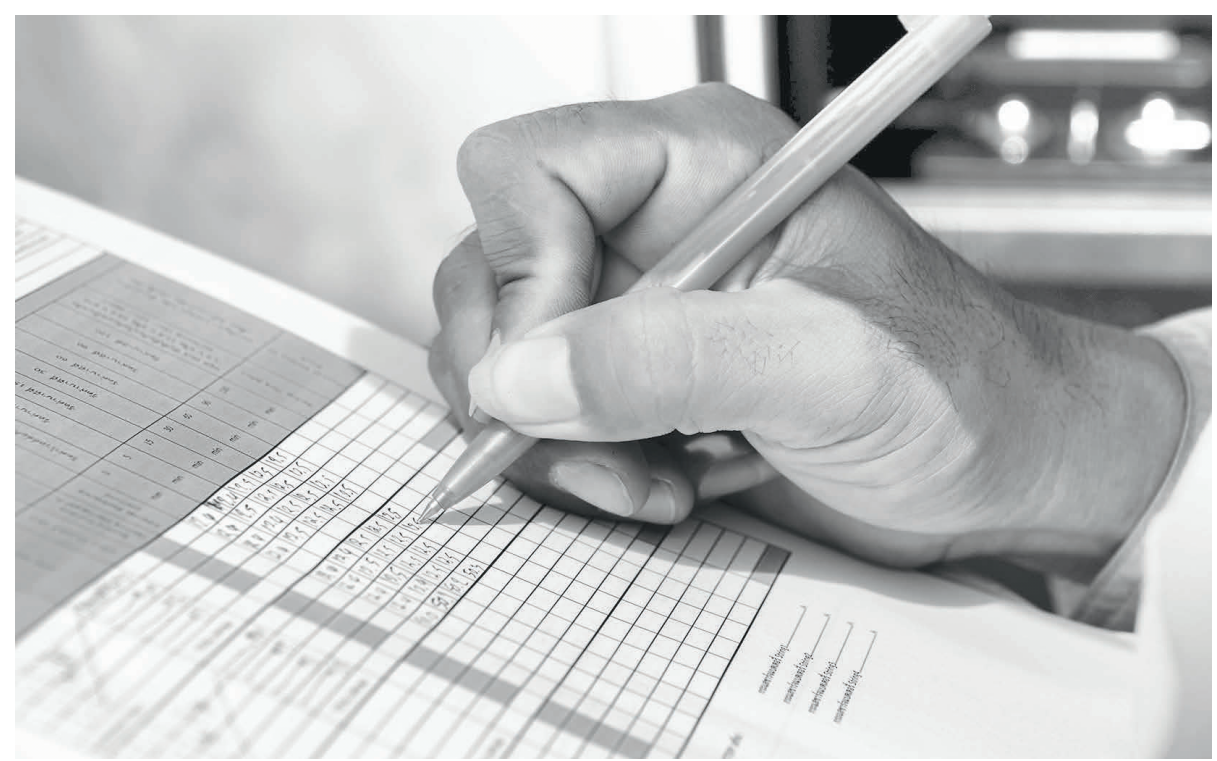

Sven Basendowski

\section{Das andere Lesen}

Das Lesen multikodaler Textualität an Einfach(st)arbeitsplätzen im Spiegel der Relation aus Bildung und Teilhabe 
Basendowski

Das andere Lesen 
Literalität und Arbeitswelt

herausgegeben von

Joachim Schroeder 


\title{
Das andere Lesen
}

Das Lesen multikodaler Textualität an Einfach(st)arbeitsplätzen im Spiegel der Relation aus Bildung und Teilhabe

\author{
Kapitel 7 unter Mitarbeit \\ von Judith Adam
}


Dieser Titel wurde in das Programm des Verlages mittels eines Peer-Review-Verfahrens aufgenommen. Für weitere Informationen siehe www.klinkhardt.de.

Bibliografische Information der Deutschen Nationalbibliothek Die Deutsche Nationalbibliothek verzeichnet diese Publikation in der Deutschen Nationalbibliografie; detaillierte bibliografische Daten sind im Internet abrufbar über http://dnb.d-nb.de.

2020.kg @ by Julius Klinkhardt.

Satz: Kay Fretwurst, Spreeau.

Bild Umschlagseite 1: @ Kitawit Jitaton / Shutterstock.com.

Druck und Bindung: AZ Druck und Datentechnik, Kempten.

Printed in Germany 2020.

Gedruckt auf chlorfrei gebleichtem alterungsbeständigem Papier.

Die Publikation (mit Ausnahme aller Fotos, Grafiken und Abbildungen) ist veröffentlicht unter der Creative Commons-Lizenz: CC BY-NC-SA 4.0 International

https://creativecommons.org/licenses/by-nc-sa/4.0/

ISBN 978-3-7815-5842-7 Digital

doi.org/10.35468/5842

ISBN 978-3-7815-2406-4 Print 


\section{Zusammenfassung}

Mit dem kulturwissenschaftlich entlehnten Ansatz von Kulturtechniken als Set sozialer literaler Praxen wird theoretisch kritisch und mittels einer Mixed-Methods-Studie empirisch befragt, welche Lesekompetenzen die Relation aus Bildung und grundlegender Teilhabe in Arbeit von sozial benachteiligten Jugendlichen bis junge Erwachsenen tatsächlich markieren. Die theoretische Auseinandersetzung reicht von einer Analyse von „Kultur“ in unterschiedlichen Lesebegriffen bis hin zur linguistischen Dekomposition der aufgefundenen multikodalen Textualität an den untersuchten Einfach(st)arbeitsplätzen, die durchaus sozialversicherungspflichtige und sichere Teilhabe in Arbeit für die Zielgruppe gewähren. Die empirischen Befunde der MixedMethods-Studie umfassen u. a. die situativen Funktionen des Lesens der aufgefundenen multikodalen Textualität an den Arbeitsplätzen, einhergehend mit der Relevanz deren Anwendung als knowing how bei der Verarbeitung. Die übergeordnete Kernaussage der Studie richtet sich kritisch an die externe Validität von Mainstream-Konzepten von Lesekompetenzen entlang von Kodalität und Funktion der zugrundeliegenden Textualität: Diese Mainstream-Konzepte haben einen blinden Fleck in der probabilistisch prognostischen Aussagekraft ihres funktionalen Mindestlevesl für den beruflichen Erfolg im Anschluss an ein (inklusives) Bildungssystem.

Dr. phil. Sven Basendowski lehrt als Juniorprofessor an der Universität Rostock am Institut für sonderpädagogische Entwicklungsförderung und Rehabilitation. Seine Arbeit-/Interessenschwerpunkte liegen in den kulturtechnischen und berufsorientierenden Bildungsprozessen unter prekären Bedingungen, (liegen) in der netzwerkanalytischen Betrachtung von sozialer Benachteiligung, Strukturen der Übergangsregimes Schule/Arbeit im Spiegel sozialer Benachteiligung sowie ihrer subjektorientierten Bearbeitung. 


\begin{abstract}
With the cultural-scientific approach of cultural techniques as a set of social literary practices, a theoretically critical and empirical study (mixed-methods study) is used to question which reading competencies mark the relation of education to fundamental participation in the work of socially disadvantaged young people to young adults. The theoretical discussion ranges from an analysis of cultural concepts in different reading approaches to the linguistic decomposition of the explored multi-codal textuality at a choice of examined simple workplaces - which grant a certain degree of social security and safe participation in work for the target group. The empirical findings of the mixed-methods study include, inter alia, the situational role of reading of the multi-codal textuality, along with the relevance of their application as knowing how in processing. The overall key message of the study is a critical view on the external validity of mainstream concepts of reading skills along with codality and social role of the underlying textuality: These mainstream concepts have a blind spot in the probabilistically prognostic significance of their functional minimum level for professional success in terms of an (inclusive) education system.
\end{abstract}

Dr. phil. Sven Basendowski teaches as a junior professor at the university of Rostock at the institute for special educational development and rehabilitation. His main areas of work/interest are the cultural-technical and vocationally oriented educational processes under precarious conditions, network-analytical consideration of social disadvantage, structures of the transitional regimes school/work in the mirror of social disadvantage as well as their subject-oriented treatment. 


\section{Inhalt}

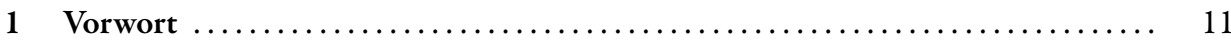

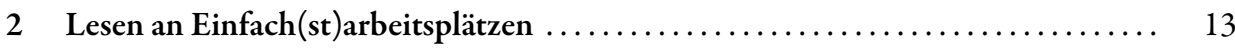

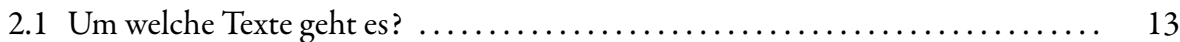

2.1.1 Erste Annäherung an Nicht-Fließtexte $\ldots \ldots \ldots \ldots \ldots \ldots \ldots \ldots \ldots \ldots$

2.1.2 Textbeispiele an Einfach(st)arbeitsplätzen .................. 15

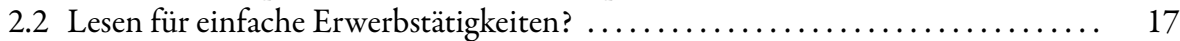

2.2.1 Mythos der grundsätzlich einfachen Arbeit .................. 19

2.2.2 Mythos der grundsätzlich prekären Arbeit .................. 20

2.2.3 Mythos der pädagogischen Determiniertheit in der Risiko-Relation von Bildung und Teilhabe in Arbeit $\ldots \ldots \ldots \ldots \ldots \ldots \ldots \ldots \ldots \ldots, 23$

3 Lesekompetenz nichtkontinuierlicher Texte $\ldots \ldots \ldots \ldots \ldots \ldots \ldots \ldots \ldots \ldots \ldots$

3.1 Lesekompetenz(en) - (funktionale) Kompetenz(en) des Lesens .......... 45

3.1.1 Kompetenzbegriffe zur Annäherung an die Handlungsfähigkeiten, -fertigkeiten und -bereitschaften des Gebrauchs von Texten (a) ...... 47

3.1.2 Lesebegriffe zur Annäherung an die Handlungsfähigkeiten, -fertigkeiten und -bereitschaften des Gebrauchs von Texten (b) ...... 48

3.1.3 Die „Kultur" der Lesebegriffe ......................... 56

3.1.4 Zeichenbegriffe zur Annäherung an die Handlungsfähigkeiten, -fertigkeiten und -bereitschaften des Gebrauchs von Texten ........ 63

3.2 Semiotische Analyse der Zeichen hinter primär kategorial-tabellarischen Texten .. 66

3.2.1 Semiotisches Kriterium der Ähnlichkeit ikonischer Darstellungen ..... 68

3.2.2 Kategoriale Tabellen: eine ikonische Repräsentation?............ 72

3.2.3 Starke Codes hinter nichtkontinuierlichen Texten ............... 76

3.2.4 Semiotische Betrachtung spezifischer Sets ikonischer Darstellungs-Kodes .. 82

3.2.5 Semiotische Betrachtung weiterer Sets nicht-arbiträrer Codes ....... 88

3.2.6 Set sprachlicher Steuerungs-Kodes ..................... 94

3.2.7 Linguistische Betrachtung der Explikation durch symbolische Zeichen .... 95

3.3 Verstehende Verarbeitung

Sets ikonischer Zeichen im Spannungsfeld des „Sozialen“ und „Situativen“....... 101

3.3.1 Spur 1: Amodale vs. schematisch-modale Makromodelle der verstehenden ikonischen Repräsentationen ..................

3.3.2 Spur 2: Amodale vs. embodied-modale Makromodelle der verstehenden ikonischen Repräsentationen .................. 110

3.3.3 Symbiose zu einem integrativen Arbeitsmodell kognitiver Verarbeitungsprozesse primär kategorial-tabellarischer Texte .........

3.4 Sets sozialer Praxen an Einfach(st)arbeitsplätzen und ihre Lesenden Verengung zu den Forschungsfragen ................................... 129

3.4.1 Primitivität der Sets sozialer Praxen an Einfach(st)arbeitsplätzen? . .... 130

3.4.2 Artefakte und lokale kulturelle Codes als Teil der Sets sozialer Praxen an Einfach(st)arbeitsplätzen? ......................... 
3.4.3 Lokale kulturelle Codes als Teil der Sets sozialer Praxen an Einfach(st)arbeitsplätzen?

3.4.4 Verkörperlicht erfahrene Verarbeitung der Lesenden der Sets sozialer Praxen an Einfach(st)arbeitsplätzen? ..................... 132

3.5 Vernetzung der Forschungsfragen $\ldots \ldots \ldots \ldots \ldots \ldots \ldots \ldots \ldots \ldots \ldots \ldots \ldots \ldots \ldots \ldots \ldots$

4 Das Historische des Lesens multikodaler Textualität $\ldots \ldots \ldots \ldots \ldots \ldots \ldots \ldots \ldots 1$

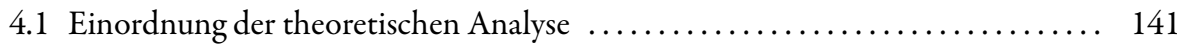

4.2 Historische Analyse der semiotischen Artefakte hinter kategorialen Tabellen .. 141

4.3 Ergebnis der Teilstudie ............................... 151

5 Das situativ Soziale des Lesens multikodaler Textualität .............. 153

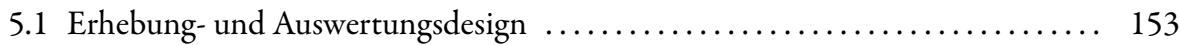

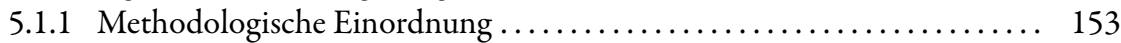

5.1.2 Erhebungsdesign von Situationen mit Texten an

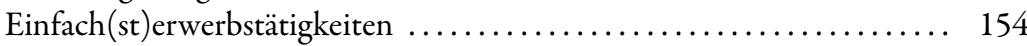

5.1.3 Auswertungsdesign entlang alltäglich-routinemäßiger und besonders herausfordernder Situationen mit Texten an Einfach(st)erwerbstätigkeiten

5.2 Qualitative Inhaltsanalyse von routinalisierten lokalen kulturellen Code in

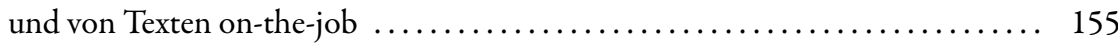

5.2.1 Regelgeleitetes Vorgehen: Strukturierende Qualitative Inhaltsanalyse ...

5.2.2 Analyseeinheiten: Das sensibilisierende Konzept ................

5.2.3 Induktive Entwicklung eines Kategoriensystems ...............

5.2.4 Finalisierung des induktiv entwickelten Kategoriensystem der situativtextuellen routinalisierten lokalen kulturellen Codes

5.3 Konfigurationen routinalisierter lokaler kultureller Codes und routinalisierter

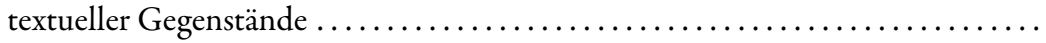

6 Primitive Kulturtechnik: Kulturell vernachlässigbar oder im ordentlichen Lesen

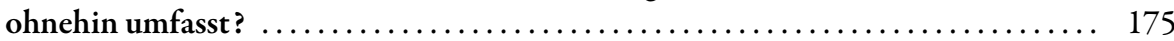

6.1 Design der quantitativen Teilstudien: Ergänzungen .................. 175

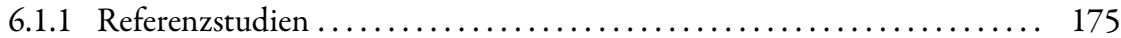

6.1.2 Methodische Schlussfolgerungen aus den Referenzstudien .......... 181

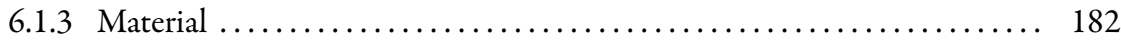

6.2 Durchführung und Stichprobe ............................. 194

6.3 Modellprüfung der standardisierten Erhebung von Lesecompetencies multikodaler Texte und Textkomplexe .......................... 195

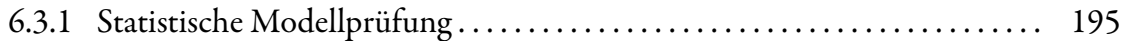

6.3.2 Inhaltliche Modellprüfung $\ldots \ldots \ldots \ldots \ldots \ldots \ldots \ldots \ldots \ldots \ldots \ldots . \ldots \ldots \ldots$

6.3.3 Deskriptive und vergleichend kriteriale Modellprüfung ........... 201

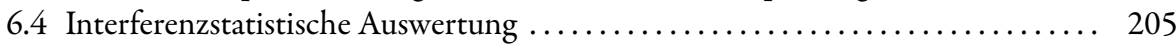

6.4.1 Statistischer Zusammenhang zwischen den Dimensionen primär ikonischen multikodalen Lesens und symbolischen Lesens .......... 206

6.4.2 Kausaler Zusammenhang zwischen den Dimensionen primär ikonischen multikodalen Lesens und symbolischen Lesens .......... 207

6.5 Ergebnis der Teilstudie . .............................. 208 
7 Empirischer Blick auf das praxeologische „Wie“ des Lesens multikodaler Texte .... 209

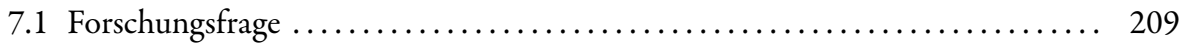

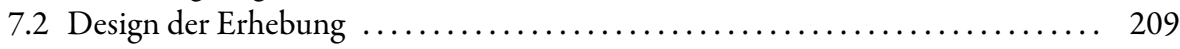

7.2.1 Teilstrukturierte Situation der Interspektion $\ldots \ldots \ldots \ldots \ldots \ldots \ldots . \ldots 211$

7.2.2 Teilstrukturierte Situation der Retrospektion ................ 212

7.2 .3 Gedächtnisprotokoll .............................. 213

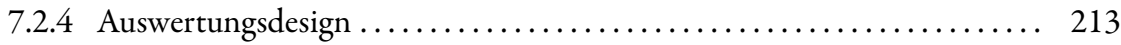

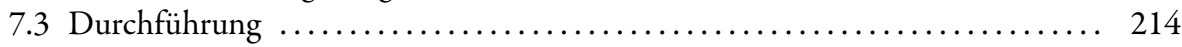

7.3.1 Feldzugang und Kriterien zur Datenerhebung ................. 214

7.3.2 Stichprobe: Idealtypen berufsfeldspezifischer Orientierung ......... 215

7.3.3 Induktive Datensichtung entlang der präzisierten Fragestellung ...... 216

7.3.4 Analyse entlang der deduktiven Kategorien ................... 219

7.3.5 Zusammenführung der drei Einzelfälle ................... 223

7.4 Auswertung der Daten: Präzisierung der Forschungsfrage ............. 226

8 Teilhabe durch multikodales Lesen $\ldots \ldots \ldots \ldots \ldots \ldots \ldots \ldots \ldots \ldots \ldots \ldots \ldots \ldots \ldots$

8.1 Theorie des multikodalen Lesens $\ldots \ldots \ldots \ldots \ldots \ldots \ldots \ldots \ldots \ldots \ldots \ldots \ldots . \ldots \ldots \ldots$

8.2 Multikodales Lesen in der Relation von Bildung und Teilhabe ............. 240

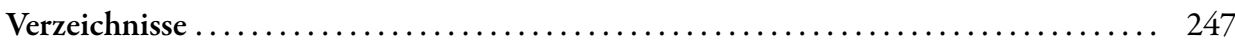

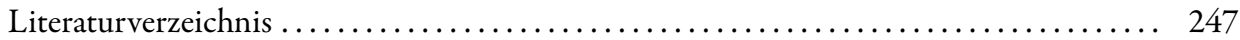

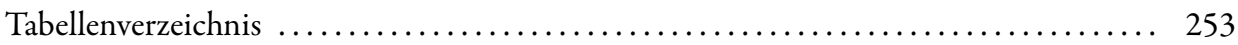

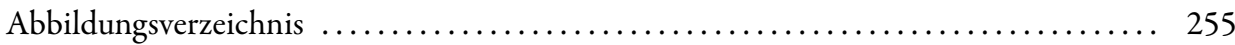

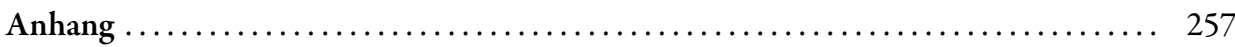

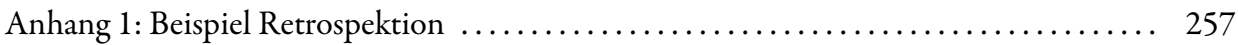





\section{Vorwort}

Im Zuge der eigenen Dissertationsstudie beschäftigte ich mich mit den mathematischen Anforderungen am Arbeitsplatz von sogenannten einfach(st)en Erwerbstätigkeiten. Die teils induktive empirische Herangehensweise sah vor, situative berufliche Handlungskompetenzen mittels problemzentrierter Interviews mit einer kontrollierten Auswahl an Mitarbeiter/innen und ihren Vorgesetzten diverser Berufsfelder aufzudecken. Eine Vielzahl der Schilderungen zu den alltäglichen und besonders herausfordernden beruflichen Situationen am Arbeitsplatz verwies immer wieder auf eine dominante Textform on-the-job: Texte, die gerade nicht in Sätzen, Absätzen oder gar Kapiteln gegliedert sind. Es sind stattdessen die Arbeitszeiten an einem gerne auch vier Meter breiten tabellarischen Aushang zu entnehmen oder Ablaufdiagramme informieren über bestimmte einzuhaltende Arbeitsabläufe. Raum- oder Lagerpläne dienen der Orientierung und Ordnung. Diese Beispiele verdeutlichen bereits die überwiegend für Arbeitsprozesse pragmatische Relevanz jener Texte. Sie dienen einer maximal schnellen Informationsentnahme einzelner Sachverhalte und ihrer Relationen in diversen anspruchsvollen beruflichen Situationen.

Ähnlich noch unstrukturierte erste Beobachtungen wurden in dem BMBF-Forschungsprojekt GRAWIRA gesammelt, welches die Anforderungen am Arbeitsplatz von Teilnehmer/innen an Literalitätskursen für Erwachsene erforschte. Gespräche mit dem Projektleiter Prof. Dr. Joachim Schroeder führten letztlich zu einem Lehr- und Forschungsprojekt, welches gezielt die Bearbeitung jener typischen Texte in Einfach(st)erwerbstätigkeiten näher erforschen sollte. Neben der Erprobung von Erhebungsmethoden bis Auswertung von Forschungsdaten offerierte das hochschuldidaktische Format den studentischen Teilnehmer/innen, ihre Abschlussarbeiten gezielt zu Teilfragestellungen des Lesens an Einfach(st)erwerbstätigkeiten zu verfassen. Die vorliegende Hauptstudie rekurriert teilweise auf jene empirischen Vorstudienergebnisse, die bis 2014 entstanden.

In diesem Zuge danke ich den Studierenden, die sich in den Veranstaltungen zum Lehr-/Forschungsprojekt an der Goethe-Universität Frankfurt und an der Universität Hamburg eingaben. Zahlreiche Diskussionen, aber letztlich auch wissenschaftliche Arbeiten deckten maßgeblich offene Fragestellungen auf bzw. führten zu einer Weiterentwicklung des erkenntnistheoretischen Ansatzes. 



\section{Lesen an $\operatorname{Einfach}(s t)$ arbeitsplätzen}

\subsection{Um welche Texte geht es?}

„Von der Wiege bis zur Bahre: Formulare, Formulare." Diese alte Redewendung spielt aufdie Menge von Formularen an, die uns im Laufe unseres Lebens immer wieder begegnen. Meldet sich jemand z.B. am neuen Wohnort an, tätigt eine Überweisung oder gibt über das Internet eine Bestellung auf, überall stößt man auf textuelle Formate, die gerade nicht durch Fließtexte charakterisiert sind. $\mathrm{Zu}$ jenen omnipräsenten Texten zählen folglich nicht nur Formulare. Entlang eines Fußweges durch die Stadt wimmelt es von Plakaten zu diversen Veranstaltungen oder Aushängen von Vereinigungen. Werbeflyer oder -beilagen, die Ergebnisse der Sonntagsumfrage bis Raumpläne sind weitere Beispiele, sowohl in Print als auch digital, die verdeutlichen, welche Relevanz ein sicherer Umgang mit denjenigen Texten - nicht nur in öffentlichen Verwaltungsangelegenheiten hat, die sich gerade nicht primär aus Wörtern, Sätzen, Absätzen, Kapiteln usw. zusammensetzen.

\subsubsection{Erste Annäherung an Nicht-Fließtexte}

Nicht-Fließtexte werden in der wissenschaftlichen Auseinandersetzung um kognitive Bearbeitungsprozesse oder linguistische bzw. graphische Differenzierungen sehr unterschiedlich bezeichnet. Die wohl prominenteste Kategorisierung als Textformat der nichtkontinuierlichen (synonym: diskontinuierlichen) in Unterscheidung zu den kontinuierlichen Texten stammt aus der PISA-Studie, respektive, basiert auf den formal-strukturellen Analysen von Kirsch/Mosenthal (1989-1990). Die kontinuierlichen Fließ- oder Prosatexte werden von den nichtkontinuierlichen Schaubildern/ graphischen Darstellungen, Tabellen/Matrizen, Diagrammen und Karten unterschieden. Jene Gliederung findet sich in weiteren wissenschaftlichen Auseinandersetzungen wieder, obgleich mit alternativen synonymen Bezeichnungen. So werden die Nicht-Fließtexte als "nichtlineare Texte " in den Bildungsstandards (KMK 2005, 21ff.) oder innerhalb von nationalen Schulleistungstests wie z. B. VERA als ,diskontinuierliche Texte" benannt (Projektgruppe VERA 2005). Die Übersicht in Abbildung 1 von Haible (2011) bezieht sich nicht zuletzt u. a. auf letztgenannte Quellen und veranschaulicht durch Beispiele neben der kategorialen Zuordnung die Bandbreite an Nicht-Fließtexten, die als nichtkontinuierlich, bzw. synonym als diskontinuierlich oder nichtlinear bezeichnet werden.

\begin{tabular}{|c|c|c|c|c|}
\hline \multirow{2}{*}{\multicolumn{2}{|c|}{ 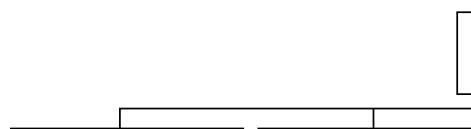 }} & $\begin{array}{l}\text { Diskontinuierliche Texte } \\
\text { (Nichtlineare Ziele) }\end{array}$ & & \\
\hline & & & 工 & \\
\hline $\begin{array}{l}\text { Tabellen und } \\
\text { Listen }\end{array}$ & $\begin{array}{c}\text { Numerische Gra- } \\
\text { phiken }\end{array}$ & Erklärgrafiken & $\begin{array}{l}\text { Topographische } \\
\text { Graphiken }\end{array}$ & Mischformen \\
\hline $\begin{array}{l}\text { z.B. Statistiken, } \\
\text { Sportergebnisse, } \\
\text { Klimatabellen, } \\
\text { Stellenausschreibun- } \\
\text { gen, Aktienkurse, } \\
\text { Fahrpläne, } \\
\text { Formulare }\end{array}$ & $\begin{array}{l}\text { z. B. alle Arten von } \\
\text { Diagrammen, } \\
\text { Umfrageergebnisse, } \\
\text { Wirtschafts- } \\
\text { entwicklungen, } \\
\text { Klimadiagramme, } \\
\text { Ländervergleiche }\end{array}$ & $\begin{array}{l}\text { z.B. schematisierte } \\
\text { Arbeitsanleitungen, } \\
\text { Gebrauchsanweisun- } \\
\text { gen, Installationsanlei- } \\
\text { tungen, Erste-Hilfe- } \\
\text { Anleitung, anatomi- } \\
\text { sche Zeichnungen, } \\
\text { und Schaubilder }\end{array}$ & $\begin{array}{l}\text { z. B. Straßenkarten, } \\
\text { Landschaftskarten, } \\
\text { Netzkarten, Relief- } \\
\text { karten, Navigations- } \\
\text { karten, Baupläne, } \\
\text { Lagepläne }\end{array}$ & $\begin{array}{l}\text { z.B. Werbung, } \\
\text { Zeitschriftenseiten, } \\
\text { Hypertexte, Lexi- } \\
\text { konartikel, Reiseka- } \\
\text { taloge, Karikaturen }\end{array}$ \\
\hline\llcorner & 1 & & 1 & 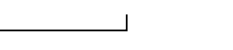 \\
\hline
\end{tabular}

Abb. 1: Deskripitiv-kategoriale Gliederung nichtkontinuierlicher Texte (Quelle: Haible 2011) 
Die Skizzierung gibt bereits eine terminologische Problematik wieder, die sich noch potenziert, wenn z.B. ferner englische Bezeichnungen einbezogen werden. Charts stehen hier einerseits für Tabellen und andererseits für Diagramme, die Zusammenhänge zwischen qualitativen Merkmalen eines Sachverhalts darstellen. Charts werden zudem neben Graphs als Diagrams bezeichnet; wobei Graphs Zusammenhänge zwischen kategorialen und intervallskalierten oder ausschließlich intervallskalierten Merkmalen eines Sachverhalts abbilden (Schnotz 1994, 97). Konsequenterweise soll für die weitere Analyse an dieser Stelle eine terminologische Setzung von Formaten hinter nichtkontinuierlichen Texten bzw. synonym logischen Bildern vorgenommen werden:

- Graphische Darstellungen basieren auf realitätsgetreuen Abbildungen eines Sachverhalts und somit auf schematischen Abbildungen, Skizzen bzw. vereinfachten Abbildungen, realistischen Abbildungen oder Fotographien (vgl. Abbildung 2). Sie können in Texten singulär, aber auch aneinandergereiht z. B. als Kompositionen (Hase: Körperteile) oder Prozesse (Hase: Sprungbewegung) repräsentiert werden.
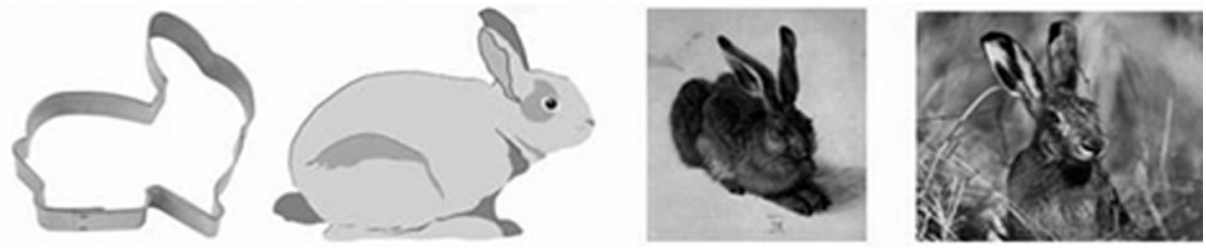

Abb. 2: Abbildungen von Hasen mit zunehmender Realitätsnähe (Quelle: Horz 2009, 110)

- Tabellen sind aus Feldern aufgebaut, die sich in der Regel aus der Kombination von Spalten und Zeilen ergeben. Die Bezeichnungsfelder, in senkrechten Tabellen üblicherweise das obere Feld einer Spalte, bzw. das linke Feld einer Zeile in waagerechten Tabellen, geben die Merkmalskategorie an, nach der die zugehörigen Textfelder der Spalte bzw. der Zeile zugeordnet sind. Die Bezeichnungs- und Textfelder können durch Zahlziffern, Wörter, Buchstaben, Piktogramme usw. expliziert sein und verweisen auf Gegenstände und Merkmale eines Sachverhalts, deren Zusammenhänge mittels Tabellen beschrieben werden können: qualitativ/qualitativ, qualitativ/ quantitativ (,informal tables'/kategoriale Tabellen) oder quantitativ/quantitativ (,mathematical or numerical tables/mathematische oder numerische Tabellen). Im einfachsten Fall besteht eine Tabelle nur aus einem Bezeichnungsfeld entweder für eine Spalte oder eine Zeile (Robson 2003).

- Diagramme eignen sich wie Tabellen, um rein qualitative oder auch/rein quantitative Zusammenhänge eines Sachverhalts abzubilden. Im ersten Fall setzen sich diese kategorialen Diagramme entweder aus Knoten, meist dargestellt durch geometrische Formen wie Rechtecke bis Ellipsen, und (un)gerichteten Kanten via Linien oder Pfeilen zusammen. Beispiele hierfür sind Struktur- oder Ablaufdiagramme. Andererseits können sie auch nur aus geometrischen Formen gebildet sein, die durch die Anordnung auf kategoriale Inklusions- oder Exklusionsbeziehungen eines Sachverhalts verweisen. Kategoriale Diagramme können folglich begriffliche, topologische oder Verlaufsstrukturen wiedergeben. Für den zweiten Fall stehen neben den „klassischen“ Kreis-, Balken-, Säulen-, Linien- und Streu- auch die sogenannten Isotyp-Diagramme, sowie diverse Zwischenformen. Jene metrischen Diagramme greifen gleichermaßen auf geometrische Formen wie Kreissegmente oder Rechtecke zurück, die durch Größe oder eingebettet in ein Koordinatensystem ordinale bis stochastische Zuordnungen veranschaulichen. Abszisse bzw. Ordinate können entsprechend kategorial bis intervallska- 
liert unterteilt sein. Den Spezialfall eines Isotyp-Diagramms machen sich Piktogramme für eine, zumeist nicht exakte und nur annähernde, Beschreibung metrischer Zusammenhänge zunutze; z. B. bei der Abbildung von Einwohnern mehrerer Städte durch Männchen-Piktogramme, wobei ein „ganzes Männchen“ für 100.000 und ein „halbes Männchen“ für 50.000 Einwohner steht (Schnotz 1994; Weidenmann 1994).

- Karten werden eingesetzt, um Zusammenhänge zwischen Gegenständen bzw. Merkmalen eines Sachverhalts mit einem Raumbezug unter Betrachtung eines weiteren Attributs darzustellen ${ }^{1}$. Jenes Attribut kann, erstens, in der Form räumlicher Diskreta (absolutes Vorkommen, relatives Vorkommen, Flächen bezogene Merkmale) oder Kontinua (reales Kontinuum, Modellkontinuum) erscheinen. Zweitens kommt ein sachlicher Bezug in Frage, je nachdem, ob die Quantität oder Qualität der Gegenstände bzw. Merkmale eines Sachverhalts besprochen wird. Drittens ist zwischen statischen und dynamischen Gegenständen und Merkmalen des Sachverhalts zu unterscheiden; es resultiert der zeitliche Bezug der Darstellung durch Karten. Unabhängig der Objektinformationen werden hierbei stets einzelne und relational zueinander in Beziehung stehende graphische Entitäten wie Punkte, Linien oder Flächen genutzt. Zur Bezeichnung der Gegenstände und Merkmale des Sachverhalts sowie als Indikator für Relationen zwischen diesen finden sich Kartenzeichen wie Signaturen, Diagramme, Halbtöne oder Schrift/Worte (Hake et al. 2002, 10ff.).

Mischformen repräsentieren primär kontinuierliche oder nichtkontinuierliche Texte, die sekundär aus mehreren bzw. verschiedenen der vorangehenden Textformate zusammengesetzt sind: Sind sie ohne Verknüpfung zusammengefügt, werden diese Kombinationen aus nichtkontinuierlichen und/oder kontinuierlichen Texten als „Gemischte Texte“ betrachtet. Liegen die jeweils zu identifizierenden und zu dekodierenden Verknüpfungen mehreren Quellen zugrunde, wird von einem „Textkomplex“ gesprochen (OECD 2010, 42).

\subsubsection{Textbeispiele an Einfach(st)arbeitsplätzen}

Mit der ersten kategorialen Annäherung sollen die aufgefundenen Texte an Einfach(st)arbeitsplätzen (Basendowski 2013; Schroeder 2011) charakterisiert werden. Der nachfolgenden Tabelle 1 sind jene textuellen Beispiele zu entnehmen, die bereits eine Akzentuierung auf bestimmte analog oder digital dargestellte Textformate erlauben, welche für diese Arbeitsplätze durch ihre Verwendung in unterschiedlichen beruflichen Kontexten als besonders relevant eingestuft werden können. Es handelt sich hierbei zuvorderst um primär oder sekundär durch kategoriale Tabellen strukturierte nichtkontinuierliche Print-Texte.

Mit Blick auf die aufgefundene Auswahl verdeutlicht sich bereits an dieser Stelle - einzelne Beispiele werden in den weiteren Kapiteln illustriert -, dass ein Großteil der insbesondere nichtkontinuierlichen Texte eng zusammenhängen. Obgleich nicht technisch und/oder über Seitenangaben verbunden, handelt es sich in diesen Fällen explizit nicht um eine lose Blättersammlung. Es sind nicht simple Kombinationen aus nichtkontinuierlichen Texten, die singulär für sich zu verarbeiten wären. Stattdessen sind mehrere nichtkontinuierliche Texte nach einer zu dechiffrierenden Kombination verknüpft, sodass sie als Textkomplexe zu interpretieren sind. Zweifelsohne sind die textuellen Anforderungen auch durch Textkomplexe aus nichtkontinuierlichen und kontinuierlichen analogen und digitalen Repräsentationen von Sachverhalten gekennzeichnet. Der Überblick verrät, dass diese im Vergleich eindeutig seltener zugeordnet werden können.

1 Diese verschiedenen Verwendungsbezüge von topographischen bis thematischen Karten wird z. B. durch die Sendung „Mit offenen Karten“ des Senders ARTE eindrücklich verdeutlicht. 
Tab. 1: Typische Texte in ausgewählten Einfach(st)arbeitsplätzen

\begin{tabular}{|c|c|c|c|c|c|c|c|}
\hline \multirow[t]{4}{*}{ Tätigkeit } & \multirow[t]{4}{*}{ Texte } & \multicolumn{6}{|c|}{ Texte } \\
\hline & & \multirow{3}{*}{$\begin{array}{l}\text { konti- } \\
\text { nuier- } \\
\text { lich }\end{array}$} & \multicolumn{5}{|c|}{ nichtkontinuierlich } \\
\hline & & & \multirow{2}{*}{$\begin{array}{l}\text { Graph. } \\
\text { Darst. }\end{array}$} & \multicolumn{2}{|c|}{ Tabellen } & \multirow{2}{*}{$\begin{array}{c}\text { Dia- } \\
\text { gramme }\end{array}$} & \multirow[t]{2}{*}{ Karten } \\
\hline & & & & kateg. & math. & & \\
\hline \multirow{5}{*}{$\begin{array}{l}\text { Container- } \\
\text { umschlag }\end{array}$} & Mitarbeiterzeitung & $\mathrm{x}$ & $\mathrm{x}$ & & & & \\
\hline & Sicherheitsvorschriften & $\mathrm{x}$ & $\mathrm{x}$ & & & $\mathrm{x}$ & \\
\hline & Crane Work List & & & $\mathrm{x}$ & & & \\
\hline & Lageplan & & & & & & $\mathrm{x}$ \\
\hline & Materialverbrauchsliste & & & $\mathrm{x}$ & & & \\
\hline \multirow{6}{*}{$\begin{array}{l}\text { Gebäude- } \\
\text { reinigung }\end{array}$} & Flyer für Kunden & $\mathrm{x}$ & $\mathrm{x}$ & $\mathrm{x}$ & & & \\
\hline & Einweisungsformular & & $\mathrm{x}$ & $\mathrm{x}$ & & & \\
\hline & Einweisung Abläufe & & $\mathrm{x}$ & $\mathrm{x}$ & & & \\
\hline & Sicherheitsinf. Reinigungsm. & & & $\mathrm{x}$ & & & \\
\hline & Sicherheitsinf. Geräte & & $\mathrm{x}$ & $\mathrm{x}$ & & & \\
\hline & Leistungsverzeichnis & & & $\mathrm{x}$ & & & \\
\hline \multirow{10}{*}{$\begin{array}{l}\text { Grünflächen- } \\
\text { und } \\
\text { Pflanzenpflege }\end{array}$} & Regelungen für Urlaub & $\mathrm{x}$ & & & & & \\
\hline & Fachzeitschriften & $\mathrm{x}$ & $(\mathrm{x})$ & $(\mathrm{x})$ & $(\mathrm{x})$ & $(\mathrm{x})$ & \\
\hline & Einweisung Geräte & & $\mathrm{x}$ & & & & \\
\hline & Einweisung Dienstleistungen & & & $\mathrm{x}$ & & & \\
\hline & Kurzbedienungsanl. Geräte & & $\mathrm{x}$ & $(\mathrm{x})$ & & & \\
\hline & Tourenplan & & & $(\mathrm{x})$ & & & $\mathrm{x}$ \\
\hline & Arbeits-/Tätigkeitsbericht & & & $\mathrm{x}$ & $\mathrm{x}$ & & \\
\hline & Aushang Geräte-Kodes & & & $\mathrm{x}$ & & & \\
\hline & Sicherheitswarnung Geräte & & $\mathrm{x}$ & & & $\mathrm{x}$ & \\
\hline & Kurzinformation Spritzmittel & & & & $\mathrm{x}$ & & \\
\hline \multirow[t]{7}{*}{ Kantine } & Hygienevorschriften HACCP & $\mathrm{x}$ & $(\mathrm{x})$ & $(\mathrm{x})$ & & & \\
\hline & Infektionsschutzgesetz & $\mathrm{x}$ & & & & & \\
\hline & Aushang Hygiene & & $\mathrm{x}$ & $\mathrm{x}$ & & & \\
\hline & Speisetemperatur & & & $\mathrm{x}$ & & & \\
\hline & Rezepte & & & $\mathrm{x}$ & & & \\
\hline & Bestellliste & & & $\mathrm{x}$ & & & \\
\hline & Verpackungsangaben & & & $\mathrm{x}$ & & & \\
\hline \multirow[t]{7}{*}{ Lagerlogistik } & Handbuch Qualitätssicherung & $\mathrm{x}$ & $(\mathrm{x})$ & $(\mathrm{x})$ & & $(\mathrm{x})$ & \\
\hline & Formular Serviceanforderung & & & $\mathrm{x}$ & & & \\
\hline & Empfangsschein & & & $\mathrm{x}$ & & & \\
\hline & Lieferschein & & & $\mathrm{x}$ & & & \\
\hline & Lagerplan & & & $(\mathrm{x})$ & & & $\mathrm{x}$ \\
\hline & Lagerplatzetiketten & & & $\mathrm{x}$ & & & \\
\hline & Logistikprogramm (PC) & & & $\mathrm{x}$ & & & \\
\hline
\end{tabular}




\begin{tabular}{|c|c|c|c|c|c|c|c|}
\hline \multirow{4}{*}{ Tätigkeit } & \multirow[t]{4}{*}{ Texte } & \multicolumn{6}{|c|}{ Texte } \\
\hline & & \multirow{3}{*}{$\begin{array}{c}\text { konti- } \\
\text { nuier- } \\
\text { lich }\end{array}$} & \multicolumn{5}{|c|}{ nichtkontinuierlich } \\
\hline & & & \multirow{2}{*}{$\begin{array}{l}\text { Graph. } \\
\text { Darst. }\end{array}$} & \multicolumn{2}{|c|}{ Tabellen } & \multirow{2}{*}{$\begin{array}{c}\text { Dia- } \\
\text { gramme }\end{array}$} & \multirow{2}{*}{ Karten } \\
\hline & & & & kateg. & math. & & \\
\hline \multirow{7}{*}{$\begin{array}{l}\text { Maschinen- } \\
\text { führung } \\
\text { (Produktion) }\end{array}$} & Sicherheitsbestimmungen & $\mathrm{x}$ & & & & & \\
\hline & Handbuch Maschine & $\mathrm{x}$ & & & & & \\
\hline & Aushang Brandverhalten & & & & & $\mathrm{x}$ & \\
\hline & Fehleranzeige Gerätedisplay & & & $\mathrm{x}$ & & & \\
\hline & Kleinstreparaturanleitung & & $\mathrm{x}$ & & & & \\
\hline & Produktionsbericht & & & $\mathrm{x}$ & & & \\
\hline & Aushang Produktionsleistung & & & $\mathrm{x}$ & & $\mathrm{x}$ & \\
\hline \multirow{2}{*}{$\begin{array}{l}\text { Möbel- } \\
\text { montage }\end{array}$} & Schulungsmappe & $\mathrm{x}$ & $(\mathrm{x})$ & $(\mathrm{x})$ & & & \\
\hline & $\begin{array}{l}\text { Montagedokumente } \\
\text { (u. a. Serviceprotokoll) }\end{array}$ & & $\mathrm{x}$ & $(\mathrm{x})$ & & & \\
\hline \multirow{5}{*}{$\begin{array}{l}\text { Service } \\
\text { Gastronomie }\end{array}$} & Arbeitsplan & & & $\mathrm{x}$ & & & \\
\hline & Tischplan & & & $(\mathrm{x})$ & & & $\mathrm{x}$ \\
\hline & Speise- und Getränkekarte & & & $\mathrm{x}$ & & & \\
\hline & Boniergerät/-kasse; Block & & & $\mathrm{x}$ & & & \\
\hline & Kassenbeleg & & & $\mathrm{x}$ & $\mathrm{x}$ & & \\
\hline \multirow{5}{*}{$\begin{array}{l}\text { Verkauf: } \\
\text { Kasse } \\
\text { Discounter }\end{array}$} & Arbeitsplan & & & $\mathrm{x}$ & & & \\
\hline & Urlaubsformular & & & $\mathrm{x}$ & & & \\
\hline & Kassenbeleg & & & $\mathrm{x}$ & $\mathrm{x}$ & & \\
\hline & Wochenprospekt & & $\mathrm{x}$ & $\mathrm{x}$ & & & \\
\hline & Formular Kassenbericht & & & $\mathrm{x}$ & & & \\
\hline \multirow{6}{*}{$\begin{array}{l}\text { Verkauf: } \\
\text { Einzelhandel }\end{array}$} & Kassenbeleg & & & $\mathrm{x}$ & $\mathrm{x}$ & & \\
\hline & Rabattliste & & & & $\mathrm{x}$ & & \\
\hline & Verkaufsflächenarrangement & & $\mathrm{x}$ & $\mathrm{x}$ & & & \\
\hline & Inventurliste & & & $\mathrm{x}$ & & & \\
\hline & Bestellschein & & & $\mathrm{x}$ & & & \\
\hline & Reklamation & & & $\mathrm{x}$ & $\mathrm{x}$ & & \\
\hline \multirow[t]{2}{*}{ Wagenpflege } & Fahrzeugprotokoll & & $\mathrm{x}$ & $\mathrm{x}$ & & & \\
\hline & Personalbogen & & & $\mathrm{x}$ & & & \\
\hline
\end{tabular}

Zuordnungsregeln: Ein Text wurde dem kontinuierlichen Typ zugeordnet, falls jener einen Absatz mit mind. drei oder mehr Sätzen beinhaltet. Die in Klammern gesetzten Zuordnungen bezeichnen, dass jene Textformate zwar eingebunden sind, aber eben sekundär: Z. B. wenn in einem längeren Fließtext einzelne graphische Darstellungen eingefügt sind, die jedoch übergeordnet der Darlegung des Sachverhalts im Fließtext folgen.

\subsection{Lesen für einfache Erwerbstätigkeiten?}

In der öffentlichen Debatte um Bildung und Arbeit findet sich wiederkehrend die Aussage, dass formale schulische, aber insbesondere berufliche Abschlüsse maßgeblich die zukünftige Teilhabe der heranwachsenden Generation in diversen Lebensbereichen prognostizieren. Die Rede um „Bildung schützt vor Arbeitslosigkeit“, d. h. die Rede um die Steigerung und Sicherstellung schulischer oder beruflicher Kompetenzoutputs aus ihrer postulierten probabilistischen Relevanz für die Teilhabe in Arbeit hat sich dabei längst von den nationalen Grenzen gelöst. Die OECD-Welt spricht hier sozusagen in einer Sprache und nicht nur alle Bildungsprogramme der 
großen bundesdeutschen Parteien, die Bildungspläne der allgemeinbildenden Schulen in den Bundesländern oder die einer zunehmenden Homogenisierung unterliegenden Abschlusstests eines Bildungsgangs. Wenn in diesem Zuge die funktionalen Lesecompetencies einer ,befriedigenden Lebensführung' bereits nahezu weltumfassend geklärt zu sein scheinen, wirkt deren kritische Befragung im Kontext von Einfach(st)arbeitsplätzen auf den ersten Blick als unnötig auf den zweiten Blick, so die bisherigen historischen und semiotischen Analysen zu Verarbeitungsprozessen, gilt es diese Position zu justieren.

Obgleich im Grunde ein probabilistisches Modell der Relation von Bildung und Teilhabe in Arbeit mit starker Kausalität beansprucht wird, in dem anerkannte Bildung wahrscheinlicher eine anerkannte Teilhabe prognostizieren lässt, verschwimmen somit die Grenzen zu einem Modell eines universellen linear-kausalen Zusammenhangs. Walther (2014) kann daher durchaus zugestimmt werden, wenn er funktionale Handlungscompetencies als determinierende Voraussetzung für Teilhabeansprüche und -chancen enttarnt. Ungeachtet dieser verschwimmenden Grenzen bleibt aus der obig dargelegten Problematik von Kausalitätsaussagen in deduktiven Modellen von Analysen von Jugendarbeitslosigkeitsstatistiken oder Studien der strukturdeterminierten Übergangsforschung der Umstand von ,unaufgeklärten Varianzen' präsent. Diese leiten über, dass diese deduktiven Modelle letztlich ,Schlussfolgerungen auf die beste Erklärung liefern, insbesondere wenn die wahrscheinlichkeitsstatistisch mehr oder weniger bedeutsamen Fälle, die gegen eine Determination von Teilhabe durch Bildung sprechen, über Alltags- und nicht empirische Kausalitäten aufgelöst werden:

Besonders anfällig für solche Fehlschlüsse des Alltagswissens sind erstens empirische Untersuchungen, bei denen Gegenstandsbereiche erforscht werden, in denen räumlich und zeitlich abgegrenzte soziale Milieus und (Sub-)Kulturen eine Rolle spielen. Die Erforschung jugendlicher Subkulturen und von deviantem Verhalten sowie berufs- und organisationssoziologische Untersuchungen, um nur einige Beispiele zu nennen, finden oft in Handlungsfeldern statt, in denen spezifische, für Außenstehende nur schwer erschließbare Handlungsregeln gelten. Mitglieder der untersuchten (Sub-)Kultur besitzen dann wesentliche größere Kompetenzen zur Erklärung der Handlungen anderer sozialer Akteure als ein sozialwissenschaftlicher Untersucher, denn sie verfügen über jenes praktische Wissen, das benötigt wird, um empirisch gehaltvolle Hypothesen darüber zu formulieren, welche Handlungsziele und Situationswahrnehmungen ein bestimmtes Handeln rational erklärbar machen (Kelle 2008, 108f.).

Jene ,Schlussfolgerungen auf die beste Erklärung' der Relation von Bildung und Teilhabe trifft für die Anforderungen on-the-job an Einfach(st)arbeitsplätzen, und letztlich auch für das Lesen von Texten und Textkomplexen zu. Dabei wird diese Form von Teilhabe in Arbeit in der pädagogischen Debatte um Lesekompetenz(en) kaum als Bewährungsfeld für eine postulierte ,befriedigende Lebensführung' mitgedacht - und dies ohne empirische Bekräftigung als wahrscheinlichkeitstheoretisch ,unbefriedigende Lebensführung. Es resultiert die Annahme, dass die Mindeststandards der funktionalen Grundbildungscompetencies daher ,ohne eine externe Validitätsprüfung' postulieren, eine Teilhabe in dieser Form von Arbeit dennoch probabilistisch prognostizieren zu können. Spätestens unter der Folie national-historisch gewachsener Systeme zwischen allgemeinbildenden Institutionen und Beschäftigungssystem, wie es die Analysen zum „deutschen Übergangsregime“ (Walter/Walther 2007) eindrücklich empfehlen, treten nicht zuletzt einige vermeintlich gesellschaftlich-konventionelle Setzungen bzw. einige Mythen zu diesem Qualifikationsniveau von Beschäftigung auf den Plan. Diese verhärten eher den determinierenden als den probabilistischen Charakter von funktionalen Grundbildungscompetencies für anerkannte Teilhabe in Arbeit: Einfach(st)arbeitsplätze gelten als grundsätzlich von schlechter Bezahlung, durch unsichere Vertragsverhältnisse, durch geringe Weiterqualifizierungsmöglichkeiten, durch ungünstige Arbeitsbedingungen und von einer schwachen Zukunftsträchtigkeit 
geprägt. Von Einfach(st)arbeit bedroht sind Übergangsbiografien zwischen Schule - (anerkannter) Ausbildung - Arbeit (mit hohem sozialem Status), die als „gescheitert“ kategorisiert werden. Infolge bspw. eines fehlenden anerkannten Schulabschlusses (vgl. z. B. Gaupp et al. 2012, 311) ist nicht die Rede von wahrscheinlichem Scheitern oder von einer Gefährdung eines Scheiterns, sondern eben einer Determinierung des „Scheiterns“. Die deduktive, strukturdeterminierende Übergangsforschung generalisiert derart nicht selten die Relevanz bspw. des in der Hierarchie untersten anerkannten Bildungsabschlusses in Deutschland - dieser ist über Rahmenlehrpläne wiederum mit den funktionalen Grundbildungscompetencies verknüpft - für den weiteren beruflichen Werdegang und eine strukturell-konventionalisiert prognostizierbare berufliche Zukunft.

Eher der determinierende als der probabilistische Charakter der Ursache funktionaler Grundbildungscompetencies in der Relation von Bildung und Teilhabe beeinflusst gleichsam die Debatte um chancengerechte inklusive Bildungssysteme; z. B. positionierte prominent die Deutsche Unesco-Kommission einen qualifizierten beruflichen Bildungsabschluss als „Voraussetzung "für das Recht an Teilhabe in Arbeit!

Für Menschen mit Behinderungen besteht das gleiche Recht auf Arbeit, einschließlich des Rechts, ihren Lebensunterhalt durch frei gewählte Arbeit zu verdienen, verankert in Art. 27 der UN-Behindertenrechtskonvention. Eine Voraussetzung zur Wahrnehmung dieses Rechts ist eine qualifizierte Berufsausbildung und/oder Hochschulbildung. Obwohl in Art. 24 der UN-Behindertenrechtskonvention der gleichberechtigte und diskriminierungsfreie Zugang zu Berufsausbildung und Hochschulbildung festgeschrieben ist, steht die Inklusionsdebatte in Deutschland in diesem Bereich noch am Anfang (DUK 2014, 16).

\subsubsection{Mythos der grundsätzlich einfachen Arbeit}

(Einfach(st)-)Arbeit ist in Deutschland eng an den Begriff „Beruf“ geknüpft. Diese Verknüpfung der beiden Begriffe, Arbeit und Beruf, führt bis heute die historischen Strukturen der tradierten Ständeordnung fort (Walter/Walther 2007). Beispielsweise verdeutlicht sich dies in den Qualifizierungsniveaus von Arbeit, welches in den offiziellen Statistiken zur Erwerbstätigkeit, Arbeitslosigkeit usw. der Bundesagentur für Arbeit folgendermaßen aufgegliedert wird: „ohne beruflichen Ausbildungsabschluss“, „Abschluss einer anerkannten Berufsausbildung“ „Meister-/Techniker- oder gleichwertiger Fachschulabschluss“, „Bachelor“, „Diplom/Magister/ Master/Staatsexamen“, „Promotion“ und „Abschluss unbekannt“. Hierbei ist eine „anerkannte Berufsausbildung “ bundesrechtlich geregelt. Sie münden in eine Sammlung von anerkannten zwei- oder dreijährigen Ausbildungsberufen², ergo von einerseits den Dualen Ausbildungen, den früher durch die Kammern und gegenwärtig teils nach dem Berufsbildungsgesetz (BBiG) geregelten anerkannten Ausbildungsberufen. Andererseits zählen zu den „anerkannten Berufsausbildungen" die nicht selten gleichermaßen dual organisierten, aber berufsschulischen Ausbildungen nach länderspezifischen (z. B. zum/r Erzieher/in) - oder bundeseinheitlichen (z.B. nach dem Krankenpflegegesetz (KrPflG)) gesetzlichen Regelungen außerhalb des Geltungsbereiches des $\mathrm{BBiG}$ oder der Handwerksordnung ( $\mathrm{HwO})$. Der dahinterliegenden Setzung von „Beruf“ geschuldet, zählen in Deutschland dagegen die folgenden Ausbildungen und Qualifizierungen nicht zu den „anerkannten Berufsausbildungen“ und stattdessen zu unqualifizierter bzw. gering-qualifizierter Arbeit: Es handelt sich dabei bspw. um weitere nach dem Bundesgesetzblatt $(\mathrm{BGBl})$ geregelte Ausbildungen - z. B. nach der „Verordnung über die Prüfung zum anerkannten Abschluss Geprüfter Taucher/Geprüfte Taucherin“, oder weiterer nach län-

2 Ein Verzeichnis der als ,anerkannt“ bezeichneten Berufsausbildungen wird, jährlich aktualisiert, vom BBiB veröffentlicht; 2015: http://www.bibb.de/dokumente/pdf/Verzeichnis_anerk_AB_2015.pdf 
derspezifischen Ausbildungsregelungen - z. B. zum/r ,staatlich geprüften Assistent/in in der Systemgastronomie“ - sowie um diejenigen nach Sach- und Fachkundeprüfungen - z. B. im Sinne des $₫ 34 \mathrm{a}$ GewO (Gewerbeordnung) für das Personal im Bewachungsgewerbe - oder auch nach berufsgenossenschaftlichen Zertifizierungen - z. B. zum/r Kranführer/in nach BGV D6 (Berufsgenossenschaftliche Vorschriften). Inwiefern Ausbildungen nach den Sonderausbildungsregelungen für Menschen mit Behinderung im Sinne des $\$ 66 \mathrm{BBiG}$ zu den „anerkannten Berufsausbildungen“ zählen, wird mit Blick auf den, auf europäische Homogenisierung und gegenseitige Anerkennung von beruflichen Ausbildungen ausgerichteten Deutschen Qualifikationsrahmen (DQR) zweifelhaft: Weder in den veröffentlichten "Listen von Qualifikationen“ noch in der Webabfrage tritt eine dieser Sonderausbildungen auf.

Es resultiert eine erste Skizzierung von Einfach(st)arbeitsplätzen als Arbeitsplätze von Beschäftigten ohne einer der als anerkannt geltenden Ausbildungen. Aus einer früheren Analyse dieses Typus von Arbeitsplätzen ging jedoch hervor, dass die Beschäftigten selbst zahlreich eine anerkannte Berufsausbildung vorweisen können. Denn an der Mehrzahl der untersuchten Arbeitsplätze derselben Arbeitsplatzprofile arbeiteten gleichsam Beschäftigte mit einer anerkannten, zumeist 2-jährigen Berufsausbildung (Basendowski 2013, 200, 242ff.). In Übereinstimmung mit Brandherm $(2007,1)$ werden im Weiteren daher Einfach(st)arbeitsplätze in Deutschland mit einer Beschäftigung verknüpft, die eine anerkannte Berufsausbildung nicht zwingend erfordert. Oder in anderen Worten, Einfach(st)arbeitsplätze konstituieren sich nicht durch die Einfachheit der zu bewältigenden Anforderungen am Arbeitsplatz, sondern aus der Setzung eines kulturellen Codes heraus, was als eine anerkannte Berufsausbildung gilt, respektive, nicht gilt. Konsequenterweise ist es nicht verwunderlich, wenn mehrerenorts wissenschaftlich festgestellt wurde, dass diese zu bewältigenden Anforderungen an Einfach(st)arbeitsplätzen alles andere als grundsätzlich „einfach“ sind (Enggruber et al. 2003, 138f.; Weinkopf/Jaehrling 2005, 17f.).

\subsubsection{Mythos der grundsätzlich prekären Arbeit}

Zusammenfassend ist festzuhalten, dass der Niedriglohnanteil in Deutschland in den vergangenen fünf Jahren zwischen gut $23 \%$ und knapp $25 \%$ schwankte. Niedriglöhne betreffen keineswegs überwiegend gering Qualifizierte oder Jüngere. Vielmehr hat die große Mehrheit der Niedriglohnbeschäftigten eine abgeschlossene Berufsausbildung und stammt aus den mittleren Altersgruppen. Im Niedriglohnsektor werden auch keineswegs ausschließlich „einfache“ Tätigkeiten geleistet (http://www.iaq.uni-due.de/ iaq-report/2014/report2014-02.pdf).

Arbeitsmarktberichte, wie der zitierte, stehen dem immerwährend von der strukturdeterminierten Übergangsforschung bis von der Bildungspolitik hervorgehobenen Zusammenhang zwischen formaler Bildung und Teilhabe in Arbeit unter gesicherten Bedingungen gegenüber. Dieser Zusammenhang drückt sich in den Analysen der Jugendarbeitslosigkeits- und Beschäftigungsstatistik aus, ergo in Analysen, die Personen z. B. eines Qualifikationsniveaus/beruflichen Rangs oder in Erwerbslosigkeit übergeordnet nach ihrem schulischen Bildungsabschluss miteinander vergleichen: Es soll an dieser Stelle weder negiert werden, dass Einfach(st)arbeit im Vergleich zu unterschiedlich qualifizierter Arbeit, ergo Arbeit, die mindestens eine anerkannte Berufsbildung zwingend voraussetzt, statistisch wahrscheinlicher mit prekären Beschäftigungsverhältnissen verbunden auftritt. Noch soll negiert werden, dass Personen ohne eine anerkannte Berufs- oder Hochschulbildung im Vergleich zu denen mit einer anerkannten Berufs- oder Hochschulbildung häufiger erwerbslos gemeldet sind. Kritisch betrachtet wird jedoch, dass zwischen den einzelnen probabilistischen Inter-Gruppenvergleichen Ableitungen auf IntraGruppenvergleiche gezogenen werden, die letztlich eine lineare Kausalität in Aussagen wie in 
(formelle) „Bildung schützt vor Arbeitslosigkeit“ verkörpern: Für ,jede/n“ lohnt ein zusätzliches anerkanntes Bildungszertifikat dahingehend, dass es linear-kausal das Risiko im Zugang zu Arbeit minimiert, und dies ohne empirische Betrachtung der Wahrscheinlichkeiten innerhalb einzelner Gruppen selbst.

Für eine empirische Überprüfung stochastischer Kausalerklärungen sozialer Handlungen (also von Hypothesen über einen probabilistisch kausalen Zusammenhang zwischen Handlungsbedingungen und Handlungen) bedeutet dies: Der Nachweis einer statistischen Assoziation zwischen unabhängigen Variablen, die Handlungsbedingungen repräsentieren, und abhängigen Variablen, die die Handlungen bzw. deren Resultate abbilden, ist für sich genommen nicht ausreichend, um eine kausale Hypothese zu stützen. Denn die statistische Assoziation kann ebenso durch bislang unbekannte, im Modell nicht berücksichtigte common causes zustande kommen, die eine gleichzeitige Veränderung sowohl der unabhängigen als auch der abhängigen Variablen bewirken (Kelle 2008, 202).

Exemplarisch kann jene ,Assoziation' zwischen probabilistischen Inter-Gruppenvergleichen in einem Intra-Gruppen-Ursachen-Wirkungs-Modell entlang der Schlussfolgerungen der BIBBErwerbstätigenbefragung 2012 (vgl. Tabelle 2) verdeutlicht werden. Wenn es hier heißt, „Eine abgeschlossene Berufsausbildung senkt das Niedriglohnrisiko deutlich, werden tatsächlich nicht die probabilistischen Zusammenhänge für einerseits Männer und andererseits Frauen betrachtet: „Die Chance, einen Bruttostundenlohn oberhalb der Niedriglohnschwelle zu beziehen, ist für Männer und Frauen mit dualer Berufsausbildung um rund das Dreifache höher als für nicht formal Qualifizierte“ (BIBB 2013, 290). Die deskriptiv-statistischen Werte verweisen zuvorderst auf statistische Zusammenhänge einerseits abhängig und andererseits unabhängig des strukturellen Gruppenmerkmals Geschlecht. Zwischen den Gruppen streuen die Verteilungswerte jedoch hoch, dass z. B. nahezu identische Anteile von Niedriglohnbeschäftigung bei Männern „ohne Berufsausbildung“" (gemäß SOEP-Definition (ebd., 287)) und Frauen „mit einer dualen Berufsausbildung“ festzumachen sind. Die kausale Ableitung eines probalistischen Inter-Gruppen-Zusammenhangs im Rahmen der Schlussfolgerung basiert weniger auf den probabilistischen Intra-GruppenZusammenhängen, sondern vielmehr auf ex post common senses. Zur Enttarnung jenes leitenden common sense im assoziierten Zusammenhang könnte die Schlussfolgerung unter einer alternativen, zugegeben provokanten, Alltagsannahme auch lauten, dass „Geschlechtsumwandlungen von Frauen deutlich das Niedriglohnrisiko senken: Die Chancen, einen Bruttostundenlohn oberhalb der Niedriglohnschwelle zu beziehen, ist bei Frauen mit einer dualen Berufsausbildung in etwa gleich hoch wie bei nicht formal qualifizierten Männern“.

Tab. 2: Niedriglohnbeschäftigung nach Geschlecht und höchstem Ausbildungsabschluss

\begin{tabular}{lccc}
\hline Ausbildungsabschluss & \multicolumn{3}{c}{ Prozentuale Anteile } \\
& Gesamt & Männer & Frauen \\
\hline ohne Berufsausbildung & 40,7 & 27,8 & 51,4 \\
mit Berufsausbildung & 16,9 & 11,0 & 23,6 \\
$\quad$ duale Berufsausbildung & 17,6 & 11,0 & 26,8 \\
schulische Berufsausbildung & 14,6 & 11,4 & 15,7 \\
sonstige Berufsausbildung & 13,9 & 10,1 & 20,0 \\
Aufstiegsfortbildung & 4,8 & 2,6 & 10,6 \\
Fachschule, Universität & 4,5 & 2,9 & 6,3 \\
\hline Gesamt & 15,5 & 9,8 & 22,2 \\
\hline (Quelle: BiBB 2013, 284) & \multicolumn{4}{|}{}
\end{tabular}


Tab. 3: Typische vs. atypische Beschäftigungsverhältnisse nach Qualifikation im zeitlichen Vergleich

\begin{tabular}{|c|c|c|c|c|c|}
\hline \multirow{2}{*}{$\begin{array}{l}\text { Qualifizierungs- } \\
\text { niveau }\end{array}$} & \multicolumn{2}{|c|}{ Anteile Bildungsstand } & \multirow{2}{*}{$\begin{array}{c}\text { Beschäftigungs- } \\
\text { merkmale }\end{array}$} & \multicolumn{2}{|c|}{ Prozentuale Anteile } \\
\hline & 2005 & 2010 & & 2003 & 2010 \\
\hline \multirow{5}{*}{$\begin{array}{l}\text { keine } \\
\text { Ausbildung }\end{array}$} & \multirow{5}{*}{29,1} & \multirow{5}{*}{26,9} & Vollzeit, unbefristet & 10 & 9 \\
\hline & & & Vollzeit, befristet & 16 & 14 \\
\hline & & & Teilzeit & 14 & 13 \\
\hline & & & geringfügig & 26 & 20 \\
\hline & & & Leiharbeit & 20 & 20 \\
\hline \multirow{5}{*}{$\begin{array}{l}\text { Berufs- } \\
\text { ausbildung }\end{array}$} & \multirow{5}{*}{59,4} & \multirow{5}{*}{57,7} & Vollzeit, unbefristet & 69 & 67 \\
\hline & & & Vollzeit, befristet & 53 & 56 \\
\hline & & & Teilzeit & 71 & 69 \\
\hline & & & geringfügig & 64 & 71 \\
\hline & & & Leiharbeit & 67 & 64 \\
\hline \multirow{5}{*}{$\begin{array}{l}\text { Fach-/ } \\
\text { Hochschul- } \\
\text { abschlusss }\end{array}$} & \multirow{5}{*}{13,1} & \multirow{5}{*}{14,8} & Vollzeit, unbefristet & 21 & 24 \\
\hline & & & Vollzeit, befristet & 31 & 30 \\
\hline & & & Teilzeit & 16 & 18 \\
\hline & & & geringfügig & 10 & 10 \\
\hline & & & Leiharbeit & 13 & 15 \\
\hline
\end{tabular}

Quellen: Keller et al. (2012, 17ff.); Genesis-Online Datenbank des Statistischen Bundesamtes (Bevölkerung nach höchstem beruflichem Bildungsabschluss in Deutschland; 2005: 15-65 Jahre, 2010: 15-65 Jahre)

Ein weiterer statistischer Einblick zu den Erwerbstätigkeiten „ohne Berufsausbildung“ macht zugleich eine weitere Unschärfe in den common senses fest und lässt die gängigen linearkausalen Assoziationen von probabilistischen Intra-Gruppenvergleichen weiterführend fraglich erscheinen: So umfasst einerseits die Schlussfolgerung bzw. der abgeleitete Inter-Gruppenvergleich die unaufgelöste Varianz, die im probabilistischen Zusammenhang verborgen liegt, dass die Mehrheit der Beschäftigten „ohne Berufsausbildung“ oberhalb des Niedriglohns entlohnt wird. Keller et al. (2012) liefern andererseits mit ihren Auswertungen der SOEP2003- und SOEP-2010-Daten nicht nur Angaben zur probabilistischen Verteilung von Beschäftigungsverhältnissen nach Qualifizierungsniveaus in einem zeitlichen Vergleich (vgl. Tabelle 3), sondern auch Anhaltspunkte für einen common sense hinter diesem Zusammenhang: Wider den immerwährenden Prognosen der gesamtwirtschaftlichen Abnahme der Relevanz von Einfach(st)arbeitsplätzen - unter der Setzung, dass Personen ohne anerkannte Berufsausbildung eben an Einfach(st)arbeitsplätzen beschäftigt sind - verharren ihre Anteile zwischen 2003 und 2010 unter sowohl den sogenannten „Normalarbeits- „als auch unter den verschiedenen Formen von „atypischen“ Beschäftigungsverhältnissen bei nahezu identischen Werten; zu diesen „atypischen“ werden hierbei neben den sozialversicherungspflichtigen geringfügigen und befristeten, der in Teilzeit und in Leiharbeit auch die außerhalb sozialer Sicherungssysteme und in der Auswertung nicht berücksichtigten Formen als Freelancer, Ein-Euro-Jobber/in oder Langzeitpraktikant/in gezählt (ebd., 1). Die anteilig größte Veränderung über diesen Zeitraum hinweg zeichnet sich unter den Personen ohne ,anerkannte Berufsausbildung" im Zugang zu einer geringfügigen Beschäftigung ab, und nicht, wie vermeintlich zu erwarten wäre, im Zugang zu einer unbefristeten Vollzeitbeschäftigung. Lag ihr Anteil 2003 unter allen Qualifizierungsniveaus noch bei $26 \%$, sank er bis 2010 auf $20 \%$. 
Zwar nahm ihr Anteil an den „Normalarbeitsverhältnissen“ durchaus zu einem Prozentpunkt $\mathrm{ab}$, doch dieser Trend verzeichnet sich gleichermaßen unter den Personen mit einer ,anerkannten Berufsausbildung“. Betrachtet man diese beiden Qualifizierungsniveaus im Spiegel ihrer ebenso zeitlichen anteiligen Abnahme über die Zeit, verliert dieser Trend an Gewicht; in der Tabelle 3 durch Angaben zum Bildungsstand des Statistischen Bundesamtes für 2005 und 2010 ergänzt. Nichtsdestotrotz gilt klarzustellen, dass v. a. unter den Beschäftigten in „Normalarbeitsverhältnissen “ diejenigen „ohne Berufsausbildung “ anteilig unterrepräsentiert sind und dies häufiger als in allen anderen Beschäftigungsformen. Allerdings nimmt jene Unterproportionalität zwischen 2003 und 2010 eher ab als zu. Überproportionale Anteile und eine überproportionale Zunahme im zeitlichen Vergleich in Beschäftigung (Ausnahme: geringfügige Beschäftigung) lassen sich überdies nur dem Niveau „Fach-/Hochschulabschluss“ zuordnen. Oder in anderen Worten, Beschäftigung über Einfach(st)erwerbstätigkeiten kann kaum in den vergangenen Jahren eine zunehmende Tendenz zur Exklusion auf den Arbeitsmärkten per se zugesprochen werden.

Entgegen der determinierenden Risiko-Relation von anerkannter Bildung und Teilhabe in Arbeit unter gesicherten Bedingungen lässt sich entlang aktueller offizieller Statistiken weiterhin verorten, ob es gefällt oder nicht, dass es durchaus keine zu vernachlässigende Zahl an Personen gibt, die in Einfach(st)erwerbstätigkeiten sowohl unbefristet als auch sozialversicherungspflichtig beschäftigt sind. Linear-kausale Ableitungen entlang probabilistischer IntraGruppen-Verteilungen nach Geschlecht, Qualifizierungsniveaus etc. reproduzieren zuvorderst ex post common senses eines generalisierbaren Zusammenhangs zwischen anerkannter Bildung und typischer Teilhabe für alle, und dies unverändert trotz einerseits z. B. der mittlerweile seit Jahren fortbestehenden Konstellation vieler junger, auch formal hoch-qualifizierter Erwachsener auf einigen „Krisenarbeitsmärkten“ der EU. Andererseits akzentuiert Mertens (1985) mit dem „Qualifikationsparadoxon“ oder Hradil (2006) mit der Methapher der „Warteschlange“ unlängst komplexere Beziehungen in dieser Relation von auch anerkannter Bildung und typischer Teilhabe in Arbeit: Formale Bildungszertifikate sichern im Wettbewerb um Arbeitsplätze höchstens eine bessere Startposition. Eine Determinierung weder des Zugangs zu diesen Arbeitsplätzen noch der „typischen“ Beschäftigungsbedingungen aller Berufstätigen können sie seriös nicht leisten. Sie garantieren folglich nicht, bzw. nicht hinreichend, den Zugang zu einer sozialversicherungspflichtigen Beschäftigung, im besten Fall mit tariflichem Kündigungsschutz, einer erweiterten Altersabsicherung durch eine Betriebsrente usw. Common senses, die im Kontext der Relation von Bildung und Teilhabe nachweislich unaufgeklärte Varianzen in ihren probabilistischen Zusammenhängen nicht aufnehmen und empirisch fortführen, sind als irrtumsanfällig zu enttarnen.

\subsubsection{Mythos der pädagogischen Determiniertheit in der Risiko-Relation von Bildung und Teilhabe in Arbeit}

Die Relation von Bildung und Teilhabe aus einer pädagogischen Perspektive betrachtet (Walther 2014), schreibt Bildung einen Voraussetzungscharakter für Teilhabe zu. Diese Richtung des Zusammenhangs verdeutlicht sich bspw. an den funktionalen Grundbildungskompetenzen im allgemeinbildenden Schulsystem und an den beruflichen berufliche Handlungskompetenzen im Berufsbildungssystem, die Teilhabeansprüche und -chancen markieren - sollen (vgl. Basendowski 2014). Die Relation wird durch den Auslösungscharakter von Teilhabe für Bildung komplettiert: In Konsequenz zeigt sich, dass offizielle Bildungsstatistiken zur sogenannten 1. oder 2. Schwelle fast ausschließlich nur erstere Wirkungsrichtung beanspruchen, um 
die Relation von Bildung und Teilhabe zu analysieren. Alsdann ist eben, erstens, im Kontext der obligatorischen Schulzeit zu gewährleisten, dass alle Jugendlichen über die festgelegten grundbildenden kognitiven competencies zu verfügen haben, „die in modernen Gesellschaften für eine Teilhabe am gesellschaftlichen, wirtschaftlichen und politischen Leben notwendig sind“ (Internetseite der PISA-Studie Deutschland). Und zweitens, im Kontext der Berufsorientierung, -auswahl, -vorbereitung und -qualifizierung wäre zu gewährleisten, dass alle über diejenigen Fach-, Sozial-, Methoden- und Selbstkompetenzen verfügen, die als berufliche Handlungskompetenzen gefasst einen reibungslosen Übergang von der Schule bis in Arbeit garantieren sollen. Wenn die Relation von Bildung und Teilhabe (in Arbeit) auf eine lineare Kausalität der Ursache (=,anerkannte“ Bildung) verengt wird, so liegt nahe, dass die dahinterliegenden funktionalen Grundbildungs- und beruflichen Handlungskompetenzen als funktionale Basiszielsetzungen fungieren. Oder in anderen Worten, die Frage um die determinierende Relation von ordentlicher Bildung und ordentlicher Teilhabe ist, im Schleier einer probabilistischen These, letztlich auch eine pädagogische.

\subsubsection{Theoretischer Ansatz zur Befragung der Determinierung der Risiko-Relation von Bildung und Teilhabe: Grundlagen einer systemtheoretischen Analyse auf der Organisationsebene}

Jenes prädikativen Zusammenhangs verdächtigt, soll an dieser Stelle die Determinierung von Teilhabe in Arbeit durch Bildung befragt werden. Es wird dieser verdichteten These mittels der Systemtheorie sensu Luhmann entlang der Differenz Inklusion/Exklusion nachgegangen. Als theoretischer Bezug empfiehlt sich dieser soziologische Ansatz, denn er eignet sich gerade bei der Beschreibung von übergeordneten strukturellen Prozessen an den Schnittstellen zwischen unterschiedlichen Systemen, wobei gesellschaftlichen Realitäten geschuldete Komplexität weder negiert noch vollumfänglich als erfassbar gilt. Auch aus der Richtung des prädikativen Zusammenhangs heraus begründet, müsste über diese soziologische Theorie des Strukuralismus darzulegen sein, dass sich eine Übernahme gewisser sozial vermittelbarer Aussagen der Institutionen der Sekundarstufe I auf Seiten der berufsbildenden Institutionen (Erziehungssystem) und weiter von den Institutionen des Wirtschaftssystems im Kontext von Beschäftigung als funktional aufdecken lassen. Oder in anderen Worten, es soll der Frage nachgegangen werden, ob die determinierte Risiko-Relation als für in einer Gesellschaft als universell gültig bezeichnet werden kann, respektive, als lokaler kultureller Code zu bezeichnen ist.

Hervorzuheben ist, dass mit der Differenz Inklusion/Exklusion systemtheoretisch keine gesellschaftlichen Zustände beschrieben werden, sondern - und im Vergleich zur politischen und ethischen Diskussion wertfrei - nicht mehr und nicht weniger die Beziehung/Kopplung zwischen psychischen und sozialen Systemen. Respektive, diese Differenz kann als ein theoretisches Schema verstanden werden, mit Hilfe dessen man die sich verändernden Relationen zwischen psychischen und sozialen Systemen in ihrer Komplexität angemessen, aber auch beobachterabhängig annähern kann. Den Veränderungen, respektive, dem sozialen Wandel geschuldet, ist diese Beschreibung der Beziehung/Kopplung temporär zu aktualisieren (Stichweh 2009, 36ff.). Hierbei gilt, dass, wenn eine Seite der Unterscheidungen zwischen Inklusion und Exklusion beobachtet wird, die andere komplementär berücksichtigt werden muss. Dies verdeutlicht sich z. B. an dem Verständnis von Exklusion in seiner Differenz zu Inklusion, dass soziale Systeme für jeweilige Kommunikation nicht erkennbar sind und infolge Kommunikation auch nicht als aussichtslos antizipiert in Gang gesetzt wird. 
Eine Analyse entlang jenem Verständnis von Inklusion/Exklusion innerhalb der Relation von Bildung und Teilhabe in Arbeit wird auf der Funktionssystemebene jedoch als wenig aufschlussreich betrachtet, wenn im schulischen Bildungskontext Stichweh (2013) pointiert, dass alsdann Kommunikation in einer Geltungsreichweite betrachtet werden müsste, die „nur auf der Ebene der Weltgesellschaft sinnvoll identifiziert werden kann“: Exklusion würde sich an einem dauerhaft und gar über alle Intra-Systemgrenzen wirkenden Ausschluss an funktionaler Kommunikation von Personen ${ }^{3}$ zeigen, d.h. eine Person wäre von jeglicher Kommunikation der intentionalen Beeinflussung auf der Welt durch die Funktionssysteme, und dies dauerhaft, nicht angesprochen bzw. mitbedacht. Kurzum, immerwährende Vollinklusion ist somit wie allgegenwärtige Vollexklusion ein nicht denkbarer Fall; mit einer Ausnahme, der Tod (ebd., 37f.; Windolf 2009, 12). Nicht systemtheoretisch formuliert, ein jeder junger Erwachsene unter „allen“kann auf der Ebene der Funktionssysteme weder gesellschaftlich vollständig drinnen noch draußen sein. Auf der Ebene der systemtheoretischen Interaktion könnte Inklusion/Exklusion eher ungefähr als ein Mitdenken der Personen in einem losen Gespräch verortet werden. Oder, wenn z. B. die Kommunikation im Unterricht die Person „Peter“ nicht mitdenkt, manifestiert sich Exklusion von Alter durch Ego - oder umgekehrt - in der Kommunikation durch wiederholende Ereignisse (Stichweh 2013), bspw., wenn die Person Peter von der Person Frau Müller - oder umgekehrt - wiederholt z. B. im Leseunterricht nicht angesprochen wird. Nicht angesprochen wird der 15-jährige Schüler einer 8. Klasse im Bildungsweg Hauptschulabschluss (geringe Lesekompetenzen; prekäres soziales Milieus) wenn symboltragende Aspekte der Körperlichkeit für die Kommunikation im Sinne „symbiotischer Mechanismen“ (Luhmann 2009, 262ff.) wiederholt im Unterricht nicht mitgedacht werden. Es verdeutlicht sich die einhergehende Komplexität für eine Analyse auf dieser Ebene sozialer Systeme, was zur Folge hat, dass diese im Weiteren ausgeblendet wird.

Für die Übertragung von Inklusion/Exklusion auf die Organisationsebene ausgehend von der Skizzierung für die systemtheoretische Interaktion gilt zu beachten, dass die Person abstrakt gefasst wird. Wenn zunehmend übergeordnete Adressatengruppeneigenschaften mitgedacht werden müssen (ebd.), könnte dies in etwa durch alle 15-jährigen Schüler/innen der 8. Klassen im Bildungsweg Hauptschulabschluss mit geringen Lesekompetenzen und/oder aus einem prekären Milieu an Sekundarstufenschulen illustriert werden; und entsprechend auch für alle Lehrer/innen dieser Schüler/innen. Gleichzeitig werden durch die Unschärfe in den Adressatenzuschreibungen zunehmend mehr Personen inkludiert, d.h. die strukturelle Differenzierung liegt asymmetrisch zur Binnendifferenzierung der funktionalen Teilsysteme. Dies hat zur Folge, dass während Systeme der Organisationsebene auf diesen Umstand mit weiteren funktionalen Ausdifferenzierungen reagieren können, um alle mitzudenken, die Möglichkeit der unmittelbaren Exklusion in den loseren systemtheoretischen Interaktionen zunimmt ${ }^{4}$.

In jenen komplexeren Teilsystemen sind entgegen einer loseren Interaktion zudem auch immer funktionale Gliederungen durch Rollen, respektive soziale Adressaten ${ }^{5}$, immanent. Organisationsspezifische Inklusion/Exklusion einer Adressatengruppe im Bildungssystem ist daher auch

3 Der Mensch in der systemtheoretischen Kommunikation ist eine Person, ein soziales Strukturbündel, und nicht das Individuum (Weinbach 2004; Fuchs 1997).

4 Beispiel: Während man z. B. in seiner Berufsrolle tolerant gegenüber diversen Adressatengruppen spricht, begrenzt sich der Kreis der Eingeladenen auf einer privaten Geburtstagsfeier.

5 In auf Adressabilität ausgerichtete Kommunikation werden der Person, am ehesten im Sinne eines sozialen Adressaten zu verstehen, Rollenerwartungen zugeschrieben (vgl. Weinbach 2004; Fuchs 1997), wobei gilt: „Eine Person wird vielmehr konstitutiert, um Verhaltenserwartungen ordnen zu können, die durch sie und nur durch sie eingelöst werden können“ (Luhmann 1984, 429). 
immer vor der Folie von primären ${ }^{6}$ Leistungs- vs. Komplementärrollen zu differenzieren. Während erstere das System sozusagen ausführen und stabilisieren - z. B. ein/e Richter/in, Arzt/Ärztin, Lehrer/in, Personalverantwortlicher/e, usw. - stellen zweitere eine/n Leistungsabnehmer/ in und/oder eine Beobachter/in im Verhältnis zu den Leistungsrollen dar - ein/e Angeklagte/r, Patient/in, Schüler/in oder eine Stellenbewerber/in. Während für die Leistungsrollen teils bestimmte Nachweise oder herausragende Leistungen im System primär differenzierend sind und ggf. stark exkludierend wirken, sind die Organisationssysteme eines Funktionssystems in den Komplementärrollen entlang ihrer Funktionsparadigma auf das Mitdenken „aller“ sozialer Adressaten bedacht. Beispielsweise richtet sich die Kommunikation privater Kliniken an die Leistungsabnehmer Patient/in mit der Krankheit $\mathrm{x}$ über medizinische Behandlungsangebote. Diese Kommunikation ist jedoch nur aussichtsvoll in Gang gesetzt für die Leistungsabnehmer Patient/in mit der Krankheit x, die sich diese Angebote leisten können. Oder, Banken eröffnen die Teilnahme am Zahlungsverkehr, und richten ihre spezifischen Angebote teils an sehr spezifische Kundengruppen. Exklusion lässt sich folglich auf der Organisationsebene nicht nur an einem Nichteinbezogensein auf der Seite des Systems in Leistungs-, sondern auch in der Berechtigung der Betreuung/Ausschluss an Möglichkeiten in Komplementärrollen - im weiteren Mitgliedsbedingungen genannt - funktional beschreiben. Exklusion einer Personengruppe lässt sich in der Differenz zur Inklusion der Organisationen des Gesundheitssystems z. B. an der Mitgliedsbedingung festmachen, dass das bei einem Patienten angenommene Krankheitsbild x nicht Teil der Kommunikation ist; Exklusion lässt sich aber auch an der Mitgliedsbedingungen feststellen, wenn schlichtweg keine Organisation die medizinischen Behandlungsangebote für Patienten/innen mit einem bestimmten ökonomischen Kapital anbietet.

\subsubsection{Systemtheoretisches Beschreibungsschema zur Befragung der Determinierung in der Risiko-Relation von anerkannter Bildung und typischer Teilhabe in Arbeit: Erziehung}

Mit der Entscheidung einer systemtheoretischen Annäherung an die ausgehende These auf der Organisationsebene wird an eine, noch als jung zu bezeichnende Diskussion in der Soziologie um die Thematik Inklusion/Exklusion angeschlossen, die sich u. a. mit der Ausdifferenzierung (Luhmann 2002, 111ff.) von Funktionssystemen beschäftigt. Dieser Ansatz bleibt nicht auf die in der Wissenschaft weithin bekannten Codes, Programm usw. von Erziehung als Funktionssystem begrenzt, sondern erweitert diese entscheidend. Vielmehr rücken Technologien entlang der Ausdifferenzierung in Organisationen der allgemeinen, beruflichen, hochschulischen, usw. Bildung in den Mittelpunkt und verrücken vermeintlich funktionale Systemparadigmen von Erziehung:

Ausdifferenzierung heißt Selbstautonomie und Selbstautonomie heißt Notwendigkeit von Selbstorganisation. Aber es kommt hinzu, daß damit ein Wachstumsprozeß eingeleitet wird, den die Gesellschaft nicht mehr kontrollieren kann. [...] Es geht nur noch zu einem geringen Teil um ,Kinder, von denen man annehmen konnte, daß sie auf Grund ihrer Natur Erziehung nötig haben. Wie in anderen Funktionssystemen muß die Semantik der Natur aufgegeben werden. [...] Sie wird durch die Semantik der ,Werte' ersetzt. Mehr und mehr Zertifikate gelten als förderlich [...] (ebd., 123).

Das Zitat verweist zum einen auf den historischen Charakter sozialer Systeme hin, bzw. die bereits angedeutete zeitliche Dynamik in organisatorischen Ausgestaltungen. Im Spannungsfeld der Funktion eines Erziehungssystems leitet sich zum anderen die Notwendigkeit der Tech-

6 Daneben kann es auch sekundäre Leistungsrollen geben, die die Systemausführung irritieren: Gutachter, engagierte Eltern im schulischen Bildungsprozess usw.; im Sinne einer Komplexitätsreduktion werden diese im Weiteren aus der Analyse herausgenommen. 
nologisierung begründet ab; eben einhergehend mit dem Zwang zur innovativen Anpassung. $\mathrm{Zu}$ den Technologisierungen zählen Bewertungsprogramme bspw. zur Gewährleistung einer chancengerechten Zertifizierung, Beschulungsprogramme z. B. zur Gewährleistung von Unterricht überhaupt oder Unterrichtsprogramme zur Gewährleistung von erfolgreichem Unterricht. Des Weiteren zählen hierzu auch die Gründung sowie Weiterentwicklung von ordentlichen Schulen/Universitäten und ihren ordentlichen Professionen zur Gewährleistung von Innovation von Unterricht, sei es ein weiterführendes, ein spezialisiertes oder ein anderes mehr an Bildung. Diese Institutionen differenzierten sich gleichwohl wie die Gesellschaft zunehmend weiter aus, nach eigenen Gesetzmäßigkeiten unter Berücksichtigung des zeitlich sich wandelnden „Wertes“ eines ordentlichen Bildungsweges. Diese Gesetzmäßigkeiten bauen aufeinander auf, z. B. zwischen den Institutionen der Primarstufe und der Sekundarstufe I, oder fungieren nebeneinander her, wie bspw. zwischen den Institutionen der Sekundarstufe I (ebd., 161ff.). Mit einer Analyse auf der Organisationsebene richtet sich das primäre Interesse nicht auf universelle, funktionale Systemparadigmen von Erziehung, so z. B. auf die Auflösung des Technologiedefizits des Erziehungssystems, welche in den vergangenen Jahrzehnten die systemtheoretische Debatte mitbestimmte. Vielmehr richtet es sich auf die gegenwärtig beobachtbare Relevanz für die Beschulungs-, Bewertungs- und Unterrichtsprogramme der Organisationen des Erziehungssystems. Entsprechend wird diesbezüglich an die bei Urban (2009, 247ff.) oder Kurtz (2006, 114ff.) verwiesenen Empfehlungen einer primären sowie einer sekundären Codierung in besser/schlechter und vermittelbar/nicht-vermittelbar übernommen.

Einerseits drängen sich die Beschulungs-, Bewertungs- und Unterrichtsprogramme im Gestus von Programmen des Erziehungssystems auf der Funktionsebene auf, als ein, sensu Stichweh, „Weltprogramm“ von Bildung; und dies insbesondere, wenn man sich bspw. der Urheber von gegenwärtigen pädagogischen Leitideen vergewissert: Kompetenz(en) lassen sich auf die OECD und Lebenslanges Lernen auf die Weltbank zurückführen (vgl. Basendowski 2013, 110ff.), gemeinhin globale, auf primär ökonomische Zusammenarbeit ausgerichtete Konsortien. Man mag suggerieren, dass mit den Homogenisierungen einhergeht, dass die sozial vermittelbaren Aussagen der Organisationen des Erziehungssystems der "Welt“ zwischen nationalen Bildungsund globalen Arbeitsmärkten moderieren. Die nationalen Bildungsmärkte würden sich demnach über Zertifikate sich selbst erhalten, indem sie ihre Funktion sichern, was eng mit einem sozial vermittelbaren Adressatenmerkmal der Komplementärrollen auf den globalen Arbeitsmärkten in Verbindung steht (Hillmert 2009, 86). Diese Outputs sind konsequenterweise maßgeblich durch die Antizipierung der Codierung von haben/nicht-haben der Organisation im System Wirtschaft beeinflusst: der Wettstreit um human capitals. Die mitschwingende „Ökonomie der Produktion von Wissens-Knappheiten“ (Bellmann/Priddat 2006, 162) versieht auf gewisse Weise den hinter den übernommenen pädagogischen Leitidee-Konzepten liegenden Fähigkeiten, Fertigkeiten und Bereitschaften mit einem Wert von human capital. Oder in anderen Worten, human capital haben/nicht-haben wird in der Wertigkeit von Zertifikaten ausgedrückt, über die das Erziehungssystem wenig Einfluss hat: Während vor einigen Jahrzehnten z. B. ein Hauptschulabschluss/Erster allgemeinbildender Schulabschluss einen aussichtsreichen Platz im Wettbewerb um den Zugang zu den Arbeitsmärkten gewährte, ist gegenwärtig das dahinterliegende Standard- und insbesondere knappe Spitzenlevel in human capital rasant angestiegen. Dass sich dieser Trend der Bildungskapitalmaximierung fortsetzt, manifestiert sich z. B. nicht zuletzt an der Anteilsverschiebung zwischen beruflicher und akademischer Berufsausbildung: „2013 gab es erstmals mehr Studienanfänger als Anfänger in der dualen Ausbildung“ ließ unlängst die deutsche Bundesregierung verlauten. Aber auch am unteren Limit, an der Grenze 
von haben/nicht-haben verfestigen sich zunehmend funktional festgelegte Grade von human capital, so bspw. auch von schriftsprachlichen Kompetenzen eines funktionalen Analphabetismus. Diese decken sich weder mit dem Programm der funktionalen Grundbildungskompetenzen der allgemeinbildenden (vgl. Grotlüschen 2011; Kretschmann 2011) noch mit dem der beruflichen Handlungskompetenzen in der Ausbildungsreife (vgl. Basendowski 2013).

Andererseits muss kritisch befragt werden, ob tatsächlich von einem „Weltprogramm“ von Bildung ausgegangen werden kann, an dem sich alle nationalen Bildungsmärkte orientieren. So kann „Unterricht“ grundsätzlich auch außerhalb von Institutionen stattfinden (Luhmann 2002, $161 \mathrm{ff}$.) und zwingt das Erziehungssystem abzusichern, dass es in seinen Organisationen überhaupt zu den für „erfolgreiche“ Erziehung notwendigen regelmäßigen Interaktionen kommt (Vanderstraeten 2006, 107). Entlang der in diesem Sinne entwickelten Technologie der kontinuierlichen und sesshaften Beschulung, in Deutschland findet sich diese als verortete Schulpflicht wieder (Schroeder 2012, 40ff.), kann eine zunehmende globale Beeinflussung zumindest festgemacht werden: Mit Ausnahme einiger Länder mit Unterrichtspflicht hat sich in den sogenannten developed countries die kontinuierliche und sesshafte Beschulung mehrheitlich durchgesetzt. Die sogenannten Entwicklungsländer werden dahingehend, mit den damit verknüpften Reproduktionen von Ungleichheit, beeinflusst, diese Technologie zu übernehmen (Windolf 2009, 14).

Als kritischer Einwurf interessiert an dieser Stelle primär, ob human capital im Sinne eines Adressatenmerkmals zwischen „allen“ Bildungs- und Arbeitsmärkten funktional moderiert. Diesbezüglich decken sich die in den vorherigen Unterkapiteln bereits zusammengetragenen Argumente mit der in der Systemtheorie begründeten Schließung der Systeme, hier der Wirtschaft und der Erziehung - übertragen somit auch auf der Organisationsebene (Hillmert 2009, 86ff.). Eine funktionale Kopplung könnte demnach höchstens innerhalb nationaler Bildungsmärkte ausgemacht werden, welche nicht unmittelbar durch Organisationen des globalen Wirtschaftssystems entschieden werden. Dieser Fall soll im Weiteren als zwischen reinen Bildungsmärkten bezeichnet und durch das Beispiel des Zugangs zum Hochschulstudium an Universitäten über bestimmte Zertifikate in Deutschland illustriert werden. Zwischen den unreinen Bildungs- sowie zu den Arbeitsmärkten ist dagegen eine gewisse Kontingenz in der Kopplung festzumachen. So wird der Zugang zu einer betrieblichen dualen beruflichen Ausbildung und die Besetzung eines Arbeitsplatzes alleinig durch ein Haben/Nicht-Haben nach Prinzipien des Wirtschaftssystems reguliert; und nicht alleinig nach den technologisierten Zertifikaten der Organisationen des Erziehungssystems (Plake 2010, 112ff.). Was für die Kopplung zwischen den unreinen Bildungs- aber auch Arbeitsmärkten gilt, müsste wiederum gleichsam die Konzepte hinter den pädagogischen Leitideen der Kompetenz(en), den funktionalen Grundbildungs- und beruflichen Handlungskompetenzen, umfassen. Das erste ohne externe Validitätsprüfung auskommen, ergo, ohne den empirischen Nachweis, dass bestimmte Fähigkeiten, Fertigkeiten und Bereitschaften explizit mit einem „erfolgreicheren“ Lebenslauf einhergehen, stützt diese Schlussfolgerung. Gleiches gilt für einige Methoden- oder Sozialkompetenzen aus der Matrix beruflicher Handlungskompetenzen für einen „erfolgreichen“ Einstieg in das Berufsleben im Anschluss an eine berufliche Ausbildung (Müller 2008).

In der Adaption des Bewertungsprogramms treten jene Kompetenz(en) in den Bildungsstandards und weiter in Deutschland in Abschlussprüfungen und Klausuren gemäß der Lehr- bzw. Bildungspläne bis hin zu den Hausaufgaben nach Schulbüchern als Maßstab für die Differenz besser/schlechter auf. Nicht zuletzt am Tag der PISA-Tests haben repräsentativ "global alle“, in den Bundesländern gleichsam am Tag der zentralen Abschlussprüfungen „landesweit alle“ in 
derselben Zeit das gleiche Wissen über dieselbe Modalität nachzuweisen. Auch die Unterrichtsprogramme sind zumindest in der Zielperspektive auf dieses Bewertungsprogramm ausgerichtet. Die selektiven Erwartungen richten sich jedoch nicht nur an den Leistungsbereich, sondern auch an angemessenes soziales bzw. normgerechtes Verhalten zur Gewährleistung von „erfolgreichem“ Unterricht. Vanderstraeten $(2006,101)$ wirft an dieser Stelle die „grammar of schooling “ ein und hebt weitere Konstrukte vor, mit denen Komplementärrollen als sozial beschreibbar gefasst werden können; z. B. durch das Konstrukt der Entwicklungstempi, der Aufmerksamkeit oder der Anstrengungsbereitschaft. Die Unterrichtsprogramme umfassen entsprechend einer zeitlichen Dynamik unterliegenden Setzungen, wo - in z. B. bestimmten Schulen, Klassen- und Fachräumen usw. -, wie - didaktische Konzepte gemäß dem Bewertungsprogramm und/oder der „grammar of schooling" - und durch wen - spezifische ordentliche Professionen/Leistungsrollen je Schulform ${ }^{7}$ - das Mitdenken von Gruppen „aller“ Schüler/innen - bestimmte Schüler/innengruppen als Komplementärrollen der Organisationen - gewährleistet wird. Dabei ist auch durch die Ausgestaltung eines Bildungssystems in Institutionen eine breite Vielfalt an Unterrichtsprogrammen denkbar, solange sie gemäß dem Bewertungsprogramm zu angemessenen erfolgreichen Effekten beitragen und das Beschulungsprogramm einhalten (Stichweh 2013; Urban 2009, 247ff.; Kurtz 2006, $114 \mathrm{ff}$.). Diese Technologien sind unter systemspezifischer Perspektive jedoch nicht „Unterricht“, sondern letztendlich als ein Evolutionsergebnis zu verstehen, welches sich entlang von Standards beobachten lässt, die zunehmend hochrangiger und parallel hierarchischer wurden. Dies verdeutlicht sich alleinig daran, dass ein Schultag nicht erst dann endet, wenn „allen“ dieselbe(n) oder ähnliche(n) bestimmte(n) Kompetenz(en) tatsächlich vermittelt wurden (Vanderstraeten 2006, 101).

Zusammenfassend lassen sich drei systemimmanente Differenzen zur Beschreibung gegenwärtiger Technologisierungen der Organisationen ausmachen, welche unter der Folie hierarchisch geordneter Institutionen der allgemeinen, beruflichen und hochschulischen Bildung zu interpretieren sind. Sie gestalten ein Schema, mit welchem entlang der standardisierten Beschulungs-, Bewertungs- und Unterrichtsprogramme eingeordnet werden kann, welche unter ,allen“" zu Betreuenden hierunter mehr oder weniger ein- oder ausgeschlossen sind, und zwar indem Mitgliedsbedingungen die Komplementärrollen

- erstens funktional durch (ordentlich) beschulbar/nicht-beschulbar charakterisieren: Es geht hierbei um die Betrachtung der Setzungen, welche Adressaten überhaupt für die Schulbildung der Organisationen ansprechbar sind, ergo betreubar und bewertbar, sind. Sowohl die allgemeinbildenden Primarstufen- und Sekundarstufenschulen kennen diese Mitgliedsbedingungen entlang dem Merkmal Alter/Schuljahre oder Wohnort, nach welchem bestimmte Adressaten inkludiert werden, um andere gleichsam zu exkludieren. Für die inkludierten Adressaten werden ein ordentlicher Ort und ordentliche professionelle Betreuung für den Unterricht usw. vorgehalten. Formen systemimmanenter Exklusion in den Organisationen von Erziehung können bei den zu Betreuenden unter „allen“ aufgedeckt werden, die der kontinuierlichen und sesshaften Beschulung nicht nachkommen, weshalb sie sich dieser aus diversen Motiven heraus verweigern (Schroeder 2012, 258ff.; Stamm 2012), weil sie z. B. keinen festen Wohnsitz haben (Schroeder 2012, 44ff.), weil sie eine Mutterschaft mit dieser nur schwer vereinen können (ebd., 365ff.), usw.

7 Daneben kann es auch sekundäre Leistungsrollen geben, die die Systemausführung irritieren: Gutachter, engagierte Eltern im schulischen Bildungsprozess usw.; im Sinne einer Komplexitätsreduktion werden diese im Weiteren aus der Analyse herausgenommen. 
- zweitens funktional durch (ordentlich) bewertbar/nicht-bewertbar charakterisieren: Bewertungsprogramme des Erziehungssystems tragen grundsätzlich zu einer Komplexitätsreduktion, der ansonsten nicht beobachtbaren Effekte der Einwirkung auf Lernende bei (Vanderstraeten 2006). Jegliche Bewertung im institutionellen Erziehungssystem führt folgerichtig zugleich zur Erzeugung einer systemimmanenten Ungleichheit, welche letztlich in Zertifikaten summiert zu einer sozialen Adressatenzuschreibung führen. Diesen Zusammenhang hatte bereits Luhmann im Zuge der „Paradoxie der Ungleichheit des Gleichen“ (Luhmann 2004, 220) herausgearbeitet. In diesem Sinne finden Adressatenmerkmale der einzelnen Komplementärrollen auf der Funktionssystemebene keine Berücksichtigung, d.h., weil hier Kommunikation alle ungeachtet von z. B. Geschlecht oder Ethnie als Gleiche mitdenkt, formieren seine standardisierten Programme aus diesen Gleichen Ungleiche. Die Reaktion auf dieses Paradoxon stellen „Regeln zur ,gerechten‘, im System kontrollierten Herstellung von Ungleichheit [dar] - also Standards für Leistungsbeurteilungen, Zensierung sowie Selektion für Schulen und Schulklassen, kurz: die Codierung (besser/schlechter) und Programmierung der Selektion“ (ebd.). Die systemimmanente Unterscheidung in bewertbar/nicht-bewertbar auf der Organisationsebene unterscheidet demgemäß, welche Lernende über Mitgliedsbedingungen in ihren Institutionen mit Bezug auf diese Standards Betreuungsangebote vorgehalten bekommen. Eine Exklusion aus den Unterrichts- und Beschulungsprogrammen geht nicht zwingend hervor. Den systemimmanenten Charakter lässt sich bspw. zwischen den allgemeinbildenden Primarstufen- und Sekundarstufenschulen verdeutlichen, denn die Sekundarstufenschulen verknüpfen ihre ordentlichen Betreuungsangebote, zumindest auf Dauer, an bestimmte Mitgliedsbedingungen. Mindeststandards (Hupka-Brunner et al. 2011) können unter diesen Bedingungen funktional die Kopplung zwischen reinen Bildungsmärkten kennzeichnen.

- drittens funktional durch (ordentlich) betreubar/nicht-betreubar charakterisieren: Die Unterrichtsprogramme machen, gemeinsam mit den Bewertungsprogrammen, erst den Kern des institutionellen Erziehungssystems der allgemeinen Schule aus (Urban 2009; Kurtz 2006). Auf der Organisationsebene rücken Standards wie der Betreuungsschlüssel Lehrende-Lernende und der ordentlichen Professionen in den Mittelpunkt. Wenn ersterer Standard in der Primarstufe bspw. bei ungefähr 1:20-1:30 sowie in der Sekundarstufe bei 1:30 fetgelegt und zweiterer im Kontext einer formalen staatlichen anerkannten Qualifizierung für jede Schulform zu positionieren ist, ergeben sich Kriterien einer Unterscheidung von betreubar/nichtbetreubar. Formen von Exklusion manifestieren sich in logischer Folge an einem verringerten Betreuungsschlüssel und anderen bzw. weiteren Professionen, die für das komplementäre Mitdenken von Adressaten steht, die von den Standardprogrammen nicht angesprochen werden können. Es ist eine soziale Realität, dass es unter „allen“ zu Betreuenden Personen gibt, denen ein mehr an Zeit sowie z. B. ausschließlich ein Sozialpädagoge als Lehrkraft vorgehalten wird (Urban 2009) und dies mit dem Ziel, u. U. über Anpassungsförderung der Verhaltensnorm, das standardisierte Bewertungsprogramm funktional anwenden zu können.

Die Schlussfolgerung, dass Adressaten, die für die allgemeine Schulbildung nach mindestens einer dieser, aber nicht allen drei Kategorien exkludiert sind, per se gleichsam durch das Erziehungssystem in ihrer Rolle als Lernende in Kommunikation nicht mitgedacht werden, ist nicht haltbar. Ein bereits angeführtes Argument verweist darauf, dass in modernen Gesellschaften in allen Systemen auf der Ebene der Organisationen, die Differenz mitdenkend, sich Subsysteme entwickelten, die Adressaten ansprechen, die gewissen Mitgliedsbedingungen an die Komple- 
mentärrollen anderer Organisationen desselben sozialen Systems nicht entsprechen (Stichweh 2013). Eine tatsächliche, andauernde Vollinklusion ist konkludierend auch auf der Organisationsebene völlig ausgeschlossen. So kann bspw. eine Person nicht zugleich als zu Betreuender von der Primar- und der Sekundarstufe angesprochen werden. Insofern Funktionssysteme, aber auch immer Supplemente für weitere Adressatengruppen (inkludierende Exklusion) als auch Gegenstrukturen (exkludierende Inklusion) zu den historisch und regional gewachsenen ordentlichen Unterscheidungen entwickeln, ist auf der Organisationsebene eine tatsächliche und andauernde Vollexklusion wenig wahrscheinlich, wenn nicht gar undenkbar. Gemeinsam ist den Institutionen dieser inkludierenden Exklusion bzw. exkludierenden Inklusion folglich, dass sie Personen mitdenken, die in anderen Institutionen als Komplementärrollenträger exkludiert wurden. Während erstere Supplemente eines Systems den Wiedereinstieg in dieses anstreben, liegt der Fokus im zweiten Fall auf der systemfremden Inklusion (Stichweh 2009, $38 \mathrm{ff}$.$) .$

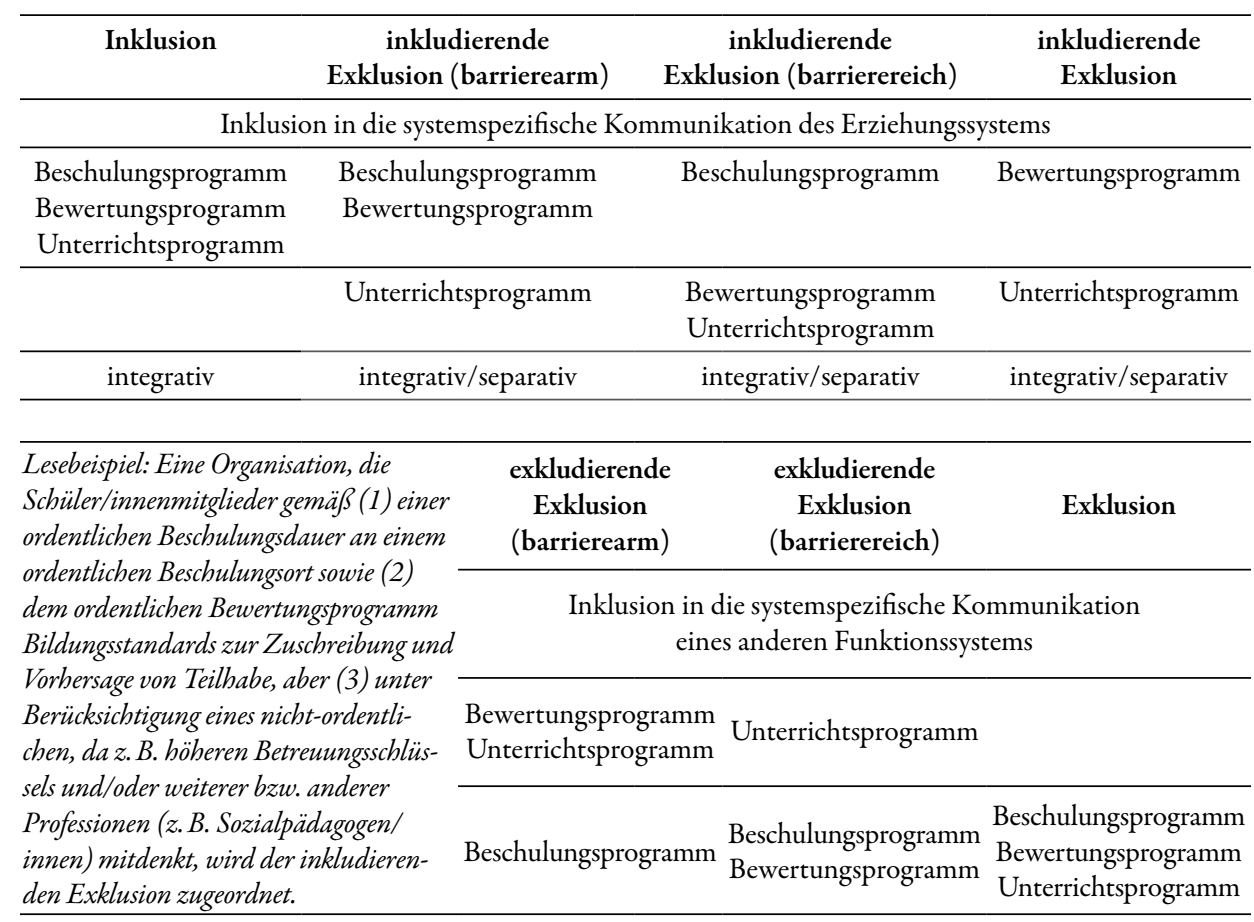

Abb. 3: Beschreibungsschema entlang von Mitgliedsbedingungen an die Komplimentärrollen der Organisationen von Bildung

Transferiert auf die nationale Organisation der allgemeinen Bildung wäre nach den systemimmanenten Differenzen in (ordentlich) beschulbar/nicht-beschulbar, bewertbar/nicht-bewertbar sowie betreubar/nicht-betreubar zu beschreiben, welche zu Betreuenden jeweils im Sinne von inkludiert, exkludiert inkludiert, inkludiert exkludiert bzw. exkludiert mitgedacht werden. Inklusion/Exklusion manifestiert sich sodann an der Berechtigung/dem Ausschluss auf der Seite der Organisationssysteme der Erziehung entlang der Mitgliedsbedingungen an ihre Komplementärrollen (vgl. Abbildung 3). 


\subsubsection{Annäherung an eine systemtheoretische Analyse auf der Organisationsebene von Bildung}

Mit dem theoretisch hergeleiteten Beschreibungsschema werden im Weiteren einige Übergänge zwischen den Subsystemen und Supplementen eines nationalen Bildungssystems befragt. Das Augenmerk liegt hierbei zwischen den Mitgliedsbedingungen der Institutionen der Sekundarstufe I bis in die berufliche Bildung, respektive, zwischen reinen, aber auch unreinen Bildungsmärkten. Konsequenterweise wird an die obig benannte Hierarchie der Institutionen der allgemeinen Schule angeschlossen (siehe Abbildung 4).

Hierzu werden, mit dem Ziel einer besseren Nachvollziehbarkeit, einige Reduktionen an der Vielfältigkeit von Subsystemen und Supplementen vorgenommen. Diese wird zum einen die Trennung zwischen integrativen und separativen Beschulungsprogrammen sensu Stichweh (2013) umfassen, respektive, zwischen räumlich inklusiven und räumlich separativen Beschulungsprogrammen. Diese Unterscheidung verdeutlicht einen zusätzlichen Standard dahingehend, was als ordentlicher Ort auf der Organisationsebene gilt. Diese Perspektive vermag in der aktuellen bildungspolitischen Diskussion um schulische Inklusion eine primäre Kategorie sein. Es entstünde ein neuer, aber inkonsequenter Standard innerhalb des Beschulungsprogramms, welches keinen an einen beliebigen ordentlichen Ort der Bildung geknüpften Raum vorsieht, sondern dessen ungeachtet einen wohnortnahen für „alle“ präzisiert. Aus der Anlage des systemtheoretischen Beschreibungsschemas geht jedoch einerseits hervor, dass hier (räumlich) separativ nicht als Differenz von inklusiv zu interpretieren ist. So bestehen im Sinne der funktionalen Ausdifferenzierung von Erziehung einzelne Organisationen, welche kaum einem nicht-ordentlichen Beschulungsprogramm zuzuordnen sind: Privatschulen und Internate umfassen Mitgliedsbedingungen, die nicht nach einer Wohnortnähe für „alle“ ausgerichtet sind. Andererseits stellt in diesem Zuge der Gegenpol integrativ keine funktionale Unterscheidung dar, die in der Kopplung mit weiteren Bildungs- und Arbeitsmärkten von Relevanz wäre. Der Besuch einer Privat- oder gar Internatschule reguliert nicht funktional den Zugang in eine weiterführende Schule oder eine schulische Ausbildung. In eine berufliche Ausbildung kann sie sowohl positive als auch negative Kopplungsmächtigkeit innehaben.

Zum Anderen sieht eine weitere Reduktion vor, den sozialen Realitäten der Schullandschaft geschuldet, die diversen „Schulen der sozialen Arbeit“ (Schroeder 2012, 436ff.) nicht in ihrer kompletten Bandbreite fortzuführen. Nichtsdestotrotz eröffnet sich an diesem Sozialatlas die Ausdifferenzierung der Organisationen von Erziehung eindrücklich. Denn es eröffnet den Blick auf die Vielfalt von Supplementen ohne eine Verengung auf die separativen Sonderschulen, die ihre Adressaten z. B. zwar primär mit Bezug auf die ordentlichen Unterrichts- und Bewertungsprgramme inkludieren, jedoch nur mittels einer Manipulation des Beschulungsprogramms und zwar in der Art, dass z. B. eine Reduktion der ordentlichen Dauer von Kommunikationen hingenommen wird. Dies trifft alsdann bspw. für die Organisation der mobilen Schulen für Zirkuskinder zu, in denen Unterricht im zeitlichen Umfang reduziert und überdies nicht regelmäßig von Montag bis Freitag vorgehalten wird. Einzelne dieser Supplemente verweisen auf manipulierte Unterrichtsprogramme hin, da die Professionen nicht den ordentlichen Leistungsträgern der als inklusiv bezeichneten Organisationen entsprechen. Andere wiederum manipulieren das Bewertungsprogramm derart, dass ein ordentlicher Schulabschluss nicht das Ziel von intentionaler Erziehung ist. 
Wie die "Schulen der sozialen Arbeit“ sind die Supplemente Sonderschulen folglich nicht der Exklusion auf der Organisationsebene zugeordnet. Diese inkludieren Schüler/innen als ordentlich beschulbar - abzulesen daran, dass nach Abschluss die Adressierung erfolgt, dass die Schulpflicht abgegolten wurde. Zu bedenken ist, dass in diesen Institutionen teils sozusagen „unter einem Dach“ einige Schüler/innen als ordentlich bewertbar und andere als nicht ordentlich bewertbar mitgedacht werden; gemessen an der Hierarchie dessen, was als ein ordentliches Bewertungsprogramm gilt. Ein Förderschulabschluss Lernen gilt so nicht nur in offiziellen Statistiken als "kein Schulabschluss“, sondern - wie sich noch zeigen wird - es erfolgt funktional eine Exklusion entlang der Mitgliedsbedingungen der inklusiven Organisationen der Sekundarstufe II im Anschluss an die Sekundarstufe I. Konsequenterweise werden die Sonderschulen in der graphischen Zusammenfassung (siehe Abbildung 4) zu den Organisationen der inkludierenden Exklusion gezählt, die zudem weiter hierarchisch unterteilt werden. Begründet durch die zentrale Relevanz des Bewertungs- nebst des Beschulungsprogramms innerhalb der Mitgliedsbedingungen an ordentliche Komplementärrollen zwischen reinen Bildungsmärkten, werden diese mit den Polen (barriere)arm/reich charakterisiert; übernommen aus der Debatte um barrierearme bzw. -reiche Zugänge in Bildung. Es verdeutlicht sich, dass Sonderschulen demnach in jene unterschiedlichen Grade exkludierend inklusiver Organisationen nach dem Beschreibungsschema zugeteilt werden, was, wie obig beschrieben, nicht dem alltäglichen Verständnis einer Organisation entspricht. Die systemtheoretische Organisation der Förder- bzw. Sonderschule Lernen ist kein Synonym der alltäglichen Institution. Gleiches gilt für die Organisation der Stadtteilschule, der Gemeinschaftsschule usw. und der Institution(en) der Stadtteilschulen, Gemeinschaftsschulen usw.

Die Organisationen dieser Supplemente des Erziehungssystems, wie z. B. Sonderschulen, denken folglich nicht einen singulären Adressaten mit, so in dem Beispiel in der schulischen Inklusion den/die nicht mehr zu exkludierende/n Schüler/in. Zusätzlich dachten sie auch seither nicht alle Adressaten mit, die entlang der Mitgliedsbedingungen der inklusiven Subsysteme exkludiert wurden. Es handelt sich vielmehr um diejenigen Adressaten, die stets als ordentlich beschulbar, jedoch nicht als ordentlich betreubar und teils zusätzlich als nicht ordentlich bewertbar mitgedacht wurden. An dieser Stelle liefert der Sozialatlas zu den "Schulen der sozialen Arbeit“ einen weiterführenden Einblick, welche in die Unterscheidung der Organisationen der exkludierenden Inklusion und Exklusion einfloss. Gemeinsam ist diesen Organisationen, dass das Beschulungsprogramm innerhalb ihrer Mitgliedsbedingungen „außer Kraft gesetzt“ wurde, denn es unterliegt der Logik eines anderen Funktionssystems. Zweiteres trifft bspw. zu, wenn Unterricht bei lebensbedrohlich Kranken nur in Abhängigkeit des Gesundheitszustandes organisierbar ist. Es lassen sich dennoch Organisationen aufdecken, in denen sowohl die ordentlichen Bewertungs- als auch Unterrichtsprogramme des Erziehungssystems zum Tragen kommen, so z. B. in der Schule im Strafvollzug oder teils bei Straßenschulen. Es empfiehlt sich analog der Organisationen der exkludierenden Inklusion auch für diese Organisationen der inkludierenden Exklusion eine Gliederung der Ausprägungsgrade durch die Pole arm/reich. Insofern keines der ordentlichen Programme bestimmte Adressaten mitdenkt, kann aus der systemspezifischen Betrachtung der Organisationen der obligatorischen Bildung davon gesprochen werden, dass diese exkludiert sind. Es handelt sich hierbei um bspw. die Jugendlichen, die anderenorts als Schulabbrecher bezeichnet werden, ergo diejenigen, die weder von den ordentlichen Beschulungs-, noch Bewertungs- und auch nicht Unterrichtsprogrammen auf Dauer mitgedacht werden. 


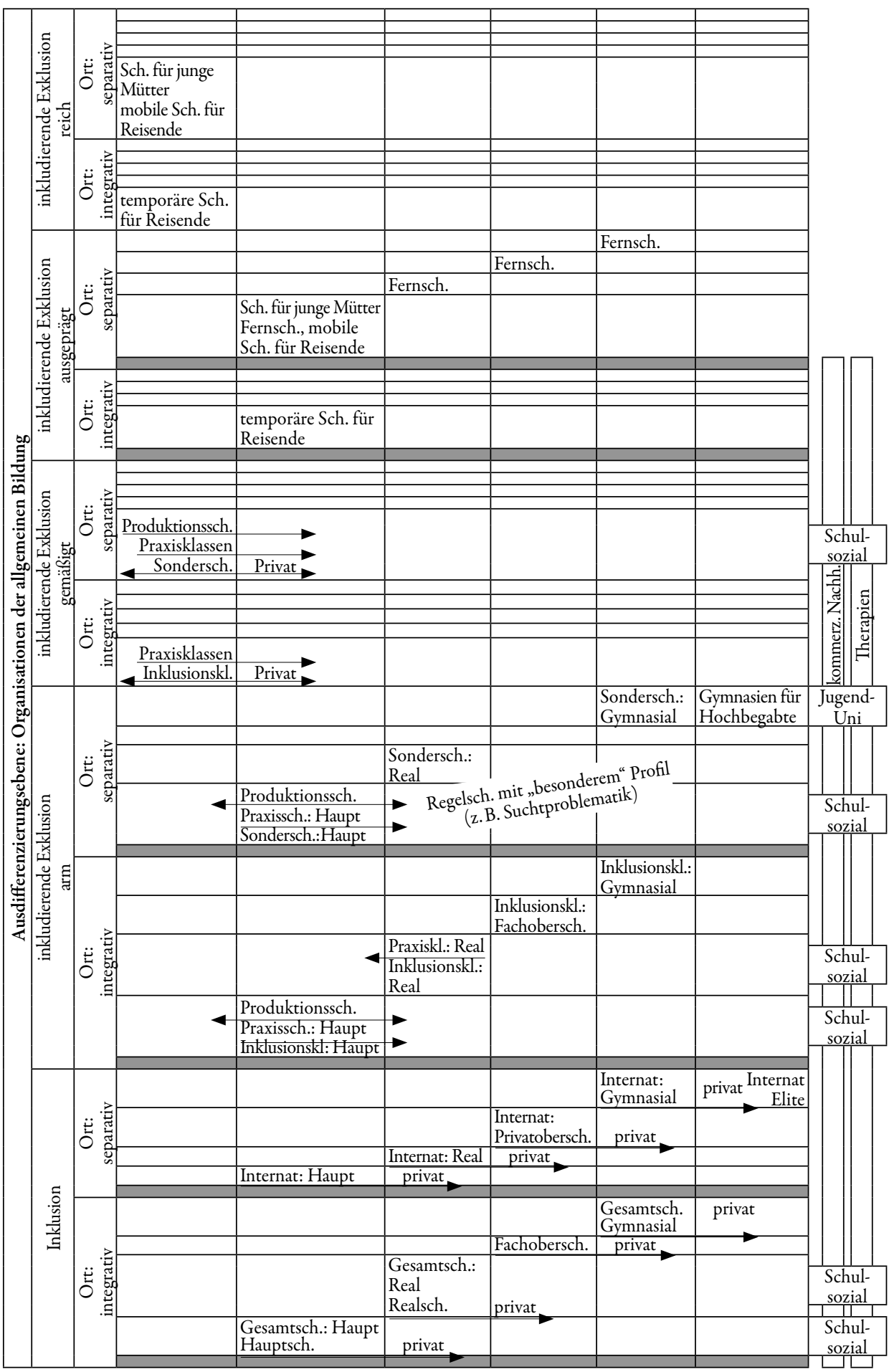

Abb. 4: Inklusive und inkludierend exklusive Mitgliedsbedingungen der Organisationen von Bildung 


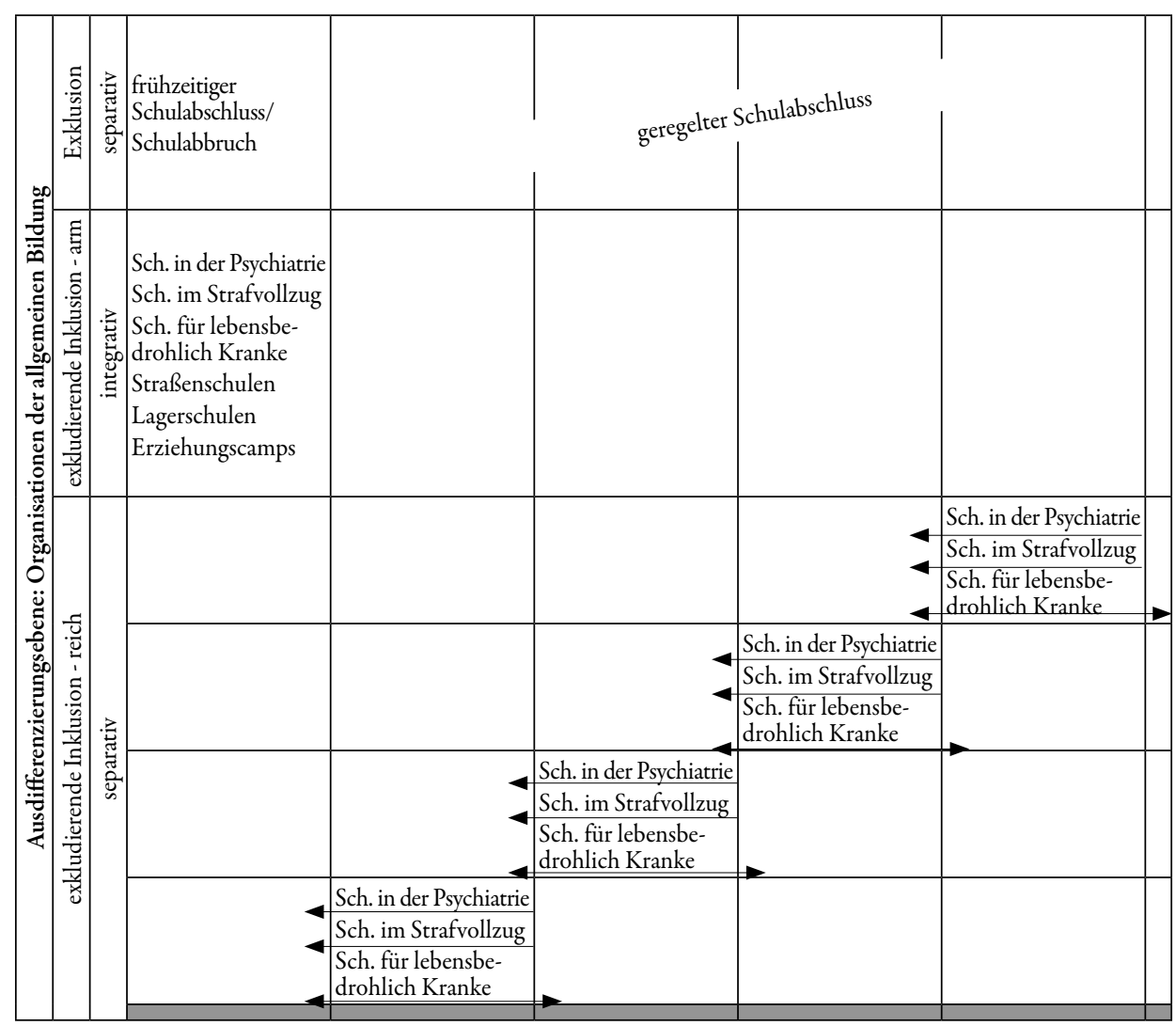

Lesehilfe: Die Organisationen prägende Hierarchie ist entlang der sozial-kommunizierbaren Zertifikate durch die Anordnung von links nach rechts gekennzeichnet.

Abb. 5: Exkludierend inklusive und exklusive Mitgliedsbedingungen der Organisationen von Bildung

\subsubsection{Systemtheoretisches Beschreibungsschema zur Befragung der Determinierung in der Relation von Bildung und Teilhabe: Arbeit}

Das Beschreibungsschema für die Übergänge zwischen den Subsystemen und Supplementen eines nationalen Bildungssystems als Grundlage übernommen, richtet sich das Interesse nun auf die Übergänge zwischen Bildungs- und Ausbildungsmärkten. Es folgt eine Analyse von inkludiert, exkludiert inkludiert, inkludiert exkludiert bzw. exkludiert mitgedachten Personen an den Übergängen zwischen Bildungs- sowie zwischen Bildungs- und Arbeitsmärkten.

Auf der Suche nach Technologien liefern u.a. einige Ergebnisse eines Sonderforschungsprogramms zu Beschäftigungsverhältnissen in Deutschland (Köhler et al. 2004) weiterführende Ansätze. Aus der Exploration sozialer Realitäten auf den Arbeitsmärkten spiegeln sich diese entgegen offizieller Statistiken nicht innerhalb eines uniformen Qualifizierungsniveaus „ohne anerkannte Berufsausbildung “wider, sondern eher entlang von Jedermann- und Tätigkeitsbezogen-qualifizierten Beschäftigungverhältnisse. Während ersterem Niveau Erwerbstätigkeiten ohne jegliche Form einer Ausbildung oder Qualifikation zuzuordnen sind, vereinen sich unter den Tätigkeitsbezogen-Qualifizierten all diejenigen mit einer nicht-anerkannten Ausbildung oder mit einer Qualifikation; hierzu zählen gleichsam betriebsinterne Fortbildungen, die mit ensprechenden Zertifikaten bestätigt werden. Eine Vielzahl der Beschäftigungsverhältnisse der 
pauschal mehr oder weniger als „beruflich Unqualifizierten“ Deklarierten weisen diese Voraussetzung auf. Die Relevanz und die Tragweite dieser Trennung innerhalb des Qualifizierungsniveaus lässt sich anhand einiger Beispiele verdeutlichen: Wenn an der Mehrheit der deutschen Flughäfen an der Sicherheitskontrolle Personal im Sinne des $\$ 34 \mathrm{a}$ GewO und nach bestandener Beleihungsprüfung durch die Bundespolizei tätig ist, so überlassen wir diese Aufgabe (außerhalb von Bayern) tatsächlich „beruflich Unqualifizierten“. Ähnlich trifft dies für die Einlasskontrolle am Deutschen Bundestag zu. Persönlichkeiten lassen sich gar von ihnen beschützen.

Neben diesem Programm entlang der Niveaus der Qualifikation eröffnet die Studie von Köhler et al. (ebd.) zudem ein weiteres Programm entlang von Niveaus der Schließung. Beide Niveaus zueinander in Beziehung gesetzt unterstreichen die bereits skizzierte Befundlage (siehe Kapitel 2.2.2): Es lassen sich einerseits mit dem Qualifizierungsniveau durchaus eine sinkende Wahrscheinlichkeit offener Beschäftigungsverhältnisse festmachen. Andererseits muss jedoch berücksichtigt werden, dass das untere Niveau der Schließung von Beschäftigung für keine zu vernachlässigende Zahl „aller“ Berufstätigen zutrifft; und dies unabhängig davon, ob mit hohen oder geringen formalen Qualifikationen. Konsequenterweise kann nicht im Sinne eines Alleinstellungsmerkmals des Personals an Einfach(st)arbeitsplätzen gelten, dass nur diese außerhalb von Tarifverträgen und/oder von Anwartschaften der sozialen Sicherungssysteme unterhalb der Niedriglohnschwelle beschäftigt werden. Somit erscheint die Sammelkennzeichnung solcher Merkmale von Beschäftigungsverhältnissen als „atypisch“ verwirrend, da sie durchaus „typisch“ für einige deutsche Arbeitsmärkte sind. Mit den Polen von offen vs. geschlossen werden diese durch Kategorien ersetzt, die sich von der hinter den Bezeichnungspaaren wie „typisch“ vs. „atypisch“, bzw. „Normal-“ vs. „atypischen Beschäftigungsverhältnissen“ liegenden konventionellen Setzung eindeutig distanzieren. Zweifelsohne gilt dies einhergehend auch für die tatsächliche Relevanz von „Beruf“ mit seinen historisch einzuordnenden Konnotationen in der sozialen Praxis der Arbeitsmärkte und verrückt die Deutungshoheit von „anerkannt“ und „normal“ innerhalb und zwischen Ausbildung und Arbeit maßgeblich.

Trotz dieser mitschwingenden Problematiken soll im Weiteren an die gängige und seither verwendete Bezeichnung von Einfach(st)arbeitsplätzen festgehalten werden. Sie greifen dennoch die kritisierte Simplifizierung durch die Ebenen der Jedermann- und Tätigkeitsbezogenen Qualifizierungsniveaus auf: Während Einfachstarbeitsplätze konsequenterweise die Arbeitsplätze der Beschäftigen ohne jegliche Form von Ausbildung, Qualifikation oder Zertifizierung repräsentieren, ist eine zumindest nicht-anerkannte Ausbildung oder eine für die Tätigkeit notwendige Qualifizierung oder Zertifizierung für Einfacharbeitsplätze konstituierend; dies umfasst zudem, aber nicht zwingend, eine ,anerkannte Berufsausbildung“.

Es lässt sich festhalten, dass die üblichen Kategorien zur Kennzeichnung des Qualifizierungsniveaus in offiziellen Statistiken soziale Realitäten nicht mehr ausreichend treffend erfassen. Mit der Matrix nach Niveau der Schließung und Qualifikation von Beschäftigung werden letztlich unterschiedliche Arbeitsmärkte ausgemacht, die nach einer kulturell vermittelten Hierarchie auf Technologien verweisen, aus denen sich „anerkannte“ und „typische“ vs. alsdann „nicht-anerkannte“ und „untypische“ Mitgliedsbedingungen an Beschäftigte ableiten lassen. Am ehesten lassen sich diese als Mitgliedsbedingungen zu unterschiedlichen Arbeitsmärkten umschreiben und nicht etwa als Einstellungspraxis eines bestimmten Unternehmens verordnen. So wie es in jedem bestimmten Unternehmen wahrscheinlich unterschiedliche Arbeitsplätze nach der Matrix der Schließung und Qualifikation gibt, so unterschiedlich sind auch die Mitgliedsbedingungen.

Als Pendant zur in Deutschland verankerten Schulpflicht tritt keine allgemeine Arbeits-, und auch keine allgemeine Erwerbsplicht auf den Plan. Alleinig beim Zugang zu bestimmten Sozialleistungen wie bspw. ALG I, und in gewissem Sinne auch bei ALG II, besteht diese Pflicht. Stattdessen wird wird innerhalb des bundesdeutschen Verständnisses von Sozialer Marktwirtschaft das Pen- 
dant in einer Marktleistungsfähigkeit als „natürliche Gründe“ systematisch verortet: Die beiden wichtigsten Kriterien sind Krankheit und Alter. So wird im deutschen Jugendarbeitsschutzgesetz (JArbSchG) die gewerbliche Beschäftigung von Kindern bis einschließlich 12 Jahren grundsätzlich und oberhalb 13 bzw. 16 Jahren unter bestimmten Einschränkungen geregelt. Hierbei knüpfen die Regelungen direkt an das Beschulungsprogramm an, denn ihr Geltungsbereich umfasst nach $\$ 2$ JArbSchG ausdrücklich „Jugendliche, die der Vollzeitschulpflicht unterliegen “. Eine Marktleistungsfähigkeit aus „natürlichen Gründen“ bemisst sich überdies am Geltungsbereich der Grundsicherung im Alter - bestimmt durch die Regelaltersgrenze im Sinne des $₫ 43$ Abs. 2 SGB VI in Verbindung mit $\$ 41$ Abs. 2 SGB XII - und bei Erwerbsminderung. Eine volle Erwerbsminderung liegt dann vor, wenn nach $\$ 43$ Abs. 2 SGB VI ansonsten Erwerbsfähige ab 16 Jahren ,wegen Krankheit oder Behinderung auf nicht absehbare Zeit [in den nächsten 9 Jahren gemäß $\$ 102$ Abs. 2 Satz 2 SGB VI] außerstande sind, unter den üblichen Bedingungen des allgemeinen Arbeitsmarktes mindestens drei Stunden täglich erwerbstätig zu sein“; festgestellt durch eine gutachterliche Einzelfallprüfung ( $\$ 45$ Abs. 1 SGB XII). Eine teilweise Erwerbsminderung bemisst sich dagegen an der täglichen Marktfähigkeit von 6 Stunden ( $\$ 43$ Abs. 1 SGB VI).

Zusammenfassend eröffnen sich als standardisierte Technologien auf der Organisationsebene der Wirtschaft zur Beschreibung des Zugangs in Beschäftigung das Qualifizierungsprogramm abgleitet aus den Niveaus der Qualifizierung -, das Vertragsprogramm - abgleitet aus den Niveaus der Schließung - und das Marktleistungsprogramm - abgleitet aus einer Marktleistungsfähigkeit unter „natürlichen Gründen“. Gemeinsam entfaltet sich entlang der ordentlichen Setzung in „anerkannt“ bzw. „typisch“ die Möglichkeit, systemimmanente Differenzierungen auf der Organisationsebene für Mitgliedsbedingungen an die Leistungsrollen vorzunehmen:

\begin{tabular}{|c|c|c|c|c|c|}
\hline Inklusion & \multicolumn{2}{|c|}{$\begin{array}{c}\text { inkludierende } \\
\text { Exklusion (barrierearm) }\end{array}$} & \multicolumn{2}{|c|}{$\begin{array}{c}\text { inkludierende } \\
\text { Exklusion (barrierearm) }\end{array}$} & $\begin{array}{l}\text { inkludierende } \\
\text { Exklusion }\end{array}$ \\
\hline \multicolumn{6}{|c|}{ Inklusion in die systemspezifische Kommunikation des Erziehungssystems } \\
\hline \multirow{3}{*}{$\begin{array}{c}\text { Marktleistungsprogramm } \\
\text { Qualifizierungsprogramm } \\
\text { Vertragsprogramm } \\
\end{array}$} & \multirow{2}{*}{\multicolumn{2}{|c|}{$\begin{array}{l}\text { Marktleistungsprogramm } \\
\text { Qualifizierungsprogramm }\end{array}$}} & \multirow{2}{*}{\multicolumn{2}{|c|}{ Marktleistungsprogramm }} & Qualifizierungsprogramm \\
\hline & & & & & $\begin{array}{l}\text { Marktleistungsprogramm } \\
\text { Vertragsprogramm }\end{array}$ \\
\hline & \multicolumn{2}{|c|}{ Vertragsprogramm } & \multicolumn{2}{|c|}{$\begin{array}{l}\text { Qualifizierungsprogramm } \\
\text { Vertragsprogramm }\end{array}$} & Unterrichtsprogramm \\
\hline integrativ & \multicolumn{2}{|c|}{ integrativ/separativ } & \multicolumn{2}{|c|}{ integrativ/separativ } & integrativ/separativ \\
\hline \multirow{4}{*}{\multicolumn{2}{|c|}{$\begin{array}{l}\text { Lesebeispiel: Eine Organisation, die } \\
\text { (im Sinne von: Ein Arbeitsmarkt, der), } \\
\text { Beschäftigte gemäß (1) einer seiner natür- } \\
\text { lichen Marktleistungsfähigkeit - z.B. der } \\
\text { Fähigkeit zur ordentlichen Bewältigung } \\
\text { eines mehr als 6h-Arbeitstages - sowie (2) } \\
\text { einer ordentlichen, ergo anerkannten Berufs- } \\
\text { oder Hochschulausbildung, aber (3) unter } \\
\text { Berücksichtigung eines nicht-ordentlichen } \\
\text { Anstellungsverhältnisses (offene Beschäfti- } \\
\text { gungsformen) mitdenkt, wird den Organisa- } \\
\text { tionen (im Sinne von: den Arbeitsmärkten) } \\
\text { der inkludierenden Exklusion zugeordnet. }\end{array}$}} & \multicolumn{2}{|c|}{$\begin{array}{l}\text { exkludierende } \\
\text { Exklusion } \\
\text { (barrierearm) }\end{array}$} & $\begin{array}{l}\text { exkludierende } \\
\text { Exklusion } \\
\text { (barrierearm) }\end{array}$ & Exklusion \\
\hline & & \multicolumn{4}{|c|}{$\begin{array}{c}\text { Inklusion in die systemspezifische Kommunikation } \\
\text { eines anderen Funktionssystems }\end{array}$} \\
\hline & & $\begin{array}{r}\text { Qualifizier } \\
\text { program } \\
\text { Vertragsprog } \\
\end{array}$ & $\begin{array}{l}\text { Ings- } \\
\text { m } \\
\text { ramm }\end{array}$ & Vertragsprogramm & \\
\hline & & \multicolumn{2}{|c|}{$\begin{array}{l}\text { Marktleistungs- } \\
\text { programm }\end{array}$} & $\begin{array}{l}\text { Marktleistungs- } \\
\text { programm } \\
\text { Qualifizierungs- } \\
\text { programm }\end{array}$ & $\begin{array}{c}\text { Marktleistungsprogramm } \\
\text { Qualifizierungsprogramm } \\
\text { Vertragsprogramm }\end{array}$ \\
\hline
\end{tabular}

Abb. 6: Beschreibungsschema entlang von Mitgliedsbedingungen an die Leistungsrollen der Organisationen von Wirtschaft 


\subsubsection{Systemtheoretische Analyse auf der Organisationsebene: Determinierung in der}

Risiko-Relation von anerkannter Bildung und typischer Teilhabe

In den nachfolgenden Abbildung 7 und 8 sind graphisch die Ergebnisse entlang der entwickelten systemtheoretischen Beschreibungsschemata zu Mitgliedsbedingungen zwischen Bildungsund Arbeitsmärkten zusammengetragen.

An dieser Stelle wird auf eine umfassende wiederholende Darlegung über einen Fließtext bewusst verzichtet und es werden stattdessen lediglich einzelne Aspekte zusammengefasst, die unter der Perspektive der einleitenden These als zentral interpretiert werden. Kurzum, das Interesse richtet sich primär an eine Kongruenz zwischen den Mitgliedsbedingungen an soziale Adressaten, die jeweils in den inklusiven, inkludierend exklusiven, exkludierend inklusiven und exklusiven Organisationen angesprochen werden.

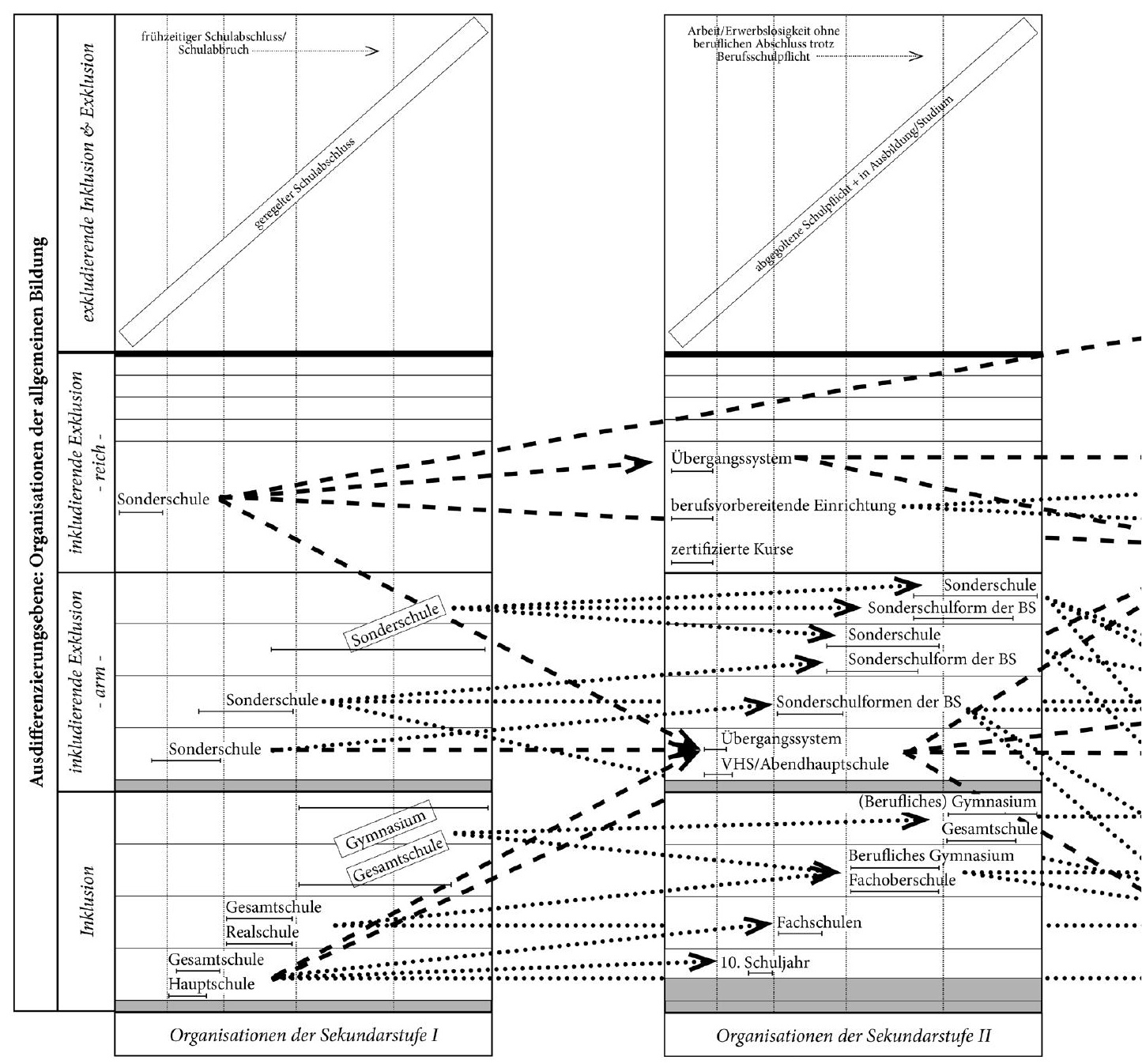

Abb. 7: Mitgliedsbedingungen an die Komplementärrollen der Organisationen von Bildung (1) 
Zwischen den inklusiven Institutionen der Sekundarstufe I und II kann demzufolge eine funktionale Kopplung primär via seiner sozial vermittelbaren Aussagen der Bewertungsprogramme zugeschrieben werden. Ein/e Schüler/in der gymnasialen Sekundarstufe II gilt als ordentlich beschulbarer, bewertbarer und betreubarer Adressat, wenn bestimmte schulische Outcomes in den Organisationen der Sekundarstufe I zugeschrieben wurden. Das notwendige Niveau der schulischen Outcomes wird zu einer Mitgliedsbedingung und verweist funktional auf Organisationen hin, die nach dem systemtheoretischen Beschreibungsschema sowohl zu den inklusiven bswp. gymnasiale Sekundarstufe I - als auch den barrierearmen exkludierend inklusiven - z. B. gymnasialer Zweig an Sonderschulen - gezählt werden können. Sie gelten alsdann für diese Institutionen als kommunikativ für die Unterrichtsprogramme der gymnasialen Sekundarstufe II anschlussfähig; und dies unabhängig der tatsächlichen Ausgestaltung des Unterrichts zuvor.

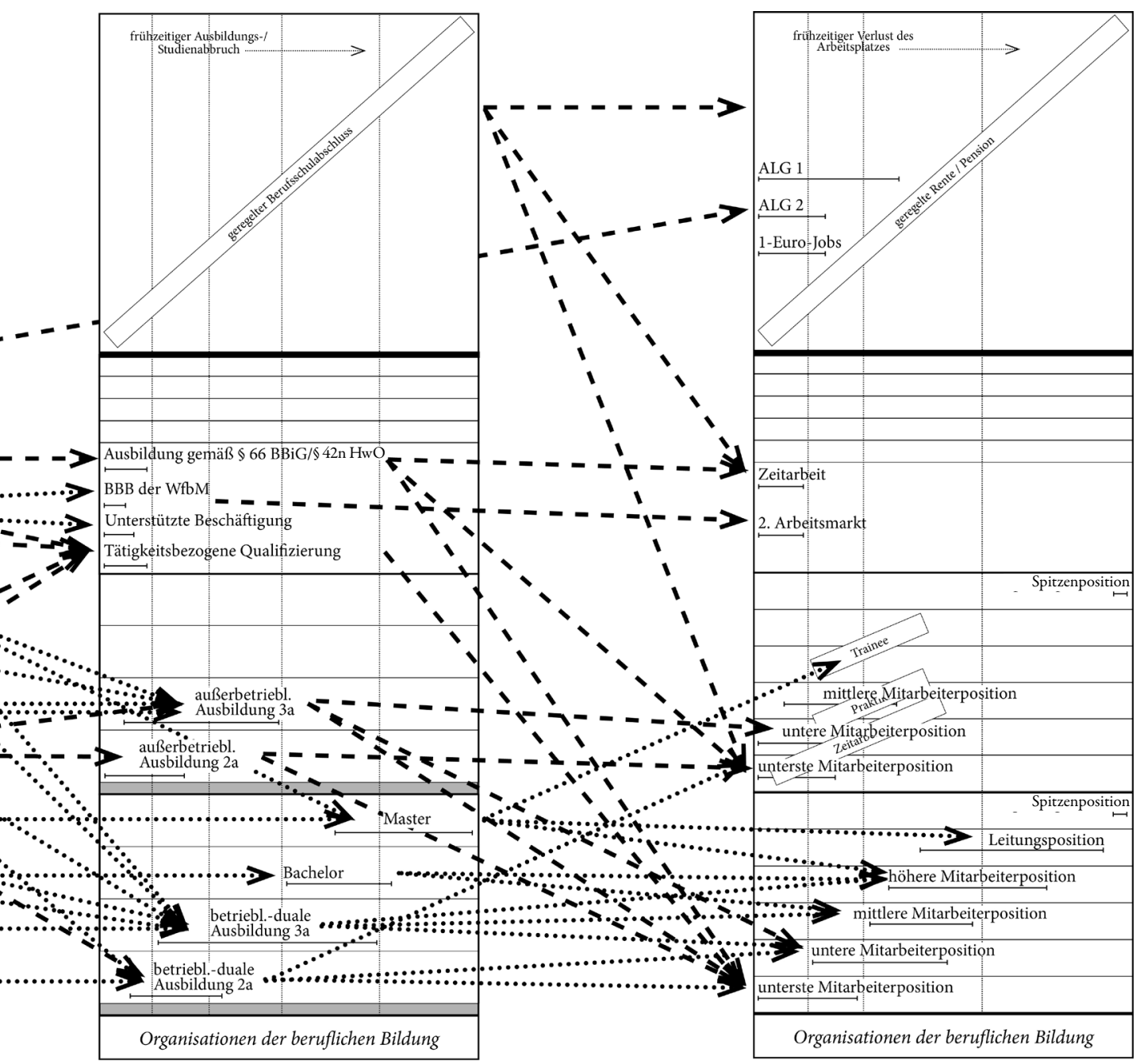

Abb. 8: Mitgliedsbedingungen an die Komplementärrollen der Organisationen von Bildung (2) 
Die in dieser ordentlichen Hierarchie niedrigeren Outcomes führen dagegen funktional zur Exklusion als ordentlich bewertbar und weiter als ordentlich betreubar.

Jene funktionale Kopplung über primär schulische Outcomes in Zertifikaten ist jedoch nur zwischen den inklusiven Organisationen der Sekundarstufe I und II funktional, respektive, sie markiert die dem Erziehungssystem zugeordnete Funktion in den Codierungen besser/schlechter und vermittelbar/nicht-vermittelbar. Daneben verdeutlicht sich, dass teilweise kein ausdifferenziertes Subsystem besteht, das eine Betreuungsberechtigung in einer inklusiven Organisation fortführt. So zeigt sich, dass die Adressaten mit einem der unterschiedlichen ordentlichen Abschlüsse der Sekundarstufe I im Zugang zur Sekundarstufe II über Werkrealschulen, Berufsfachschulen usw. mitgedacht werden, aber diejenigen Adressaten ohne jenen ordentlichen Abschluss jedoch nicht. Es gibt - mit Ausnahmen weniger Bundesländer - keine inklusive Organisation von Erziehung, die Personen ohne einen Hauptschulabschluss/Ersten allgemeinbildenden Schulabschluss (ESA) als Adressaten Schüler/in mitdenken. Eindrücklich zeichnet sich diese Dichotomie auch bei den Schüler/innen in Bildungsgängen ab dem Niveau Hauptschulabschluss/ESA ab, welche ihre Betreuungsberechtigung verlieren: Sie verlieren entgegen von Schüler/innen in Bildungsgängen oberhalb dieses Niveaus zugleich die Betreuungsberechtigung in den inklusiven Organisationen als ordentlich beschulbar, bewertbar und betreubar. Die Reproduktion der Ungleichen verstärkt sich folglich und prägnant in der funktionalen Ausgrenzung von Adressaten ohne Hauptschulabschluss/ESA, darunter sowohl für die zuvor inklusiv oder inkludierend exklusiv als beschulbar, bewertbar und betreubar mitgedachten. Das heißt jedoch nicht, dass diese Schulabsolventen auf der Organisationsebene vollständig exkludiert werden würden. An deren Stellen treten u. a. die Organisationen der inkludierenden Exklusion. Institutionell repräsentiert, handelt es sich hierbei um die Organisationen von Bildung, welche u. a. Angebote des Übergangssystems wie das Berufsvorbereitungsjahr oder auch alternativ um Abendhauptschulen, Fernschulen und Volkshochschulkurse prägen. Diese Organisationen der exkludierend inklusiven Sekundarstufe II manipulieren überwiegend und einschneidend das ordentliche Beschulungsprogramm. In mehreren Bundesländern werden über diese Angebote zwar die Berufsschulpflicht über ordentliche Zeiten von Unterricht abgegolten, aber eben nicht an ordentlichen Orten für Unterricht - z.B. in Werkstattklassen - und/oder von nicht ordentlichen Professionen der Bildung: An den Schulen der Übergangsmaßnahmen unterrichtet weder eine spezifische Lehrer/in-Profession wie dies für Primar-, Sekundar- und selbst Sonderschulen über eigene Studiengänge und Vorbereitungsdienste/Referendariate geregelt ist und teils überhaupt kein/e Lehrer/in. Die systemimmanente Adressierung von hierarchisierbaren Ungleichen durch Ausschluss der Betreuungsberechtigung in inklusiven Organisationen der Sekundarstufe II pointiert sich folglich innerhalb reiner Bildungsmärkte an den Absolventen/ innen ohne Hauptschulabschluss/ESA.

Vergleichbar zur Organisation von Erziehung auf der Sekundarstufe I und II entstanden in der beruflichen Bildung neben Subsystemen einige Supplemente der inkludierenden Exklusion. Diese reichen einerseits von den Organisationen betrieblicher und andererseits außerbetrieblicher anerkannter Berufsausbildungen bis nicht-anerkannten Qualifizierungen - z. B. im Bilde der Sonderausbildungsregelungen für Menschen mit Behinderung, der beruflichen Qualifizierung im Berufsbildungsbereich der Werkstätten bzw. der Unterstützten Beschäftigung. Eine Hierarchisierung in arm/reich innerhalb der inkludierend exklusiven beruflichen Bildung setzt sich ebenso fort: Die Adressaten, die zuvor von den inklusiven oder inkludierend exklusiven Organisationen der Bildung teils als nicht (mehr) ordentlich beruflich beschulbar mitgedacht wurden, können nicht per se auf der Organisationsebene von Arbeit als exkludiert betrachtet 
werden. Teils finden sie sich auch als ordentlich beruflich beschulbare Auszubildende wieder; und dies auch ohne Hauptschulabschluss/ESA.

Inkludierend exklusive Ausschlüsse finden sich folglich gleichsam in den Organisationen der beruflichen Bildung, jedoch mit einer Besonderheit: Eine Person, die nicht durch eines der bundeseinheitlich geregelten Rahmenlehrpläne auf Dauer als bewertbar und betreubar angesprochen werden kann, auch nicht durch z. B. einen Wechsel des Betriebs, wird sodann von der Betreuung ohne inklusive Alternative funktional ausgeschlossen. Es handelt sich um eine Besonderheit, die mit dem Beschulungsprogramm der inklusiven betrieblichen Bildung einhergeht. Während in rein schulischen allgemeinen bzw. beruflichen Bildungsmärkten primär entlang der Bewertungsprogramme funktional entschieden wird, welche Adressaten als Mitglieder angesprochen werden, wirkt hier durch die organisatorische Kopplung primär die Systemspezifik der Organisationen des Wirtschaftssystems, die entscheidet, wer über die Mitgliedsbedingungen als ordentlicher Auszubildender beschulbar gilt. Darüber hinaus werden die Bewertungs- und Unterrichtsprogramme parallel und voneinander getrennt praktiziert. So gibt es nicht wenige Fälle an Auszubildenden, denen aus der Systemlogik der Organisationen der Wirtschaft äußerst ansprechende Leistungen attestiert werden und im Gegensatz dazu durch die Organisationen der Bildung die Adressierung nicht-vermittelbar gepaart mit schlechter zugeschrieben bekommen. Es verdeutlicht sich, dass mit dem standardisierten Bewertungsprogramm ein funktionales Kriterium innerhalb der Mitgliedsbedingungen an die Schüler/innen der - und auch nur der inklusiven Organisationen herausgearbeitet wurde, das innerhalb der Mitgliedsbedingungen an Auszubildende und weiter an Beschäftige entlang des Qualifikationsprogramms höchstens als kontingent zu interpretieren ist. Anders ist nicht zu erklären, warum Personen, die zuvor aus den inklusiven Organisationen der Bildung inkludierend exkludiert wurden, nun als ordentliche Auszubildende und/oder als ordentliche Berufstätige wiederzufinden sind.

Zweifelsohne irritiert das Erziehungssystem maßgeblich das Wirtschaftssystem hin zu einer Übernahme ihrer sozial vermittelbaren Zeugnisse, gleichzeitig muss, ob es gefällt oder nicht, die Irritation bidirektional gefasst werden: Human capital zu haben/nicht-haben vollzieht sich bereits an jedem institutionellen inklusiven Übergang zwischen Primarstufe, Sekundarstufe I/ II, beruflicher Bildung/Qualifizierung bis hin zum Einstieg in die Arbeitsmärkte, wenn auch unterschiedlich im Zuge der spezifischen Funktionslogiken beider Systeme. Oder in anderen Worten, mit der Adressierung durch schulische Zertifikate liegen je unterschiedliche Quantitäten an human capital vor, die auf virtuellen (Aus-)Bildungs- und Arbeitsmärkte "gehandelt“ werden können. Je nach Markt moderieren diese Quantitäten über Haben/Nicht-Haben den Zugang, eingesetzt versprechen sie, vergleichbar den virtuellen Handelsmärkten des Wirtschaftssystems, jedoch keine Garantie einer Rendite (Bellmann/Priddat 2006). Einzelne Märkte „akzeptieren“ ordentliche schulische bzw. berufliche formale Abschlüsse mehr, andere weniger, andere Märkte gleichzeitig aber auch nicht-anerkannte Abschlüsse, Zertifikate und informelle Bescheinigungen über Netzwerke (Flap/Boxman 2001). Findet sich die Kommunikationsantizipation anerkannter Berufs- und Hochschulausbildungen in geschlossene Beschäftigungsverhältnisse eher in formal höher qualifizierten Arbeitsmärkten, ist dies Ausdruck jener „Akzeptanz“, aber nicht der Garantie der Rendite. Hier hat die Kommunikation der inklusiven Organisationen der Erziehung "gelernt“, dass die Kommunikation der inklusiven Organisationen der Wirtschaft am ehesten ihre Mitteilungen annimmt - und vice versa. In diesem Sinne ist auch einzuordnen, dass die Hierarchie der Schulabschlüsse der Organisationen des Erziehungssystems von den Organisationen des Wirtschaftssystems nicht monokausal identisch fortgeführt wird, wie sich dies zwischen Organisationen des Erziehungssystems beobachten lässt. 
Diese Inkongruenzen manifestieren sich sowohl im Vergleich der grundständig gegenüber den nicht grundständig erworbenen Schulabschlüssen, als auch zwischen betrieblichen und außerbetrieblichen Ausbildungsabschlüssen (Hupka-Brunner et al. 2011; Autorengruppe Bildungsberichterstattung 2008).

Wenn die einleitend zur These pointierte Risiko-Wahrscheinlichkeit innerhalb der Relation von Bildung und Teilhabe in Arbeit aufgelöst werden soll, kann diese aus der vorliegenden systemtheoretischen Analyse am ehesten in den „Gesetzen von haben/nicht-haben der sozialen Märkte" verortet werden: Lediglich zwischen reinen und überdies inklusiven Bildungsmärkten konnte die postulierte funktionale Determination aus Bewertungs- und Unterrichtsprogramm herausgearbeitet werden. Zugleich repräsentiert werden solche „Gesetze bestimmter sozialer Märkte“: Hin zu hierarchiehöheren im Vergleich zu hierachieniedrigeren Organisationen der Sekundarstufe I erfährt man, aus dem Unterrichtsprogramm abgleitet, das betreubar/nicht-betreubar in den Mitgliedsbedingung eine gleichsam höhere Relevanz, jedoch nicht von Organisationen der Sekundarstufe II hin zu denen der akademischen Hochschulbildung. Für die unreinen (Aus-)Bildungs- und Arbeitsmärkte ist in erster Linie abzuleiten, dass diese je nach Markt unterschiedlich zu fassen sind und daher auch nach ihren eigenen "Gesetzen“ von haben/nicht-haben zu beschreiben sind. Einzelne "Gesetze“ weisen auf eine dominantere "Akzeptanz" des Habens von sozial vermittelbarer schulischer Bildung hin; in diesen Fällen zumeist von einem hohen Grad dieses human capitals - z. B. in höheren, aber nicht Führungs-Positionen. Andere gestalten sich dagegen derart, dass Arbeitszeugnisse oder die informelle Auskunft über die Passung eines Adressaten die dominante „Währung“ darstellen. Aus dem Prinzip des Selbsterhalts der Systeme zu erklären, knüpft die Kommunikation der ordentlichen Organisationen der Erziehung letztlich an die Organisationen von Wirtschaft an und umgekehrt, in denen sie am ehesten Adressibilität antizipieren kann, oder in anderen Worten, in denen sie am ehesten Rendite versprechen können. Auf diese Weise kann zugleich erklärt werden, warum statistisch ein Hauptschulabschluss/ESA die Chancen für einen Ausbildungsplatz innerhalb anerkannter dualer Berufsausbildungen teils erhöht, teils senkt und teils verhindert. Des Weiteren erscheint das Ergebnis des benannten zugangsbestimmenden Wirtschaftssystems für die beruflich Beschulbaren hochgradig anschlussfähig an einzelne Arbeitsmärkte zu sein: Wer in einzelnen Berufsfeldern seine betrieblich-duale Ausbildung besteht, hat im Vergleich zu allen anderen beruflichen Ausbildungswegen die höchsten Chancen als junger Erwachsener im Anschluss eine Beschäftigung im geschlossenen Beschäftigungssystem zu erhalten (Kock 2008).

Eine Konsequenz dieser Betrachtung wäre, dass die Relation zwischen Bildung und Teilhabe in Arbeit maßgeblich durch das Programm der Knappheiten zu denken wäre; spezifisch je System. Auf der einen Seite verdeutlicht sich Knappheit eindrücklich in den Innovationen von Arten der Beschäftigung, die eher mit einer übergeordnet anteiligen Abnahme von „Normalarbeitsverhältnissen“, bzw., „typischer“ Teilhabe in Arbeit einhergehen; dies auch während vermeintlich sehr guten ökonomischen Bedingungen. Auf der anderen Seite vollziehen sich Innovationen in der ordentlichen Ausdifferenzierung im Erziehungssystem in erster Linie in den Organisationen mit Programmen spezialisierten Wissens und in einem deutlich geringen Maße, wenn nicht gar gegensätzlichem Sinne entlang grundbildenden Wissens. Beobachtungen reihen sich unproblematisch ein, denn institutionelle Zusammenlegungen im Zuge der schulischen Inklusion oder von der Hierarchie niedrigen Sekundarstufen I in Stadtteil-/Gemeinschaftsschulen spiegeln diese Tendenz zur Innovation wider. Gleiches gilt für die in der Anzahl nur spärlichen Weiterentwicklung von „anerkannten“ 
Berufsausbildungsgängen unterhalb der dreijährigen Vollausbildungen. Im Gegensatz dazu differenzieren sich organisatorisch die hierarchiehöhere Sekundarstufe II mit immer neuen Schwerpunkten oder die akademische Berufsbildung durch Neuschöpfungen an dualen Studiengängen immer weiter aus. Die zunehmend eine, gegenwärtig noch ordentliche Sekundarstufenschule unterhalb der Gymnasien droht konsequenterweise, sich mit der Zeit zu einer Organisation der exkludierenden Inklusion - Stichwort „Rest-Schule“ - zu verwandeln. Diese Innovationstendenz weitergedacht, wäre die Auflösung eines Bildungsgangs mit dem Ziel Hauptschulabschluss/ESA eine logische Folge, sowie damit einhergehend das Dilemma der Aberkennung seines gegenwärtig noch anerkannten Schulabschlusses. Analog zeichnen sich, wie bereits angedeutet, erste Indizien der Tendenz hin zu einer „Rest-Berufsbildung“ von gegenwärtig noch ordentlichen dualen Berufsausbildungen unterhalb von akademischen Berufsausbildungen $\mathrm{ab}$.

Im Sinne eines Zwischenfazits kann systemtheoretisch aus den Informationen zur einleitenden These folgende Mitteilung dechiffriert werden: Nur hierarchiehöhere formelle schulische Abschlüsse eröffnen einen Zugang zu hoch-qualifizierten formellen beruflichen Bildungsabschlüssen. Nur hoch-qualifizierte Arbeitsplätze wahren am ehesten vor Arbeitslosigkeit im Spiegel geschlossener Beschäftigungsverhältnisse.

\subsubsection{Systemtheoretische Analyse auf der Organisationsebene: Determinierung in der Risiko-Relation von Lesekompetenz und typischer Teilhabe}

Wird aus dieser Information eine vermeintlich determinierende Risiko-Relation abgeleitet, so werden Personen nicht mitgedacht, bzw. exkludiert, die weder in hoch-qualifizierter formeller schulischer und beruflicher Bildung in der Komplementärrolle noch in hoch-qualifizierter Arbeit in der Leistungsträgerrolle angesprochen werden. Dieses Nicht-Mitdenken manifestiert sich daran, dass die sozial vermittelbaren Outputs Personen entlang des Leitmediums Bildungsstandards bzw. funktionale Grundbildungskompetenzen als nicht weiter ordentlich beschulbar antizipiert werden. Insbesondere manifestiert es sich bei denjenigen Personen, an den sämtliche Stufen jener funktionalen Grundbildungskompetenzen, so auch von Lesekompetenzen als Teil einer sozial vermittelbaren Handlungsfähigkeit: Keine Stufe ermöglicht es, irgendwelche kognitiven competencies zuzuschreiben. Diejenigen, die dieses Level nicht „erreichen“, formieren folglich funktional die von den Bewertungs- und Unterrichtsprogrammen nicht-mitbedachten Adressaten - ungleich z. B. des Ortes von Erziehung. Das es sich hierbei nur um eine sozial vermittelbare Handlungsfähigkeit zwischen reinen Bildungsmärkten handelt, wird deutlich, wenn eine Person im Übergang in eine anerkannte berufliche Ausbildung im Zuge der Feststellung einer Ausbildungsreife eingeschätzt wird: Innerhalb dieser Mitgliedsbedingung an Auszubildende wird keine Handlungsfähigkeit im schulischen Sinne entlang von funktionalen kognitiven Grundbildungskompetenzen herangezogen, sondern entlang des Konzepts von beruflicher Handlungsfähigkeit. Übertragen auf Lesekompetenzen wird keine psychometrisch messbare universelle Handlungsfähigkeit mit oder entlang von Texten mehr beansprucht, sondern kategorial eine Methodenkompetenz, welche in der Performanz innerhalb von Berufsfeld unabhängigen Situationen von Relevanz ist. Dies führt dazu, dass eine Person einerseits keine Lesecompetencies sozial vermittelbar zugeschrieben bekommen kann und anderseits gleichzeitig durchaus Lesekompetenzen. Funktionale Grundbildungskompetenzen und berufliche Handlungskompetenzen sind weder konzeptionell noch inhaltlich abgestimmt. Insofern das Mindestlevel ersteres weitaus höher ansetzt, justiert sich 
der Blick auf die Relevanz des Lesens als Handlungsfähigkeit, welche mit einer ,befriedigenden Lebensführung' in der einen Gesellschaft unmittelbar verknüpft ist.

Angesichts des immer weiter verhärteten Widerspruches in der Prägnanz und Relevanz von funktionalen Grundbildungskompetenzen als universell gültiges symbolisches Zuschreibungskriterium von wahrscheinlichen Teilhabechancen muss dieses Kriterium gar über eine fehlende externe Validitätsbestätigung hinaus als gewisse Adressatengruppen funktional exkludierend enttarnt werden. Mit Verweis auf die Schweizer TREE-Studie (Hupka-Brunner et al. 2011; Buchholz et al. 2012) kann eine Einschätzung jener externen Validität der Risiko-Relation zwischen Lesecompetencies der formalen Grundbildungskompetenzen und Teilhabe in Arbeit zwischen unreinen Bildungs- und Arbeitsmärkten vorgenommen werden. Die Ergebnisse müssen jedoch unter dem Vorbehalt gelesen werden, dass sie nur eingeschränkt auf Deutschland übertragbar sind, da sie auf nur bedingt ähnlichen Ausbildungs- und Arbeitsmarktbedingungen beruhen (vgl. Basendowski 2013): Mit der Studie konnte nur die Wahrscheinlichkeit der Einmündung in „höher qualifizierte“ Ausbildungen vier Jahre nach der obligatorischen Schulzeit über Lesecompetencies statistisch signifikant vorhergesagt werden. Dieser gar hoch signifikante Effekt löst sich allerdings auf, wenn der Faktor einer ausgeprägten regionalen Dichte an klein- und mittelständigen Ausbildungsbetrieben ergänzt wird. Über die Wahrscheinlichkeit für den Zugang zu einer Ausbildung unterhalb dieses Levels bis gar alternativ in Arbeit ohne Ausbildung erlaubt das Konzept der funktionalen Grundbildungskompetenzen des Lesens letztlich keine wissenschaftlich soliden Aussagen. Diese Schlussfolgerung deckt sich mit dem untersuchten Prädiktor funktionaler Grundbildungsbildungskompetenzen unter Beschäftigten unterschiedlicher Qualifizierungsniveaus im Rahmen der PIAAC-Studie (OECD 2013): Den Ergebnissen zufolge lassen Lese- und mathematische Kompetenzen lediglich seriöse Vorhersagen für „hochqualifizierte“ vs. „einfachen“ und „qualifizierten Arbeiter-“Berufen zu, aber nicht zwischen den beiden letztgenannten Niveaus. Solange nur für Personen ab höher-qualifzierten unreiner Bildungs- und Arbeitsmärkte bislang eine Handlungsfähigkeit ausgemacht werden konnte, hier im Sinne eines sozial vermittelbaren Zuschreibungskriteriums, welches wiederum eine sozial vermittelbare Zuschreibung von Teilhabechancen in Arbeit empirisch solide ermöglicht, unterliegt dieses Konzept der Handlungsfähigkeit eindeutig dem Verdacht der Exklusion von bestimmten Adressatengruppen aus ihren Bewertungs- und Unterrichtsprogrammen.

Aus der Erforschung von Einstellungsvoraussetzungen an Einfach(st)arbeitsplätze (Basendowski 2013, 232ff.) lässt sich ein weiteres symbolisches Zuschreibungskriterium ableiten, welches gerade nicht an Zertifikate des Bildungssystems als Vorschussaussage über die Passung eines/r Bewerbers/in anschließt. An deren Stelle tritt das erfolgreiche Bewältigen von Anforderungen in beruflichen Handlungssituationen on-the-job. Hierunter zählen auch Situationen, in denen Zeichensysteme integriert sind. Ob im Rahmen von Praktika, welche einer Anstellung vorausgehen oder innerhalb der ersten Wochen der Probezeit rückt vielmehr ein „kompetentes“ Lesen von typischen Texten, eingebettet in berufliche Anforderungsbedingungen an Einfach(st) erwerbstätigkeiten in den Mittelpunkt. Vor diesem Hintergrund werden im Weiteren diverse Konzepte von Lesekompetenzen dahingehend befragt, inwiefern sie diese sozialen Realitäten an ein „kompetentes“ Lesen auch an Einfach(st)arbeitsplätzen dezidiert mitdenken. 


\section{Lesekompetenz nichtkontinuierlicher Texte}

„In der internationalen Bildungspolitik hat sich in den letzten zwei Jahrzehnten ein tiefgreifender Paradigmenwechsel vollzogen. [...] Da Wissensinhalte in einer globalisierten Wissensgesellschaft schnell veralten und der beschleunigte gesellschaftliche Wandel auch berufliche Qualifikationen rasch veralten lässt, heißt die Devise, die „Schule könne unter diesen Umständen nur noch Basiskompetenzen vermitteln“ (Garbe 2009, 19). Große Einigkeit in der wissenschaftlichen Diskussion herrscht überdies darüber, dass grundsätzlich Lesekompetenz(en) zu diesen Basiskompetenzen zählen, unabhängig, ob im Sinne von literacy, literacies, basic education oder Grundbildung.

\subsection{Lesekompetenz(en) - (funktionale) Kompetenz(en) des Lesens}

Jene Einigkeit umfasst jedoch nicht das, was (eine) Lesekompetenz(en) ist/sind und nicht ist/ sind und weiter, welche Lesekompetenz(en) Teil jener Basiskompetenzen ist/sind, oder nicht ist/ sind. Entlang einer linguistischen Betrachtung von „Lesekompetenz(en) als ein endozentrisches Determinativkompositum (vgl. Kapitel 3.2.7) empfiehlt sich die folgende Annäherung an diese Differenzen: Die zweite Konstituente Kompetenz/en bestimmt semantisch die erste Konstituente Lesen. Der Geltungsbereich von Lesen reduziert sich konsequenterweise auf die Teilklasse, die durch Kompetenz/en definiert ist. In das Zentrum der Betrachtung rückt folglich das zugrundeliegende Verständnis dessen, (a) was als Kompetenz(en) im Gestus eines Konglomerats gilt, das „über kognitive und nicht-kognitive Fähigkeiten sowie spezifisches Wissen zur Verwirklichung von Handlungszielen hinaus auch volitionale, motivationale und emotionale Elemente, sowie Wertorientierungen und soziale Einstellungen einschließt“" (Weinert 2001, 27f.). Oder in anderen Worten, wie das „Fähigkeitspotenzial [theoretisch fundiert wird], das einen Menschen in Bezug auf relevante Anforderungen und Ziele handlungsfähig macht“ (Hurrelmann 2010, 20f.). Erst in Abhängigkeit des jeweiligen handlungstheoretischen Verständnisses von Kompetenz(en) kann betrachtet werden, was alsdann Lesen ist - Lesebegriff -, einschließlich der Klasse von Zeichen, auf die sich dieses Lesen beziehen - Zeichenbegriff. Leitend für jene Auseinandersetzung mit den Handlungsfähigkeiten, -fertigkeiten und -bereitschaften des Gebrauchs von Texten ist die referierte Setzung, das mit dem Lesen als Basiskompetenz(en) die Befähigung zu einer befriedigenden, gegenwärtigen und zukünftigen Lebensführung in sowohl privaten, öffentlichen als auch beruflichen Kontexten in modernen Mediengesellschaften verknüpft wird (ebd., 20).

Es wird sich im Weiteren zeigen, dass sich die unterschiedlichen Begriffe zu Polen von Lesekompetenz(en) verschmelzen, die von einer explizierbaren Universalität bis hin zu einer Implizität geprägt sind. Insofern absolute Universalität beansprucht wird, so bezieht sich diese auf die Grundlegung als hierarchisierbare kognitive Fähigkeit im semiotischen und propositionalen Dekodieren, unabhängig sowohl des Textes als auch der Situation, in welcher der Text gelesen wird - handlungstheoretisch-kognitive competencies des Lesens als Informationsverarbeitung/Kulturtechnik. Jene kognitiven Fähigkeiten stellen Entitäten im Lesenden dar, die die Ausführung einer Handlung, in der das Lesen der Ausdruck einer ,befriedigenden Lebensführung ist, ermöglicht. Für die Auswahl der Texte gilt jedoch der Anspruch der Referenz zur Kultur und der Gesellschaft jeweils im Singular. Gleichzeitig wird eine Grundlage konstruiert, um neben Graden einer ,befriedigenden Lebensführung' eine untere Grenze dessen festlegen zu können, 
respektive, eine Dichotomie hin zu einer nicht mehr ,befriedigenden Lebensführung؛. Diese verweist auf bestimmte kognitive Fähigkeiten, festgehalten durch funktionale Grundbildungskompetenzen des Lesens, die alsdann eng mit der gegenwärtigen und insbesondere zukünftigen Befähigung zum Lernen durch die Informationsverarbeitung von, im Grunde kontinuierlichen, Texten verknüpft ist.

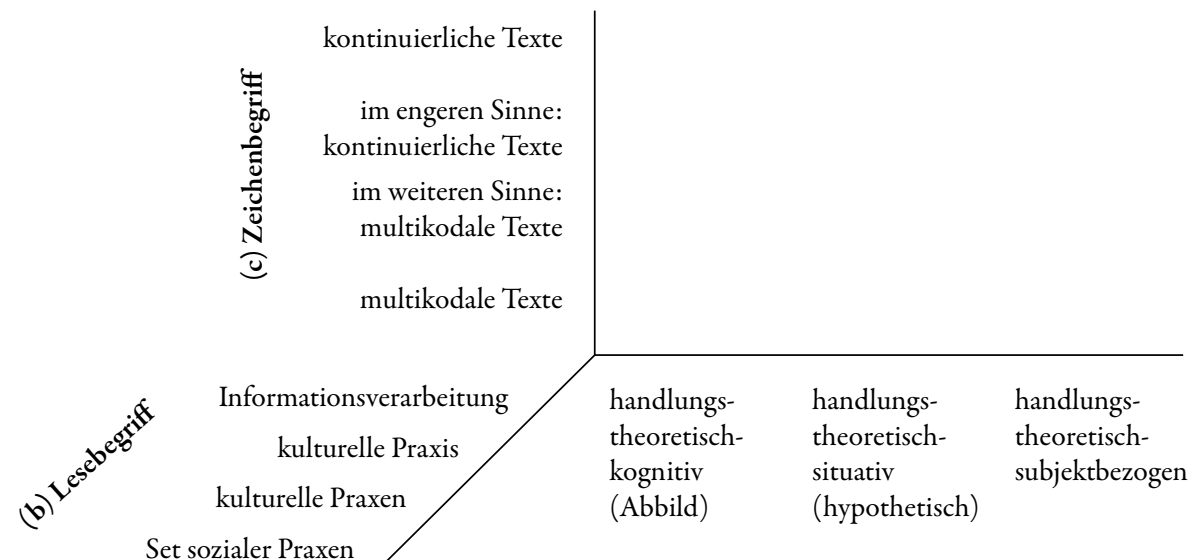

(a) Kompetenzbegriff

Abb. 9: Determinierung des textuellen Lesens im Spannungsfeld von Kompetenz/en

Dieser Ansatz aus der Verknüpfung eines bestimmten Lesebegriffes in der Geltungsreichweite des referentiellen Kompetenzbegriffes wird sich als nur bedingt geeignet auszeichnen, die textuellen Anforderungen an Einfach(st)erwerbstätigkeiten darzulegen, gleichermaßen wie die Kombination, welche die Ideen einer kollektiven Universalität verfolgen. Diese schließen an Texte in unterschiedlichen sozialen Kontexten an und rücken die Frage, was grundbildende Fähigkeiten, Fertigkeiten und Bereitschaften des Lesens darstellen in eine plurale Perspektive einer ,befriedigenden Lebensführung' - handlungstheoretisch-kognitive competencies des Lesens als kulturelle Praxen. Gemein ist, dass die Textwahl entgegen der einen Kultur der einen Gesellschaft das Nebeneinander entlang von Klassen annimmt. Diese Klassen rekurrieren entweder auf Kollektive hinter Schicht spezifischen Lebensweisen oder von Kollektiven entlang von Generationen. So ergeben sich für jede Schicht spezifizierbare Anforderungen an grundbildende Lesekompetenzen, ebenso wie für heranwachsende Generationen entgegen ihren Eltern usw. im Zuge der zunehmenden Technologisierung. Diese werden in Ausschnitte des Kontinuums von hierarchisierbaren kognitiven Fähigkeiten oberhalb einer Dichotomie konstruiert, welche über alle Klassen hinaus gilt.

Vom gegenüberliegenden Pol der Implizität ausgehend, stellt dagegen jegliche semiotische und propositionale Dekodierung eine durch Sinn geprägte Handlung einer Person dar. Abgeschwächte Nuancen jener Implizität spiegeln sich überdies in Ansätzen von „subjektiv“ relativierender universeller Lesekompetenzen wider - handlungstheoretisch-kognitive competencies in der Geltungsreichweite subjektiver Kompetenz des Lesens als kulturelle Praxis. Weniger die Setzung des Lesens als Teil eines normativen Verständnisses von befriedigender Lebensführung umfas- 
send und somit auch weniger die Relevanz von bestimmten Texten als Ausdruck der Kultur der Gesellschaft in Frage stellend, werden hier, nebst der kognitiven, weitere Dimensionen des Lesens in den Mittelpunkt gerückt: Die emotional-motivationalen und sozial-kommunikativen Dimensionen innerhalb der Handlungsfähigkeiten, -fertigkeiten und -bereitschaften des Gebrauchs von Texten werden jedoch letztlich als kaum beschreibbar verortet.

Die Explizierungdes Impliziten prägtgleichermaßen die letzte Linie der Lesekompetenzansätze, die der handlungstheoretisch-situativen Kompetenzen des Lesens als Set sozialer Praxen. Gleichermaßen wider die Reduktion von Lesen als rein kognitives semiotisches und propositionales Dekodieren richtet sich die Explizierung hier auf die „Regeln“ im Gebrauch von letztlich der Repräsentationsmächtigkeit von Zeichensystemen in bestimmten soziokulturell gerahmten und verketteten Situationen. Diese „Regeln“ rekurrieren nicht auf der normativen Idee, welche im Gebrauch eines bestimmten Texttyps einer bestimmten Textsorte eine wiederum bestimmte Funktion des Gebrauchs in der einen Gesellschaft zuordnen. Vielmehr konstituieren sich diese, teils durch mehr oder weniger sozial konventionalisierte Praxen oder in anderen Worten, nicht in dem vermeintlich universellen Gebrauch kategorialer Tabellen einer Gesellschaft, sondern bspw. in den Praxen der Dokumentation eines KleinbetragKaufvertrags (vgl. Abbildung 21) oder von geleisteten Arbeitszeiten (vgl. Abbildung 12). Jene Texte, respektive, „Spielweisen“ mit Zeichensystemen aus einzelnen Praxen in Arbeit heraus verortet, verfolgt dieser Lesekompetenzansatz die Idee, die hier inhärenten impliziten lokalen „Regeln“, dem knowing how auch zum Gebrauch von Zeichensystemen in miteinander soziokulturell verketteten Situationen zu explizieren, die für teilnehmende Personen von Bedeutung $\mathrm{zu}$ sein scheinen. Insbesondere in jener praxeologischen Justierung der Relation von einer weder universellen noch kollektiv-universellen ,befriedigenden Lebensführung' wird sich im Weiteren jener Lesekompetenzansatz des Lesens als Set sozialer-literaler Praxen im Vergleich als am ehesten kongruent herausstellen, um die tatsächlichen textuellen Anforderungen an Einfach(st)arbeitsplätze stimmig zu betrachten.

\subsubsection{Kompetenzbegriffe zur Annäherung an die Handlungsfähigkeiten, -fertigkeiten und -bereitschaften des Gebrauchs von Texten (a)}

An dieser Stelle soll auf eine detaillierte erziehungswissenschaftliche Dekomposition verschiedener Kompetenzbegriffe unterschiedlicher Disziplinen im Rahmen einer früheren Arbeit verwiesen werden (vgl. Basendowski 2013, 113ff.). Das Analyseergebnis leitet über zu einer Gegenüberstellung von Kompetenzbegriffen entlang dreier handlungstheoretischer Stränge, dem handlungstheoretisch-kognitiven von competencies, dem handlungstheoretisch-situativen von Kompetenzen und dem handlungstheoretisch-subjektbezogenen von Kompetenz (vgl. Tabelle 4). Demzufolge unterscheiden sich die funktional-deskriptiven Kompetenzen - z. B. in den beruflichen Handlungskompetenzen - von den funktional-messbaren competencies - z. B. in den Bildungsstandards für allgemeinbildende Schulen - eindrücklich darin, dass die beobachtbare Performanz bei Aufgaben nicht als direktes Abbild eines universellen Fähigkeitspotenzials interpretiert wird. Universell wäre es daher, da competencies alleinig über die kognitiven Dispositionen erfassbar sind: Unter der Setzung von kulturell einvernehmlichen sozialen, motivationalen und volitionalen Dispositionsräumen wird deren empirische Eliminierung begründet. Im Gegensatz dazu stellen Kompetenzen lediglich ein hypothetisches Konstrukt dar, auf welches über die Performanz in einer Situation im Sinne von "erfolgreiches Handeln erklären“ (Sloane 2007, 120) geschlossen werden kann. Sozio-kontextuell zu unterscheidenden Situationen verweisen auf mehr oder weniger dominante symbolische Orientierungssysteme hin. Diese 
moderieren entlang z. B. konventioneller Normen und Werte, welche Performanz erwartet wird und geben vor, welche kognitiven, motivationalen, volitionalen und sozialen Facetten von Kompetenzen als solche beurteilt werden. Innerhalb jener Akzentuierung rückt folglich die Analyse von symbolischen Orientierungssystemen, in welche die Situation einer Performanz verwoben ist, in den Mittelpunkt. Infolge bleiben Kompetenzen einerseits nach diesem handlungstheoretisch-situativen Ansatz zweifelsohne an kollektiven Erwartungen gebunden und sind folglich gleichermaßen als funktional zu bezeichnen. Andererseits können sie entgegen competencies konzeptionell berücksichtigen, dass sich das, was kompetentes Handeln ausmacht, in Situationen stark variieren, wenn nicht gar widersprechen kann.

Von diesen beiden Ansätzen unterscheidet sich der dritte in einer handlungstheoretisch-subjektbezogenen Grundlegung, welches einer pädagogisch-anthropologischen Tradition entlehnt, jegliche Kategorisierung und Einstufung negiert. Mit Kompetenz wird jenem Verständnis von Handlung Rechnung getragen, dass in jeder Performanz einer Person sowohl kognitiv als auch per se empraktisch eine Sinnhaftigkeit für ihn/sie zugrunde liegt. Als Handlungsfähigkeit verhärtet sich eine nur singulär zu positionierende Kompetenz als etwas orginär dem Menschen Innenliegendes und nie Explizierbares, welches lediglich im Sinne einer humanistischen Anwendung pädagogisch begrenzt wird.

Tab. 4: Kontrastierung von Kompetenzbegriffen entlang ihrer handlungstheoretischen Annahmen

\begin{tabular}{l}
\hline competencies \\
\hline handlungstheoretisch-kognitiv \\
fundiert \\
\hline Handeln als Folge von funktio- \\
nalem Wissen \\
\\
\hline psychometrisch messbar: \\
competencies als Abbild aus der \\
Performanz in Aufgaben \\
\hline universeller Kontext der Auf- \\
gaben infolge der Setzung von \\
sozial einvernehmlich konstru- \\
ierten motivationalen, volitiona- \\
len und sozialen Dispositionen \\
\hline Output-Orientierung: durch \\
die empirische Vergleichbarkeit \\
kognitiven Wissens
\end{tabular}

\begin{tabular}{l}
\hline Kompetenzen \\
\hline handlungstheoretisch-situativ \\
fundiert \\
\hline funktionales Wissen als Index \\
von Handeln
\end{tabular}

kategorisierbar: Kompetenzen als hypothetisches Konstrukt aus der Performanz in Situationen situationsspezifischer Kontext entlang dominanter symbolischer Orientierungssysteme (Soziokontextualität)

Input-Orientierung: durch soziokulturell gerahmten SollIst-Vergleich
Kompetenz
handlungstheoretisch-subjektiv fundiert
Handeln gerahmt durch pädagogisch-anthropologisch „rechtem" Gebrauch von Wissen und Erfahrung

Kompetenz implizit und nicht beschreibbar: Subjektivität in jeder Situation/Aufgabe

Quelle: modifizierte Darstellung nach Basendowski (2013, 113ff.)

\subsubsection{Lesebegriffe zur Annäherung an die Handlungsfähigkeiten, -fertigkeiten und -bereitschaften des Gebrauchs von Texten (b)}

Nach Hurrelmann $(2010)$ und Philipp $(2011,68)$ dominieren gegenwärtig zwei Modelle des Lesebegriffs die erziehungswissenschaftliche Diskussion auf der schulischen Ebene: das kognitionstheoretisch orientierte Modell und das kulturwissenschaftlich orientierte Modell. Ersteres 
interpretiert Lesen primär als Informationsverarbeitung, die einer zielorientierten und flexiblen Wissensaneignung dient. Es basiert auf international bewährten Ansätzen sowohl zur Textverarbeitung in der Psycholinguistik als auch auf empirisch-statistischen Dimensionen des Lesens. Hierbei wird eine prinzipielle Zweigliederung vorgenommen, durch welche textimmanente von wissensbasierten Verstehensleistungen unterschieden werden. Im ersten Fall sind demzufolge die im Text enthaltenen Informationen ausreichende Grundlage für das Verständnis (Hurrelmann 2010; 2002). Als statistisch bedeutsame Faktoren gelten hier z. B. die „kognitive Grundfähigkeit“ als eine Intelligenzvoraussetzung, die „Dekodierfähigkeit“ als die lesespezifische Fähigkeit die richtige Bedeutung des Textes schnell erfassen zu können oder das „Lernstrategiewissen“ als Zugang zum Lernen aus Texten (ebd., 9). Im zweiten Fall muss dagegen eine Aufgaben adäquate Interpretation unter Rückgriff auf ein nicht im Text enthaltenes Vorwissen geleistet werden. Das kognitionstheoretische Modell des Textverstehens umfasst üblicherweise diese beiden Subdimensionen (Hurrelmann 2010, 23). So sollen z. B. innerhalb von PISA mit den Teilkompetenzen „Informationen ermitteln“ und „Textbezogenes Interpretieren“ die textimmanente Informationsverarbeitung charakterisiert werden. Die dritte Subdimension „Reflektieren und Bewerten" kombiniert die textimmanente mit der wissensbasierten Informationsverarbeitung. Diese erfordert die Verknüpfung von im Text repräsentierten Informationen mit textexternem Wissen (Garbe 2009, 23). Jene drei Subdimensionen kennzeichnen universell sowohl das Lesen von unterschiedlichen Texttypen kontinuierlicher als auch nichtkontinuierlicher Texte in sowohl privaten, wie öffentlichen und beruflichen Kontexten (OECD 2002, 27ff.), weshalb der dahinter liegende Lesebegriff im Weiteren mit Lesen als Kulturtechnik bezeichnet wird:

- „Informationen ermitteln“: Informationen eines Sachverhalts, die mehr oder weniger tief in den Text oder Textkomplex eingebettet sind und mehr oder weniger klar differenzierbar innerhalb des Textes oder Textkomplexes lokalisierbar sind, sind zu identifizieren und zu dekodieren. Diese Informationen sind folglich dem Text oder Textkomplex inhärent und bedürfen nicht zwingend weder externes Faktenwissen noch einer tiefen Auseinandersetzung mit dem Text (= im Sinne der Herstellung „lokaler Kohärenz“).

- „Textbezogenes Interpretieren“: Zusammenhänge zwischen Sachverhalten (Begriffe, Konzepte usw.), die mehr oder weniger mehrdeutig im Text oder Textkomplex und mehr oder weniger differenziert und strukturiert repräsentiert werden, sind zu identifizieren, dekodieren und zu deuten. Diese Zusammenhänge sind folglich dem Text oder Textkomplex inhärent und bedürfen nicht zwingend externes Faktenwissen, jedoch einer mehr oder weniger tiefen Auseinandersetzung mit dem Text oder Textkomplex als Ganzes (= im Sinne der Herstellung "globaler Kohärenz").

- „Reflexion und Bewertung“ von Informationen eines mehr oder weniger umfangreich textuell repräsentierten Sachverhalts oder von Zusammenhängen zwischen mehr oder weniger umfangreich textuell repräsentierten Sachverhalten unter Rückgriff auf mehr oder weniger spezifisches externes Faktenwissen (= im Sinne der Herstellung „lokaler“ und „globaler Kohärenz“)

Jenem „dominant[en] kognitive[n] Lesebegriff in verschiedenen Leistungs-Studien“ (Garbe 2009, 30f.) steht ein kulturwissenschaftlicher Lesebegriff der Lesesozialisationsforschung gegenüber, der sowohl seinen deskriptiven Dimensionen, den Motiven für das Lesen, den Emotionen während dem Lesen und nicht zuletzt der Kommunikation im Anschluss an das Lesen neben den messbaren kognitiven Dimensionen ein hierarchiegleiches Gewicht beimisst. Nach Hurrelmann (2002) sind zur Teilnahme an der Lesekultur Fähigkeiten, Fertigkeiten und Bereitschaften in allen diesen Bereichen nötig, denn „erst in ihrer Gesamtheit gewährleisten sie das, 
was das Lesen auch im Medienzeitalter als zentrale Bedingung fur die Teilhabe an kultureller und gesellschaftlicher Praxis unverzichtbar macht“ (ebd., 14). Als „Moment realer kultureller Praxis" (ebd., 13) lässt sich daher das Lesen nur durch ein Nebeneinander dieser kognitiven, emotional-motivationalen und sozial-kommunikativen Facetten als sich gegenseitig beeinflussende, zeitlich parallel verlaufende und hierarchiegleiche Dimensionen des Lesens fassen. Gleichzeitig markiert jenes Nebeneinander den wohl zentralen Unterschied jenes Verständnisses im Begriff des Lesens als kulturelle Praxis zu dem des Lesens als Informationsentnahme/ Kulturtechnik. Gemeinsam ist beiden, dass sie durch einen Charakter einer normativen Leitidee geprägt sind, an welcher sich „pädagogische Prozesse der Bildung schriftsprachlicher Kommunikationsfähigkeit“ letztlich orientieren (ebd., 17).

Die skizzierten drei Dimensionen des Lesens im Lesebegriff als kulturelle Praxis sind insbesondere in dem Mehrebenenmodell basierend auf dem DFG-Schwerpunkt „Lesesozialisation in der Mediengesellschaft" und rezitiert durch Rosebrock/Nix (2008) einsichtig dargelegt: In ihm sind die kognitive Dimension über die Prozessebene des Lesens, die emotional-motivationale Dimension über die Subjektebene und die sozial-kommunikative Dimension über die soziale Ebene integriert. Innerhalb der Prozessebene werden die bereits thematisierten kognitiven textimmanenten und wissensbasierten Leistungen wieder aufgenommen und hierarchisch geordnet: Sie umfassen das Dekodieren von Graphemen bis zum Wort, welche über arbiträre symbolische Zeichen repräsentiert werden, einhergehend mit Konventionen zu festgelegten Relationen zu Phonemen, auch als Teil von Morphemen, des deutschen Schriftsystems. Darüber hinaus reicht die Prozessebene über eine mentale Textoberflächenrepräsentation hin zu mikropropositionalen Repräsentationen von Satzphrasen und deren Vernetzung bzw. Verdichtung zu makro-propositionalen Repräsentationen mehrere Sätze, Absätze und Texte. Infolge dessen lassen sich textstrukturell die repräsentierten Relationen eines/von Sachverhalt/en durch die Art und Tiefe von Interferenzen zwischen den Propositionen hierarchisch ordnen. Die Herstellung sowohl der lokalen als auch der globalen Kohärenz wird jedoch nicht ausschließlich als sequenzielle propositionale Extraktion der vorliegenden Propositionen verstanden, sondern als sich wechselseitig beeinflussende und interaktive bottom-up- und top-down-Prozesse, bei denen einem online-Charakter gleich metasprachliches Literatur- und Welt-Wissen textbasiert, aber auch intensional oder extensional mit einfließt - u.a. des Wortschatzes, von Superstrukturen wie z. B. von Schemata von Textgattungen, von Darstellungsstrategien wie z. B. Rhetorik und/oder von Erwartungen des Rezipienten an den Text. Über das Konzept der Leseflüssigkeit/ reading fluency wird innerhalb der Prozessebene u. a. eine angemessene Lesegeschwindigkeit und die ausdruckstarke, sinngemäße Betonung beim Lesen bedacht (ebd., 18ff., 38).

Das Selbstkonzept einer Person als (Nicht-)Leser/in macht nach dem Mehrebenenmodell eine weitere, eben hiercharchiegleiche Dimension des Lesens aus (ebd., 20ff.). Damit werden durch die Subjektebene u. a. motivationale Teilfacetten betont, wie z. B. die Bereitschaften, „die komplizierten und vielschichtigen Denkakte beim Lesen langwierig einzuüben“ (ebd., 21) oder diejenigen, Schwierigkeiten beim Textverstehen zu überwinden. Eine hohe Lesemotivation wird sodann mit der Mobilisierung positiver Erwartungen an die schriftliche Kommunikation verbunden (Hurrelmann 2009a, 30). Die Subjektebene umfasst darüber hinaus „prototypisch“ die Fähigkeiten, Texte bedürfnisentsprechend auszuwählen, Bezüge zu den eigenen Erfahrungen herzustellen, Gefühle mit dem Gelesenen zu verbinden als auch das ästhetische Wahrnehmen und Genießen (Hurrelmann 2009b, 277ff.). Darüber hinaus kann der subjektive Bezug zu einem Text durch das (Welt-)Wissen des/r Lesenden moderiert werden (Rosebrock/Nix 2008, 21). 


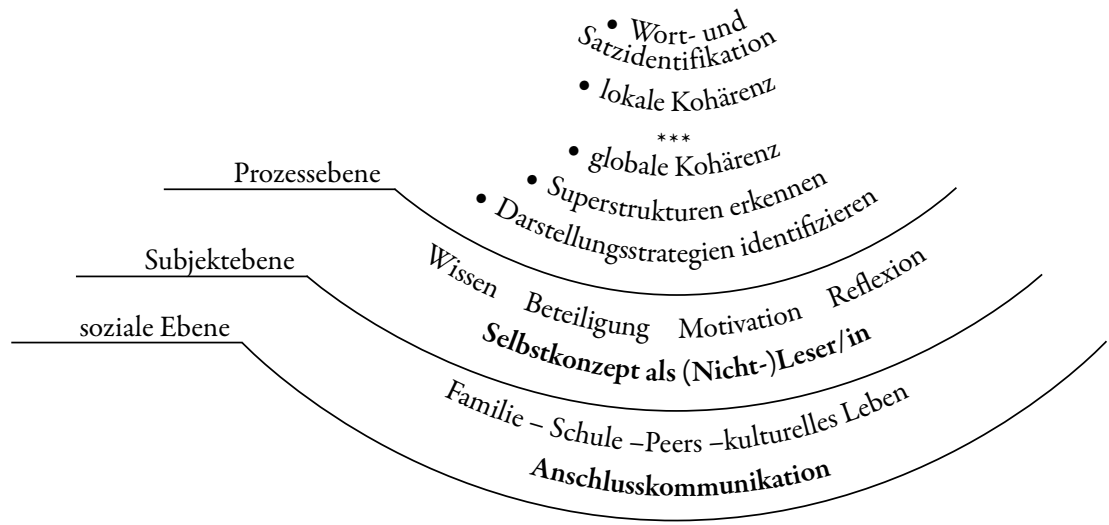

Abb. 10: Mehrebenenmodell des Lesens als Beispiel des Lesebegriffs als kulturelle Praxis (Quelle: Rosebrock/Nix 2008)

Die Subjektebene, die dritte Dimension des Mehrebenenmodells, rückt mit der Akzentuierung von Anschlusskommunikation eine interaktive bis hin zu einer partizipativen Funktion des Lesens in den Mittelpunkt (Hurrelmann 2002, 14). Dass die zeitliche und hierarchische Parallelität der Ebenen des Lesens nicht ausschließlich im Verhältnis zu den kognitiven Prozessen zu verstehen ist, verdeutlicht sich. Die Anschlusskommunikation bezieht sich weder ausschließlich auf z. B. die Rückmeldung zu korrekt dekodierten Wörtern beim lauten Vorlesen oder im Zuge von Leseverständnisaufgaben, noch schließt sie ausschließlich an die Sachverhalte an, die in dem Text repräsentiert wurden und zu „Reflektieren und Bewerten“ auffordern. Vielmehr kann sie den subjektiven Bezug zum Text und somit die Motive des Leseanlasses beeinflussen und so zu einer „Intensivierung des Textverstehens“ (Rosebrock/Nix 2008, 23) beitragen - gerne wird an dieser Stelle zur Illustration auf das Phänomen „Harry Potter“ verwiesen - oder dieser entgegenwirken. Überdies konstituiert sich die soziale Dimension in der Reflexion über das Gelesene im Austausch mit anderen: Diese schlägt sich im Aushandeln von Bedeutungskonsensen und -unterschieden, in der Toleranz bei unterschiedlichen Interpretationen sowie in der Selbstreflexion durch Rückbezug des Gelesenen auf die eigene Lebenssituation nieder (Hurrelmann 2009b, 277ff.). Übertragen auf Basis-Lesekompetenzen relativiert dieser Lesebegriff den Blick darauf, ob eine Person als grundlegend kompetente/r Leser/in eingeschätzt wird: Nicht ausschließlich von den kognitiven Facetten auch im Zuge mehr oder weniger komplexer Texte abhängig, sondern ebenso maßgeblich von den situativen Bedingungen, die dem Lesen jener Texte erst eine Relevanz geben; diese werden als nicht universell und zugleich nicht beliebig verstanden.

Kurzum, aus der Erweiterung der kognitiven, bzw. aus der Spezifizierung der emotional-motivationalen und sozial-kommunikativen Dimension des Lesens verdeutlichen sich nicht zuletzt die kaum haltbaren „universelle“ Dispositionsräume: Die Eliminierung dieser beiden Dimensionen aus einem Lesebegriff negiert bspw. die Vielfalt an Erwartungshaltungen an einen persönlichen Gewinn zu einem Text, welcher nicht auf Wissen, auch nicht auf reflektiertes und bewertetes Wissen, reduziert werden kann. Diese zeigen sich im schulischen Kontext letztlich 
an der Auseinandersetzung mit bestimmten kontinuierlichen Texten des „Literaturunterrichts“ (Hurrelmann 2002, 8f.). Darüber hinaus liefern die zunehmend in das Interesse der Lesesozialisationsforschung rückenden biographischen Bezüge von Schüler/innen unterschiedliche Motive für das Lesen und manifestieren weiterführend die bereits angerissene Relevanz von Facetten der Lebenssituation der/des Lesenden ${ }^{8}$. Entsprechende Arbeiten von Volz (2005), Bertschi-Kaufmann (2004) oder Pieper et al. (2004) zeigen letztlich die Problematik bei einem Lesebegriff als universeller Kulturtechnik oder einer (singulären) kulturellen Praxis auf, die sich sowohl auf vermeintlich prototypische Situationen begrenzen - eben zuvorderst das Lesen in der Schule -, als auch entlang einer protonormalen Lesesozialisation von Kindern und Jugendlichen pädagogisch umrahmt werden. Wird die Perspektive auf das Lesen außerhalb der Schule gerichtet, so auch u. a. spezifisch von medial dargebotenen Texten an Computer bis Smartphone, können maßgebliche Inkongruenzen zu den zuvor skizzierten Konzeptionen aufgedeckt werden, die alle drei Dimensionen des Lesens umfassen. So weist z. B. Bertschi-Kaufmann (2010) auf folgende Erkenntnisse ihrer interessanten Befragung über SMS von Jugendlichen zu Lesegewohnheiten bis -konzepten hin: Das Lesen in der Freizeit vollzieht sich zu einem beträchtlichen Anteil entlang interaktiver Formen der Textverwendung - Kommunikation über social network Plattformen, Smartphone-Apps usw. Die Diskongruenzen manifestieren sich u.a. bei der Mehrheit der Jugendlichen, für die Lesen in der Freizeit infolge inkohärenter Texttypenreferenz nicht mit dem Lesen in der Schule zusammenzuführen ist. Ihnen fällt es schwer, das Lesen innerhalb jener multimedial interaktiven Formen überhaupt als Lesen zu erfassen, sowie dessen „Stellenwert“ als auch dessen „Funktionalität“ zu beschreiben (ebd., 351f.):

Mit ,richtigem ' Lesen kann unter diesen Voraussetzungen dann nur das Lesen von informativ oder ästhetisch gehaltvollen Texten gemeint sein - eine Einschränkung, welche den heranwachsenden Leserinnen und Lesern den Blick auf ihre eigene, tatsächliche Lesepraxis verstellt, zumindest diesen nicht freigibt (ebd., 351).

Neben diesen Referenzen zur motivational-emotionalen und sozial-kommunikativen Dimension des Lesens werden notwendige Neujustierungen auch der kognitiven angedeutet. Gemeinsamkeiten reduzieren sich auf „basale Fähigkeiten“, welche als Basis für sowohl die Praxis des Lesens in und für die Schule als auch für die Praxis des Lesens in neuen Medienwelten außerhalb der Freizeit bezeichnet werden können. Hierbei handelt es sich „um basale Lese- und Schreibkompetenzen [...], um die Fähigkeit und den Wunsch, in Textwelten Erfahrungen zu sammeln, Informationen zu finden und zu nutzen, Texte zu dechiffrieren“, die allesamt „Voraussetzung zur Teilhabe an der partizipatorischen Medienkultur“ sind (ebd., 346). Aus den referierten Erkenntnissen wird geschlussfolgert, dass die eine kulturelle Praxis des Lesens kaum haltbar ist: Der dahinter liegende alternative Lesebegriff wird im Weiteren durch die Bezeichnung Lesen als kulturelle Praxen markiert.

Zweifelsohne finden sich Überschneidungen im Lesen als kulturelle Praxen und im Lesen als kulturelle Praxis. Wenn, wie bereits dargelegt, das „handlungsfähige Subjekt“ mittels der Fähigkeiten, Fertigkeiten und Bereitschaften als teils explizit messbar und teils implizit beschreibbar definiert ist, die „erst in ihrer Gesamtheit gewährleisten [...], was das Lesen auch im Medienzeitalter als zentrale Bedingung für die Teilhabe an kultureller und gesellschaftlicher Praxis unverzichtbar macht" (ebd., 14), stellt sich die Frage, was diese kulturelle Praxis im Singular mit Verweis für die Teilhabe an der einen Gesellschaft im Singular sein kann? Verbirgt sich dahinter

8 Unterschiedliche Motive im und zur Förderung des Lesen finden sich gleichermaßen bei Lehrer/innen biographisch begründet (vgl. Scherf 2013). 
die Konzeption einer Schnittmenge zwischen der kulturellen Praxis X, Y und Z oder eher die Summe aus X, Y und Z? Umfassten alsdann die übernommenen Ausprägungen der kognitiven Dimension des Lesens jene Schnittmenge oder Summe? Aber auch: Verändern sich diese nur aufgrund des Aufkommens neuer Medien und somit der Veränderung des Gebrauchs von Schrift? Gibt es neben der kuturellen Praxis des Lesens in Bildungs- und Nicht-Bildungskontexten weitere Praxen des Lesens? Müssen diese kulturellen Praxen nicht noch differenzierter erfasst werden?

Es sind u. a. jene Fragen, die letztlich zu einem alternativen Lesebegriff geführt haben: zu dem des (grundbildenden) Lesens als Set sozialer Praxen. Dieser basiert auf den „New Literacy Studies“, einem primär in Großbritannien entwickeltem interdisziplinärer Ansatz bspw. der Linguistik, Sozialgeschichte, Psychologie und Kulturwissenschaften. In Deutschland findet er v. a. in Diskursen der Erwachsenenbildung Referenz, jedoch kaum im Kontext schulischer Bildung (Linde 2007, 96). Arbeiten zu „Multiple Literacies“ (Street 1991, 1993, 1999) und im Besonderen zu „Local Literacies“ (Barton/Hamilton, 1998), weiterentwickelt zu „Situated Literacies“ (Barton et al. 2000), (reinschauen Kapitel 2 zu literacy event und practices) kritisieren zuvorderst eine singuläre auf kognitive und technisch-funktionale Aspekte reduzierte Sichtweise (auch) auf das Lesen, respektive, die Verengung auf die „Konsequenzen von Lesen und Schreiben für individuelle, kognitive Prozesse“ sowie auf deren „funktionale[n] Wirksamkeit“ (Linde 2007, 95). Dem gegenüber steht das von Street entwickelte sogenannte ideologische Modell, in welchem (auch) das Lesen als kulturell geformte soziale Praxen verstanden wird und die Vielfalt literaler Praxen sowie deren „sozialen Beschaffenheit“ Berücksichtigung erfährt. Die in einzelne Differenzlinien, nach Ethnie oder sozialer Lage usw. gegliederte Forschung zu Lesegewohnheiten von Erwachsenen unterstreicht die sozio-kontextuellen Unterschiede in den Situationen, in denen gelesen wird, als auch in den Bedeutungen und Gebrauchsformen des Lesens. Diese Kritik umfasst somit auch die Negierung einer immanenten Funktion jedes Texttyps, ergo einer universellen Funktion aller narrativer, einer universellen Funktion aller appellativen, einer universellen Funktion aller expositorischen Texte usw. (vgl. Garbe 2009, 22). Konsequenterweise erweitert dieser Lesebegriff auch den als kulturelle Praxen/kulturelle Praxis, die in den Freizeittexten jene Gemeinsamkeit im Sinne einer Schnittmenge oder als Summe noch postulieren; denn als Kulturtechnik im Zuge der übergeordneten universellen Berücksichtigung innerhalb der kognitiven Subdimensionen allemal.

Zur Annäherung an das Lesen als Set sozialer Praxen erfolgt eine Trennung seiner Komponenten von „litercy practices“ und „literacy events“: Diese „literacy events“ sind die Orte der Produktion und Nutzung von semiotischen Systemen (Barton/Hamilton 2000, 8), darunter primär Texte, und sind als empirisch beobachtbar und explizierbar gekennzeichnet. Jeder „literacy event “ steht zugleich in Beziehung mit spezifischen, aber nie spezifizierbaren „literacy practices“:

We have adapted the term literacy events, derived from the sociolinguistic idea of speech events [...]. Shirley Brice Heath characterised a ,literacy event' as , any occasion in which a piece of writing is integral to the nature of the participants' interactions and their interpretative processes' (Heath 1982, S. 93). Brian Street has employed the phrase, literacy practices' (Street 1984, S. 2000) as a means of focusing on ,social practices and conceptions of reading and writing' and the models of literacy that participants bring to bear upon those events and that give meaning to them. Consideration of the relation between literacy events and literacy practices is a useful basis from which to engage in field research on literacy as a social issue (Tomlin et al. 2002, 1f.).

Hinter „literacy practices“ verbergen sich implizit in etwa sämtliche Werte, Einstellungen und soziale Beziehungen, die einen „literacy event“ situativ kontextualiseren. Durch die Akzentu- 
ierung auf „social literacy events“ erfährt diese Rahmung von „literacy practices“ eine gewisse, sicherlich pragmatische Explizierbarkeit; so sensu Linde (2008, 66, 73) zu der Beziehung zwischen beiden Komponenten:

Das beinhaltet das Bewusstsein über Literalität, Deutungen und Diskurse, d.h. Reflexionen über Literalität und deren Sinnhaftigkeit. Diese innerlichen Prozesse sind bei der Erforschung der Literalitätspraxen ebenso zu berücksichtigen wie die Tatsache, dass Praxen soziale Prozesse sind, durch die Menschen miteinander verbunden sind. Geteilte Erfahrungen und Erkenntnisse sind in Weltanschauungen und sozialen Identitäten repräsentiert. Die Praxen sind durch soziale Regeln geteilt, die den Gebrauch und die Verteilung von Text regulieren und bestimmen, wer Texte produziert und wer Zugang zu ihnen haben kann. [...] Verschiedene Institutionen unterstützen und strukturieren Aktivitäten besonderer Lebensbereiche wie Familie, Religion und Erziehung - allesamt soziale Institutionen mit unterschiedlichem Regulierungsgrad. Einige sind formaler strukturiert als andere; reguliert durch den Druck sozialer Konventionen und Einstellungen.

Mit der Komponente (social) „literacy event“ wird letztlich deutlich, dass die Fähigkeiten, Fertigkeiten und Bereitschaften jener Produktion oder Nutzung von semiotischen Zeichen auf der Mikro-Ebene, der Ebene der Individuen, zugunsten ihrer Betrachtung auf der Makro-Ebene, der Ebene der Gemeinschaft, zurückgestuft werden (Barton/Hamilton 2000, 7ff.). Darüber hinaus stellen sich weitere deutliche Unterschiede im Vergleich dieses Lesebegriffs zu dem als kulturelle Praxen, kulturelle Praxis und als Kulturtechnik heraus: Semantisch übertragen liegt eine je bestimmbare Kontingenz an diversen emotional-motivationalen und sozial-kommunikativen Anforderungen an einem Text in Abhängigkeit der diskursiven Struktur der Situation auf Seiten des/r Lesenden vor, in welchem der Text dargeboten wird. Ein weiterer auffälliger Unterschied bezieht sich auf die Integration einer bestimmten Kontingenz der Funktionen eines Textes, wiederum in Abhängigkeit des sozialen Kontextes und deren Rezeption durch den Leser/die Leserin. Gleichwohl gibt es eine gewisse Kontingenz an Texten, die für jede, teils mit den Lebensbereichen assoziierte (social) „literacy practice“, bzw. für die Teilhabe an jeder dieser Praxen von Bedeutung sind, jedoch einem Wandel unterliegen (können).

Diese Kontingenzen samt ihrer Wandelbarkeit im Zuge sozialer Dynamiken sind primär auf der Makro-Ebene zu verordnen, auch abzulesen an den Forschungsaktivitäten zu diesem pluralen Begriff von Lesen als Set sozialer Praxen: Diese orientieren sich bspw. an dem „how literacy is a communal resource utilized by families, by community groups, and by individuals“ (Barton/ Hamilton 2000, 5), oder an dem ,how they [people in various communities and walks of life] use their knowledge of reading and writing, to what tasks they apply it, and how they accomplish these tasks" (Scribner/Cole 2001, 197, zit. nach Linde 2008, 67). Hierbei stehen weniger die Abgrenzungen, sondern vielmehr die Überlappungen, so z. B. der diversen Funktionen der Nutzung von Texten, im Vordergrund. Es gilt zeitlich und räumlich begrenzt aufzuzeigen, welche Praxen in einzelnen Gemeinschaften „stärker, sichtbarer und einflussreicher" sind (Barton/ Hamilton 1998, 7) und wie sich die je historische (kollektive) Situiertheit von Praxen auch vor der Folie von Machtbeziehungen fortsetzen, so u. a. durch soziale Institutionen und „größere soziale Ideen“ (ebd., 7):

The growing entanglement of literacy with economic producitivity not only affects how reading and writing are learned and practiced. It also shapes the rationales for acquiring literacy, how it is understood, valued, and evaluated. When Calvin Lockett, for example, learned handwrititng in a rural school in the late-nineteenth century, having a ,fine hand' was associated with decorum, discipline, character, in other words, moral qualities that linked mass literacy with its original of assimilation and civic training. But in rail depots and Western Union offices of the early twentieth century, handwriting was redeployed 
and redefined as a production skill, valued now for clarity, efficiency, and especially speed. Some telegraphers even learned to write with both hands at the same time, so that they could decode a message coming over the wire while simultaneously ticking off boxes and entering data on the message forms! Of course, moral connotations still cling to handwriting as they do to other elements of literacy. But in the transformation from a moral imperative to a production imperative, what was ,good 'about literacy underwent shifts (Brandt 2009, xii).

Der Grad an Literalität wird oftmals als Indiz für Intelligenz aufgefasst. Obwohl diese Auffassung unter linguistischen, psychologischen und pädagogischen Gesichtspunkten kaum haltbar ist, ist sie dennoch stark verbreitet und beeinflusst die subjektive Bedeutung, die dem Beherrschen, vor allem aber dem Nicht-Beherrschen des Lesens und Schreibens beigemessen wird (Kamper 1997, 10 zit. nach Linde 2008, 61).

Sekundär können diese Kontingenzen auf der Mikro-Ebene verortet werden. Alsdann rückt eine Art Biographie der Beziehungen zwischen den (social) „literacy practices“ mit den „literacy events" einer Person, oder in anderen Worten, mit seiner/ihrer Geschichte aller Situationen der Produktion und Nutzung von semiotischen Zeichen im Spannungsfeld der skizzierten mal stärker, mal weniger dominanten sozialen Vorgaben auf der Makro-Ebene. Über jene Beziehungen, die das knowing how von Lesen erfassen, liefern die bisherigen Forschungsaktivitäten zu diesem Lesebegriff keine Aussagen über die kognitive Dimension des Lesens, weder auf der Makro- noch auf der Mikro-Ebene. Mit Verweis auf Street $(1995,149 ; 1993,9)$ lässt sich dieser Umstand dadurch aufklären, dass die kognitive Dimension (auch) des Lesens in diesem theoretischen Ansatz nicht grundsätzlich negiert wird, jedoch nicht zu deren Hauptinteresse zählt. Hier kann zuvorderst auf das aktuelle Forschungsprojekt lea - Literalitätsentwicklung von Arbeitskräften - verwiesen werden. Unter Verweis auf diverse kulturwissenschaftliche Untersuchungen zu unterschiedlichen Typen des textuellen Sprachgebrauchs wird eine Unterscheidung in eine „Basale Literalität der Unterschicht“, eine „Mainstream-Literalität der Mittelschicht“ und eine „Legitime Literalität der Oberschicht“ empfohlen (Grotlüschen 2011, 30). Zentrale Merkmale zur Differenzierung bilden bestimmte Texte und Funktionen des Gebrauchs von Texten. Für die „Basale Literalität“ ist demgemäß z. B. das auf unmittelbaren Nutzen im Alltag einzuordnende Lesen von verschriftlichten Repräsentationen mit einer überschaubaren Anzahl symbolischer kombiniert mit ikonischen Zeichen charakteristisch. Texte der Wettervorhersage oder zum Fernsehprogramm entsprechen dieser ersten Funktion. Eine zweite kann in einer ritualisierten Abgrenzung zu anderen gesellschaftlichen Schichten verortet werden (ebd., 30f.). Im Gegensatz dazu ist die „Mainstream-Literalität“ mit den folgenden realitätsbezogenen bis fiktionalen Textformaten verknüpft, die wiederum mittels Funktionen ihres Gebrauchs gekennzeichnet werden: „instrumentelle Gründe (Etiketten, Uhren etc)“, „nachrichtenbezogenen Gründe (Berichte, Nachrichten)“, „Erholungsgründe (Comics, Sportmagazine, Einladungen, Romane)“, „soziale Gründe (Briefe, Alumni-Magazin)“ oder „bestätigende Gründe (Steuerformular, Geburtsurkunde)“ (ebd., 31). Werden „kritische bzw. bildungsbezogene Gründe (Lesen mit dem Ziel, politische, ästhetische oder soziale Sachverhalte diskutieren zu können) [...] mit der akademischen Literalitätstradition verknüpf“ (ebd., 31f.), liegen dagegen Beispiele der „Legitimen Literalität“ vor. Werden jene drei Typen des textuellen Sprachgebrauchs im Spiegel des empirischen Messkonzepts von lea diskutiert, welches ausschließlich die kognitive Dimension (auch) des Lesens behandelt und in Alpha-Levels gegliedert überführt (vgl. Tabelle 5), justiert sich die Relevanz der damit verknüpften Auswahl an Texten und Funktionen dieser Texte. Einerseits werden entlang jener Dreier-Gliederung „multiple und situierte Handlungsweisen mit Schrift“ als nicht defizitär zu wertend gegenüber gestellt (ebd., 26ff.). Andererseits ,jedoch sind diese vor der Folie einer hierarchischen Gesellschaft auch ungleich verteilt, ungleich komplex und 
ungleich durchsetzugsstark“ (ebd., 34). Wird die Produktion und Nutzung von semiotischen Zeichen auf die Prozessebene verengt, so stellt die „Basale Literalität“ eine Referenz von basalen kognitiven Fähigkeiten und Fertigkeiten des Lesens für ,jedermann“ in dieser hierarchischen Gesellschaft dar. Inwiefern die Texte und die Funktionen ihres Gebrauchs, die der „Basalen Literalität“ zugeordnet wurden, jedoch mit den Alpha-Levels z. B. über die dahinterliegenden Items verknüpft sind, bleibt in der Darlegung der Studie unklar: Die als Testitems eingekleideten Texte verweisen zwar durchaus auf eine „alltägliche Nützlichkeit“, diese Nützlichkeit beruht jedoch auf keiner empirischen Analyse, respektive, deren Nachweis auf externe Validität mit der „Basalen Literalität" wird keine Bedeutung beigemessen. Kurzum, wenn hier kognitive Fähigkeiten und Fertigkeiten des Lesens benannt und hierarchisiert werden, so handelt es sich um universelle, unabhängig des Textes und unabhängig der Funktionen im Gebrauch des Textes. Die Nähe zum Lesebegriff als Informationsentnabme/Kulturtechnik verdeutlicht sich jedoch in ihrer Geltungsreichweite auf die in der Hierarchie niedrigsten in einem häufig postulierten Kontinuum von Fähigkeiten und Fertigkeiten des Lesens. Diese verschieben, respektive, differenzieren die Grenze der Dichotomie dessen aus, welche kognitiven Fähigkeiten und Fertigkeiten des Lesens im Sinne eines symbolischen knowing that als universell notwendige kognitive Fähigkeiten und Fertigkeiten des Lesens „vor der Folie einer hierarchischen Gesellschaft“"verstanden werden.

Tab. 5: Hierachisches Modell der kognitiven Anforderungen von Texten „Basaler Literalität“

\begin{tabular}{ll}
\hline Stufe 1 & $\begin{array}{l}\text { K1V2K, VKV; max. } 2 \text { Silben, gebräuchliche Grapheme (außer Q, X, Y), } \\
\text { 1-2-gliedrige Grapheme, gebräuchliche Wörter }\end{array}$ \\
\hline Stufe 2 & KKV, KVK KVK; KVKK bis max. 2 Silben, alle Grapheme, gebräuchliche Wörter (auch Q, X, Y) \\
\hline Stufe 3 & $\begin{array}{l}\text { KVK, VKV, KKV, KVK KVK-Wörter, max. } 3 \text { Silben, alle Grapheme, gebräuchliche Wörter, } \\
\text { einschließlich eingedeutschte Fremdwörter und gebräuchliche Anglizismen }\end{array}$ \\
\hline Stufe 4 & Wörter beliebiger Länge und Struktur, einschließlich eingedeutschte Fremdwörter und Anglizismen \\
\hline Stufe 5 & Wörter beliebiger Länge und Schwierigkeit, Fremdwörter außer Fachvokabular \\
\hline
\end{tabular}

(Quelle: Kretschmann 2011, 48)

\subsubsection{Die „Kultur“ der Lesebegriffe}

Als ein stark verkürztes Zwischenfazit der Gegenüberstellung kann pointiert werden, dass ein Begriff des Lesens zu kurz greift, wenn er nur auf seinen kognitiven, nur seinen motivationalemotionalen oder nur seinen sozial-kommunikativen Anteilen beruht. Konsequenterweise rücken u. a. die Folien von Kultur in den Mittelpunkt, welche jeweils maßgeblich die Trias von Dimensionen setzen. Im Weiteren erfolgt mit Reckwitz (2003; 2010) eine demgemäß sozialtheoretische Annäherung. Eine detaillierte Analyse und Einordnung der dahinterliegenden Begriffe von Kultur werden hierbei weder angestrebt noch postuliert.

Unter dieser Perspektive kann zum einen im Lesen als Informationsentnahme/Kulturtechnik und im Lesen als kulturelle Praxis im Vergleich zu den anderen beiden Begriffen die größte, unterschwellige Nähe zu dem normativen Verständnis von Kultur postuliert werden. Diese leitet sich unmittelbar aus der jeweils dargelegten formalistisch-universellen Funktion des Lesens ab: Gemäß der Debatte der Lesesozialisationsforschung zu dem Lesen als kulturelle Praxis verdeutlicht sich diese innerhalb der „Erweiterung der kognitiven Lese-Leistungsperspektive“ um die deskriptiven Dimensionen Motivation, Emotion, Reflexion und Anschlusskommunikation, mit welcher die dem Leseprozess vorausgehenden Bedingungen und nachfolgenden Wirkun- 
gen beachtet werden, als auch die normative Leitidee verortet wird, an welcher sich „pädagogische Prozesse der Bildung schriftsprachlicher Kommunikationsfähigkeit“ letztlich orientieren (Hurrelmann 2002, 17). Als oberste normative Leitidee gilt hierbei das „gesellschaftlich handlungsfähige Subjekt“, welches den Anforderungen unserer schriftbasierten Mediengesellschaft gewachsen ist und für dessen Umgang mit der Welt die Teilhabe an schriftsprachlicher Kommunikation große Bedeutung hat (vgl. Garbe 2009, 32f.). Wenn mit der normativen Kultur im Singular Kultur als eine menschliche Lebensform verstanden wird, respektive, „eine ausgezeichnete, letztlich für ,jedermann' erstrebenswerte Lebensform“ (Reckwitz 2010, 20), verhärten sich universelle Geltungsansprüche hinter dem Lesen als Informationsentnahme/Kulturtechnik bzw. als kulturelle Praxis. Diese normative Kultur ist dabei eine kollektive, d. h. einzelne Kollektive verfügen über keine eigene kollektive Form von Kultur, sondern ihnen lassen sich Grade der einen, hier als formalistisch-universell zu verstehenden, normativen Kultur zuschreiben:

Der Universalismus geht davon aus, dass [...] auf der Ebene der Gesellschaft und Kultur, den normativen, symbolischen und systemischen Ordnungen, allgemeine, für alle Menschen oder Lebensformen geltende Strukturen existieren. Ein Spezialfall des Universalismus ist der Formalismus: Ausgehend von der Unterscheidung zwischen Form und Inhalt, wird hier vorausgesetzt, dass auf der Ebene der ,Inhalte kulturelle Variationen existieren mögen, dass jedoch die ,formalen Strukturen', die diesen inhaltlichen Differenzen vorausliegen sollen, allgemeiner, kulturübergreifender Art sind (ebd., 29).

Dagegen weist der Begriff von Lesen als kulturelle Praxen unterschweillig die größte Nähe zu einem totalitätsorientierten Ansatz von Kultur auf (ebd., 32ff.), indem es unterschiedliche Funktionen von Kulturtechniken für einzelne Kollektive aufgliedert, und dies gebunden an einen geographischen Raum und an eine historische Tradition, hier z. B. an die Schicht des deutschen bzw. „westlichen“ „Industriearbeiters“ (Grotlüschen 2011,26) oder zwischen Generationen im Zuge der rasant zunehmenden Technologisierung von Kommunikation:

[Der totalitätsorientierte Kulturbegriff] entuniversalisiert das Kulturkonzept, er kontextualisiert und historisiert es. Kultur ist keine ausgezeichnete Lebensform mehr, Kulturen sind vielmehr spezifische Lebensweisen einzelner Kollektive in der Geschichte, und der Kulturbegriff kommt konsequenterweise im Plural vor, er bezieht sich auf Diversitäten der Totalitäten menschlicher Lebensformen in verschiedenen ,Völkern', ,Nationen', ,Gemeinschaften', Kulturkreisen' (Reckwitz 2005, 95).

Über die Erklärung mittels „Macht“ lassen sich zudem Gemeinsamkeiten mit einem bedeutungstheoretisch-strukturalistischem Vokabular von Kultur auffinden. Kultur konstituiert sich „als eine Konfiguration von übersubjektiven symbolischen Strukturen“ und das „Subjekt“ gewissermaßen „als Produkt oder Exekutor kultureller Strukturen“ (Reckwitz 2010, 39). Die drei schichtenspezifischen Literalitäten explizieren demgemäß Sinnoptionen auf der MakroEbene, welche eben im Spannungsfeld von „Macht“ einer räumlich, zeitlich und historisch annäherbaren hierarchischen Ordnung unterliegen:

Als Strukturen können die symbolischen Ordnungen eine historisch-spezifisch distinkte Form besitzen, aber im Zeitraum ihrer historischen Wirksamkeit stellen sie bestimmte Unterscheidungsmuster auf Dauer [...] [, wobei] symbolische Ordnungen als unüberschreitbare Voraussetzungen, als Bedingungen verstanden [werden], die vorgeben, welche Ereignisse, welche Formen des Subjekts, welche Handlungsund Diskurspraktiken möglich sind: sie erscheinen als Sinnmuster, die den Möglichkeitsspielraum von Sinnzuschreibungen in einzelnen Situationen durch die einzelnen Individuuen bestimmen. [...] [Die] symbolischen Codes [sind] der Ort, an dem Macht ihre subtilste Wirkung entfalten kann: durch die symbolischen Codes wird eingeschränkt, was überhaupt denkbar, sagbar, wünschbar ist. Gleichzeitig entfaltet die Macht der symbolischen Codes eine produktive Qualität: sie limitiert nicht nur, sondern bringt auch bestimmte Denk-, Wahrnehmungs- und Handlungsweisen hervor (ebd., 39f.). 
Demgegenüber betont der praxeologische Kulturbegriff in seiner „bottom up-Perspektive“ nicht die „Analyse der übersubjektiven und bestimmte Kontexte transzendierenden kulturellen Reproduktion“, sondern die Analyse der situationsspezifischen Mikroprozesse, „,in denen individuelle oder kollektive Akteure routinisiert, innovativ oder konflikthaft Interpretationsleistungen vollziehen“ (ebd., 41). Jene synthetisierend skizzierende Perspektive auf das Soziale und Handeln auf einzelne, für die vorliegende Studie zentrale Aspekte von einer (sozialen) Praktik reduziert, die ausdrücklich auch „,anspruchsvolle‘ Tätigkeiten wie die des Lesens, Schreibens und Sprechens" (Reckwitz 2003, 290) umfassen, ergeben sich einige Charakterisierungen, aber auch Erklärungsproblematiken für die Trias an Dimensionen des Lesens.

Eine Praktik ist, erstens, weder über von außen beobachtbaren Aktivitäten auf stets explizierungsfähiges oder explizierungsbedürftiges Wissen gebunden. Eine Praktik stellt ein demgemäß zu verstehender „Nexus von wissensabhängigen Verhaltensroutinen“ (ebd., 291) dar. Zweitens verbirgt sich hinter der Routinemäßigkeit einer Praktik durchaus ein enger Bezug zu sozialen Kriterien. Jene fließen bei dem Vollzug implizit ein, indem sie ermöglichen, situativ eine „Sinnwelt“ zu erzeugen, „in denen Gegenstände und Personen eine implizit gewusste Bedeutung besitzen“ (ebd., 292). Dieses mobilisierte implizite Wissen umfasst

ein Wissen im Sinne eines interpretativen Verstehens, d. h. einer routinemäßigen Zuschreibung von Bedeutungen zu Gegenständen, Personen, abstrakten Entitäten, dem ,eigenen Selbst' etc.; ein i. e. S. methodisches Wissen, d. h. script-förmige Prozeduren, wie man eine Reihe von Handlungen ,kompetent ' hervorbringt; schließlich das, was man als ein motivational-emotionales Wissen bezeichnen kann, d.h. ein implizierter Sinn dafür ,was man eigentlich will', ,worum es einem geht' und was ,undenkbar' wäre (ebd.).

Als soziale Praktik ist folglich eine Praktik an eine „skillful performance“ rückgebunden, eine, die „potentiell intersubjektiv als legitimes Exemplar der Praktik X verstehende Praktik“ (ebd., 290). Konstituieren überdies Artefakte eine soziale Praktik, so ist diesen hierbei weder einerseits ein eigenständiger „materieller noch kulturell-symbolischer Stellenwert“ zuzuschreiben, noch sind sie handlungsdeterminierend zu interpretieren. Diese Relevanz von Artefakten kann am Beispiel des Kommunikationsmediums Buchdruck verdeutlicht werden, ohne welche einige Praxen weder entstehen, kontextuell-historisch reproduziert und routinalisiert werden konnten. Andererseits verdeutlichten sich in den Artefakten, eben in Kommunikationsmedien wie z. B. dem Buchdruck, von Kleidung, von motorisierten Fahrzeugen usw. spezifische Begrenzungen, die durch seine Beschaffenheit gegeben sind:

Artefakte erscheinen weder ausschließlich als Objekte der Betrachtung noch als Kräfte eines physischen Zwangs, sondern als Gegenstände, deren sinnhafter Gebrauch, deren praktische Verwendung Bestandteil einer sozialen Praktik oder die soziale Praktik selbst darstellt. In diesem sinnhaften Gebrauch behandeln die Akteure die Gegenstände mit einem entsprechenden Verstehen und einem know how, das nicht selbst durch die Artefakte determiniert ist. Andererseits und gleichzeitig erlaubt die Faktizität eines Artefakts nicht beliebigen Gebrauch und beliebiges Verstehen (ebd., 291).

Wenn die praxeologische Perspektive jene „kulturellen Codes“ auch z. B. im Kontext von Kommunikationsmitteln per se nicht negiert, jedoch im Sinne von etwas systematisch Aufgebautem, und v. a. als etwas Universellem, so gilt das übertragen auch, drittens, für die Vorstellung, dass „kulturelle Codes“ hinter diskreten und expliziten „Zwecken“ und „Interessen“ einer Handlung aktualisiert werden. Diese Dualität konstituiert sich letztlich durch die „Logik der Praxis“: Sie bewegt sich zwischen „einer relativen Geschlossenheit“ in der Wiederholung und einer relativen Offenheit für Misslingen, Neuinterpretation und Konflikthaftigkeit „des alltäglichen Vollzugs“, welche nicht einem Subjekt im Sinne von Autonomie, Reflexivität usw. zugeordnet werden (ebd., 294f.): 
Diese enthält die beiden Momente der Zukunftsgewissheit und des Potenzials der Sinnverschiebung. Das seine ,soziale Praktik' einen kollektiven und ,die Zeit bindenden' Typus darstellt, ändert nichts daran, dass der Vollzug [...] aus der Sicht des Akteurs in der Sequenz der Zeit stattfindet und in jedem Moment - wie routinisiert auch immer - erneut hervorgebracht werden muss. [...] Die, immer wieder neue' Anwendung einer Praktik ist nur im Grenzfall eine identische Wiederholung - gleich der Generierung kopienhafter ,tokens' aus einem ,type - zu denken; sie enthält vielmehr das Potenzial ,zufälliger - sprunghafter oder schleichender - Verschiebungen im Bedeutungsgehalt der Praktik und ihres Wissens, die sich in bestimmten Kontexten ereignen (ebd., 295).

„Zwecke“ und „Interessen“, respektive, „vermeintlich ,allgemeingültige‘ Eigenschaften des ,Menschen“" sind alsdann nicht mehr als Quelle von sozialem Wandel zu verstehen. Subjekte agieren in einer Praktik, die mit anderen lose gekoppelt ist, ergo in Praxiskomplexen im Spannungsfeld unterschiedlicher - auch konkurrierender oder widersprüchlicher - möglicher implizierter bis - z. B. durch formalisierte Kataloge von Normen - mehr oder weniger expliziter Sinnoptionen. Das, was als „Zweck“ und „Interesse“ in der Praktik übernommen erscheint, „ergibt sich [...] aus der praktischen Notwendigkeit, mit verschiedenartigen Verhaltensroutinen und deren heterogenen Sinngehalte umzugehen" und dies aktualisiert in der Praktik (ebd., 296). Jegliche Explizierung, so auch was „Wissen“ im Kontext einer Handlung sein kann, vermag das Feld des Impliziten nicht zu repräsentieren:

Die Praxistheorie betont die Implizitheit dieses Wissens, das kein explizierbares Aussagewissen (knowing that) von Überzeugungen darstellt, sondern einem ,praktischen Sinn 'ähnelt; sie hebt hervor, dass die ,expliziten Regeln', die in einem Handlungsfeld als relevant angegeben werden, diesen impliziten Kriterien in keiner Weise entsprechen müssen; sie betont schließlich auch, dass das Wissen nicht als ein ,theoretisches Denken' der Praxis zeitlich vorausgeht, sondern als Bestandteil der Praktik zu begreifen ist [...]. Dies hat zur Konsequenz, dass aus Sicht der Praxistheorie und im Gegensatz zum Mentalismus Wissen und seine Formen nicht, praxisenthoben' als Bestandteil und Eigenschaften von Personen, sondern immer nur in Zuordnung zu einer Praktik zu verstehen und zu rekonstruieren sind: Statt zu Fragen, welches Wissen eine Gruppe von Personen, d.h. eine Addition von Individuen, ,besitzt', lautet die Frage, welches Wissen in einer bestimmten sozialen Praktik zum Einsatz kommt (und erst darauf aufbauend kann man auf die Personen als Träger der Praktiken rückschließen) (ebd., 292).

Im Zuge dieser beiden leitenden Fragen praxeologischer Ansätze und dezidiert innerhalb von sozialen Praktiken, die sich entlang von Artefakten vollziehen, konkretisieren sich jedoch letztlich ungeklärte Bezüge: Wenn, wie skizziert, Artefakte einerseits einen Teil einer sozialen Praktik darstellen, respektive, jene Praktik historisch-kontextuell erst ermöglichten und, wenn andererseits hierbei jeweils der Gebrauch nicht an explizierbaren und universellen Maßstäben aus der Faktizität von Artefakten abgeleitet werden kann, so gilt ein nicht beliebiger Gebrauch dieser Artefakte im Zuge einer sozialen Praktik zu berücksichtigen. Wenn nicht zuletzt diverse technische (Medialität), sensorische (Modalität) und semiotische (Kodalität) Zeichensysteme als solche Artefakte verstanden werden, so rückt sozusagen über die Hintertüre gleichsam die Frage, wie deren nicht beliebiger Gebrauch gekennzeichnet werden kann, in den Mittelpunkt. Jene ungeklärten Bezüge, die ggf. auf ein knowing that im Gebrauch von Artefakten, respektive, auf kognitive Anteile des Lesens von Zeichensystemen hindeuten, bleiben dennoch an eine soziale Praktik bzw. mehrere soziale Praxen gebunden, an denen handelnde Subjekte teilnehmen. Zur Verhärtung und Illustrierung dieser These soll auf eine der wenigen praxeologischen Erklärungsmodelle einer sozialtheoretischen Vermittlung verwiesen werden: Schmidt (2012, 217ff.) konkretisiert am Beispiel der Praktik des Fußballspielens, wie über die Teilnahme und den praktischen Vollzug von - immer wieder anderen - Spielern zugleich reproduziert und neu erschaffen wird, was „ein gemeinsames Verständnis über die richtige Ausführung des Fuß- 
ballspieles“ (ebd., 218) ist. Der praktische Vollzug, einschließlich seinen Ebenen von Kritik, Korrektur und Übernahme, vermittelt demnach die relevanten Schemata des Fußballspielenkönnens wie z.B. „Bewegungsmuster, Techniken der Ballbehandlung, dem Geschehen folgende Aufteilung im Raum etc." (ebd., 219) als etwas nicht Kognitives sondern der Praktik Immanentes. Hierdurch vermittelt es den Teilnehmern „fortlaufend praktisches Know-how [...] und macht sie auf diese Weise zu kompetenten Teilnehmern beziehungsweise Mitspielern“ (ebd., 218) und kann als „Transmission von Schemata als ein lokales, konkret situiertes, öffentliches, dargestelltes, immer wieder in ähnlicher Weise wiederholtes intelligibles Tun und Können“ (ebd.) näher charakterisiert werden. Aber wie kann alsdann erklärt werden, wenn zwischen einem ,lokalen, konkret situierten, öffentlichen, dargestellten, immer wieder in ähnlicher Weise wiederholten intelligiblen Tun' zu einem demgemäßen ,Können` Diskongruenzen auftreten, die auch trotz jeglicher Korrektur, Kritik und Übernahme im praktischen Vollzug bspw. in den nicht beliebigen Bereich des Gebrauchs des Artefaktes Ball reichen? Einerseits vermag der praktische Vollzug die Vermittlung eines knowing how, bspw. der „richtigen Ausführung" der Balltechnik, treffend charakterisieren. Andererseits bleiben, jedoch die Prozesse davon unberührt, die erklären, wenn trotz des wiederholten praktischen Vollzugs z.B. ein Teilnehmer mit dem Fuß nie den Ball trifft, respektive, wenn das skizzierte Tun mit dem skizzierten Können in vermeintlich keiner sozialen Praxis des Fußballspielens entlang eines nicht beliebigen Gebrauchs des Artefaktes Ball vereinbar ist. An den zentralen Fragen praxeologischer Ansätze verhärtet sich eine Art blinder Fleck, der durch das Interesse auf ein in gewisser Hinsicht bereits könnendes Tun rekurriert.

Vor diesem Hintergrund verwundert es kaum, dass weder das Forschungsinteresse noch die Forschungstradition hinter dem Lesebegriff als Set sozialer Praxen, welcher eben auf praxeologischen Ansätzen beruht, bspw. dezidiert den Erwerb des Gebrauchs von verschiedenen Zeichensystemen behandelt (vgl. Grotlüschen 2011, 22). Weitet man den Blick, so bedarf es der Berücksichtigung einer kognitiven Dimension des Lesens einerseits innerhalb der Akzentuierung auf das knowing how von Zeichen, und dies illustriert sich an den genannten Beispielen: $\mathrm{Ob}$ in den Praktiken der Dokumentation eingehender Nachrichten der Western Union oder in der Praktik des Unterrichts zu dieser Zeit, so verweist die Produktion und Nutzung von Zeichen im Grunde auf die mehr oder weniger gute Beherrschung deren Repräsentationsmächtigkeit hin. Diese umfasst nicht nur das knowing how, was es heißt, die Zeichen zu produzieren und zu gebrauchen, sondern konstituiert sich vielmehr auch aus der Faktizität der referierten Zeichensysteme auf stärker und schwächer arbiträren Strukturen entlang unterschiedlicher schriftlicher Notationssysteme. Anderenfalls wäre im Grunde ,jede lautierte Verschriftlichung [...] eben eine Variante aus vielen möglichen Literalitäten“, eine Annahme, die treffend „als zu pluralistisch und auf problematische Weise postmodernistisch und beliebig“ (ebd.) interpretiert abzulehnen ist. Jeglicher Gebrauch eines Zeichens, ergo auch ein Gebrauch, welcher die Repräsentationsmächtigkeit der Zeichen nicht umfasst, die bspw. eine Praktik X erst ermöglichten, wären dennoch - und unlogisch - zugleich Teil dieser Praktik X. Gleichermaßen wäre das knowing how einer Praktik des (physischen) Fußballspielens, ob die des freizeitlichen, des wettbewerblichen bis gar des professionellen Fußballspielens, sozialtheoretisch-praxeologisch nicht zu erklären, wenn es nicht auf einem knowing that der Teilnehmenden beruht, dass grundsätzlich mit dem Fuß der Ball gespielt wird. Dieses ist letztlich durch die Grenzen des nicht-willkürlichen Gebrauchs des Balles vorgegeben.

Andererseits, aus der skizzierten Beziehung heraus begründet, werden im Zuge des Rückschlusses eines ,lokalen, konkret situierten, öffentlichen, dargestellten, immer wieder in ähnlicher 
Weise wiederholten intelligiblen Tuns und Könnens' auf der Makro-Ebene jeweils bestimmbare Konzepte eines knowing that beansprucht. Dies erfolgt in dem Sinne, dass das „Mentale [...] als ein Bestandteil von Praktiken aufgefasst [wird], der diese mit organisiert, und nicht als eine geistige Entität, die Handeln ,verursacht““, respektive, „keine soziale Existenz [haben], solange sie sich nicht in Praktiken ausdrücken und dort - auf der Grundlage sozialer Identifikationskriterien - ,sichtbar' werden“ (ebd., 221). Nur in einer konkreten lokalen Situation eröffnen sich Einblicke in das Tun und Können von Teilnehmenden „als performativer Effekt des je spezifischen Zusammenwirkens körperlicher und mentaler, beobachtbarer und nichtbeobachtbarer, gedanklicher und sichtbarer Vollzüge (ebd., 222).

Tab. 6: Gegenüberstellung der vier Begriffe des Lesens nach Kulturkonzepten (a)

\begin{tabular}{|c|c|c|c|c|}
\hline & $\begin{array}{l}\text { Informations- } \\
\text { verarbeitung/ } \\
\text { Kulturtechnik }\end{array}$ & kulturelle Praxis & kulturelle Praxen & Set sozialer Praxen \\
\hline $\begin{array}{l}\text { Forschungs- } \\
\text { interesse }\end{array}$ & $\begin{array}{l}\text { forschungsprag- } \\
\text { matisches Kon- } \\
\text { zept zur Messung } \\
\text { der Leseleistung } \\
\text { Jugendlicher }\end{array}$ & $\begin{array}{l}\text { pädagogisch-pragma- } \\
\text { tisches Konzept zur } \\
\text { Förderung des Lesens } \\
\text { unter Bedingung der } \\
\text { an der Sozialisation } \\
\text { von Lesekompetenz } \\
\text { beteiligten Struktu- } \\
\text { ren und Prozesse in } \\
\text { einer Mediengesell- } \\
\text { schaft }\end{array}$ & $\begin{array}{l}\text { forschungspragma- } \\
\text { tisches Konzept zur } \\
\text { Ausdifferenzierung } \\
\text { der Dichotomie- } \\
\text { grenze von Literalität } \\
\text { und Illiteralität in } \\
\text { einer hierarchischen } \\
\text { Gesellschaft/in einer } \\
\text { Gesellschaft aus Me- } \\
\text { diengenerationen }\end{array}$ & $\begin{array}{l}\text { in Praxen lokali- } \\
\text { sierbares und - u. a. } \\
\text { an Artefakte und } \\
\text { Teilnehmenden - } \\
\text { gekoppeltes Wissen }\end{array}$ \\
\hline $\begin{array}{l}\text { Konzeption des } \\
\text { Kollektivs }\end{array}$ & universell & $\begin{array}{l}\text { graduell gleich } \\
\text { handlungsfähige } \\
\text { Personen }\end{array}$ & $\begin{array}{l}\text { Personen einer Schicht } \\
\text { in einer hierarchischen } \\
\text { Gesellschaft/einer } \\
\text { Mediengeneration }\end{array}$ & $\begin{array}{l}\text { durch eine Praktik } \\
\text { lose gekoppelte } \\
\text { Teilnehmende/ } \\
\text { Mitglieder }\end{array}$ \\
\hline $\begin{array}{l}\text { Konzeption der } \\
\text { Zeitlichkeit }\end{array}$ & $\begin{array}{l}\text { objektiv: Epoche } \\
\text { der modernen } \\
\text { Mediengesell- } \\
\text { schaft }\end{array}$ & $\begin{array}{l}\text { objektiv: Epoche der } \\
\text { modernen Medien- } \\
\text { gesellschaft }\end{array}$ & $\begin{array}{l}\text { objektiv: Epoche } \\
\text { der (hierarchischen) } \\
\text { modernen Medien- } \\
\text { gesellschaft }\end{array}$ & $\begin{array}{l}\text { lokal (Praktik/ } \\
\text { Teilnehmende) }\end{array}$ \\
\hline $\begin{array}{l}\text { kognitive } \\
\text { Dimension des } \\
\text { Lesens }\end{array}$ & $\begin{array}{l}\text { symbolisch: } \\
\text { universelle } \\
\text { Fähigkeiten und } \\
\text { Fertigkeiten als } \\
\text { Eigenschaften von } \\
\text { Personen }\end{array}$ & $\begin{array}{l}\text { symbolisch: (teils } \\
\text { Textsorten spezifi- } \\
\text { sche) Fähigkeiten } \\
\text { und Fertigkeiten } \\
\text { als Eigenschaften } \\
\text { von Personen; über } \\
\text { gemeinsame basale } \\
\text { Basis verknüpft }\end{array}$ & $\begin{array}{l}\text { symbolisch: } \\
\text { Fähigkeiten und Fertig- } \\
\text { keiten als Eigenschaften } \\
\text { von Personen, die sich } \\
\text { aus der semiotischen } \\
\text { Komplexität von den } \\
\text { Texten hierarchischer } \\
\text { Literalitäten ableiten } \\
\text { lassen }\end{array}$ & \multirow{3}{*}{$\begin{array}{l}\text { knowing that der Re- } \\
\text { präsentationsmäch- } \\
\text { tigkeit von Zeichen } \\
\text { der Praktik - auch in } \\
\text { Artefakten - und ih- } \\
\text { ren Teilnehmenden } \\
\text { inhärentes knowing } \\
\text { what; sowohl } \\
\text { Voraussetzung als } \\
\text { auch Resultat einer } \\
\text { skillful performance } \\
\text { durch attunement in } \\
\text { - lokalen kulturellen } \\
\text { Codes (soziale } \\
\text { Identifikationsmerk- } \\
\text { male) }\end{array}$} \\
\hline $\begin{array}{l}\text { emotional-motiva- } \\
\text { tionale Dimension } \\
\text { des Lesens }\end{array}$ & $\begin{array}{l}\text { symbolisch: } \\
\text { universell }\end{array}$ & $\begin{array}{l}\text { symbolisch-deskriptiv: } \\
\text { Textsorten immanen- } \\
\text { te Motive }\end{array}$ & $\begin{array}{l}\text { symbolisch: Schichten } \\
\text { spezifischem Sprach- } \\
\text { gebrauch immanent }\end{array}$ & \\
\hline $\begin{array}{l}\text { soziale Dimensi- } \\
\text { on des Lesens }\end{array}$ & $\begin{array}{l}\text { symbolisch: } \\
\text { universell }\end{array}$ & $\begin{array}{l}\text { symbolisch-deskriptiv: } \\
\text { Textsorten immanen- } \\
\text { te Funktion }\end{array}$ & $\begin{array}{l}\text { symbolisch: Schichten } \\
\text { spezifischem Sprach- } \\
\text { gebrauch immanent }\end{array}$ & \\
\hline
\end{tabular}


Eine universelle Hierarchisierbarkeit der kognitiven Prozesse des Lesens als einer Handlung vorausgehend sind konsequenterweise per se unhaltbar und zugleich treten sie innerhalb einiger Praxen - z. B. der schulischen Bildung - als hierarchisches, soziales Identifikationskriterium 'von kompetenten Lesenden im Sinne eines knowing how wieder auf den Plan. Hier sind sie zugleich soziale Voraussetzungen und Resultate in praktischen Vollzügen einhergehend mit praktischen Kritiken, praktischen Korrekturen und praktischen Bestätigungen im Gebrauch des Artefakts Zeichensysteme. In diesem Sinne strahlt das „Soziale“ auf alle drei Dimensionen des Lesens aus. Wenn in der Forschung zum Lesen als Set sozialer Praktiken sensu Street, innerhalb seines ideologischen Modells, die Trennung der Komponente „literacy event“ von der Komponente „literacy practices“ praktiziert wird, kann hierin ein gewissermaßen forschungspragmatischer Ansatz entlang der für die praxeologische Forschungstradition charakteristischen beiden Leitfragen entdeckt werden: Die Annäherung an das Wissen über historisch situativ-kontextuelle gerahmte Beobachtungen von Situationen dieser einen Praktik, ein ,Wissen, das in einer bestimmten sozialen Praktik zum Einsatz kommt', ein Wissen, das m. E. - hier die Repräsentationsmächtigkeit von Zeichensystemen - als von Grund auf implizit zu interpretieren ist. Sie zielen auf die Aufdeckung von lokalen kulturellen Codes, die der Praktik schon immer inhärent sind und zugleich von den Teilnehmenden reproduziert und verändert werden. Oder in anderen Worten, es handelt sich um ein empirisches Programm der Aufdeckung einer immer wieder in Situationen aktualisierten praktischen „Angleichung und Abstimmung (attunement)“ dessen, was eine skillful performance auch mit Zeichensystemen ausmacht. Während die lokalen kulturellen Codes folglich die Sinnoptionen in sich tragen, die über die Voraussetzungen und die Relevanz einer skillful performance routinalisiert entscheiden, gilt dies für die mehr oder weniger in der Faktizität verborgene Arbitrarität des Artefakts, sprich des routinalisiert verborgenen Zeichensystems. Eine skillful performance wird sozusagen vor, während und im Zuge der literalen Praktik immer wieder aufs Neue erzeugt, wobei es routinalisiert auf die Repräsentationsmächtigkeit der eingebetteten Texte zugreift.

An dieser Stelle wird deutlich, dass Sinnoptionen z. B. eines Motivs im, vor oder während des Lesens in der sozialen Institution Schule eher konventionalisierter geprägt sind als in Praxen mit Zeichensystemen in anderen verketteten Situationen und zugleich auf „andere“ routinalisierte lokale kulturelle Codes verweist. Wenn „literacy practices“ außerhalb der Schule auf anderen lokalen kulturellen Codes beruhen, konkretisiert sich dies an „anderen“ Kriterien einer ,anderen' skillful performance des Lesens des jeweils aktualisierten Zeichensystems.

Theoriebasiert ergibt sich für die Analyse von „literacy practices“ jedoch, dass die mit den lokalen kulturellen Codes charakterisierten Sinnoptionen im Grunde nie in ihrer Bandbreite explizierbar sind. Forschungspragmatisch eröffnen dagegen die miteinander verketteten „literacy events“ einer literalen Praktik einen Zugang zu den im Hier und Jetzt „explizit aktualisierten“ Sinnoptionen zum referentiellen Zeichensystem. Diese „explizit aktualisierten“ Sinnoptionen wiederum eröffnen den ,Rückschluss auf die Personen als Träger der Praxen“, sprich die routinalisierte Funktion vor und hinter einer skillful performance unter Berücksichtigung der Arbitrarität des referentiellen Zeichensystems. „Literacy events" sind folglich immer auf das Hier und Jetzt begrenzt. Allerdings geben sie einen Ausschnitt der übergeordneten literalen Praktik Preis. Die theoretischen Kongruenzen eines solchen forschungspragmatischen Lesebegriffs als Set sozialer Praxen mit einem funktional handlungsorientiert-situativen Kompetenzbegriff konkretisieren sich (vgl. Basendowski 2013, 135ff.): Eine skillful performance basiert auf einem „Handeln als Index für Wissen“, welches im Sinne von „,als-ob'-Klassifizierungen empirischer Beobachtungen“" sozio-kontextuell situativ rekonstruiert wird; und ausdrücklich 
nicht - alleinig - als Folge von Wissen im Sinne eines knowing that, respektive, nicht im Sinne von mentalen Entitäten im Subjekt als Quelle jeglicher Handlung. Zugleich begrenzt dieser Kompetenzbegriff den praxeologischen Lesebegriff: Er begrenzt ihn einerseits für die soziokulturell verketteten Situationen (events) mit stark routinalisierten lokalen kulturellen Codes. Andererseits begrenzt er ihn durch den Fokus auf demgemäß als-ob-klassifizierbare kognitive, emotional-motivationale und sozial-kommunikative Anteile, die entlang einer skillful performance in einer Praktik beobachtbar sind. In das empirische Interesse rücken konsequenterweise zuvorderst die - hypothetischen - routinalisierten lokalen kulturellen Codes in einer Praktik der Dokumentation eines Kleinbetrag-Kaufvertrags (Kassenbeleg), von geleisteten Arbeitszeiten (Arbeitsprotokoll) usw. in beruflichen Kontexten. Erst darauf aufbauend ist ein Rückgriff auf einzelne Teilnehmende einer Praktik möglich. Hierbei gilt wieder zu beachten, dass ein nicht beliebiger funktionaler Gebrauch des referentiellen Zeichensystems als lokale Kriterien einer skillful performance mitgedacht werden. Dieser nicht beliebige funktionale Gebrauch ist nicht nur dem Zeichensystem inhärent, sondern eröffnet Zugang - im Sinne einer hypothetischen Konstruktion - zu den alsdann kognitiven, emotional-motivationalen und sozial-kommunikativen Performanzen eines/r Teilnehmers/in in den verketteten literalen events zur Annäherung an literale Praxen.

\subsubsection{Zeichenbegriffe zur Annäherung an die Handlungsfähigkeiten, -fertigkeiten und -bereitschaften des Gebrauchs von Texten}

Weder im Kompetenz- noch im Lesebegriff wurde seither im engeren Sinne auf die Zeichensysteme eingegangen, auf welche sich (eine) Lesekompetenz(en) bezieht. Zweifelsohne wurde diesbezüglich bereits eindeutig die Position eingenommen, dass die entlang einer technischen (Medialität), der sensorischen (Modalität) und der semiotischen (Kodalität) Klassifizierung von Zeichen eben auch diejenigen zu umfassen hat, welche die häufig an Einfach(st)arbeitsplätzen aufgefundenen Texte formieren. In der Mehrheit sind diese über Papier medialisiert, visuell modalisiert und primär kategorial-tabellarisch kodal in Textkomplexen dargelegt.

Ausgehend von den referierten vier Lesebegriffen kann alsdann für den als Informationsverarbeitung/Kulturtechnik, z.B. mit der Referenz PISA-Studie, aufgezeigt werden, dass hier einerseits neben Texten aus rein symbolischen Zeichen (kontinuierliche Printtexte) durchaus auch nichtkontinuierliche Printtexte als Lesen interpretiert werden. In PISA 2000 sollen 89 Aufgaben auf kontinuierlichen und 52 Aufgaben auf nichtkontinuierlichen Printtexten basieren (Kirsch et al., 2002, 30). Unter den Aufgaben auf der Basis nichtkontinuierlicher Printtexte werden dabei überwiegend Tabellen (15 Aufgaben) und graphische Darstellungen (16 Aufgaben) herangezogen. Die übrigen Aufgaben verteilen sich auf Formulare, schematische Zeichnungen, Anzeigen oder Karten, welche die 15-Jährigen lesen und benutzen können sollen (ebd., 32). Andererseits handelt es sich mit Blick auf die veröffentlichen tabellarischen Beispielaufgaben stets um einseitige Printtexte, die mittels einzelner Sätze eingeführt und via Legenden spezifiziert werden.

Auch unter Bezug kultureller Praxen finden sich in den letzten Jahren Anhaltspunkte in Diskussionen, die nach Niederhaus (2011) verdeutlichen, dass sich die damit verknüpfte Handlungsfähigkeit im Zuge des gesellschaftlichen Wandels durchaus sowohl entlang kontinuierlicher als auch nichtkontinuierlicher Texte vollzieht. „Als Folge der wachsenden Informationsflut in modernen Gesellschaften spielt [...] auch die Bildkommunikation eine zunehmend wichtige Rolle. Sowohl in Printmedien als auch in elektronischen Medien hat der Bildanteil in den letzten Jahrzehnten deutlich zugenommen“"(Schnotz/Dutke 2004, 63). Mit Blick auf die For- 
schungsaktivitäten verhärtet sich jedoch, dass auch mit Referenz zu diesen beiden Lesebegriffen insbesondere graphische Darstellungen und Diagramme und weniger Tabellen von Bedeutung sind, die in - auch didaktischen - Sach- und Informationstexten oder innerhalb von Hypertexten eingebunden sind. Primär sind diese Texte jedoch eindeutig als kontinuierliche Text(-komplexe) zu kategorisieren und die informierenden schematischen Abbildungen und Diagramme letztlich immer eine Repräsentations-Dopplung. Des Weiteren liegt das Interesse an jenen primär kontinuierlichen und sekundär nichtkontinuierlichen Texten, in deren Funktion für das Lernen, welche gleichsam typisch für Schulbücher ist: So richten sich die Forschungsaktivitäten zuvorderst an die Prozesse der Koordination und Integration von alsdann multiplen Repräsentationen eines Sachverhalts (vgl. Seufert 2003; Christmann/Groeben 2002). Im Gegensatz zu künstlerischen, realitätsnäheren sind logische Abbildungen wie Diagramme hochgradig funktionalisiert und sollen einer effektiveren Lernvermittlung dienen, welche schlussendlich primär über kontinuierliche Texte samt ihrer Repräsentationsstrukturen initiiert entfaltet wird. Neben Schulbüchern lassen sich jene Sach- und Informationstexte mit multiplen Repräsentationen vor allem in den Lehrmaterialien höherer Klassenstufen der allgemeinbildenden sowie der berufsbildenden Schulen auffinden; ihre Relevanz variiert zudem in ihrer Anzahl und Auswahl in Abhängigkeit des Fachs (vgl. Niederhaus 2011, 2; Hiller 2010, 30f., 43ff.; Christmann/Groeben 2002; Schnotz 1994, 95; Weidenmann 1994, 9f.). Im Kontext der Auseinandersetzung mit Hypertexten dominieren zuvorderst die für diese Textsorte spezifischen Strukturen im Zuge von nicht-linear thematischen Netzen (Schmidt 2010; Kamil/Kim Chou 2009).

Als Zwischenfazit kann gezogen werden, dass die nichtkontinuierlichen Texte, die exemplarisch als Grundlage des universellen Lesens als Informationsverarbeitung/Kulturtechnik gefasst und innerhalb des Lesens als kulturelle Praxis bzw. kulturelle Praxen zuvorderst behandelt werden - aufgenommen als Deskriptoren von Bildungsstandards -, sich auf der semiotischen Ebene maßgeblich von den aufgefundenen nichtkontinuierlichen Texten und Textkomplexen an Einfach(st)arbeitsplätzen unterscheiden.

Tab. 7: Gegenüberstellung textueller Beispiele und Strukturen ikonischer Zeichen

\begin{tabular}{|c|c|c|}
\hline $\begin{array}{l}\text { textuelle Beispiele: } \\
\text { PISA }\end{array}$ & $\begin{array}{l}\text { Gegenstand des Lesens: } \\
\text { Strukturen ikonischer Zeichen }\end{array}$ & $\begin{array}{l}\text { textuelle Beispiele: } \\
\text { Bildungsstandards }\end{array}$ \\
\hline $\begin{array}{l}\text { - „Das implizierte Ausgangsda- } \\
\text { tum einer Graphik identifizie- } \\
\text { ren.“ } \\
\text { - „Numerische Informationen } \\
\text { in einem Baumdiagramm } \\
\text { lokalisieren.” } \\
\text { - „Informationen, die in einer } \\
\text { Kurvengraphik und deren ein- } \\
\text { leitenden Text enthalten sind, } \\
\text { lokalisieren und miteinander } \\
\text { verknüpfen, um Rückschlüsse } \\
\text { auf einen fehlenden Wert zu } \\
\text { ziehen." }\end{array}$ & $\begin{array}{l}\text { Informationen eines Sachverhalts, } \\
\text { die mehr oder weniger tief in den } \\
\text { Text oder Textkomplex eingebet- } \\
\text { tet sind und mehr oder weniger } \\
\text { klar differenzierbar innerhalb } \\
\text { des Textes oder Textkomplexes } \\
\text { lokalisierbar sind, sind zu identi- } \\
\text { fizieren und zu dekodieren. Diese } \\
\text { Informationen sind folglich dem } \\
\text { Text oder Textkomplex inhärent } \\
\text { und bedürfen nicht zwingend } \\
\text { weder externes Faktenwissen noch } \\
\text { einer tiefen Auseinandersetzung } \\
\text { mit dem Text (,lokale Kohärenz“ } \\
\text { im Sinne von „Informationen } \\
\text { ermitteln“). }\end{array}$ & $\begin{array}{c}\text { - „Informationen zielgerichtet } \\
\text { entnehmen, ordnen }[. . .]^{4}\end{array}$ \\
\hline
\end{tabular}




\begin{tabular}{|c|c|c|}
\hline $\begin{array}{l}\text { textuelle Beispiele: } \\
\text { PISA }\end{array}$ & $\begin{array}{l}\text { Gegenstand des Lesens: } \\
\text { Strukturen ikonischer Zeichen }\end{array}$ & $\begin{array}{l}\text { textuelle Beispiele: } \\
\text { Bildungsstandards }\end{array}$ \\
\hline $\begin{array}{l}\text { - „Die Struktur eines Baumdia- } \\
\text { gramms verstehen.“ } \\
\text { - „Mehrere kurz beschriebene } \\
\text { Fälle analysieren und den } \\
\text { in einem Baumdiagramm } \\
\text { abgebildeten Kategorien } \\
\text { zuordnen, wobei ein Teil der } \\
\text { erforderlichen Informationen in } \\
\text { Fußnoten zu finden ist.“ } \\
\text { - „Die Hauptidee eines einfachen } \\
\text { Balkendiagramms aus seinem } \\
\text { Titel ableiten.“ }\end{array}$ & $\begin{array}{l}\text { Zusammenhänge zwischen } \\
\text { Sachverhalten (Begriffe, Konzepte } \\
\text { usw.), die mehr oder weniger } \\
\text { mehrdeutig im Text oder Text- } \\
\text { komplex und mehr oder weniger } \\
\text { differenziert und strukturiert } \\
\text { repräsentiert werden, sind zu } \\
\text { identifizieren, dekodieren und } \\
\text { zu deuten. Diese Zusammen- } \\
\text { hänge sind folglich dem Text } \\
\text { oder Textkomplex inhärent und } \\
\text { bedürfen nicht zwingend externes } \\
\text { Faktenwissen, jedoch einer mehr } \\
\text { oder weniger tiefen Auseinan- } \\
\text { dersetzung mit dem Text oder } \\
\text { Textkomplex als Ganzes (globale } \\
\text { Kohärenz im Sinne „Textbezoge- } \\
\text { nes Interpretieren“). }\end{array}$ & $\begin{array}{l}\text { - „,erschiedene Textfunktionen } \\
\text { und Sorten unterscheiden: } \\
\text { informieren (z. B. Lexikontext), } \\
\text { appellieren (z. B. Werbetext), } \\
\text { regulieren (z. B. Jugendschutz- } \\
\text { gesetzt, Arbeitsvertrag), } \\
\text { instruieren (z. B. Bedienungsan- } \\
\text { leitung)“ } \\
\text { - „,nichtlineare Texte (auch im } \\
\text { Zusammenhang mit linea- } \\
\text { ren Texten) auswerten: z. B. } \\
\text { Schaubilder } \\
\text { - „Intention(en) eines Textes } \\
\text { erkennen } \\
\text { - ,aus Sach- und Gebrauchstex- } \\
\text { ten begründete Schlussfolge- } \\
\text { rungen ziehen“ }\end{array}$ \\
\hline $\begin{array}{l}\text { - „Über ein unerwartetes Phä- } \\
\text { nomen Hypothesen aufstellen, } \\
\text { indem externes Wissen mit } \\
\text { allen relevanten Informationen } \\
\text { verknüpft wird, die in einer } \\
\text { komplexen Tabelle über ein } \\
\text { nicht sonderlich vertrautes } \\
\text { Thema enthalten sind.“ } \\
\text { - „Über ein unerwartetes Phä- } \\
\text { nomen Hypothesen aufstellen, } \\
\text { indem externes Wissen mit } \\
\text { einigen relevanten Informa- } \\
\text { tionen verknüpft wird, die in } \\
\text { einer komplexen Tabelle über } \\
\text { ein nicht sonderlich vertrautes } \\
\text { Thema enthalten sind.“ } \\
\text { - „Zwischen Variablen und } \\
\text { strukturellen Merkmalen eines } \\
\text { Baumdiagramms unterscheiden. }\end{array}$ & $\begin{array}{l}\text { Reflexion und Bewertung von } \\
\text { Informationen eines mehr oder } \\
\text { weniger umfangreich textuell } \\
\text { repräsentierten Sachverhalts oder } \\
\text { von Zusammenhängen zwischen } \\
\text { mehr oder weniger umfangreich } \\
\text { textuell repräsentierten Sachver- } \\
\text { halten unter Rückgriff auf mehr } \\
\text { oder weniger spezifisches externes } \\
\text { Faktenwissen (lokale Kohärenz } \\
\text { im Sinne von „Reflektieren und } \\
\text { Bewerten“). }\end{array}$ & $\begin{array}{l}\text { - „Informationen zielgerichtet } \\
\text { entnehmen, ordnen, prüfen und } \\
\text { ergänzen“ } \\
\text { - „Information und Wertung in } \\
\text { Texten unterscheiden: z. B. in } \\
\text { Zeitungen }\end{array}$ \\
\hline
\end{tabular}

Der zugrunde liegende Zeichenbegriff umfasst hier folglich nur unter diesem Primat symbolischer Zeichen auch die Zeichen, welche nichtkontinuierliche Texte beanspruchen. Wenn mit Schnotz (2006) Texte als „Leitmedium in Lehr- und Lernsituationen“ bezeichnet werden können, muss zusammenfassend das tatsächliche Forschungsinteresse hinsichtlich nichtkontinuierlicher Texte als verengt bezeichnet werden. Demgemäß „eigenständige“ (Adamzik 2011, 367) nichtkontinuierliche Texte und Textkomplexe, die eben primär über Tabellen, graphische Darstellungen, Diagramme und Karten Sachverhalte repräsentieren und ggf. sekundär über kontinuierliche Texte in z. B. Legenden, werden nicht berücksichtigt - und dies unabhängig ob als Print- oder digitales Medium. Konsequenterweise wären die semiotischen Zeichen, welche jene nicht berücksichtigten primär kategorial-tabellarischen Texte charakterisieren, keine 
Zeichen, die in dem Lesen im Singular einer Kultur im Singular für eine Gesellschaft im Singular von Relevanz sind, respektive, begrenzt für ein Lernen in der schulischen Bildung und für das lebenslange Lernen. Oder im besten Fall sind es schlichtweg primitive Zeichen, welche in der einen Kulturtechnik, der einen kulturellen Praxis, oder über alle kulturellen Praxen hinweg per se schon immer berücksichtigt werden; jeweils in Referenz zum Lernen sowohl in als auch außerhalb von Bildungsinstitutionen. Es manifestiert sich die übergeordnete Problemstellung in einem Nicht-Mitdenken von Zeichensystemen der primär nichtkontinuierlichen Texte und Textkomplexe in schulischen Leseprogrammen, welche sich im Übergang Schule/Arbeit an der Textualität in Einfach(st)erwerbstätigkeiten pointiert und von der These geleitet ist, dass diese Texte nicht als „einfach" bzw. "primitiv“ eingestuft und daher in institutionellen Bildungsprozessen auch nicht vernachlässigt werden können.

Innerhalb der Darlegung des Ansatzes des Lesens als Set sozialer Praxen wurde mehrfach betont, dass die Forschungstradition seither im engeren Sinne auf der „Explizierung“ von lokalen kulturellen Codes lag, so z. B. wenn in der Lebensstilforschung „die scheinbare - leicht reifizierbare - Totalität einer ,ganzen Lebensform in die Heterogentität präzise bestimmbarer Alltagspraktiken, die ein ,bürgerliches Leben“ ausmachen, exploriert wird: [...] „Praktiken des Selbst, etwa im Schreiben von Tagebüchern, in der Lektüre von Romanen und Sachliteratur, im „Kunstgenuss““ (Reckwitz 2003, 293). Entsprechende Ergebnisse wurden in der Zusammenfassung des Forschungsprojektes lea bereits referiert. Die Forschungstradition akzentuiert seither, wie bereits hervorgehoben, einerseits nicht dezidiert die Zeichensysteme hinter den Texten, die in bestimmten Praxen einfacher Erwerbstätigkeiten routinalisiert eingebundenen sind und daher eben auch funktional nicht willkürlich zu gebrauchen sind. Andererseits setzen die bisherigen Forschungsprojekte im Grunde die kognitive Verarbeitung dieser semiotischen Zeichen bereits voraus, respektive, bemühten sich seither nicht, den Ansatz des Lesens als Set sozialer Praxen im Spannungsfeld der verstehenden Verarbeitung der Zeichen selbst zu befragen. Ausnahme der erstgenannten Leerstelle bildet die Arbeit von Schroeder (2011), in welcher dezidiert auch kategorial-tabellarische Texte an Einfach(st)arbeitsplätzen erstmalig semiotisch näher betrachtet wurden. Aufbauend auf diese Vorarbeit, die bereits nichtkontinuierliche Texte dem Verdacht von Primitivität entrückten, wird im Weiteren, wie von Schroeder empfohlen, eine vertiefte semiotische Analyse von Zeichensystemen erfolgen, welche zur Charakterisierung der aus der Routinalisiertheit in Praxen abzuleitenden textuellen Anforderungen einer skillful performance mit bestimmten Texten u. a. für notwendig erachtet werden kann. Zuvorderst soll mit einer Annäherung an jene Routinalisiertheit verdeutlicht werden, dass letztlich bestimmte semiotische Codes hinter kategorial-tabellarischen Texten durch einzelne Praxen heraus evolutionär entwickelt wurden; einhergehend mit der Vermutung, dass sie letztlich auch nur aus einzelnen Praxen heraus zu interpretieren sind. Diese vermutete Relevanz des „Sozialen“ und „Situativen“ gilt es im Spannungsfeld eines Erklärungsmodells der kognitiven Verarbeitung der analysierten, dahinterliegenden semiotischen Zeichen aufzuspüren.

\subsection{Semiotische Analyse der Zeichen hinter primär kategorial-tabellarischen Texten}

Aus der ersten Annäherung an die arbeitsweltliche Textualität konnte bereits ein Primat des technischen Zeichenträgersystems Print und der visuellen Darstellungsmodalität erschlossen werden. Nichtsdestotrotz ist nicht zu vernachlässigen, dass in einigen Tätigkeiten, wie z.B. im Verkauf oder der Maschinenführung, die Bedienung von Geräten allgegenwärtig sind. Ein- 
hergehend wird die Steuerung jener Geräte verlangt und somit neben der Einsicht in die teils interaktive Menüsteuerung bzw. der sorgfältigen und exakten Bedienung auch das Lesen von technisch dargestellten Texten und Audiosignalen; wie die beiden angefügten Situationsbeschreibungen on-the-job verdeutlichen:

Die Servicechefin hebt die Notwendigkeit einer sorgfältigen Einarbeitung der Mitarbeiterin beim Bonieren mit der Kasse hervor. Seither hat sie noch wenig Umgang mit dem Bonieren, da es ihr noch nicht zugetraut wird. Gegenwärtig schreibt sie jede Bestellung auf einen Zettel und lässt es von einer erfahrenen Kraft bonieren. Nur wenn es die Zeit zulässt, darf sie diese unter Beobachtung in die Kasse selbst eingeben. Die Mitarbeiterin ist dabei sehr interessiert bei der Sache. Das Problem ist jedoch, dass sich die Servicechefin sicher sein muss, dass die Bonierung exakt ausgeführt wird und hebt in diesem Zuge hervor, dass noch viele Fehler passieren, wie z. B., dass Getränke auf einen falschen Tisch eingebucht wurden („Wenn das Bonieren mal abgegeben wird, dann muss ich mir auch sicher sein, dass es hundertprozentig funktioniert. “; Deskription nach Transkription, Service Gastronomie, ländliches Restaurant).

Die Mitarbeiterin erklärt ein mobiles MDE-Gerät, welches sie für die ständige Erfassung des Warenbestands der Warengruppe im Supermarkt oder für Bestellungen im Großlager einsetzt, für die sie verantwortlich ist. Am oberen Rand des Gerätes, welches an den Paketboten erinnert, ist ein Scanner eingebaut. Auf der Vorderseite befindet sich ein großes monochromes Display oberhalb eines numerischen Tastenfeldes und einer auffälligen gelben Taste. Nimmt man das Gerät in die Hand und fährt von rechts nach links über das Etikett eines Artikels bestellt man eine Einheit des Artikels. Möchte man mehr als eine Einheit bestellen, so muss man von links nach rechts das Etikett einscannen und im Anschluss die gewünschte Anzahl manuell eintippen und mit der gelben Taste bestätigen. Das Gerät gibt sodann einen markanten Ton ab, einen anderen bei einem Fehler. Im Büro gibt es eine Dockingstation. Das Gerät eingesetzt, öffnet sich automatisch ein Fenster am angeschlossenen Computer. Man muss nur noch die Angaben kontrollieren und die Bestellung bestätigen (Deskription nach Transkription, Verkäufer Einzelhandel; ländlicher Supermarkt).

Es muss mit Blick auf die gefundenen Texte folgerichtig postuliert werden, dass Einfach(st)arbeitsplätze von diversen multimedialen und multitechnischen Repräsentationen zur Beschreibung bestimmter Sachverhalte geprägt sind. Infolgedessen musste aus der ersten Annäherung an die gesichteten Texte an Einfach(st)arbeitsplätzen eine Reduktion vorgenommen werden. Da es sich in der Mehrheit um primär kategorial-tabellarische Texte oder Textkomplexe handelt, richtet sich das spezifische Interesse in erster Linie nicht an Fließtexte (symbolische Zeichen), in die sodann verschiedene Tabellen und vereinzelt graphische Darstellungen, Karten oder Diagramme (ikonische Zeichen) eingebunden sind.

Erschwerend kommt mit dieser Setzung hinzu, dass sich nahezu alle Referenzstudien zum Lesen nichtkontinuierlicher Texte oder zu didaktischen Konzepten der letzten Jahre nur eingeschränkt eignen, um sich semiotisch dieser Textualität anzunähern. Hier liegt das Augenmerk fast ausschließlich, wie dargelegt, in den Möglichkeiten und der Optimierung der Unterstützung bei der Bildung von Kohärenzen in Fließtexten durch Einbindung von graphischen Darstellungen. Es werden daher die Arbeiten von Weidemann (1994) und Schnotz (1994) zum Lesen von Ikonen herangezogen, die angesichts ihrer zahlreichen Rezeption bei z. B. Horz (2009), Niederhaus (2011), Ulrich et al. (2012) oder Horn et al. (2012) als aktuell bezeichnet werden können. Obgleich die beiden Autoren in ihren semiotischen Analysen nicht konkret auf kategoriale Tabellen eingehen, liegt hierin die Vermutung begründet, jenes Textformat zumindest als von Prinzipien ikonischer Darstellungen maßgeblich geprägt zu bezeichnen. Mit dem semiotischen Kriterium der inhärenten und mehr oder wenigen abstrakten Ähnlichkeit für ikonische Zeichen soll an dieser Stelle jedoch keine im engeren Sinne wissenschaftlich-semiotische Einordnung 
folgen. Vielmehr liegt das Interesse an diesem Kriterium darin, sich begründet der graphischen Strukturierung der aufgefundenen Texte an den untersuchten Einfach(st)arbeitsplätzen weiter anzunähern.

\subsubsection{Semiotisches Kriterium der Ähnlichkeit ikonischer Darstellungen}

Jene semiotische Einordnung der Ikonizität von u.a. einem Diagramm kann bereits mit Rückgriff auf Peirce $(1991,320)$ vorgenommen werden, denn es kann als ein „Ikon einer Menge von relational aufeinander bezogenen Objekten“ umschrieben werden; daneben zählt der Mitbegründer der modernen Semiotik Fotographien bis graphische Darstellung zu den Ikonen. An jene Einordnung innerhalb der Objektdimension Peirces schließt u.a. Schnotz (1994) an, die im engeren Sinne innerhalb der triadischen Zeichenrelation und somit in der weiteren Unterscheidung zur Zeichendimension und Interpretantendimension zu betrachten ist. Es geht folglich verkürzt um die Analyse, ob ein Objekt in die triadische Zeichenrelation ikonisch (erstheitlich) oder symbolisch (drittheitlich) eingebunden ist. Die weitere übernommene Reduktion um die indexikalische Zeichenrelation begründet sich hier aus deren kausalen Bedingtheit in der Verknüpfung mit dem Bezeichneten (zweiheitlich). Es würde ansonsten kaum eine eindeutige semiotische Trennung zu den anderen beiden Kategorien resultieren. Diagramme sind demzufolge den Ikonen zuzuordnen, und dies unabhängig davon, ob sie Sachverhalte mit Zusammenhängen zwischen rein quantitativen oder quantitativen und qualitativen Merkmalen beschreiben, sowie unabhängig davon, welche graphischen Entitäten vom Kreissegment bis Piktogramm oder auch symbolischen Bezeichnungen sie hierbei nutzen. Maßgeblich ist, dass sie unter bestimmten Umständen beim Interpretanten einen Eindruck der Ähnlichkeit zwischen Zeichen und Bezeichnetem wachrufen können.

Diese $\ddot{A}$ hnlichkeit zwischen Zeichen und Bezeichnetem begrenzt sich jedoch nicht auf alle äußeren Strukturmerkmale. Verdeutlichen lässt sich dies innerhalb der Kategorisierung unterschiedlich realitätsgetreuer graphischer Darstellungen: schematische Abbildung, Skizze/vereinfachte Abbildung, realistische Abbildung, Fotografie (Kapitel 2.4.1). Übertragbar auf die schematische Tankanzeige einem aufgefundenen nichtkontinuierlichen Text in der Wagenpflege (Abbildung 11) lässt sich somit festhalten, dass die Merkmale einer ikonischen Darstellungen nicht alle visuell ähnlichen Aspekte eines Sachverhalts repräsentieren müssen. Es handelt sich vielmehr um eine gezielte Auswahl übereinstimmender repräsentierender und repräsentierter Merkmale eines Sachverhalts in einer graphischen Darstellung.

Die zweidimensionale, flächige Tankanzeige trägt darüber hinaus bereits eine funktionale Analogie des Sachverhalts in sich: Die Relation des Füllstands bei Abgabe und Annahme des Fahrzeugs aus Sicht eines/r Mitarbeiters/in. Es beschreibt diesen Sachverhalt mittels bestimmter Merkmale, hier mittels eines Kreissegments, von Linien, von Zahlenziffern und eines Piktogramms. Zweifelsohne könnte dieser auch über einen Fließtext dargelegt werden, ergo mittels des Repertoires an symbolischen Elementarzeichen, die nach bestimmten (syntagmatischen) morphologisch-syntaktischen, semantisch-lexikalischen und pragmatischen Regeln kombiniert, interpretiert und verwendet werden können. Solche symbolischen Repräsentationen unterscheiden sich insbesondere von der schematischen Tankanzeige darin, dass die repräsentationsrelevanten Struktur- und Funktionsanalogien nicht in ihnen inhärent sind. Für die Beschreibung der skizzierten relationalen Elemente zwischen Abgabe und Annahme des Fahrzeugs benötigt man u. a. Adjektive, Präpositionen und Verben, ergo, wiederum symbolische 
HuberAutoRent

Fahrzeugprotokoll

Mietvertrag Nr.:

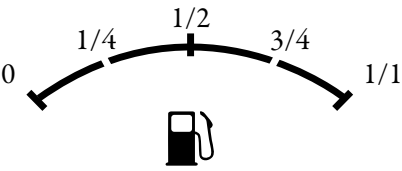

\begin{tabular}{|l|l|l|l|l|}
\hline Kennzeichen & CO-Datum & CO-Kilometer & Cl-Datum & Cl-Kilometer \\
\hline & & & & \\
\hline
\end{tabular}

Funktionsprüfung \& Austattung/Zubehör

\begin{tabular}{|l|c|c|l|l|l|l|c|c|}
\hline & ja & nein & & ja & nein & & ja & nein \\
\hline Außenspiegel & & & Licht & & & Verbandkasten/Warndreieck & & \\
\hline Reifen OK & & & Instrumente & & & Funktion Ladebordwand & & \\
\hline Schweibenwischer & & & Signalhorn & & & Ersatzrad & & \\
\hline Bremsen & & & Bordwerkzeug & & & Laderaumabdeckung & & \\
\hline
\end{tabular}

!! Bei Fahrzeugen in der Halle bitte Gang \& Handbremse Raus, Radio leise, Sitz zurück!!

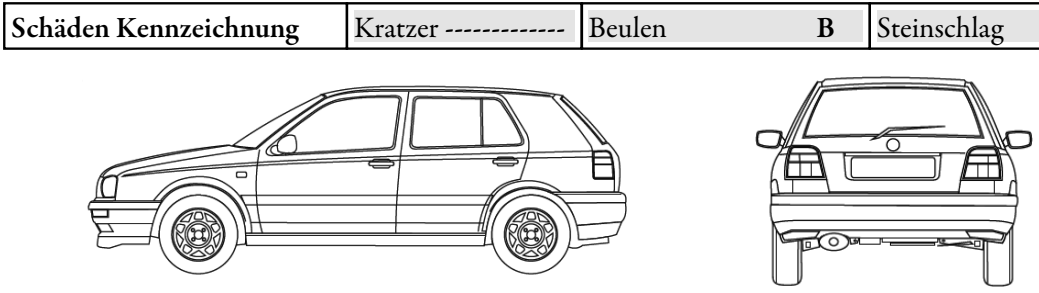

Bitte alle Schäden mit den entsprechenden Symbolen kennzeichnen
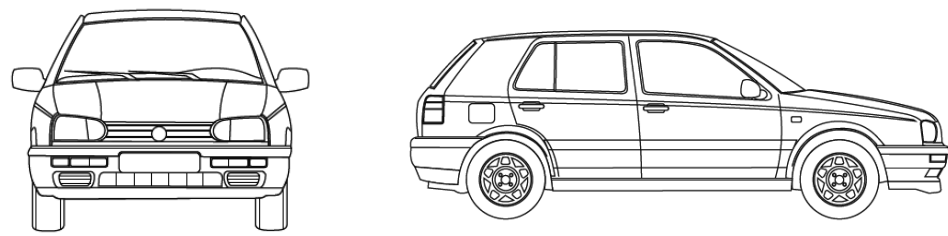

\begin{tabular}{|l|}
\hline Sonstige Schäden z. B.: Riss Frontscheibe, fehlende Fahrzeugteile Bitte beschreiben in kurzen Worten \\
\hline \\
\hline \\
\hline
\end{tabular}

Unbedingt beachten:

- Alle unsere Fahrzeuge sind Nichtraucherfahrzeuge

- Bei jedem Unfallschaden die Polizei hinzuziehen

- Bei Nachtabgabe kann die Rücknahme (Schadenmeldung) erst bei Geschäftsöffnung erfolgen.

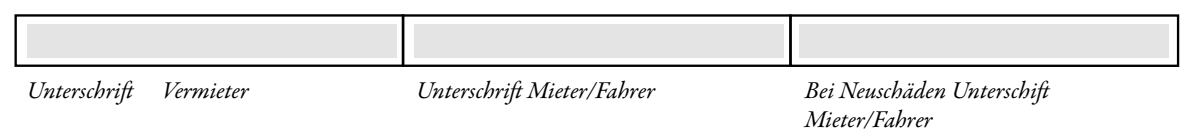

Abb. 11: Fahrzeugprotokoll in der Wagenpflege ${ }^{9}$

9 Aus Datenschutz rechtlichen Gründen wurde die fotografierten oder kopierten Originaldokumente am Computer nachgebildet, wobei eine möglichst exakte Nachbildung intendiert war. Graphische oder sprachliche Einheiten, die auf die Quelle verweisen könnten, so z. B. Firmenlogos, wurden durch fiktive Angaben ersetzt. 
Zeichen, die für bestimmte Relationen mit bestimmten Eigenschaften (z. B. Transitivität, Symmetrie usw.) stehen. Da hier die repräsentationsrelevanten Struktureigenschaften gewissermaßen von außen ,eingebaut werden, bezeichnet [...] [man] solche symbolischen Repräsentationen als extrinsisch (ebd., 107).

Depiktionen sind [dagegen] durch die inhärenten Struktureigenschaften immer vollständig. Stellt man ein Objekt bildhaft dar, so kann man neben der Form immer auch dessen Größe, Position, Farbe etc. ablesen. Eine Deskription ermöglicht hingegen die Darstellung nur einer dieser Eigenschaften (Seufert 2003, 16).

Graphische Darstellungen enthalten entgegen symbolischen Darstellungen folgerichtig immer bereits explizite relationale Elemente, welche auf einer inhärenten Ähnlichkeit zwischen Zeichen und Bezeichnetem beruhen. Diese müssen durch das Lesen identifiziert und dekodiert werden, weshalb sie gleichermaßen wie symbolische als intrinsische Repräsentationen bezeichnet werden. Jene inhärente Ähnlichkeit lässt sich gleichermaßen in logischen Bildern aufzeigen: In bspw. einem Liniendiagramm liegt sie darin begründet, dass die räumliche Veränderung eines „Punktes“ automatisch zu einer Modifikation der Relationen dieses „Punktes“ zu allen anderen „Punkten“ führt. Gleiches gilt für die qualitative Relation von Begriffen, die in einem Strukturdiagramm durch Visualisierung einzelner Kategorien von bestimmten Attributen präsentiert werden. In Ablaufdiagrammen verändern sich z.B. die Beziehungen zwischen den einzelnen dargestellten Zuständen.

Zweifelsohne unterscheidet sich die Art der inhärenten Ähnlichkeit in Diagrammen von der in graphischen Darstellungen, da sie

eine besondere Form ikonischer Zeichen sind, die durch eine abstraktere Form der Ikonizität gekennzeichnet sind und sich dementsprechend auch für die Darstellung von Sachverhalten eignen, die der Wahrnehmung nicht unmittelbar zugänglich sind. Zeichen bedeuten jedoch nur dann etwas, wenn sie auch als Zeichen verwendet werden (Schnotz 1994, 109).

In Diagrammen werden, entgegen graphischen Darstellungen, keine visuell beobachtbaren Objekte und bestimmte Beziehungen zwischen diesen Objekten mittels Konturen, Schattierungen, Perspektiven, Farbgebung oder Proportionen realitätsgetreu im engeren Sinne inhärent abgebildet. Vielmehr werden sie verwendet, um bestimmte Relationen zwischen Merkmalen (auch von Objekten) eines Sachverhalts zu beschreiben, die mittels bspw. unterschiedlicher Stärke, Farbe, Form und räumlicher Lage von Linien oder Flächen dargestellt werden. Bei Diagrammen als Ganzes (=Globalzeichen) handelt es sich um abstrakte Ikone, die grundsätzlich auf arbiträren Repräsentationsanalogien beruhen. Gemeint ist damit, dass Zeichen und Bezeichnetes nur durch bestimmte Konventionen strukturell miteinander verknüpft sind. Innerhalb dieses abstrakten ikonischen Globalzeichens können einzelne Zeichen (=Lokalzeichen) durchaus auf „erfahrungsnahe Analogien und räumliche Metaphern“ zurückgreifen (Weidenmann 1994, 21f.):

Die sequenzielle Anordnung der Kästen eines Flußdiagramms (sic!) ist analog zur Reihenfolge der Handlungsschritte. Bei [Liniendiagrammen zu Umsatzwerten] verlaufen die Kurven [...] analog zur Entwicklung des Umsatzes, es gibt ein ,Auf' und ,Ab'. [...] [Ein häufig so benanntes gestapeltes Säulendiagramm] stellt eine Analogie zum Vorgang des Stapelns oder Auftürmens her. [...] Weitere Beispiele sind die Stammbaum-Analogie bei schematischen Darstellungen und die Torten-Analogie (einschließlich der Metapher des Aufschneidens) beim Kreisdiagramm. Selbst Einzelzeichen in logischen Bildern sind nicht immer völlig arbiträr und ohne Ähnlichkeit mit dem Bezeichneten, sondern stellen teilweise ebenfalls Analogien dar, etwa der Pfeil, der eine Richtung anzeigt und den Rezipienten anregt, eine Bewegung in Verlängerung der Pfeilspitze zu imaginieren. 
Jene arbiträren Repräsentationsanalogien kommen zwar spezifisch auch bei symbolischen Zeichen zum Tragen, z.B. innerhalb von syntagmatischen Beziehungen einzelner symbolischer Entitäten zu komplexen Ausdruckseinheiten oder in den Regeln der Graphem-PhonemKorrespondenzen in morphophonemischen Schriften wie dem Deutschen. Nichtsdestotrotz unterscheiden sich diese abstrakten ikonischen Zeichensysteme hinter Diagrammen in den semiotischen Bedingungen der inhärenten Äbnlichkeit und mehr oder weniger abstrakten Äbnlichkeit; letzteres hier im Sinne von ,erfahrungsnahen Analogien und räumlichen Metaphern: In der Form „der strukturellen Übereinstimmung zwischen Repräsentation und repräsentierendem Sachverhalt, wo repräsentierende und repräsentierte Merkmale voneinander verschieden sind, [die auch] [...] als natürliche Isomorphie“ (Schnotz 1994, 108) bezeichnet wird, unterscheiden sich diese ikonischen Zeichensysteme hinter logischen Bildern von denen hinter graphischen Darstellungen, welche eine „pyhsikalische Isomorphie“ kennzeichnen.

Tab. 8: Semiotische Aspekte des Zusammenhangs zwischen Repräsention und repräsentiertem Sachverhalt bei graphischen Darstellungen, Karten und Diagrammen

\begin{tabular}{|c|c|c|c|c|}
\hline Repräsentation & \multicolumn{2}{|c|}{ repräsentierter Sachverhalt } & Isomporhie & Ähnlichkeit \\
\hline $\begin{array}{l}\text { Graphische } \\
\text { Darstellungen }\end{array}$ & \multirow{2}{*}{$\begin{array}{l}\text { Darstellung eines } \\
\text { visuell wahrnehmba- } \\
\text { ren Sachverhalts }\end{array}$} & & \multirow[t]{2}{*}{ physikalisch } & \multirow{2}{*}{$\begin{array}{l}\text { inhärent; } \\
\text { konkrete } \\
\text { Ikonizität }\end{array}$} \\
\hline Karten & & mit Raumbezug & & \\
\hline $\begin{array}{l}\text { Struktur- und } \\
\text { Ablaufdiagramme }\end{array}$ & \multirow{3}{*}{$\begin{array}{l}\text { Darstellung eines } \\
\text { visuell nicht } \\
\text { wahrnehmbaren } \\
\text { Sachverhalts }\end{array}$} & $\begin{array}{l}\text { im Sinne terminologischer, } \\
\text { kategorialer, topologischer } \\
\text { oder von Verlaufsstrukturen }\end{array}$ & \multirow{3}{*}{ natürlich } & \multirow{3}{*}{$\begin{array}{l}\text { inhärent; } \\
\text { abstrakte } \\
\text { Ikonizität }\end{array}$} \\
\hline $\begin{array}{l}\text { Kreis-, Balken-, } \\
\text { Säulen- und Iso- } \\
\text { Typdiagramme }\end{array}$ & & $\begin{array}{l}\text { im Sinne von Zusammenhän- } \\
\text { gen zwischen qualitativen und } \\
\text { quantitativen Merkmalen }\end{array}$ & & \\
\hline $\begin{array}{l}\text { Linien- und Streu- } \\
\text { diagramme }\end{array}$ & & $\begin{array}{l}\text { im Sinne von Zusammenhän- } \\
\text { gen zwischen quantitativen } \\
\text { Merkmalen }\end{array}$ & & \\
\hline
\end{tabular}

Neben Diagrammen beruhen auch Karten teils auf jener natürlichen Isomorphierelation zwischen Zeichen und Bezeichnetem und können in diesem Zuge der abstrakten Form von Ikonizität zugeordnet werden (vgl. Tabelle 8). Einfache topologische Karten, die im Grunde an ,erfahrungsnahen Analogien und räumlichen Metaphern' zwischen visuell wahrnehmbaren Raumbezügen mit durchaus visuell wahrnehmbaren Objekten darzustellen intendieren und hierbei v. a. ohne eine festgelegte Skalierung durch Achsen auskommen, stellen nicht nur historisch-evolutionär eine Zwischenstufe dar. Vergleichbar der bereits angerissenen historischen Zusammenhänge zwischen Karten mit wie auch immer skalierten Längen- und Breitengraden und ersten Linien- oder Streudiagrammen, kann die Geschichte nichtkontinuierlicher Textformate auch als sukzessive Distanzierung ikonischer Darstellungen von der konkreten Ikonizität interpretiert werden. Diese Distanzierung umfasst jedoch nicht eine grundsätzliche Ablösung von jeglicher Ikonizität. Linien- und Streudiagramme haben sich jedoch gegenüber ihren Vorreitern als geeignet erwiesen, wenn nicht ausschließlich Zusammenhänge mit Raumbezug analog textuell beschrieben werden sollen. Sie wurden zunehmend in diesen Kontexten weiterentwickelt (Playfair 1786; zit. nach ebd., 109) und präferiert beansprucht, wenn der seman- 
tische Fokus in der quantitativen/qualitativen und v.a. quantitativen/quantitativen Relation mehrerer Objekte innerhalb eines nicht direkt beobachtbaren Sachverhalts liegt.

Es manifestieren sich jeweils spezifische standardisierte Repräsentationsanalogien: Es sind die „arbiträren analytischen Darstellungscodes“(Weidenmann 1994) von bzw. die „starken Codes“ (Schnotz 1994) hinter Balken-, Kreis- bis Struktur- oder Ablaufdiagrammen sensu Weidemann (1994), die zwischen Zeichen und Bezeichnetem über Konventionen moderieren und hierbei auf bestimmte graphische Entitäten wie Punkte/Knoten, Linien/Kanten, Formen usw. verweisen; teils sind diese heutzutage durch DIN-Normen ${ }^{10}$ festgelegt. Jene graphischen Entitäten unterliegen andererseits bestimmten räumlichen Anordnungen nach graphischen Schemata und ermöglichen die Identifikation eines Diagramms, einer graphischen Darstellung, einer Karte und eben auch einer Tabelle. So werden in Karten die Linien, Formen, Schraffuren usw. so arrangiert, dass sie die fehlende Räumlichkeit bei analogen, flächigen schematischen Realitätsausschnitten kompensieren. Aber erst durch die spezifischen starken Codes von Karten tragen die bekannten Arrangements jener graphischen Entitäten inhärent die relationalen räumlichzeitlichen Informationen zum repräsentierenden Sachverhalt in sich. Berücksichtigt man sie als Autor/in, so z. B. eine der konventionalisierten Projektionstechniken visuell wahrnehmbarer Räume einhergehend mit besonderen Kartenzeichen, ist es wahrscheinlicher, dass Lesende die Aussage identifizieren können. Starke Codes und graphische Schemata greifen auf jeweils typische graphische Entitäten zurück und sind evolutionäres Ergebnis kultureller Praktiken in spezifischen sozialen Kontexten; eingebunden durch diverse Funktionen, die sie zu erfüllen haben. An dieser Stelle sei nochmals auf die Historie der analogen Kartographie im Spiegel der Sozialisation zu verweisen (Hake et al. 2002; Weidenmann 1994).

\subsubsection{Kategoriale Tabellen: eine ikonische Repräsentation?}

Mit den Kriterien der inhärenten und mehr oder wenigen abstrakten Ähnlichkeit für ikonische Repräsentationen lassen sich einige Strukturrelationen zwischen Zeichen und Bezeichnetem innerhalb kategorialer Tabellen begründet charakterisieren. Diese Parallelen basieren rein auf struktureller Übereinstimmung der darzustellenden Sachverhalte. Jene Übereinstimmung mit v. a. Kreis-, Balken-, und Säulendiagrammen werden offensichtlich, wenn man die gegenwärtige Praxis der computergestützten Gestaltung dieser Diagramme bedenkt. In sogenannten Tabellenkalkulationsprogrammen ordnet man jeweils den zuvor festgelegten qualitativen Kategorien quantitative Werte zu. Man füllt nichts anderes als eine kategoriale Tabelle aus und das Computerprogramm transferiert diese Werte, unter Berücksichtigung der arbiträren Repräsentationsanalogien sowohl einer Tabelle als auch eines Diagramms, in eine alternative nichtkontinuierliche Textform. Dass jener Transfer mit bestimmten Algorithmen überhaupt möglich ist, macht offensichtlich, dass bei beiden nichtkontinuierlichen Texten starke Codes vorliegen müssen, einhergehend mit eindeutigen graphischen Schemata der jeweiligen Entitäten. Insofern kategoriale Tabellen in Kreis-, Balken- und Säulendiagramme wechselseitig direkt übertragbar sind, überschneiden sich gleichsam die Sachverhalte, die sie beschreiben. Für die Variante von Diagrammen mit rein quantitativen Merkmalen, die wie bereits begründet nicht im Fokus dieser semiotischen Betrachtung stehen, begrenzt sich die Überschneidung der Sachverhalte mit tabellarischen Darstellungen: eine Auswahl bestimmter Objekte/Werte. Ob bei Linien- bzw. Streudiagrammen oder skalierten Karten, es werden bei einer entsprechenden mathematischen Tabelle lediglich einzelne Zusammenhänge akzentuiert. Die intendierte Aussage mittels einer mathematischen Tabelle kann, muss aber folglich nicht identisch sein. 
Diese nichtkontinuierlichen Textformen als ikonische Zeichen einzuordnen, wird in den theoretischen Grundlagen der PISA-Studie über weitere Argumentationslinien begründet. So basiert die Festlegung von nichtkontinuierlichen Texten in Differenz zu den kontinuierlichen Texten zum einen auf den formal-strukturellen Analysen von Kirsch/Mosenthal (1989-1990):

[...] wie der Satz die kleinste Organisationseinheit eines kontinuierlichen Textes ist, kann aufgezeigt werden, dass sich alle nichtkontinuierlichen Texte aus einer Reihe von Listen zusammensetzen. Manchmal handelt es sich um eine einzige einfache Liste, in den meisten Fällen jedoch um Zusammenstellungen mehrerer einfacher Listen (OECD 2010).

Unterschiedliche Listen, ergo Tabellen, sind demnach formal-strukturell lediglich eine alternative Repräsentation zu u.a. diversen Diagrammen. Die Einzelanalysen greifen darüber hinaus auch „locative documents“ wie Landkarten, piktionale Abbildungen bis Raumpläne auf und positionieren, dass diese gleichsam auf Listen zurückzuführen sind. Veranschaulichte Kategorien sind entsprechend quantitative Werte von Flächen oder Abständen, aber auch kategoriale von Materialbeschaffenheiten und topologischen Beziehungen (Mosenthal/Kirsch 1998, 649).

Zum anderen stützt sich PISA auf die klassische funktionale Texttypenanalyse sensu Werlich (1975) im Sinne von Kriterien der Textauswahl (OECD 2002, 32ff.; OECD 2010, 43ff.). Nach dem Prinzip der Vertextungsstrategie werden hier Texttypen zusammengefasst, die auf ähnliche Kommunikationssituationen verweisen; konkretisiert für das nichtkontinuierliche Textformat sind es:

- Formulare sind strukturierte und formatierte Texte, in denen Leser spezifische Fragen auf spezifische Weise beantworten sollen. Viele Organisationen verwenden Formulare, um Daten zu erheben. Oft enthalten sie strukturierte oder vorkodierte Antwortformate. Typische Beispiele sind Steuererklärungen, Einwanderungsanträge, Visa-Anträge, Bewerbungsbögen, statistische Fragebögen usw.

- Informationsblätter unterscheiden sich insofern von Formularen, als sie eher der Weitergabe als der Erhebung von Informationen dienen. Sie fassen Informationen strukturiert und formatiert zusammen, sodass der Leser bestimmte Einzelinformationen einfach und schnell finden kann. Informationsblätter können verschiedene Textformen enthalten sowie Listen, Tabellen, Abbildungen und graphisch besonders aufbereitete Textteile (Überschriften, Schrifttypen, Einrückungen, Rahmen usw.), durch die die Informationen zusammengefasst und hervorgehoben werden. Fahrpläne, Preislisten, Kataloge und Programme sind Beispiele für diese Art nichtkontinuierlicher Texte.

- Aufrufe und Anzeigen sind Dokumente, mit denen der Leser aufgefordert wird, etwas zu tun, z. B. Güter oder Dienstleistungen zu kaufen, Versammlungen oder Treffen zu besuchen, eine bestimmte Person für ein öffentliches Amt zu wählen usw. Der Zweck dieser Dokumente ist, die Leser von etwas zu überzeugen. Sie bieten etwas an und fordern sowohl Aufmerksamkeit als auch Handlungen. Beispiele für dieses Format sind Anzeigen, Einladungen, Aufrufe, Warnungen und Bekanntmachungen.

- Gutscheine dienen dem Nachweis, dass ihr Besitzer Anspruch auf bestimmte Dienstleistungen hat. Aus den darin enthaltenen Informationen muss hervorgehen, ob der Gutschein noch gültig ist oder nicht. Typische Beispiele sind Fahrscheine, Rechnungen usw.

- Bescheinigungen sind schriftliche Bestätigungen über die Gültigkeit einer Vereinbarung oder eines Vertrags. Bei ihnen ist eher der Inhalt als das Format formalisiert. Sie müssen von einer oder mehreren autorisierten und kompetenten Personen unterschrieben sein, die den Wahrheitsgehalt der Angaben bezeugen können. Garantien, Schulzeugnisse, Diplome, Verträge usw. sind Dokumente, die diese Eigenschaften aufweisen (ebd., 43ff.; OECD 2002, $31 \mathrm{f}$.). 
Insbesondere innerhalb der in ihrer gesellschaftlichen Funktion konventionalisierten Informationsblätter sowie der Aufrufe und Anzeigen finden sich sowohl kategoriale Tabellen als auch ihre verwandten Diagramme und Karten wieder. Es sind wahrscheinlich eher die technischen Bedingungen als auch der sozio-kulturelle Kontext des Autors und/oder Adressat, ggf. in Kombination mit persönlichen Vorlieben, die für eine Textualisierung in der Struktur einer Tabelle oder einem Diagramm sprechen. In Formularen, Gutscheinen und Bescheinigungen sind dagegen kaum bis gar keine Diagramme oder Karten anzutreffen, stattdessen fast ausschließlich Tabellen.

Die arbiträren Repräsentationsanalogien der Darstellungs-Kodes von Tabellen zwischen repräsentierten und repräsentierenden Merkmalen liegen dabei irgendwo zwischen denen von logischen Bildern und symbolischen Zeichensystemen: Sie konstituieren sich aus spezifisch räumlich angeordneten Feldern (Bezeichnungs- und Textfelder), „an deren Stelle“ auch Piktogramme, Wörter oder Zahlziffern stehen können. Dass in der räumlichen Anordnung der Felder über Konventionen bestimmte Relationen eines Sachverhalts dargelegt werden, verdeutlicht sich bspw. darin, dass jene Bezeichnungs- oder Textfelder nicht expliziert sein müssen. Und dennoch haben die referentiellen Text- oder Bezeichnungsfelder keine beliebige „Bedeutung“. Hierbei handelt es sich um das Spektrum an semantischen Strukturanalogien der verwandten Diagramme, kurzum, um begriffliche, kategoriale, topologische oder Verlaufsstrukturen zwischen Objekten entlang einzelner ihrer Merkmale bzw. Attribute. Exemplarisch verdeutlicht sich jene Relevanz von konventionellen Repräsentationsanalogien entlang der nummeriert gekennzeichneten Spalten in einem textuellen Auszug aus dem aufgefundenen ,Bautagebuch (Abbildung 12) aus einer Einfach(st)erwerbstätigkeit in der Grünflächenpflege:

Das graphische Schemata sieht vor, dass Angaben zum ,Maschineneinsatz je Mitarbeiter/in am Tag X` über die zugehörigen Bezeichnungsfelder (hier der Spalten) ,Personal-Nr.', ,Name', ,Facharbeiter', ,Vorarbeiter ' mit den freien Textfeldern für ,Geräte zusammenhängen. In letzteres haben die Mitarbeiter/ innen das ID-Kürzel jedes elektronischen oder motorisierten Gerätes einzutragen und anzukreuzen, welches sie am entsprechenden Tag X eingesetzt haben. Das ID-Kürzel selbst ist bei jedem dieser Geräte wetter- und belastungsbeständig eingraviert; zudem hängt in der Werkstatt eine wiederum tabellarische Übersicht aller Geräte aus. Funktionaler Hintergrund dieses Auszugs aus dem kategorial-tabellarischen „Bautagebuch` ist, erstens, dass die Beanspruchung einzelner Geräte auch mit Blick auf deren Wartung administriert werden muss. Zweitens, Verantwortlichkeiten können dokumentiert werden, die sich aus der personenbezogenen Nutzung ergeben: Vorarbeiter/innen haben überdies zu gewährleisten, dass der Einsatz der Geräte aller zugeordneter Mitarbeiter/innen sachgemäß erfolgt. Mit der Intention, jenen Sachverhalt der personenbezogenen Nutzung von Geräten an einem bestimmten Tag standardisiert zu textualisieren, sind jene hierarchischen Bezeichnungsfelder für die Spalten und Zeilen gestaltet (Deskription nach Transkription, Mitarbeiter/in in der Gartenpflege; städtischer Bauhof).

Werden in diesem Auszug aus dem ,Bautagebuch bspw. über „ein Kreuz“ in den Textfeldern der hier nicht explizierten Bezeichnungsfelder der ,eingesetzten Geräte` Kategorien von Verantwortlichkeiten dargelegt, so wird hierbei eine semantische Strukturanalogie referiert, die auf einer abstrakten Ähnlichkeit beruht. Unter Berücksichtigung der folgenden angenommenen ,erfahrungsnahen Analogien und räumlichen Metaphern' ist diese als eine mebr oder weniger abstrakte Ähnlichkeit zu konkretisieren: Nach dem Gestaltungsgesetz der gemeinsamen Region liegt es nahe, Elemente als zusammengehörig wahrzunehmen, die in abgegrenzten topologischen oder räumlichen Bereichen angeordnet sind (Inventar in Schränken oder Besteckkästen). Das Gestaltungsgesetz der verbundenen Elemente ist Hinweis dafür, dass verbundene Elemente als ein Objekt wahrzunehmen sind (die Stehlampe mit samt ihren Bestandteilen wie z. B. Netzkabel, Glühbirne, Lampenschirm etc.; Foto sich an den Händen haltender Personen einer Familie). Für die Präferenz der Wahrnehmung von einprägsamen und einfachen Anordnun- 
gen von Elementen spricht das Gestaltungsgesetz der Prägnanz. Letztlich stellen sich kreuzende Bewegungen von zwei Objekten aus einer horizontalen sowie einer vertikalen Richtung eine dieser denkbaren ,erfahrungsnahen Analogien und räumlichen Metaphern' im Sinne von „trifft zusammen" dar.

Bezeichnungsfeld M1: Geräteeinsatz je Mitarbeiterin eines bestimmten Arbeitstages

Bezeichnungsfeld M1.1: Angaben zum Mitarbeiter Bezeichnungsfeld M1.2: eingesetzte Geräte

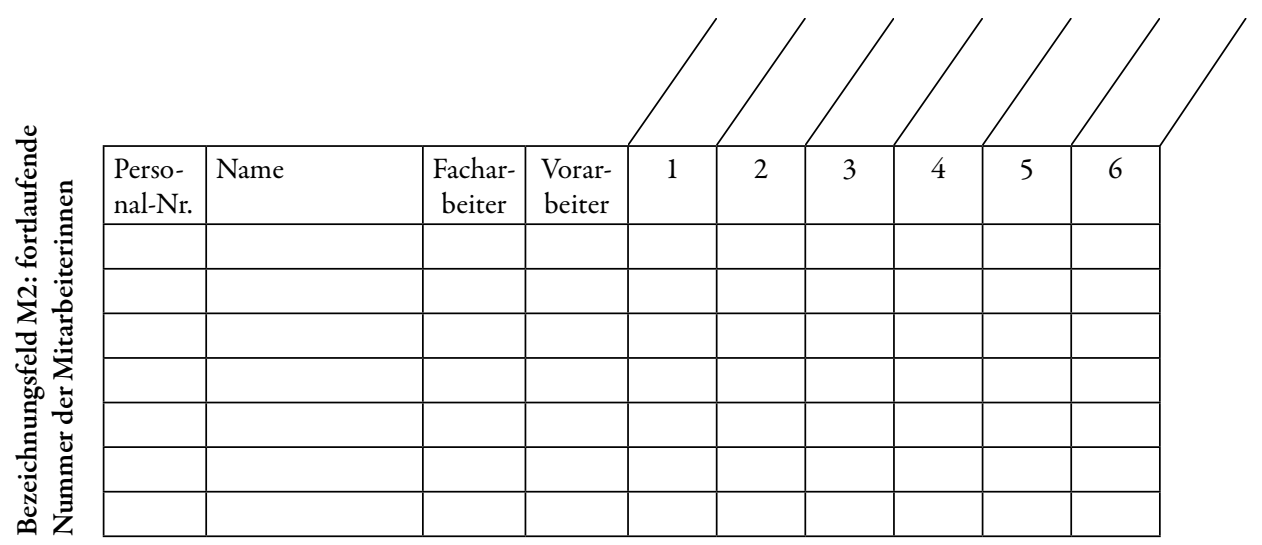

Abb. 12: Bautagebuch in der Gartenpflege (Quelle: Imitation GRAWIRA)

Am ,Bautagebuch` lässt sich überdies illustrieren, dass es sich auch bei kategorialen Tabellen um eine inhärente Ähnlichkeit zwischen Zeichen und Bezeichnetem handelt: Manipuliert man eines der soeben thematisierten „Kreuze“ so werden „automatisch“ auch die dargelegten Verantwortlichkeiten für die einzelnen Geräte manipuliert. Oder in anderen Worten, auch in einer Tabelle wird nicht nur ein Merkmal eines Objekts/Sachverhalts bezeichnet, sondern dieses ist neben weiteren Merkmalen, wenn nicht gar auch Attributen, von Objekten eines Sachverhalts bereits immer inhärent.

Tab. 9: Semiotische Aspekte des Zusammenhangs zwischen Repräsentation und repräsentiertem Sachverhalt bei Tabellen

\begin{tabular}{|c|c|c|c|c|}
\hline Repräsentation & \multicolumn{2}{|c|}{ repräsentierter Sachverhalt } & Isomporhie & Ähnlichkeit \\
\hline $\begin{array}{l}\text { kategoriale Tabellen } \\
\text { (qualitativ/qualitativ) }\end{array}$ & \multirow{3}{*}{$\begin{array}{l}\text { Darstellung eines } \\
\text { visuell nicht } \\
\text { wahrnehmbaren } \\
\text { Sachverhalts }\end{array}$} & $\begin{array}{l}\text { im Sinne terminologischer, } \\
\text { kategorialer, topologischer oder } \\
\text { von Verlaufsstrukturen }\end{array}$ & \multirow[t]{3}{*}{ natürlich } & \multirow[t]{3}{*}{$\begin{array}{l}\text { inhärent; } \\
\text { abstrakte } \\
\text { Ikonizität }\end{array}$} \\
\hline $\begin{array}{l}\text { kategoriale Tabellen } \\
\text { (quantitativ/qualitativ) }\end{array}$ & & $\begin{array}{l}\text { im Sinne von Zusammenhän- } \\
\text { gen zwischen qualitativen und } \\
\text { quantitativen Merkmalen }\end{array}$ & & \\
\hline $\begin{array}{l}\text { mathematische Tabellen } \\
\text { (quantitativ/quantitativ) }\end{array}$ & & $\begin{array}{l}\text { im Sinne von Auszügen aus } \\
\text { Zusammenhängen zwischen } \\
\text { quantitativen Merkmalen }\end{array}$ & & \\
\hline & \multicolumn{2}{|c|}{$\begin{array}{l}\text { eingebunden in Kommunikationsanlässe in der } \\
\text { Form von Formularen, Informationsblättern, Aufru- } \\
\text { fen/Anzeigen, Gutscheinen oder Bescheinigungen }\end{array}$} & & \\
\hline
\end{tabular}


Jener vollzogene Transfer der Darstellungsprinzipien einer inhärent abstrakten Ähnlichkeit lässt sich gleichermaßen für Tabellen nachzeichnen, die Sachverhalte beschreiben, die sich entlang des Zusammenhangs eines quantitativen und eines qualitativen Merkmals vollziehen. In einem anderen Betrieb der Grünflächenpflege wurde eine ähnliche Vorlage ,Bautagebuch genutzt, hier jedoch zur Dokumentation, wie lange welcher/e Mitarbeiter/in in einer Woche an einem bestimmten Auftragsort gearbeitet hat. Anstelle der zusammengesetzten Spalten mit dem Bezeichnungsfeld ,eingesetzte Geräte‘ fand sich lediglich eine „einfache“ Spalte ,Arbeitszeit in Minuten'. Das Mähen großflächiger Wiesen beansprucht mehrere Stunden, ist zudem u. a. wetterabhängig und kann auch daher von mehreren Mitarbeitern/innen anteilig erledigt werden. Wie bei der alternativen Darstellung desselben Sachverhalts mittels z. B. eines Balkendiagramms geht der Abzug der Arbeitszeit von 10 Minuten bei eine/r Mitarbeiter/in in der Tabelle sowohl mit einer um 10 Minuten verringerten Gesamtarbeitszeit einher, als auch mit einer veränderten Relation der Arbeitszeiten der einzelnen Mitarbeiter/innen untereinander, die diese Grünfläche anteilig gemäht haben.

Aus den Analysen zur inhärenten abstrakten Ähnlichkeit bei kategorialen Tabellen geht die Vermutung hervor, dass die dahinterliegende Kodalität letztlich ikonischer Natur ist. Dies wird im Weiteren insofern von Bedeutung sein, um Transfers zum Lesen ikonischer Zeichen anzustellen. Nicht etwa die Wörter in den Bezeichnungsfeldern werden folgerichtig in den Mittelpunkt gestellt, sondern die spezifischen starken Codes hinter kategorialen Tabellen.

\subsubsection{Starke Codes hinter nichtkontinuierlichen Texten}

Starke Codes spiegeln Konventionen in einem Kulturraum wider, die vorgeben, wie Texte mit einer abstrakten Ikonizität verfasst oder gelesen werden können. Sie setzen sozusagen voraus, wie Objekte bzw. Merkmale (und ggf. zusätzlicher Attribute) in Tabellen, Diagrammen und Karten graphisch abgebildet und angeordnet werden können, sodass sie zugleich relative Aussagen zwischen diesen Objekten bzw. Merkmalen (und ggf. zusätzlicher Attribute) inhärent erzeugen. Sie sind es, die es vermuten lassen, dass Lesende jene Ähnlichkeit zwischen dem - zumeist nicht direkt wahrnehmbaren - Sachverhalt einschließlich seiner spezifischen semantischen Strukturanalogien (=Bezeichnetes) und seiner nichtkontinuierlichen Darstellung (=Zeichen) erkennen können.

Während Schnotz oder Weidemann die primär interessierenden kategorialen Tabellen, wie bereits erwähnt, in ihren semiotischen Betrachtungen nicht berücksichtigen, finden sich zu den verwandten Diagrammen mit rein qualitativen bzw. quantitativen/quantitativen Aussagen Charakterisierungen der dahinterliegenden starken (Darstellungs-)Codes. Entlang von unterschiedlich komplexen Strukturdiagrammen eröffnet sich, ohne einen Anspruch auf Vollständigkeit, jedoch die Möglichkeit, die arbiträren Repräsentationsanalogien zwischen Zeichen und Bezeichnetem in kategorialen Tabellen im Anschluss abzuleiten.

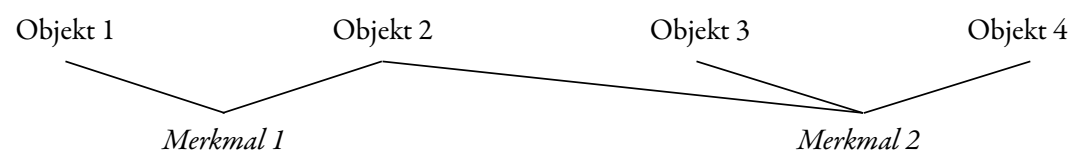

Abb. 13: Starke Codes in einem Strukturdiagramm (4. Ebene)

Streudiagramme bestehen formal betrachtet „aus Knoten und aus ungerichteten und gerichteten Kanten“ (ebd., 97). In einem „einfachen“ Streudiagramm (Abbildung 13) werden die Kno- 
ten durch z. B. Piktogramme, Wörter oder Zahlziffern dargestellt und repräsentieren Begriffe oder Sachverhalte. Mittels ungerichteter Kanten werden sodann die intendierten terminologischen, kategorialen oder topologischen Relationen mit den Merkmalen erzeugt. Zudem bedingt das Prinzip offener Knoten, dass es keine festgelegte Schreib- und Leserichtung gibt. Man kann ausgehend von einem der beiden Merkmale entnehmen, welche Objekte zugeordnet sind; die repräsentierten Relationen verändern sich im Zuge einer umgekehrten Leserichtung nicht. Auch kann die topologische Anordnung der Objekte und Merkmale variieren.

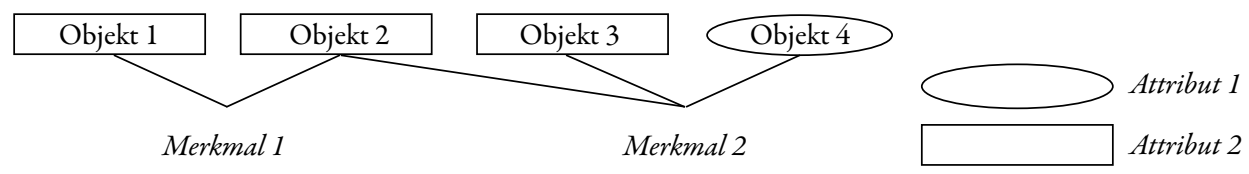

Abb. 14: Starke Codes in einem Strukturdiagramm (2. Ebene)

Bei einem komplexeren Streudiagramm (Abbildung 14) setzen sich die Knoten neben ihrer Objektrepräsentation zusätzlich aus Ellipsen, Rechtecken oder anderen geometrischen Formen zusammen. Jene Ergänzung ermöglicht es, die Objekte durch Attribute weiter zu differenzieren. Auf diese Weise können z.B. Unter- und Oberkategorien für terminologische, kategoriale oder topologische Zusammenhänge beschrieben werden. Die ungerichteten Kanten erzeugen wiederum keine vorgegebene Schreib- und Leserichtung. Eine Legende kann die Darstellung ergänzen, ist allerdings nicht zwingend Bestandteil zur Repräsentation des relationalen Sachverhalts auf zwei Ebenen und kann folglich nicht dem Gestaltungsprinzip eines starken Codes zugeordnet werden.
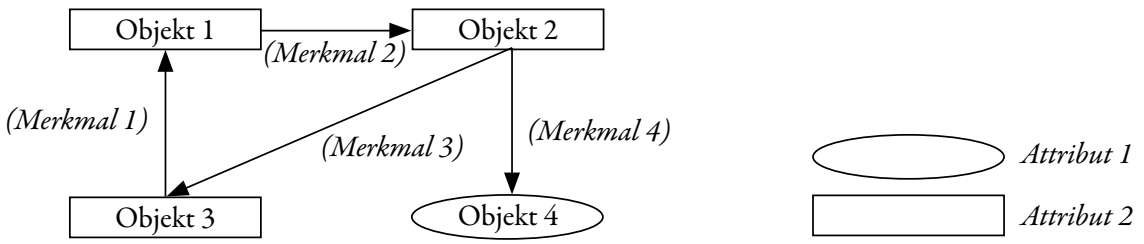

Abb. 15: Starke Codes in einem Strukturdiagramm (3. Ebene)

Gerichtete Kanten zwischen den Knoten sollen beim Rezipienten den Eindruck von Relationen zwischen Objekten eines Sachverhalts erwecken; in „unseren“ sozial literalen Praxen hat sich dieses Streudiagramm insbesondere zur Repräsentation von topologischen Strukturen innerhalb eines sozialen, biologischen oder technischen Systems durchgesetzt. Prozesskomponenten können durch gerade oder bogenförmige Pfeile berücksichtigt werden. Infolgedessen geben sie bestimmte Leserichtungen des nichtkontinuierlichen Textes vor, die nicht unbedingt mit dem „von links oben nach rechts unten“ der deutschen Schrift übereinstimmen müssen. Terminologische oder kategoriale Zusammenhänge zwischen den Objekten werden durch geometrische Formen integriert. Merkmale der Prozesskomponenten können durch Bezeichnungen in Wort oder mittels Piktogramme einfließen; sie führen, hinsichtlich der repräsentierten Relationen auf drei Ebenen, über zu der komplexesten Struktur eines Streudiagramms. Insofern diese Beschreibung räumlich eindeutig am nächsten an der gerichteten Kante visualisiert wird, kann der/die Lesende nach dem Gesetz der Nähe dieses als Merkmal der entsprechenden Prozesskomponente entnehmen. 
Angesichts dessen, dass verschieden komplexe Diagramme Repräsentationen von Sachverhalten mit Relationen auf zunehmend mehr Ebenen umfassen können, wird im Weiteren von einem jeweils spezifischen Set von starken Codes ausgegangen. Die Anzahl der berücksichtigenden Objekte, Merkmale und Attribute kann ansteigen, beansprucht aber weiterhin dieselben Konventionen von Zeichen und Bezeichnetem einerseits und der Anforderung der graphischen Entitäten andererseits: den sogenannten graphischen Schemata. Unabhängig der symbolischen oder ikonischen Beschriftungen der Objekte selbst zählen zu dem Set starker Codes von Strukturdiagrammen die Kombinationen aus Knoten und Kanten. Je nach Komplexität der darzustellenden Beziehungen zwischen Objekten sowie der dahinter liegenden relationalen Merkmale müssen graphisch angeordnete geometrische Formen und Kantenbeschriftungen ergänzt werden; unter Einhaltung von spezifischen Konventionen. Sie erlauben es erst, die terminologischen, kategorialen Beziehungen diverser Objekte einerseits und deren topologischen Beziehungen andererseits zu beschreiben. Zudem sind die Lese-/Schreibrichtungen den Streudiagrammen innerhalb der Gestalt von gerichteten und ungerichteten Kanten der Abbildung inhärent.

In Tabelle 10 werden weitere spezifische Sets von starken Codes von Diagrammen zusammengetragen (Schnotz 1994), wobei aus der eher geringen textuellen Relevanz auf Varianten für rein quantitative Zusammenhänge verzichtet wird. Hierbei verdeutlichen sich u. a. innerhalb von Balken- oder Säulendiagramme die Bandbreite an möglichen Repräsentationen für terminologische und kategoriale Relationen zwischen den dargestellten Objekten mittels z. B. Schraffierung, Farbe, Bezeichnungen (Wörter, Piktogramme) oder im Sinne von Venn-Diagrammen. Auch bedürfen die Attribute der quantitativen Relationen nicht zwangsweise eine entsprechend skalierte Achse, alternativ können Bezeichnungen (Prozentwerte, Maßzahlen und mathematische Größen, usw.) auch diesen Zweck erfüllen. Sie sollten daher nicht den spezifischen starken Codes subsumiert werden.

Die diversen graphischen Schemata, ergo die konventionellen Anorderungen von durch Sets starker Codes vorgegebener graphischer Entitäten, vermitteln in einigen Fällen zumindest eine Funktionsanalogie zum dargestellten Sachverhalt. Unabhängig der Strukturanalogien zwischen Zeichen und Bezeichnetem erinnern Kreisdiagramme mit ihren Sektoren einer skizzierten Darstellung aufgeschnittener Gegenstände mit Zylinderform. Auch mittels Pfeilen (gerichtete Kanten) kann man jene „Einbindung von erfahrungsnahe[n] Analogien und räumliche[n] Metaphern" in einzelnen Diagrammen positionieren (Weidenmann 1994, 22).

Die vorgenommene Betrachtung der Sets von starken Codes der Strukturdiagramme entlang der Graden an Komplexität von Abbildung inhärenten Relationen erinnert an die aus der formalstrukturellen Analyse von Tabellen bekannten Hierarchie der Listenstruktur sensu Mosenthal und Kirsch (Mosenthal/Kirsch 1998). Eine einzelne einfache Liste, eine „simple-list“, ist hierbei dadurch gekennzeichnet, dass mehrere einem Merkmal zugehörige Objekte eingetragen oder einzutragen sind; nach dem Gesetz der Nähe in einer senkrechten oder waagerechten Ausrichtung. Im Weiteren werden gemäß Mosenthal und Kirsch die senkrechten kategorialen Tabellen weiter thematisiert. Das Set von starken Codes hinter dem waagerechten Pendant kann direkt übertragen werden. Über diese Anordnung der Objekte werden Felder generiert, die weder ein Bezeichnungsfeld, ergo den Verweis auf das gemeinsame Merkmal mittels z.B. eines Wortes oder Piktogramms, noch Linien zur Begrenzung der Felder untereinander bedürfen. Sie formieren das graphische Schemata dieser Tabelle, welche als To-do-Liste u. a. im Verkauf oder am Arbeitsplatz der Beiköche/Kochhilfen innerhalb der betrachteten Einfach(st)erwerbstätigkeiten vereinzelt aufgefunden werden konnten. Im ersten Fall war diese Tabelle Teil der Anleitung zum Aufbau eines Verkaufsarrangements nebst einem Foto bzw. mehreren Fotos. Die unterei- 
nander aufgeführten Materialien waren zu beschaffen bzw. bereitzulegen, um die anhand von Fotos visualisierte Produktpräsentation nachzubauen.

Tab. 10: Set starker Codes hinter Diagrammen (qualitativ/qualitativ; qualitativ/quantitativ)

\begin{tabular}{|c|c|c|c|}
\hline Repräsentation & Set $s$ & starker Codes & Leserichtung \\
\hline $\begin{array}{l}\text { Struktur- } \\
\text { diagramme }\end{array}$ & $\begin{array}{l}\text { - Knoten (Objekte) } \\
\text { - gerichtete/ungerichtete Kanten } \\
\text { (terminologische, kategoriale, } \\
\text { topologische Relationen) }\end{array}$ & $\begin{array}{l}\text { - geometrische Formen: Merkmale der } \\
\text { Objekte (Knoten) } \\
\text { - Beschriftung nach dem Gesetz der Nähe: } \\
\text { Attribute der Relationen (Kanten) }\end{array}$ & $\begin{array}{r}\text { - inhärent } \\
\text { (Kanten) }\end{array}$ \\
\hline $\begin{array}{l}\text { Ablauf- und } \\
\text { Flussdia- } \\
\text { gramme }\end{array}$ & $\begin{array}{l}\text { - Knoten (Systemzustände) } \\
\text { - gerichtete Kanten (Relatio- } \\
\text { nen: Verlaufsstrukturen) }\end{array}$ & $\begin{array}{l}\text { - geometrische Formen: Merkmale der Sys- } \\
\text { temzustände (Knoten); Konventionen } \\
\text { für Anfangs-, End-, Entscheidungs- und } \\
\text { Operationszustände } \\
\text { - Beschriftung nach dem Gesetz der } \\
\text { Nähe: Attribute der Relationen } \\
\text { (Kanten) }\end{array}$ & $\begin{array}{l}\text { - inhärent } \\
\text { (i.d.R. von } \\
\text { oben links } \\
\text { nach unten } \\
\text { rechts) }\end{array}$ \\
\hline $\begin{array}{l}\text { Venn- } \\
\text { Diagramme }\end{array}$ & $\begin{array}{l}\text { - geometrische Formen (Objekt), } \\
\text { menge, Vereinigungsmenge, Dif } \\
\text { Repräsentation von Inklusions- } \\
\text { Objekten; folglich entlang eines }\end{array}$ & $\begin{array}{l}\text {, angeordnet nach dem Prinzip der Schnitt- } \\
\text { ifferenzmenge, Symmetrische Differenz zur } \\
\text { - und Exklusionsbeziehungen zwischen den } \\
\text { s Merkmals }\end{array}$ & - beliebig \\
\hline $\begin{array}{l}\text { Kreisdia- } \\
\text { gramme }\end{array}$ & $\begin{aligned} \text { - Kreissektoren (Objekte) } \\
\text { - Anteilige Fläche eines Kreissek } \\
\text { Häufigkeiten, Rangfolgen) }\end{aligned}$ & stors (quantitative Relation: Verteilung, & - beliebig \\
\hline $\begin{array}{l}\text { Balken-/ } \\
\text { Säulen- } \\
\text { diagramme }\end{array}$ & $\begin{array}{l}\text { - Balken waagerecht/Säulen } \\
\text { senkrecht (Objekte) } \\
\text { - Höhe/Breite der Balken/Säulen } \\
\text { von einem waagerechten/senk- } \\
\text { rechten Startpunkt (quantitative } \\
\text { Relation: Verteilung, Häufigkei- } \\
\text { ten, Rangfolgen, Zeitreihen) }\end{array}$ & $\begin{array}{l}\text { - Sonderform Abweichungsdiagramm: } \\
\text { Differenzierungsmerkmale werden } \\
\text { durch entgegengesetzte Ausrichtung } \\
\text { der Balken/Säulen dargestellt (z.B. } \\
\text { Verlust zu Gewinn) }\end{array}$ & $\begin{array}{l}\text { - inhärent } \\
\text { (waagerecht } \\
\text { senkrecht) }\end{array}$ \\
\hline $\begin{array}{l}\text { Iso-Typ- } \\
\text { Diagramme }\end{array}$ & $\begin{array}{l}\text { - Piktogramme (Objekte) } \\
\text { - Anzahl der Piktogramme nach } \\
\text { tion: Verteilung, Häufigkeiten, }\end{array}$ & $\begin{array}{l}\text { dem Gesetz der Nähe (quantitative Rela- } \\
\text {, Rangfolgen, Zeitreihen) }\end{array}$ & $\begin{array}{l}\text { - inhärent } \\
\text { (waagerecht } \\
\text { senkrecht) }\end{array}$ \\
\hline
\end{tabular}

Werden mehrere „simple-lists“ auf einer Seite abgebildet, so entsteht eine „list-of-simple-lists“ (Abbildung 16), insofern die Merkmale hinter den in Feldern angeordneten Objekten in keinem Zusammenhang stehen. Es resultiert, dass in beiden Varianten hinter der Reihenfolge der Objekte keine Relationen verborgen liegen.

\begin{tabular}{|c|c|c|c|c|}
\hline (Merkmal) & (Merkmal 1) & (Merkmal 2) & (Merkmal 3) & (Merkmal 4) \\
\hline Objekt 1 & Objekt 1 & Objekt 5 & Objekt 9 & Objekt 13 \\
\hline Objekt 2 & Objekt 2 & Objekt 6 & Objekt 10 & Objekt 14 \\
\hline Objekt 3 & Objekt 3 & Objekt 7 & Objekt 11 & Objekt 15 \\
\hline Objekt 4 & Objekt 4 & Objekt 8 & Objekt 12 & Objekt 16 \\
\hline
\end{tabular}

Abb. 16: Starker Kode hinter einer (senkrechten) simple-list bzw. list-of-simple-lists (4. Ebene) (Quelle: adaptiert nach Mosenthal/Kirsch 1998) 
Auch vereinzelt sind die sogenannten „combined-lists“ (Abbildung 17) als primäre graphische Schemata an den untersuchten Einfach(st)arbeitsplätzen aufzufinden. Sie unterscheiden sich von den „list-of-single-lists“ darin, dass die Merkmale, nach denen die Objekte zugeordnet werden, untereinander nach einem weiteren Attribut in Beziehung stehen. So lag z. B. im Service in der Gastronomie eine Vorlage den Mitarbeitern vor, der sie wichtige Vorbereitungen vor den Mittag- und Abendöffnungszeiten entnehmen können: Die erste Spalte enthielt Vorgaben zu den Vorbereitungen vor Öffnung des Gastraums zur Reinigung bzw. der Dekoration, der Bestuhlung und der Bedeckung der Tische für den Mittag und die zweite analog für den Abend. „Combined-lists" können auch innerhalb von Formularen eingebunden sein, wie die Tabelle zum Eintrag von ,Kennzeichen', ,CO-Datum` usw. in der Wagenpflege (Abbildung 11).

\begin{tabular}{llll}
\hline & \multicolumn{2}{c}{ (Attribut 1) } & \\
Merkmal 1 & Merkmal 2 & Merkmal 3 & Merkmal 4 \\
\hline Objekt 1.1 & Objekt 2.1 & Objekt 3.1 & Objekt 4.1 \\
Objekt 1.2 & Objekt 2.2 & Objekt 3.2 & Objekt 4.2 \\
Objekt 1.3 & Objekt 2.3 & Objekt 3.3 & Objekt 4.3 \\
Objekt 1.4 & Objekt 2.4 & Objekt 3.4 & Objekt 4.4 \\
\hline
\end{tabular}

Abb. 17: Starker Kode hinter einer (senkrechten) combined-list (2. Ebene) (Quelle: adaptiert nach Mosenthal/Kirsch 1998)

Exemplarisch verdeutlicht sich, dass die Reihenfolge der Objekte weiterhin nicht Teil der abgebildeten Relation darstellt. Wenn vor der Mittagszeit zuerst der Eingangsbereich gekehrt wird, ergibt sich hieraus nicht unmittelbar, dass auch vor der zweiten Öffnungszeit wiederum dieser Vorgabe zuerst genüge getan werden muss. Auch bedarf es nicht mindestens zweier Objekte je Merkmal, um von einer „combined-list“ zu sprechen, sodass Abfragen von Personenmerkmalen zu einem typischen Beispiel dieser Listenstruktur werden. Zur Differenzierung von der „list-ofsimple-lists" hat sich bewährt, dass die Merkmale durch die Bezeichnungsfelder benannt werden. Mittels z. B. Wörtern, Piktogrammen usw. wird auf die Merkmale verwiesen. Das diesen Merkmalen übergeordnete Attribut bedarf dagegen nicht zwingend einer Benennung. Mit den nicht notwendigen Linien zur Abgrenzung der erzeugten Felder zählen die Bezeichnungsfelder des übergeordneten Attributs weiterhin nicht zum Set von starken Codes hinter Tabellen. Weitaus häufiger fanden sich die sogenannten ,intersected-lists“ (Abbildung 18) in den textuellen Anforderungen an Einfach(st)erwerbstätigkeiten wieder. In ihnen tauchen erstmalig die Zusammenhänge der Felder aus Spalten und Zeilen in dem Sinne auf, dass die Reihefolge der Objekte je Merkmal beim Verfassen und Lesen berücksichtigt werden muss. In senkrechten kategorialen Tabellen erfüllt in der Regel die linke Spalte diesen Zweck. Preisaushänge, Speiseoder Getränkekarten ordnen einer Dienstleistung oder Ware genau einen Preis zu. Einigen Listen lässt sich der spezifische Code von Geräten, Zubehörteilen, usw. entnehmen; sie dienen häufig als Referenz in weiteren Tabellen. Aber auch die unter der Überschrift ,Funktionsprüfung und Ausstattung/Zubehör' geführte Tabelle innerhalb des Formulars ,Fahrzeugprotokoll' (Abbildung 11) in der Tätigkeit der Wagenpflege ist eine solche „intersected-list“; hier als sekundäres graphisches Schemata als Teil des Formulars eingebettet. Das Attribut, hier im Grunde ein Verweis auf das bipolare Verhältnis zwischen den Merkmalen ,ja und ,nein', muss, wie das Merkmal für die Zeilenrelation nicht in jedem Fall durch Wörter, Piktogramme oder (ordinalen, kardinalen, ...) Zahlziffern ausgeführt werden. Dass man jenes Bezeichnungsfeld 
nicht benötigt und dennoch mittels der starken Codes aus Zeile zu Spalte die Objekte zuordnen kann, lässt vermuten, dass sich jene Tabellen durch zahlreiche Praktiken verstetigen konnten.

\section{(Attribut 1)}

\begin{tabular}{cccc} 
(Merkmal 1$)$ & Merkmal 2 & Merkmal 3 & Merkmal 4 \\
\hline Objekt 1 & Objekt $1 \times 2$ & Objekt $1 \times 3$ & Objekt $1 \times 4$ \\
Objekt 2 & Objekt $2 \times 2$ & Objekt $2 \times 3$ & Objekt $2 \times 4$ \\
Objekt 3 & Objekt $3 \times 2$ & Objekt $3 \times 3$ & Objekt $3 \times 4$ \\
Objekt 4 & Objekt $4 \times 2$ & Objekt $4 \times 3$ & Objekt $4 \times 4$ \\
\hline
\end{tabular}

Abb. 18: Starker Kode hinter einer (senkrechten) intersected-list (3. Ebene) (Quelle: adaptiert nach Mosenthal/ Kirsch 1998)

Folgt die Anordnung der Objekte nicht nur in Zeilen und Spalten, sondern zugleich verschränkt im Zuge eines den Merkmalen übergeordneten Attributs, liegt nach Mosenthal und Kirsch die komplexeste Listenstruktur vor. Um sich diese Besonderheit der Verschränkung der Objekte in „nested-lists“ zu vergewissern, kann der aufgefundene Arbeitsplan in der Gastronomie (Abbildung 19) herangezogen werden. Wie in einer ,intersected-list“ sind hier jedem/r Mitarbeiter/in (linke Spalte) an bestimmten Tagen (weitere Spalten) Einsatzzeiten zugeordnet. Diese Einsatzzeiten sind dem Umstand geschuldet, dass es an den einzelnen Wochentagen einen bestimmten Personalbedarf in den unterschiedlichen Schichten in der Küche sowie im Service an der Bar bzw. in den einzelnen Gasträumen gibt. Jenes Attribut der Verschränkung gilt es zu bedenken, falls Mitarbeiter/innen einmal ihre Einsatzzeiten (Objekte) tauschen möchten bzw. müssen.

\section{Zeitplan (30.6. - 12.7.) \\ Sa So}

Sa So

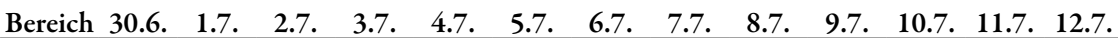

\begin{tabular}{|c|c|c|c|c|c|c|c|c|c|c|c|c|c|c|}
\hline nne & $S$ & $\mathrm{f}$ & $\mathrm{f}$ & $\mathrm{f}$ & $17-1$ & $17-1$ & $9-15$ & $\mathrm{f}$ & $\mathrm{f}$ & $\mathrm{f}$ & $\mathrm{f}$ & $17-1$ & $17-1$ & $9-1$ \\
\hline Brigitte & $S$ & $\mathrm{f}$ & $\mathrm{f}$ & $\mathrm{f}$ & $9-15$ & $9-15$ & $9-15$ & $\mathrm{f}$ & $\mathrm{f}$ & $\mathrm{f}$ & $9-15$ & $9-15$ & $9-15$ & $\mathrm{f}$ \\
\hline hristine & $\mathrm{K}$ & -14 & $5-14$ & $5-14$ & -14 & $\mathrm{f}$ & $\mathrm{f}$ & $5-14$ & $5-14$ & $5-14$ & $5-14$ & $\mathrm{f}$ & $\mathrm{f}$ & $5-1$ \\
\hline ena & $S$ & $17-1$ & $\mathrm{f}$ & $\mathrm{f}$ & $17-1$ & $17-1$ & $17-1$ & $17-1$ & $\mathrm{f}$ & $\mathrm{f}$ & $17-1$ & $17-1$ & $17-1$ & $17-1$ \\
\hline oria & B & $\mathrm{f}$ & $\mathrm{f}$ & s & $17-1$ & $17-1$ & $\mathrm{f}$ & $\mathrm{f}$ & $\mathrm{f}$ & $\mathrm{f}$ & $\mathrm{f}$ & $17-1$ & $17-1$ & $\mathrm{f}$ \\
\hline ori & $S$ & $17-1$ & $\mathrm{f}$ & $\mathrm{f}$ & $\mathrm{f}$ & $\mathrm{f}$ & $17-1$ & $17-1$ & 1 & $\mathrm{f}$ & 1 & $\mathrm{f}$ & $17-1$ & $17-1$ \\
\hline $\mathrm{anz}$ & S & $17-1$ & $17-1$ & $17-1$ & $\mathrm{f}$ & $\mathrm{f}$ & $17-1$ & $17-1$ & $17-1$ & $17-1$ & $\mathrm{f}$ & $\mathrm{f}$ & $17-1$ & $17-1$ \\
\hline auk & $S$ & $\mathrm{f}$ & $17-1$ & $17-1$ & $17-1$ & $17-1$ & $\mathrm{f}$ & $\mathrm{f}$ & $17-1$ & $17-1$ & $17-1$ & $17-1$ & $\mathrm{f}$ & $\mathrm{f}$ \\
\hline$n$ & B & $17-1$ & $\mathrm{f}$ & $\mathrm{f}$ & $\mathrm{f}$ & $\mathrm{f}$ & $\mathrm{f}$ & $17-1$ & $17-1$ & $\mathrm{f}$ & $\mathrm{f}$ & $\mathrm{f}$ & f & $f$ \\
\hline laus & $\mathrm{K}$ & 5-14 & $5-14$ & $\mathrm{I}$ & $\mathrm{f}$ & 5-14 & 5-14 & 5-14 & $5-14$ & $\mathrm{f}$ & $\mathrm{f}$ & 5-14 & 5-14 & $5-14$ \\
\hline icha & $\mathrm{K}$ & $14-23$ & $\mathrm{f}$ & $14-23$ & $\mathrm{f}$ & $14-23$ & $\mathrm{f}$ & $14-23$ & $\mathrm{f}$ & $14-23$ & $\mathrm{f}$ & $14-23$ & $\mathrm{f}$ & $14-2$ \\
\hline ira & $S$ & $9-15$ & $9-15$ & $9-15$ & $\mathrm{f}$ & $\mathrm{f}$ & $\mathrm{f}$ & $9-15$ & $9-15$ & $9-15$ & $\mathrm{f}$ & $f$ & $\mathrm{f}$ & $9-15$ \\
\hline irja & $\overline{\mathrm{K}}$ & $14-23$ & $14-23$ & $14-23$ & $14-23$ & $\mathrm{f}$ & $\mathrm{f}$ & $14-23$ & $14-23$ & $14-23$ & $14-23$ & $\mathrm{f}$ & f & $14-23$ \\
\hline ter & $\mathrm{K}$ & $\mathrm{f}$ & $14-23$ & $f$ & $14-23$ & $14-23$ & $14-23$ & $\mathrm{f}$ & $14-23$ & $\mathrm{f}$ & $14-23$ & $14-23$ & $14-23$ & $\mathrm{f}$ \\
\hline id & $S$ & $f$ & $f$ & $\mathrm{f}$ & $9-15$ & $9-15$ & $9-15$ & 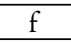 & $\mathrm{f}$ & $\mathrm{f}$ & $9-15$ & $9-15$ & $9-15$ & $\mathrm{f}$ \\
\hline bas & & $\mathrm{f}$ & $\mathrm{f}$ & $\mathrm{f}$ & $\mathrm{f}$ & $\mathrm{f}$ & $\mathrm{f}$ & $\mathrm{f}$ & $\mathrm{f}$ & $\mathrm{f}$ & $\mathrm{f}$ & $\mathrm{f}$ & $\mathrm{f}$ & $f$ \\
\hline tan & K & $\mathrm{f}$ & f & $5-14$ & $5-14$ & $5-14$ & $5-14$ & $\mathrm{f}$ & t & 5-14 & $5-14$ & $5-14$ & $5-14$ & $\mathrm{f}$ \\
\hline nja & $S$ & $9-15$ & $9-15$ & $9-15$ & $f$ & $\mathrm{f}$ & $\mathcal{f}$ & $9-15$ & $9-15$ & $9-15$ & $\mathrm{f}$ & $\mathrm{f}$ & $\mathrm{f}$ & $9-1$ \\
\hline mas & & $\mathcal{f}$ & $\mathrm{f}$ & $\mathrm{f}$ & $17-1$ & $17-1$ & f & 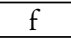 & 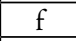 & $\mathrm{f}$ & $\mathrm{f}$ & $17-1$ & $17-1$ & \\
\hline & B & $\mathrm{f}$ & $17-1$ & $17-1$ & $\mathrm{f}$ & $\mathrm{f}$ & & $\mathrm{f}$ & & $17-1$ & $17-1$ & 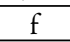 & 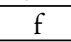 & \\
\hline & & 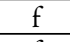 & $17-1$ & $17-1$ & $17-1$ & $17-1$ & $\mathrm{f}$ & $\mathrm{t}$ & $17-1$ & $17-1$ & $17-1$ & $17-1$ & 1 & $\mathrm{f}$ \\
\hline orben & B & $\mathrm{f}$ & $\mathrm{f}$ & $\mathrm{f}$ & $17-1$ & $17-1$ & $\mathrm{f}$ & $\mathrm{f}$ & $\mathrm{f}$ & $\mathrm{f}$ & $\mathrm{f}$ & $17-1$ & \begin{tabular}{|l|}
$17-1$ \\
\end{tabular} & $\mathrm{f}$ \\
\hline
\end{tabular}

\begin{tabular}{c|c|c|c|c|}
\hline $9-15$ & \multicolumn{2}{|c|}{ Türseite \& Mitte \& Buffet } & \multicolumn{2}{c|}{ Stammtischseite \& Terrasse } \\
\hline $17-1$ & Türseite \& Mitte & Stammtischseite \& Buffet & Terrasse \\
\hline
\end{tabular}

Abb. 19: Arbeitsplan in der Gastronomie (Quelle: Basendowski 2013; Imitation) 
Weitere typische Beispiele von „nested-lists“ sind tabellarische Auflistungen nach ,Produkten“, ,Anzahl', ,Stückpreis‘ und ,Gesamtpreis'. Verschränkt sind hier nicht nur die offensichtlichen Gesamtpreise aus Anzahl und Stückpreis, sondern zugleich der Stückpreis mit einem einzelnen Produkt. Jene „nested-lists“ sind konsequenterweise nicht auf eine analoge Repräsentation, oder in anderen Worten, auf eine Seite begrenzt. Wenn innerhalb des bereits besprochenen ,Bautagebuchs' (vgl. Abbildung 12) die Codes der eingesetzten Geräte in der Grünflächenpflege einer zweiten Tabelle entnommen werden müssen, fusionieren beide nach Mosenthal und Kirsch zu jener komplexesten Listenform. Das Attribut der Verschränkung umfasst entsprechend diverse Relationen und darunter bei weitem nicht ausschließlich mathematisch-operationale.

Die in Abbildung 20 den beiden Autoren entlehnte formal-strukturelle Listenstruktur von „nested-lists“ ist konsequenterweise nicht derart zu interpretieren, dass die konventionelle Anordnung seiner Entitäten auf einer Seite zu erfolgen hat; gleiches gilt für die zuvor besprochenen weniger komplexen Tabellen. Der Skizzierung des spezifischen Sets starker Codes berücksichtigt wieder, dass diverse Bezeichnungsfelder nicht zwingend notwendig sind, um die repräsentierten Relationen eines Sachverhalts zu textualisieren bzw. zu lesen. Dies führt zweifelsohne zu einem bekannten Problem bei nested-lists, wenn sozusagen selbstsagend davon ausgegangen wird, dass der,Geräte-Kode‘ sich durch den Aushang erst zu einem Text ergänzt, oder dass der,Gesamtpreis sich aus ,Produkt', ,Anzahl', ,Stückpreis‘ unter Verweis auf die Tabelle Produkt-Stückpreis ergibt. Die Tabellen inhärente Leserichtung kann in diesem Zuge nicht auf eine Seite begrenzt bleiben, sondern kommt am ehesten einem Hypertext nahe, ergo einem Text mit nicht-linearem Aufbau.

\begin{tabular}{|c|c|c|c|c|}
\hline \multirow[b]{3}{*}{ (Merkmal1) } & \multicolumn{4}{|c|}{ (Bezeichnungsfeld) } \\
\hline & \multicolumn{2}{|c|}{ (Attribut 1) } & \multicolumn{2}{|c|}{ (Attribut 2) } \\
\hline & (Merkmal 2) & (Merkmal 3) & (Merkmal 4) & (Merkmal 5) \\
\hline Objekt 1 & Objekt $1.1 \times 2$ & Objekt $1.1 \times 3$ & Objekt $2.1 \times 4$ & Objekt $2.1 \times 5$ \\
\hline Objekt 2 & Objekt $1.2 \times 2$ & Objekt $1.2 \times 3$ & Objekt $2.2 \times 4$ & Objekt $2.2 \times 5$ \\
\hline Objekt 3 & Objekt $1.3 \times 2$ & Objekt $1.3 \times 3$ & Objekt $2.3 \times 4$ & Objekt $2.3 \times 5$ \\
\hline Objekt 4 & Objekt $1.4 \times 2$ & Objekt $1.4 \times 3$ & Objekt $2.4 \times 4$ & Objekt $2.4 \times 5$ \\
\hline
\end{tabular}

Abb. 20: Starker Kode hinter einer (senkrechten) nested-list (4. Ebene)

\subsubsection{Semiotische Betrachtung spezifischer Sets ikonischer Darstellungs-Kodes}

Mehrfach wurde seither postuliert, dass die Texttypen ,Formulare' und ,Gutscheine' nichtkontinuierlicher Texte kategorial-tabellarisch aufgebaut sind. Mit dem erarbeiteten Instrumentarium unterschiedlicher Sets ikonischer Codes soll nicht nur der Versuch unternommen werden, jenes Postultat zu bekräftigen. Vielmehr dienen die Transfers der Annäherung an die spezifischen textuellen Anforderungen jener Texttypen. ,Informationsblätter', die zweifelsohne gleichermaßen häufig in den untersuchten Einfach(st)erwerbstätigkeiten aufgefunden wurden, werden hier nicht weiter betrachtet, da sie sich nahezu in allen Fällen aus nur einer Tabelle, einem Diagramm, einer Karte oder einer graphischen Darstellung zusammensetzen, gepaart mit den (expliziten) sprachlichen Steuerungs-Kodes in der Form einer Überschrift und Legende. Die aufgefundenen ,Formulare' und ,Gutscheine' setzen sich dagegen aus Kombinationen zusammen und können daher als einseitige nichtkontinuierliche Textkomplexe betrachtet werden.

Formulare im Sinne von analogen Kopiervorlagen, die innerhalb von Arbeitsprozessen wiederkehrend ausgefüllt werden müssen, treten in jedem untersuchten Berufsfeld auf: Die Mitarbeiter/innen in der Grünflächenpflege haben ihre Tätigkeiten bzw. die eingesetzten Geräte mit 
einem ,Bautagebuch' (Abbildung 12) zu dokumentieren, in der Wagenpflege den Funktionszustand eines Fahrzeugs mittels eines ,Fahrzeugprotokolls (Abbildung 11) oder in der Möbelmontage eine zu bearbeitende Reklamation durch eine ,Serviceanforderung' (Abbildung 24). Ein Formular wird u. a. in der Kantine eingesetzt, um die Temperatur jeder hergestellten Sauce festzuhalten, bevor sie ausgeliefert wird. Wie bereits der Deskription der Funktion von Formularen zu entnehmen war, werden hier, strukturiert' und ,formatiert' über ,spezifische Fragen' „Daten' erhoben. Dass in diesen sich wiederholenden Arbeitsprozessen nicht jeweils auf schriftlich ausgeschriebene Fragen mit erklärendem Fließtext gesetzt wird, kann durch deren Bedingungen der Ökonomisierung und des Controllings, dem ,berechnenden 'Überwachen der Arbeits- oder Produktionsleistung usw., begründet werden. Formulare bedienen diesen Zweck im hohen Maße, repräsentieren sie semantisch fokussiert die jeweils interessierenden Merkmale und Attribute des Sachverhalts.

Tab. 11: Spezifische ikonische Codes in Formularen

\begin{tabular}{llcl}
\hline Repräsentation & \multicolumn{2}{c}{ Set starker Codes } & Leserichtung \\
\hline einzelne Tabelle & - Bezeichnungsfelder & - leeres Textfeld oberhalb, unterhalb & - inhärent (i.d. R. \\
& - Textfelder & oder neben einem Bezeichnungs- & von links nach \\
& - Markierung von & feld markiert die Frageform des & rechts und \\
& zusammenhängenden & Bezeichnungsfeldes und offene & von oben nach \\
& Objekten nach einem & Antwortoptionen & unten) \\
& Merkmal oder Attribut & - ausgefüllte Textfelder oberhalb, & \\
& je nach Struktur der & unterhalb oder neben einem & \\
& Tabelle & Bezeichnungsfeld markieren die & \\
& & Frageform des Bezeichnungsfeldes & \\
& & und geschlossene Antwortoptionen & \\
\hline Repräsentation & - Set schwacher Codes & \multicolumn{3}{c}{ - Leserichtung } \\
\hline graphische Enti- & & - Markierung eines Textfeldes & - inhärent (i.d. R. \\
täten im Textfeld & & - alternative Repräsentation der & von links nach \\
& & tabellarischen Antwortmöglich- & rechts und von \\
& & keit ,ja “/“nein“ & oben nach unten) \\
\hline
\end{tabular}

Jene ,spezifische Fragen' in den Bezeichnungsfeldern einer Tabelle sind mehrheitlich gar auf einzelne Begriffe reduziert: ein ,Personal-Nr. steht anstelle von z. B. „Wie lautet Ihre Personalnummer?"“, oder ein ,Außenspiegel' für „Haben die Außenspiegel die Funktionsprüfung bestanden?“. Es verdeutlicht sich eine Besonderheit von Formularen, denn hinter der ikonischen Repräsentation von einem freien Textfeld oberhalb, unterhalb oder neben einem Bezeichnungsfeld werden nicht nur die typischen Relationen durch das Set der starken Codes von Tabellen beschrieben, sondern die Bezeichnungsfelder tragen zugleich eine Frageform in sich. Häufig durch symbolische Einträge konkretisiert, können diese eine, müssen aber keine Markierung durch ein Fragezeichen enthalten. Die freien Textfelder verkörpern folgerichtig geschlossene bis offene Antwortoptionen. Eine weitere Besonderheit in Formularen liegt in der Verwendung der graphischen Entität Quadrat verborgen. In Abbildung 24 vielfach aufzufinden, wird mit einem Quadrat im Textfeld eine alternative zweispaltige geschlossene Antwortmöglichkeit im Sinne von „ja“ und „nein“ ersetzt. Parallel können ähnliche geometrische Formen in diesem ,Serviceformular" lediglich ein freies Textfeld markieren. 
Der nichtkontinuierliche Texttyp ,Gutscheine‘ erfüllt, wie bereits charakterisiert, die Funktion von rechtlich gültigen Ansprüchen auf bestimmte Dienstleistungen. Unter den genannten Beispielen sind es v. a. unterschiedliche Arten von Rechnungen, ergo, schriftlichen Quittungen eines Kaufvertrags im Sinne des $₫ 433$ BGB, die in den untersuchten Einfach(st)erwerbstätigkeiten und hierunter insbesondere im Berufsfeld Dienstleistungen aufgefunden wurden. Einerseits handelt es sich um Kassenbons, respektive, Kleinbetrag-Rechnungen, die in Deutschland grundsätzlich nach dem $\$ 33$ der Umsatzsteuer-Durchführungsverordnung (UStDV) folgende Informationen enthalten müssen - und in der Regel auch nur wenige weitere Informationen repräsentieren:

- Vollständiger Name und Anschrift des leistenden Unternehmers (Tabelle 1),

- Ausstellungsdatum (Tabelle 2),

- Menge und Art der gelieferten Gegenstände bzw. Art und Umfang der sonstigen Leistung (Tabelle 3),

- Entgelt für die Lieferung oder sonstige Leistung in einer Summe (Tabelle 4)

- und der darauf entfallende Steuerbetrag sowie den anzuwendenden Steuersatz; oder im Fall einer Steuerbefreiung einen Hinweis darauf, dass für die Lieferung oder sonstige Leistung eine Steuerbefreiung gilt (Tabelle 5).

Obgleich nicht gesetzlich zwingend, so erfüllen die meisten Kassenbons mit der Angabe der Steuernummer, bzw. in der Regel der Umsatzsteuer-Identifikationsnummer, zusätzlich den \$14 UStG. Ein Kassenbon verweist jeweils nur einmal auf die aufgeführten Informationen, obgleich diese teils sehr unterschiedlich nach Lage angeordnet sind, wenn mehrere Kassenbons miteinander verglichen werden (vgl. Abbildung 21). Durch die referentiell rechtlichen Konventionen beziehen sich dennoch die angegebene Adresse und Umsatzsteuer-Identifikationsnummer dieser einseitigen nichtkontinuierlichen Textkomplexe stets auf das leistende Unternehmen. Diese Zuordnungen bedürfen demnach keiner weiteren spezifizierenden Erklärung der Bezeichnungsfelder dieser Merkmale. Gleiches gilt für die weiteren Objekte, ergo hier die rechtlich verbindlichen Informationen, auch wenn diese durch mehrere Tabellen strukturiert verteilt abgedruckt sind, obgleich nicht konventionalisiert räumlich angeordnet. Die referentiell rechtliche Konvention leistet, dass wie im Beispiel zu Tabelle 3 bei einer combined-list nicht explizit erklärt werden muss, dass es sich um die ,Menge und Art der Gegenstände‘ eines Kaufvertrags handelt, die mit dem Kassenbon quittiert werden. Das Ausstellungsdatum (,Tabelle 2‘; single-list) bedarf keiner spezifizierenden Notation. ,Tabelle $3^{\prime}$ und ,Tabelle $5^{\prime}$ müssten durch ihre Verknüpfung der ,anfallenden Steuerbeträge‘ jeweils der höchsten Komplexitätsstufe einer Tabelle, einer nested-list, zugeordnet werden. Insbesondere in der symbolischen Bezeichnung von Steuersätzen sind Variationen in der Verwendung von Abkürzungen bzw. Zahlen- bzw. Buchstabencodes festzustellen; gleiches gilt bei der Auflistung der, Gegenstände ${ }^{{ }^{11}}$.

Neben den verpflichtenden Informationen auf Kassenbons können, müssen aber nicht, auf der Vorderseite Angaben zu z B. Öffnungszeiten, dem/der Verkäufer/in, einer Kassen- und/oder Belegnummer bzw. einer Tischnummer in der Gastronomie gemacht werden. Wie auch ein Logo, ein Barcode oder eine Dankesfloskel sind auch diese zusätzlichen Tabellen des je Unternehmen typischen Kassenbons zu diskriminieren. Auch für diese gilt, dass es keiner Bezeichnungsfelder für die symbolischen und ikonischen Zeichen in den Feldern bedarf. Auf der Rückseite finden sich zudem zusätzlich Auszüge aus den Geschäftsbedingungen für den elektronischen Zahlungsverkehr. 

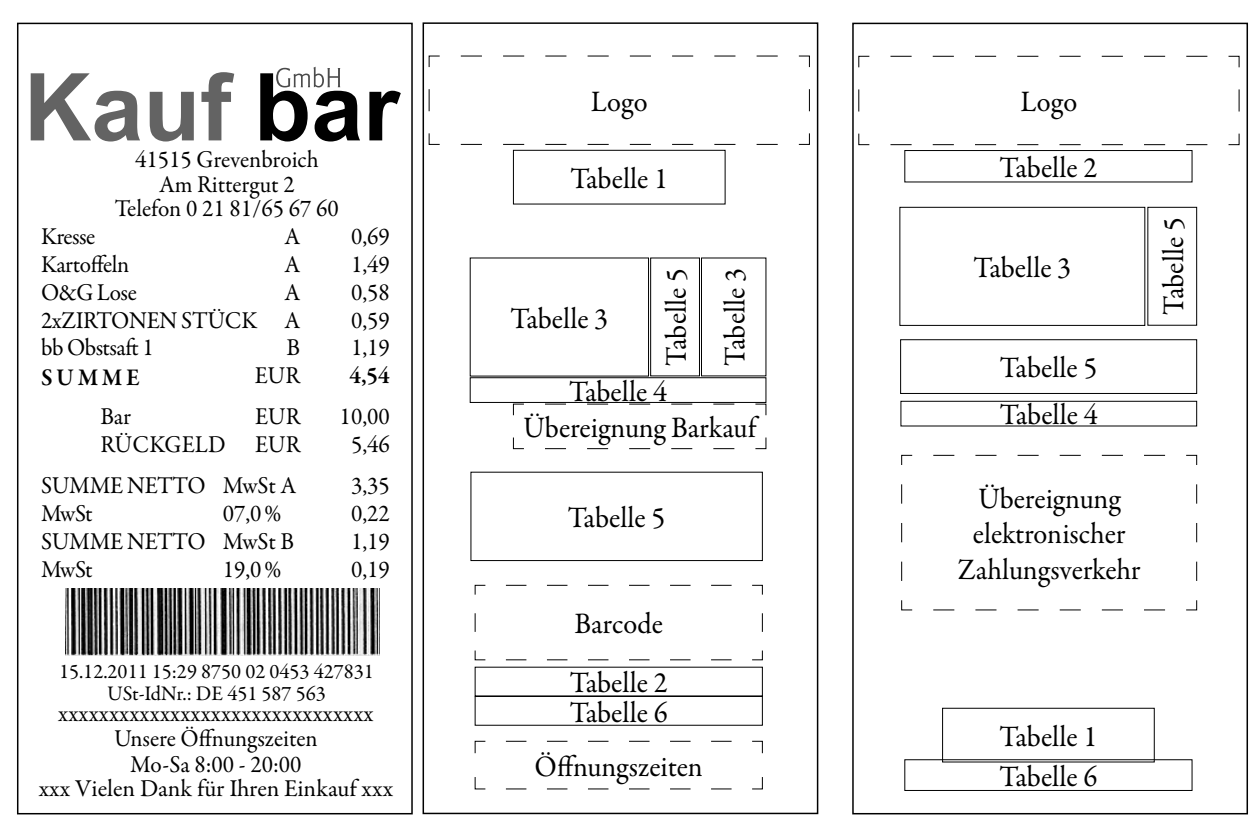

Abb. 21: Kassenbon und Variation seiner tabellarischen Felder

Für Rechnungen oberhalb der Kleinbetragsumme sieht die deutsche Steuerverwaltung gegenwärtig ${ }^{12}$ nicht nur zwingend die zusätzliche Information zu der Steuer- oder UmsatzsteuerIdentifikationsnummer des leistenden Unternehmens vor, sondern auch Informationen zu dem ,vollständigen Namen und Anschrift des Leistungsempfängers', der unikaten ,Rechnungsnummer', der mindestens auf den Monat genauen getroffenen Regelung der ,Lieferung ' und den ggf. ,vereinbarten Minderungen des Entgelts wie Rabatte, Boni und Skonti etc.. Darüber hinaus muss eine Rechnung die Überschrift, Rechnung' tragen und ist in der Regel nach der Form B der DIN 676 und der DIN 5008 für Geschäftsbriefe gestaltet ${ }^{13}$; im Falle von in das Handelsregister eingetragenen Unternehmen gelten hierbei zusätzlich die Impressumpflichten nach den unterschiedlichen gesetzlichen Bestimmungen.

Für die textuelle Anforderung dieses Beispiels des Texttyps ,Gutschein' ist zu schlussfolgern, dass hier ein spezifischer starker Kode zugrunde liegt. Entgegen einem Kassenbon ist die räumliche Lage vieler Tabellen, aus denen sich eine Rechnung zusammensetzt, derart konventionalisiert, dass die meisten Bezeichnungsfelder für Merkmale bzw. Attribute nicht explizit benannt werden müssen: Dies gilt u. a. für die Kontodaten bis zur Umsatzsteuer-Identifikationsnummer im ,Impressumblock' oder für den ,Informationsblock', ergo die Anschrift des leistenden Unternehmens. Durch diesen starken Kode in Geschäftsbriefen kann letztere Anschrift eindeutig von der zweiten, auf einer Rechnung zwingend aufzuführenden Adresse differenziert werden, der des Kaufvertragspartners in dem ,Anschriftblock'. Die Überschrift, Rechnung ' findet sich in der ,Bezugzeichenzeile‘ und ist höchstens durch einen expliziten Steuerungs-Kode hervorgehoben,

12 http://www.ihk-berlin.de/linkableblob/bihk24/recht_und_steuern/Steuern_und_Finanzen/Download/ 817632/.47./data/Merkblatt_Pflichtangaben_fuer_Rechnungen-data.pdf

13 http://www.hk24.de/recht_und_steuern/wirtschaftsrecht/unternehmensgruendung-und-fuehrung/362918/ pflichtangaben_briefe.html 
d. h. mittels typologogischer Variationen aus einer größeren Schriftart, Fett- und/oder Kursivschrift. Der Gegenstand des Vertrags in Menge, Art, Einzel- und Gesamtpreise und Steuersätze, der hiermit quittiert wird, findet sich in einer verschränkten Tabelle im ,Textblock'. Auch hier bedarf es keines expliziten verschriftlichten Hinweis.

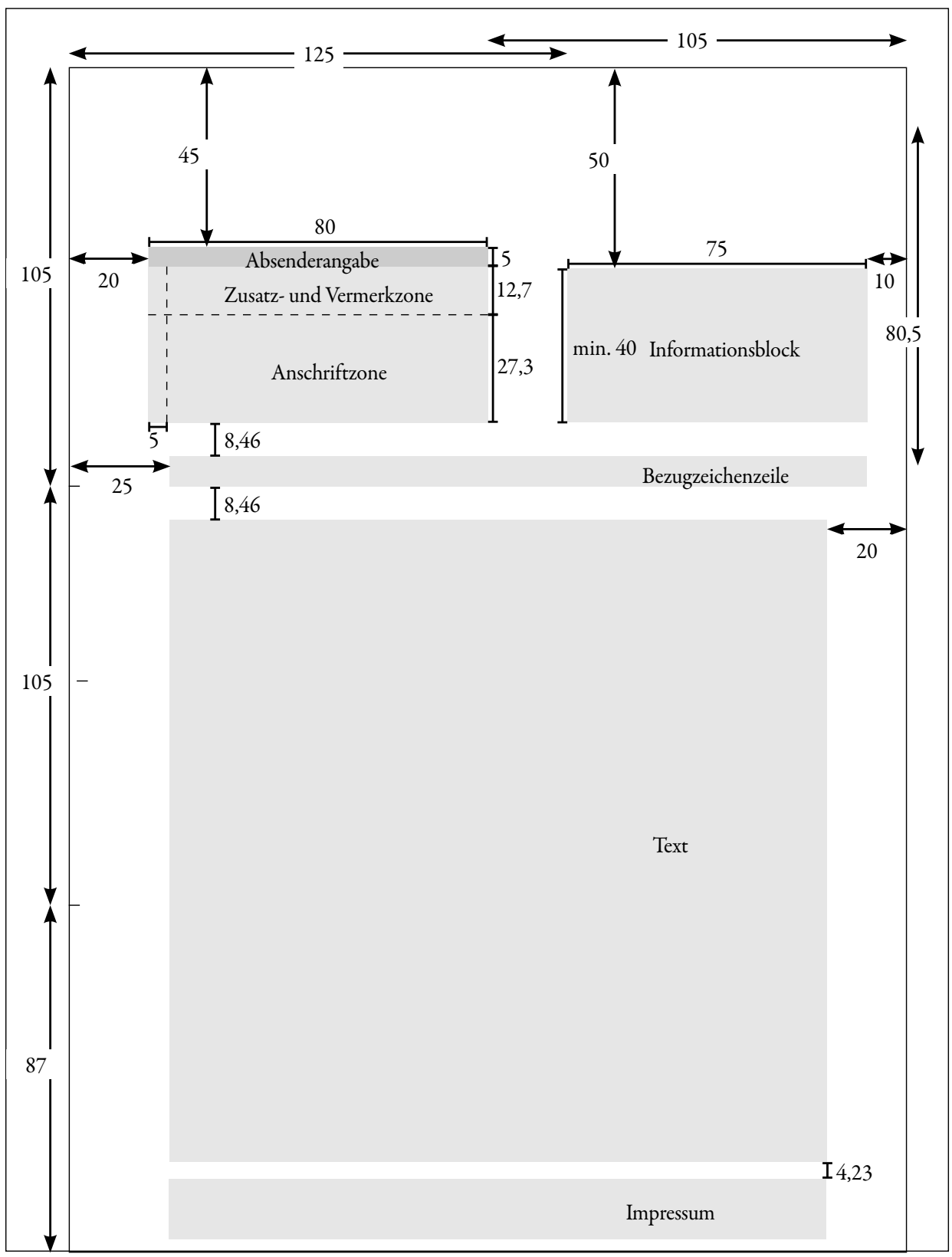


Ausnahmen des starken Codes in Rechnungen bilden die verpflichtenden Angaben zur fortlaufenden ,Rechnungsnummer', dem ,Ausstellungsdatum' und dem ,Lieferdatum'. Diese können im ,Informationsblock', in der ,Bezugszeichenzeile' oder auch im ,Textblock' aufgeführt sein. Durch diese somit schwache Kodierung werden Bezeichnungsfelder notwendig, um z.B. die Daten voneinander zu unterscheiden.

\section{Electron Europa}

Electron Industries Europe GmbH, Rheinstr. 15, 65760 Eschborn

Herr

Otto Weber

Schillerstraße 232

69117 Heidelberg

Ihr Auftrag : Abgabel. Electron-Shop Auftrag vom : 06.03.2011

Rechnungs-Nr. : 554891

Auftragsdatum : 06.03.2011

Kunden-Nr. : 568759

Sachbearbeiter : Kundendienst-Team

Telefon : :06196/937-200

Fax : :06196/937-289

Datum : 23.03.2011

Seite $\quad: 1$

Rechnung

\begin{tabular}{lllllr} 
Pos & Artikel-Nr. & Bezeichnung & Menge & Preis & Positionswert \\
\hline 1 & $5687600045-$ V 69 KF & V 69 KF & 1,00 ST & 239,10 EUR & $239,10 \mathrm{EUR}$ \\
& & Akku-Handkreissäge ELEC & & & \\
2 & \multirow{2}{*}{28883716} & 12 Zähne, 160 x 20 mm & $2,00 \mathrm{ST}$ & $15,20 \mathrm{EUR}$ & $30,40 \mathrm{EUR}$ \\
3 & & Verpackung \& Versand & pauschal & & $5,90 \mathrm{EUR}$ \\
\hline & & Nettobetrag & $:$ & $269,50 \mathrm{EUR}$ \\
& & $19 \%$ Mwst. & $:$ & $51,21 \mathrm{EUR}$ \\
& & Versand & $:$ & $5,90 \mathrm{EUR}$ \\
& & & Gesamtbetrag & $:$ & $326,61 \mathrm{EUR}$ \\
\hline
\end{tabular}

Versandart : KLF Paket-Dienst

Lieferung : ab Werk

Zahlung : : Sofort netto Kasse ohne jeden Abzug

Lieferung am: 24.03.2011

Vielen Dank für Ihren Auftrag.

Es gelten unsere allgemeinen Geschäftsbedingungen (AGB): Unsere AGB sehen Sie

bitte auf unserer Homepage unter www.electron-europa.com ein.

Electron Industries Europe $\mathrm{GmbH}$

\begin{tabular}{llll}
\hline Rheinstr. 15 & Email & Internet & Geschäfsführer: Friedrich Teichert, John Taride \\
65760 Eschborn & eleckundendienst@electron-europe.com & http://www.electron-europe.com & Sitz: 52255 Frankfurt am Main (HRB 87569)
\end{tabular}

Abb. 22: Beispiel einer Rechnung nach DIN 676 und DIN 5008 
Tab. 12: Spezifische ikonische Codes in Gutscheinen

\begin{tabular}{|c|c|c|c|}
\hline Repräsentation & & starker Codes & Leserichtung \\
\hline $\begin{array}{l}\text { Geschäftsbrief: } \\
\text { Rechnung }\end{array}$ & $\begin{array}{l}\text { - konventionalisierte } \\
\text { ikonische Anordnung } \\
\text { einzelner Tabellen } \\
\text { (verbindliche Informa- } \\
\text { tionen) }\end{array}$ & $\begin{array}{l}\text { - vollständiger Name und Anschrift } \\
\text { des leistenden Unternehmers im } \\
\text { Informationsblock } \\
\text { - Menge und Art der geliefer- } \\
\text { ten Gegenstände bzw. Art und } \\
\text { Umfang der sonstigen Leistung im } \\
\text { Textblock } \\
\text { - Entgelt für die Lieferung oder } \\
\text { sonstige Leistung in einer Summe } \\
\text { im Textblock } \\
\text { - Steuerbetrag und Steuersatz bzw. } \\
\text { Steuerbefreiung im Textblock } \\
\text { - Kontodaten, Handelregisterver- } \\
\text { weis und Umsatzsteuer-Identifika- } \\
\text { tionsnummer im Impressumblock } \\
\text { - Verweis als „Rechnung“ in der } \\
\text { Bezugszeichenzeile }\end{array}$ & - inhärent \\
\hline Repräsentation & - Set schwacher Codes & & - Leserichtung \\
\hline $\begin{array}{l}\text { Geschäftsbrief: } \\
\text { Rechnung }\end{array}$ & $\begin{array}{l}\text { - ikonische Anordnung } \\
\text { einzelner Tabellen } \\
\text { (verbindliche Informa- } \\
\text { tionen) }\end{array}$ & $\begin{array}{l}\text { - Ausstellungsdatum im Informati- } \\
\text { onsblock, in der Bezugszeichenzeile } \\
\text { oder im Textblock } \\
\text { - Angaben zum Lieferungsdatum im } \\
\text { Informationsblock, in der Bezugs- } \\
\text { zeichenzeile oder im Textblock } \\
\text { - fortlaufende Rechnungsnum- } \\
\text { mer im Informationsblock, in } \\
\text { der Bezugszeichenzeile oder im } \\
\text { Textblock }\end{array}$ & $\begin{array}{l}\text { - inhärent (spezi- } \\
\text { fisch je leistenden } \\
\text { Unternehmens) }\end{array}$ \\
\hline $\begin{array}{l}\text { Kassenbon: } \\
\text { Kleinbetragrech- } \\
\text { nung }\end{array}$ & $\begin{array}{l}\text { - ikonische Anordnung } \\
\text { einzelner Tabellen } \\
\text { (verbindliche Informa- } \\
\text { tionen) }\end{array}$ & $\begin{array}{l}\text { - eigene Tabelle je verpflichtender } \\
\text { Information } \\
\text { - zusätzliche Tabellen (Barcode, } \\
\text { Öffnungszeiten, usw.) }\end{array}$ & $\begin{array}{l}\text { - inhärent (spezi- } \\
\text { fisch je leistenden } \\
\text { Unternehmens) }\end{array}$ \\
\hline
\end{tabular}

\subsubsection{Semiotische Betrachtung weiterer Sets nicht-arbiträrer Codes}

Möchte man einen Sachverhalt mit einem nicht unmittelbar wahrnehmbaren, rein qualitativen oder qualitativen/quantitativen Zusammenhang textuell darstellen, kann eine Tabelle anstelle eines Fließtextes gewählt werden. Seine graphischen Entitäten, in erster Linie die Felder, sind in diesem Fall so anzuordnen, dass sie den graphischen Schemata einer Tabelle genügen. Unter Einhaltung dieser Konvention ist es naheliegend, dass Rezipienten mittels der dahinterliegenden Sets von starken Codes von Tabellen die antizipierten Aussagen extrahieren können. Mit Tabellen vertraute Rezipienten bedürfen hierfür lediglich einer auf das Minimum reduzierten Anordnung seiner Felder erkennen, wie in den vorausgehenden Abschnitten mehrfach thematisiert wurde. Es eröffnet sich zugleich die Frage nach der Funktion der isolierten Bezeichnungsfelder, Linien, Schraffierungen, Legenden, Überschriften usw., die häufig bei der Textualisierung in Tabellen auftauchen. Wiederum aus den semiotischen Analysen von insbesondere Diagram- 
men und graphischen Darstellungen abgeleitet (Weidenmann 1994; Schnotz 1994), können diese zwei unterschiedlichen Gattungen nicht-arbiträrer Codes zugeordnet werden. Infolge ist ihnen gemeinsam, dass sie, wenn überhaupt, konventionell keine eindeutige Relation zum Bezeichneten inhärent in sich tragen.

Set nicht-arbitärer Darstellungs- und Set nicht-arbiträrer Steuerungs-Kodes: Schraffierungen können bspw. den Zweck erfüllen, die Lesenden auf bestimmte Objekte, Zeilen oder Spalten hinzuweisen. In z. B. dem bereits besprochenen ,Fahrzeugprotokoll' dienen die Schraffierungen zur Hervorhebung der Felder, die gemäß Vorgabe des Arbeitsplatzes unbedingt ausgefüllt werden müssen. Im ,Arbeitsplan' in der Gastronomie (vgl. Abbildung 19) repräsentieren diese graphische Entitäten dagegen die Verknüpfungen einzelner Einsatzzeiten mit einem der Arbeitsorte. Während im ersten Beispiel eine Schraffierung als Steuerungs-Kode „die Rezeption des visuellen Arguments lenken“ soll, dient es im zweiten Beispiel als Darstellungs-Kodes „der Visualiserung des Arguments" selbst (Weidenmann 1994, 23).

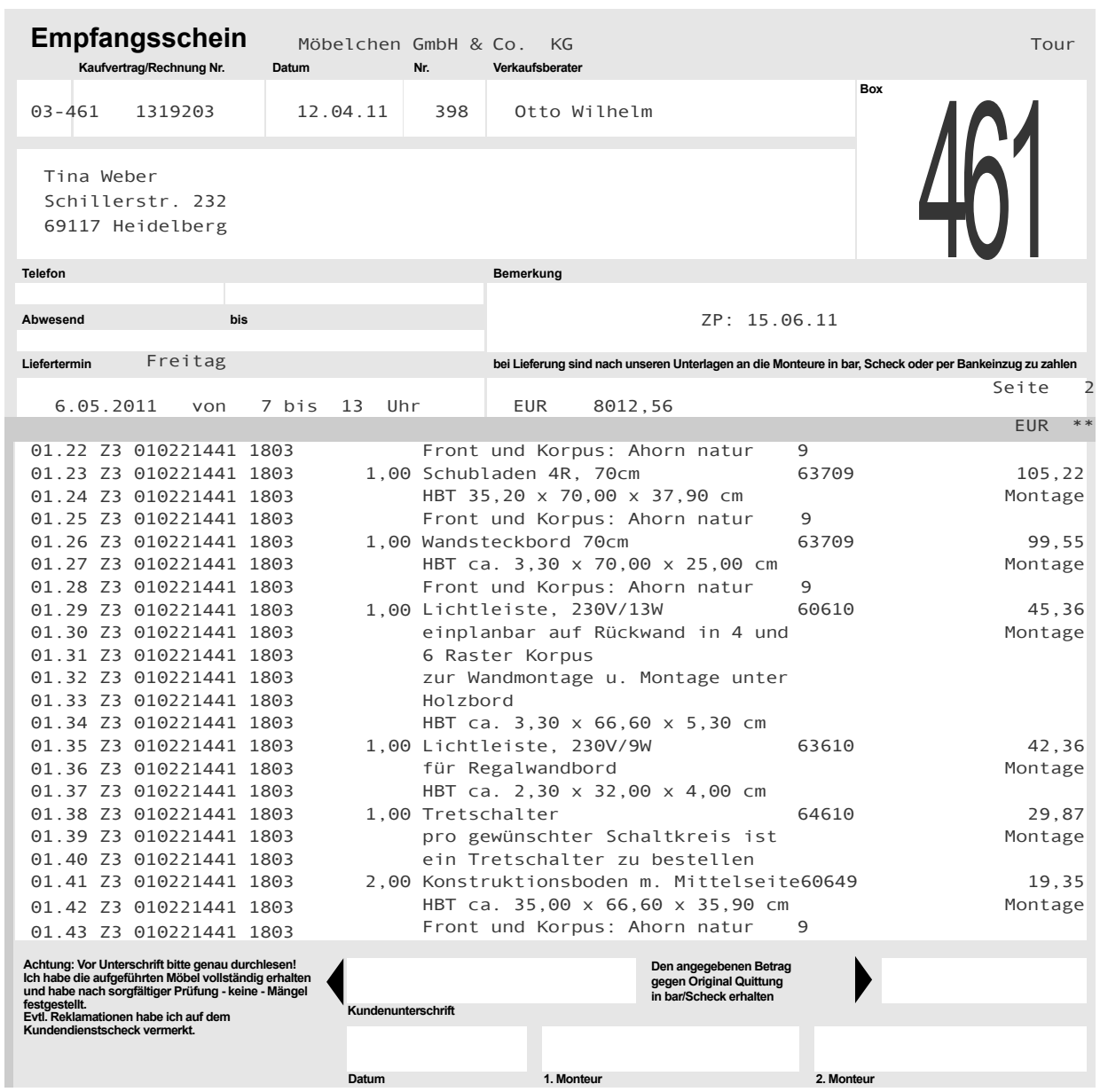

Abb. 23: Empfangsschein in der Möbelmontage (Quelle: Imitation; GRAWIRA) 
Mit der Intention, die graphische Schemata von kategorialen Tabellen zu verdeutlichen, werden häufig z.B. Linien mit identischer Stärke zur Abgrenzung seiner Felder ergänzt. Diese Linien verdeutlichen lediglich den visuellen Eindruck einer Tabelle, heben jedoch keinen bestimmten Zusammenhang zwischen den Objekten in den Feldern hervor. Gleiches gilt für Schraffierungen von Zeilen und/oder Spalten, die nicht bedeutungstragend sind, respektive, mehrere Tabellen lediglich voneinander visuell abtrennen (Abbildung 23). Mit diesen Steuerungs-Kodes gehen somit Modifikationen der graphischen Schemata einer kategorialen Tabelle oder eines Diagramms einher und zwar durch Linien in unterschiedlicher Kontur, d. h. Dicke, Farbe und Gestalt (gestrichelt, gepunktete, durchgezogen, doppelt). Es sind folglich auch nicht Linien einer bestimmten Kontur, die mit der Funktion als Steuerungs- oder auch als Darstellungs-Kode per se einhergehen. Auf der Vorderseite der aufgefundenen, Serviceanforderung' in der Möbelmontage (Abbildung 24) sticht über die Variation der Linienstärke eine der Tabellen, die dieses Formular strukturieren, hervor. In Abbildung 26, entdeckt am Einfach(st)arbeitsplatz eines Anlagenführers in der produzierenden Industrie, repräsentieren Linien dagegen Hierarchien in der Abfolge von Handlungen ausgehend von zwei Zuständen. Auch die gerichtete Version von Linien impliziert nicht die Funktionen als ikonische Steuerungs- oder Darstellungs-Kode.

Wenn in den vorausgehenden Abschnitten mehrfach auf einzelne Abschnitte eines beispielhaft aufgefundenen nichtkontinuierlichen Textes sprachlich hingewiesen wurde, hätte zur Hervorhebung ein in der Kontur graphisch abgegrenzter Pfeil gezielt integriert werden können. In den untersuchten arbeitsweltlichen Texten fand sich diese lenkende Funktion immer als Mittler zwischen einzelnen besonders wichtigen Feldern und einer Kurzerklärung mit symbolischen Zeichen, so z. B. in Abbildung 23. Besonders auffällig ist diese intendierte Referenz mittels schematisierter Pfeile innerhalb der Schulungsmappe zu diesen tabellarischen Formularen in der Möbelmontage (Abbildung 25). Die „Lenkung“ neben Wörtern und kurzen anleitenden Sätzen über Pfeile in der Schulungsmappe beruht dagegen auf einem Untertypus von graphischen Steuerungs-Kodes: dem impliziten. „Implizite“ Steuerungs-Kode sind nach Weidenmann „absichtsvolle Variationen“ (ebd., 23f.) der ikonischen Repräsentationen selbst, so z. B. die Vergrößerung oder der Detailreichtum eines Ausschnitts. Dies ist in graphischen Darstellungen oder Karten ein häufig genutztes Mittel, um das Argument auf einen bestimmten Bereich zu lenken; ein bekanntes Beispiel ist die „Lupe“ in der Gallienkarte in den Asterix-Heftchen. Mit der Schulungsmappe ist jedoch eine andere „absichtsvolle Variation“ gemeint. Werden zwei Tabellen „nebeneinander“ betrachtet, welche sich zum einen möglichst offensichtlich und zum anderen in wenigen Elementen der intendierten Aussage gezielt unterscheiden, „dann ist zu erwarten, daß die Rezipienten die unterschiedlichen Merkmale auch als bedeutsam für das zu extrahierende Argument bewerten“ (ebd., 24). Auf diese Weise impliziert ein ausgefülltes neben einem Blanko-Formular, welche Felder durch Zahlziffern oder Wörter ausgefüllt werden müssen. Neben ikonischen Zeichen können auch symbolische Zeichen jene Funktion erfüllen. 


\section{Serviceanforderung zu KV}

\section{I}

Sehr geehrter Kunde, wir wünschen Ihnen schon heute viel Freude mit Ihren neuen Einrichtungsgegenständen. Wir hoffen, dass diese Ihren Wünschen entsprechen. Sollte sich wider Erwarten eine Beanstandung ergeben, nutzen Sie bitte diese Serviceanforderung und geben Sie diese in einem unserer Geschäfte ab oder schicken Sie sie einfach per Fax an uns zurück.

Fax Nr.

Wir werden Ihr Anliegen kurzfristig bearbeiten.

Ihre

LT: $\quad$ BOX: LP.:

Welches Teil wird beanstandet? (siehe Rückseite)

Teil Nr.

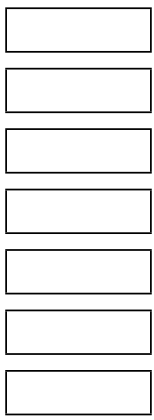

Welcher Fehler trat auf? (siehe Rückseite)

Fehler Nr.

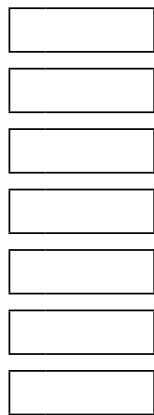

$\mathrm{Zu}$ welcher Vertragsposition? Ggf. Skizze

(Pos./BKZ/Liefernr./Artikelnr.)

Was wünschen Sie zur Reklamationserledigung?

Kunde montiert selbst $\square$

Versicherungsschaden

Montageänderung

Zeit der Reklamationserledigung $\square \square \square$ Minuten

AnzahlColli Rückware

Art der Rückware:

Zustand der Rückware:

$\square$ in Ordnung

$\square$ beschädigt

$\square \mathrm{KD}$

$\square$ AV teilw.

$\square$ Ware komplett

$\square$ Ware unvollständig

$\square$ Rückholung

$\square$ AV gesamt

$\square$ Ware montierbar

$\square$ Ware nicht montierbar

$\square$ AÜr

$\square$ NA

Verwendungszweck der Rückware:

Lagerplatz:

Unterschrift RB/SW

$\square$ nicht geschafft

Unterschrift Kunde

Unterschrift Monteur

Abb. 24: Serviceanforderung in der Möbelmontage (Quelle: Imitation; GRAWIRA) 


\section{Serviceanforderung}

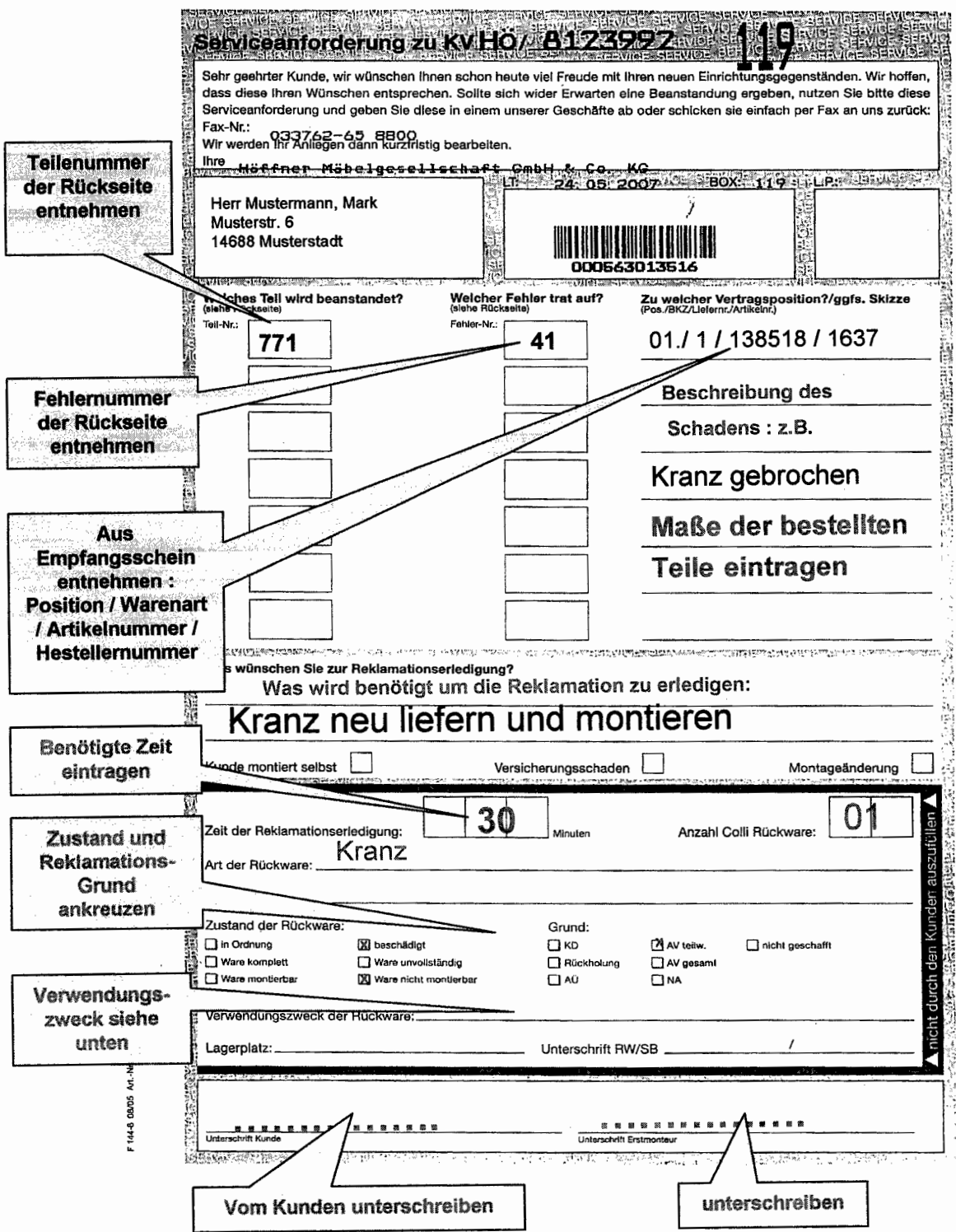

Verwendungszwecke : ZZW = zurück zum Werk, FEK = freier Einkauf, Stellplatz = Lager,

a. RW W. $v_{0}=$ auf Rückware wird verzichtet

Abb. 25: Schulungsmappe zu den Formularen in der Möbelmontage (Quelle: Kopie; GRAWIRA) 


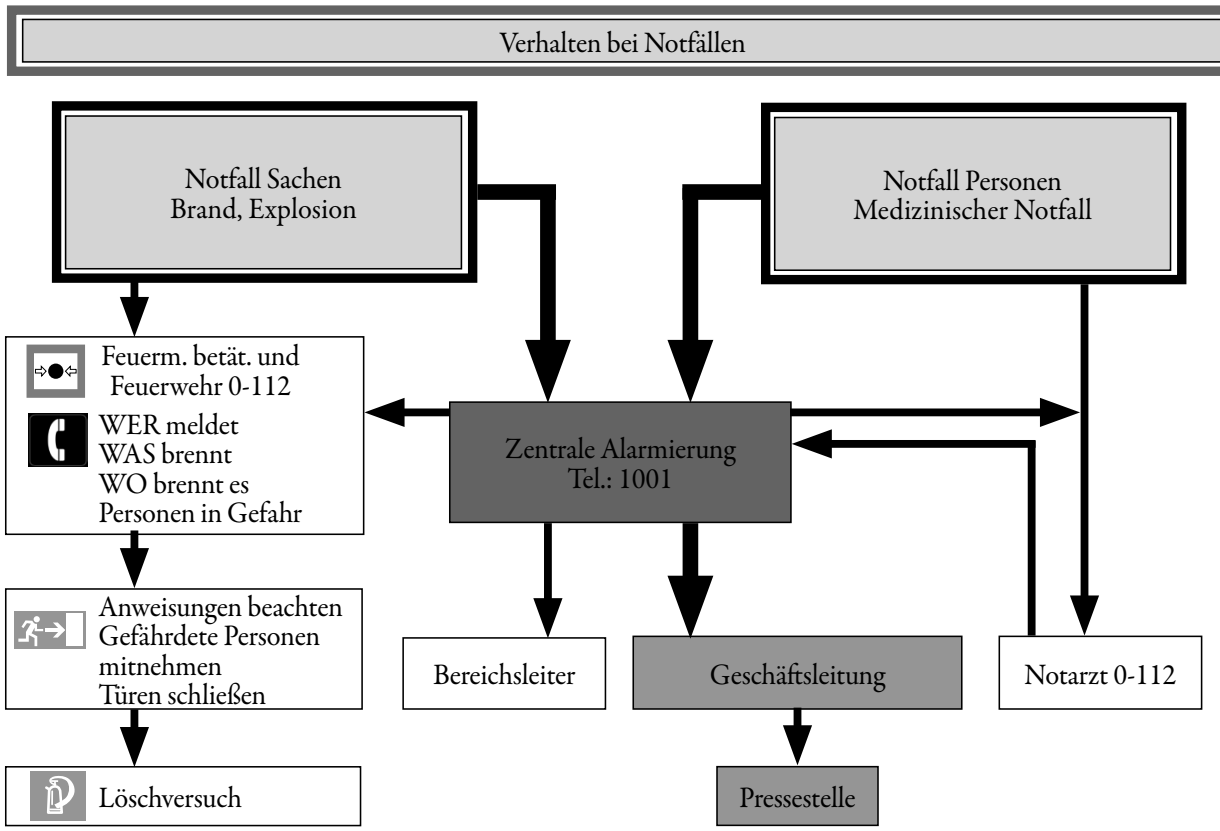

Abb. 26: Aushang Brandverhalten in der Produktion (Quelle: Imitation; Studie Basendowski)

Tab. 13: Ikonische Steuerungs-Kodes

\begin{tabular}{|c|c|c|c|}
\hline Zeichen & & Bezeichnetes (Lenkung) & \\
\hline \multirow[b]{2}{*}{$\begin{array}{l}\text { Variationen der } \\
\text { Schraffierung } \\
\text { (explizit) }\end{array}$} & $\begin{array}{l}\text { - Hervorhebung von } \\
\text { zusammenhängenden } \\
\text { Objekten, Merkmalen } \\
\text { oder Attributen } \\
\end{array}$ & $\begin{array}{l}\text { - Hervorhebung von kontex- } \\
\text { tuell erwünschten } \\
\text { Anwendungen des nicht- } \\
\text { kontinuierlichen Textes }\end{array}$ & $\begin{array}{l}\text { - Repräsentation von } \\
\text { Hierarchien (inhärente } \\
\text { primäre Leserichtung) }\end{array}$ \\
\hline & $\begin{array}{c}\text { - „in Gestalt aneinander- } \\
\text { gereihter paralleler oder } \\
\text { engmaschig gekreuzter } \\
\text { Striche wird zum Hell- } \\
\text { Dunkel-Verlauf, der in } \\
\text { der realen Wahrneh- } \\
\text { mung Vertiefung oder } \\
\text { Wölbung signalisiert.“ }\end{array}$ & & \\
\hline
\end{tabular}




\begin{tabular}{|c|c|c|c|}
\hline Zeichen & \multicolumn{3}{|c|}{ Bezeichnetes (Lenkung) } \\
\hline & $\begin{array}{l}\text { - Hervorhebung von } \\
\text { zusammenhängenden } \\
\text { Objekten, Merkmalen } \\
\text { oder Attributen }\end{array}$ & $\begin{array}{l}\text { - Hervorhebung von kon- } \\
\text { textuell erwünschten } \\
\text { Anwendungen des Textes } \\
\text { als Mittler sprachlicher } \\
\text { Ausführungshinweise }\end{array}$ & $\begin{array}{l}\text { - Repräsentation von } \\
\text { Hierarchien (inhärente } \\
\text { primäre Leserichtung) }\end{array}$ \\
\hline $\begin{array}{l}\text { Variationen von } \\
\text { Linien/Pfeile } \\
\text { (explizit) }\end{array}$ & $\begin{array}{l}\text { - „Eine Linie wird als } \\
\text { Kontur zum Index für } \\
\text { den Umriß eines Objek- } \\
\text { tes, wie ihn die reale } \\
\text { Wahrnehmung aufgrund } \\
\text { von Prozessen der Figur- } \\
\text { Grund-Unterscheidung } \\
\text { identifiziert.“ }\end{array}$ & $\begin{array}{l}\text { - „Geschlossene Linien } \\
\text { neben unterbrochenenen } \\
\text { Konturen kommunizieren } \\
\text { Schlüsselinformationen zur } \\
\text { Platzierung von Objekten; } \\
\text { das geschlossen konturierte } \\
\text { Objekt erscheint räumlich } \\
\text { vor dem unterbrochen } \\
\text { konturierten Objekt } \\
\text { angeordnet." }\end{array}$ & $\begin{array}{l}\text { - „Sich auf einen ,Flucht- } \\
\text { punkt' hin gleichförmig } \\
\text { zubewegende Linien } \\
\text { sind Hinweise für } \\
\text { Zentralperspektive und } \\
\text { Staffelung von Objekten } \\
\text { im imaginierten Raum.“ }\end{array}$ \\
\hline $\begin{array}{l}\text { typographische } \\
\text { Variationen } \\
\text { symbolischer } \\
\text { Schriftzeichen } \\
\text { (explizit) }\end{array}$ & $\begin{array}{l}\text { - Hervorhebung von } \\
\text { zusammenhängenden } \\
\text { Objekten, Merkmalen } \\
\text { oder Attributen }\end{array}$ & $\begin{array}{l}\text { - Hervorhebung von kon- } \\
\text { textuell erwünschten } \\
\text { Anwendungen des Textes } \\
\text { als Mittler sprachlicher } \\
\text { Ausführungshinweise }\end{array}$ & $\begin{array}{l}\text { - Repräsentation von } \\
\text { Hierarchien (inhärente } \\
\text { primäre Leserichtung) }\end{array}$ \\
\hline $\begin{array}{l}\text { Variationen der } \\
\text { ikonischen } \\
\text { Repräsentation } \\
\text { selbst } \\
\text { (implizit) }\end{array}$ & $\begin{array}{l}\text { - Hervorhebung von } \\
\text { zusammenhängenden } \\
\text { Objekten, Merkmalen } \\
\text { oder Attributen durch } \\
\text { kompensatorische } \\
\text { Extraktion }\end{array}$ & & \\
\hline
\end{tabular}

(Zitationen aus Weidenmann 1994, 15)

\subsubsection{Set sprachlicher Steuerungs-Kodes}

Eine Einbindung von Tabellen, Diagrammen und graphischen Darstellungen (oder auch Karten) in einen Fließtext zur Verdeutlichung von Aussagen, wie in diesem Buch praktiziert, konnte, wie bereits erwähnt, nur sehr vereinzelt an den Arbeitsplätzen der untersuchten Einfach(st) erwerbstätigkeiten entdeckt werden. Die textuelle Praxis unterscheidet sich folglich mehrheitlich von der, die für die (Berufs-)Schule zutrifft: Hier fungieren nichtkontinuierliche Textteile als visualisierte Dopplung der Argumente eines Sachverhalts, die innerhalb der dominierenden Struktur eines kontinuierlichen Textes bereits mittels Wörtern, Sätzen usw. beschrieben wurden (Niederhaus 2011; Seufert 2003; Blömeke 2003; Weidenmann 1994).

Umgekehrt heißt das jedoch auch für die somit eigenständigen nichtkontinuierlichen Texte, dass sie nahezu ohne verschriftlichte Anleitung auskommen müssen, wie sie denn zu lesen oder zu bearbeiten sind. Ausnahme bilden lediglich die sprachlichen Steuerungs-Kodes wie Überschriften oder unterschiedliche Formen von Legenden. Mittels einzelner Begriffe, Satzphrasen oder maximal kurzer Sätze kann der geringen „Ausdrucksmächtigkeit“ (Seufert 2003, 17) von graphischen Steuerungs-Kodes entgegengewirkt und können die Rezeptionsprozesse des Lesenden gelenkt werden:

Mithilfe von Deskriptionen lassen sich sehr einfach allgemeine Negationen oder allgemeine Disjunktionen formulieren, wohingegen Depiktionen spezifischer sind und somit meist mehrere Bilder notwendig sind, um die gleiche Ausdrucksmächtigkeit zu erzielen. Möchte man bspw. den einfachen Satz „Tiere 
sind nicht erwünscht" bildhaft verdeutlichen, kann man zwar einen Hund abbilden und durch eine Durchstreichung die Negation anzeigen, es wird, aber damit letztlich nur dargestellt, dass Hunde nicht erwünscht sind (ebd.).

Ein Beispiel eines sprachlichen Steuerungs-Kodes findet sich in der obig vorgestellten Schulungsmappe. Wenn in Abbildung 24 mittels der Linienkontur eine einzelne Tabelle gegenüber anderen Tabellen visuell hervorgehoben wird, erfährt man durch den Eintrag „nicht durch den Kunden auszufüllen“ mehr über deren dahinterliegenden Intention. Mit Arrangements graphischer Entitäten allein lässt sich diese Lenkung nur schwer erzielen, insofern diese nicht im sozialen Kontext einer eindeutigen Konvention unterliegen.

Sprachliche Steuerungs-Kodes sind konsequenterweise dann immer bevorzugtes Repräsentationsmittel, wenn Bearbeitungsprozesse gelenkt werden sollen, die außerhalb der ausdrucksmächtigen, weil durch starke Codes verknüpften, Möglichkeiten der Anordnung von ikonischen Zeichen liegen. Wörter, Satzphrasen oder einzelne Sätze in den Überschriften und Legenden sind zu dechiffrieren und mit den nichtkontinuierlichen Texten zu verknüpfen. Hervorzuheben ist an dieser Stelle, dass sie mit der primär ikonischen Struktur der Texte untergeordnet interagieren. Die einhergehende textuelle Anforderung umfasst das Lesen dieser sprachlichen Einheiten, ist aber ausdrücklich nicht mit dem Pendant gleichzusetzen, wenn Tabellen, Diagramme usw. komplementär in Fließtexte eingebunden werden.

\subsubsection{Linguistische Betrachtung der Explikation durch symbolische Zeichen}

Mit den bisherigen semiotischen Betrachtungen konnte herausgearbeitet werden, dass Tabellen und Diagramme auf, erstens, typische Anordnungen ihrer Entitäten (graphische Schemata) und, zweitens, gewissen damit verknüpften Konventionen der inhärenten Relation zwischen diesen Zeichen und dem Bezeichneten (starke Codes) beruhen. Nichtkontinuierlichen Textualisierungen können in diesem Sinne auf diverse Sachverhalte angewandt werden, begrenzt lediglich durch die Art von Zusammenhängen, die sie abzubilden im Stande sind: z. B. terminologische, kategoriale, topologische, verlaufsstrukturelle usw. Während jeweils spezifisch visualisierbaren Zusammenhänge eines Sachverhalts innerhalb dieser Bedingungen grundsätzlich als inhärent gelten, so gilt dies jedoch nicht für den Sachverhalt selbst, aus dem diese Zusammenhänge stammen. Kurzum, der Sachverhalt ist durch seine Merkmale oder gar Attribute markiert, welche nicht alleinig in den ikonischen Repräsentationen abgebildet sind und müssen daher expliziert werden. In den aufgefundenen Texten an Einfach(st)arbeitsplätzen finden sich aus diesem Grund an den Knoten, Kanten, Bezeichnungsfelder der Merkmale bzw. Attribute usw. mehrheitlich ausgeschriebene oder abgekürzte Begriffe. Diese Explikationen intendieren gezielte „Element-Element-“ oder „Relation-Relation“-Interrelationen des Sachverhalts in der textuellen Repräsentation mit denen der beruflichen Handlungssituation selbst. Grundsätzlich verbirgt sich dahinter der Erklärungsansatz, dass einerseits einzelne Merkmale oder Attribute (Element) und deren Zusammenhänge (Relation) getrennt in beiden Systemen vorliegen. Andererseits sind jeweils die hierunter ausgewählten, da visualisierten, Merkmale und Attribute und deren Zusammenhänge beider Systeme miteinander zu verknüpfen (ebd., 27ff.).

In einer Verkaufsniederlassung eines Herstellers für gewerbliche Elektrogeräte (z. B. Schlagbohrmaschinen, Schweißgeräte, ...) ist eine Reparaturwerkstatt angebunden. Im Lager treffen daher in erster Linie defekte Elektrogeräte ein; mehrere Paketdienste liefern täglich aus. Der Ablauf sieht vor, dass diese Einsendungen im Lager zuerst ausgepackt werden, wobei die Kundendaten und die eingesendeten Teile mittels einer Maske eines computergestützten Logistikprogramms eingetragen werden. Die Kundendaten sind in der Regel über eine Kundennummer bereits im System hinterlegt. Das zu reparierende Gerät wird durch die Produkt- und 
Seriennummer und weiteres Zubehör durch den Eintrag der Menge verbucht; es erfolgt die Lieferung in den Originalkoffern und nicht jede/r Kunde/in entfernt aus diesen Verpackungen Ladegeräte, Akkus, Bohrer, Bits usw. Während das defekte Gerät selbst in einer blauen Kunststoffwanne deponiert wird, wird das Zubehör an einzelnen Lagerplätzen zwischenzeitlich verstaut; die Koffer getrennt von den weiteren Einzelteilen. Jeder dieser Lagerplätze trägt einen Code, der zusätzlich in die Maske eingetragen werden muss. Bevor die blaue Kunststoffwanne auf einem Lieferband ihren Weg in die Reparaturwerkstatt findet, muss noch ein Etikett mit einem Bar-Kode ausgedruckt und an die dafür vorgesehene Stelle an der Wanne eingeschoben werden. Ihren Rückweg treten die Geräte in gelben Wannen zusammen mit einem ausgedruckten Lieferschein an [Beispiel siehe Abbildung 27]. Der/Die Mitarbeiter/in in der Lagerlogistik entnimmt zuerst diesen Lieferschein und scannt ihn über den Bar-Kode ein. Auf dem Bildschirm erscheinen die Kundendaten und hinterlegt sämtliche Aufträge. Dem beigefügten Lieferschein kann der/die Mitarbeiter/in den vorliegenden Auftrag entnehmen und den Eintrag in der Maske öffnen. Diese Maske beinhaltet jedoch keine Details des Auftrags selbst. Es ist der Lieferschein, der die weiteren Arbeitsschritte anleitet, respektive, einzelne Felder dieses Lieferscheins. Es ist anhand der Produkt- und die Seriennummer das vorliegende Gerät zu überprüfen, bevor es gemäß dem ,Reparaturstatus ' bei der Phrase (a), Altgerät Retoure' für den Rückversand an den/ die Kunden/in vorbereitet wird, oder (b) bei ,Neugerät mit Verschrotten' ersetzt wird; eine dritte Variante ist durch (c) ,Verschrotten' gekennzeichnet. Die Entscheidung wird in der Reparaturabteilung zumeist in Absprache mit dem Kunden getroffen; in die Prozesse bei Garantie- bis Reparaturfällen mit Kostenvoranschlag sind die Mitarbeiter/innen in der Lagerlogistik nicht involviert. Den Geräten selbst ist der Reparaturstatus zudem in der Regel nicht anzusehen. Im Fall (a) muss nun vom angegebenen ,Lagerplatz' der Koffer und ggf. weiteres Zubehör entnommen werden. In diesem bzw. mit diesen sicher verstaut, wird der Auftrag in der Maske bestätigt; es erfolgt ein Ausdruck der ,Auftragsbestätigung 'mit Adresskopf des Kunden. Nach der ,Versandart' werden die Formulare entweder in einem Couvert zur Abholung im Shop bereitgestellt, oder in einem passenden Karton mit oder ohne Füllmaterial versandbereit verpackt. Die ,Auftragsbestätigung wird hierfür in einer Versandtasche angebracht und der Karton auf einer Palette deponiert. Im Fall (b) muss dagegen das Gerät in der Wanne im entsprechenden Müllcontainern entsorgt werden. Stattdessen ist ein Neugerät nach den Angaben der Serien- und Produktnummer im unteren Kasten des ,Lieferscheins aus den Regalen zu holen und analog für die Abholung oder den Versand vorzubereiten. Was mit dem eingesandten Koffer und Zubehör passieren soll, ist auch dort angegeben. (c) sieht dagegen vor, dass lediglich die Entsorgung aller eingesandten Teile vorgenommen wird und dies dem Kunden mit der ,Auftragsbestätigung ' postalisch bestätigt wird. Die jeweilige ,Auftragsbestätigung' der drei Varianten wurde in der Reparaturabteilung vorbereitet, die zugleich Einblick in die Lagerbestände der Neugeräte haben und ggf. über das Zentrallager eine Ersatzbestellung beauftragen können; in Garantiefällen erfolgt der Versand in der Regel über das Zentrallager. Die beiden Formulare sind codetechnisch über die ,Auftragsnummer' verknüpft, die automatisch bei der Eingabe in der Maske bei der Reparatureinbuchung vergeben wird (Deskription nach Transkription, Lagerlogistik; Elektrohandel).

Die Skizzierung eines beruflichen Handlungsfeldes am Arbeitsplatz einer Einfach(st)erwerbstätigkeit in der Lagerlogistik kann letztlich in die hier miteinander verknüpften Situationen zergliedert werden. In den einzelnen Situationen mit textuellen Anforderungen können zum einen diverse Element-Element-Interrelationen aufgedeckt werden: Im Beispiel ist dies der Fall, wenn die einzelne Produkt- oder Seriennummer in der Warenannahme oder in der Zustellungsvorbereitung verglichen wird, bzw., die Codes der Lagerplätze eingetragen oder abgelesen werden. Der/Die Mitarbeiter/in muss hier auf der einen Seite auf dem ,Lieferschein' die Objekte ,Seriennummer‘ oder ,Lagerplatz' identifizieren, auf der anderen Seite die ,Seriennummer' auf dem Gerät und den ,Lagerplatz` am Regal. Entscheidend für die Element-Element-Interrelation ist, die relevante Beziehung zwischen diesen zugehörigen Merkmalen des Sachverhalts zu erkennen und herzustellen und dies auch, wenn am Gerät anstelle der ,Seriennummer' ein ,SN' eingraviert ist und man vergeblich am Regal den Begriff, Lagerplatz‘ suchen würde. 


\section{Electron Europa}

Electron Industries Europe GmbH, Rheinstr. 15, 65760 Eschborn

Lieferschein-Nr. : $\quad 524873$

|IIIIIII

\begin{tabular}{lll} 
& $:$ \\
\hline Sachbearbeiter & $:$ & Kundendienst-Team \\
Telefon & $:$ & $06196 / 937-200$ \\
Fax & $:$ & $06196 / 937-289$ \\
Datum & $:$ & 23.03 .2011 \\
Seite & $:$ & 1
\end{tabular}

Lieferschein Nr. 524873

(Bitte bei Rückfragen angeben)

\begin{tabular}{|c|c|}
\hline $\begin{array}{ll}\text { Auftragsnr.: } & 554891 \\
\text { Annahmedatum: } & 06.03 .2011 \\
\text { Referenz: } & \text { Abgabe l. Electron-Shop } \\
\text { Referenzdatum: } & 06.03 .2011 \\
\text { Gerätedaten: } & \left.5689500014 \text { V69KF_T }{ }^{* * *}\right) \\
\text { Seriennr:: } & 98567 \\
\text { Herstellerdatum: } & \text { F2008 }\end{array}$ & $\begin{array}{ll}\text { Reparaturstatus: } & \text { Neugerät mit Verschrotten } \\
\text { Lagerplatz: } & \begin{array}{l}06-02-01-02 \\
\text { K_02-C-01 }\end{array} \\
\text { Versandart: } & 02 \text { Abholung Electron-Shop } \\
\text { Zahlungsbedingung: } \begin{array}{ll}\text { Z1 Sofort netto Kasse ohne } \\
\text { jeden Abzug }\end{array}\end{array}$ \\
\hline 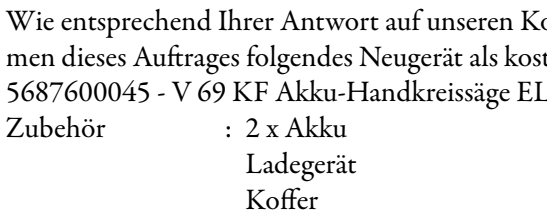 & $\begin{array}{l}\text { oranschlag 987567gewünscht, erhalten Sie im Rah- } \\
\text { ichtige Ersatzlieferung: }\end{array}$ \\
\hline
\end{tabular}

Mitfreundlichen Grüßen

Electron Werkzeuge

Service-Team

Es gelten unsere allgemeinen Geschäftsbedingungen (AGB): Unsere AGB sehen Sie bitte auf unserer Homepage unter www.electron-europa.com ein.

Electron Industries Europe $\mathrm{GmbH}$

\begin{tabular}{llll}
\hline Rheinstr. 15 & Email & Internet & Geschäfsführer: Friedrich Teichert, John Taride \\
65760 Eschborn & eleckundendienst@electron-europe.com & http://www.electron-europe.com & Sitz: 52255 Frankfurt am Main (HRB 87569)
\end{tabular}

Abb. 27: Lieferschein in der Lagerlogistik (Quelle: Imitation; Studie Basendowski) 
Übertragen auf eine Relation-Relation-Interrelation können die Phrasen des Textfeldes zum Merkmal ,Reparaturstatus' herangezogen werden. Sie geben nicht nur in beiden Systemen, der Handlung und der ikonischen Repräsentation, vor, welcher der routinierten Arbeitsschritte eingeleitet werden soll, sondern besetzen weitere Felder zugleich mit unterschiedlicher Bedeutung: Eine Phrase mit „Neugerät“ führt zum Eintrag der Serien- und Produktnummer im unteren Kasten des ,Lieferscheins'. In Kombination mit „Verschrotten“ wird in einem weiteren Arbeitsschritt die Prüfung des eingesandten Zubehörs verlangt. Es werden die Teile im selben Kasten erwähnt, die zurück an den Kunden gehen. Kurzum, das Merkmal ,Reparaturstatus` unterliegt Zusammenhängen mit weiteren Attributen, die Verlaufsstrukturen repräsentieren; der Sachverhalt dieser Situation ließe sich alternativ durch ein Ablaufschema darstellen. Der Begriff, Reparaturstatus impliziert in diesem Sinne kein „Element“, sondern eine „Relation“. Dass Begriffe in Tabellen beide Interrelationen ansprechen, lässt sich gleichermaßen an klassischen Rechnungen aufzeigen: Steht das Bezeichnungsfeld ,Gesamtpreis‘ (Relation) im Zentrum der Situation und formiert somit den Sachverhalt so verweist dieses auf den Zusammenhang zur ,Stückzahl' (Element) des ,Artikels' (Element) mit dem ,Einzelpreis' (Element); richtet sich die Betrachtung dagegen auf die ,Stückzahl' (Relation), so setzt sich diese aus der ,Verpackungseinheit' des ,Artikels' zusammen, welche z. B. einer anderen Tabelle entnommen werden kann.

Beide Beispiele verdeutlichen, was sich auch an den anderen bereits vorgestellten untersuchten Texten an Einfach(st)erwerbstätigkeiten fortzieht. Substantive stehen an der Stelle von Bezeichnungsfeldern, die „Elemente“ oder auch „Relationen“ beschreiben. In Tabellen ist hierbei in der Regel visuell nicht direkt ableitbar, auf welche „Elemente“ sie sich im Falle von „Relationen“ beziehen. Der ,Gesamtpreis'steht neben ,Stückzahl', ,Artikel', und ,Einzelpreis' und ist, wenn überhaupt, lediglich durch den implizierten graphischen Steuerungs-Kode der Kontur der Schrift (z. B. Fettschrift) hervorgehoben. Der hier inhärente multiplikative Zusammenhang in der Darstellung einer Tabelle begründet sich maximal in der Differenz von ,Einzelpreis‘ und ,Gesamtpreis` und leitet über zur Lexik, die nichtkontinuierliche Texte und hierunter insbesondere Tabellen, primär prägen: Begriffe „einzelsprachlicher Fachsprachen“ der Institutionen (Roelcke 2001).

Im allgemeinen gilt der Wortschatz als diejenige Ebene, auf der fachsprachliche Besonderheiten besonders deutlich zutagetreten. Zu diesen Besonderheiten zählen zum einen die intra- und interfachliche Bestimmtheit von Fachwörtern sowie zum anderen einige weitere Eigenschaften wie deren Definiertheit, Exaktheit, Eindeutigkeit und Metaphorik die jedoch je nach theoretischer und empirischer oder auch nach deskriptiver und präskriptiver Grundlage eine recht unterschiedliche Bewertung erfahren [...]. Grammatische Besonderheiten der deutschen Fachsprachen finden sich nur zum geringen Teil in qualitativer, sondern vor allem in quantitativer Hinsicht. Die Fachsprachengrammatik des Deutschen besteht in einer Selektion grammatischer Muster aus dem allgemein- oder standardsprachlichen Bereich, wobei sowohl Erscheinungen der Wort- und Formbildung auch solche der Syntax eine Rolle spielen; darunter: Kompositionsneigung, Derivations- und Abkürzungsmöglichkeiten, Neigung zu Nominalisierung und Funktionsverbgefügen, Erhöhung der Satzglied- und der Gliedsatzkomplexität. [...] Neben der Bestimmung und Gliederung von Fachsprachen sowie deren Eigenschaften auf den Ebenen Wortschatz, Grammatik und Text gehören schließlich auch Form und Funktion verschiedenartiger fachkommunikativer Hilfsmittel wie Graphiken (Tabelle, Matrix, Strukturbaum, Diagramm, Bild usw.), Wörterbücher [...] oder Normung [...] (ebd., 227).

Innerhalb einiger der diskutierten analogen Schriftstücke an Einfach(st)erwerbstätigkeiten als textuelles Mittel unter Beanspruchung von fachsprachlichen Begriffen einzelner Institutionen hat Schroeder (2011) hinsichtlich ihrer Wortbildung die Präferenz von mehrgliedrig zusammengesetzten Substantiven hervorgehoben. Diese stellen zwar grundsätzlich im Deutschen den „Proto- 
typ der Komposition "14 (Römer/Matzke 2005, 72) dar, unterliegen jedoch in ihren semantischen Wortgruppe-Wort- (endozentrisch) oder pars-pro-toto-Beziehungen (exozentrisch) der besagten intra- bzw. interfachlichen Bestimmtheit des einzelnen Arbeitsplatzes bzw. Berufsfeldes.

Insbesondere innerhalb der Analyse der Einfach(st)arbeitsplätze in der Gebäudereinigung verdeutlicht Schroeder (Schroeder 2011, 230f.) die Dominanz der endozentrischen Determinativkomposita; interessant vermag an dieser Stelle sein, dass die Bezeichnung, Gebäudereinigung selbst auf einer Metapher des Berufsfeldes beruht, denn die damit bezeichnete Bandbreite an Dienstleistungen lässt sich kaum mit der Paraphrasierung „Reinigung eines Gebäudes“ wirklich sämtlich erfassen. Das Leistungsverzeichnis umfasst nach dem Flyer einer Firma u.a. zusammengesetzte Substantive mit der finalen Konstituente „-reinigung“:

Fensterreinigung - Teppichbodenreinigung - Polstereinigung - Wohnungsreinigung - Wohnungsgrundreinigung - Wohnungsstaubreinigung - Treppenhausreinigung - Bauschlussreinigung - Baufeinreinigung - Fräsgutreinigung - Fassadenreinigung - Kunstrasenreinigung - Industrieflächenreinigung - Sonderflächenreinigung - ...

Die erste Konstituente umreißt oder die vorderen Konstituenten umreißen hier die relevante ,Wortgruppe' oder in anderen Worten, das, was gereinigt werden kann. In der Fachsprache im Berufsfeld sind jedoch die Kombinationen auch dahingehend begrenzt, dass es sich nur um Flächen usw. in, an oder um bestimmte Gebäude handelt. Es resultieren Fachbegriffe für spezifische Dienstleistungen mit Verweis auf teils sehr unterschiedliche Arbeitsschritte bzw. -anforderungen. Begrifflich erfasst werden sie mittels alltagssprachlicher Substantive nach einem ,grammatikalischen Muster aus dem allgemein- und standardsprachlichen Bereich'.

Die aufgefundene Textualität an Einfach(st)erbwerstätigkeiten, die primär auf kategorial-tabellarischen Repräsentationen reduziert werden konnte, hat hinsichtlich der darin verborgenen Anforde-

14 Es wird auf einige Oberkategorien in der linguistischen Betrachtung der Wortbildung nach Römer und Matzke (2005, 61ff.) eingegangen; auf diverse Sonderformen und teils unterschiedliche Kategorisierungen soll an dieser Stelle bewusst verzichtet und auf die Quelle verwiesen werden:

- Komposition: „Die Komposition ist eine Worbildungsart, bei der durch die Verbindung von mehreren, mindestens aber zwei Basismorphemen oder Stämmen ein neues Wort (Kompositum) entsteht“" (ebd., 71f.). „Unter Stamm soll hier immer eine Kombination von Morphemen verstanden werden, bestehend aus Basismorphem(en) und Wortbildungsmorphem(en)“ (ebd., 66), so z. B. bei Gleich/heit, und Flexionsmorpheme miteingeschlossen werden (Simme/n). Sie formieren Konstituenten bei einer nominalen Komposition, zwischen die ein Fugenelement zur Verknüpfung eingeschoben sein kann, welches jedoch keine bedeutungstragende Einheit darstellt (Wohn/ung-s-reinig/ung) (ebd. 66).

- Endozentrische Determinativkomposita: „Sie repräsentieren die umfangreichste und produktivste Gruppe unter den Komposita. Durch weitere Kombination zweigliedriger Komposita kann man theoretisch zu unendlichen Gebilden gelangen. Die semantisch-hypotaktische Relation zwischen den beiden Kompositionsgliedern zeigt sich darin, dass die 4. UK [ gemeint ist die 4. unmittelbare Konstituente, das erste Basismorphem oder der erste Stamm] die 2. UK semantisch determiniert, d. h. in ihrem Geltungsbereich, ihrer Extension einschränkt: Der Geltungsbereich von Fenster ist in Kellerfenster auf eben diese Art reduziert und bezieht sich nicht auf irgendein Fenster, sondern nur auf eine Teilklasse“ (ebd., 73). Es besteht „zwischen den UK eine Wortgruppe-Wort-Relation“" (ebd., 74).

- Exozentrisches Determinativkomposita: „Das was bezeichnet werden soll, wird durch das Kompositum selbst nicht erfasst. Löwenmaul kann sich als endozentrisches Determinativkompositum auf das Maul eines Löwen beziehen; als exozentrisches Determinativkompositum oder Possessivkompositum benennt es eine Blume, deren Blütenform vergleichbar mit dem Körperteil dieses Tieres ist. Der Terminus ,Possessivkompositum' verweist darauf, dass jeweils ein besitzendes Merkmal (z. T. als Metapher) ausschlaggebend für die Benennung ist. Possessivkomposita liegt eine pars-pro-toto Relation (ein Teil steht für das Ganze) zugrunde“ (ebd., 75). 
rungen folglich auch das Lesen von in erster Linie nominal zusammengesetzten Fachbegriffen an der Stelle von Bezeichnungsfeldern einzugehen: Sie zu lesen bedarf es, erstens, einer Einsicht in die Muster von (nominalen) Determinativkomposita. Das finale,Wort' oder Grundwort ist als solches mit seiner für das Deutsche üblichen tragenden semantischen Funktion für den Fachbegriff, als „morphologischer Head“ (Römer/Matzke 2005, 68), herauszuarbeiten; ggf. unter Berücksichtigung der Identifikation des Basis- und Wortbildungsmorphems (reinig/ung). Zudem ist zwischen bedeutungstragenden Flexionsmorphemen und nicht bedeutungstragenden Fugenelementen zu differenzieren: ,Sonder/fläch/en-reinig/ung'vs. ,Auftrag-s-nummer'. Die voranstehenden Wortteile in nominalen Komposita sind, zweitens, als, Wortgruppe (Auftrag-s-nummer) oder im Sinne von „pars-pro-toto“ (Sonder/fläch/en-reinig/ung), entgegen der konventionellen Leserichtung zu ergänzen. Sie sind es, die einen bestimmten aus möglichen Bezügen des Grundwortes explizieren; hierbei zweifelsohne nicht frei der semantischen Besetzungen von Fachbegriffen in einem Betrieb oder gar Berufsfeld. Drittens, markieren die von Roelcke bereits erwähnten Abkürzungsmöglichkeiten im Deutschen einen weiteren Anforderungscharakter: In Kompositionen können „Partielle Kürzwörter" (ebd., 71) einfließen, sodass im Grunde an der Stelle von ,Sonder/fläch/en-reinig/ ung' ein ,besondere/Fläch/en-reinig/ung'verborgen liegt.

Fachbegriffe gemäß der Wortbildung nach dem Muster der Determinativkomposita an der Stelle von Bezeichnungsfeldern leiten über zu einer gewissen Wahrscheinlichkeit, dass hier Relationen und nicht Elemente bezeichnet werden; respektive, „Relation-Relation-Interrelationen “ hergestellt werden müssen. Diese Wahrscheinlichkeit liegt in den vorgestellten „Wortgruppe-Wort“oder „pars-pro-toto“-Prinzipien begründet:

- Gesamtpreis (Lagerlogistik: Lieferschein) - Preis, der sich aus der Anzahl der Produkte und ihrem Preis, je nach der Einheit, in der sie verkauft wird, gesamt ergibt

- Reparaturstatus (Lagerlogistik: Lieferschein) - Status innerhalb der Status des Verlaufs einer Reparatur

- Seriennummer (Lagerlogistik: Lieferschein) - Nummerischer Code, der kontextuell dem Eigennamen eines Produktes entspricht

Im Falle von „Rektionskomposita“ bilden die Konstituenten des Fachbegriffs gar vergleichbar einer Satzphrase eine eindeutige Argumentenstruktur, „die ein Agens (,jemand'), einen Adressaten (,jemanden') und ein Thema (,etwas') als Argumente enthält" (ebd., 76). Dies ist der Fall, wenn die finale Konstituente aus einem verbalen Basismorphem besteht; und unabhängig davon, ob jenes Basismorphem durch ein Wortbildungsmorphem z. B. substantiviert wird. Die dem verbalen oder deverbalen morphologischen Head voranstehende Konstituente/n formiert/en deren Argument:

- Sonderflächenreinigung (Gebäudereinigung: Verzeichnis) - eine besondere Fläche reinigen; das Besondere kann sich z. B. durch die Beschaffenheit der Oberfläche oder infolge spezifischer Hygienebedingungen kontextuell ergeben

- Laderaumabdeckung (Wagenpflege: Fahrzeugprotokoll) - etwas, das den Laderaum eines Fahrzeugs abdeckt

- Fahrzeugprotokoll (Wagenpflege: Fahrzeugprotokoll) - der Zustand eines Fahrzeugs ist zu protokollieren

In corpore, wenn in kategorial-tabellarisch gegliederten Texten für Bezeichnungsfelder „einfache“ bis auf Satzphrasen zurückführende zusammengesetzte Begriffe stehen, so handelt es sich hierbei zuvorderst um einzelne Elemente (Merkmale, Attribute) oder eine einzelne Relation (zwischen Attributen eines Merkmals) aus einem Sachverhalt. Nur vereinzelt sind Fachbegriffe einzelner Betriebe oder eines Berufsfeldes nach dem Muster von Determinativkomposita imstande, auch 
auf darüberhinausgehende Beziehungen zwischen gar mehreren Elementen und Relationen zu vermitteln, die den übergeordneten semantischen Kern der textuellen Repräsentation des Sachverhalts ausmachen. In einem Fließtext leisten wiederum symbolische Einheiten die Verknüpfung mehrerer Satzphrasen; sie gestalten die Aneinanderreihung von Satzphrasen und Sätzen unter Einhaltung mehrerer arbiträrer Regeln. An diese Stelle treten in kategorial-tabellarisch gegliederten Texten nunmehr die mehr oder minder standardisierten analogen Arrangements ikonischer Entitäten und der damit verknüpften Sets an mehr oder minder starken Codes.

\subsection{Verstehende Verarbeitung Sets ikonischer Zeichen im Spannungsfeld des „Sozialen“ und „Situativen“}

Gemäß Kapitel 3.4.2 werden die Prozesse der (symbolischen) Textverarbeitung einem onlineCharakter gleich skizziert. Dieser Charakter beruht auf einer intensiven wissenschaftlichen Forschung insbesondere der Textlinguistik der vergangenen Jahrzehnte und ist im Spiegel der (Weiter-)Entwicklung von Makromodellen zu betrachten, in welchen eine große Bandbreite an Einflussfaktoren Berücksichtigung finden sollen, die ein Textverstehen beeinflussen können (vgl. Tabelle 14-16, linke Spalte). Demnach initiieren vorliegende Texte einerseits eine Art textstrukturellen Möglichkeitsraum von Relations- bzw. Interferenztypen und -tiefen zwischen einzelnen Konstituenten (Buchstabe, Wort, Satz und Text; des jeweils verwendeten symbolischen Zeichen- und Sprachsystems). Diese Initiierung führt zu fortlaufenden, zirkulären Evaluierungen einer konsistenten Kohärenz mittels (Vor-)Wissensstrukturen, Zielsetzungen und Erwartungen.

Die zahlreichen Arbeiten [...] verdeutlichen, dass die situative Textrepräsentation multidimensional ist. Dies bedeutet, dass sowohl Informationen über räumliche und zeitliche Aspekte der Situation, über handelnde Personen, deren Charakteristika, Emotionen, Ziele als auch über Kausalzusammenhänge enthalten sind. Das Situationsmodell ist notwendigerweise eine inhaltlich korrekte Abbildung der im Text enthaltenen Informationen. [...] Die Bildung eines Situationsmodells ist also flexibel, vorwissensund zielabhängig" (Deutsches PISA-Konsortium 2001, 72).

Entgegen diesen im Weiteren als explizit-konzeptuell bezeichneten Makromodellen der Textverarbeitung verorten die als implizit-embodied/inkorporiert bezeichneten Makromodelle Relationsbzw. Interferenztypen und -tiefen nicht in die Textstruktur, sondern vielmehr in die von Erfahrung geprägten Interpretation der Lesesituation der/des einzelnen Rezipienten/in. Während ihnen mit den explizit-konzeptuellen Makromodellen gemein ist, dass eine Evaluierung von Kohärenz via mentale Modelle konzeptioniert wird, beruhen sie auf konkurrierende Sichtweisen und Ansätze, die teils mit spezifischen Akzentuierungen - und somit Forschungsinteressen - einhergehen und teils durch eine „Menge von Forschungsarbeiten mit Einsichten, darunter auch Unklarheiten und Widersprüchen ,begleitet' [werden], die etliche Kontroversen produziert haben“ (Leopold 2009, 166). In corpore, ermöglichen jene vorliegenden umfassenden Theoriemodelle kaum „ein integriertes Bild der beteiligten Prozesse beim Verstehen eines Textes aufzubauen. Es fällt jedoch auch auf, dass sich bisher kaum um eine Integration der Befunde bemüht wurde“ (ebd.).

Erschwerend kommt hinzu, dass, wie bereits erwähnt, im Vergleich zu der Verarbeitung symbolischer Repräsentationen für die ikonischen sowohl eine deutlich geringere Forschungsaktivität als auch Konzeptionstiefe besteht. Durch das Beschreibungsmodell von Schnotz (1994) zur Verarbeitung von primär logischen Bildern als auch durch die Beschreibungsmodelle von Seufert (2003) und Rouet (2006) zur Verarbeitung von multiplen Repräsentationen liegen jedoch recht tragfähige Ansätze vor, welche den Aufbau von Verstehensstrukturen insbesondere auf der Textoberfläche nichtkontinuierlicher Repräsentationen behandeln. 
Vor diesem Hintergrund resultiert konsequenterweise für eine Annäherung an die kognitiven Verarbeitungsprozesse ikonischer Repräsentationen eine theoretische konzeptionelle Symbiose. In einem ersten Schritt werden für diesen Zweck aus dem Stand der Forschung zu jenen Verarbeitungsprozessen entlang von amodalen bis strukturell-modalen Makromodellen beider Zeichensysteme dekomponiert. In einem zweiten Schritt erfolgt eine Zusammenführung auf dieser Ebene von Makromodellen. Die Darlegung jener Symbiose gliedert sich hierbei ausgehend von den Konstituenten beider Zeichensysteme: von der Identifikation einzelner Entitäten aus semiotischen Zeichen - Graphem und Lokalzeichen (vgl. Tabellen 14 \& 15) - bis hin zur Dekodierung ganzer Sets dieser Entitäten semiotischer Zeichen - Text und Globalzeichen (vgl. Tabelle 16). Bei den ,Leerstellen' hinsichtlich der Verarbeitung ikonischer Texte und Textkomplexe werden theoretische Konzepte und empirische Befunde auf der jeweiligen Ebene symbolischer Zeichen zu Rate gezogen. Darüber hinaus werden Ergänzungen via Transfers aus den bisherigen semiotischen Analysen zu diesem Zeichensystem bemüht. Ein dritter Schritt zielt auf die Dekomposition eines Arbeitsmodells bzw. hypothetischen Makromodells der Verarbeitung multikodaler Texte und Textkomplexe primär ikonischer Kodalität.

\subsubsection{Spur 1: Amodale vs. schematisch-modale Makromodelle der verstehenden ikonischer Repräsentationen}

Innerhalb der Beschreibungsmodelle zur Identifikation und Verarbeitung von ikonischen Zeichen werden diese in Lokal- und Globalzeichen untergliedert - analog zur Gliederung von Graphem, Wort, Satz, Absatz, usw. bei symbolischen Zeichen. Globalzeichen beruhen hierbei entgegen Lokalzeichen auf bestimmte konventionalisierte „globale“ graphische Schemata, respektive, auf bestimmte Anordnungen von Lokal- zu Globalzeichen, welche über spezifische arbiträre Analogien auf Relationen eines Sachverhalts im Bezeichneten und in der Repräsentation verschränkt sind. Während ein Globalzeichen somit auf graphische Schemata höherer Ordnung im Vergleich zu Lokalzeichen beruhen (Schnotz 1994, 123), ist darüber hinaus die weitere Kategorisierung je nach Text als Pol in einem Kontinuum zu interpretieren (Seufert 2003, 24): Auch mit Blick auf die, an einfachen Erwerbstätigkeiten aufgefundenen mehrseitigen nichtkontinuierlichen Textkomplexe, welche hinsichtlich ihrer textstrukturellen Besonderheiten in den Vorarbeiten wie bereits erwähnt keine Berücksichtigung finden, wird auch mit dem Ziel der Gegenüberstellung mit symbolischen Repräsentationen im Weiteren die folgende Untergliederung berücksichtigt (vgl. Kapitel 2.3.2.3 \& 2.3.2.4):

- Globalzeichen höherer Ordnung - bezieht sich auf einen, auf der Textoberfläche vorliegenden nichtkontinuierlichen Textkomplex, der sich aus mindestens zwei nichtkontinuierlichen Texten basierend auf je eigenständigen graphischen Schemata - Globalzeichen mittlerer Ordnung - zusammensetzt (Beispiel: Textkomplex aus Empfangsschein und Serviceanforderung)

- Globalzeichen mittlerer Ordnung - bezieht sich auf einen, auf der Textoberfläche vorliegenden nichtkontinuierlichen Textkomplex, der aus mindestens zwei nichtkontinuierlichen Texten - Globalzeichen niedriger Ordnung - basierend auf einem gemeinsamen graphischen Schema zusammensetzt (Beispiel: Rechnung)

- Globalzeichen niedriger Ordnung - bezieht sich auf einen, auf der Textoberfläche vorliegenden nichtkontinuierlichen Text aus ikonischen Lokalzeichen (Beispiel: Bautagebuch)

- Lokalzeichen höherer Ordnung - bezieht sich auf mehrere, auf der Textoberfläche repräsentierte Merkmale oder Attribute innerhalb einer einzelnen Tabelle, einer einzelnen graphischen Darstellung, eines einzelnen Diagramms oder einer einzelnen Karte, die auf einem graphischen Schemata mehrerer Lokalzeichen niedriger Ordnung aufbauen (Beispiel: „eine Spalte“ im Bautagebuch) 
- Lokalzeichen niedriger Ordnung - bezieht sich auf ein, auf der Textoberfläche repräsentiertes Merkmal oder Attribut innerhalb einer einzelnen Tabelle, einer einzelnen graphischen Darstellung, eines einzelnen Diagramms oder einer einzelnen Karte (Beispiel: ein Textfeld in „einer Spalte“ im Bautagebuch)

Die Bedeutungseinheit zu einer identifizierten, ikonischen Textoberfläche wird innerhalb eines imaginalen Verarbeitungssystems konzeptioniert: Ikonische Repräsentationen stellen externalisiert einen Sachverhalt dar, deren Kohärenz lediglich auf der Textoberfläche textstrukturell als mehr oder weniger hierarchisch komplex kategorisiert werden kann. Diese Kohärenz ist von der internalisierten zu differenzieren, sodass sich die Einheit aus beiden Seiten „keine statische Eigenschaft" besitzt und "sich im Kommunikationsprozess zwischen Autor und Leser" manifestiert (Seufert 2003, 23f.). Es wird angenommen, dass hierbei initiiert durch die textuelle Repräsentation ikonischer Zeichen zuvorderst ein analoges, quasi-räumliches mentales Modell aus „hypothetische[n] mentale[n] Quasi-Objekte[n], die in einer Struktur- oder Funktionsanalogie zu dem dargestellten Gegenstand stehen“, konstruiert werden (Schnotz 1994, 127).

Tab. 14: Hierarchie-niedrige Prozessebene: interaktive Identifikation von Lokalzeichen

\begin{tabular}{|c|c|}
\hline symbolische Repräsentationen & ikonische Repräsentationen \\
\hline $\begin{array}{l}\text { - Die Buchstaben- und Wortidentifikation wird } \\
\text { nicht singulär erklärt, sondern über den „direkte[n] } \\
\text { visuelle[n] Zugang der Aktivationsausbreitung“ und } \\
\text { den sowohl „indirekte[n] Zugang über das phono- } \\
\text { logische System“ als auch „über die morphologische } \\
\text { Struktur“ (Christmann/Groeben 1999, 151). Gemäß } \\
\text { dem ,direkten visuellen Zugang' werden im Gedächtnis } \\
\text { neben den ,abstrakten Buchstabeneinheiten ' gleicher- } \\
\text { maßen Buchstabenfolgen in diesem Sinne gespeichert } \\
\text { und infolge der Identifikation einzelner Buchstaben } \\
\text { gehemmt oder aktiviert wird. Hierbei sind ,graphische } \\
\text { Elemente“ von Buchstaben, bzw. ,abstrakte Buch- } \\
\text { stabeneinheiten“ und nicht etwa „,konkrete Buchsta- } \\
\text { benformen“ im Gedächtnis gespeichert (ebd., 149). } \\
\text { Die interaktive visuelle Identifikation erfolgt durch } \\
\text { Erkennen jener graphischen Elemente von Buchstaben } \\
\text { und Wörtern einhergehend u. a. mit der Aktivierung } \\
\text { des Gedächtniseintrags durch „,Vorliegen bzw. Fehlen } \\
\text { bestimmter Merkmale“ (ebd.). Die, ,indirekte[n] } \\
\text { Zugänge“ verdeutlichen einen in der Wortidentifika- } \\
\text { tion inhärenten interaktiv-konstruktiven Charakter } \\
\text { und beschreiben das sukzessive Analysieren und } \\
\text { Synthetisieren durch die Relation von Graphem und } \\
\text { Phonem - einzelheitlich oder als Entität eines Mor- } \\
\text { phems - einschließlich Aktivierungen und Hemmun- } \\
\text { gen (ebd., 150f.). Jener „Wortüberlegenheitseffekt“ bei } \\
\text { der Buchstabenidentifikation gilt übertragen auch für } \\
\text { einen „Satzüberlegenheitseffekt“, insofern hierdurch } \\
\text { ein referentieller Kontext für die Wortbedeutung } \\
\text { vorliegt, der als stark relevant sowie häufig beansprucht } \\
\text { bezeichnet werden kann (ebd., 152). }\end{array}$ & $\begin{array}{l}\text { - Die Lokalzeichenidentifikation wird als attentiv } \\
\text { bezeichnet, da es sich um eine, auch durch Vor- } \\
\text { wissen und Zielsetzung der Verarbeitung beein- } \\
\text { flusste, bewusste konzeptgeleitete Analyse der } \\
\text { wahrgenommenen, visuellen Rohdaten handelt } \\
\text { (Schnotz 1994, 122). Zu diesen Konzepten zäh- } \\
\text { len u. a. graphische Schemata von Lokalzeichen, } \\
\text { wobei mehrere, aber zugleich eine begrenzte } \\
\text { Anzahl von Entitäten dieser graphischen Sche- } \\
\text { mata auf einen Blick selegiert erfasst werden. } \\
\text { - Demgegenüber wird die Identifikation von Ele- } \\
\text { mentarzeichen als „kaum willentlich beeinfluß- } \\
\text { bar (sic!)“ über Automatismen im „Erkennen } \\
\text { bestimmter visueller Eigenschaften der Objekt- } \\
\text { welt“ (ebd., 120) beschrieben. Elementarzeichen } \\
\text { stellen hierbei bestimmte Gruppierungen der } \\
\text { graphischen Entitäten von Punkte, Linien, } \\
\text { Flächen dar und umfassen sowohl „,distinktive } \\
\text { Merkmale“ wie Höhe, Breite, Strichstärke, } \\
\text { Textur usw. als auch „,integrierte Merkmale“ wie } \\
\text { z. B. Farbe, Sättigung. Jene Automatismen beim } \\
\text { Erkennen beruhen zuvorderst auf den Prinzipien } \\
\text { der sogenannten Gestaltgesetze, bspw. dem } \\
\text { Gesetz der Nähe, der Ähnlichkeit, Geschlossen- } \\
\text { heit oder der guten Fortsetzung. Beispiel eines } \\
\text { Elementarzeichens ist eine Linie, die sich mit } \\
\text { anderen Linien schneider; sie wird dennoch als } \\
\text { Linie erkannt (ebd., 120f.). }\end{array}$ \\
\hline
\end{tabular}




\begin{tabular}{ll}
\hline symbolische Repräsentationen & ikonische Repräsentationen \\
\hline - Als interaktiv muss die Buchstaben- und Wortidenti- & - Diese konzeptgeleitete Analyse konstituiert sich \\
fikation interpretiert werden, weil hierbei neben den & aus sich wechselseitig beeinflussende bottom-up- \\
umschriebenen bottom-up- auch top-down-Prozesse & und top-down-Prozessen (ebd., 122ff.). \\
einflußnehmend sind: durch semantische Textbedeu- & \\
tungsstrukturen, durch Vorwissen, durch Erwartun- & \\
gen und Zielsetzungen an einen Text usw. & \\
\hline
\end{tabular}

Hierbei wird betont, dass „repräsentierte Merkmale und repräsentierende Merkmale [...] durchaus voneinander verschieden sein können“ (ebd.). Jene somit bei ikonischen Repräsentationen zeichencodeabhängigen, primär initiierten quasi-räumlichen mentalen Modellierungen können sowohl zu einzelnen Lokalzeichen als auch zu einzelnen Globalzeichen auf der Textoberfläche konstruiert werden. Für die Kohärenzbildung gilt vielmehr, dass sie einerseits sequenziell bottom-up erfolgen kann, d. h. ausgehend von Extraktionen mentaler Modelle zu Lokalzeichen, die verknüpft oder verdichtet zu einem mentalen Modell des Globalzeichens komponiert werden. Andererseits kann man „ebenso mit eher globaleren Verarbeitungsprozessen beginnen, bevor [...] einzelne lokalere Details intensiver verarbeitet" werden (Seufert 2003, 24f.) oder in anderen Worten, gemäß einer Verarbeitung top-down, ausgehend von einer globalen/globaleren Kohärenzbildung hin zu lokalen/lokaleren Kohärenzbildungen. Übertragen aus der Kohärenzbildung entlang von Mikro- und Makro-Propositionen bei symbolischen Repräsentationen könnte man folglich gleichermaßen von Mikro- und Makro- (quasi räumlichen) Modellen bei der verstehenden Verarbeitung ikonischer Zeichen ausgehen.

Tab. 15: Hierarchie-niedrige Prozessebene: interaktive Verarbeitung von Lokalzeichen

\begin{tabular}{ll}
\hline symbolische Repräsentationen & ikonische Repräsentationen \\
\hline - Mikro-Propositionen stellen eine quasi- & - (Mikro-)Mentale quasi-räumliche Modelle zu Lokalzeichen \\
sprachliche Bedeutungseinheit zu einer & umfassen die verstehende Verarbeitung ikonisch repräsen- \\
vorliegenden Satzphrase dar, welche über & tierter Merkmale und ihrer Relationen (bspw. auch zwischen \\
Argument-Prädikat-Strukturen die seman- & bestimmten Objekten eines Merkmals oder Attributs). \\
tischen Relationen zwischen den hierbei & Diese lässt sich textstrukturell entlang von syntaktischen \\
eingebundenen Wortkonzepten - und eben & und/oder semantischen Relationen zwischen den Entitäten \\
nicht von Worten selbst - repräsentieren & differenzieren (Seufert 2003, 28ff.): Erstere beziehen sich \\
(Christmann/Groeben 1999, 152f.). & auf Oberflächenmerkmale wie eine identische Texturfarbe/ \\
- Jene Mikro-Proposition zu extrahieren wird & -schraffierung, Hintergrundfarbe/-schraffierung, Größe, \\
als Kern der verstehenden Verarbeitung & Form (und letztlich auch räumlichen Lage) dieser Entitäten \\
einer vorliegenden Satzstruktur - als Teil & (,welche im Grunde als Darstellungs- und Steuerungs- \\
der Herstellung einer lokalen Kohärenz - & Kodes zu differenzieren sind; vgl. Kapitel 2.3.2.4 - 2.3.2.6). \\
gefasst (Kintsch 2012, 26; Christmann/ & Zweitere referieren eine identische Repräsentationsanalogie, \\
Groeben 1999, 153). Jene Extraktion der & welche mit der graphischen Repräsentation der Entitäten \\
semantischen Sinnstrukturen können & verknüpft ist (vgl. ebd., 30f.). (Übertragen auf kategoriale \\
durch syntaktische Identifikationen und & Tabellen handelt es sich um die semantische Beziehung \\
Segmentierungsstrategien, ,parser', - bspw. & zwischen jenen Objekten eines Merkmals oder Attributs, \\
,kanonische Sentoid-Strategie “,minimal & welche gemäß Kapitel 2.3.2.2 als eine terminologische, \\
attachment', ,late closure', gestützt werden & kategoriale, topologische oder Verlaufsstruktur bzw. als ein \\
(ebd., 154f.). & quantitativ-qualitativer Zusammenhang begrenzt werden \\
& kann: ein „Mehr“, ein „Höher“, ein ,Synonym“, ein „Vorher“ \\
& usw. des einen Merkmals im Bezug zum zweiten Merkmal.) \\
\hline & \\
\hline
\end{tabular}


Tab. 16: Prozessebene: interaktive Verarbeitung von Globalzeichen

\begin{tabular}{|c|c|}
\hline symb & xon \\
\hline $\begin{array}{l}\text { Makro-Propositionen stellen abstraktere und globale } \\
\text { quasi-sprachliche Bedeutungseinheiten über mehrere } \\
\text { Sätze und Absätze hinweg her und rekurrieren auf } \\
\text { eine Verknüpfung oder Verdichtung von mikro- } \\
\text { propositionalen Repräsentationen. Entgegen dem } \\
\text { Verständnis als eine simple Addition einzelner } \\
\text { Bedeutungseinheiten je Satzphrase, sind Interferen- } \\
\text { zen zwischen diesen Mikro-Propositionen zu konst- } \\
\text { ruieren, zu selegieren, zu generalisieren, zu integrieren } \\
\text { oder auszulassen (Kintsch 2012, 26; Christmann/ } \\
\text { Groeben 1999, 166). Jene Interferenzbildung wird } \\
\text { in (a) semantisch-syntaktische Relationen - bspw. } \\
\text { gemäß der Koreferenzarten, dem Thema/Rhema- } \\
\text { Konzept - in (b) semantisch-pragmatische Relatio- } \\
\text { nen - bspw. gemäß dem Konzept-Evidenz-Konzept } \\
\text { - in (c) textorganisatorisch-rhetorische Relationen - } \\
\text { bspw. durch an der Textoberfläche hervorgehobenen } \\
\text { Funktion von Absätzen im Sinne einer Einleitung, } \\
\text { eines Vergleichs usw. - und in (d) über textstrukturell } \\
\text { hinausreichenden Konzeptrelationen - dem „Wissen } \\
\text { über die nicht-sprachliche Realität sowie die Kennt- } \\
\text { nis des situativen Zusammenhangs, in dem die Sätze } \\
\text { eingebettet sind“ (ebd., 157f.). } \\
\text { - Beim Verstehen eines Textes ist die Extraktion von } \\
\text { amodalen (Stichwort: symbol grounding problem) } \\
\text { Mikro-/Makropropositionen schlussfolgernd als } \\
\text { interaktiv verknüpft mit nicht zwingend textuell } \\
\text { repräsentierten bzw. nicht-sprachlichen Bedeutungs- } \\
\text { einheiten zu interpretieren. Jene zweite Ebene des } \\
\text { Verstehensprozesses wird in Metatheorien innerhalb } \\
\text { einer mentalen Modelltheorie verortet: Neben } \\
\text { einer propositionalen Ebene wird eine Ebene eines } \\
\text { mentalen Modells - oder weitgehend synonym eines } \\
\text { „referentiellen Modells“, eines „Situationsmodells“, } \\
\text { eines „discourse models“ oder eines „Szenarioreprä- } \\
\text { sentation“ (Leopold 2009, 149ff.) - positioniert, } \\
\text { welche „represent what the text is about, not the text } \\
\text { itself (Glenberg et al. 1987,,70 zit. nach ebd., 149). In } \\
\text { einigen Makromodellen werden diese beiden Ebenen } \\
\text { zudem durch die Ebene der Textoberflächenrepräsen- } \\
\text { tation ergänzt (Kintsch 2012), der Repräsentation } \\
\text { des exakten Wortlyuts eingehettet indie vorliegende }\end{array}$ & $\begin{array}{l}\text { räumliche Modelle stellen } \\
\text { sseinheit von Relationen } \\
\text { okalzeichen eines Global- } \\
\text { er Ordinate und Abszisse } \\
\text { (oder der Merkmale und } \\
\text { orialen Tabelle, oder der } \\
\text { betrag-Rechnung usw.). } \\
\text { tischen und semantischen } \\
\text { rren lassen sich auch für } \\
\text { elle syntaktische und } \\
\text { eelation-Strukturen ausma- } \\
\text { er Oberflächenmerkmale } \\
\text { äsentationsanalogien dieser } \\
\text { t 2003, 30f.). (Als Beispiel } \\
\text { pographische Textur der } \\
\text { er identische Steuerungs- } \\
\text { alen Tabelle, bzw., die über } \\
\text { en ikonischen Darstel- } \\
\text { alen Tabellen repräsentier- } \\
\text { irt werden; zweitere lassen } \\
\text { 2.2 in terminologische, } \\
\text { ne oder Verlaufsstrukturen } \\
\text { ttitativ-qualitativen Zusam- } \\
\text { len Merkmalen und/oder } \\
\text { en.) } \\
\text { ggen, dass die Verarbeitung } \\
\text { nplementär über proposi- } \\
\text { lierungen erfolgen kann: } \\
\text { Verstehensprozesses wird } \\
\text { (Mayer } 1997,5 \text { zit. nach } \\
\text { ugt. } \\
\text { der Bearbeitung logischer } \\
\text { zegenüber „Novizen“ ver- } \\
\text { ber elaboriertere Strategien } \\
\text { wwohl Global- als auch } \\
\text { stellungs- und Steuerungs- } \\
\text { erten [sind] besser in der } \\
\text { ifenden Mustern zu suchen, } \\
\text { eine lokal begrenzte Suche } \\
\text { ten vornehmen“ (Schnotz } \\
\text { erschied manifestiert sich } \\
\text { lleren“ Identifikation der } \\
\text { eten globalen und lokalen } \\
\text { als Darstellungs- aber auch } \\
\text { lererseits wird der Zusam- } \\
\text { ieften konzeptgeleiteten } \\
\text { tisch) Kohärenzbildung } \\
\text { ohl die Dekodierung der } \\
\text { ken Repräsentationsanalo- }\end{array}$ \\
\hline
\end{tabular}




\begin{tabular}{|c|c|}
\hline symbc & ionen \\
\hline 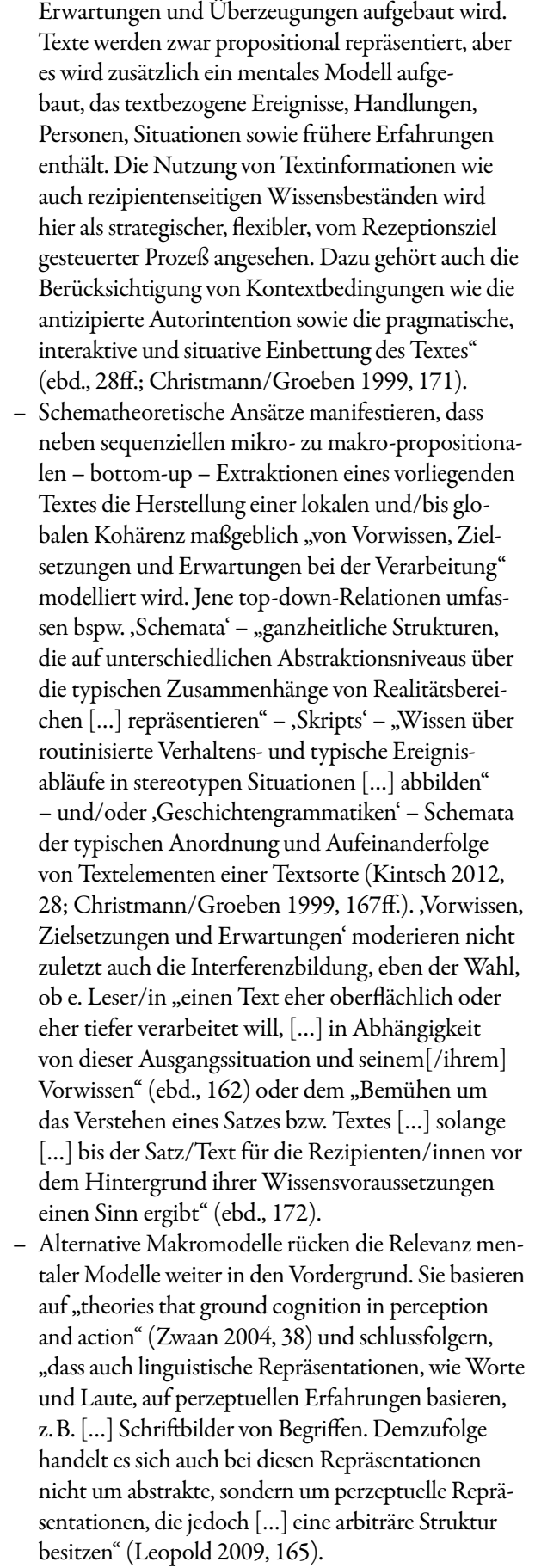 & 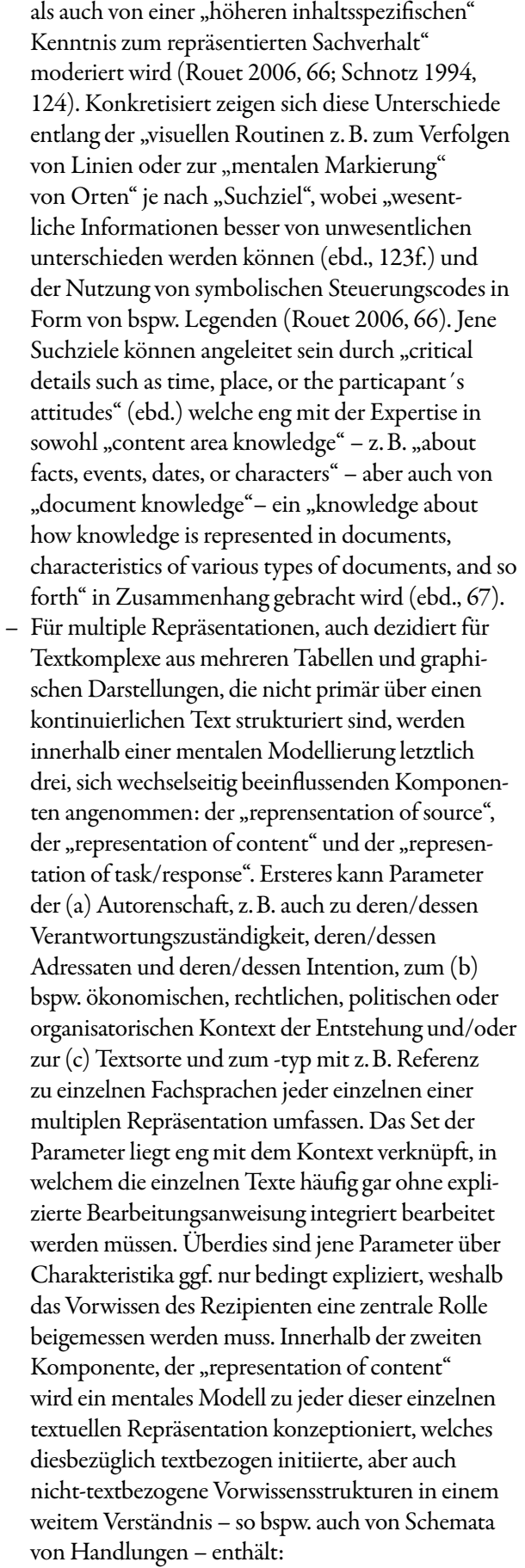 \\
\hline
\end{tabular}




\begin{tabular}{|c|c|}
\hline symbolische Repr & ikonische Repräsentationen \\
\hline $\begin{array}{l}\text { Demgemäß wird beim Lesen eines visuellen } \\
\text { symbolischen Inputs eine „experiential representa- } \\
\text { tion of words (lexical, grammatical, phonological, } \\
\text { motoric, tactile) as well as assocciated experiential } \\
\text { representations of their referents [aktiviert] - } \\
\text { motor, perceptual, and emotional representations, } \\
\text { and often combinations of these [...]. These } \\
\text { traces [...] enable reconstitution of experience. In } \\
\text { this sense, then, comprehension is the vicarous } \\
\text { experience of the described events through the } \\
\text { integration and sequencing of traces from actual } \\
\text { experience cued by the linguistic input (Zwaan } \\
\text { 2004, 38). Konzeptioniert, werden mentale } \\
\text { Modelle über die nicht sequenziell sondern sich } \\
\text { wechselseitig beeinflussenden Komponenten } \\
\text { „Aktivierung“ auf der Wortebene, „Eingrenzung“ } \\
\text { auf der Satzebene und „Integration“ auf der Tex- } \\
\text { tebene konstruiert, welche auf jeweils perzeptuelle } \\
\text { Simulationen von „experimental traces“ beruhen } \\
\text { (Leopold 2009, 157ff.; Zwaan 2004, 38ff.): } \\
\text { bspw. zu einem Begriff(Wortebene), der nach } \\
\text { Zeit, Raum und Perspektive eingegrenzt wird } \\
\text { (Satzebene) UnD mit weiteren über Transitionen } \\
\text { integriert wird (Textebene), wie „das Heran- und } \\
\text { Herauszoomen, das Abscannen oder das Fixieren } \\
\text { auf ein bestimmtes Objekt“ bzw. ,zwischen senso- } \\
\text { rischen Modalitäten, z. B. wenn [...] plötzlich auf } \\
\text { die Emotionen der handelnden Person eingegan- } \\
\text { gen wird“ (Leopold 2009, 158). Innerhalb jener } \\
\text { Konstruktion von mentalen Modellen verliert } \\
\text { die komplexe Interferenzbildung, der Kern der } \\
\text { propositionalen Extraktion auf der Textebene an } \\
\text { Bedeutung, ,weshalb Johnson-Liard (1983) auch } \\
\text { von expliziten oder Pseudo-Interferenzen spricht“ } \\
\text { (ebd., 151). }\end{array}$ & $\begin{array}{l}\text { In der „representation of content“ werden einerseits } \\
\text { mehrere Bedeutungseinheiten „direkt“ aus der Text- } \\
\text { oberfläche extrahiert, verknüpft und verdichtet und } \\
\text { andererseits ,,indirekt“ über sogenannte „,source- } \\
\text { content-links“, ergo „indirekt“ über die mentale } \\
\text { Modellierung der ,sources“. Letztlich gilt für die } \\
\text { Kohärenzbildung über mehrere dieser einzelnen } \\
\text { Repräsentationen auf der Textoberfläche hinweg, } \\
\text { dass die expliziten und/oder impliziten Interferen- } \\
\text { zen zwischen diesen einzelnen Repräsentationen } \\
\text { auf der Textebene verarbeitet werden müssen - } \\
\text { bspw. im Sinne von einer Referenz, einer Abfolge, } \\
\text { einer Relevanz, im Geiste von, im Widerspruch } \\
\text { zu, in Bekräftigung gegenüber usw. Diese werden } \\
\text { sowohl in der Komponente der „representation } \\
\text { of source“ über sogenannte source-to-source- } \\
\text { links mental konstruiert - mithilfe eines Sets an } \\
\text { Parametern - als auch auf der Bedeutungsebene } \\
\text { innerhalb der „representation of content“ (ebd., } \\
71 f f .) \text {. Der nicht-lineare Struktur sowie der häufig } \\
\text { nicht explizierten Beziehungen innerhalb multipler } \\
\text { Repräsentationen geschuldet, kommt der dritten } \\
\text { Komponente des zu konstruierenden mentalen } \\
\text { Modells eine besondere Rolle zu: der „,represen- } \\
\text { tation of task/response“. Innerhalb dieser gilt es } \\
\text { sowohl die (a) textuellen, aber auch kontextuellen } \\
\text { Ressourcen (z. B. Zeitraum für die Bearbeitung, } \\
\text { verbalisierte Arbeitsaufträge, Charakter des End- } \\
\text { produkts) zu berücksichtigen, als auch die (b) Vor- } \\
\text { wissensstrukturen der Rezipienten (z. B. Schemata } \\
\text { von Handlungen, situativ-kontextuelle Erfahrun- } \\
\text { gen, thematisches Vorwissen) zu berücksichtigen, } \\
\text { die mit der und während der Verarbeitung eines } \\
\text { multiplen Textes zu konzeptionierenden und ggf. } \\
\text { modifizierenden „internal representation of the } \\
\text { search task to be completed“ und der zirkulär ver- } \\
\text { knüpften ,internal representation of the response } \\
\text { to be output“ (ebd., 106) einhergehen. }\end{array}$ \\
\hline
\end{tabular}

Aus den Gegenüberstellungen des state of the art zur Identifikation und verstehenden Verarbeitung von symbolischen Zeichen und ikonischen Lokal- bis Globalzeichen entfalten sich zuvorderst Parallelen, die sich kaum mit einer eindimensionalen Sichtweise auf das Lesen vereinen lassen. Hinter dieser Sichtweise verbirgt sich, die traditionelle Sichtweise der psychometrischen Textforschung auf eine generalisierbare kognitive Verarbeitung entlang der Performanz von Behaltensleistungen und einer Verarbeitungstiefe basierend auf einer objektiv hierarchisierbaren Komplexität auf der Textoberflächenstruktur (Kintsch 2012, 34): Wenn hierin auf der Textbasis propositional-textstrukturell die Identifikationen eines Graphems - einer Entität eines symbolischen Zeichensystems - bis hin zum Verstehen eines kontinuierlichen Textes Set aus Entitäten eines symbolischen Zeichensystems - als ein sich wechselseitiges Ineinander- 
greifen von mikro- zu makropropositionalen bottom-up-Prozessen mit von metalinguistischen (syntaktisch, semantisch, rhetorisch, pragmatisch) und metatextuellen Konzepten begleiteten top-down-Prozessen erklärt werden, spricht im Übertrag vieles für ebenso wechselseitige Prozesse bei der Dekodierung eines depiktionalen Lokalzeichens bis hin zum Verstehen eines nichtkontinuierlichen Textes oder Textkomplexes. Wenn hierin propositional-textstrukturell in der Art und Tiefe von Interferenzen zwischen Propositionen jene hierarchisierbare Komplexität für herzustellende Relationen auf der Satzebene und von Interferenzen auf der Textebene postuliert werden, spricht auch diesbezüglich vieles dafür, dass innerhalb der repräsentierten Relationen auf der Ebene von Lokalzeichen und Interferenzen auf der Ebene von Sets aus Lokalzeichen durchaus hierarchisierbare Anforderungsgrade angenommen werden müssten. Diese liegen aus den bisherigen Darlegungen eng mit der explizit-konzeptionellen Verarbeitung jeglicher semantischer und syntaktischer Relationen zwischen Lokalzeichen und Interferenzen aus Sets von Lokalzeichen verknüpft, deren Spektrum textstrukturell in der ikonischen Darlegung eines Sachverhalts via Diagramme oder kategorialer Tabellen beansprucht wird (vgl. Kapitel 3.2.2); einschließlich sämtlicher arbiträren Repräsentationsanalogien in ihren Lokalzeichen wie bspw. Knoten, Kanten, Formen usw. bei Diagrammen oder wie Bezeichnungs- und Textfelder bei kategorialen Tabellen (vgl. Kapitel 3.2.3). Diese Anforderungsgrade würden neben der explizit-konzeptionellen Verarbeitung sämtlicher vorliegenden semantischen, syntaktischen und pragmatischen semiotischen Strukturen in den depiktionalen Repräsentationen via starke Darstellungs-Kodes gleichermaßen auch sämtliche nicht-arbiträren Darstellungs- und Steuerungs-Kodes zu berücksichtigen haben, die in den Kapiteln 3.2.5 und 3.2.6 thematisiert wurden. Dieser Übertrag ist insofern theoretisch belegbar, wenn jene hypothetisch angenommene explizit-konzeptionelle Verarbeitung ikonischer Zeichen im Kontext von „mikro- und makrotypographischen“ (Christmann/Groeben 1999, 159) Interferenzstrategien bei kontinuierlichen Texten diskutiert werden. Dahinter verbirgt sich z. B. die Einflussgröße der gezielten Variationen der Textur für die Kohärenzbildung.

Innerhalb der Gegenüberstellung wurden dagegen Erklärungsansätze der verstehenden Verarbeitung von primär ikonischen Repräsentationen favorisiert, die jene Verarbeitung als interaktiv zwischen sowohl bottom-up- als top-down-Prozessen positionieren. Hierüber werden insbesondere die Grenzen der postulierten, universellen Bestimmbarkeit von textuellen Komplexitätsgraden aufgegriffen. Denn aus der Leseforschung symbolischer Zeichen liegen eher Befunde vor, dass „sie [die amodalen Textbedeutungsstrukturen] ihrem Anspruch, eine völlig objektive Beschreibung der jeweiligen Textstruktur zu liefern, nicht gerecht werden (können). [...] Leser/innen erzeugen eine Textbedeutungsstruktur nicht ausschließlich auf der Grundlage koreferentieller Bezüge, sondern auch mit Hilfe von Relationen, die sich aus ihrem Vor- und Situationswissen ableiten" (Christmann/Groeben 1999, 164). Lediglich für die Interferenzbildungsart der referentiellen Identität konnten empirische Belege für jene universelle Bestimmbarkeit gefunden werden.

Textbasierte analoge Dekodierungen referieren demzufolge einerseits eine Art textstrukturell vorgegebenen Möglichkeitsraum über Relations- und Interferenzbeziehungen und -tiefen zwischen einzelnen Konstituenten - hier die Lokal-/Globalzeichen. Welche dieser Beziehungen andererseits wann aktualisiert werden und in eine fortlaufende, zirkuläre Evaluierung einer konsistenten Kongruenzbildung einfließen sowie moderieren, lässt sich weder zwingend auf ausschließlich textbasierte noch ausschließlich nicht-textbasierte (Vor-)Wissensstrukturen, Zielsetzungen und Erwartungen reduzieren. Es werden für das nichtkontinuierliche wie für das kontinuierliche Textverstehen folglich kognitive Prozesse angenommen, die durch explizit-konzeptionelle - semantische, syntaktische, pragmatische, rhetorische und metatextuelle - 
Verarbeitungsstrategien nur ausreichend charakterisiert werden können. Oder skizzenhaft und exemplarisch auf das Beispiel des Kassenbons (vgl. Kapitel 3.2.4) übertragen, pointiert sich die Relevanz einer multidimensionalen Sichtweise auf das Lesen auch nichtkontinuierlicher Texte, welche das Soziale und das Situative der Lesesituation dezidiert berücksichtigt: Hier anhand eines einseitigen Textkomplexes aus primär kategorialen Tabellen manifestieren sich singulär textstrukturell abgeleitete, als objektiv postulierte Komplexitätsgrade einer Kohärenzbildung dahingehend als nicht objektiv, da diese weder unabhängig von (Vor-)Wissensstrukturen, Erfahrungen und Zielsetzungen der Rezipienten noch von situativ-kontextuellen Bedingungen an ein Lesen dieses Kassenbons getroffen werden können: Müssen in einer Situation mit diesem Text für ein kompetentes Lesen die entfallenen Steuerbeträge und auch anzuwendenden Steuersätze je Gegenstand des Kaufvertrags in Beziehung gesetzt werden? Müssen zudem die Gegenstände des Kaufvertrags stets kontrolliert werden? Bedarf es immer einer Verarbeitung der Steuer-Identifikationsnummer? Verlangt die Situation zudem grundsätzlich ein Lesen mit dem Ziel bspw. der Behaltensleistung sämtlicher textuell vorliegender Informationen? Wird die Komplexität der Kohärenzbildung nicht maßgeblich von diversen Erfahrungen mit Kassenbons beeinflusst? Grenzt nicht vielmehr die Situation, in welche der Text eingebunden ist, entscheidend die Kohärenzbildung über die Auswahl innerhalb des Möglichkeitsraums an herzustellenden Bedeutungsstrukturen ein, die im Text stets in einer größeren Bandbreite vorliegen?

Ein Makromodell, welches versucht, jene sozialen und situativen Faktoren einer Lesesituation eines nichtkontinuierlichen Textes zu berücksichtigen hat Rouet $(2006,105 \mathrm{ff}$.) mit dem schemaorientierten TRACE-Modell of Document Processing vorgelegt, welches sich aus den folgenden drei ineinander greifenden „central components of text processing “ interaktiv zusammensetzt: „the construction of a task model“, „the assessment of document relevance“, „[the construction of an] internal response model“. Hierüber greift es die Bandbreite von „relevancebased“ initiierenden, begleitenden und evaluierenden (ebd., 102ff.) ,internal needs and environmental constraints“ deskriptiv auf, einhergehend mit den situativen Rahmenbedingungen von „available information resources and search tools“ (ebd., 105). Das Textverstehen als „cyclical extraction and integration of content information“ - respektive, die Konzeption des "content model“ - findet sich entsprechend im TRACE-Modell einerseits gerahmt von den mentalen Modellierungen des „task-models“ und des „response model“ - welche man ungefähr mit der konstruierten Idee der Interaktion mit einem vorliegenden Text und der konstruierten Idee eines, auch sozial, angemessen antizipierten Textverständnisses übersetzen könnte. Andererseits wird die mentale Modellierung des „content model“ von der eines „source models“ moderiert, welche in etwa mit der konstruierten Idee dessen umschrieben werden kann, welche Relevanz einzelnen Repräsentationen bei einer multiplen Darbietung im Verhältnis zueinander zugeschrieben werden. Mittels der Konkretisierung entlang eines Sets aus den Parametern „author“/ Autorenschaft, „setting“ und "form of the document" werden keine systematischen oder gar prädikativ-messbaren Einflussvariablen benannt. Stattdessen sind diese von der bei ExpertiseRezipienten diverser multipler Repräsentationen aufgedeckten Nutzung semantischer Bedeutungsstrukturen auf der metatextuellen Ebene abgeleitet, die bei der zirkulären Evaluation des „content" beansprucht wurden. Sie haben folglich einen deskriptiv-klassifizierenden Charakter, ergo, sie sind einerseits aus ihrer Abhängigkeit des "Sozialen“ und der "Situation“ heraus nicht als generalisierbare und somit stets notwendige explizit-konzeptuelle Verarbeitungsstrategien zu interpretieren. Experten von historischen Textkomplexen nutzen bspw. das folgende Set an Parametern - welche sich deutlich von denen unterscheiden, die Experten mit domänen-alternierenden Textkomplexen anführen (ebd., 63ff.): 
Author identification variables include the name of the author (whether an individual or an organization), his or her credentials (e. g., status, experience, reputation), and means as regards access to the information reported (e. g., witness, participant, student-of, individual, or teamwork). Information about the author also includes his or her motivations conveying the content (e. g., career, career, posterity), intended audience (students, colleagues, customers), and communication purposes (e. g., to inform, persuade, sell). [...] Source information also include the setting or context of production (i. e., place in which the document is created, date and historic period, economic, political, or cultural context). In addition, document form variables characterize the type (e. g., treaty, letter, textbook, magazine, essay, and so forth) and the language style (e. g., legal, diplomatic, conversational) of the document (ebd., 72f.)

Wenn sich eine Kohärenzbildung innerhalb dieses schemaorientierten TRACE-Modells über die zirkuläre Extraktion, Integration und Evaluation von Bedeutungseinheiten nicht alleinig auf den "content" bezieht, sondern gleichermaßen die moderierenden Beziehungen aus den konzipierten Ideen der "task“/,response“ und der „,sources“ umfasst, verdeutlicht sich nicht zuletzt eindrücklich das zu berücksichtigende „Soziale“ und „Situative“ einer Lesesituation. Zugleich erfährt der initiierende Charakter der Kohärenzbildung alleinig aus der vorliegenden Textstruktur eine Relativierung, welche von den explizit-konzeptionellen Erklärungsansätzen postuliert wird. Oder in anderen Worten, die mentale Konzeption jeder dieser konzipierten Ideen nebst dem „content" kann eine Auseinandersetzung mit einem Text initiieren, sprich alsdann einer Identifikation und Dekodierung einer ersten semiotischen Entität bereits vorausgehen; und trägt insofern der jeweiligen sozialen und situativen Einbettung Rechnung. Verdichtet in der Auseinandersetzung mit dem jeweils konkreten Text wird eine zirkuläre Extraktion, Integration und Evaluationen in Gang gesetzt, u. U. modifiziert, verworfen, neu konstruiert, usw. Mit diesem schematischen Ansatz geht jedoch nicht indirekt eine eindimensionale Sicht auf das Lesen nichtkontinuierlicher Texte und Textkomplexe alleinig über die „source information“ einher, die entlang der Parameter ,Autorenschaft' („,author“), ,Entstehungskontext“ (,situation“) und ,Textsorte und -typ („,form of document") vergleichbar den Superstrukturen bei kontinuierlichen Texten nach dem Mehrebenenmodell von Rosebrock und Nix (2006) strukturiert werden: Getrennt vom „content" würde mittels dieser Parameter keine stets objektiven Beziehungsstrukturen zwischen den „sources“, bzw. den Lokal- bis Globalzeichen bestehen, da die Kohärenzbildung darüber hinaus maßgeblich von der Komponente "task“/,response“ beeinflusst werden; so bspw. auch nicht zwischen einer Tabelle auf Seite $\mathrm{x}$ und einer Tabelle auf Seite y eines nichtkontinuierlichen Textkomplexes (vgl. Kapitel 3.2.4). Die vom Lesenden sowohl konstruierte Idee der Interaktion mit einem vorliegenden Text als auch die konstruierte Idee eines, auch sozial, angemessen antizipierten Textverständnisses rücken maßgeblich in den Mittelpunkt dieses schematisierten Makromodells des Lesens nichtkontinuierlicher Texte.

\subsubsection{Spur 2: Amodale vs. embodied-modale Makromodelle der verstehenden ikonischen Repräsentationen}

Die „embodied-theory“ des Textverständnisses setzt sich gleichermaßen mit den textuellen und nicht-textuellen initiierenden Bedingungen auseinander, indem es die kognitive Verarbeitung von Zeichen aus der ,assumption that thoughts, feelings, and behaviors are grounded in bodily interaction with the environment" (Meier et al. 2012, 705) betrachtet. Diese fundamental unterschiedliche Herangehensweise verdeutlicht sich insbesondere in den Arbeiten zum „Immersed Experiencer Framework“" nach v. a. Zwaan, welche für die vorliegende Auseinandersetzung dahingehend von besonderem Interesse sind, da sie sich dezidiert auf Sets symbolischer Zeichen, auf Wörter, auf Sätze und auf Texte, beziehen (Leopold 2009, 156): 
In short, the basic assumption is reading or hearing a word activates experiential representations of words (lexical, grammatical, phonological, motoric, tactile) as well as associated experiential representations of their referents - motor, perceptual, and emotional representations, and often combinations of these (see also Sadoski \& Paivio, 2001). These traces can be activated by verbal input and as such enable the reconstitution of experience. In this sense, then, comprehension is the vicarious experience of the described events through the integration and sequencing of traces from actual experience cued by the linguistic input" (Zwaan 2004).

Wie sich im Weiteren zeigen wird, liegen über diesen Theorieansatz des Textverständnisses, welcher die Konstruktion mentaler Modelle via die Aktivierung, Eingrenzung und Integration perzeptueller Simulationen von Erfahrungen verortet, fundierte Annahmen vor, welche zuvorderst die Extraktion, Integration und Evaluation von Bedeutungseinheiten der Kohärenzbildung im Kern „neu“ justieren: Diese akzentuieren insbesondere die Annahmen, die in stärker explizitkonzeptionellen Makromodellen das Textverstehen konzeptionieren, ergo, aus dem Primat von Extraktionen semantischer, syntaktischer, pragmatischer und rhetorischer Bedeutungsstrukturen aus einem vorliegenden Text innerhalb einer amodalen mentalen Modellierung von mikro-propositionalen Relationen und von makro-propositionalen Interferenzen, die alsdann komplementäre modale mentale Modellierungen initiieren und moderieren. Gleichermaßen aufbauend auf der semiotischen Identifikation eines Wortes verortet der „Immersed Experiencer Framework“ die Verarbeitung von Relationen auf der Ebene lokaler Kohärenz und die von Interferenzen auf der Ebene globaler Kohärenzen zuvorderst innerhalb der modalen mentalen Modellierung.

Gemäß diesem Framework prozessiert das mentale Modell räumlich-zeitlich aufgebaut, in sogenannten „events“. Grundsätzlich von der Körperlichkeit von Erfahrungen geschuldet werden diese „events“ entlang der „embodied tripartite classification of space“ unterschieden:

- „personal space“: dynamische mentale Modelle von Erfahrungen im Raum, der z. B. mit den Armen und Beinen direkt unmittelbar erreichbar ist;

- „action space“: dynamische mentale Modelle von Erfahrungen im Raum, der via Sprache, Wurf bzw. Schuss eines Gegenstands oder schnellen Laufwegen indirekt unmittelbar erreichbar ist;

- „vista space“: dynamische mentale Modelle von Erfahrungen im Raum, der über den „action space" hinaus noch über die Sinne erreichbar ist (mittelbar).

Oder in anderen Worten, es sind aus diesen Trias von Raum mannigfaltige dynamische mentale Modelle bspw. zu einem bestimmten Gegenstand oder Geräusch (= "focal entity") bei einer Person hypothetisch anzunehmen. Während ein dynamisches Modell, ein „event“, auf eine der diversen direkt unmittelbaren Erfahrungen mit dieser „focal entity“ aus der körperlichen Perspektive dieser Person beruhen, referieren andere „events“ auf eine der diversen indirekt unmittelbaren oder eine der diversen darüber hinaus noch sinnlich wahrnehmbaren Erfahrungen. Diese Referenz der Person selbst stellt jedoch lediglich eine der „perspectival components“ dar, die der (a) „location“, welche erst zusammen einen detaillierten Einblick in den Aufbau der angenommenen modalen mentalen Modelle liefern. Weitere sind (ebd., 44f.):

- (b) „distance“: Mit dieser Komponente werden Bedingungen aus der räumlichen Beziehung der Person zu der „focal entity“ akzentuiert. Diese umfassen einerseits, dass sich mit der räumlichen Beziehung die Gestalt der „focal entity“ verändert. Beispielsweise „sieht“ ein „Flugzeug“ oder ein "Sofa“ unterschiedlich aus, wenn es sich im Vergleich zur unmittelbaren (die Person sieht in der Ferne das Flugzeug) oder indirekt unmittelbaren (die Person sieht am Flughafen durch einen Zaun das Flugzeug oder im Nachbarraum einer Wohnung das Sofa) oder in direkt unmittelbarer Nähe einer Person befindet (die Person sitzt in dem Flugzeug, auf dem Sofa oder steht vor dem Sofa). Mit diesem Beispiel verdeutlicht sich zudem, dass komplementär unterschiedliche akustische, olfaktorische und (bedingt) haptische Gestalten mit einem Flugzeug 
oder Sofa einhergehen. Andererseits schränken räumliche Beziehungen die Vielfalt der wahrnehmbaren Gestalten ein. Beispielsweise kann eine Person kaum ein Sofa in 100m Entfernung weder „sehen“, „hören“, „riechen“ noch „fühlen“. Sozialisatorische Faktoren bedingen, welche räumlichen Beziehungen eine Person mit Objekten herstellen kann.

- (c) „orientation“: Diese Komponente bezieht sich auf moderierende Bedingung aus der räumlichen Beziehung der Person zu einer „focal entity“. Beispielsweise hat eine Person, die selbst noch nie geflogen ist, höchstwahrscheinlich ein „Flugzeug“ aus der Ferne ausschließlich „,vor den Wolken“ und nicht „hinter den Wolken“, sowie „von unten“ und nicht „von innen“ „gesehen“. Ein „Flugzeug“ in indirekt unmittelbarer Nähe „sieht“ diese Person dagegen „hinter dem Zaun“ und nicht „vor dem Zaun“. Aber auch ein „Sofa“ „sehen“ wir eher „vor einer Wand“, ,hinter einem Tisch" usw.

Modale mentale Modelle prozessieren folglich in „events“, für die gilt: Ein „event takes place at a certain time and in a certain spatial region. Within this spatio-temporal framework [...], there is a perspective. Within this perspective are a focal entity, a relation, and a Background entity " (ebd., 46). Hierbei handelt es sich jedoch nicht um dynamische wahrnehmungsähnliche visuell, akustisch, olfaktorisch, gustatorisch und/oder taktil/haptisch ,anschauliche events“, sondern vielmehr um dynamische mehr oder weniger „schematic“ quasi-visuelle, quasi-akustische, quasi-olfaktorische, quasi-gustatorische und/oder quasi-taktile/haptische Simulationen:

Confined to a single body and brain, we only experience the world from one vantage point at any moment in time. From this vantage point, we can perceive a region limited by the range of our sensory organs. If we want to change our vantage point, we need to move, theeby continously updating our vantage point, usung vestibular, proprioceptive, and optic flow information. If we want to learn more about an entitiy, we can visually scan it in a systematic sequence of fications and saccades, we can move closer to it, touch, manipulate, and smell or even taste it (ebd., 48).

Jene Simulationen werden für die Bedeutungsbildung zu Symbolen - hier zuvorderst von sprachlichen Begriffen - bis hin zum Aufbau eines Textverständnisses - unter Berücksichtigung auch abstrakter Konzepte - als relevant erachtet.

The contention is that mental processes involve simulations of body-related perceptions and actions, for which a variety of different, not mutually exclusive, conceptualizations has been offered. Some note that we ,evolved from creatures whose neural resources were devoted primarily to perceptual and motoric processing' (Wilson, 2002, 625) and suggest that higher mental processes reuse evolutionarily older programs $[\ldots]$; others emphasize developmental processes and suggest that our early experiences with the physical world (e.g., moving around in space) structure our later understanding or representation of more abstract concepts (e.g., likes and dislikes) (Meier et al. 2012, 706)

Gemäß des „Immersed Experiencer Framework“ für kontinuierliches Textverständnis wird eine Lesesituation grundsätzlich als in der jeweiligen Zeit, im jeweiligen Raum und der jeweiligen Perspektive „neue“ mentale modale Modellierung gefasst, respektive, von quasi-simulierten „event-sequences“. Mit einem visuellen Input eines Wortes kann demgemäß keine Enkodierung gemeint sein. Vielmehr werden im Hier und Jetzt diverse sich überlappende, sogenannte „functional webs“ zu diesem Referenten aktiviert, die ebenso aktiviert werden, wenn „wir“ die Entität direkt unmittelbar, indirekt unmittelbar oder mittelbar „sehen“: „Functional webs“ können als schematische Muster von Quasi-Simulationen verkörperlichter experiences mit diesem Referenten im Sinne einer „focal identity“ oder „background identity“ interpretiert werden; stets inhärent komplementiert mit einer „focal identity“ bzw. „background identity“, einer bestimmten Relation zwischen diesen und aus einer bestimmten Perspektive (vgl. Abbildung 28). Der Grad 
der Diffusität unbewusster und „immediately“ aktivierter „functional webs“ unterscheidet sich hierbei jeweils in Abhängigkeit der Auftretenshäufigkeit und der zeitlichen-räumlichen Nähe einzelner experiences mit dem Referenten/der Entität. Zudem ist davon auszugehen, dass über Zielsetzungen oder emotionale Besetzungen und somit auch der übergeordnete situative und soziale Kontext der aktuellen Lesehandlung bestimmte Aktivierungen implizit präferiert werden; ein Einfluss, welcher auch - ohne weitere Erklärung der theoretischen Einbindung - dem „knowledge“ zugesprochen wird (vgl. Zwaan 2004, 45). Je schwächer dieser Grad der Diffusität, so die Annahme, desto schematischer sind die Quasi-Simulationen von experiences in „functional webs“, oder im Umkehrschluss, je stärker der Grad, desto „konkreter“ sind die aktivierten „functional webs“, respektive, desto stärker voreingenommen wird eine zweite Entität, deren Relation und eine Perspektive mit aktiviert (bspw. zu einem „functional web“ von „Flugzeug über sich vor den Wolken“ samt seiner visuellen Gestalt von „Flugzeugen von unten“).

Auf der Ebene von Sätzen bzw. Satzphrasen sind jene mehr oder weniger diffusen, aktivierten „functional webs" mehrerer Referenten in die modale mentale Modellierung im Hier und Jetzt zu bezeichnen „by way of a constraint-satisfaction mechanism“ (="construal“). Beispielsweise verdeutlicht sich in einer angenommenen vorliegenden Satzphrase „Der Pilot betritt das Flugzeug ..., dass letztlich die „functional webs“ zum Referenten „Flugzeug“ aktiviert werden, die aus der räumlich-zeitlichen Perspektive konsistenter sind: Eine adäquate visuelle Gestalt des „Flugzeuges“ wäre hier bspw. nicht „Flugzeuge von unten“ und setzt voraus, dass der/die Rezipient/in „requisite visual experiences“ gesammelt hat. Wenn für die „construal“, die modale mentale Modellierung zu dieser Beispielsatzphrase gilt, the „more specific, or ,articulated“, the initial representation, the stronger the constraints it exerts on the subsequent activation“, würde sie im Idealfall der „articulation“ von in diffusen aktivierten Erfahrungen mit einem „Piloten“ („,focal identity“) weniger schematisch anschließen, die aus der Perspektive des/r Beobachters/in („location“) - z. B. sitzend im Flugzeug oder beobachtend am Zaun des Flughafens („distance“) - vor („orientation“) dem Eingangsbereich des Flugzeugs („,background identity“) erscheint oder hinter („orientation“) dem Eingangsbereich des Flugzeugs („background identity“) verschwindet - einhergehend mit weniger schematischen, ,articulated“ visuellen, aber auch akustischen usw. Gestalten von „Piloten“ und „Flugzeugen“. Alternative „functional webs“ mit alternativen visuellen usw. Gestalten „remain unarticulated“ (bspw. von den „Flugzeugen von unten aus der Ferne“, „von Geschwindigkeit während des Starts eines Flugzeugs“, usw.). Außerhalb dieses Idealfalls wären diffuse aktivierte Erfahrungen zuvorderst „Pilot“, „betritt“ und „Flugzeug“ in der modalen mentalen Modellierung im Hier und Jetzt, in der Quasi-Simulation eines „events“ gleichermaßen „immediately“ zu verdichten: d. h. höhergradig schematisch.

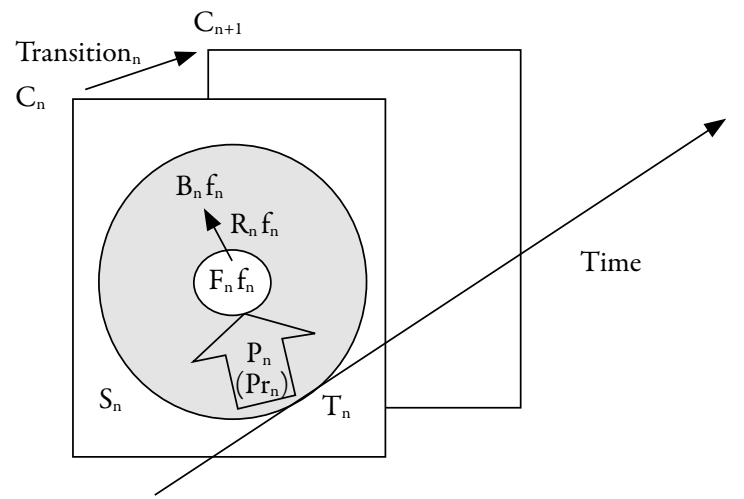

$$
\begin{aligned}
\mathrm{C}= & \text { construal } \\
\mathrm{T}= & \text { time } \\
\mathrm{S}= & \text { spatial region } \\
& \text { (personal, action, vista) } \\
\mathrm{P}= & \text { perspective } \\
\mathrm{F}= & \text { focal entity } \\
\mathrm{R}= & \text { relation } \\
\mathrm{B}= & \text { backround enity } \\
\mathrm{f}= & \text { feature }
\end{aligned}
$$

Abb. 28: Functional webs im Immersed Experiencer Framework (Quelle: ebd., 42) 
Es liegt insbesondere aus dem ersten Fall nahe, dass anschließende „event sequences“ bereits aktiviert, bezeichnet und integriert werden, respektive, sogenannte „experiental traces“ im Übergang von der Satz- zur Textebene antizipiert konstruiert werden. Gleiches gilt jedoch, erstens, aus der „Continuity Assumption“ heraus auch für zweiteren Fall. Diese besagt, dass die Integration von „construals“ zu „experiental traces“ grundsätzlich an die Körperlichkeit von Erfahrungen anschließt, ergo, an eine zeitlich-sukzessive Kontinuität zur „focal identity“ oder der „background identity“ mit einer räumlichen Diskontinuität in den perspektivischen Relation zwischen beiden Entitäten, einhergehend mit ggf, alternierenden Gestalten - und erklärt hierüber mögliche Schwierigkeiten im Textverstehen, wenn ein vorliegender Text bestimmte Diskontinuitäten insbesondere zur Zeit und zum/r Beobachter/in („location“) erzwingt oder auch nur eine bestimmte Kontinuität in der perspektivischen Relation zwischen zwei Referenten verlangt: bspw. eine Modifikation hinsichtlich der „distance“ (u. a. Heran- oder Herauszoomen), oder hinsichtlich der „orientation“ (u.a. eine spezifische vor-hinter-Relation der Entitäten).

It is perhaps not too much of simplification to assume that the human experience involves continuity of time, space, and perspective. Confined to a single body and brain, we only experience the world from one vantage point at any moment in time. From this vantage point, we can perceive a region limited by the range of our sensory organs. If we want to change our vantage point, we need to move, thereby continuously updating our vantage point, using vestibular, proprioceptive and optiv flow information. If we want to learn more about an entity, we can visually scan it in an systemtic sequence of fixations and saccades, we can move closer to it, touch, manipulate, and smell or even taste it.

Zweitens, die „Overlap Assumption“ besagt, dass jene antizipierten „experiental traces“ „more specific predictions“ gestalten, wenn „focal entity“, „background entity“ und „perspective components“ zwischen den „construals“ stärker, eben, überlappen; eine Deckungsgleichheit aus den Bedingungen von Zeit, Raum und Perspektive ist hierbei auszuschließen. Drittens, eine „Predictability“ erschließt, dass Kausalität und weiter ,Zielsetzungen' körpergebunden in Sequenzen von Erfahrungen erfahren werden. Demgemäß ist davon auszugehen, dass antizipierte „experiental traces“ somit Interferenzen von einem „event“ auf nachfolgende „events“ bereits integrieren.

We often experience effects before causes. We become aware of a disturbance in our environment and then look for or infer a cause. The cause is then either perceived in the case of a continous force [...], or inferred in the case of a punctate force [...]. Also, according to the ideomotor theory of action [...] a mental representation of an action's intended effect is assumed to be the cause of the effect. Thus, we are accustomed to construing effects before causes.

Viertens, wird verkörperlichten Erfahrungen in einzelnen „liguistic cues“ Relevanz in der Integration von „events“ (Satzebene) mit „sequences of events“ (Textebene) innerhalb der modalen mentalen Modellierung von „experiental traces“ beigemessen.

Syntactic cues such as word order or case cue the comprehender with respect to the elements of the construal. Word order or case provide cues as to that the focal and backgrounded entities are. Tense markers provide information on where the event should be placed on the timeline, whereas aspect markers indicate whether the event should be conceptualized as punctual, or temporally unbounded. The indefinite article indicates that a new entity is introduced, whereas the definite article is a cue to carry the entity over from one construal to the next. [...] Prepositions can be viewed as instrutions on the placement of and distribution of attention over entities in the mental simulation.

Für den Erklärungsansatz des verstehenden Lesens über modale dynamische mentale Modellierungen kristallisiert sich heraus, dass dieser an den über amodale explizit-konzeptionelle 
Makromodelle insofern anschließen, dass „the information captured by amodal propositions [derart eingeordnet werden, dass sie] forms a subset of information captured by a perceptual analysis“. D. h., aber auch, dass einerseits sich die modalen mentalen Modellierungen während des Textverständnisses strukturell von dem des linguistischen Inputs unterscheiden und andererseits, dass auch amodale mentale Modellierungen als Teil einer "the victorious experience“ in den umschriebenen implizit-embodied Verarbeitungsprozessen eines Textverständnisses zu positionieren sind. Entgegen den statischen explizit-konzeptionellen Makromodellen, die den innitiierenden Charakter des Textverständnisses primär im textuellen Input verorten, werden insbesondere maßgeblich „perspective changes“ auf allen Ebenen theoretisch ergänzt. Wie hierbei statische modale mentale Modellierungen von Entitäten, die als syntaktische, rhetorische oder pragmatische Relationsstrukturen bzw. Interferenzstrukturen anderenorts bezeichnet wurden, als Teil von dynamischen modalen mentalen Modellen theoretisch gefasst werden können, bleibt an dieser Stelle in letzter Instanz jedoch als unklar zu markieren. Erste Forschungsbefunde deuten, aber zumindest darauf hin, dass hierbei der theoretischen Verortung über verkörperlichte Erfahrungen besondere Aufmerksamkeit geschenkt werden müsste. So konnte aufgezeigt werden, dass Personen mit einer Abneigungstendenz für Abstraktes bzw. Komplexes dazu tendieren, begrifflich eigene Handlungen mit „embodied methapors“ zu beschreiben. Ein weiterer Befund unterstreicht jene „embodied-metaphors“-Konsistenz in Handlungen aus der Tendenz von Personen mit einer starken Vorliebe für physische Sauberkeit in „stronger automatic associations" zwischen Moral/Amoral und den Farben weiß/schwarz gegenüber Personen, für die diese Vorliebe nicht zutrifft (Meier et al. 2012, 712f.).

\subsubsection{Symbiose zu einem integrativen Arbeitsmodell kognitiver Verarbeitungsprozesse primär kategorial-tabellarischer Texte}

\subsubsection{Interaktive, verstehende Verarbeitung eines initialen Sets ikonischer Zeichen}

Innerhalb der Positionierung, dass die Repräsentationsanalogien hinter kategorialen Tabellen grundsätzlich auf einer abstrakten, inhärenten Ähnlichkeit beruhen, wurde die Annahme begründet, dass durchaus, wie bei Diagrammen oder Karten, einzelne Facetten einer „physikalischen Isomorphie“ festzumachen sind. In diesem Kontext wurden bereits einige dieser inhärenten ,erfahrungsnahen Analogien und räumlichen Metaphern' herausgearbeitet: „mehr/ weniger“, „vorher/nachher“, „auf/ab“, „hinter/vor“, „zusammengehörend/nicht-zusammengehörend“ usw. (vgl. Kapitel 2.3.2.1 für Diagramme; 2.3.2.2 für kategoriale Tabellen). Innerhalb dieser ,erfahrungsnahen Analogien und räumlichen Metaphern' liegen Verarbeitungsprozesse begründet, welche durch die „embodied theory of text comprehension“ erklärt werden können: Nach diesem dynamischen Modellierungsansatz wird bei einem event die Identifikation eines Referenten einer Zeichensystem-Entität - hier eben eines ikonischen Lokal- oder Globalzeichens - in dem Sinne interpretiert, dass hierzu mehr oder weniger abstrakte Quasi-Simulationen erzeugt werden. Wie skizziert verbirgt sich hinter diesen events, dass im Hier und Jetzt eine oder mehrere jeweils perspektivische Relationen zu dem identifizierten Zeichen in der Interaktion mit dem Text konstruiert werden (= functional webs). Bei jedem im Hier und Jetzt konstruierten functional web tritt das identifizierte Zeichen entweder als (a) focal entity oder (b) background entity auf, einhergehend mit einem jeweiligen Pendant (a) eine background entity oder (b) eine focal-entity einschließlich einer jeweils perspektivischen Relation, welche auf verkörperlichte Erfahrungen beruhen. Auf diese Weise können bei der Identifikation unterschiedliche quasi-sensorischen Gestalten einer identifizierten Entität einhergehen, unter denen bspw. die Relationen „mehr/weniger“, „vorher/nachher“, „auf/ab“, „hinter/vor“, „zusammengehö- 
rend/nicht-zusammengehörend“ zum bereits immer schon dynamisch komplementär modellierten Pendant inhärent sind. Jene ,erfahrungsnahen Analogien und räumlichen Metapher werden jedoch weder wahrnehmungsähnlich visuell, akustisch, olfaktorisch, gustatorische und/ oder taktil/haptisch im Sinne von anschaulichen dynamischen Modellen erzeugt, noch handelt es sich ausschließlich um quasi-visuelle oder quasi-taktile/haptische Gestalten. Die bei den im Zuge der Identifikation eines ikonischen Zeichens im Hier und Jetzt konstruierten, konkurrierenden functional webs, können „mehr/weniger“, „vorher/nachher“, „auf/ab“, „hinter/vor“, „zusammengehörend/nicht-zusammengehörend“ innerhalb der Trias aus focal idenity, background identity und Relation zwischen diesen, auch quasi-akustisch, quasi-olfaktorisch und/ oder quasi-gustatorisch geprägt sein.

Welche functional webs, erstens, zum Referenten eines identifizierten ikonischen Zeichens mit welchen Relationen überhaupt aktiviert werden, ist, wie innerhalb der embodied theory dargestellt, grundsätzlich davon abhängig, welche verkörperlichten Erfahrungen der/die Lesende machen konnte. Unter jenen Relationen sind folglich im Weiteren diejenigen von besonderem Interesse - im Kontext kategorial-tabellarischer Texte und Textkomplexe - die verkörperlichten ,erfahrungsnahen Analogien und räumlichen Metaphern“ „entsprechen“. Einzelne verkörperlich erfahrungsnahe Analogien und räumliche Metaphern, die als quasi-simulierte Relationen innerhalb functional webs im Zuge der Identifikation von ikonischen Lokal- oder Globalzeichen zum Tragen kommen könnten, sind exemplarisch:

- „mehr/weniger“: z. B. verkörperlichte quasi-gustatorische Erfahrungen;

- „vorher/nachher“: z. B. verkörperlichte quasi-akustisch Erfahrungen;

- „auf/ab“: z. B. verkörperlichte quasi-visuelle Erfahrungen;

- „hinter/vor“: z. B. verkörperlichte quasi-olfaktorische Erfahrungen;

- „zusammengehörend/nicht-zusammengehörend“: bspw. verkörperlicht erfahrene Bewegungen mit bspw. den Fingern horizontal und vertikal zu einem Punkt (quasi-haptisch/taktil).

Jene Querverbindungen zu verkörperlichten erfahrungsnahen Analogien und räumlicher Metaphern eines repräsentierten Sachverhalts via starke Darstellung-Kodes sind gleichermaßen bei Steuerungs-Kodes auszumachen. Ein ikonisch inhärent repräsentierter „cause“ oder "effect“ kann über körpergebundene Erfahrungen mit bspw. einem „Pfeil“ in Beziehung gebracht werden, welcher wiederum auf die Relationen vorher/nachher oder ,zusammengehörend/nichtzusammengehörend" verweist.

Welche functional webs, zweitens, stärker als andere in einer bestimmten Lesesituation eines nichtkontinuierlichen Textes bzw. Textkomplexes articulated werden, würde analog der Identifikation der Referenten rein symbolischer Entitäten von der Konsistenz mit den aktivierten functional webs abhängen (=construal). Diese Konsistenz wird im Sinne eines sogenannten constraint-satisfaction mechanism angenommen, wonach jene functional webs bei der Identifikation eines ikonischen Zeichens besonders stark im Hier und Jetzt angesprochen werden, bei denen sich die Trias aus der Relation zwischen der focal und background idenity ausgehend von location, distance und orientation (= perspectival components) mit verkörperlichten Erfahrungen insbesondere decken. Der hierbei hervorgebrachte Grad der Diffusität entscheidet darüber, ob die einsetzenden antizipierenden mentalen Modellierungen eher konkreter oder schematischer Art sind. Wie dargelegt, sind sie umso konkreter körpergebunden erfahrungsnah einzuschätzen, je weniger überlappende articulated functional webs involviert sind. Wenn im Umkehrschluss zunehmend mehr überlappende articulated functional webs involviert sind, so sind die einsetzenden antizipierenden mentalen Modellierungen ebenso als schematischer zu interpretieren 
oder in anderen Worten, desto unvoreingenommener wird eine komplementäre Identität (focal oder background identity) einschließlich sowohl deren Relation zueinander als auch der Perspektive auf diese Relation kognitiv verarbeitet. Durch den constraint-satisfaction mechanism auf der Ebene construal und analog über das Prinzip des Overlap Assumption auf der Ebene der integration können zwar höhergradig schematische dynamische mentale Modellierungen erklärt werden, jedoch wird von dem Extremfall einer amodalen verstehenden Verarbeitung innerhalb des theoretischen Konzeptes „Immersed Experiencer Framework“ an dieser Stelle nicht gesprochen. Jede einsetzende antizipierende mentale Modellierung eines identifizierten Lokal- oder Globalzeichens positioniert. Wie dargelegt, gilt dies auch für die weiteren Prozesse des sukzessiven Leseverständnisses eines kompletten nichtkontinuierlichen Textes oder Textkomplexes, welcher als victorious experience eindeutig diese Handschrift trägt. Erst durch diese Positionierung knüpft das symbiotische Erklärungsmodell der verstehenden Verarbeitung kongruent an den Ansatz von „cognition as social, situated and embodied“ (ebd.) an. Dieser ist zweifelsohne kaum mit kognitiv-generativen (Chomsky) oder kognitiv-strukturellen (de Saussure) vereinbar, sowie nur bedingt mit kognitiv-funktionalen Theorien (Lakoff). Der gebrauchsbezogene Ansatz aus der kognitiven Linguistik, zu dem der „Immersed Experienced Framework“ gezählt werden kann, verweist vielmehr auf „Kandidaten“ wie Schematisierung, Habituation, Automatisierung, die die Entwicklung von letztlich hochgradig schematischen dynamischen Modellierungen einer verstehenden Verarbeitung semiotischer Zeichen erklären. Entscheidend bleibt jedoch, dass das Leseverständnis stets im Hier und Jetzt aktualisiert wird und somit auch im Extremfall von hochgradig schematisierten, habitualisierten und automatisierten dynamischen mentalen Modellierungen stets der Rekontextualisierung unterliegen (Schneider-Wiejowski et al. 2013, 328ff.). Vor diesem Hintergrund wird im Weiteren die victorious experience entlang eines nichtkontinuierlichen Textes bzw. Textkomplexes als dynamische mentale Modellierung interpretiert, welche sich zwischen den Polen „konkret körpergebunden erfahren“ und „abstrahiert körpergebunden erfahren“ - im Sinne einer rekontextualisierten Schematisierung, Habituation und Automation bewegt.

Welche einzelnen konkreten bis abstrahiert körpergebunden erfahrenen articulated functional webs, drittens, bei der einsetzenden antizipierenden mentalen Modellierung (= construal of experiental traces) in einer bestimmten Lesesituation mit einem nichtkontinuierlichen Text oder Textkomplex letztlich beansprucht werden, hängt überdies nach dem „Immersed Experiencer Framework“ ebenso von der symbolischen Kohärenzbildung kleinerer Sets symbolischer Entitäten und somit übertragen von emotionalen Besetzungen, von knowledge, von der Zielsetzung oder von der Häufigkeit sowie der zeitlichen Nähe der referentiellen verkörperlichten Erfahrung ab. Ein nachvollziehbares Modell dessen, was sich hinter diesen einzelnen Einflussfaktoren verbirgt, bleibt dieser Erklärungsansatz jedoch schuldig. Das schemaorientierte TRACE-Modell liefert vor diesem Hintergrund insofern Erweiterungen für die Einflussfaktoren Zielsetzung und knowledge, indem es innerhalb des seither beschriebenen Transfers der verstehenden Verarbeitung aus der „embodied theory“ ab der Ebene construal einige Konkretisierungen vornimmt: Es stellt dem verarbeitenden Lesen eines textuellen Inputs, respektive, der mentalen Modellierung eines content models, einerseits komplementär die Erzeugung einer Art $\mathrm{zu}$ initiierenden, begleitenden und evaluierenden Bedingungen zur Seite, welche die aktuelle Lesesituation prägen (=task/response model). Dies ist vor dem Hintergrund der Lesesituationen on-the-job von Bedeutung, welche nicht durch einen textuellen Input selbst initiiert werden, sondern bspw. durch eine bestimmte Anforderung on-the-job, die verbal angewiesen werden oder Arbeitsroutinen inhärent sind. Andererseits ist die Konstruktion des content models von 
einer Art Idee zur Relevanz begleitet, welche der einzelnen ikonischen Zeichen bzw. Relationen zwischen Sets aus ikonischen Zeichen zukommt (=source model). Bei der weiteren Symbiose mit dem TRACE-Modell ist daher die Annahme leitend, dass sich bei diesen drei komplementären mentalen Modellierungen jeweils um konkret bis abstrabiert körpergebunden erfabrene handelt. Oder in anderen Worten, auch Zielsetzung und knowledge werden nicht als amodale, explizitkonzeptionelle Konstrukte verstanden, sondern vielmehr im Hier und Jetzt rekontextualisierte dynamische Modelle, die der/die Lesende aktiviert. Die zeitliche Reihenfolge, mit dem die komplementären task/response model und/oder source model mit dem content model interagiert, greift zudem die differenzierten bottom-up- und top-down-Verarbeitungsprozesse auf.

\subsubsection{Interaktive, verstehende Verarbeitung entlang des task/response models der Teilnehmenden sozialer literaler Praxen}

Die verstehende Verarbeitung entlang eines textuellen Inputs aus einem primär kategorialtabellarischen Text oder Textkomplex muss die herausgearbeitete Initiierungsproblematik berücksichtigen: Wie dargelegt, kann die Interaktion mit Texten grundsätzlich nur in seinem Wechselverhältnis aus der sozialen und situativen Einbettung der Lesesituation heraus betrachtet werden. Die Identifikation und die verstehende Verarbeitung der ikonischen Lokal- bis Globalzeichen - die Konstruktion eines source models und content models - in Abhängigkeit von der abgetrennten aber komplementären Idee des task/response models zu setzen, positioniert jedoch nicht nur die Einflussgröße Zielsetzung, die einen Leseprozess initiiert. Es eröffnet zudem zu erklären, dass der Leseprozess durch ein weites Verständnis von Zielsetzung zugleich initiiert, begleitet und evaluiert wird. Das task/response model greift übertragen auf das Konzept des Lesens als literale Praxen die in den Personen - als Träger der literalen Praktik - (re)produzierten routinalisierten lokalen kulturellen Codes einer „skillful performance“ auf: Die „explizit aktualisierten" lokalen kulturellen Codes in literalen events eröffnen annäherungsweise den ,Rückschluss auf die Personen als Träger der Praxen', sprich die routinalisierte Funktion vor und hinter einer „skillful performance“ unter Berücksichtigung der Arbitrarität des referentiellen Zeichensystems“" vgl. Kapitel 3.4.3).

Die mentale Modellierung dieser komplementären Idee von im Grunde situativen und sozialen Kriterien einer „skillful performance“ wird hierbei analog dem content als dynamisch zwischen den Polen „konkret körpergebunden erfahren“ und „abstrahiert körpergebunden erfahren“ betrachtet. Oder in anderen Worten, das task/response model aktiviert rekontextualisierte verkörperlichte Erfahrungen, die die Bandbreite von quasi-,räumlich-zeitlich' konkret bis schematisiert, habitualisiert und automatisiert abdecken können. Dem Umstand Rechnung tragend, dass insbesondere innerhalb jener nichtkontinuierlichen Textkomplexe häufig keine Instruktion präsent ist, respektive, aus den Grenzen ikonischer Repräsentationen auch nicht dezidiert hervorgehen kann (Stichwort „Ausdrucksmächtigkeit“; Seufert 2003, 16), muss der, eine soziale Lesesituation moderierenden Idee einer Art ,routinalisierten lokalen kulturellen Codes', eine entscheidende Bedeutung beigemessen werden.

Eine komplementäre Idee einer Art ,routinalisierten lokalen kulturellen Codes' erweitert derart deren im „Immersed Experience Framework“ dargelegte inhärente Wechselbeziehung innerhalb der Ebenen construal und integration. Sie begleitet die Auswahl der „functional webs“ zu einer referentiellen Entität indem verengt wird, welche der darunter aktivierten bei der einleitend antizipierenden dynamischen mentalen Modellierung präferiert werden. Sie beeinflusst jedoch nicht nur begleitend, welche focal/background identity und/oder Relation präferiert wird. Der Einfluss kann sich gleichermaßen auf die Perspektive dieser Relation beziehen, welcher zuvor als differenzierendes Kriterium der mentalen dynamischen Modellierung zwischen 
den Polen „konkret körpergebunden erfahren“ und „abstrahiert körpergebunden erfahren“ ausgemacht wurde. Oder in anderen Worten, die komplementäre Idee einer Art ,routinalisierten lokalen kulturellen Codes kann innerhalb des „Immersed Experience Framework“ auf der Ebene construal begleitend moderieren, ob eher eine Rekontextualisierung quasi-,räumlichzeitlich' konkrete bis gar eher schematisierte, habitualisierte und automatisierte verkörperlichte Erfahrungen angesprochen werden. Aussagen darüber, inwiefern jedoch bspw. eine Zielsetzung überhaupt einen Leseprozess über die Prozesse der Ebene activation initiiert, lassen sich jedoch nicht festmachen. Die initiierende Relevanz der Zielsetzung anerkennend, vermag diese das Lesen eines nichtkontinuierlichen Textes oder Textkomplexes a priori maßgeblich zu beeinflussen: Die verstehende Verarbeitung des contents erfolgt im Hier und Jetzt im Spannungsfeld eines sodann bereits praktizierten oder konzipierten task/response models. In diesem Fall werden bestimmte Komponenten aus der Trias focal/background identity, Relation und Perspektive beim einleitenden Leseprozess a priori präferiert und moderieren deren sukzessive Verknüpfung mit weiteren Lokal- und Globalzeichen.

Weitere Hinweise dahingehend, dass eine komplementäre Idee einer Art ,routinalisierten lokalen kulturellen Codes' gleichermaßen das weitere sukzessive Erlesen des textuellen Inputs moderieren kann, begründet sich zuvorderst über die Prinzipien Continuity Assumption und Predictability innerhalb des „Immersed Experiencer Framework“. Ausgehend von der Identifikation und einleitend antizipierenden verstehenden Verarbeitung eines ersten ikonischen Lokal- oder Globalzeichens auf der Ebene construal werden auf der Ebene integration die präferierten, noch antizipierenden experimental traces zu event sequences sukzessive mental verarbeitet. Wie für symbolische Zeichen dargelegt, sind u. a. wahrnehmbare Erfahrungen mit Kontinuitäten und Diskontinuitäten in Zeit, Raum und Perspektive (=Continuity Assumption) einflussnehmend für diese sukzessive Verarbeitung. Ein Beispiel dieser verkörperlichten Erfahrungssequenzen stellt das Herauszoomen dar, welches übertragen auf nichtkontinuierliche Texte und Textkomplexe die integrierend-antizipierende verstehende Verarbeitung (=experimental traces) ausgehend von einem Textfeld hin zu einer gesamten Tabelle illustriert werden kann. Das Herauszoomen knüpft folglich an verkörperlichten hier quasi-visuellen Erfahrungssequenzen an und erklärt aus der embodied theory das Leseverständnis von einem Lokal- bzw. Globalzeichen niedriger Ordnung hin zu dem Pendant höherer Ordnung. Vor dem Hintergrund, dass ikonische Repräsentationen sowohl initial über ein Lokal- oder über ein Globalzeichen gelesen werden können, gilt dies im Umkehrschluss auch für die verkörperlichten Erfahrungssequenzen des Hineinzoomens. Wenn in der Leseforschung nichtkontinuierlicher Texte „visuelle[n] Routinen z. B. zum Verfolgen von Linien oder zur ,mentalen Markierung'von Orten“ (Rouet 2006, 66; Schnotz 1994, 124) als Kennzeichen von Lese-Experten vs. -Novizen aufgedeckt wurden, so kann dieser Befund innerhalb der embodied theory mittels Ergänzung des Prinzips Predictability bei der intergration von experimental traces zu event sequences erklärt werden: Predictability filtert demgemäß aus den verkörperlichten Erfahrungssequenzen nach Zeit, Raum und Perspektive diejenigen heraus, die auf Kontinuität beruhen. Hierdurch lässt sich eine Art auf Kausalität beruhende Antizipation festmachen, genauer gesagt, eine Art von Kausalität im Sinne einer Alltags- und nicht wissenschaftlichen Kausalität, sowie eine Art von Kausalität, die nicht als amodales kognitives Konstrukt im Sinne eines rational-choiceParadigmas zu positionieren ist. Wenn „wir“ auf in diesem Sinne von ,more specific predictions“ ein bestimmtes ,Suchziel' zurückgreifen, unterstützt die aufgefundene Lesesituation mit einer „Rechnung “ im Verkauf die Vermutung eines komplementären task/response models, welche das Erlesen der repräsentierten Sachverhalte a priori steuert. Ohne sich explizit-konzeptionell der ikonischen Repräsentations-Kodes kategorialer Tabellen zwangsweise bedienen zu müssen, können Experten 
dieser „Rechnungen“ an eine verkörperlichte Routine anknüpfen, dass sie im Impressum-Feld die für die Buchhaltung zentrale Umsatzsteuer-Identifikationsnummer finden. Über die „embodied theory“ ist es alsdann möglich, die verstehende Verarbeitung dieser „Rechnung“ gemäß der antizipierten Zielsetzung samt einer Art Kausalität zu erklären, auch, wenn hierbei der/die Lesende/n den textstrukturellen Aufbau im Sinne eines starken Darstellungs-Kodes weder explizit-konzeptuell benennen noch indirekt referieren kann. Ein von außen beobachtbarer routinisierter Umgang entlang bestimmter Sets ikonischer Zeichen kann zu einem Hinweis werden, dass das Lesen topdown durch ein rekontextualisertes task/response model moderiert wird.

Dem interaktiven Verhältnis von top-down- und bottom-up-Verarbeitungsprozessen geschuldet, ist, aber auch in dieser exemplarischen Lesesituation eine Evaluation, sprich ein Verwerfen oder ein Modifizieren des task/response models oder content models, naheliegend. Dies ist bei den Lesesituationen insbesondere zu vermuten, bei denen eine Antizipation der Kontinuität auf der Grundlage körpergebundender Erfahrungssequenzen bei der intergration von experimental traces zu event sequences auf Diskontinuitäten stößt. Die innerhalb des TRACE-Modells positionierte begleitende oder finale Evaluation für die Interaktion der beiden komplementären dynamischen Modellierungen findet sich in dem Wechselspiel von Kontinuität und Diskontinuität zwischen den komplentären mentalen Modellen des Antizipierten im task/response model und des verstehenden Lesens mehrerer Lokal- und/oder Globalzeichen (=content model).

Mit dem quasi-visuellen Hinein- und Herauszoomen oder anderen verkörperlicht erfahrenen rountinierten Blicksequenzen werden zugleich die Beziehungen zwischen den Relationen des Sachverhalts sukzessiv mental verknüpft, die mittels der jeweiligen referentiellen Lokal- und Globalzeichen niedriger und höherer Ordnung repräsentiert werden. Jene Beziehungen zwischen Relationen des repräsentierten Sachverhalts werden innerhalb der explizit-konzeptionellen Erklärungsansätze der Kohärenzbildung symbolischer Zeichen als Interferenzen bezeichnet (vgl. Kapitel 3.3.1). Das Hinein- und Herauszoomen bis visuelle Routinen sind demzufolge verkörperlichte Erfahrungssequenzen, die die Interferenzen innerhalb nichtkontinuierlicher Texte und Textkomplexe herstellen können, welche zuvorderst auf terminologische, kategoriale auf Verlaufsstrukturen eines repräsentierten Sachverhalts verweisen. Es ist daher naheliegend, dass für das Lesen dieser Texte insbesondere ein Hinein- und Herauszoomen oder eine weitere visuelle Routinen mit denjenigen verkörperlichten Erfahrungssequenzen verknüpft ist, welche quasi-zeitlich-räumlich auf ein „mehr/weniger“, „,vorher/nachher“, „auf/ab“, „hinter/vor“ und „einschließend/ausschließend“ lenken und charakterisieren letztlich Sequenzen aus verkörperlicht erfahrungsnahen Analogien und räumlichen Metaphern. Neben diesen verkörperlichten quasi-visuellen Erfahrungssequenzen sind gleichsam die weiteren quasi-sensorischen Pendents mitzudenken, so z.B. wenn quasi-haptisch erfahrene Handlungsabfolgen mit den Händen rekontextualisiert werden. Hintergrund dessen, dass sowohl für die Kohärenzherstellung der Relationen und Interferenzen zwischen diesen Relationen ähnliche verkörperlicht erfahrungsnahe Analogien und räumliche Metapher für nichtkontinuierliche Texte und Textkomplexe von Relevanz sind, begründet sich aus der Bedingung, dass deren Darstellungs-Kodes bereits sämtliche terminologische, kategoriale sowie Verlaufsstrukturen des repräsentierten Sachverhalts inhärent sind. Gleiches gilt für die externen Steuerungs-Kodes wie Pfeile, durch die die Richtung einer repräsentierten Kausalität und Effekte von Kausalitäten hervorgehoben werden können.

Diese Skizzierung des Prinzips des Continuity Assumption und der Predictability soll in erster Linie verdeutlicht haben, wie die Idee einer Art ,routinalisierten lokalen kulturellen Codes im Sinne eines komplementären dynamischen task/response models die verstehende Verarbeitung von Interferenzen über die Konstruktion von experimental traces sowohl a priori als auch beglei- 
tend maßgeblich beeinflussen kann. Zentral hierbei ist die herausgearbeitete Antizipation auf der Grundlage von „konkret körpergebunden erfahrenen“ sequenziellen Kontinuitäten. Den anderen Pol der "abstrahiert körpergebunden erfahrenen“ dynamischen Modellierungen des task/response models mitbedacht, können auch quasi-sensorische Erfahrungssequenzen dem Prinzip der Continuity Assumption und Predictability entsprechen, die eher als schematisiert, habitualisiert und automatisiert zu charakterisieren sind. Oder in anderen Worten, das task/ response model kann auch über die rekontextualisierte Aktivierung von „abstrakt körpergebunden erfahrene“ Sequenzen - insbesondere entlang der „critical details such as time, place, or the participant's attitutes“ (Rouet 2006, 66) - die verstehende Verarbeitung des textuellen Inputs initiieren, begleiten sowie evaluieren, respektive, die Rahmenbedingungen einer victorious experience konstruieren. Konkretisiert handelt es sich nach dem TRACE-Modell hierbei um folgende situativ-kontextuelle Ideen entlang der wechselseitig verknüpften internal representation of the task to be completed und of the response to be output:

- Rahmenbedingungen wie z. B. Zeitraum für die Bearbeitung oder Medialität eines ggf. vorliegenden Arbeitsauftrages bzw. Endprodukts

- typische funktionale Einbettung in charakterisitischen Handlungsroutinen einschließlich Erkennen atypischer funktionaler Einbettungen

- typische interessierende Sachverhalte einschließlich Erkennen atypischer Sachverhalte

Die skizzierten situativ-kontextuellen Ideen konkretisieren einerseits das komplentäre task/response model, welches Personen als Träger literaler Praxen vor, während und mit dem „Lesen“ praktizieren bis konzipieren. Aus der Herleitung über die embodied theory quer zum TRACE-Modell ergänzen sie den Leseansatz als Set sozialer Praxen entscheidend als die Personen bezogene Seite ,routinalisierter lokaler kultureller Codes', welche dieser Praxen schon immer inhärent sind. Das task/response model basiert nach diesem Verständnis und nicht auf ein explizierbares, situativ unabhängiges Wissen in den Dispositionen der Personen, sondern verortet sie im Spiegel des „Sozialen" und "Situativen“, aktualisiert in der jeweiligen Praktik. Andererseits offenbaren die skizzierten situativ-kontextuellen Ideen dezidiert eine Problematik in einer Engführung auf das amodal „Kognitive" insbesondere in Praxen des Lesens in der Sekundarstufe I, die zudem einer Bewertung von Lesecompetencies als hierarchisches ,soziales Identifikationskriterium' (vgl. Kapitel 3.4.3) bei den Teilnehmenden verknüpft sind. Mit einem Verweis auf die Leseforschung auf diesem Niveau umfassen die interessierenden ausschließlich diejenigen, die sich auf die ,routinalisierten lokalen kulturellen Codes'von literalen Praxen im Sinne einer Behaltensleistung oder eine Einstellungsänderung reduzieren lassen (vgl. Tabelle 17). Oder in anderen Worten, das Lesen auf Sekundarstufenniveau I unterliegt dem Verdacht nur sehr elektiv literale Praxen der routinalisierten lokalen kulturellen Codes entlang der Behaltensleistung und Einstellungsänderung mitzudenken und somit weitere literale Praxen zu ignorieren. Während die literalen Praxen an den untersuchten Einfach(st)erwerbstätigkeiten noch einer Analyse bedürfen, liefert die Textforschung bereits Hinweise auf Alternativen, die durchaus im schulischen Alltag aktualisert werden. Jedoch unterliegen die Praktiken des Lesens zur Erinnerung bzw. zur Überwindung räumlicher und zeitlicher Distanz keiner Einschätzung als Lesecompetencies (vgl. Tabelle 18).

Dabei ist für die Lektürewirkung allerdings eine deutliche Getrennheit der Forschungsentwicklung zu literarischen vs. pragmatischen (Informations-) Texten festzustellen [...]. Das hat vor allem mit der Unterschiedlichkeit der untersuchten Wirkeffekte zu tun. Bei den pragmatischen oder InformationsTexten gibt es, der (kommunikativen) Funktion dieser Texte entsprechend, zwei zentrale Wirkeffekte, die untersucht worden sind: Das eine ist - gemäß der Funktion der Informationsvermittlung - das 
Kriterium des Behaltenserfolgs, das im Mittelpunkt der angewandten Leseforschung steht [...]; zum anderen handelt es sich - gemäß der (potentiellen) Überzeugungsfunktion pragmatischer Texte - um das Kriterium der Einstellungsänderung (Christmann/Groeben 1999, 172f.).

Tab. 17: Routinalisierte lokale kulturelle Codes in sozialen Lesepraxen der Sekundarstufe I

\begin{tabular}{lc}
\hline $\begin{array}{l}\text { Routinalisierte lokale kulturelle Codes entlang der } \\
\text { Behaltensleistung }\end{array}$ & $\begin{array}{l}\text { Routinalisierte lokale kulturelle Codes entlang der } \\
\text { Einstellungsänderung }\end{array}$ \\
\hline- ... des Lernens explizit-konzeptuellen Wissens & $-\ldots$ des Lernens entlang der Reflexion bedeutungsoffe- \\
des repräsentierten Gegenstands (Stichwort: & ner und mehrdeutiger Auslegungen von Texten und \\
Behaltensleistung). Hierunter auch die Identifika- & Textkomplexen (Stichwort: Einstellungsverände- \\
tion von Darstellungsstrukturen insbesondere bei & rung) begrenzt auf politische, ästehetische und soziale \\
Informations- und Sachtexten primär kontinuierli- & Sachverhalte: Hierunter auch die Identifikation von \\
cher Struktur. & Darstellungsstrukturen insbesondere bei literarischen \\
- verknüpft mit der mnemonischen Funktion bezo- & kontinuierlichen Texten und Textkomplexen \\
gen auf die Speicherung und Überlieferung sowie & - verknüpft mit der sozialen Kontrollfunktion bezogen \\
Akkumulation von Wissensbeständen & auf die Höherwertung des Geschriebenen vs. das \\
- verknüpft mit der Funktion der Verdinglichung & Gesprochene \\
bezogen auf eine Entkontextualisierung und & - verknüpft mit der ästhetischen Funktion \\
Verselbstständigung & \\
\hline
\end{tabular}

Tab. 18: Routinalisierte lokale kulturelle Codes in sozialen Lesepraxen der Primarstufe und außerhalb von Lesecompetencies

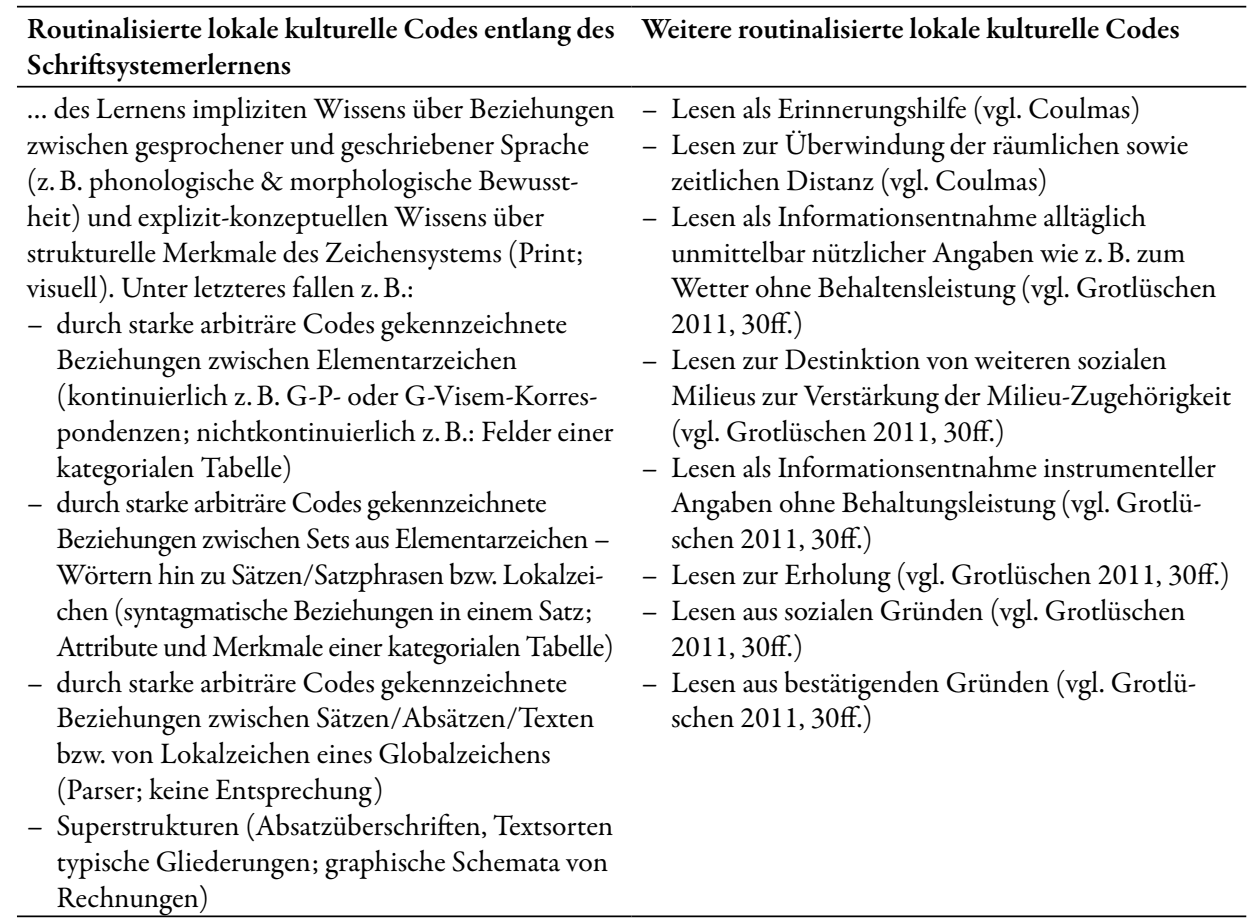


Aus der theoretischen Herleitung ergibt sich aus der Vernachlässigung weiterer literaler Praxen schlussfolgernd, dass die dahinterliegenden routinalisierten lokalen kulturellen Codes und nicht-symbolischen Zeichensysteme in der schulischen Markierung des Lesens außen vor bleiben. Diese Problematik wurde bereits in Kapitel 3.4.2 innerhalb der Debatte um die ,Kultur in der Kulturtechnik Lesen augegriffen und verhärtet sich nun innerhalb des aus der embodied theory erschlossenen TRACE-Verarbeitungsmodells als integrale Ergänzung des Lesens als Set sozialer Praxen seiner Teilnehmenden. Provokant formuliert heißt dies, dass nicht nur alternative literale Praxen des Lesens außen vor bleiben, sondern auch die Relevanz des Lesens für die Teilnahmen an sozialen Praxen außerhalb der Schule. Insbesondere ist dies kritisch für soziale Praxen des Lesens anzumerken, die auf Texten beruhen, die weder routinalisiert auf symbolische Zeichen noch auf die lokalen kulturellen Codes entlang der Behaltensleistung oder Einstellungsänderung beruhen.

\subsubsection{Interaktive, verstehende Verarbeitung entlang des source models der Teilnehmenden sozialer literaler Praxen}

Die sukzessive Konstruktion einer victorious experience selbst, respektive, die mentale dynamische Modellierung des contents eines textuellen Inputs, erfolgt sowohl nach der embodied theory als auch nach dem TRACE-Modell unter Einfluss einer noch zu spezifizierenden Art textstrukturellen Wissens. Bei zweiterem wird das komplementäre mentale source model zu einem ,Wissen' content area knowledge, document knowledge und ein knowledge about how content area knowledge and document knowledge is represented in texts - z. B. ,in documents, characteristics of various types of documents, and so forth““ innerhalb des Leseverständnisses propagiert Rouet (ebd., 67). Das benannte ,Wissen' ist als kognitives Konzept zu grundlegenden semantischen oder syntaktischen Segmentierungsstrukturen zu interpretieren, welche bei nichtkontinuierlichen Texten über die Bandbreite an starken bis schwachen ikonischen Darstellungs- und Steuerungs-Kodes ausgemacht wurden (vgl. Kapitel 3.2.3).

knowledge about how content area knowledge is represented:

- zu typischen Darstellungsstragien zum Sachverhalt

- zu typischen Darstellungsstragien von Merkmalen und Attributen des Sachverhalts

knowledge about how document knowledge is represented:

- ,Wissen über Autorenschaft des Textes bzw. Textkomplexes, einschließlich der jeweiligen Verantwortungszuständigkeit, der jeweiligen Addressaten sowie der jeweiligen Intention und/ oder

- ,Wissen ' zu typischen Darstellungsstragien in ökonomischen, rechtlichen, politischen, organisatorischen usw. Kontexten der Entstehung des Textes bzw. Textkomplexes und/oder

- ,Wissen' zu charakteristischer Textsorte und -typ mit z.B. Referenz zu einzelnen Fachsprachen jeder einzelnen einer multiplen Repräsentation

- weitere Superstrukturen (vgl. Kapitel 3.4.2).

Die angedeutete konzeptuelle Kongruenz erschließt entlang jenes ,kowledge‘ beider Dimensionen über die linguistic cues auf der Ebene construal und integration des „Immersed Experience Framework“. Jene linguistic cues wurden neben der Overlap Assumption, der Continuity Assumption und der Predictability als Prinzipien beim sukzessiven Leseverständnis mehrerer events im Kontext eines bestimmten vorliegenden Textes (=sequence of events) dargelegt. Wenn sich hinter den lingistic cues semantische und syntaktische Segmentierungsstrukturen verbergen, so lässt sich ein knowledge about how knowledge is represented mit Blick auf nichtkontinuierliche Texte 
und Textkomplexe als Teil der verstehenden Verarbeitung einordnen. Oder in anderen Worten, jenes knowledge umfasst rekontextualisierte verköperlichte Erfahrungen zu den ikonischen Darstellungs- und Steuerungskodes, welche wiederum durch die Bandbreite von quasi-,räumlichzeitlich' konkret bis schematisiert, habitualisiert und automatisiert charakterisiert werden kann. Als eigenes, aber komplementäres dynamisches source model knüpfen sie an die Sets starker bis schwacher Darstellungs- und Steuerungskodes des Textinputs an und können derart die sukzessive integration von events - Lokal-/Globalzeichen niedriger Ordnung - bis hin zu sequences of events - Lokal-/Globalzeichen höherer Ordnung - moderieren:

(I) Den vorausgehenden semiotischen Analysen entsprechend können kategoriale Tabellen textstrukturell in unterschiedliche Repräsentationstiefen entlang ihrer Sets starker Darstellungs-Kodes - von single-lists bis nested-lists - unterteilt werden. Diese verweisen zum einen auf textuell vorliegende Bedeutungsstrukturen (Ia) in

- erstens, syntaktischen Lokalzeichen-Lokalzeichen-Relationen, die ungeachtet des repräsentierten Sachverhalts eine grundsätzlich gemeinsame Referenz zweier Lokalzeichen in einem Merkmal/Attribut anzeigen und demgemäß auf eine ggf. zu differenzierende Komplexität verweisen: In Abbildung 29 ist demnach im linken Beispiel ein knowledge zu aktualisieren, dass „Objekt 2.“ als auch „Objekt 3““ „irgendwie via ,Merkmal 1“ zusammengehören“; im rechten Beispiel, dass „Objekt 2x“ und „Objekt 3x“ „irgendwie via ,Merkmal 2“ zusammenund ,via Merkmal 1' nicht zusammengehören“. Jenes ,irgendwie` verweist auf die mögliche Relevanz von starken Darstellungs-Kodes der list-of-single-lists links und der intersected-lists rechts im Sinne eines verkörperlichten knowledge about how knowledge is represented.

\begin{tabular}{|cc|}
\hline \multicolumn{2}{|c|}{ (Attribut 1) } \\
Merkmal 1 & Merkmal 2 \\
$\mid$ & Objekt 1. \\
Objekt 1. & Objekt 2. \\
Objekt 2. & Objekt 3. \\
Objekt 4. & Objekt 4.
\end{tabular}

Abb. 29: Beispiele textstrukturell syntaktischer Lokalzeichen-Lokalzeichen-Relationen

- zweitens, syntaktischen Globalzeichen-Globalzeichen-Interferenzen, die ungeachtet des repräsentierten Sachverhalts eine grundsätzlich gemeinsame Referenz eines Sets aus Lokalzeichen anzeigen und demgemäß auf eine ggf. ebenso zu differenzierende Komplexität verweisen: In Abbildung 30 ist demnach im linken Beispiel ein knowledge zu aktualisieren, dass „Merkmal 2“ aus seiner Relation mit „Attribut 1“ und „Merkmal 3“ aus seiner Relation mit „Attribut 1“ „irgendwie, aber anders als zu ,Merkmal 1' in Beziehung steht“; und analog im rechten Beispiel, dass der ,Block“ „Anschriftzone“ als eine verbindliche Angabe einer Rechnung und der „Informationsblock“ als eine andere verbindliche Angabe einer Rechnung ,irgendwie zueinander in Beziehung steht“. Jenes „irgendwie“ verweist auf die mögliche Relevanz von starken Darstellungs-Kodes der intersected-list links und der von standardisierten Rechnungen rechts im Sinne eines verkörperlichten knowledge about how knowledge is represented. 


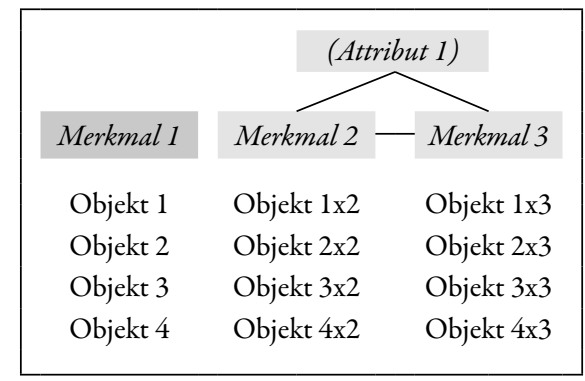

verbindliche Angaben und konventionelle Anwendung (Rechnung in D)

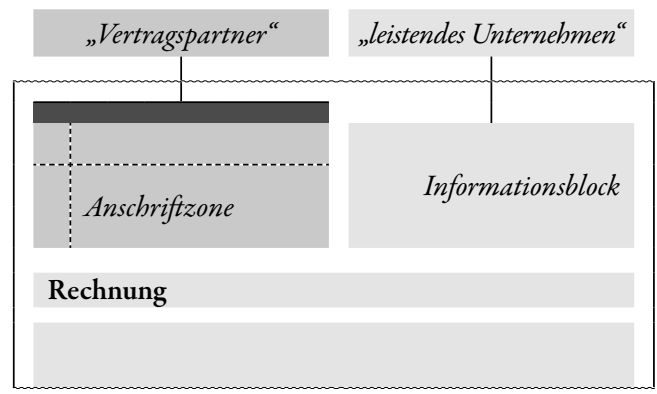

Abb. 30: Beispiele textstrukturell syntaktischer Globalzeichen-Globalzeichen-Interferenzen

- drittens, in semantischen Lokalzeichen-Lokalzeichen-Relationen, die eine der inhärenten, konkreten terminologischen, kategorialen, topologischen oder Verlaufs-Repräsentationsanalogien zwischen Merkmalen/Attributen eines Sachverhalts berücksichtigt; und demgemäß referentielle Felder in ihrer semantischen Komplexität charakterisieren können: Wenn in Abbildung $31 \mathrm{im}$ linken Beispiel dargelegt ist, dass „Objekt .2“ sowie „Objekt .3“ angenommen und jeweils eine Verlaufsstruktur des Sachverhalts spezifiziert über „Merkmal 1“ abbilden, sodann ist diese Tabelle inhärent, dass „Objekt.2“ und „Objekt. .“ im Sinne eines Verlaufs vorher oder nachher „zu erledigen, zu erreichen, zu befolgen usw.“ sind. Im rechten Beispiel, angenommen, wird jenes ,vorher' und ,nachher über „Merkmal 1“ spezifiziert.

\begin{tabular}{|c|c|}
\hline \multicolumn{2}{|c|}{ (Attribut 1) } \\
\hline $\begin{array}{c}\text { Merkmal } 1 \\
\vdots\end{array}$ & Merkmal 2 \\
\hline \begin{tabular}{c} 
Objekt.1 \\
\hdashline
\end{tabular} & Objekt .1 \\
\hline Objekt .2 & Objekt .2 \\
\hline Objekt.3 & Objekt.3 \\
\hline Objekt .4 & Objekt .4 \\
\hline
\end{tabular}

\begin{tabular}{|cccc|}
\hline & & \multicolumn{2}{c|}{ (Attribut 1) } \\
(Merkmal 1) & \multicolumn{1}{c|}{ Merkmal 2 } & Merkmal 3 \\
Objekt 1 & $\vdots$ & \\
& & Objekt x2 & Objekt x3 \\
Objekt 2 & $\ldots$ & Objekt x2 & Objekt x3 \\
Objekt 3 & $\ldots \ldots$ Objekt x2 & Objekt x3 \\
Objekt 4 & & Objekt x2 & Objekt x3 \\
\hline
\end{tabular}

Abb. 31: Beispiele textstrukturell semantischer Lokalzeichen-Lokalzeichen-Relationen

- viertens, in semantischen Globalzeichen-Globalzeichen-Interferenzen, die akzentuieren, dass die inhärenten, konkreten terminologischen, kategorialen, topologischen oder Verlaufs-Repräsentationsanalogien zwischen Merkmalen/Attributen eines Sachverhalts zu verdichten sind, respektive - vergleichbar bei kontinuierlichen Texten (siehe Kapitel 2.3.3) - „zu konstruieren, zu selegieren, zu generalisieren, zu integrieren oder auszulassen “ sind: Das linke Beispiel in Abbildung 32 soll illustrieren, dass „Merkmal 2“ und „Merkmal 3“ jeweils eine Ausgangsbedingung eines Sachverhalts mit Verlaufs-Struktur repräsentieren, die über das „Attribut 1“ = „Adressat eines Beschwerdeschreibens“ spezifiziert wird. „Merkmal 1“ bezeichnet „Personen, die nacheinander zu kontaktieren sind“. Demgemäß wäre z. B. einerseits die Relevanz zwischen „Merk- 
mal 2“ und „Merkmal 3“ via „Attribut 1“ ikonisch repräsentiert, und weiter zwischen „Objekt $1 \times 2$ “ und „Objekt $1 \times 3$ “. Andererseits gilt dies für das Selegieren der Abfolge zwischen „Merkmal 2“/,Merkmal 2“ zu „Merkmal 1“, und weiter zwischen „Objekt 1 2 2“ und „Objekt „2x2“. Im rechten Beispiel der Abbildung 32 handelt es sich ggf. um eine zu generalisierende Referenz zwischen „der Anschriftzone “ und „des Informationsblock“, und weiter der Angaben in den beiden „Blöcken“, unter Bezug der konventionalisierten Annordnung von verpflichtenden Angaben in Rechnungen von umsatzsteuerpflichtigen Unternehmen.

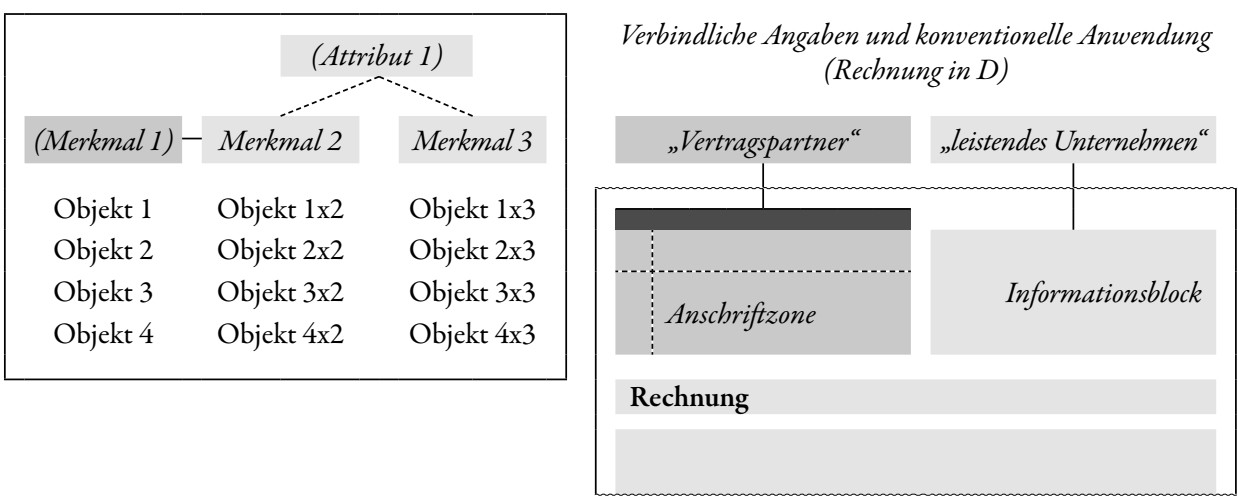

Abb. 32: Beispiele textstrukturell semantischer Globalzeichen-Globalzeichen-Interferenzen

Teils wie in den Beispielen beansprucht, sind textstrukturell die synatktischen und semantischen Bedeutungsstrukturen jedoch nicht ausschließlich den starken Darstellungs-Kodes inhärent. Vielmehr sind weitere Bedeutungsstrukturen einzubeziehen, die folglich kaum hierarchische Komplexitätsgrade vermuten lassen; übergeordnet zudem wechselseitig moderiert über die Idee der routinalisierten lokalen kulturellen Codes innerhalb des task/response models. Zu den weiteren textstrukturell beanspruchten Bedeutungsstrukturen zählen aus den bisherigen Analysen die

(Ib) ggf. verwendeten Sets schwacher Darstellungs-Kodes

- durch Linien zur Markierung der Felder in Tabellen;

- durch Einbindung graphischer Entitäten (z. B. anstelle einer zweispaltigen/-zeiligen Ja/ Nein-Tabelle);

- von graphischen Darstellungen (z. B. Skizzen von Fahrzeugen) und/oder;

- von Karten (z. B. Raum- oder Lagerpläne);

(Ic) und/oder ggf. verwendeten Sets ikonischer Steuerungs-Kodes, die dezidiert einzelne oder zusammenhängende Lokal-/Globalzeichen hervorheben (sollen)

- mittels Variationen an z. B. Schraffierungen oder Farben der Felder;

- mittels Linien/Pfeile zwischen z. B. Feldern;

- mittels Variationen der Typographie symbolischer Zeichen (z. B. mittels Fettschrift) in den Feldern.

(Id) Bedeutungsstrukturen auf der Ebene der Lokalzeichen können zudem mittels der einzelnen Begriffe oder Satzphrase dubliziert sein, welche einzelne Bezeichnungs- oder Textfelder repräsentieren. Und dennoch, sowohl die Relationen zwischen diesen einzelnen Merkmalen/ Attributen als auch die Interferenzen über Merkmale und Attribute hinweg sind rein über ikonische Repräsentationsanalogien dargelegt. Symbolische Explikationen dienen auch im Falle von Komposita letzlich zuvorderst der ausdrücksmächtigen Bezeichnung von Merkmalen und Attributen des dargelegten Objektes/Sachverhalts. 
Aus der Symbiose mit dem TRACE-Modell beschreibt alsdann das source model, wie in der Interaktion mit einem nichtkontinuierlichen Text oder Textkomplex Modifikationen, Verwerfungen, Neufassungen usw. über verkörperlichtes knowledge zu semantischen und syntaktischen Bedeutungsstrukturen erklärt werden können. Ausgehend von dem Ansatz einer komplementären Konstruktion der sources wird die Kohärenzbildung des contents multikodaler Texte und Textkomplexe über sogenannte „source-content-links“ im Sinne von Referenz, Abfolge, Relevanz, im Geiste von, im Widerspruch zu, in Bekräftigung gegenüber usw moderiert. Diese akzentuieren semantische oder syntaktische Bedeutungsstrukturen, die auch auf Schematisierungen, Habitalisierungen und Automatisierungen innerhalb dynamischer mentaler Modelle verweisen können, welche keine bestimmten perspektivischen Relationen innerhalb der inhärent repräsentierten terminologischen, kategorialen oder Verlaufsstrukturen hinter den textuell repräsentierten Interferenzen zwischen Relationen von Lokal- und/oder Globalzeichen mehr umfassen.

\subsubsection{Das Arbeitsmodell der Verarbeitung multikodaler Texte}

In den vorausgehenden Abschnitten wurde über eine dekonstruierenden Analyse (Tenorth 2010, 93) aus TRACE-Modell und Immersed Experiencer Framework mit großen Übereinstimmungen dargelegt, dass die verstehende Verarbeitung des content eines vorliegenden multikodalen Textes gerahmt von den sich wechselseitig initiierend oder begleitend beeinflussenden konzipierten Ideen der Zielsetzung eines task/response models verknüpft mit emotionalen Besetzungen, mit Antizipationen im Zuge bspw. routinalisierter Rahmenbedingungen der Lesesituation sowie mit der Idee von einem textuellen knowledge eines source models interpretiert werden kann (vgl. Abbildung 33). Insbesondere die textuell vorliegende semantischen und syntaktischen Bedeutungsstrukturen - Stichwort source model und linguistic cues werden in den beiden dekonstruierten Makromodellen unterschiedlich verortet. So ist beiden Makromodellen einerseits gemeinsam, dass sich eine Kohärenzbildung nicht alleinig über bottom-up-Verarbeitungsprozesse im Sinne von sukzessiven Verknüpfungen von Relationen und Interferenzen zwischen Sets semiotischer Zeichen bzw. ausschließlich auf der Textoberflächenstruktur erklären lassen. Eine rein textstrukturell generalisierbare Hierarchie ist im Zuge der interaktiv sowohl bottom-up- als auch top-down-Verarbeitungsprozessen eindeutig zurückzuweisen dies auch vor dem Hintergrund des Einflusses sozio-kontextueller Bedingungen der Lesesituation, aber auch zurückliegender Handlungserfahrungen der Lesenden. Andererseits ist die Identifikation als auch die Verarbeitung ikonischer Repräsentationen nicht unabhängig der beanspruchten semiotischen Strukturen des textuellen Inputs zu interpretieren. Im vorliegenden Arbeitsmodell der Verarbeitung multikodaler Texte (vgl. Abbildung 33) werden die semantischen und syntaktischen Bedeutungsstrukturen aus dem Erklärungsansatz von ,cognition as social, situated and embodied in service of action' präferiert. Via die Rekontextualisierung von konkret bis abstrahiert körpergebundenen Erfahrungen ist gleichsam davon Abstand zu nehmen, dass die soziale und situative Komponente kein komplementärer Teil einer kognitiven Verarbeitung von ikonischen Zeichen darstellt.

Für diese verstehende Verarbeitung werden demzufolge in dem vorliegenden Arbeitsmodell komplementäre dynamische Modellierungen angenommen, die durch die Pole von jeweils eher rekontextualisierten konkreten bis eher schematisiert, habitualisiert und automatisiert verköperlichten Erfahrungen weiter zergliedert werden können. Oder in anderen Worten, die komplementären Konzeptionen der Idee der task/response, der sources und des content führen einerseits die Annahme mit sich, dass diese in der Aktualisierung im Hier und Jetzt unterschiedlich auf weniger überlappende und somit konkreter körpergebunde erfahrungsnahe oder mehr überlappende quasi-räumlich-zeitlich schmatisierte, habitualisierte und automatisierte dynamische Modellierungen basieren. 


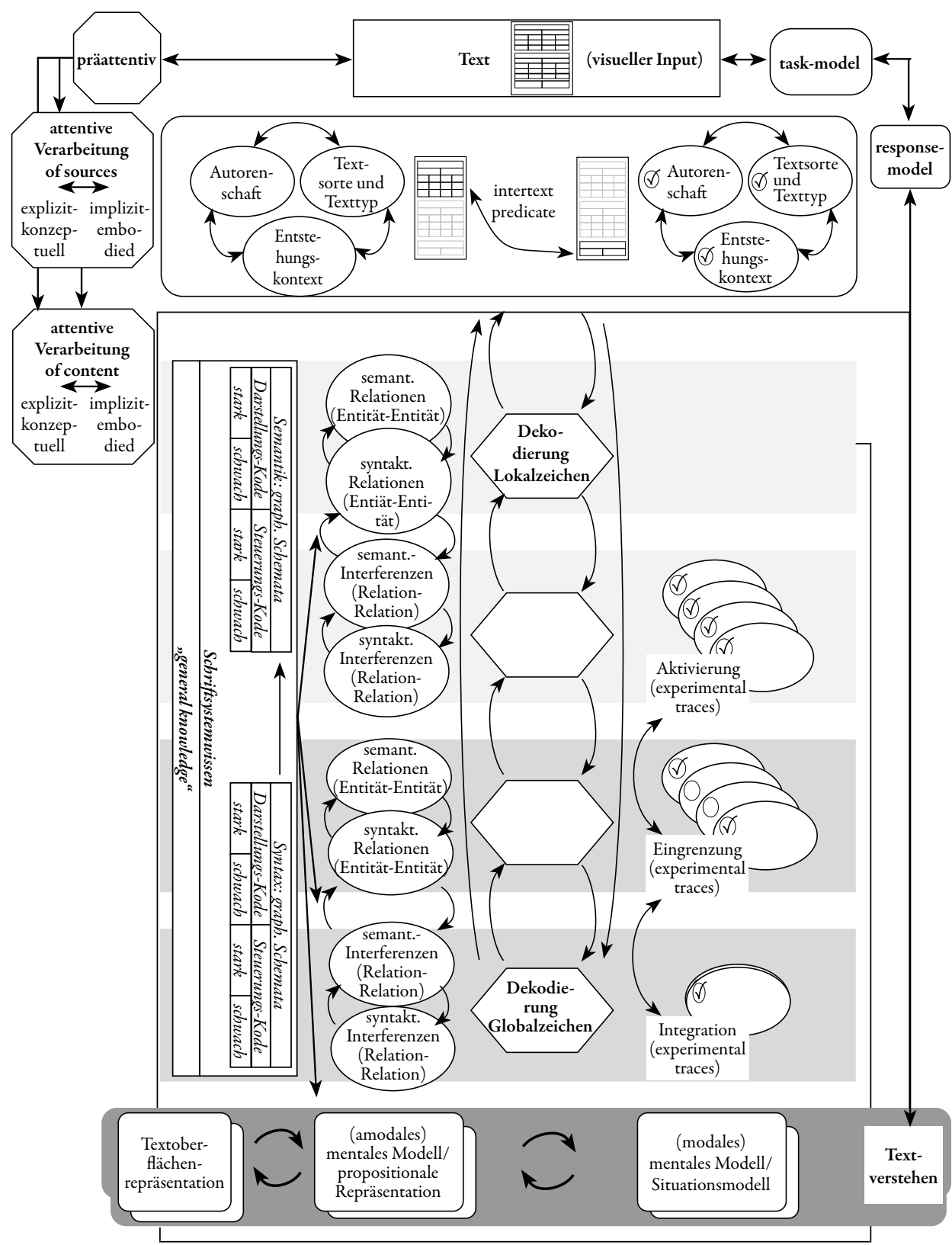

Abb. 33: Arbeitsmodell der attentiven (implizit-embodied bis explizit-konzeptionellen) Verarbeitung von primär ikonisch repräsentierten Texten und Textkomplexen

Andererseits führt die Integration der drei komplementären mentalen Modellierungen als Einflussfaktoren des verstehenden Lesens die Annahme mit sich, dass sich diese im Sinne einer interaktiven bottom-up- und top-down-Verarbeitung eines textuellen Inputs interpretieren lassen, wobei die zeitliche Reihenfolge der beteiligten komplementären Modellierungen der task/ 
response, der sources und des content als Ausgangspunkt erachtet wird. Insofern die Modellierung von task/response und/oder die der sources der Modellierung des content u. a. vorausgehen, ergo vor der Identifikation eines semiotischen Zeichens einsetzen, kann von einer initiierenden topdown-Verarbeitung gesprochen werden. Charakteristisch für initiierende top-down-Verarbeitungen sind bspw. die Ideenkonstruktion zur Autorenintention, die thematische Einordnung der über den Text repräsentierten Sachverhalte, die gegebenen zeitlichen und medialen Bedingungen der Lesesituation usw. Insbesondere für diesen Typus der verstehenden Verarbeitung rücken innerhalb des Ansatzes von, cognition as social, situated and embodied in service of action' nicht zuletzt die sozio-kontextuellen Bedingungen der aktuellen Lesesituation in den Mittelpunkt. Zweifelsohne erfahren aber auch die sozialen Kontexte sämtlicher zurückliegender Handlungserfahrungen an Relevanz, die sich verkörperlicht in den/die Lesende eingeschrieben haben und in der aktuellen Lesesituation ihre Aktualisierung finden.

Von einer verstehenden bottom-up-Verarbeitung eines Textes kann demgegenüber gesprochen werden, wenn die mentale Modellierung des content vor der ersten Idee der task/response und/oder der sources erfolgt. Sowohl im TRACE-Modell als auch innerhalb des „Immersed Experiencer Framework" können die beteiligten Modellierungen des Lesens mit der Identifikation eines semiotischen Zeichens in Gang gesetzt werden, welche jedoch deren prä-attentiven Erkennens von semiotischen Entitäten aus Punkten, Linien usw. zu Elementarzeichen voraussetzen, welche, wie dargelegt, auf den Gestaltungsgesetzen sozusagen auf den ersten Blick beruhen; oder übertragen das prä-attentive Erkennen von Punkten und Linien zu Graphemen singulär oder als Teil von Morphemen im deutschen Schriftsystem. Im Kontext der Identifikation erfährt nicht zuletzt deren sozial-historische Dimension über z. B. die diversen Typographien von Graphemen eines Schriftsystems oder diverser graphischer Schemata der räumlichen Anorderung von Bezeichnungsfeld-Textfeld(ern) bei kategorialen Tabellen an Relevanz. Für den/die Lesende resultiert folglich, dass auch die verstehende bottom-up-Verarbeitung nach dem Ansatz von ,cognition as social, situated and embodied in service of action' von den sozio-kontextuellen Bedingungen der aktuellen Lesesituation sowie zurückliegender Handlungserfahrungen maßgeblich beeinflusst wird.

\subsection{Sets sozialer Praxen an Einfach(st)arbeitsplätzen und ihre Lesenden - Verengung zu den Forschungsfragen}

In dem vorausgehenden Kapitel wurde insbesondere verdeutlicht, dass innerhalb der Relation von Bildung und Teilhabe in Arbeit die Mindeststandards des Lesens nach dem Konzept der funktionalen Grundbildungskompetenzen weder als notwendiges noch als hinreichendes Kriterium verstanden werden kann, um die Teilhabechancen derjeniger zu bestimmen, die durch Einfach(st)arbeit inkludiert sind: Sie haben sich seither empirisch als nicht geeignet herausgestellt, probabilistische Prognosen für Beschäftigung in unterschiedlichen Qualifizierungsniveaus unterhalb des hoch-qualifzierten seriös anzustellen. Des Weiteren eignen sie sich nicht, um zwischen den eher offenen oder eher geschlossenen Beschäftigten an Einfach(st)arbeitsplätzen zu differenzieren.

Diese Schlussfolgerung ist jedoch primär auf die Analyse einer externen Validität der mit diesem Konzept verknüpften Eigenschaft als soziales Identifikationskriterium zurückzuführen, welches universell Aussagen über die Handlungsfähigkeit im Kontext von Texten in einer Gesellschaft trifft. Darüber hinaus verhärtete sich, dass jene Analyse über die inhaltliche Ebene jener Handlungsfähigkeit im Kontext von Texten kaum Auskunft geben kann: Es erklärt kaum, ob jenes Konzept der funktionalen Grundbildungskompetenzen daran „scheitert“, da es eine typische Textualität an bspw. Einfach(st)arbeitsplätzen dezidiert nicht umfasst. Der Hintergrund könnte 
in der vermeintlich konventionellen Setzung von ordentlichen Berufen in der einen Gesellschaft gesehen werden, welche innerhalb der Relation von anerkannter Bildung und typischer Teilhabe auf ein Nicht-Mitdenken dieser Form von Arbeit schließen lässt - ein Einflussfaktor der jedoch durch national-traditionelle Übergangsregimes maßgeblich beeinflusst wird. Respektive allgemeiner, dieses „Scheitern“ könnte in der Eliminierung der Relevanz von bestimmten sozio-kulturellen Kontexten begründet liegen, in welche das Lesen eingebunden ist. Darüber hinaus schwingt aus dem Ansatz eines hierarchischen Modells von Lesefähigkeit, -fertigkeiten und -bereitschaften für die eine Handlungsfähigkeit in der einen Weltgesellschaft die Vermutung mit, dass die - auch textuellen - Anforderungen an Einfach(st)erwerbstätigkeiten nicht spezifiziert betrachtet werden müssen, da diese durch das Grundbildungslevel innerhalb dieses Modells - die Mindeststandards - bereits ausreichend erfasst sind. Für eine erziehungswissenschaftliche Auseinandersetzung im Spannungsfeld der Relation von Bildung und Teilhabe in Arbeit rücken im Zuge eines Verstehens dieser Handlungsfähigkeit, die in den nachfolgenden Unterkapiteln skizzierte Fragen in den Mittelpunkt. Eine Annäherung im Rahmen dieser Arbeit richtet sich dabei explizit an ein Mitdenken von Teilhabe in Arbeit in Formen der Beschäftigung außerhalb anerkannter Berufe.

\subsubsection{Primitivität der Sets sozialer Praxen an Einfach(st)arbeitsplätzen?}

Entlang der theoretischen und semiotischen Analysen ikonischer Textualität wurde bereits eindeutig die Positionierung vorgenommen, dass deren Verarbeitung nicht als etwas „Primitives“ und somit auch für institutionelle Bildungsprozesse „Vernachlässigbares“ interpretiert werden kann. Nichtdestotrotz konnte die in der Mainstream-Lesekompetenzdebatte mitschwingenden Setzung als ein ebensolches enttarnt werden. Aus dieser Setzung leitet sich einerseits der Verdacht der Reduktion von in Deutschland mit Teilhabe - zweifelsohne getragen von protonormalisitischen ständeorientierten Gliederungen - verknüpften Qualifizierungsniveaus ab, die sich entlang anerkannter beruflicher Bildung erst konstituieren. Andererseits drängt sich wissenschaftlslogisch die Problematik auf, dass Einfach(st)arbeit nicht entlang der auch textuellen Anforderungen markiert wird, sondern ausschließlich nach der protonormalistischen, ständeorientierten Anerkennung. Einfach(st)arbeit macht sich folglich nicht an einfachen Anforderungen im Sinne beruflicher Handlungsfähigkeit fest und somit auch nicht an beruflichen Handlungsanforderungen entlang primitiver Texte. Die nachfolgende empirischen Befragungen zielen daher auf die explorative Befragung, ob die an Einfach(st)arbeitsplätzen aufgefundene Textualität durch die in der Konzeption von funktionalen Lesecompetencies als Leitsemantik jener Teilhabe voraussetzenden Bildung des Lesens in der allgemeinbildenden Schule Berücksichtigung findet: Der Gegenstand der interessierenden Textualität akzentuiert weder die Dekodierung innerhalb funktionaler mathematischer competencies aufgeführten quantitativen Diagramme und Tabelle noch isolierter bzw. in kontinuierliche Texte sekundär eingebundener kategorialer Diagramme und Tabellen innerhalb der funktionalen Lesecompetencies (OECD 2002, 43; KMK 2005).

Unter der erziehungswissenschaftlichen Perspektive auf die Relation von Bildung und Teilhabe in Arbeit erwächst aus den bisherigen Analysen zuvorderst in der primär beanspruchten ikonischen Textualität und negiert keineswegs Relativierungen gegenüber den im Sinne von voraussetzungsrelevanten Fähigkeiten, Fertigkeiten und Bereitschaften entlang des primär symbolischen Zeichensystems des deutschen Schriftsystems. Es wurde eine semiotische Spezifik der Textualität offengelegt, die nichtkontinuierliche Textkomplexe entlang ihrer primären Ikonizität positionierten. Kurzum, ihr Lesen sollte entgegen der Setzung einer „primären Kulturtechnik“ - so die übergeordnete These - eine Aufwertung als zu berücksichtigender Teil in der Relation von Bildung und Teilhabe erfahren, insofern Einfach(st)erwerbstätigkeiten dezidiert als Formen von Arbeit anerkannt werden, die auch mit Teilhabe grundsätzlich verknüpft sind: 
(Quant_I) Sind die Anforderungen einer Handlungsfäbigkeit entlang der an Einfach(st)arbeitsplätzen aufgefundenen ikonischen Textualtität zu vernachlässigen, da die funktionalen Lesecompetencies im Sinne eines sozialen Identifikationskriteriums bereits eine Teilhabewabrscheinlichkeit in auch Einfach(st)arbeit ausreichend determinieren?

\subsubsection{Artefakte und lokale kulturelle Codes als Teil der Sets sozialer Praxen an Einfach(st) arbeitsplätzen?}

Für die aufgefundenen Texte und Textkomplexe an Einfach(st)arbeiten wurde eine multikodale Textualität herausgearbeitet (vgl. Kapitel 3.2). Angesichts der dahinterliegenden semiotisch analysierten Bandbreite an ikonischen Zeichensystemen mit einer starken bis schwachen Darstellungs- und Steuerungs-Kodierung in Kombination mit symbolischen Entitäten zuvorderst auf der Wort- bis Satzphrasenebene kann eine Konkretisierung der Multikodalität in einer primär divers ikonischen und sekundär symbolischen Textualität festgemacht werden. Diesbezüglich kann Schroeder (2011) hinsichtlich der textuellen Anforderungen an Einfach(st)arbeitsmärkten nur zugestimmt werden, dass diese

„weniger lexikalischer als vielmehr ikonischer Natur sind: Was die dort Beschäftigten ,lesen (dekodieren) müssen, sind überwiegend Zeichen und Symbole, Diagramme und Piktogramme, Icons, Pics und Farbcodes" (ebd., 215).

Aus den bisherigen theoretischen Betrachtungen (Tenorth 2010, 94ff.) zur einleitend dargelegten Problematik um einen Lesekompetenzbegriff, welcher die textuellen Anforderungen an Einfach(st) erwerbstätigkeiten ausgehend der befragten Relation von Bildung und Teilhabe (in Arbeit) mitdenkt, manifestiert sich mit einem multikodalen Zeichenbegriff, ein Zeichenbegriff, der sich nicht mehr auf Sets symbolischer Entitäten begrenzen lässt. Eine skillful performance entlang der soziokontextuell gerahmten Anforderungen eines Lesens an Einfach(st)arbeitsplätzen verlangt neben dieser Sensibilität auf einen mulitkodalen Zeichenbegriff auch eine Sensibilität hinsichtlich eines erweiterten Lese- und Kompetenzbegriffs. Ein Konglomerat, welches mit Lesekompetenzen allgemeingültige Fähigkeiten und Fertigkeiten und additiv emotional-motivationaler und sozialer Bereitschaften für die, normativ-kulturell theorisiert, eine Gesellschaft postuliert - im Sinne der Zuschreibung eines, für Teilhabe formalistisch-universell ausreichenden Grades -, muss angesichts der seither gescheiterten externen Validätsprüfung als nicht vielversprechend zurückgewiesen werden. Die im Zuge der Enttarnung diverser Mythen aufgedeckte, dahinter verborgene monokausale Beziehung von Bildung für Teilhabe (in Arbeit) manifestierte, dass der Ansatz von (symbolischen) Lesekompetenzen im Geltungskreises von handlungstheoretisch-kognitiven competencies mit Blick auf das Mitdenken von Einfach(st)erwerbstätigkeiten als konzeptuell unsensibel zurückzuweisen ist.

Mit der aufgenommenen „Spurensuche“ nach einem alternativen Konglomerat aus Lese- und Kompetenzbegriff kristallisierte sich eine Favorisierung heraus: das Lesen als Set sozialer Praxen im Geltungskreis von handlungstheoretisch-situativ fundierten Kompetenzen. Jene Favorisierung begründet sich neben einer - zumindest größtenteils - linguistischen, auch aus einer sozialtheoretischen Stringenz. Die Relativierung liegt in der aufgedeckten Problematik begründet, welche sich zuvorderst aus der verstetigten Forschungsprogrammatik und weniger aus der theoretischen Funderierung hinter dem Lesebegriff als Set sozialer Praxen ergibt. Wie bereits dargelegt, befasst sich diese im Grunde ausschließlich mit der Annäherung an das implizite, Wissen, das in einer bestimmten sozialen Praktik zum Einsatz kommt. Was eine skillfulperformance in einer bestimmten sozialen Praktik mit mulitkodalen Texten oder Textkomplexen ausmacht, bezieht sich jedoch seither fast immer ausschließlich auf die routinalisierten lokalen kulturellen Codes entlang jener 
Texte oder Textkomplexe. Mit der Frageabfolge der praxeologischen Forschungsprogrammatik wird suggeriert, dass Analysen von „Praktiken des Selbst, etwa im Schreiben von Tagebüchern, in der Lektüre von Romanen und Sachliteratur, im ,Kunstgenuss “ (Reckwitz 2003, 293) und nicht mit dem , praktischen Sinn' der hierbei somit unhinterfragt übernommenen grundsätzlichen Repräsentationsanalogien der routinalisiert eingebundenen Artefakte, ergo der routinalisiert eingebundenen semiotischen Zeichensysteme befassen müsste. Pointiert durch die Kritik von Grotlüschen $(2011,22)$ muss gar konstatiert werden, dass in diesen Analysen das ,zum Einsatz kommende Wissen' über Bedingungen des nicht willkürlichen Gebrauchs von Zeichensystemen, welche jene sozialen Praktiken erst ermöglichten bzw. Teil derer sind, schlichtweg ausgeblendet werden.

(Theorie_I) Welche fundamentale Repräsentationsmächtigkeit lässt sich hinter ikonischen und hierunter insbesondere kategorial-tabellarischen ikonischen Zeichen historisch aufdecken?

\subsubsection{Lokale kulturelle Codes als Teil der Sets sozialer Praxen an $\operatorname{Einfach}(s t)$ arbeitsplätzen?}

Aus dem favorisierten Lesekompetenzansatz resultiert hinsichtlich der Exploration von kognitiven, emotional-motivationalen und sozial-kommunikativen Fähigkeiten, Fertigkeiten und Bereitschaften des Lesens einer Person - oder in anderen Worten, von Lesekompetenzen einer Person, diese als an eine soziale Praktik gebundene zu betrachten. Demgemäß rückt eine zumindest annähernde Explikation von Facetten des ,zum Einsatz kommenden Wissens' in bestimmten sozialen Praxen des Lesens an Einfach(st)arbeitsplätzen in den Mittelpunkt, das in dem favorisierten Ansatz der dynamischen Verarbeitung semiotischer Zeichen innerhalb des goal/task-models verortet wurde: Hierunter für die vorliegende Arbeit zuvorderst von Interesse sind die routinalisiert lokalen kulturellen Codes, die sich an literalen events der untersuchten Einfach(st)erwerbstätigkeiten überwiegend aufdecken lassen:

(Qual_I) Unter welchen situativ-kontextuellen Bedingungen einer skillful performance sind die primär ikonischen Texte und Textkomplexe an Einfach(st)erwerbstätigkeiten zu lesen? Oder: Woran bemisst sich eine skillful performance in sozialen Praxen mit ikonischen Textkomplexen an Einfach(st)arbeitsplätzen?

Übergeordnet schwingt hier die seither generell vernachlässigte Befragung des intersubjektiv explorierten ,praktischen Sinns' von Artefakt und lokalen kulturellen Codes für die Personen, die Träger einer Praktik erst werden sollen mit; oder in anderen Worten, die erziehungswissenschaftliche Interpretation des Ergebnisses um die Relation von Bildung und Teilhabe (in Arbeit) an Relevanz gewinnt.

\subsubsection{Verkörperlicht erfahrene Verarbeitung der Lesenden der Sets sozialer Praxen an Einfach(st)arbeitsplätzen?}

Die situativ-kontextuellen Bedingungen von Lesesituationen mitbedacht, können aus den Bedingungen einer skillful performance auf kognitive, emotional-motivationalen und sozialkommunikativen Fähigkeiten, Fertigkeiten und Bereitschaften des Lesens einer Person somit - theoretisch stringent - nicht durch den Anspruch gekennzeichnet sein, dass es sich um universelle und somit für die Teilnahme an „allen“ sozialen Praxen „notwendige“ handelt. Konsequenterweise konkretisiert sich das Interesse auch nicht an der Fundierung von funktionalen Lesecompetencies für auch nichtkontinuierliche Texte und Textkomplexe. Anstelle eines Abbilds von einer demgemäßen Handlungsfähigkeit aus der Performanz tritt vielmehr das Interesse an der Exploration der kognitiven, emotional-motivationalen und sozial-kommunikativen Fähigkeiten, Fertigkeiten und Bereitschaften in der von einer skillfulperformance gerahm- 
ten Interaktion mit nichtkontinuierlichen Texten und Textkomplexen an den untersuchten Einfach(st)arbeitsplätzen im Sinne einer hypothetischen Konstruktion, welche entlang dem theoretisch abgeleiteten Makromodell als kategorisierbar angenommen werden.

Im Anschluss an die praxeologische Forschungslogik dient dieses aus der embodied theory maßgeblich erweiterte TRACE-Verarbeitungsmodell des Lesens zum hypothetisch konstruierten Rückschluss auf Lesekompetenzen der skillful performenden Teilnehmenden. Eine Interaktion mit auch nichtkontinuierlichen Texten und Textkomplexen zu beschreiben bedarf aus den theoretischen Herleitungen nebst impliziten Anteilen eines, zum Einsatz kommenden Wissens' die Offenheit gegenüber auch ,explizierbarem Faktenwissen', respektive, nebst implizt-embodied auch die Offenheit gegenüber explizit-konzeptionellen Verarbeitungsprozessen. Die Analysen legten ein wechselseitiges Verhältnis von statitischen und dynamischen Modellierungen von task/goal-, source- und content-model offen, um symbiotisch eine Bandbreite an Befunde zum Textverstehen zu integrieren. Jenes in diesem Sinne theoretisch abgeleitete Makromodell zur Beschreibung der Interaktion einer an einer sozialen Praktik an Einfach(st)erwerbstätigkeiten teilnehmenden Person mit ikonischen Textkomplexen hat sich jedoch empirisch zu bewähren.

(Qual_II) Teilhabe an sozialen Praxen mit nichtkontinuierlichen Texten und Textkomplexen an Einfach(st)arbeitsplätzen eröffnen Lernprozesse auch explizit-konzeptionellen Wissens über semiotische Strukturen? (Können diese Unterschiede oder Gemeinsamkeiten mittels des theoretisch abgeleiteten Makromodells abgebildet werden? Respektive, zeigen sich eindeutig abzugrenzende Präferenzen oder eher wechselseitig sich beeinflussende implizit-embodied oder explizit-konzeptionelle kogntivite Verarbeitungsprozesse bei den Teilnehmenden?)

\subsection{Vernetzung der Forschungsfragen}

Als für die Annäherung an spezifische Anforderungen des Lesens nichtkontinuierlicher Textkomplexe aus Einfach(st)erwerbstätigkeiten rahmend wurde die Bedeutung einer übergeordneten Positionierung innerhalb der Relation von Bildung und Teilhabe in Einfach(st)arbeit herausgearbeitet. Mit den Forschungsfragen wird ein erziehungswissenschaftliches Spannungsfeld aufgerissen, dass in einer intendierten externen Validitätsprüfung der Determinierung von Teilhabechancen und -prognosen im Geltungskreis von Einfach(st)arbeit und somit am Rande und unterhalb von in Deutschland anerkannten beruflichen Ausbildungswegen und Beschäftigungsformen über funktionale Grundbildungscompetencies mündet. Auf dem ersten Blick erscheint die Mehrheit der Studienergebnisse der insbesondere strukturdeterminierten Übergangsforschung diese prominente Ursache-Wirkung-Relation zwischen einer bestimmten psychometrisch messbaren Bildung für eine bestimmte kategorisch definierte Teilhabe bereits geklärt zu haben. Mit dem zweiten Blick auf die einschlägige strukturdeterminierte Forschung drängt sich jedoch insbesondere das Desiderat auf, welches tatsächlich diese Determination aufnimmt und bearbeitet. Vielmehr werden als Ursachefaktoren überwiegend Schulnoten bzw. Schulabschlüsse herangezogen und eben nicht funktionale Grundbildungscompetencies. Es manifestiert sich eine pragmatische Problematik zur Durchführung einer somit notwendigen externen Validitätsprüfung hinsichtlich Teilhabechancen und -prognosen entlang für die science community in der Regel nicht zugänglichen Items und/ oder von pseudo-anonymisierten personenbezogenen Daten der PISA-Studie, welche auch für die vorliegende Arbeit zutrifft. Da weder eine empirische Rekonstruktion möglich noch über einen direkten Quellenzugriff die zu betrachtenden funktionalen Grundbildungscompetencies befragt werden können, begründet sich die Entscheidung für eine theoretische und gegen eine empirische externe Validitätsprüfung der interessierenden Relation. 


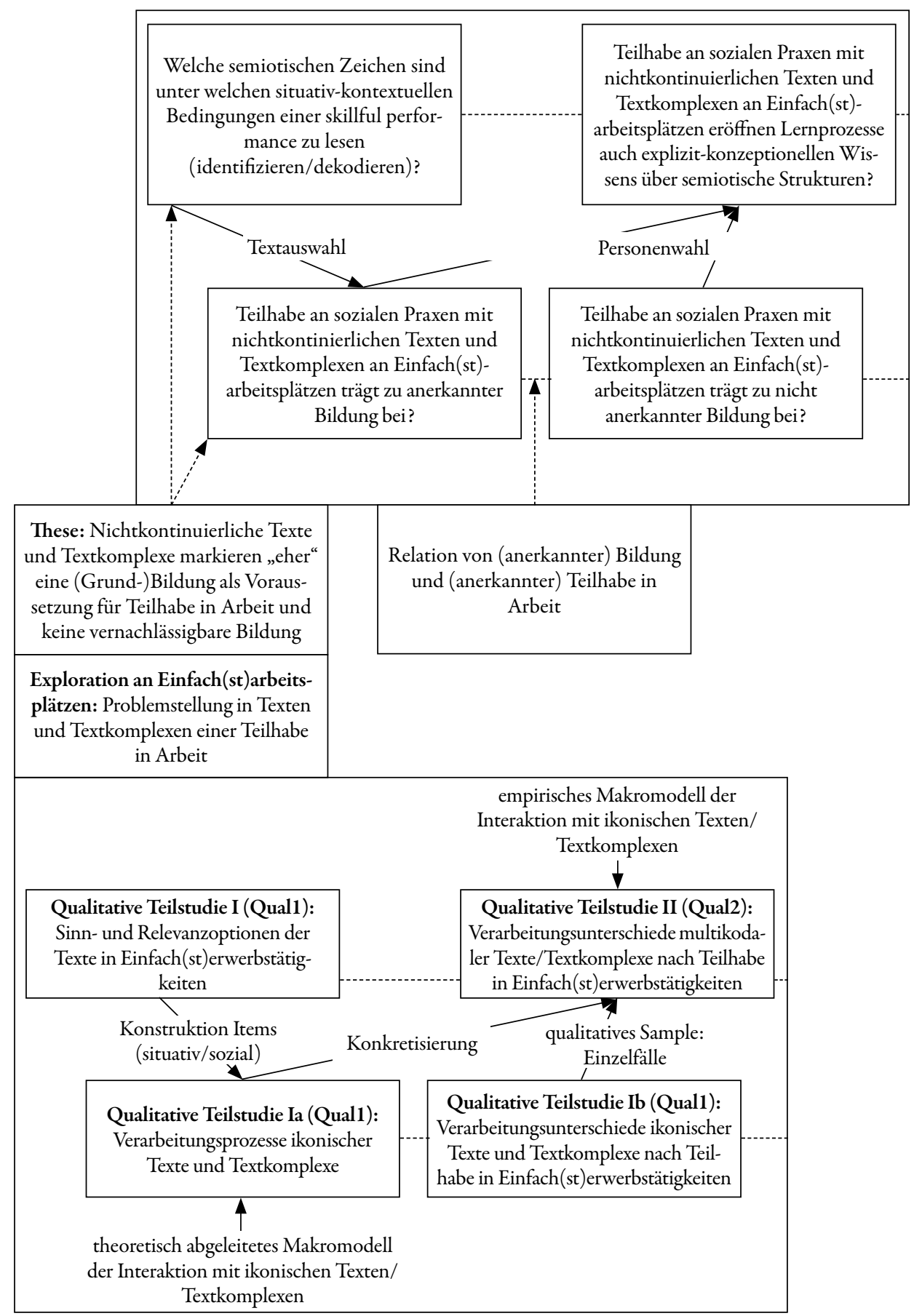

Abb. 34: Struktur der Vernetzung (unten) der einzelnen Forschungsfragen (oben) 
Die quantitative Forschungsfrage (Quant1; Kapitel 6) führt daher im Rahmen einer Theoriearbeit zu einer Recherche von einzelnen relevanten Referenzstudien, von denen insbesondere diejenigen von Interesse sein werden, die dezidiert mehr oder weniger auf jenen empirischen PISA-Quellen aufbauen, aber auch Einfach(st)erwerbstätigkeiten in ihren Betrachtung überhaupt aufnehmen - wie sich noch zeigen wird, werden diese ohne weitere Spezifizierung und empirischer Prüfung häufig als per se "gescheiterte" Teilhabe in Arbeit der heranwachsenden Generation mitgedacht. Im Rahmen einer sozialwissenschaftlichen-statistischen Analyse werden diese Studienergebnisse „wissenschaftslogisch“ kritisch befragt und im Sinne eine „Umgewichtung" (re)konstruiert (Tenorth 2010, 94ff.). Diese Art der wissenschaftstheoretischen Analyse ermöglicht darzulegen „dass sich auch Theorien, Argumentmuster oder Methoden [...] zwischen Kulturen und bei bestimmten [...] Gruppen ausbilden und variieren“, sowie, „dass Theorien [...] ihre eigene [...] Kulturalität besitzen“ (ebd., 94): im vorliegenden Fall für die Ursache anerkannter vs. nicht-anerkannter Bildung und die Wirkung in der Form von anerkannter vs. nicht-anerkannter Beschäftigung bzw. beruflicher Wege. Mit der Auswahl der Referenzstudien geht die Bestrebung einer Relativierung der Problematik einher, dass mit einer theoretischen Kritik, Analyse und Konstruktion kein universeller Geltungsanspruch postuliert werden kann, wenn in deren Zuge Determinationen zwischen anerkannter Bildung für anerkannte Teilhabe in Arbeit negiert, bekräftigt oder aufgelöst werden.

Für die explorative Annäherung an spezifische Anforderungen des Lesens nichtkontinuierlicher Textkomplexe aus Einfach(st)erwerbstätigkeiten im Spannungsfeld der Relation von Teilhabe und Bildung entlang der weiteren herausgearbeiteten qualitativen Fragestellungen empfiehlt sich ein sukzessives Modell eines qualitativ-quantitativ-qualitativ Mixed-Methods-Designs:

Will man auf die kausale Erklärung sozialen Handelns nicht verzichten (und es sprechen [...] gute Gründe gegen den Verzicht auf einen sozialwissenschaftlichen Kausalitatsbegriff), so reicht die Verwendung eines statistischen Instrumentariums, das Abweichungen von hypothetisch postulierten kausalen Zusammenhängen als Fehler toleriert und damit die Modellierung nicht-deterministischer Zusammenhänge erlaubt, nicht aus. Zusätzlich müssen Werkzeuge zur Verfügung stehen, mit denen bislang unbekannte kausale Pfade und [...] Bedingungen [Handlung in einer Dualität von Struktur] identifiziert und beschrieben werden können, die in kausalen Modellen von Sozialwissenschaftlern deswegen nicht auftauchen, weil sie keinen Zugang zu dem sich ändernden Handlungswissen der Akteure haben. Insbesondere in solchen Handlungsfeldem, die durch eine Pluralität von Handlungsmustern und kausalen Pfaden und durch sozialen Wandel gekennzeichnet sind, erfordert die empirische Untersuchung kausaler Zusammenhänge die Kombination quantitativer und qualitativer Methoden. Quantitative Verfahren dienen dabei zur Entwicklung von Modellen schwacher Kausalität und qualitative Methoden zur Entdeckung bislang unbekannter Handlungsbedingungen, durch deren Einbeziehung die quantitativen Modelle an Erklärungskraft gewinnen können (Kelle 2008, 200).

Dieses Vorgehen begründet sich zum einen aus dem favorisierten Lesekompetenzansatz und der damit einhergehenden praxeologischen Forschungstradition zu entlehnenden Implizität der verkörperten sozialen und situativen Rahmung der routinalisierten Interaktionen mit Texten. Für den analytischen Rückschluss auf Fähigkeiten, Fertigkeiten und Bereitschaften von an Praktiken mit diesen Texten teilhabenden Personen wurde von kaum denkbar explizierbaren Werten, Einstellungen und sozialen Beziehungen ausgegangen, die eine Lesesituation (literacy event) als Teil einer sozialen Praktik (literacy practice) situativ kontextualisieren. Mit der übergeordneten erziehungswissenschaftlichen Befragung der Relation von Bildung und Teilhabe wird unter jener Bandbreite des ,zum Einsatz kommenden Wissens' beim Gebrauch von ikonischen Zeichensysteme in sozialen Praxen an Einfach(st)arbeitsplätzen deren Kontrastierung mit den Funktionen des Lesens in sozialen Praxen der obligatorischen Schulbildung eine Akzentuierung 
gesetzt (QualI; Kapitel 5). Dieser erste Forschungsbefund wird für die weitere empirische Arbeit an einem hypothetischen Konstrukt zur Beschreibung von kognitiven, motivational-emotionalen und sozial-kommuikativen Dimensionen des Lesens von auch nichtkontinuierlichen Texten und Textkomplexen einfließen. Die herausgearbeitete These, dass eine Nichtberücksichtigung der dahinterliegenden ikonischen Textualität in üblichen Lesekompetenzansätzen als ein nicht zu vernachlässigender Teil der Relation von Bildung und Teilhabe mitzudenken ist, konkretisiert sich im Kontext sozialer Praxen in Einfach(st)erwerbstätigkeiten auch an einer gegebenfalls aufzudeckenden Bandbreite der Funktionen des Lesens über den Schriftspracherwerb, die Behaltensleistung und die Einstellungsänderung hinaus.

Wider einer Legitimation des Lesens dieser Texte und Textkomplexe als „primitive Kulturtechnik“ wird es daher dahingehend befragt, ob andere und somit spezifische dahinterliegende Verarbeitungsprozesse angenommen werden können, die nicht ohnehin durch die funktionalen Lesecompetencies ausreichend erklärt werden. Angesichts dieser letztlich empirischen Kontrastierung erscheint es unabdingbar, eine vergleichbar von einem probabilistischen Kausalitätsparadigma geleitete quantitative Teilstudie anzuschließen (QuanI; Kapitel 6). Für diese gilt gemäß dem favorisierten Lesekompetenzansatz jedoch eine eben ,schwache Kausalität', die sich bspw. in keiner vollständigen und umfassenden Auflösung von generalisierbaren Dispositionen manifestiert, welche auf explizit-konzeptionellen kognitiven Verarbeitungsprozesse entlang vermeintlich textstrukturell hierarchisierbarer Komplexitätsgrade rückgeführt werden könnte. Oder in anderen Worten, aus dem favorisierten Lesekompetenzansatz können nur die Performanz in den damit empirisch beobachtbaren sozialen Situationen erfasst werden (literacy event) und nicht erklärt werden, welche Dispositionen tatsächlich eine Handlungsfähigkeit kennzeichnen. Dem Explorationsgedanken folgend werden hierfür Texte und Textkomplexe zuvorderst gemäß ihrer Sinn- und Relevanzoptionen in sozialen Praxen an Einfach(st)erwerbstätigkeiten ausgewählt (Forschungsbefunde aus QualI) und sekundär nach jenen inhärenten semiotischen Textstrukturen hin kategorisiert. Die „Nähe“ zu den authentischen situativen Situationen ist folglich vorrangig und es wird bewusst auf eine konformative Nachbildung von umfassend universellen textstrukturellen Komplexitätsgrade durch die Selektion von Items abgelehnt und somit gleichermaßen bewusst probabilistische Erklärungsmodelle mit schwacher Kausalität in Kauf genommen.

Methodologische Probleme, die durch akteursinduzierte Diversitat von Handlungsmustern und sozialen Wandel erzeugt werden, lassen sich aber trotzdem mit statistischen und probabilistischen Konzepten allein nicht lösen. Die Frage, was sich hinter unerklärten Varianzen, Fehlertermen und der Unvorhersagbarkeit von Einzelereignissen in stochastischen Kausalmodellen verbirgt, lässt sich mit dem Verweis auf Handlungs- und Willensfreiheit nicht hinreichend beantworten. Wahrscheinlichkeitstheoretische Konzeptionen von Zufall sind zwar hilfreich, um Handlungen, die auf freien Entscheidungen beruhen, in statistische Modelle einzubeziehen, wenn nicht genügend Informationen über die Gründe der Handelnden bzw. über deren Handlungsbedingungen vorliegen. Theoretisch lässt sich menschliche Entscheidungsfreiheit aber keinesfalls durch ein (wahrscheinlichkeitstheoretisches) Konzept von Zufall substituieren. In handlungstheoretischer Hinsicht sind Handlungsfreiheit und Zufall eher Gegensätze, denn eine Entscheidung, die durch einen Zufall erzeugt wurde, lässt sich kaum sinnvoll als freie Entscheidung bezeichnen. [...] Weil auch ein Handeln, das auf freien Entscheidungen beruht, begründet werden kann, Regeln folgt und deswegen rational rekonstruierbar (wenn auch oft nicht prognostizierbar) ist, stellt das Konzept des Zufalls oftmals nur einen ,Lückenbüßer für das unvollständige Wissen eines sozialwissenschaftlichen Untersuchers über die faktischen Handlungsgründe der sozialen Akteure dar (ebd., 199f.).

Das theoretisch abgeleitete Makromodell des Textverstehens greift nicht nur diese Grundannahme von nicht umfassend generalisierbaren Fähigkeiten, Fertigkeiten und Bereitschaften des Lesens auf. Mit seinem Erklärungsansatz negiert es weder explizit-konzeptionelle Verarbeitungs- 
prozesse beim Lesen, noch vernachlässigt es Einflussfaktoren, die in den Erfahrungen mit und aus Texten als Artefakte unterschiedlicher sozialer Praxen verortet wurden: dem knowing how über die soziale Kontextualität dessen Gebrauchs. Wenn über an sozialen Praktiken mit auch nichtkontinuierlichen Texten und Textkomplexen teilhabenden Personen auf deren knowing how rückgeschlossen werden kann, diese Träger jenes Wissens sind, so müsste sich entlang des Rückschlusses auf Fähigkeiten, Fertigkeiten und Bereitschaften des Lesens einzelner Personen auch Bedingungen dessen/deren Teilhabe an unterschiedlichen sozialen Praxen niederschlagen. Oder in anderen Worten, wenn eine Person an unterschiedlichen sozialen Praxen mit nichtkontinuierlichen Texten und Textkomplexen teilnimmt, so kann von einer impliziten Bildung durch den praktischen Vollzug ausgegangen werden; und dies im Sinne einer ,Transmission von Schemata als ein lokales, konkret situiertes, öffentliches, dargestelltes, immer wieder in ähnlicher Weise wiederholtes intelligibles Tun und Können' (siehe Kapitel 2.3.3.). Eine rückschließende Annäherung an jenes knowing how von Personen, bzw. von insbesondere implizit-embodied Verarbeitungsprozesse beim Gebrauch von ikonischen Texten und Textkomplexen müsste sich insbesondere mit Formen von der Teilhabe an sozialen Praxen aufzeigen lassen; jedoch begrenzt durch die jeweilig routinalisiert kontextualisierten lokalen kulturellen Codes.

Vor diesem Hintergrund sieht das weitere Forschungsprogramm vor, die explorative Annäherung an spezifische Anforderungen des Lesens nichtkontinuierlicher Textkomplexe aus Einfach(st)erwerbstätigkeiten über die Kontrastierung der Alternierung von Teilhabe an sozialen Praxen mit diesen Texten fortzuführen. In einem ersten Schritt wird über statistische Verfahren untersucht werden (Quant1), ob in der situativ-kontextuellen Performanz überhaupt Unterschiede zwischen Schüler/innen primär entlang schulorganisatorischer Bedingungen der zeitlichen Einbindung von Langzeitpraktika in beruflichen Kontexten festzustellen sind; und somit auch nicht primär entlang der Kontrastierung nach Bildungsgängen. Diese Untersuchung kann Erklärungen liefern, ob jene Schüler/innengruppen bei bestimmbaren Lokal- bis Globalzeichen den lokalen kulturellen Codes entsprechend probabilistisch häufiger skillful performen. Diese leiten über zu Einstufungen, welche Lokal- bis Globalzeichen für bestimmte Schüler/innen gegenüber anderen Schüler/ innen einfacher und welche schwieriger zu identifizieren und dekodieren sind.

Welche expliziten Fähigkeiten, Fertigkeiten und Bereitschaften, die probabilistisch häufiger skillful performten Lokal- bis Globalzeichen jedoch aufklären, können statistische Verfahren im Sinne eines Modells schwacher Kausalität nicht leisten. Hier kann eine wissenschaftslogische Übereinstimmung innerhalb der dargelegten theoretischen Analysen zum Lesebegriff hervorgehoben werden: Die problematische Annahme, dass probabilistisch einfachere oder schwierigere semiotische Anforderungen über textstrukturell objektive Komplexitätsgrade aufgeklärt werden können. Solche deduktiven Erklärungen können die Gefahr unterlaufen,

den Umstand [zu] verdecken, dass es sich [...] [hierbei] nicht um Deduktionen handelt, sondern um „Hypothetische Schlussfolgerungen 'bzw. um ,Schlussfolgerungen auf die beste Erklärung': es wird nicht ausgehend von einer allgemeinen Gesetzmäßigkeit und der Beobachtung von Antezedensbedingungen auf das zu erklärende Phänomen geschlossen, sondern es wird unter Zuhilfenahme einer allgemeinen Gesetzmäßigkeit vom Bestehen eines Sachverhalts ex post auf dessen Antezedensbedingungen geschlossen. Diese Form der Schlussfolgerung ist nicht nur riskant und irrtumsanfällig (und muss deshalb eine empirische Prüfung nach sich ziehen, ob denn die Antezedensbedingungen in dem konkreten Fall tatsächlich vorliegen), sie erfordert auch die Kenntnis allgemeiner Gesetzmäßigkeiten, deren Geltung für den Gegenstandsbereich problemlos vorausgesetzt werden kann (ebd., 103).

Oder in anderen Worten, es kann nicht aufgeklärt werden, ob und welche eher explizitkonzeptionelle als auch eher implizit-embodied Verarbeitungsprozesse der ,Zielsetzung;, 
der ,sources' und des ,content' hierbei einflussnehmend sind - nach dem hypothetisch abgeleiteten Makromodell des Lesens ikonischer und symbolischer Textualität. Wenn mit der abschließenden Forschungsfrage die Exploration eben jener nicht aufgeklärten Bandbreite an expliziten und impliziten kognitiven, emotional-motivationalen und sozialkommunikativen Dimensionen des Lesens in das Zentrum des Interesses rückt (Qual2; Kapitel 7), steht gleichsam dieses Makromodell auf dem Prüfstand. Jenes hat sich in qualitativen kontrastierenden Einzelfallanalysen der Bearbeitung von Texten und Textkomplexen aus Einfach(st)erbwerbstätigkeiten zu bewähren: Das theoretical sampling (Kelle 2008, 140ff.) zur Kontrolle der Geltungsreichsweite der qualitativen Analyse soll auf destinktive Kategorien der vorausgehenden quantitativen Teilstudie zurückgreifen und vereint somit beide Stärken eines sequenziellen quantiativen-qualitativen Mixed-Methods-Designs,

1. der Identifikation von (ggfs. schwer interpretierbaren) statistischen Zusammenhängen und unaufgeklärten Varianzen, die als Explananda die Suche nach sozialwissenschaftlichen Erklärungen anregen sollen, und

2. der Identifikation von Kriterien für die Fallauswahl für den qualitativen Anteil der Studie und der Entwicklung eines Stichprobenrahmens für diesen Zweck (ebd., 286).

Ergänzt um z. B. die theoretisch analysierten, bei kategorial-tabellarischen Darstellungen textstrukturell dominierenden Strukturen von zuvorderst combined- und nested-lists verhärtet sich mit ihrer Kategorisierung der primär ikonischen Textualität zudem die Position, dass ihr Lesen im Vergleich zu kontinuierlichen Texten nicht nur „anders“, sondern gleichsam auch nicht als „einfach“ und schon gar nicht als „selbstverständlich“ einzuordnen ist. Diese These soll im Weiteren empirsch befragt werden, tangiert sie nicht zuletzt Schlussfolgerungen für deren Thematisierung in schulischen Settings.

Das Lesen multikodaler Texte und Textkomplexe markiert, erstens, jene Anforderungen der Identifikation und Dekodierung von textuell dargelegten Sachverhalten, die durch das Spektrum von kategorialen, terminologischen oder Verlaufsstrukturen auf der Ebene von Lokalzeichen und von zuvorderst Referenz, Relevanz, Widerspruch, Bekräftigung und Abfolge auf der Ebene von eher Globalzeichen eingegrenzt werden kann. Hierin ist die Identifikation und Dekodierung von symbolischen Zeichen zuvorderst als Explizierung von Merkmalen oder Atrributen jener kategorialen, terminologischen oder Verlaufsstrukturen einzuordnen und im Falle der linguistischen Gestalt von Komposita als Dopplung jener relationalen Strukturen. Die Dekodierung ist in den sich wechselseitig beeinflussenden primär explizit-konzeptuellen bis implizit-embodied Segmentierungsprozessen, respektive, in statischen und dynamischen mentalen Modellierungen zu verorten. Ein Makromodell der Interaktion mit multikodalen Texten und Textkomplexen hat jedoch, zweitens, die Idee einer ,Zielsetzung' an prominenter Stelle aufzunehmen: Einerseits begründet angesichts der nicht vorauszusetzenden textuell explizierten ,Zielsetzung' für die Interaktion und andererseits im Zuge des Vollständigkeitsprinzip, wonach bereits sämtliche Relationen zwischen Lokalzeichen und Interferenzen zwischen eher Globalzeichen den unterschiedlichen Sets an ikonischen Darstellungs- und Steuerungs-Kodes von und innerhalb in Tabellen, graphischen Darstellungen, Karten usw. stets inhärent sind. Jenes Makromodell hat, drittens, keinem Primat einer chronologischen Verarbeitung geschuldet, diverse Segmentierungsstrategien abzubilden, die eine ,Idee der Relevanz' aller Relationen zwischen Lokalzeichen und Interferenzen zwischen eher Globalzeichen umfasst („source model“). In corpore, es resultiert konsequenterweise die These, dass ein Makromodell der Interaktion mit einem multikodalen Text oder Textkomplex grundsätzlich seine situative und soziale Rahmung 
zu bezeichnen hat und diese durch eine Offenheit gegenüber eher explizit-konzeptuellen als auch eher implizit-embodied Segementierungsstrategien auf der Ebene des „content" und der „sources" fortführt.

Die thematisierten Makromodelle verdeutlichten, dass die einzelnen Entitäten und Sets von Entitäten aus primär ikonischen und sekundär symbolischen Zeichensystemen Grundlage von dynamischen mental modellierbaren Bedeutungsstrukturen sein können. Obgleich keine umfassendenen - auf der Ebene der dekodierenden Verarbeitung - generalisierbaren Komplexitätsgrade in dieser Darlegung festzumachen sind, so ist die Bandbreite an aus diesem Möglichkeitsraum aktualisierten Bedeutungsstrukturen jedoch nicht gänzlich frei von der dargebotenenen Textoberflächenstruktur. Hinter bestimmten arbiträren starken Codes von Entitäten können einige fundamentale Repräsentationsanalogien vermutet werden. Diese haben sich in zahlreichen sozialen Praxen eines semiotischen Kulturraums evolutionär derart verstetigt, dass eine konventionalisierte Repräsentationsanalogie zwischen Zeichen und Bezeichnetem angenommen werden kann, und dies einschließlich generalisierbarer Komplexitätsgrade auf der Ebene der identifizierenden Verarbeitung: von Elementarzeichen als z. B. Grapheme im Sinne von Referenten von Entitäten eines bestimmten Sprachsystems, als z. B. Lokalzeichen im Sinne von Referenten von Entitäten von Tabellen usw. Diese müssen - nahezu - unabhängig jeglicher sozialen und situativen Rahmung im referentielle Zeichensystem Anwendung finden.

Jene Texte, respektive, „Spielweisen“ mit Zeichensystemen aus einzelnen Praxen in Arbeit heraus verortet, verfolgt dieser Lesekompetenzansatz die Idee, die hier inhärenten impliziten lokalen „Regeln“, dem knowing how auch zum Gebrauch von Zeichensystemen, in miteinander soziokulturell verketteten Situationen zu explizieren, die für teilnehmende Personen von Bedeutung $\mathrm{zu}$ sein scheinen. Insbesondere in jener praxeologische Justierung der Relation von einer weder universellen noch kollektiv-universellen ,befriedigenden Lebensführung' und dem Gebrauch von Zeichensystemen wird sich im Weiteren jener Lesekompetenzansatz im Vergleich als am ehesten kongruent herausstellen, die textuellen Anforderungen an Einfach(st)arbeitbeitsplätze stimmig zu betrachten. 



\title{
4 Das Historische des Lesens multikodaler Textualität
}

\author{
The list is the origin of culture. \\ Whereever you look in cultural history, you will find lists
}

(Eco 2009).

\subsection{Einordnung der theoretischen Analyse}

Das entwickelte Arbeitsmodell der Verarbeitung multikodaler Texte und Textkomplexe warf die Frage nach dem semiotischen Artefakt auf: Das Arbeitsmodell befragt Lesekompetenzen als hypothetisches Konstrukt über die Handlungsfähigkeit einer Person und versteht hieraus die Verarbeitung als historisch- und situativ-sozial eingebettet. Wie und warum semiotische Zeichen in Texte und wie innerhalb sozialer Praxen eingebunden sind, ist demgemäß weder als beliebig noch trotz aller Routinalisiertheit als zeitlich keinen Veränderungen unterliegend zu betrachten. Sie sind als literacy practices somit integraler Bestandteil der Interaktionen und interpretierenden Prozesse der teilnehmenden Personen zu verorten, wobei jene Personen eher implizit und nur bedingt explizit über diese soziale Beschaffenheit in der einzelnen Lesesituation (=situated literacy event) Kenntnis haben. Dies gilt umso mehr für die semiotischen Artefakte hinter den insbesondere interessierenden kategorialen Tabellen, die diesen Praxen bereits inhärent sind. Die explikative Annäherung an diese Artefakte erfolgt daher über eine theoretische Arbeit im Sinne einer historischen Analyse (Tenorth 2010, 94f.).

\subsection{Historische Analyse der semiotischen Artefakte hinter kategorialen Tabellen}

Nichtkontinuierliche Texte mit einer primär oder sekundär kategorial-tabellarischen Struktur haben entgegen kontinuierlichen Texten gemeinsam, dass sie eben nicht primär nach Sätzen, Absätzen oder gar Kapiteln gegliedert werden. Wenn im Weiteren mittels einer historischen Spurensuche Praxen aufgedeckt werden, welche eine Routinalisiertheit von Zeichensystemen verdeutlichen sollen, so richtet sich diese ausdrücklich nicht an primär kontinuierliche Texte, in welche kategorial-tabellarische Texte eingebunden sind. Mit dieser Akzentuierung liefert eine etymologische Annäherung der deutschen „Tabelle“ und des englischen „table“ eine vermutete Nähe zu Praxen von Spielen und Darstellungen von Zahlen.

Tabelle f. ,listenförmige Zusammenstellung, Übersichtstafel, Verzeichnis', entlehnt (2. Hälfte 16. Jh.) aus lat. tabella ,Brettchen, Gemälde, Schreibtafel, Brief, Stimmtäfelchen, Urkunde, Akte, Register, Liste, Deinutivum von lat. tabula [...]. Eine davon unabhängige frühere Entlehnung aus lat. tabella liegt vor in ahd. tabel(l)a, auch tavela, tafela ,Tafel, Täfelchen' (um 1000, Notker). - tabellarisch Adj. ,in Form einer Tabelle, listenförmig geordnet, in übersichtlicher Zusammenstellung dargetan ' (2. Hälfte 18. Jh.); vgl. lat. tabellārius ,zu den Briefen, Stimmtäfelchen, Dokumenten gehörig.' ${ }^{15}$

table ate 12c., „board, slab, plate," from Old French table „,board, plank, writing table, picture“ (11c.), and late Old English tabele, from West Germanic *tabal (cf. Old High German zabel, German Tafel), both the French and Germanic words from Latin tabula „a board, plank, table," originally „small flat slab or piece“ usually for inscriptions or for games, of uncertain origin, related to Umbrian tafle „on the board.“

15 http://www.dwds.de/?qu=tabelle 
The sense of "piece of furniture with the flat top and legs" first recorded c.1300 (the usual Latin word for this was mensa [...]. Old English writers used bord [...]). The meaning ,arrangement of numbers or other figures for convenience" is recorded from late $14 \mathrm{c}$. (e.g. table of contents, mid-15c.). ${ }^{16}$

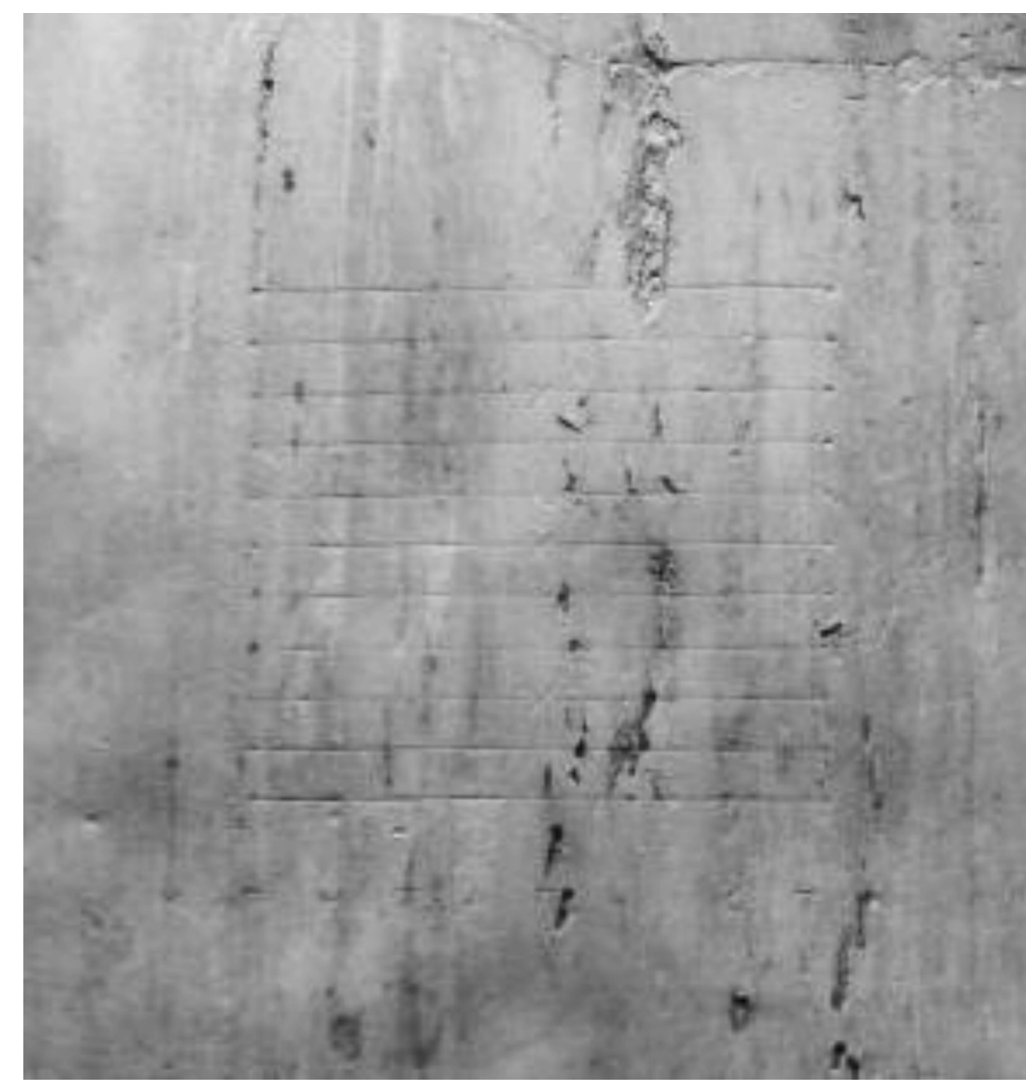

Abb. 35: Salaminischer Abacus

Zweitere Referenz fortgeführt, müsste sich bspw. in Praxen mit historischen Rechentafeln bzw. -tischen, heutzutage auch Abacus genannt, aufzeigen lassen. Mit den Linien auf dem Salaminischen Abacus (vgl. Abbildung 35) formiert sich einerseits noch keine vermeintlich vollständige Tabelle, wie wir sie heute aus unterschiedlichen Kontexten kennen.

Andererseits verhärten sich mit diesem auf ca. das 4. Jh. v. Chr. datierte Fundstück benannte Bezüge zu einem historisch abgeleiteten nicht-beliebigen Gebrauch von Tabellen - Stichwort „arrangement for convenience“: Die abendländische Wiederentdeckung nach der Reconqista des griechisch-römischen Abacus (Euklid 300 v. Chr.; Anicius Manlius Severinus Boëthius, ca. 480-520) wird häufig in den Zusammenhang mit Gerbert von Aurilac, später Papst Silvester II (946-1003), gebracht. Seine Weiterentwicklung sah u. a. vor, dass man apices, kleinen Hornscheiben mit eingeritzten oder beschriebenen arabischen Zahlenziffern, auf den horizontalen 
Linien des Rechenbrettes verschob. In Kombination mit einer Multiplikationstafel (vgl. Abbildung 36) waren mit diesem optimierten Abacus auch komplexe Berechnungen möglich; u.a. indem die zweckmäßige Anordung der Apices beschrieben wurde.

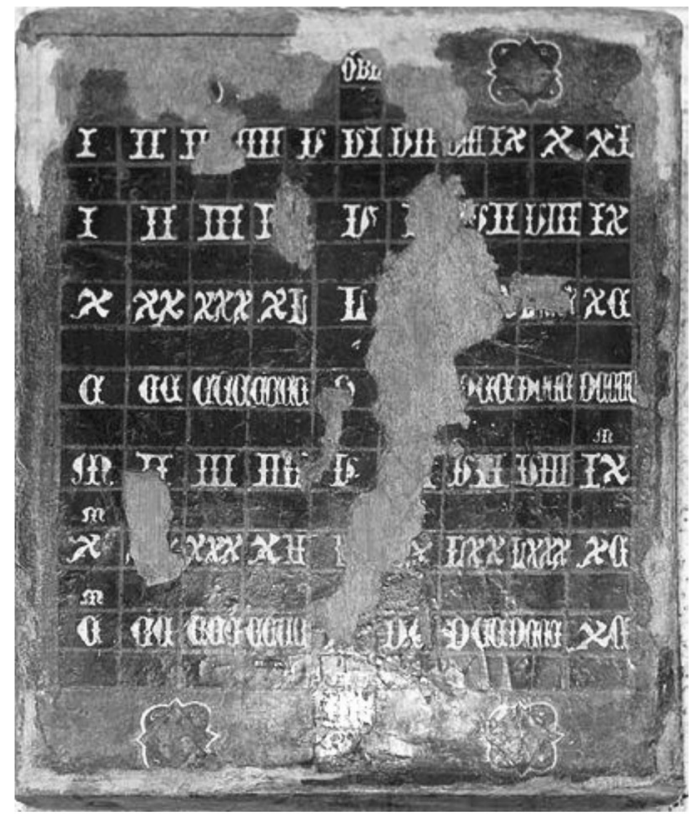

Abb. 36: Multiplikationstafel/Abacus-Tabelle aus dem europäischen Mittelalter

Die aufgestellte Vermutung der etymologischen Nähe der Tabelle/table zu bestimmten „Täfelchen“, „Brettchen“ usw. kann zudem durch Abhandlungen zur Geschichte der Mathematik konkretisiert, respektive, genährt werden; genauer in den terminologischen Verstrickungen der mensa pythagorica und dem abacus pythagoricus im Mittelalter:

Ich muss mich hier ganz allein auf die Untersuchung der Stelle in der Geometrie von Boëthius einschränken [...]. Eine ziemlich wörtliche Übersetzung, welche uns den Sinn der Stelle wieder zu geben scheint, ist folgende: [...] ,Die Pythagoräer hatten, um Irrthümer in ihren Multiplicationen, Divisionen und Messungen zu vermeiden [...], zu ihrem Gebrauch eine Tafel erdacht, die sie zur Ehre ihres Meisters pythagoraäische Tafel nannten; weil sie zu dem, was sie entwarfen, die erste Idee von diesem Philosophen erhalten hatten'. ,Diese Tafel wurden den Neuern abacus genannt. Durch dieses Mittel, welches sie durch ihre Geistesanstrengung erfunden haben, konnten sie die Kenntnisse leichter zu gebräuchlicher und allgemeiner machen, indem sie es, so zu sagen, dem Auge darlegten. Sie gaben dieser Tafel eine sehr vorzügliche Gestalt, welche hier unten dargestellt wird: [...] Hier findet sich die Tafel der Multiplication in den Ausgaben des Boëthius und wahrscheinlich in den Manuscripten [...]. Diese Tafel des Pythagoras findet sich aber nicht in einem sehr vorzüglichen Manuscript aus dem 11ten Jahrhundert, welches der Bibliothek zu Chartes gehört und welches oft correcter ist, als die Ausgabe von 1570. Dieser Umstand hat bei mir die Idee erzeugt, dass es vielleicht nicht die Tafel der Multiplication (welcher man, auf die Autorität dieser Stelle hin, den Namen des Pythagoras gegeben hat) war, von der Boëthius wirklich gesprochen hat. Und ich bin seitdem zu dem Glauben gekommen, dass die Schwierigkeit, welche man darin gefunden hat, den Aussprüchen des Verfas- 
sers einen Sinn unterzulegen, nur daher kam, dass man dieselben auf diese Tafel der Multiplication anwenden wollte. [...] Vor Boëthius ist durchaus nicht, weder von der Tafel der Multiplication noch von der Tafel des Pythagoras gesprochen; es begründet sich nur auf die Autorität dieser Stelle seiner Geometrie, wo sich in den Manuscripten die Tafel der Multiplication findet, dass man seitdem auf diese Tafel die Namen mensa pythagorica und abacus pyhtagoricus angewandt hat. [...] Der Sinn, welchen wir der Stelle des Boëtius gegeben haben, beruht auf der doppelten Annahme, dass das Wort abacus, welches dabei gebraucht wird, sich nicht auf die Tafel der Multiplication anwendet, wie man es bisher angenommen hat, sondern vielmehr auf ein Tableau einer besondern Disposition [...].Vor Boëthius ist durchaus nicht, weder von der Tafel der Multiplication noch von der Tafel des Pythagoras gesprochen; es begründet sich nur auf die Autorität dieser Stelle seiner Geometrie, wo sich in den Manuscripten die Tafel der Multiplication findet, dass man seitdem auf diese Tafel die Namen mensa pythagorica und abacus pyhtagoricus angewandt hat (Chasles 1839, 528ff.).

In der Glarean'schen Ausgabe des Boëthius, und wahrscheinlich in den moisten Manuscripten, ist unsere Stelle insbesondere noch dadurch entstellt, dass da, wo die Figur des Abacus im Texte als eingeschaltet erwähnt wird, statt einer irgendwie zur Beschreibung passenden Tabelle, die allbekannte, gewöhnlich Muliplicationstafel, das Ein mal Eins, sich eingeschoben findet. [...] Nur auf Autorität unserer Stelle und der erwähnten Beschaffenheit der Manuscripte hin, haben seitdem die Ausdrück abacus pythagoricus und mensa pythagorica die Bedeutung: Multiplikationstafel, oder Ein mal Eins, angenommen. [...] In zwei werthvollen, dem 14. oder möglicher Weise dem 10. Jahrhunderte angehörigen Manuscripten, deren eines der Bibliothek zu Altdorf angehörte, das andere zu Chartes bewahrt wird, findet sich an der hier fraglichen Stelle des Textes eine von gedruckten Ausgaben des Boëthius wesentlich abweichende Darstellung des Abacus. [...] So möchten denn auch die Abbildungen in den beiden vorgenannten Manuscripten des Boëthius so wenig wie der erläuternde Text des letzteren uns erschärfende und sichere Auskunft darüber zu geben geeignet sein, wie die zum esoterischen Gebrauchs rechte Ausführung der Abacus-Tabelle - die wahre F üll u n g derselben [...] beschaffen gewesen sein mag (Thimus 1868, 146ff.).

Die beiden Quellen verweisen zum einen darauf, dass die kreuztabellarischen Multiplikationstabellen (vgl. Abbildung 36) wahrscheinlich keinen antiken Ursprung haben und folglich im abendländischen Mittelalter entwickelt wurden; die zudem zunehmend komplexere Aufgaben umfassten; vgl. z. B. Athanasisus Kirchers Mensa Pythagorica expansa. Zum anderen deuten Chasles und von Thimus letztlich im Sinne des Nebeneinanders der Rechnung auf den Linien und der sich zunehmend ausweitenden Rechnung auf der Feder in dieser Epoche auf eben auch das Nebeneinander von tabellarischen Multiplikationstafeln mit indo-arabisch entlehnten Algorithmen. Diese Tendenz ist letztlich durch diverse Rechenbücher bekannt, die spätestens zwischen 1400 und 1600 für insbesondere die Söhne von Kaufleuten vermehrt entstanden. Die beiden von Adam Ries sind hier wahrscheinlich die am weitläufigsten vertrautesten. Hervorzuheben ist an dieser Stelle jedoch das Werk „Behende und hubsche Rechenung auff allen Kauffmannschaft“ von 1489 von Johannes Widmann (ca. 1460-1498) ${ }^{17}$ : In diesem Rechenbuch findet sich die erste gedruckte tabellarische Einmaleinstafel (vgl. Abbildung 37). Der folglich vermutete, nicht vom Abacus und seinem Gebrauch in der finanzmathematischen Buchhaltung im ausgehenden Mittelalter zu trennende Wortursprung würde letztlich mit einigen Praxen übereinstimmen, in denen Tabellen auch heute noch - Anwendung finden. Tabellen wie auch nichtkontinuierliche Texte bedienen sich nicht Worten, sondern „belehrenden“, sprich informierenden Bildern. Jene basieren auf graphischen Entitäten im Sinne von „vielfach verflochtenen Merkmalen“, die zugleich der Bedingung von spezifischen, zweckmäßigen Anordnungen unterliegen. Aber nicht nur die Rechenverfahren auf den Linien bewahrten noch Jahrhunderte ihre Popularität. Auch finden sich Belege, dass sich der

17 Durch den Erfolg dieses Rechenbuches zum schriftlichen Rechnen, so vermutet man, haben sich die Additions- und Substraktionssymbole „+/-“ ausgebreitet. 
damit u. a. verknüpfte Ausdruck Abacus synonym für die aufkommende Rechenkunst aufder Feder, ergo Algorithmus, erhielt. So nannte bspw. Leonardo von Pisa, bekannt auch unter dem Namen Fibonacci, sein Werk zur, aus dem indo-arabischen Raum entlehnten, Arithmetik, Liber Abaci'. Die folglich historisch nicht aus der heutigen Verwendung von Abacus und Multiplikationstafel abzuleitenden Handlungen verweisen auf terminologische Annäherungen von mensa (pythagorica) mit abacus (pythagoricus):

Auch die sprachliche Ableitung des Wortes abacus deutet eine semitische Herkunft an. Abach, Abak [...], i n e i n a n d e r w i n d e n, in intransitiver Bedeutung ve r w i c k e l t s e i n, in einander verfloch te n se in [...], entspricht vollkommen der Zusammensetzung des Abacus aus vielfach verflochtenen Reihen von Zahlen. [...] Das griechische $\alpha \beta \alpha \xi$ liesse sich vielleicht in Verbindung bringen mit dem Adjektiv $\ddot{\alpha} \beta \alpha \xi$, s t u m m, s p r a ch los ( $\beta a ́ \zeta \omega$, r e d e n, s p r e ch e n [...]); und würde der Name des geheimnisvollen Diagramms dann so viel bedeuten als: ,nicht in Worten redend, sondern in belehrenden Bildern reden', oder wie es in dem sogleich zu erwähnenden merkwürdigen Briefe Papst Silvesters II heißt: ,Vie rationis, bevis verbis, prolixa sententiis'. Im gewöhnlichen Sprachgebrauche wurde das Wort, griechisch wie lateinisch, für: T i s ch, B r e t t (insbesondere das mit Kugelschnüren bespannte Rechenbrett), T a f e l (daher auch in der Architectur die viereckige, den obersten Theil des Säulencapitels bildende Platte so heißt) gebraucht (ebd., 150).

Der folglich vermutete, nicht vom Abacus und seinem Gebrauch in der finanzmathematischen Buchhaltung im ausgehenden Mittelalter zu trennende Wortursprung würde letztlich mit einigen Praxen übereinstimmen, in denen Tabellen - auch heute noch - Anwendung finden. Tabellen wie auch nichtkontinuierliche Texte bedienen sich nicht Worten, sondern „belehrenden“, sprich informierenden Bildern. Jene basieren auf graphischen Entitäten im Sinne von „vielfach verflochtenen Merkmalen“, die zugleich der Bedingung von spezifischen, zweckmäßigen Anordnungen unterliegen.

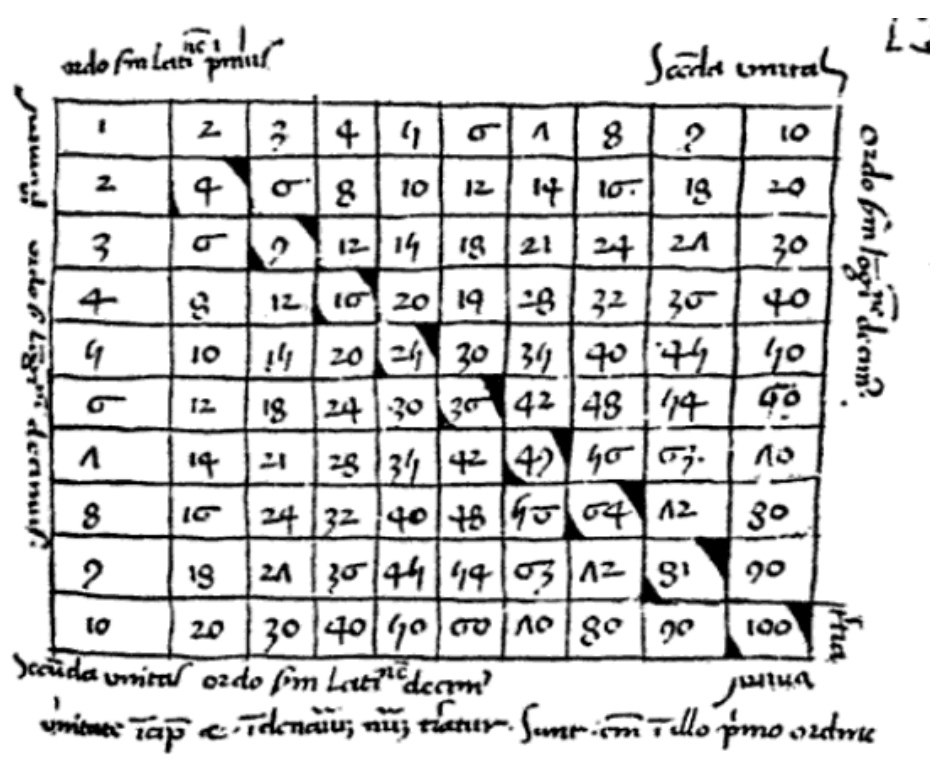

Abb. 37: Erste bekannte gedruckte Fassung einer Multiplikationstafel 
Die wenigen wissenschaftlichen Abhandlungen zur Datierung der Erfindung jener „belehrenden Bilder“ aus „,vielfach verflochtenen Merkmalen“ erlauben gleichermaßen über sozio-kulturelle Bedingungen letztlich lediglich Hypothesen aufzustellen. Sie verweisen auf chronologische Abhandlungen in bestimmten historischen Kulturräumen, können aber kaum Aspekte des Transfers zwischen mehreren dieser historischen Kulturräume nur ansatzweise aufdecken. Nichtsdestotrotz manifestieren die wenigen Arbeiten, welche primär den Ursprung mathematischer Tabellen hin zu Tabellenkalkulationen (Campbell-Kelly et al. 2003) bzw. Diagrammen (Tobies/Tournès 2011) aufzudecken intendieren, dass es sich auch bei den kategorialen Tabellen um Errungenschaften des „arrangement of numbers or other figures for convenience“ handelt, die Jahrtausende zurückliegen. Mit Eco (2009) können entlang des 2. Gesangs der Ilias von Homer die Darstellung qualitativen/qualitativen oder qualitativen/quantitativen Sachverhalte ${ }^{18}$ historisch differenziert werden. Sie folgen nach dem italienischen Schriftsteller, Philosoph, aber auch Semiotiker dem menschlichen Bedürfnis der Komplexitätsreduktion in der „unity of a set of objects". Oder in anderen Worten, in den - mehr die metrischen, aber auch die kategorialen - tabellarischen Darstellungen kann die Bestrebung der Kontrolle über Begriffe, Mengen, Zeiten, Tätigkeiten, Verhältnisse usw. gesehen werden. Bishops (1988) ethnomathematische Analysen unterstreichen hier ergänzend, dass sich auch in ,isolierten“ Stämmen Afrikas oder Ozeaniens kardinale Ordnungsprinzipien auffinden lassen.

Das älteste Fundstück einer kategorialen Tabelle erlaubt über diese Funktionen hinaus eine ungefähre Einordnung dieses Zeichensystems innerhalb „der Erfindung der Schrift“. Aus den bürokratischen Archiven der am Euphrat gelegenen mesopotamischen Stadt Ur wurde eine Vielzahl von textuellen Dokumentationen archäologisch gesichert, so u. a. auch mehrere Tafeln zu Mengen von Nutztieren. Es wird vermutet, dass diese der zentralen Verwaltung des damaligen Herrschaftsgebietes entstammen. Während die früheren, auf das späte 4000 v. Chr. datierten Tafeln ausschließlich kontinuierlich beschrieben sind (vergleichbar der linke und mittleren Skizze in Abbildung 38), finden sich ab ca. 2030 v. Chr. erste vereinzelte Belege auch in einer tabellarischen Notation (rechte Skizze in Abbildung 38). Die nicht-tabellarischen Fundstücke sehen keine visuell gestützte Trennung zwischen der Anzahl (quantitativ) nach Nutzviehgattungen (qualitativ) vor, was sich mittels eines Übersetzungsbeispiel der mit Keilschrift unter- und nebeneinander festgehaltenen Daten verdeutlichen lässt (Robson 2003, 21):

7 nanny goats 3 billy goats day 10

5 sheep, 5 kids, 5 billy goats

7 nanny goats: day 13

4 kids, 1 nanny goat 1 billy goat: day 14

2 sheep, 3 kids 2 nanny goats

4 billy goats: day 15

3 sheep, 15 kids 3 billy goats, 9 nanny goats: day 16

3 sheep, 5 kids, 1 billy goat

6 nanny goats: day 18

12 sheep, 11 billy goats, 7 nanny goats:

day 20

18 Anhand z. B. des etwa 350-zeiligen Katalog zu den eingesetzten 4.186 Schiffen bei der Invasion Trojas, sortiert nach regionalen Clans, ihren eingebrachten Schiffen, ihren Anführern sowie den Heimatorten der Krieger im 2. Gesang der Ilias von Homer markiert Eco die Differenzierung von ,finite‘ und ,indefinite' ,lists': Während mit der Aufzählung von Anführern oder Schiffen versucht wird, jene Objekte der Umwelt ,finite‘ festzuhalten, bleibt die Anzahl von Kriegern je Anführer oder Schiff, indefinite. 

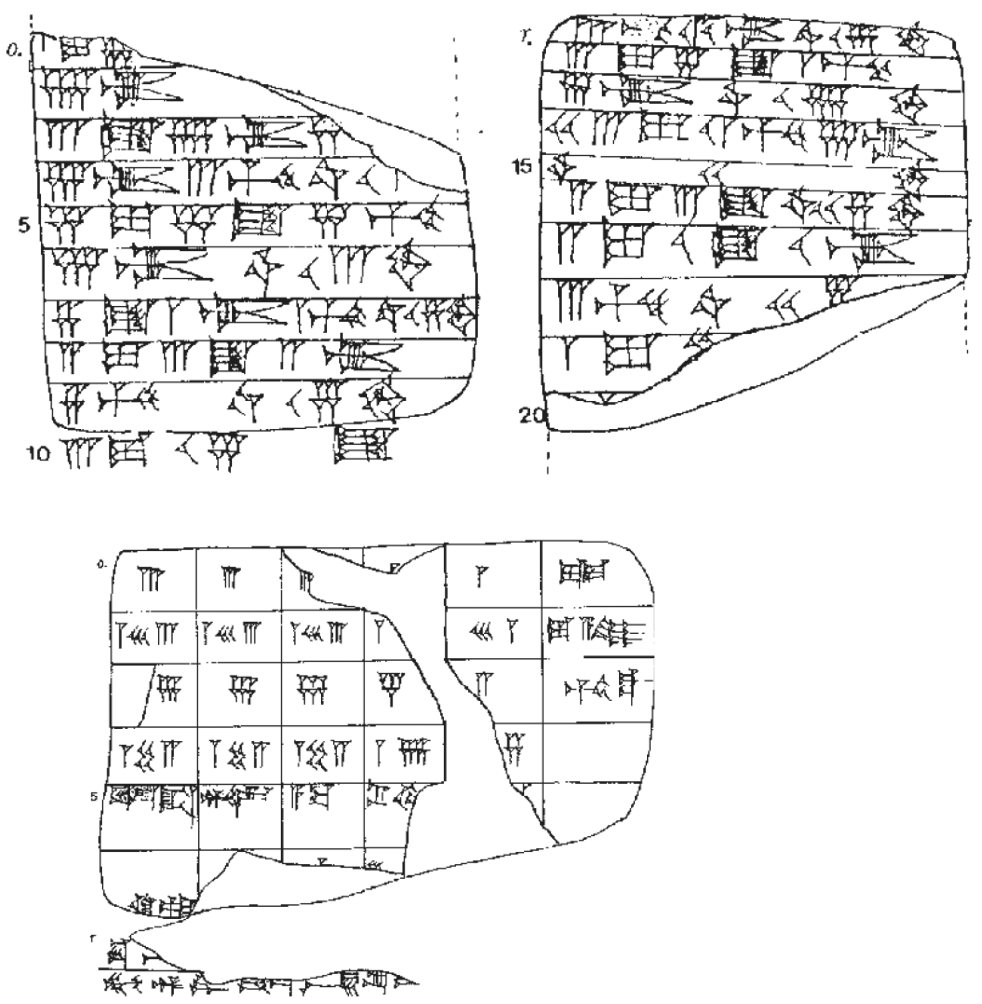

Abb. 38: Bürokratische Archivierung einer Anzahl von Schafen und Ziegen (Quelle: Robson 2003, 24)

Die aufgefunde kategorial-tabellarische Variante gliedert sich dagegen entlang der in der rechten Spalte angeordneten Bezeichnungsfelder der Nutzviehgattungen und gibt in den jeweiligen Zeilen die zugehörige Anzahlen von „lambs“, „first-rate sheeps“ und „billy goats“ an. In der vierten Zeile wurden die Gesamtzahlen je Spalte dokumentiert und in der fünften Zeile durch ein Namenkürzel jeweils offiziell bestätigt; unterhalb der Tabelle finden sich zudem Datumsangaben. Die Spalten selbst sehen keine Bezeichnung vor, weshalb die Intention der Gliederung unklar bleibt. Nichtsdestotrotz kann dieses historische Fundstück angesichts der parallel ausgegrabenen, hunderten von kontinuierlichen Dokumentationen rund um Sachverhalte mit Anzahlen von Nutztieren als ein erster Versuch interpretiert werden, Verwaltungsprozesse zu optimieren. Eine tatsächliche Standardisierung weisen die ersten tabellarischen Fundstücke jedoch noch nicht auf. Erst ab Mitte des 1900 Jh. v. Chr., so Robson (ebd.), kann ein konsistenter, eben routinalisierter Gebrauch von Tabellen aus den Sammlungen festgemacht werden, die Einblicke in immer nur kurze zeitliche Abschnitte von 10 bis 80 Jahren erlauben. Ob der Dokumentation der Lebensmittel, die an Tempelmitarbeiter ausgegeben wurden, der Einkünfte aus Steuern und/oder Mieten eines Stadtteils, der Handelstransaktionen eines Lagers oder der Löhne, die an Beschäftigten eines Lagers oder eines Tempels ausbezahlt wurden, zeigen die über mehrere Jahrhunderte verteilten, stets administrativen Zwecken dienenden Tabellen einige identische, wiederkehrende kategoriale Anordnungen ihrer Elemente: 
- Bezeichnungsfelder,

- Zwischensummen und/oder Endsummen,

- Nutzung von Spalten quer zu Zeilen bei Berechnungen,

- eine übergeordnete Überschrift oder Einleitung,

- erklärende Einfügungen,

- ein den Bezeichnungsfeldern der Reihen oder Spalten übergeordnetes Bezeichnungsfeld „MU. BI.IM“, welches den Feldeintrag der Person vorsah, die die Tabelle ausfüllte (Robson 2003).

Diese identischen, wiederkehrenden Anordnungen seiner Entitäten verhärten nicht zuletzt die Repräsentationsanalogien hinter primär kategorialen Tabellen, welche bereits zuvor als starke Darstellungs-Kodes (Kapitel 3.2.3 \& Kapitel 3.3.3.3) bezeichnet wurden. Diese Konventionen zwischen Zeichen und Bezeichnetem mittels bestimmter räumlicher Anordnungen graphischer Entitäten wie Punkte/Knoten, Linien/Kanten, Formen usw. markieren die evolutionär dargelegte Faktizität dieses speziellen, eben textuellen Artefakts, welches Rechwitz (2003) in seiner Definition hervorhebt:

Artefakte erscheinen weder ausschließlich als Objekte der Betrachtung noch als Kräfte eines physischen Zwangs, sondern als Gegenstände, deren sinnhafter Gebrauch, deren praktische Verwendung Bestandteil einer sozialen Praktik oder die soziale Praktik selbst darstellt. In diesem sinnhaften Gebrauch behandeln die Akteure die Gegenstände mit einem entsprechenden Verstehen und einem know how, das nicht selbst durch die Artefakte determiniert ist. Andererseits und gleichzeitig erlaubt die Faktizität eines Artefakts nicht beliebigen Gebrauch und beliebiges Verstehen (Reckwitz 2003, 291).

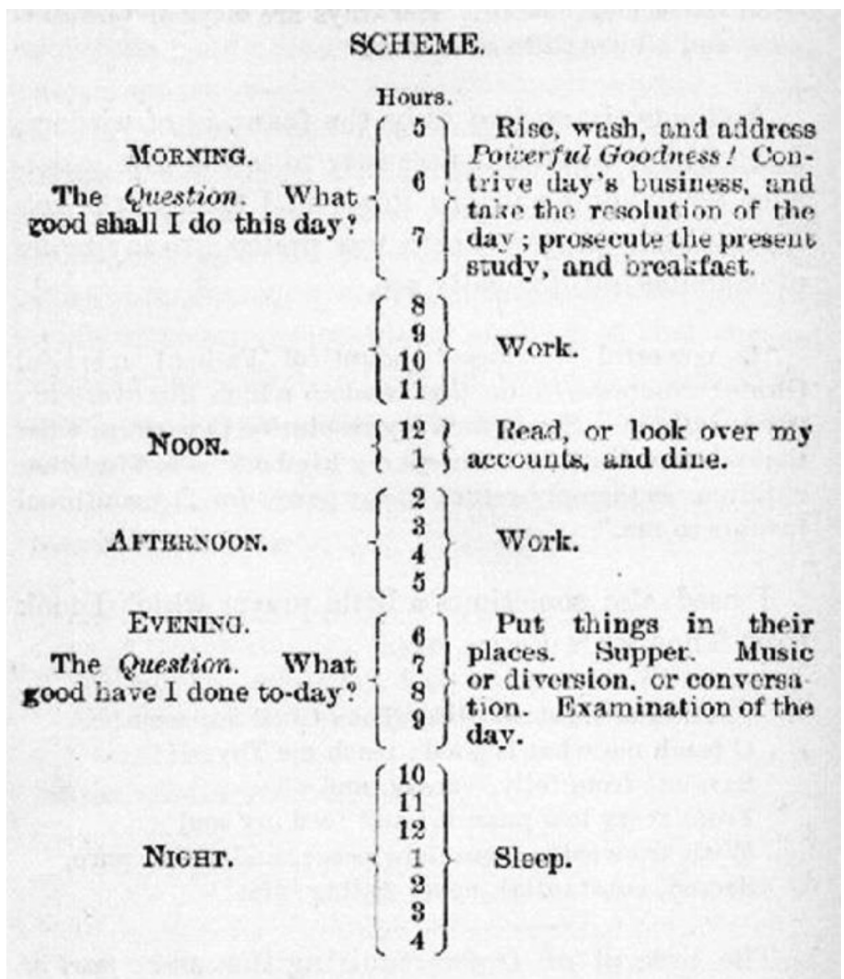

Abb. 39: To-do-Liste von Benjamin Franklin (Quelle: Franklin 1916) 
In der weiteren nur bruchstückartig bekannten Herkunft der kategorialen Tabelle lässt sich entlehnen, dass es zu einer Ausweitung dieses textuellen Artefakts in weiteren Verwendungsbezügen in unterschiedlichen Herrschaftsgebieten kam und dies mit routinierter Einbindung der dargelegten starken Darstellungs-Kodes. Zusammenfassend auf jene Ausweitung sozialer Praxen mit kategorialen Tabellen entlang alt-ägyptischer ${ }^{19}$, alt-indischer (Sankrit) oder babylonischer astronomischer Texte bis hin zu ersten Navigationstabellen der Schifffahrt oder der Venezianischen Buchhaltung (Tobies/Tournès 2011; Robson 2003) hingewiesen werden kann. Exemplarisch verhärtet sich die Ausweitung dieses textuellen Artefakts in weiteren Verwendungsbezügen in der Erfindung der To-do-Liste: Benjamin Franklin zugeordnet und in seiner Autobiographie im Zusammenhang mit seinem „Plan for attaining moral perfection“ gemäß dem Motto „Let all your things have their places; let each part of your business have its time“ (Franklin 1916, 146ff.) erstmalig textuell festgehalten, lässt sich die Erkenntnis spätestens an dieser Stelle begründen, dass unterschiedliche Verwendungsbezüge zweckmäßig angeordneter, vielfach verflochtener Merkmale zu Tabellen auch immer eng mit kulturellen Umbrüchen in bestimmten sozialen Kontexten zu interpretieren sind (vgl. Abbildung 39).

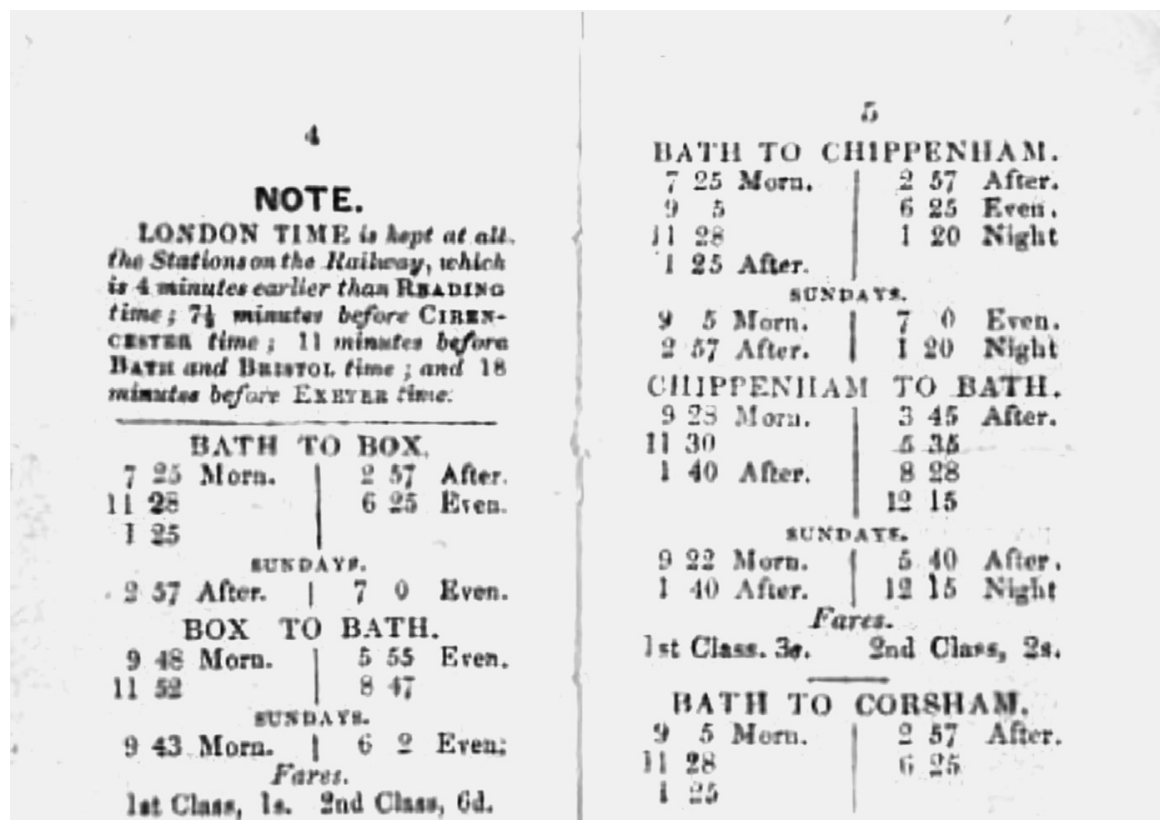

Abb. 40: Historische Fahrpläne im Eisenbahnpersonenverkehr (1839) (Quelle: 1839, http://www.scienceandsociety. co.uk/results.asp?image $=10562991$ )

19 „The graphical tables used in Egyptian star clocks present an interesting middle ground between the two approaches. First, the measurement of the stellar position with reference to bodily measurements challenges a strict defintion of numeric tables, but even overlooking this difficulty, the tables challenge classification. Because the horizontal axis represents successive portions of the ecliptic and the vertical axis represents time, the table lacks a true index and forms a (possibly compressed) list. As a further complication, some scribes working in this genre have indicated vertical displacement from some line by vertical displacement on the temporal axis" (Tobies/Tournès 2011, 663). 


\section{LONDON AND BIRMINGHAM RAILWAY.}

From London to Birmingham.

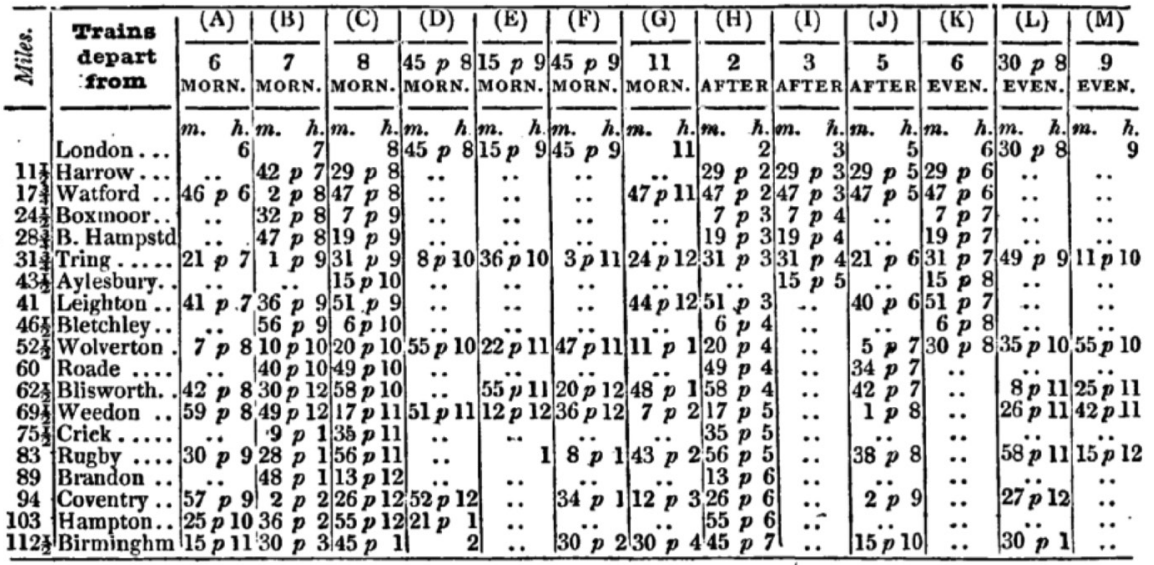

(A) is a mlxed train, in connexion with the Grand Junction | tion, \&c. lines, and the North Union and Lancaster and Presand the Liverpool and Manchester lines; also with the Bir- ton Jnnction.

mingham and Derby Junction; with the North Midland, and $(G)$ is a mixed train, calling at frst class stations, and is in with the Midland Counties, Leicester, Nottingham and Derby. connexion with the Grand Junction, with the North Midland, (B) is - 1 third class train, by which horses and carriages can be oon reyed.

(C) is a mixed train, in connexion with the Grapd Junction and the Manchester and Liverpool lines.

(D) is a first class train, in connexion with the Grand Junetion, \&c. lines; also with the North Union and the I.ancaster and Preston Junction, \& the Birmingham \& Derby Junction.

(E) is a mixed train, calling at first class stations, and is in conjunction with the North Midland and the Midland Counties failways.

and the Midland Connties' Railways.
(H) is a inixed train, in connexion with the Birmingham and Derby Junction.

I) is a mixed train, unconnected with any other line.

$(J)$ is a mixed train, calling at first class stations, and is in conjunction with the Midland Counties' Railway.

$(\mathbf{K})$ is a mixed train, unconnected with any other line.

(L) is a mixed train, with mail, and is in connexion with the Graud Junction and Liverpool and Manchester lines.

F) is a mail train, and in connexion with the Grand Jane- (M) is a mail train, in conjunction with the North Midland
aidland Counties' Railways.

Sunday Trains.

Mixed train, morning at 8 and $\ddagger$ before 10 (mail), and mixed trains, evening $\frac{t}{2}$ past 8 and at mine; also a mixed train to Wolverton, at 6 in the evening.

The mail train at $\frac{1}{4}$ before 10 morning, and the mixed train at $\frac{5}{2}$ past 8 evening, are in connexion with the Grand Junction, and the North Union, and Lancaster and Preston Junction.

The mixed trains, at eight morning and nine at night, are in conjunction with the North Midland and the Midland Counties' Railways.

FARES.

\begin{tabular}{|c|c|c|c|c|c|c|c|c|c|c|}
\hline \multirow[b]{2}{*}{ 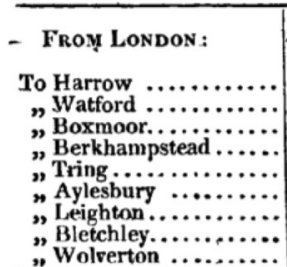 } & (A) & (B) & (C) & (D) & \multirow[b]{2}{*}{ 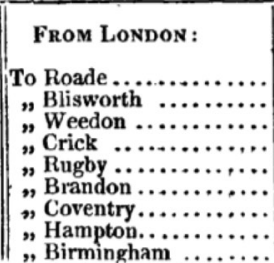 } & (A) & \multicolumn{2}{|c|}{ (B) } & (C) & (D) \\
\hline & $\begin{array}{cc}s & d \\
3 & 6 \\
5 & 0 \\
7 & 0 \\
8 & 0 \\
9 & 6 \\
12 & 0 \\
13 & 6 \\
15 & 6\end{array}$ & $\begin{array}{rr}8 . & d \\
3 & 0 \\
4 & 6 \\
6 & 6 \\
7 & 6 \\
8 & 6 \\
8 & 6 \\
11 & 0 \\
12 & 6 \\
14 & 0\end{array}$ & \begin{tabular}{cc}
8. & $d$ \\
$\mathbf{2}$ & 6 \\
$\mathbf{4}$ & $\mathbf{0}$ \\
$\mathbf{5}$ & $\mathbf{6}$ \\
$\mathbf{6}$ & $\mathbf{6}$ \\
$\mathbf{7}$ & $\mathbf{0}$ \\
\hdashline & 0 \\
9 & 0 \\
10 & $\mathbf{6}$ \\
11 & $\mathbf{6}$
\end{tabular} & $\begin{array}{ll}8 & d \\
2 & 0 \\
3 & 0 \\
4 & 6 \\
5 & 0 \\
5 & 6 \\
5 & 6 \\
7 & 6 \\
8 & 6 \\
9 & 6\end{array}$ & & $\begin{array}{ll}s . & d . \\
17 & 6 \\
18 & 6 \\
20 & 6 \\
22 & 0 \\
24 & 6 \\
26 & 0 \\
27 & 6 \\
30 & 0 \\
32 & 6\end{array}$ & $\begin{array}{l}8 . \\
16 \\
17 \\
18 \\
20 \\
22 \\
24 \\
25 \\
27 \\
30\end{array}$ & $\begin{array}{l}d . \\
0 \\
0 \\
6 \\
0 \\
0 \\
0 \\
0 \\
6 \\
0\end{array}$ & $\begin{array}{ll}8 . & d . \\
13 & 6 \\
14 & 0 \\
15 & 6 \\
17 & 0 \\
18 & 6 \\
20 & 0 \\
21 & 0 \\
23 & 0 \\
25 & 0\end{array}$ & $\begin{aligned} & 8 . \\
& 10 \\
& 11 \\
& 12 \\
& 13 \\
& 15 \\
& 16 \\
& 16 \\
& 18 \\
& 20\end{aligned}$ \\
\hline
\end{tabular}

Abb. 41: Historische Fahrpläne im Eisenbahnpersonenverkehr (1841) (Quelle: 1841, http://leicester.contentdm. oclc.org/cdm/ref/collection/p15407coll9/id/1264) 


\subsection{Ergebnis der Teilstudie}

(Theorie_I) Welche fundamentale Repräsentationsmächtigkeit lässt sich hinter ikonischen und hierunter insbesondere kategorial-tabellarischen ikonischen Zeichen historisch aufdecken?

Zusammenfassend erscheint die "Geschichte der Tabellen“ als ein Siegeszug, übertragbar auch auf Karten und graphischen Darstellungen - gemeinsame Entwicklungslinien lassen sich vermuten $^{20}$ - in zunehmend mehr öffentlich-institutionellen und letztlich auch privaten Verwendungsbezügen zu auch immer detailreicheren Sachverhalten zu immer kleineren Objekten der Welt, respektive, sensu Elias einer zunehmenden Berechenbarkeit dienlichen Explizierung der Welt. Diese umfasst u. a. Praxen zur Darlegung von Herrschaftsgebieten oder der Erschließung der Welt in der analogen Kartographie. Gleiches gilt für Diagramme, deren repräsentierte qualitativen/quantitativen bzw. quantitativen/quantitativen Sachverhalte gänzlich bzw. in Auszügen alternativ auch in Tabellen dargestellt werden können. An dieser Stelle kann empfohlen werden, sich der Beziehung des Normalen mit der Statistik in u. a. der Form der Normalverteiligungskurve in der Praxis der Konstruktion eines vermeintlich selbstverständlichen Orientierungsmaßstabes moderner Subjekte im Alltag „der westlichen Welt“ weiter auseinanderzusetzen (Link 1999). Jeder Sachverhalt, der mittels nichtkontinuierlicher Texte in unterschiedlichen Praxen beschrieben wird, unterliegt daher auch dem Verdacht, dass er über diese Repräsentation von seiner Unendlichkeit befreit werden könnte. Diese Beispiele verweisen überdies auf den Siegeszug der Konventionen zu zweckmäßigen Anordnungen zunehmend vielfach verflochtener Merkmale in einzelnen Praxen. Eindrücklich bspw. in den Regeln zu Tabellen oder Graphiken der Gesellschaft für Psychologie illustriert, handelt es sich hierbei um durchaus historisch verortbare, in einzelnen Praktiken sich routinalisierten Konventionen.

Paradoxerweise, kann hier Umberto Eco nur zugestimmt werden. Dennoch können jene nichtkontinuierlichen Texte lediglich als limitierter textualisierter Ausdruck von Sachverhalten, wenn nicht gar deren Lesen als eine primitive Kulturtechnik betrachtet werden. In diesem Sinne ist seine Gegenposition nur zu unterstützen:

The list doesn't destroy culture; it creates it.

(Umberto Eco)

20 Geographische Landkarten aus China in der zeitlichen Epoche des Mittelalters zeigen bereits die Prinzipien von Längen- und Breitengraden auf. Die beiden Achsen, mit denen man die Lage von Orten mittels Repräsentation inhärenten Relationen darlegen kann, werden als Vorläufer von Streudiagrammen gesehen werden. 



\section{Das situativ Soziale des Lesens multikodaler Textualität}

\subsection{Erhebung- und Auswertungsdesign}

\subsubsection{Methodologische Einordnung}

Zur Bearbeitung der Forschungsfrage nach Funktionen von Texten in sozialen Praxen an Einfach(st)arbeitsplätzen, anhand derer eine skillful performance routinalisiert situativ kontextualisiert wird, empfehlen sich grundsätzlich qualitative Verfahren. Diese Empfehlung rekurriert einerseits auf dem explorativen Charakter angesichts des dargelegten Desiderats innerhalb der Lesebegriffsdebatte (siehe Kapitel 3.1). Andererseits begründet sich diese methodologische Grundpositionierung aus dem favorisierten Lesekompetenzansatz: Es befragt gerade Lesekompetenzen als hypothetisches Konstrukt über die Handlungsfähigkeit einer Person, inwiefern hierbei Kognition als historisch- und situativ-sozial sowie embodied/verkörperlicht erfahren sich wechselseitig beeinflussend betrachtet werden kann. Wie und warum Texte innerhalb sozialer Praxen eingebunden sind, ist demgemäß weder als beliebig noch trotz aller Routinalisiertheit entlang von Zeit und Raum einem Wandel unterliegend. Sie sind als literacy practices somit als integraler Bestandteil der Interaktionen und interpretierenden Prozesse der teilnehmenden Personen zu verorten, wobei jene Personen eher implizit und nur bedingt explizit über deren routinalisierte lokalen kulturellen Codes in der einzelnen Lesesituation (=situated literacy event) Kenntnis haben. Die explikative Annäherung dieser überwiegend impliziten situativ aktualisierten Werte, Einstellungen und sozialen Beziehungen, die eine Lesesituation sozial im Hier und Jetzt markieren, können über die deduktiven Methoden und Techniken des quantitativen Paradigmas kaum methodologisch schlüssig operationalisiert werden. Diese Explikationen zielen dagegen auf Erklärungen begrenzter Reichweite, die jene raumzeitlichen Bedingungen jener zuvorderst in der sozialen Realität implizit aktualisierten Werte, Einstellungen und sozialen Beziehungen gegenstandsbezogen verankert und zu einem gewissen Grad verallgemeinert wissen (Lamnek 2005, 117ff.): Umfassender Art basieren diese auf dem knowing how der teilnehmenden Personen, deren Regel- und/oder Sinnhaftigkeit dem Forschenden eher verborgen als zugänglich ist, dies umso mehr ,je tiefer diese in ihrer habitualisierten, routinemäßigen Handlungspraxis verankert sind“ (Bohnsack 2001, 336f. zit. nach Lamnek 2005, 225).

Vor dem pragmatischen Hintergrund von Untersuchungsdesigns im Spiegel klassischer praxeologischer Betrachtungen des Lesens, war eine Fokussierung aufgrund eingeschränkter finanzieller Ressourcen auf den wesentlichen Kern des Erkenntnisinteresses zu legen. Anstelle der Explikation einer großen Bandbreite an Motiven, Einstellungen und sozialer Beziehungen wurde bereits mit der Akzentuierung auf routinalisierte lokale kulturelle Codes der Texte in sozialen Praxen an Einfach(st)arbeitsplätzen eine Vorauswahl getroffen, die eine weniger ,tiefe Verankerung' prägt. Hierdurch eröffnen sich einerseits methodologisch begründbar Erhebungund Auswertungsmethoden, die der theoretischen Perspektive erwachsen, die routinemäßige Handlungspraxis mit Texten im Sinne von praktischem Wissen zu verorten: Es rekurrieren Erhebungsmethoden innerhalb der Vielfalt qualitativer Interviews zu Situationen mit Texten an Einfach(st)arbeitsplätzen und Auswertungsmethoden der induktiven qualitativen Inhaltsanalyse von aktualisierten Regel- und Sinnhaftigkeiten einer skillful performance in den zugehörigen literacy events entlang von routinalisierten lokalen kulturellen Codes (ebd., 224, 478ff.). Andererseits gilt es mit dieser Reduktion hervorzuheben, dass keine umfassende Annäherung 
an die literacy practices an Einfach(st)arbeitsplätzen geleistet werden kann. Diese kann als Gegenstand für zukünftige Forschungsaktivitäten empfohlen werden.

\subsubsection{Erhebungsdesign von Situationen mit Texten an Einfach(st)erwerbstätigkeiten}

Es wird auf ein Datenmaterial zurückgegriffen, welches im Rahmen einer vorausgegangenen explorativen qualitativen Studie zu insbesondere herausfordernden beruflichen Situationen an Einfach(st)erwerbstätigkeiten gewonnen wurde (Basendowski 2013). Diese beziehen sich auf eine für die Region Mannheim-Heidelberg valide Auswahl von Arbeitsplätzen: Beikoch/ köchin, Fachlagerist/in, Metallfeinbearbeiter/in und Holzbearbeiter/in aus dem Berufsfeld des produzierenden Gewerbes; Gartenbauwerker/in und Baufachwerker/in aus dem Berufsfeld Bau/Landwirtschaft; Fachkraft im Gastgewerbe und Verkäufer/in aus dem Berufsfeld Dienstleistung (ebd., 169f.). Die skizzenhafte Darlegung hat daher zuvorderst zum Ziel, die Eignung dieses Datenmaterials für eine Sekundäranalyse im Rahmen der vorliegenden Studie zu begründen.

Mit der Ausrichtung an der Criticial Incident Technique orientierte sich das Erhebungsdesign an einem in der z. B. Organisationspsychologie bewährten Verfahren zur Exploration von Handlungssituationen, welche für die Performanz in beruflichen Kontexten von besonderer Bedeutung sind (Butterfield et al. 2005; Stitt-Gohdes et al. 2000): Kern der qualitativen Erhebung sind demgemäß Situationen am Arbeitsplatz, welche nicht unmittelbar sowie unter Zuhilfenahme von Unterstützung alsdann gelöst oder nicht gelöst werden können. Demgemäß basiert das Ausgangsmaterial der Primärstudie auf problemzentrierte Sammlungen und episodische Beschreibungen jener als besonders herausfordernd bezeichneten beruflichen Handlungssituationen. Diesen vorgeschaltet und abweichend zur Grundanlage gemäß der Critical Incident Technique wurde eine episodische Thematisierung von Handlungssituationen vorgenommen, welche aus einem routinemäßigen Arbeitsalltag erwachsen. Diese dienten insbesondere neben Begehungen des Arbeitsplatzes vor, während oder nach den Interviews der Annäherung. Der Vorteil dieses Vorgehens gegenüber z. B. teilnehmenden Beobachtungen an Einfach(st)arbeitsplätzen liegt insbesondere in der Kontrolle einer berücksichtigten Bandbreite an herausfordernden Handlungssituationen angesichts von anzunehmenden jahreszeitzyklischen bis einmaligen Besonderheiten (Basendowski 2013).

Die Relativierung, dass die situativen Schilderungen an die Perspektive der befragten, an diesen Handlungssituationen teilhabenden Personen gebunden ist (= Experten; vgl. Gläser/Laudel 2008, 12), ist hervorzuheben, wird im Vergleich jedoch als akzeptabel gedeutet, auch da in der Primärstudie hierzu einige, im Sinne eines theoretical sampling kontrollierende Maßnahmen (Lamnek 2005, 265f.; Kelle 2008, 87, 249) vorgenommen wurden. Es wurden jeweils (mit einer Ausnahme) nur Tandems aus Mitarbeiter/innen und unmittelbaren Vorgesetzten als Experten für die interessierenden Einfach(st)arbeitsplätze interviewt, deren Spezial-Wissen sowohl den Kriterien der typischen Betriebsgröße als auch der typischen Branche entsprachen. Als Experte auf der Ebene der Mitarbeiter/innen galt zudem nur, wer gemäß der Definition von Einfach(st)arbeit (vgl. Kapitel 4.1) über keinen anerkannten beruflichen Abschluss verfügt (Basendowski 2013, 189ff.).

Das Ausgangsmaterial umfasst folglich keine allumfassende, aber dennoch kontrollierte Auswahl an Schilderungen zu alltäglich-routinemäßigen und zu besonders herausfordernden beruflichen Handlungssituationen on-the-job aus der Perspektive beider Expertengruppen: Es setzt sich aus der Transkription (gemäß Gläser/Laudel 2008, 193ff.) der Interviews vor Ort, deren paraphrasierenden Aufbereitung nebst Einbettung von Kopien bzw. Fotographien von thema- 
tisierten Texten in Situationen mittels des Computerprogramms MaxQDa zusammen; Kopien und Fotographien nur insofern dies gestattet wurde. Obgleich in der Primärstudie insbesondere diejenigen Situationen mit mathematischen Problemkonstellationen - dem Forschungsinteresse der Primärstudie geschuldet - thematisch vertieft analysiert wurden, wird dieses Material angesichts der Anlage der Erhebung ausdrücklich auch für eine Sekundäranalyse derjenigen Situationen, in denen Texte eingebunden sind, für geeignet eingeschätzt. Es liefert dezidiert keine problemzentrierte Thematisierung auf ausschließlich das vergewisserte, explizite Wissen über die Regeln einer skillful performance an diesen Einfach(st)arbeitsplätzen, sondern der in Routinen mit Texten inhärenten impliziten und expliziten Wissensbeständen, welche auf aktualisierte Regel- und Sinnhaftigkeiten einer skillfulperformance in den zugehörigen literacy events entlang von routinalisierten lokalen kulturellen Codes schließen lassen.

\subsubsection{Auswertungsdesign entlang alltäglich-routinemäßiger und besonders herausfordernder Situationen mit Texten an Einfach(st)erwerbstätigkeiten}

Für die Analyse jener inhärenten, nicht als tiefgreifende Strukturen positionierten Regel- und Sinnhaftigkeiten einer skillful performance mit Texten in alltäglich-routinemäßigen und besonders herausfordernden Situationen an bestimmten Einfach(st)erwerbstätigkeiten wird im Weiteren aus der Auswahl an induktiven Verfahren der Inhaltsanalyse (Lamnek 2005, 513ff.) die der Qualitativen Inhaltsanalyse sensu Mayring favorisiert. Diese Präferierung begründet sich, erstens, aus ihrer Regelgeleitetheit in der Verdichtung von Datenmengen auf einen interessierenden Gegenstand, welche maßgeblich u. a. einer Transparenz dienlich ist (ebd., 146ff.). Zweitens, ist durch seine relative Theoriegeleitetheit nicht nur offen gegenüber sensibilisierenden Konzepten, sondern integriert diese innerhalb des Verfahrens im Sinne einer Positionierung der Erwartungen des Forschenden; und knüpft somit an der Kritik entgegen einer „naiv-empiristischen Tabula-rasa-Vorstellung [an], wonach zu Beginn einer Untersuchung begrifflich-theoretische Konzepte quasi aus dem Datenmaterial emergieren [...], [obgleich] es keine Wahrnehmung gibt, die nicht von Erwartungen durchsetzt ist“ (ebd., 115). Zweifelsohne ist dem Prinzip der Offenheit innerhalb der Analyse eines interessierenden Gegenstands besondere Aufmerksamkeit zu schenken, um letztlich auch nicht das Postulat einer Kontextungebundenheit zu strapazieren, welche mit dem Forschungsinteresse aus der begründeten Perspektive entlang des interpretativen Paradigmas heraus nicht vereinbar wäre (ebd., 21ff., 169). Drittens, begründet sich die Auswahl nicht zuletzt pragmatisch aus Bedingungen der Bearbeitungszeit und der Zusammensetzung der Forschergruppe, welche für weniger regel- und (relativ) theoriegeleitete Verfahren der Inhaltsanalyse kritisch angemerkt werden; exemplarisch für die Grounded Theory als Kodierverfahren von Mey/Mruck $(2011,34)$.

\subsection{Qualitative Inhaltsanalyse von routinalisierten lokalen kulturellen Code in und von Texten on-the-job}

\subsubsection{Regelgeleitetes Vorgehen: Strukturierende Qualitative Inhaltsanalyse}

Die Qualitative Inhaltsanalyse des Ausgangsmaterials nach lokalen kulturellen Codes in und von Texten in sozialen Praxen an Einfach(st)arbeitsplätzen, anhand derer eine skillfulperformance routinalisiert situativ kontextualisiert wird, zielt auf eine typisierende Strukturierung jener lokalen kulturellen Codes. Entgegen des klassischen Ablaufmodells der strukturierenden qualitativen Inhaltsanalyse (Lamnek 2005, 526; Mayring 2010, 75ff.) wird bewusst - auch mit Blick auf das Kriterium der Offenheit - von der einer späteren Revision unterliegenden Anwendung eines rein 
theoriegeleitet entwickelten Kategoriensystems zu routinalisierten lokalen kulturellen Codes Abstand genommen. An die Stelle jener deduktiven treten induktive Zwischenschritte, welche dem Verfahren der zusammenfassenden Qualitativen Inhaltsanalysen entlehnt sind (ebd., 74f.). Hierbei handelt es sich um die Paraphrasierung, Generalisierung und Reduktion mit dem Ziel der kategorialen Kennzeichnung und Beschreibung von routinalisierte lokalen kulturellen Codes im Sinne eines überschaubaren Corpus, „der immer noch Abbild des Grundmaterials ist“ (ebd., 58). Demgemäß wurde das Ausgangsmaterial, Empfehlungen von Mayring (ebd., 75) zur Aufteilung folgend, für die mittels der zusammenfassenden Qualitativen Inhaltsanalyse angestrebten Entwicklung eines Kategoriensystems (Zyklus 1) und anschließenden Prüfung und ggf. Revision mittels der strukturierenden Qualitativen Inhaltsanalyse (Zyklus 2) aufgeteilt: Für den Zyklus 1 wurden als Ausgangsmaterial insgesamt 16 Interviews ausgewählt, wobei auf die Berücksichtigung aller Wirtschaftssektoren im dem Kontext der untersuchten Einfach(st)erwerbstätigkeiten Wert gelegt wurde (vgl. Tabelle 19). Für den Zyklus 2 stehen folglich Quellen im Zuge weiterer Quellen von Arbeitsplätzen derselben Wirtschaftssektoren zur Verfügung. Diese dienen dezidiert der sättigenden Prüfung und ggf. Überarbeitung bis Revision der induktiv entwickelten Kategorien von routinalisierten lokalen kulturellen Codes, praktiziert durch einen zweiten Coder.

Tab. 19: Niedriglohnbeschäftigung nach Geschlecht und höchstem Ausbildungsabschluss

\begin{tabular}{|c|c|c|c|}
\hline \multirow{2}{*}{$\begin{array}{l}\text { Einfach(st)arbeitsplatz eines/ } \\
\text { einer (deckungsgleich mit dem } \\
\text { beruflichen Aufgabenspektrum } \\
\text { der Mitarbeiter/innen mit Tätig- } \\
\text { keitsbezogenen Qualifizierungen) }\end{array}$} & \multirow[t]{2}{*}{ Wirtschaftssektor/Branche } & Zyklus 1 & Zyklus 2 \\
\hline & & $\begin{array}{r}\text { Anzal } \\
\text { Mitarb } \\
\text { Vo }\end{array}$ & $\begin{array}{l}\text { nterviews } \\
\text { er/innen - } \\
\text { setzte }\end{array}$ \\
\hline Beikoch/köchin & Produktion, Logistik/Mensen, Kantinen & $1-$ & \\
\hline Fachlagerist/in & $\begin{array}{l}\text { Produktion und Logistik } \\
\text { Großhandel mit Nahrungsmitteln, } \\
\text { Getränken und Tabakwaren } \\
\text { Großhandel mit Holz, Baustoffen, } \\
\text { Anstrichmitteln und Sanitärkeramik } \\
\text { Großhandel mit elektrischen Geräten }\end{array}$ & & $\begin{array}{l}1-0 \\
1-2 \\
0-1\end{array}$ \\
\hline Metallfeinbearbeiter/in & $\begin{array}{l}\text { Produktion und Logistik } \\
\text { Montage (Metall, Anlagenbau) } \\
\text { Maschinenbau } \\
\end{array}$ & $\begin{array}{l}2- \\
2- \\
\end{array}$ & \\
\hline Holzbearbeiter/in & $\begin{array}{l}\text { Produktion und Logistik } \\
\text { Holzwirtschaft }\end{array}$ & $1-($ & \\
\hline Gartenbauwerker/in & $\begin{array}{l}\text { Bau und Landwirtschaft } \\
\text { Allgemeiner Gartenbau }\end{array}$ & $2-$ & \\
\hline Baufachwerker/in & $\begin{array}{l}\text { Bau und Landwirtschaft } \\
\text { Trockenbau }\end{array}$ & & $1-2$ \\
\hline Fachkraft im Gastgewerbe & $\begin{array}{l}\text { Dienstleistung } \\
\text { Pensionen und Gasthäuser } \\
\text { Restaurants mit herkömml. Service }\end{array}$ & $\begin{array}{l}1- \\
0-\end{array}$ & \\
\hline Verkäufer/in & $\begin{array}{l}\text { Dienstleitung } \\
\text { Discounter } \\
\text { kleiner Verbrauchermarkt } \\
\text { Kaufhaus (Non-Food) } \\
\text { großer Verbrauchermarkt }\end{array}$ & & $\begin{array}{l}1-1 \\
1-2 \\
1-1 \\
1-1\end{array}$ \\
\hline
\end{tabular}

(Quelle: Basendowski 2013, 189ff.; modifizierte Aufbereitung) 
Für die Darlegung der regelgeleiteten Entwicklung und Prüfung des Kategoriensystems wird die von Gläser/Laudel (2008, 270ff.) befürwortete Strategie übernommen. Als Kompromiss zwischen lückenloser schrittweiser Darstellung und Präsentation der Kategorien werden exemplarisch Auszüge eingebunden, anhand derer gewonnene Schlussfolgerungen veranschaulicht werden.

\subsubsection{Analyseeinheiten: Das sensibilisierende Konzept}

Tab. 20: Sensibilisierendes Konzept

\begin{tabular}{|c|c|c|c|}
\hline Gegenstand des Lesens & ... mit dem Ziel ... & & \\
\hline $\begin{array}{l}\text { Informationen eines Sachverhalts, die } \\
\text { mehr oder weniger tief in den Text oder } \\
\text { Textkomplex eingebettet sind und mehr } \\
\text { oder weniger klar differenzierbar inner- } \\
\text { halb des Textes oder Textkomplexes } \\
\text { lokalisierbar sind, sind zu identifizieren } \\
\text { und zu dekodieren. Diese Informatio- } \\
\text { nen sind folglich dem Text oder Text- } \\
\text { komplex inhärent und bedürfen nicht } \\
\text { zwingend weder externen Faktenwissens } \\
\text { noch einer tiefen Auseinandersetzung } \\
\text { mit dem Text („lokale Kohärenz“ im } \\
\text { Sinne von „Informationen ermitteln“). } \\
\text { Zusammenhänge zwischen Sachver- } \\
\text { halten (Begriffe, Konzepte usw.), die } \\
\text { mehr oder weniger mehrdeutig im } \\
\text { Textkomplex inhärent und bedürfen } \\
\text { nicht zwingend externen Faktenwissens, } \\
\text { jedoch einer mehr oder weniger tiefen } \\
\text { Auseinandersetzung mit dem Text } \\
\text { oder Textkomplex als Ganzes (globale } \\
\text { Kohärenz im Sinne „textbezogenes } \\
\text { Interpretieren“) } \\
\text { Reflexion und Bewertung von Infor- } \\
\text { mationen eines mehr oder weniger } \\
\text { von Zusammenhängen zwischen mehr } \\
\text { oder weniger umfangreich textuell } \\
\text { repräsentierten Sachverhalten unter } \\
\text { Rückgriff auf mehr oder weniger spezifi- } \\
\text { sches externes Faktenwissen (lokale } \\
\text { Kohärenz im Sinne von „Reflektieren } \\
\text { und Bewerten“). }\end{array}$ & $\begin{array}{l}\text {...des Lernens explizit-konzeptu- } \\
\text { ellen Wissens über strukturelle } \\
\text { Merkmale des Zeichensystems } \\
\text { (Print; visuell). Hierunter fallen } \\
\text { z.B.: } \\
\text { - durch starke arbiträre Codes } \\
\text { gekennzeichnete Beziehungen } \\
\text { zwischen Elementarzeichen } \\
\text { (kontinuierlich z.B. G-P oder } \\
\text { G-Visem-Korrespondenzen; } \\
\text { nicht kontinuierlich z. B. Fel- } \\
\text { der einer kategorialen Tabelle) } \\
\text { - durch starke arbiträre Codes } \\
\text { gekennzeichnete Beziehungen } \\
\text { zwischen Sets aus Elemen- } \\
\text { tarzeichen - Wörtern hin } \\
\text { zum Sätzen, Satzphrasen bzw. } \\
\text { Lokalzeichen (syntagmatische } \\
\text { Beziehungen in einem Satz; } \\
\text { Attribute und Merkmale einer } \\
\text { kategorialen Tabelle) } \\
\text { - durch starke arbiträre } \\
\text { Codes gekennzeichnete } \\
\text { Beziehungen zwischen } \\
\text { Sätzen/Absätzen/Texten } \\
\text { bzw. von Lokalzeichen eines } \\
\text { Globalzeichens (Parser, keine } \\
\text { Entsprechung) } \\
\text { - Superstrukturen (Absatzüber- } \\
\text { schriften, textartentypische } \\
\text { Gliederungen; Graphische } \\
\text { Schemata von Rechnungen) }\end{array}$ & $\begin{array}{l}\text {... des Lernens } \\
\text { explizit- } \\
\text { konzeptuellen } \\
\text { Wissens des } \\
\text { repräsentierten } \\
\text { Gegenstands } \\
\text { (Stichwort: } \\
\text { Behaltens- } \\
\text { leistung). } \\
\text { Hierunter auch } \\
\text { die Identifi- } \\
\text { kation von } \\
\text { Darstellungs- } \\
\text { strukturen ins- } \\
\text { besondere bei } \\
\text { Informations- } \\
\text { und Sachtexten } \\
\text { primär kon- } \\
\text { tinuierlicher } \\
\text { Struktur. }\end{array}$ & $\begin{array}{l}\text {... des Lernens } \\
\text { entlang der } \\
\text { Reflexion } \\
\text { bedeutungs- } \\
\text { offener und } \\
\text { mehrdeutiger } \\
\text { Auslegungen } \\
\text { von Texten und } \\
\text { Textkomplexen } \\
\text { (Stichwort: } \\
\text { Einstellungs- } \\
\text { veränderung). } \\
\text { Hierunter auch } \\
\text { die Identifi- } \\
\text { kation von } \\
\text { Darstellungs- } \\
\text { strukturen } \\
\text { insbesondere } \\
\text { bei literarischen } \\
\text { kontinuierli- } \\
\text { chen Texten } \\
\text { und Textkom- } \\
\text { plexen. }\end{array}$ \\
\hline
\end{tabular}

(Quelle: Kapitel 2.2.2; 2.3.3; 3.4.2 OECD 2002, 38ff.)

Aus dem Ausgangsmaterial wurden entlang der Analyseeinheit (Mayring 2010, 46f.) die interessierenden Bestandteile selegiert: Es handelt sich hierbei um Situationsschilderungen zu einer im Weiteren über routinalisierte lokale kulturelle Codes zu spezifizierenden Einbindung eines Zeichensystems, welches technisch auf das Medium Papier und sensorisch auf die visuelle Modalität begrenzt wird (vgl. Kapitel 2.2.4). 
Hinsichtlich des hierbei angestrebten Abstraktionsniveaus der Analyseeinheiten im Zuge der zusammenfassenden Qualitativen Inhaltsanalyse ist das folgende sensbilisierende Konzept leitend, welches aus der theoretischen Analysen rekurriert und eine Gegenüberstellung mit routinalisierten lokalen kulturellen Codes von primär kontinuierlichen Texten in den lokalen kulturellen Codes von sozialen Praxen der allgemeinbildenden Schulbildung mit Referenz der funktionalen Grundbildungscompetencies ermöglichen soll (siehe Tabelle 20): In ihm ist auf der einen Seite die Unterscheidung in die drei herausgearbeiteten dominanten Codes aufgenommen, die ein soziales Identifikationskriterium inhärent in sich tragen (vgl. Kapitel 3.3.3.2). Auf der anderen Seite wurde entlang der Debatte um Makromodelle des Lesens von sowohl primär kontinuierlichen als auch nichtkontinuierlichen Texten und Textkomplexen die mögliche Differenzierung in Informationen innerhalb eines (= lokale Kohärenz) oder zwischen Sachverhalten (=globale Kohärenz) als textseitige Struktur unterstrichen - und dies mittels in der Faktizität des Zeichensystems evolutionär verborgenen Repräsentationsanalogien (vgl. Kapitel 3.3.3.3 \& Kapitel 4). Diese Differenzierung wird mit dem Ziel der Markierung der Relevanz von explizierbarem Faktenwissen in den lokalen kulturellen Codes der obligatorischen Schule mit Referenz der funktionalen Grundbildungscompetencies weiter unterteilt; und zwar insofern die Interaktion mit dem Text oder Textkomplex explizit-konzeptionelles Wissen verlangt oder nicht verlangt, welches nicht durch den Text oder Textkomplex repräsentiert wird (vgl. Kapitel 3.2.2; 3.3.3.2; 3.3.3.3).

Tab. 21: 4. Materialdurchlauf: Generalisierung

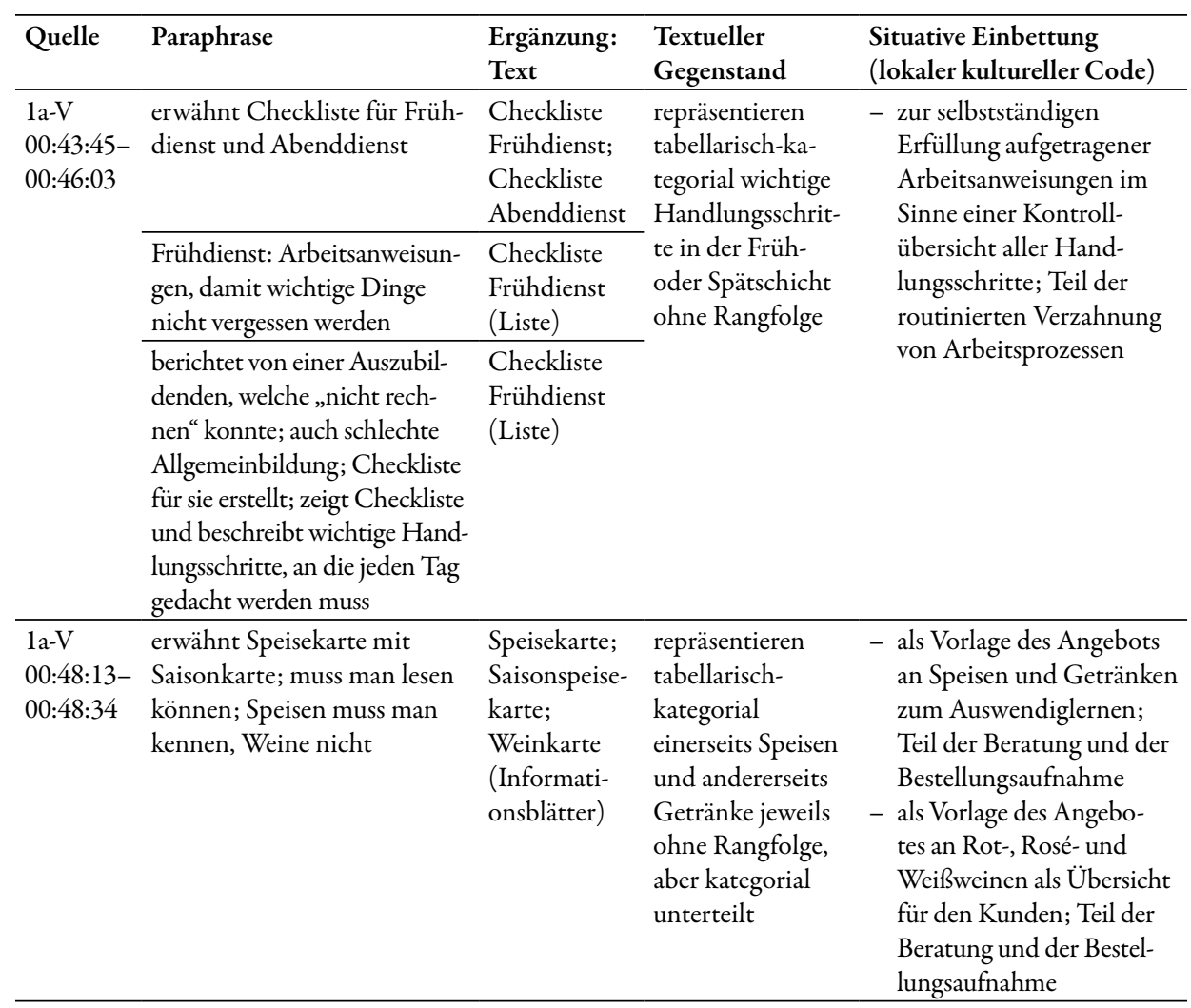




\begin{tabular}{|c|c|c|c|c|}
\hline Quelle & Paraphrase & $\begin{array}{l}\text { Ergänzung: } \\
\text { Text }\end{array}$ & $\begin{array}{l}\text { Textueller } \\
\text { Gegenstand }\end{array}$ & $\begin{array}{l}\text { Situative Einbettung } \\
\text { (lokaler kultureller Code) }\end{array}$ \\
\hline $\begin{array}{l}1 \mathrm{a}-\mathrm{V} \\
00: 51: 06- \\
00: 52: 30\end{array}$ & $\begin{array}{l}\text { haben einen Arbeitsplan; } \\
\text { dieser hängt unten [Mitarbei- } \\
\text { tereingang]; haben sie wegen } \\
\text { Auszubildenden gemacht; } \\
\text { Auszubildende wurden in } \\
\text { Plan eingeteilt; Stammmit- } \\
\text { arbeiter haben ihre festen } \\
\text { Arbeitszeiten }\end{array}$ & $\begin{array}{l}\text { Arbeitsplan } \\
\text { (Tabelle) }\end{array}$ & $\begin{array}{l}\text { repräsentieren } \\
\text { tabellarisch- } \\
\text { kategorial die } \\
\text { Arbeitstage und } \\
\text {-zeiten je Mitar- } \\
\text { beiter/in und ihre } \\
\text { Einsatzbereiche }\end{array}$ & $\begin{array}{l}\text { - zur selbstständigen } \\
\text { Orientierung zu Team- } \\
\text { zusammensetzungen und } \\
\text { von Aufgabenbereichen; } \\
\text { Teil der routinierten Ver- } \\
\text { zahnung unterschiedlicher } \\
\text { Aufgabenbereiche } \\
\text { - zur selbstständigen Orien- } \\
\text { tierung über die zu leisten- } \\
\text { den Arbeitszeiten; Teil des } \\
\text { privaten Zeitmanagements }\end{array}$ \\
\hline
\end{tabular}

\subsubsection{Induktive Entwicklung eines Kategoriensystems}

Neben jenem sensibilisierenden Konzept zum angestrebten Abstraktionsgrad der zu bildenden Kategorien von routinalisierten lokalen kulturellen Codes ist zuvorderst die Regel deren induktiven Entwicklung aus der zusammenfassenden Qualitativen Inhaltsanalyse leitend. In einem ersten Durchlauf ist jedes zur Sprache gekommene social literacy event zu erfassen und möglichst dicht am Ausgangsmaterial zu generalisieren; wie bereits erwähnt kann auf eine Paraphrasierung der situativen Schilderungen samt frei codierter Zuordnung zu einem Text aus der Primärstudie zurückgegriffen werden - diese Auflistung wurde in Kapitel 2.4.2 thematisiert. Aus dem sensibilisierenden Konzept wurde daher lediglich a priori übernommen, dass die Generalisierung jeweils einen textuellen Gegenstand mit seinen situativ-kontextuellen Anforderungen einer skillfulperformance kennzeichnet, basierend auf eher explizite Bezeichnungen in den einzelnen diesbezüglichen Aussagen oder auf eher Ableitungen inhärenter Bedin-

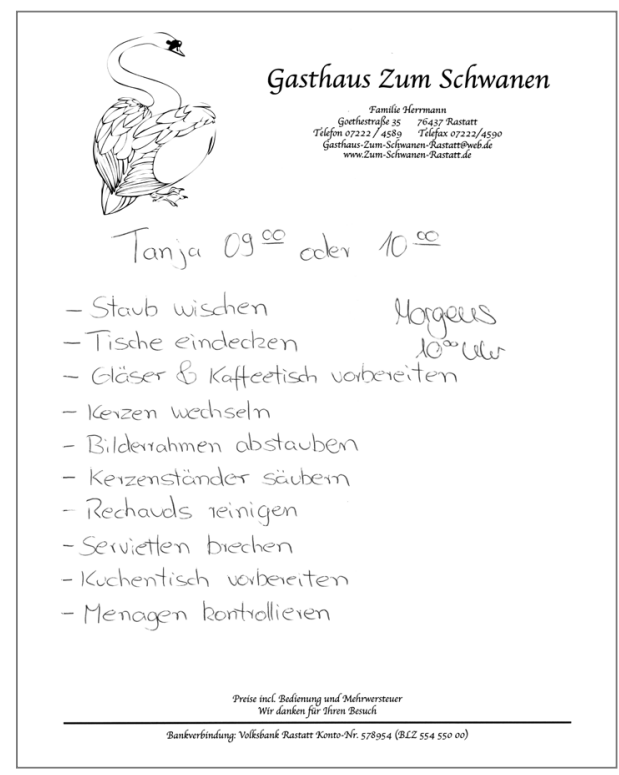

Abb. 42: Checkliste Frühstücksdienst (Quelle: Imitation) gungen in den Situationsschilderungen. Zur Illustrierung jenes ersten Durchgangs durch das in etwa hälftige Ausgangsmaterial werden in Tabelle 22 exemplarisch vorgenommene Generalisierungen im Kontext des Arbeitsplatzes einer Fachkraft im Gastgewerbe dargelegt, die noch möglichst konkret die situativen Anforderungen einer Text- oder Textkomplexanwendung, hier z.B. einer Checkliste umreißen (vgl. Abbildung 42).

Insgesamt konnten im ersten Durchlauf derart 121 textuelle Gegenstände samt situativkontextueller Anforderungen einer skillful performance exploriert werden, unter denen lediglich drei primär kontinuierlicher Sorte sind. Aufgefunden an den Einfach(st)arbeitsplätzen im Wirtschaftssektor Produktion/ Logistik entsprechen sie nach der Texttypenunterteilung gemäß PISA einem Textkomplex aus „Anweisungen“ und „Dokumenten“. Diese Arbeitsplätze sind durch Sicherheitsbestim- 
mungen beim Führen von Maschinen oder hinsichtlich der Einhaltung von spezifischen HPCD bei der Zubereitung von Speisen gekennzeichnet. Rechtliche Bedingungen werden über entsprechende Handbücher oder Merkblätter fixiert, die in der überwiegenden Mehrheit der untersuchten Arbeitsplätze jedoch über mündliche Einweisungen flankiert bis gar ersetzt werden. In der Produktion/Logistik müssen die Mitarbeiter/innen per Unterschrift oder Stempel auf einem standardisierten Formular (kontinuierlicher Text) die Kenntnis juristiziabel bestätigen.

Bei den drei primär kontinuierlichen Texten handelt es sich entsprechend um den Typ, „der sagt, was zu tun ist. Darunter fallen Verfahren, Regeln, Vorschriften und Gesetze, die Anforderungen für bestimmte Verhaltensweisen festlegen" (OECD 2002, 30). Im Zuge der geringen Häufigkeit, aber auch angesichts der Relativierungen eines tatsächlich notwendigen Studiums im Spannungsfeld der Kontextbedingungen on-the-job (vgl. Tabelle 19) wird der kontinuierliche Texttyp im Weiteren aus den Analysen ausgeklammert.

Tab. 22: Erster Materialdurchlauf mit Bezug auf kontinuierliche Texte

\begin{tabular}{|c|c|c|}
\hline Quelle & araphrase & Ergänzung: Text \\
\hline $\begin{array}{l}2 \mathrm{a}-\mathrm{V} \\
00: 15: 22- \\
00: 16: 04\end{array}$ & $\begin{array}{l}\text { ein Koch muss nicht alle Rezepte kennen, sondern schlägt auch nach; muss } \\
\text { aber Grundbegriffe kennen; Beispiel blanchieren; Umgang mit Rezept } \\
\text { bedeutet Rezept lesen, verinnerlichen und dann ohne Kochbuch kochen: } \\
\text { „Dann hast du } 5 \text { bis } 10 \text { Minuten Zeit und dann kannst du dir das einprägen } \\
\text { und dann kannst du das übernehmen“. }\end{array}$ & $\begin{array}{l}\text { Kochbuch/ } \\
\text { Rezepte } \\
\text { (Anweisungen) }\end{array}$ \\
\hline $\begin{array}{l}00: 17: 24- \\
00: 19: 49\end{array}$ & $\begin{array}{l}\text { Beispiel selbstständiger Aufgabenbereich Salattheke; Rezepte werden gelesen } \\
\text { und müssen mit der Zeit auswendig beherrscht werden; Änderungen möglich; } \\
\text { Bedingung ist der gute Geschmack aus der Sicht der Gäste: „Er kann letztlich } \\
\text { machen, was er will, es muss schmecken“; „,Also die Leute müssen es mögen!“; } \\
\text { Rezepte für Dressings sind grob und ohne Liter- und Grammangaben; Her- } \\
\text { stellung erfolgt in großen Mengen: „Da wird der Behälter gefüllt ... eine Hand } \\
\text { voll Salz, eine Hand voll Zucker ...”; „... wir reden von } 15 \text { Litern!“ }\end{array}$ & $\begin{array}{l}\text { Rezepte } \\
\text { (Anweisungen) } \\
\text { [teilweise auch } \\
\text { rein tabellarisch] }\end{array}$ \\
\hline $\begin{array}{l}3 \mathrm{c}-\mathrm{M} \\
00: 11: 15- \\
00: 12: 56\end{array}$ & $\begin{array}{l}\text { Handbuch gibt mögliche Fehlerfälle und das jeweils richtige Verhalten vor; } \\
\text { zu manchen Arbeitsschritten gibt es auch Zeichnungen, die vor Verletzun- } \\
\text { gen warnen sollen; kleinere Reparaturen dürfen von Mitarbeitern selbst } \\
\text { erledigt werden; diese kleineren Reparaturen werden lediglich in einem Text } \\
\text { ohne Bilder erklärt }\end{array}$ & ngen) \\
\hline $\begin{array}{l}\text { 4c-M2 } \\
00: 00: 10- \\
00: 01: 05\end{array}$ & $\begin{array}{l}\text { gibt wöchentlich Qualitätsgespräche; Fehler und Verantwortlichkeiten } \\
\text { werden besprochen; Gruppe als Kollektiv wird verantwortlich gemacht; } \\
\text { Besprechung in der Gruppe über Fehler }\end{array}$ & \\
\hline $\begin{array}{l}00: 16: 09- \\
00: 17: 58\end{array}$ & $\begin{array}{l}\text { neben Qualitätsgesprächen gibt es halbjährliche Sicherheitseinweisungen; } \\
\text { Themen z. B. Verhalten im Notfall oder Arbeitsschuhe; Anwesenheit an } \\
\text { Sicherheitseinweisung ist mit Unterschrift auf einem Formular einzeln zu } \\
\text { bestätigen, an Qualitätsgesprächen als Gruppe mit Stempel; Stempel zeigt } \\
\text { Abteilung und Personalnummer }\end{array}$ & $\begin{array}{l}\text { Formular } \\
\text { (Dokument) } \\
\text { Formular } \\
\text { (kategoriale } \\
\text { Tabelle) } \\
\end{array}$ \\
\hline $\begin{array}{l}6 \mathrm{a}-\mathrm{V} \\
00: 25: 05- \\
00: 26: 01\end{array}$ & $\begin{array}{l}\text { Einweisung in die Maschinen muss es immer wieder geben, schon aus versiche- } \\
\text { rungsrechtlichen Gründen; Einschätzung, dass die Mitarbeiter gut geschult } \\
\text { seien; bei eventuellen Fragen können sich Mitarbeiter aber an ihn wenden, da er } \\
\text { Sicherheitstechnik-Beauftragter ist; es gibt auch Merkblätter, die gelesen werden } \\
\text { können: „[...] zur Sicherheit für sich selbst und zur Sicherheit von den Gegen- } \\
\text { ständen und Personen darum herum“; auf den Merkblättern stehen Hinweise } \\
\text { bspw. zur Benutzung von Leitern; Beispiel: letzte Woche gab es eine Einweisung } \\
\text { zum Thema Radlader, vermutlich weil es neue Regelungen gibt; in die Merkblät- } \\
\text { ter kann man jederzeit hineinschauen: „[...] wenn sie interessiert sind.“ }\end{array}$ & $\begin{array}{l}\text { Merkblätter } \\
\text { (Anweisungen) }\end{array}$ \\
\hline
\end{tabular}


In einem anschließenden zweiten Materialdurchlauf wird die Erhöhung des Abstraktionsgrads der textuellen Gegenstände und situativen Einbettung einer skillful performance und die Entwicklung erster vorläufiger Kategorien im Sinne einer vierten Reduktion angestrebt, die parallel hinsichtlich Selektionen und Streichungen zu prüfen sind. Dabei gilt vorrangig, dass dieser Prozess unter Kontrolle von „so konkret wie möglich, so abstrakt wie nötig“ abermals wiederholt werden kann, um sich dem Abstraktionsniveau des sensibilisierenden Konzepts anzunähern. Wiederum über die exemplarische Darstellungsstrategie wird über die Tabelle 23 \& Tabelle 24 das regelgeleitete Vorgehen illustriert.

Tab. 23: Zweiter Materialdurchlauf: situative Einbettung

\begin{tabular}{|c|c|c|c|}
\hline \multirow[b]{2}{*}{ Quelle } & \multirow[b]{2}{*}{ Paraphrase } & \multicolumn{2}{|c|}{ situative Einbettung (lokaler kultureller Code) } \\
\hline & & Generalisierung & Reduktion \\
\hline $\begin{array}{l}1 \mathrm{a}-\mathrm{V} \\
00: 51: 06- \\
00: 52: 30\end{array}$ & $\begin{array}{l}\text { haben einen Arbeitsplan; dieser hängt } \\
\text { unten [Mitarbeitereingang]; haben sie } \\
\text { wegen Auszubildenden gemacht; Aus- } \\
\text { zubildende wurden in Plan eingeteilt; } \\
\text { Stammmitarbeiter haben ihre festen } \\
\text { Arbeitszeiten }\end{array}$ & $\begin{array}{l}\text { zur selbstständigen Orientie- } \\
\text { rung zu Teamzusammenset- } \\
\text { zungen und von Aufgabenbe- } \\
\text { reichen; Teil der routinierten } \\
\text { Verzahnung unterschiedlicher } \\
\text { Aufgabenbereiche }\end{array}$ & $\begin{array}{l}\text { selbstständige Orien- } \\
\text { tierung hinsichtlich } \\
\text { Zuständigkeiten und } \\
\text { Integration in routinier- } \\
\text { te Handlungsfelder }\end{array}$ \\
\hline \multirow[t]{2}{*}{$\begin{array}{l}2 \mathrm{a}-\mathrm{V} \\
00: 09: 22- \\
00: 11: 55\end{array}$} & \multirow{2}{*}{$\begin{array}{l}\text { Selbstständiges Arbeiten bedeutet auch, } \\
\text { dem Speiseplan zu entnehmen, welche Vor- } \\
\text { bereitungen noch vor Feierabend im zuge- } \\
\text { teilten Aufgabenbereich möglich sind; z. B. } \\
\text { Gefrierwaren herauslegen; sieht es ungern, } \\
\text { wenn vor Feierabend ständig auf die Uhr } \\
\text { geschaut wird; „Das ist gar nicht so einfach, } \\
\text { weil wenn man fast Feierabend hat, nicht } \\
\text { an den Feierabend zu denken, sondern an } \\
\text { den nächsten Tag vorzudenken“. }\end{array}$} & $\begin{array}{l}\text { zur selbstständigen Ori- } \\
\text { entierung zu erledigenden } \\
\text { Vorarbeiten im eigenen Ein- } \\
\text { satzbereich; Teil routinier- } \\
\text { ter Handlungsfelder }\end{array}$ & $\begin{array}{l}\text { selbstständige Orien- } \\
\text { tierung hinsichtlich } \\
\text { Zuständigkeiten und } \\
\text { Integration in routinier- } \\
\text { te Handlungsfelder } \\
\end{array}$ \\
\hline & & $\begin{array}{l}\text { zur selbstständigen Aus- } \\
\text { wahl von Zutaten; Teil rou- } \\
\text { tinierter Handlungsfelder }\end{array}$ & $\begin{array}{l}\text { selbstständige Orien- } \\
\text { tierung hinsichtlich zu } \\
\text { bearbeitender Produkte } \\
\text { und Integration in routi- } \\
\text { nierte Handlungsfelder }\end{array}$ \\
\hline $\begin{array}{l}\text { 4d-M } \\
00: 02: 32- \\
00: 05: 29\end{array}$ & $\begin{array}{l}\text { Tankdeckel und Stecköffnung sind bei } \\
\text { allen Modellen gleich; gibt aber verschie- } \\
\text { dene Seitenverkleidungen; kann man } \\
\text { von dem Zettel unter der Motorhaube } \\
\text { ablesen; beschreibt relevante und irrele- } \\
\text { vante Angaben auf dem Zettel: „Dann } \\
\text { verschiedene Nummern, wo ich nichts } \\
\text { damit anfangen kann“. }\end{array}$ & $\begin{array}{l}\text { zur selbstständigen } \\
\text { Auswahl von Teilen in der } \\
\text { Fertigung; Teil routinierter } \\
\text { Handlungsfelder }\end{array}$ & $\begin{array}{l}\text { selbstständige Orien- } \\
\text { tierung hinsichtlich zu } \\
\text { verarbeitender Teile und } \\
\text { Integration in routinier- } \\
\text { te Handlungsfelder }\end{array}$ \\
\hline
\end{tabular}

Tab. 24: Zweiter Materialdurchlauf: situative Einbettung und textueller Gegenstand

\begin{tabular}{|c|c|c|c|c|}
\hline \multirow[b]{2}{*}{ Quelle } & \multirow[b]{2}{*}{ Paraphrase } & \multirow{2}{*}{$\begin{array}{l}\text { situative Ein- } \\
\text { bettung (lokaler } \\
\text { kultureller Code) }\end{array}$} & \multicolumn{2}{|l|}{ textueller Gegenstand } \\
\hline & & & Generalisierung & Reduktion \\
\hline $\begin{array}{l}1 \mathrm{a}-\mathrm{V} \\
00: 51: 06- \\
00: 52: 30\end{array}$ & $\begin{array}{l}\text { haben einen Arbeitsplan; } \\
\text { dieser hängt unten [Mit- } \\
\text { arbeitereingang]; haben } \\
\text { sie wegen Auszubildenden } \\
\text { gemacht; Auszubildende } \\
\text { wurden in Plan eingeteilt; } \\
\text { Stammmitarbeiter haben } \\
\text { ihre festen Arbeitszeiten }\end{array}$ & $\begin{array}{l}\text { Selbstständige } \\
\text { Orientierung } \\
\text { hinsichtlich } \\
\text { Zuständigkeiten } \\
\text { und Integration in } \\
\text { routinierte Hand- } \\
\text { lungsfelder }\end{array}$ & $\begin{array}{l}\text { repräsentieren } \\
\text { tabellarisch-katego- } \\
\text { rial die Arbeitstage } \\
\text { und -zeiten je Mit- } \\
\text { arbeiter/in und ihre } \\
\text { Einsatzbereiche }\end{array}$ & $\begin{array}{l}\text { Interferenz zwischen } \\
\text { hinter Einzelinfor- } \\
\text { mationen stehenden } \\
\text { Sachverhalten aus } \\
\text { kategorialen Tabellen } \\
\text { und knowing how/ } \\
\text { that über routinierte } \\
\text { Handlungsfelder }\end{array}$ \\
\hline
\end{tabular}




\begin{tabular}{|c|c|c|c|c|}
\hline \multirow[b]{2}{*}{ Quelle } & \multirow[b]{2}{*}{ Paraphrase } & \multirow{2}{*}{$\begin{array}{l}\text { situative Ein- } \\
\text { bettung (lokaler } \\
\text { kultureller Code) } \\
\text { Reduktion }\end{array}$} & \multicolumn{2}{|c|}{ textueller Gegenstand } \\
\hline & & & Generalisierung & Reduktion \\
\hline \multirow[t]{2}{*}{$\begin{array}{l}2 \mathrm{a}-\mathrm{V} \\
00: 09: 22- \\
00: 11: 55\end{array}$} & \multirow{2}{*}{$\begin{array}{l}\text { Selbstständiges Arbeiten } \\
\text { bedeutet auch, dem Speise- } \\
\text { plan zu entnehmen, welche } \\
\text { Vorbereitungen noch vor } \\
\text { Feierabend im zugeteilten } \\
\text { Aufgabenbereich möglich } \\
\text { sind; z. B. Gefrierwaren he- } \\
\text { rauslegen; sieht es ungern, } \\
\text { wenn vor Feierabend stän- } \\
\text { dig auf die Uhr geschaut } \\
\text { wird; „Das ist gar nicht so } \\
\text { einfach, weil wenn man fast } \\
\text { Feierabend hat, nicht an } \\
\text { den Feierabend zu denken, } \\
\text { sondern an den nächsten } \\
\text { Tag vorzudenken“. }\end{array}$} & $\begin{array}{l}\text { selbstständige } \\
\text { Orientierung } \\
\text { hinsichtlich } \\
\text { Zuständigkeiten } \\
\text { und Integration in } \\
\text { routinierte Hand- } \\
\text { lungsfelder }\end{array}$ & $\begin{array}{l}\text { repräsentieren } \\
\text { tabellarisch-ka- } \\
\text { tegorial überge- } \\
\text { ordnet zukünftige } \\
\text { Arbeitsergebnisse } \\
\text { on-the-job }\end{array}$ & $\begin{array}{l}\text { Interferenz zwischen } \\
\text { hinter Einzelinfor- } \\
\text { mationen stehenden } \\
\text { Sachverhalten aus } \\
\text { kategorialen Tabellen } \\
\text { und knowing how/ } \\
\text { that über routinierte } \\
\text { Handlungsfelder }\end{array}$ \\
\hline & & $\begin{array}{l}\text { selbstständige } \\
\text { Orientierung } \\
\text { hinsichtlich zu } \\
\text { bearbeitender Pro- } \\
\text { dukte und Integra- } \\
\text { tion in routinierte } \\
\text { Handlungsfelder }\end{array}$ & $\begin{array}{l}\text { repräsentieren ta- } \\
\text { bellarisch-kategorial } \\
\text { bereitzustellende } \\
\text { Zutaten }\end{array}$ & $\begin{array}{l}\text { Relation zwischen } \\
\text { Einzelinformationen } \\
\text { eines Sachverhalts aus } \\
\text { kategorialen Tabellen } \\
\text { und knowing how/ } \\
\text { that über routinierte } \\
\text { Handlungsfelder }\end{array}$ \\
\hline $\begin{array}{l}4 d-M \\
00: 02: 32- \\
00: 05: 29\end{array}$ & $\begin{array}{l}\text { Tankdeckel und Steck- } \\
\text { öffnung sind bei allen } \\
\text { Modellen gleich; gibt aber } \\
\text { verschiedene Seitenverklei- } \\
\text { dungen; kann man von dem } \\
\text { Zettel unter der Motor- } \\
\text { haube ablesen; beschreibt } \\
\text { relevante und irrelevante } \\
\text { Angaben auf dem Zettel: } \\
\text { „Dann verschiedene Num- } \\
\text { mern, wo ich nichts damit } \\
\text { anfangen kann“. }\end{array}$ & $\begin{array}{l}\text { selbstständige } \\
\text { Orientierung } \\
\text { hinsichtlich zu ver- } \\
\text { arbeitender Teile } \\
\text { und Integration in } \\
\text { routinierte Hand- } \\
\text { lungsfelder }\end{array}$ & $\begin{array}{l}\text { repräsentieren } \\
\text { tabellarisch-kate- } \\
\text { gorial auszuwäh- } \\
\text { lende Teile für die } \\
\text { Fertigung }\end{array}$ & $\begin{array}{l}\text { Relation zwischen } \\
\text { Einzelinformationen } \\
\text { eines Sachverhalts aus } \\
\text { kategorialen Tabellen } \\
\text { und knowing how/ } \\
\text { that über routinierte } \\
\text { Handlungsfelder }\end{array}$ \\
\hline
\end{tabular}

Jener zweite Materialdurchlauf reduziert das bis dato erschlossene etwa hälftige Ausgangsmaterial, wobei, wie oben beschrieben, identische und bedeutungsgleiche, vorläufige Kategorien von sozio-kontextuellen Relevanz- und Sinnoptionen aus textuellem Gegenstand und situativer Einbettung subsumiert werden konnten. Diese induktiv entwickelten vorläufigen Kategorien distanzierensicheinerseitsvonihremursprünglichen KontextspezifischerEinfach(st)erwerbstätigkeiten, markieren andererseits jedoch soziale Praxen an diesen Arbeitsplätzen nach Berufsfeldern. Soziale Praxen unterliegen routinalisiert ähnlichen lokalen kulturellen Codes: Ein je textstrukturell ähnlicher Gegenstand ist hinsichtlich einer skillful performance unter je ähnlichen situativ-kontextuellen Anforderungen zu bearbeiten (vgl. Kapitel 3.3.3.1). Während für diese Teilkategorie der situativen Einbettung nach dem zweiten Materialdurchlauf noch eine weitere Reduktion möglich war (vgl. Tabelle 25), ist der Abstraktionsgrad der Teilkategorie des textuellen Gegenstands mit Blick auf das durch das sensibilisierende Konzept anvisierte Niveau bereits erreicht und von einer weiteren Reduktion wird abgesehen.

Das regelgeleitete Auswertungsverfahren sieht vor, die explorierten Kategorien zur situativen Einbettung (lokale kulturelle Codes) und zum textuellen Gegenstand in einem weiteren Zyklus zu prüfen (vgl. Kapitel 5.2.1). Gemäß der Empfehlungen von Mayring (2010, 75) wird die zweite Hälfte des Ausgangsmaterials zu diesem Zweck von einem zweiten Coder analysiert. 
Tab. 25: Zweiter Materialdurchlauf: einige Kategorien situativer Einbettungen im Produktions-/Logistik- und Dienstleistungssektor

\begin{tabular}{|c|c|}
\hline Produktion/Logistik & Dienstleistung \\
\hline $\begin{array}{l}\text { selbstständige Orientierung hinsichtlich Zuständig- } \\
\text { keiten und Integration in routinierte Handlungs- } \\
\text { felder }\end{array}$ & $\begin{array}{l}\text { selbstständige Orientierung hinsichtlich Zuständig- } \\
\text { keiten und Integration in routinierte Handlungs- } \\
\text { felder }\end{array}$ \\
\hline $\begin{array}{l}\text { selbstständige Orientierung hinsichtlich zu bearbei- } \\
\text { tender Produkte u. I. i. r. H. }\end{array}$ & $\begin{array}{l}\text { selbstständige Orientierung hinsichtlich von Kun- } \\
\text { denwünschen und Integration in zu modifizierende } \\
\text { routinierte Handlungsfelder }\end{array}$ \\
\hline $\begin{array}{l}\text { selbstständige Orientierung hinsichtlich zu verar- } \\
\text { beitender Teile u. I.i.r. H }\end{array}$ & $\begin{array}{l}\text { selbstständige Orientierung über die zu leistenden } \\
\text { Arbeitszeiten und Integration in das berufliche und } \\
\text { außerberufliche Zeitmanagement }\end{array}$ \\
\hline $\begin{array}{l}\text { selbstständige Orientierung hinsichtlich der Lage- } \\
\text { rung zu verarbeitender Teile u. I. i.r. H }\end{array}$ & $\begin{array}{l}\text { selbstständige Orientierung hinsichtlich des Raum- } \\
\text { managements u. I. i. r. H }\end{array}$ \\
\hline \multicolumn{2}{|l|}{$\begin{array}{l}\text { formale Bestätigung der Anwesendheit an routi- } \\
\text { nierten innerbetrieblichen Fortbildungen }\end{array}$} \\
\hline \multicolumn{2}{|l|}{$\begin{array}{l}\text { formale Bestätigung der Arbeitszeit in außerbe- } \\
\text { trieblichen Einsatzorten }\end{array}$} \\
\hline \multicolumn{2}{|l|}{$\begin{array}{l}\text { formale Bestätigung des geleisteten Arbeitsergeb- } \\
\text { nisses in außerbetrieblichen Einsatzorten }\end{array}$} \\
\hline \multicolumn{2}{|l|}{$\begin{array}{l}\text { formale Bestätigung quantitativ messbarer Arbeits- } \\
\text { ergebnisse }\end{array}$} \\
\hline \multicolumn{2}{|l|}{$\begin{array}{l}\text { Kontrolle der Einhaltung von sequenziell (nicht) } \\
\text { vorgegebenen Handlungsschritten u. I. i. r. H }\end{array}$} \\
\hline $\begin{array}{l}\text { gezielte Überprüfung der quantitativen Vollständig- } \\
\text { keit einer Lieferung u. I. i.r. H }\end{array}$ & $\begin{array}{l}\text { gezielte Überprüfung bei der Weitergabe von Kauf- } \\
\text { verträgen u. I.i.r.H }\end{array}$ \\
\hline \multicolumn{2}{|l|}{$\begin{array}{l}\text { gezielte Überprüfung der qualitativen Vollständig- } \\
\text { keit von Arbeitsergebnissen u. I. i. r. H }\end{array}$} \\
\hline Merkhilfe seltener Handlungsschritte u. I. i. r. H & Merkhilfe seltener Handlungsschritte u. I. i. r. H \\
\hline $\begin{array}{l}\text { Merkhilfe seltener Produkt- oder Teile-Kodes u. } \\
\text { I.i.r. H }\end{array}$ & $\begin{array}{l}\text { Merkhilfe seltener, auch saisonaler Speise-oder } \\
\text { Getränke-Kodes u. I.i.r. H }\end{array}$ \\
\hline \multirow[t]{2}{*}{$\begin{array}{l}\text { Lernvorlage für Faktenwissen über gängige } \\
\text { Produkt- oder Teile-Kodes und Integration in zu } \\
\text { routinisierende Handlungsfelder }\end{array}$} & $\begin{array}{l}\text { Lernvorlage für Faktenwissen über gängige Speise-, } \\
\text { Getränke oder Raummarkierungs-Kodes und Integ- } \\
\text { ration in zu routinisierende Handlungsfelder }\end{array}$ \\
\hline & $\begin{array}{l}\text { Lernvorlage für Faktenwissen über Inhaltsstoffe } \\
\text { und Zutaten und Integration in zu routinalisierende } \\
\text { Handlungsfelder }\end{array}$ \\
\hline
\end{tabular}

Sowohl zum textuellen Gegenstand noch zur situativen Einbettung ist eine zusätzliche Kategorie notwendig. Da sich die in der ersten Hälfte explorierten Kategoriensysteme somit als erschöpfend erweisen, kann qualitativ von einer erreichten Sättigung gesprochen werden.

\subsubsection{Finalisierung des induktiv entwickelten Kategoriensystem der situativ-textuellen routinalisierten lokalen kulturellen Codes}

Nachdem die Kategorien des Kategoriensystems vollständig ausdifferenziert und damit voneinander unterscheidbar auf das Ausgangsmaterial anwendbar gemacht wurden, folgt nun der letzte abschließende Durchgang durch das Interviewmaterial. Das Vorgehen sah vor, dass das 
Material erneut je Tätigkeitsfeld durchgearbeitet wird, wobei den einzelnen Analyseeinheiten Kategorien zugeordnet werden. Der hierbei angestrebte Abstraktionsgrad der routinalisierten lokalen kulturellen Codes ist theoriebasiert vom situated-literacy-Ansatz geprägt und somit u. a. von einer Ablösung der bis dato diversifizierenden Berufsfeld- bzw. Wirtschaftssektorspezifik. Darunter bedeutungsgleiche bzw. ähnliche situativ-kontextuelle Relevanz- und Sinnoptionen sind unter dieser Zielsetzung weiter zu subsumieren, wobei Kontextbedingungen auf der Ebene von Deskriptoren der finalen Kategorien übernommen werden. Mit der zunehmenden Abstraktion rücken folglich insbesondere vergleichbare situativ-textuelle routinalisierte lokale kulturelle Codes ins Blickfeld, welche teils die lokalen kulturellen Codes repräsentieren, die eine skillful performance mit multikodalen Texten und Textkomplexen erst kontextualisieren. Bei der Sichtung dieser situativ-textuellen Relevanz- und Sinnoptionen wird bereits deutlich werden, dass sich diese von den routinalisierten lokalen kultutellen Codes entlang der Behaltensleistung und der Einstellungsänderung unterscheiden, die von Christmann/Groeben (1999, 172f.) mit Blick auf symbolische Texte in der Sekundarstufe I ausgemacht wurden (vgl. Kapitel 3.3.3.2).

Tab. 26: Situativ-textuelle routinalisierte lokale kulturelle Codes zur Annäherung an eine skillful performance mit nichtkontinuierlichen Texten und Textkomplexen

\begin{tabular}{|c|c|}
\hline \multicolumn{2}{|l|}{ Kategorie: punktuelle textuelle Organisationshilfe } \\
\hline \multicolumn{2}{|c|}{$\begin{array}{l}\text { Definition: Texte und Textkomplexe dienen der unmittelbaren, einmaligen Entnahme von für routinierte } \\
\text { Handlungsfelder aktuell relevanter Informationen ohne Bedarf der v. a. längerfristigen Einprägung }\end{array}$} \\
\hline Deskriptoren & Ankerbeispiele \\
\hline $\begin{array}{l}\text { - für Einhaltung einzelner Vorgaben zu Arbeitsprozes- } \\
\text { sen relevant } \\
\text { - Informationen beziehen sich auf zu be-/verarbeitende } \\
\text { Produkte, Teile oder leistende Dienstleistungen } \\
\text { - Informationen beziehen sich auf Raummarkierungen } \\
\text { der Lagerung von Produkten/Teilen oder auf den Ort } \\
\text { von Dienstleistungen } \\
\end{array}$ & $\begin{array}{l}\text { - zwingend ist z. B. das Kochen von Milchreis } \\
\text { nach Rezept, oder von Gemüsesuppe, einer } \\
\text { Sauce; richtige Reissorte aus dem Lager holen } \\
\text { (Kantine: Rezept, Lagerplan, Etikett; } 2 \text { b-V) } \\
\text { - Tankdeckel und Stecköffnung sind bei allen } \\
\text { Modellen gleich; gibt aber verschiedene Seiten- } \\
\text { verkleidungen; kann man von dem Zettel unter }\end{array}$ \\
\hline $\begin{array}{l}\text { Auswahl an Texten/Textkomplexen: Tabellen zu Pro- } \\
\text { dukten und Dienstleistungen (Preis-/Code-Liste Akti- } \\
\text { onswoche, spezielle Getränke); handschriftliche oder } \\
\text { formale Arbeitsaufträge (Sonderwünsche; Lieferschein, } \\
\text { Produktblatt); Etiketten }\end{array}$ & $\begin{array}{l}\text { der Motorhaube ablesen; beschreibt relevante } \\
\text { und irrelevante Angaben auf dem Zettel: } \\
\text { „Dann verschiedene Nummern, wo ich nichts } \\
\text { damit anfangen kann“ [...] Zeitdruck v. a. zu } \\
\text { Beginn an einer neuer Station; Ablauf auto- }\end{array}$ \\
\hline $\begin{array}{l}\text { Codierregeln: } \\
\text { - werden mehrere, in Handlungsfelder verkettete } \\
\text { Handlungsschritte behandelt, sodann der prozessua- } \\
\text { len textuellen Organisationshilfe ggf. zuordnen } \\
\text { - gibt es keinen situativ-kontextuellen Verweis eines } \\
\text { einmaligen Lesens unter Zeitdruck, sodann der punk- } \\
\text { tuellen textuellen Merkhilfe ggf. zuordnen } \\
\text { - Texte/Textkomplexe dieser Kategorien dienen nicht } \\
\text { der Bearbeitung durch Schreiben }\end{array}$ & $\begin{array}{l}\text { matisiert sich aber mit der Zeit: „Du bist halt } \\
\text { nicht mitgekommen. (Fertigung; Produktblatt } \\
4 \mathrm{~d}-\mathrm{M})\end{array}$ \\
\hline
\end{tabular}


Kategorie: punktuelle textuelle Merkhilfe

Definition: Texte und Textkomplexe dienen der ggf. mehrfachen Entnahme aktuell relevanter, aber nicht einzuprägender Informationen

\begin{tabular}{|c|c|}
\hline Deskriptoren & \\
\hline $\begin{array}{l}\text { - für Einhaltung der Vorgaben zu Arbeitsprozessen } \\
\text { relevant } \\
\text { - Informationen beziehen sich auf zu be-/verarbeitende } \\
\text { Produkte, Teile oder Dienstleistungen } \\
\text { - Informationen beziehen sich auf Raummarkierungen } \\
\text { der Lagerung von Produkten/Teilen oder auf den Ort } \\
\text { von Dienstleistungen }\end{array}$ & \multirow{3}{*}{$\begin{array}{l}\text { - beim Bonieren muss man für Getränke und } \\
\text { Gerichte einen Code eingeben; Codes der } \\
\text { Speise- und Getränkekarte sollte man mit } \\
\text { der Zeit kennen; für spezielle Getränke und } \\
\text { Gerichte hängt eine Liste mit Codes an der } \\
\text { Bonierkasse (Gastgewerbe: tabellarischer } \\
\text { Aushang zu Codes spezieller Gerichte und } \\
\text { Getränke; 1b-V) } \\
\text { - Öl und Treibstoff sind selbst nachzufüllen; mit } \\
\text { der Zeit ist ein Gefühl zu entwickeln, wann } \\
\text { Füllstand zu kontrollieren ist; benötigter Öltyp } \\
\text { steht auf dem Etikett der Maschine und dem } \\
\text { Ölkanister (Gartenbau: Etikett Maschine, } \\
\text { Etikett Ölkanister; 6a-M) }\end{array}$} \\
\hline $\begin{array}{l}\text { Auswahl an Texten/Textkomplexen: Tabellen zu Produk- } \\
\text { ten und Dienstleistungen (Preis-/Code-Liste Aktionswo- } \\
\text { che, spezielle Getränke); Checkliste in der Einlernphase; } \\
\text { handschriftliche oder formale Arbeitsaufträge (Sonder- } \\
\text { wünsche; Lieferschein, Produktionsschein); Etiketten }\end{array}$ & \\
\hline $\begin{array}{l}\text { Codierregeln: } \\
\text { - im Gegensatz zur prozessualen Organisationshilfe } \\
\text { werden hier einzelne zu verknüpfende Informationen } \\
\text { eines Sachverhalts (Handlungsfeld) betrachtet } \\
\text { - Texte/Textkomplexe dieser Kategorien dienen nicht } \\
\text { der Bearbeitung durch Schreiben }\end{array}$ & \\
\hline
\end{tabular}

\section{Kategorie: prozessuale textuelle Organisationshilfe}

Definition: Texte und Textkomplexe dienen der einmaligen Entnahme von für routinierte Handlungsfelder mittelbar hinter Informationen oder unmittelbar repräsentierten, aktuell relevanten Sachverhalten ohne Bedarf der v. a. längerfristigen Einprägung

\begin{tabular}{|c|c|}
\hline TIS & \\
\hline $\begin{array}{r}\text { - Infor } \\
\text { inner } \\
\text { - für } \mathrm{E} \\
\text { ten } \\
\text { - für } \mathrm{E} \\
\text { lungs }\end{array}$ & \multirow{3}{*}{$\begin{array}{l}\text { - ein Koch in der Kantine muss nicht alle } \\
\text { Rezepte kennen, sondern schlägt nach; muss } \\
\text { aber Grundbegriffe kennen; Beispiel blan- } \\
\text { chieren; Umgang mit Rezept bedeutet Rezept } \\
\text { lesen, verinnerlichen und dann ohne Kochbuch } \\
\text { kochen: „Dann hast du } 5 \text { bis } 10 \text { Minuten Zeit } \\
\text { und dann kannst du dir das einprägen und } \\
\text { dann kannst du das übernehmen“ (Beikoch: } \\
\text { Rezept, Kochbuch-Register; 2b-V) } \\
\text { - selbstständiges Arbeiten bedeutet, dass man } \\
\text { dem Speiseplan die zu erledigenden Aufgaben } \\
\text { entnimmt; wenn man für Beilagen verantwort- } \\
\text { lich ist, sämtliche Vorbereitungen und Zuberei- } \\
\text { tung entnimmt; mit der Zeit muss ein Gespür } \\
\text { für die Menge entstehen; Mengenangabe gibt } \\
\text { es nicht (Beikoch: Speiseplan; 2a-V) }\end{array}$} \\
\hline & \\
\hline $\begin{array}{r}\text { Codi } \\
- \text { we } \\
\text { on } \\
\text { on } \\
\text { gib } \\
\text { ein } \\
\text { zes } \\
\text { - Te } \\
\text { de }\end{array}$ & \\
\hline
\end{tabular}




\section{Kategorie: prozessuale textuelle Merkhilfe}

Definition: Texte und Textkomplexe dienen der ggf. mehrfachen Entnahme aktuell relevanter, aber nicht einzuprägender, mittelbarer hinter Informationen oder unmittelbar repräsentierter Sachverhalte, auch im Zuge besonderer Handlungsbedingungen

\begin{tabular}{|c|c|}
\hline Deskriptoren & Ankerbeispiele \\
\hline $\begin{array}{l}\text { - für Einhaltung der Vorgaben zu Arbeitsprozessen nur } \\
\text { unter bedingtem Zeitdruck relevant } \\
\text { - Informationen beziehen sich auf Zuständigkeiten } \\
\text { - Informationen beziehen sich auf verkette Handlungs- } \\
\text { schritte in Handlungsfeldern }\end{array}$ & \multirow{3}{*}{$\begin{array}{l}\text { - täglich zu Arbeitsbeginn liegen im Regal die } \\
\text { Aufträge; Aufträgen liegt technische Zeich- } \\
\text { nung bei; kann man eigenen Arbeitsauftrag } \\
\text { und Kunden entnehmen; Zeichnungen und } \\
\text { Auftrag müssen gelesen werden: welches der } \\
\text { unterschiedlichen Rohmaterialien zu verar- } \\
\text { beiten ist, kann man Zeichnung entnehmen } \\
\text { und hängt von der Menge ab; enthalten auch } \\
\text { Maße; Stückzahl steht auf dem Auftragszettel; } \\
\text { [...] richtiges Programm an der Maschine muss } \\
\text { nach Maßen ausgewählt werden; einzelne } \\
\text { Maße wie z. B. Durchmesser müssen ergänzt } \\
\text { werden; pro Auftrag werden meist 30-40 Teile } \\
\text { gefertigt; unterschiedliche Produktionsschritte } \\
\text { aufgeteilt auf mehrere Mitarbeiter/innen } \\
\text { (Produktion: Auftragsformular; technische } \\
\text { Zeichnung; 4b-M) } \\
\text { - Kontrolle der Tiefkühlgeräte: Teil von Vor- } \\
\text { schriften; gibt einen Ordner mit Checkliste } \\
\text { auch hierfür; Kühlraum wird mit Messgerät } \\
\text { gemessen; wenn Temperatur nicht stimmt ruft } \\
\text { man Vorgesetzte/n; er/sie eine/n Elektriker/ } \\
\text { in (Verkauf: Checkliste zur Einhaltung von } \\
\text { Vorschriften, Display Messgerät; 8d-M) }\end{array}$} \\
\hline $\begin{array}{l}\text { Auswahl an Texten/Textkomplexen: Arbeitspläne, } \\
\text { Raumpläne, Produktionspapiere }\end{array}$ & \\
\hline $\begin{array}{l}\text { Codierregeln: } \\
\text { - im Gegensatz zur prozessualen textuellen Organisati- } \\
\text { onshilfen gibt es hier keinen Verweis von Zeitdruck, } \\
\text { der ein wiederholtes Lesen situativ-kontextuell nicht } \\
\text { erlaubt würde } \\
\text { - Texte/Textkomplexe dieser Kategorien dienen nicht } \\
\text { der Bearbeitung durch Schreiben }\end{array}$ & \\
\hline
\end{tabular}

\section{Kategorie: prozessuale textuelle Lernvorlage (Behaltensleistung)}

Definition: Texte und Textkomplexe dienen der Behaltensleistung von repräsentierten Sachverhalten, die in routinierten Handlungsfeldern von Relevanz sind

\begin{tabular}{|c|c|}
\hline Deskriptoren & Ankerbeispiele \\
\hline $\begin{array}{l}\text { - Einprägen im Sinne eines Ergebnisses intensionalen } \\
\text { Lernens } \\
\text { - Relevanz des Lesens dieser Texte löst sich mit Einprä- } \\
\text { gen der Informationen auf }\end{array}$ & \multirow{3}{*}{$\begin{array}{l}\text { - sind eine Weinstube; haben diverse Weine im } \\
\text { Angebot; zumindest über die offenen Weine } \\
\text { sollte man Auskunft geben können: über die } \\
\text { Rebsorte, Prädikatsstufe, trocken usw. (Gast- } \\
\text { gewerbe: tabellarische Datenblätter zu offenen } \\
\text { Weinen; 1b-V) } \\
\text { - man muss die Werbung [des eigenen Arbeit- } \\
\text { gebers] lesen; man muss wissen, welche Ware } \\
\text { gerade im Angebot ist; man muss aber auch } \\
\text { den Lagerort [im Laden] kennen } \\
\text { - „Die müssen die Karte kennen!“: mit der } \\
\text { Zeit ist es wichtig, alle Gerichte auf der Karte } \\
\text { auswendig zu kennen; dazu gehört auch, was } \\
\text { in den Gerichten enthalten ist; z. B. was ein } \\
\text { Trüffel ist, wie ein Trüffel aussieht und wo ein } \\
\text { Trüffel im Gericht zu finden ist (Gastgewerbe: } \\
\text { Speisekarte, Rezepte; 1b-V) }\end{array}$} \\
\hline $\begin{array}{l}\text { Auswahl an Texten/Textkomplexen: Textkomplexe } \\
\text { setzen sich ggf. aus losen, aber Arbeitsplatz relevanten } \\
\text { Texten zusammen }\end{array}$ & \\
\hline $\begin{array}{l}\text { Codierregeln: } \\
\text { - Kern der Behaltensleistung richtet sich an Sachverhalte, } \\
\text { ergo gegebener Informationen einschließlich ihrer reprä- } \\
\text { sentierten Relationen zueinander }\end{array}$ & \\
\hline
\end{tabular}




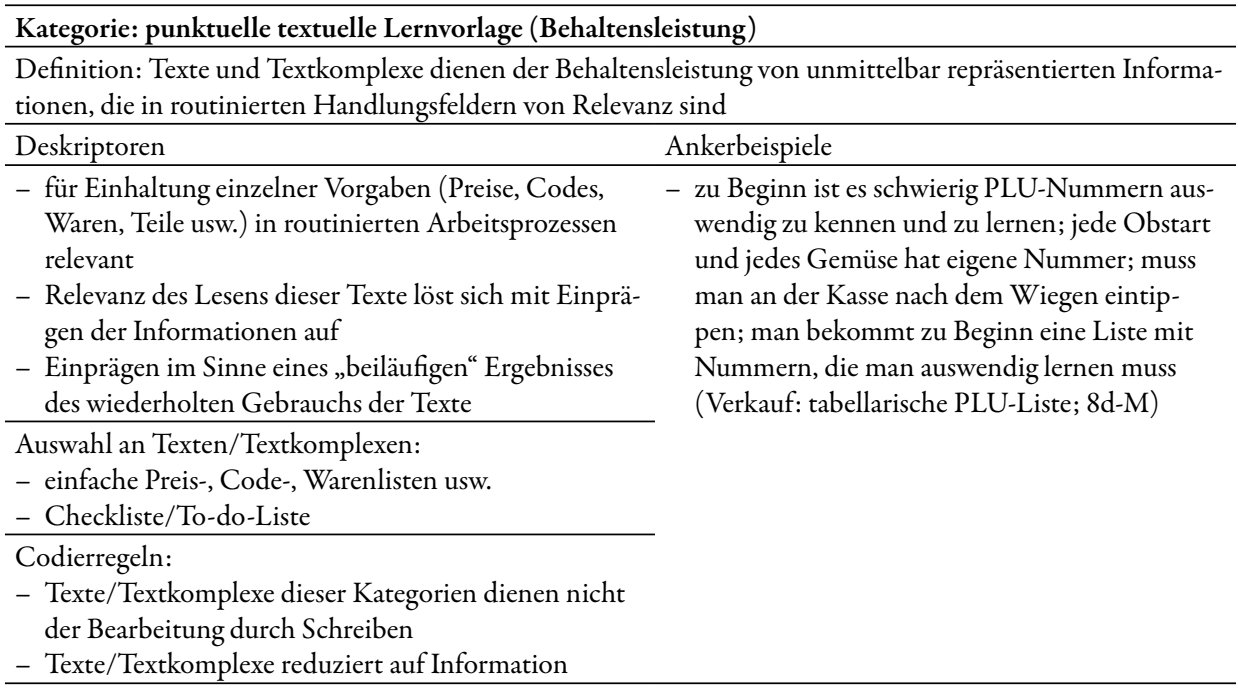

\section{Kategorie: prozessualer Textgebrauch (Lernen semiotischer Strukturen)}

Definition: Texte und Textkomplexe dienen der Erläuterung des Gebrauchs dieser Texte bis Textkomplexe, die für routinierte Handlungsfelder von Relevanz sind

\begin{tabular}{ll}
\hline Deskriptoren & Ankerbeispiele \\
\hline $\begin{array}{l}\text { - Texte/Textkomplexe mit Eintragungen oder Markie- } \\
\text { rungen, die den Gebrauch illustrieren oder anleiten }\end{array}$ & $\begin{array}{l}\text { Pflanzenschutz ist ein wichtiges Thema; } \\
\text { Arbeitsfeld Unkrautbefreiung auf dem }\end{array}$ \\
$\begin{array}{l}\text { - Erläuterungen können durch verbale Anweisungen } \\
\text { ergänzt sein }\end{array}$ & $\begin{array}{l}\text { Friedhof: Spritzmittel wird durch ein Fass } \\
\text { auf die Wege gespritzt; Verhältnis ist auf dem }\end{array}$ \\
$\begin{array}{ll}\text { - Texte/Textkomplexe können durch den/die Mitar- } \\
\text { beiter/in selbst erzeugt worden sein }\end{array}$ & $\begin{array}{l}\text { Etikett angegeben; Spritzfläche muss geschätzt } \\
\text { werden; Menge an Spritzmittel muss berechnet }\end{array}$ \\
$\begin{array}{ll}\text { Auswahl an Texten/Textkomplexen: Duplikate in Schu- } \\
\text { lungsmappen; eigene Duplikate mit ggf. handschriftli- } \\
\text { chen Anmerkungen }\end{array}$ & $\begin{array}{l}\text { übrig bleibt; darf nicht einfach ausgeleert } \\
\text { werden; das ist Vorschrift; war Inhalt einer }\end{array}$ \\
& $\begin{array}{l}\text { Fortbildung um mit Spritzmitteln arbeiten zu } \\
\text { dürfen (Gartenbau: Etikett; 6a-M) }\end{array}$ \\
\hline
\end{tabular}

\section{Kategorie: Kontrolle in der non-formalen textuellen Kommunikation}

Definition: Texte und Textkomplexe dienen der routinierten Prüfung aktuell relevanter, aber nicht einzuprägender Informationen

\begin{tabular}{|c|c|}
\hline Deskriptoren & Ank \\
\hline $\begin{array}{l}\text { - Texte/Textkomplexe mit Eintragungen oder Markie- } \\
\text { rungen, die für die Organisation des Arbeitsplatzes } \\
\text { von Bedeutung sind } \\
\text { - Texte/Textkomplexe können durch den/die Mitar- } \\
\text { beiter/in selbst erzeugt worden sein } \\
\end{array}$ & \multirow{3}{*}{$\begin{array}{l}\text { - neben Krankmeldungen müssen auch } \\
\text { Urlaubsanträge ausgefüllt werden; es hängt ein } \\
\text { Kalender aus, auf dem man nachschauen kann, } \\
\text { wann Urlaub noch möglich ist (Produktion: } \\
\text { Kalender; 4b-M) } \\
\text { - „Die müssen die Karte kennen!“: mit der } \\
\text { Zeit ist es wichtig, alle Gerichte auf der Karte } \\
\text { auswendig zu kennen; dazu gehört auch, was } \\
\text { in den Gerichten enthalten ist; z. B. was ein } \\
\text { Trüffel ist, wie ein Trüffel aussieht und wo ein } \\
\text { Trüffel im Gericht zu finden ist; (Gastgewerbe: } \\
\text { Rezepte; } 1 \text { b-V) }\end{array}$} \\
\hline $\begin{array}{l}\text { exen: „Schmierzettel“; } \\
\text { enbestand) }\end{array}$ & \\
\hline $\begin{array}{l}\text { Codierregeln: } \\
\text { - Texte/Textkomplexe dieser Kategorien dienen nicht } \\
\text { der Bearbeitung durch Schreiben }\end{array}$ & \\
\hline
\end{tabular}




\begin{tabular}{|c|c|}
\hline \multirow{2}{*}{\multicolumn{2}{|c|}{$\begin{array}{l}\text { Kategorie: Kontrolle in der formalen textuellen Kommunikation } \\
\text { Definition: Texte und Textkomplexe dienen der routinierten Prüfung aktuell relevanter, aber nicht einzu- } \\
\text { prägender Informationen mit einem rechtlichen oder offiziellen Charakter }\end{array}$}} \\
\hline & \\
\hline Deskriptoren & Ankerbeispiele \\
\hline $\begin{array}{l}\text { Texte/Textko } \\
\text { Markierunger } \\
\text { Tätigkeiten a } \\
\end{array}$ & \multirow{3}{*}{$\begin{array}{l}\text { - Bestellungen werden am Tisch aufgenommen; } \\
\text { als Kürzel; Bestellung wird an der Kasse über } \\
\text { Codes boniert; gehen automatisch in die Küche; } \\
\text { Sonderwünsche müssen auf Bedienerblock notiert } \\
\text { werden; Verdeutlichung der Relevanz zur Kennt- } \\
\text { nis über Zutaten in Gerichten (Gastgewerbe: } \\
\text { Rezepte, Bedienerblock; 1b-V) } \\
\text { - zum Beispiel muss eine Anlieferung von Beton } \\
\text { angenommen werden; Lieferschein ist zu unter- } \\
\text { schreiben; man hat Lieferschein vorher flüchtig zu } \\
\text { prüfen; zu prüfen ist, dass es der richtige Liefer- } \\
\text { schein ist: Name der Firma und die Art der Ware; } \\
\text { Menge der Ware muss nicht geprüft werden (Bau: } \\
\text { Lieferschein; 9b-M) }\end{array}$} \\
\hline & \\
\hline $\begin{array}{l}\text { Codierregeln: } \\
\text { - Texte/Textkomplexe dieser Kategorien dienen } \\
\text { nicht der Bearbeitung durch Schreiben Texte/ } \\
\text { Textkomplexe sind nicht durch den/die Mitarbei- } \\
\text { ter/in selbst erzeugt worden }\end{array}$ & \\
\hline
\end{tabular}

\section{Kategorie: formale textuelle Weitergabe}

Definition: Texte und Textkomplexe dienen der betriebsinternen Kommunikation (Voraussetzung für Dokumentation) mit rechtlichem Charakter

\begin{tabular}{|c|c|}
\hline Deskriptoren & Ankerbeispiele \\
\hline $\begin{array}{l}\text { - Texte/Textkomplexe verlangen Eintragungen oder } \\
\text { Markierungen, die Tätigkeiten am Arbeitsplatz } \\
\text { dokumentieren, prüfen oder nachweisen } \\
\text { - Dokumentation, Prüfung und Nachweis sind } \\
\text { rechtlich notwendig }\end{array}$ & $\begin{array}{l}\text { - zwingend Protokollierung der Produktions- und } \\
\text { Abfülltemperatur mittels Formvorlagen des } \\
\text { betriebsinternen HDCP-Konzeptes: „Das ist } \\
\text { eine Standardanforderung bei uns.“ (Kantine: } \\
\text { tabellarisches HDCP-Prüfprotokoll; 2b-V) }\end{array}$ \\
\hline $\begin{array}{l}\text { Auswahl an Texten/Textkomplexen: Arbeitszeiten; } \\
\text { Arbeitsleistung; beanspruchte Maschinen/Geräte }\end{array}$ & $\begin{array}{l}\text { - Checkliste existieren für alle diese Vorgänge } \\
\text { [der Protokollierung der Produktionszahlen je }\end{array}$ \\
\hline $\begin{array}{l}\text { Codierregeln: } \\
\text { - Texte/Textkomplexe dieser Kategorien dienen der } \\
\text { Bearbeitung durch Schreiben } \\
\text { - Texte/Textkomplexe sind nicht durch den/die } \\
\text { Mitarbeiter/in selbst erzeugt worden }\end{array}$ & $\begin{array}{l}\text { für [steuerrechtlich relevante] Banderolen; } \\
\text { wichtig bei Produktionsfehlern; Checkliste } \\
\text { gibt an, was nacheinander genau zu tun ist, z. B. } \\
\text { Nachprüfen, ob der Anfangsbestand stimmt; } \\
\text { zweites Formular für Produktionsmenge der } \\
\text { Schicht; angegeben nach Einheiten ganze } \\
\text { Palette und Packungen; drittes Formular [als } \\
\text { Teil der Protokollierung der Produktionszahlen } \\
\text { je Schicht] ist der Gesamtauftragsschein; wird } \\
\text { von Schicht zu Schicht weitergegeben; gibt ver- } \\
\text { bleibende Produktion nach Auftrag an; Menge } \\
\text { in Paletten; Schichtführer teilen sich das gegen- } \\
\text { seitig mit (Fertigung: Produktionsprotokoll im } \\
\text { Sinne einer Checkliste; Banderolen-Protokoll } \\
\text { im Sinne einer Checkliste; 3c-M) }\end{array}$ \\
\hline
\end{tabular}




\subsection{Konfigurationen routinalisierter lokaler kultureller Codes und routinalisierter textueller Gegenstände}

Die qualitative Inhaltsanalyse bestätigt die theoretische Herleitung, dass die einzelnen multikodalen Texte und Textkomplexe nicht ausschließlich einer situativ-kontextuellen Relevanz- und Sinnoption zuzuordnen sind. Insbesondere eine skillful performende Verarbeitung derjeniger Texte und Textkomplexe, die auf Repräsentationsanalogien entlang lokaler oder globaler Kohärenzbeziehungen zwischen Lokal- oder Globalzeichen und Fakten- bis praktischem Wissen referieren, kann durch die routinalisierten lokalen kulturellen Codes entlang des Textgebrauchs (Lernen semiotischer Struktureinheiten), der Lernhilfe (Behaltensleistung), der Merkhilfe, der Organisationshilfe, der Weitergabe und der Kontrolle (re)kontextualisiert sein. Diese und weitere situativ-kontextuellen Relevanz- und Sinnoptionen und routinalisiert eingebundene Repräsentationsanalogien können der Tabelle 27 und der Tabelle 28 entnommen werden. Die Ergebnisse, ergo die Sachverhalte sind hier primär ikonisch repräsentiert und sollen an dieser Stelle nicht via eine primär symbolische Repräsentation lediglich dupliziert werden. Als zentrales Ergebnis soll im Sinne eines Belegs und nicht eines gar allgemeingültigen Beweises festgehalten werden, dass sich eine Bildung als Voraussetzung für die berufliche Teilhabe an den untersuchten Einfach(st)arbeitsplätzen eher entlang (hypothetischer) Lesekompetenzen primär ikonischer Textualität ableiten lässt, als entlang (funktionaler) Lesecompetencies primär symbolischer Textualität.

Vielmehr soll auf eine zweite Ebene von Ergebnissen weitergehend eingegangen werden, die sich aus der Gegenüberstellung von Praxen an Einfach(st)erwerbstätigkeiten und an schulischen Bildungssettings der Sekundarstufe I mit (Sach-)Texten ergeben. Diese sind via den SteuerungsKode Schraffierung in den Tabelle 27 \& Tabelle 28 eingepflegt und zeigen, erstens, eine anteilige Deckung hinsichtlich der routinalisierten lokalen kulturellen Codes. Beispielsweise zeigt sich eine skillfulperformance on-the-job ebenso entlang der Behaltensleistung von Faktenwissen, vergleichbar wie für schulische Settings (vgl. in Kapitel 3.3.3.2). Maßgeblicher Unterschied ist jedoch, dass Reflexion und Bewertung von Faktenwissen keine textuelle Herausforderung der literalen Praxen ausmachen. Zweitens kann eine bedingte Deckung zwischen Lesesituationen an Einfach(st)arbeitsplätzen und in der Sekundarstufe I analysiert werden, welche sich auf das Lernen semiotischer Strukturen bezieht. An Einfach(st)arbeitsplätzen konnten hinter der Kategorie „Textgebrauch“ Lesesituationen aufgedeckt werden, in denen das Erlernen der routinalisiert eingebundenen Repräsentationsanalogien den Kern ausmachten, in diesen Situationen kompetent beruflich zu handeln. Interessant ist, dass sich dieses ,Erlernen' der Repräsentationsanalogien auf multikodale Textkomplexe und nicht einen isolierten einseitigen Text bezog, ergo, auf der Ebene der Globalzeichen einzuordnen war. Gleiches gilt für primär symbolische Texte, respektive, Fließtexte oder Sachtexte, die vereinzelt on-the-job von Einfach(st)erwerbstätigkeiten aufgefunden wurden. Die für diese spezifische Arbeitswelt relevanten Textkomplexe mit insbesondere starken Darstellungs-Kodes, wie z. B. Rechnungen, prägen die literalen Praxen mit diesem routinalisierten lokalen kulturellen Code. Während die Bearbeitung von einseitigen multikodalen Texten eher vorausgesetzt wird, spielen primär symbolische Texte, wie bereits enttarnt, keine entscheidende Rolle, um die gestellten Anforderungen kompetent zu meistern.

„Das ist jetzt krass [formuliert], egal wie blöd[, dass] / [...] einer ist, aber wenn einer etwas öfters macht, dann muss er es irgendwann können. Nicht?"

(Beikoch; IIa-V). 
Tab. 27: Gegenüberstellung der routinalisierten lokalen kulturellen Codes in und von Texten in sozialen Praxen an der obligatorischen Schule und an den untersuchten Einfach(st)arbeitsplätzen

\begin{tabular}{|c|c|c|c|c|c|c|c|}
\hline $\begin{array}{l}\text { Gegenstand des } \\
\text { Lernens }\end{array}$ & $\begin{array}{l}\text { Lernen se- } \\
\text { miotischer } \\
\text { Struktur- } \\
\text { einheiten }\end{array}$ & $\begin{array}{l}\text { Behaltens- } \\
\text { leistung }\end{array}$ & $\begin{array}{l}\text { Einstel- } \\
\text { lungs- } \\
\text { änderung }\end{array}$ & Merkhilfe & $\begin{array}{l}\text { Organisa- } \\
\text { tionshilfe }\end{array}$ & Kontrolle & $\begin{array}{l}\text { Weiter- } \\
\text { gabe }\end{array}$ \\
\hline $\begin{array}{l}\text { lokale Kohärenz } \\
\text { zwischen Lokal- } \\
\text { zeichen }\end{array}$ & & $\begin{array}{l}\text { punktuelle } \\
\text { textuelle } \\
\text { Lern- } \\
\text { vorlage }\end{array}$ & & & & & \\
\hline $\begin{array}{l}\text { globale Kohärenz } \\
\text { zwischen (eher) } \\
\text { Globalzeichen }\end{array}$ & & $\begin{array}{l}\text { prozessua- } \\
\text { le textuelle } \\
\text { Lern- } \\
\text { vorlage }\end{array}$ & & & & & \\
\hline $\begin{array}{l}\text { lokale Kohärenz } \\
\text { zwischen Lokal- } \\
\text { zeichen und prak- } \\
\text { tischem Wissen/ } \\
\text { Faktenwissen }\end{array}$ & & & & $\begin{array}{l}\text { punktuelle } \\
\text { textuelle } \\
\text { Merkhilfe }\end{array}$ & $\begin{array}{l}\text { punktuelle } \\
\text { textuelle } \\
\text { Organisa- } \\
\text { tionshilfe }\end{array}$ & & \\
\hline $\begin{array}{l}\text { globale Kohärenz } \\
\text { zwischen (eher) } \\
\text { Globalzeichen } \\
\text { und praktischem } \\
\text { Wissen/Praxis- } \\
\text { wissen }\end{array}$ & $\begin{array}{l}\text { prozedua- } \\
\text { ler Text- } \\
\text { gebrauch }\end{array}$ & & & $\begin{array}{l}\text { prozessuale } \\
\text { textuelle } \\
\text { Merkhilfe }\end{array}$ & $\begin{array}{l}\text { prozessuale } \\
\text { textuelle } \\
\text { Organisa- } \\
\text { tionshilfe }\end{array}$ & & $\begin{array}{l}\text { formale } \\
\text { textuelle } \\
\text { Weitergabe }\end{array}$ \\
\hline $\begin{array}{l}\text { Reflexion und/ } \\
\text { oder Bewertung: } \\
\text { Kohärenz und } \\
\text { praktisches Wis- } \\
\text { sen/Fachwissen }\end{array}$ & & & & & & $\begin{array}{l}\text { Prüfung in der } \\
\text { formalen und } \\
\text { non-formalen } \\
\text { textuellen } \\
\text { Kommunika- } \\
\text { tion }\end{array}$ & \\
\hline
\end{tabular}

Tab. 28: Gegenüberstellung von textuellen Gegenständen des Lesens einer skillful performance entlang sozialer Praxen der obligatorischen Schulbildung (mit Referenz der funktionalen Grundbildungscompentencies) und an Einfach(st)arbeitsplätzen

\begin{tabular}{|c|c|}
\hline Gegenstand - obligatorische Schulbildung & Gegenstand - Einfach(st)erwerbstätigkeiten \\
\hline $\begin{array}{l}\text { lokale Kohärenz zwischen Lokalzeichen } \\
\text { (Informationen eines Sachverhalts, die mehr } \\
\text { oder weniger tief in den Text oder Textkomplex } \\
\text { eingebettet sind und mehr oder weniger klar } \\
\text { differenzierbar innerhalb des Textes oder Textkom- } \\
\text { plexes lokalisierbar sind, sind zu identifizieren und } \\
\text { zu dekodieren. Diese Informationen sind folglich } \\
\text { dem Text oder Textkomplex inhärent und bedürfen } \\
\text { nicht zwingend weder externes Faktenwissen noch } \\
\text { einer tiefen Auseinandersetzung mit dem Text) }\end{array}$ & $\begin{array}{l}\text { lokale Kohärenz zwischen Lokalzeichen } \\
\text { (Informationen eines Sachverhalts, die mehr oder } \\
\text { weniger tief in den Text oder Textkomplex eingebet- } \\
\text { tet sind und mehr oder weniger klar differenzierbar } \\
\text { innerhalb des Textes oder Textkomplexes lokalisierbar } \\
\text { sind, sind zu identifizieren und zu dekodieren. Diese } \\
\text { Informationen sind folglich dem Text oder Textkom- } \\
\text { plex inhärent und bedürfen nicht zwingend weder } \\
\text { externes Faktenwissen noch einer tiefen Auseinander- } \\
\text { setzung mit dem Text) }\end{array}$ \\
\hline
\end{tabular}




\begin{tabular}{ll}
\hline Gegenstand - obligatorische Schulbildung & Gegenstand - Einfach(st)erwerbstätigkeiten \\
\hline globale Kohärenz zwischen (eher) Globalzeichen & globale Kohärenz zwischen (eher) Globalzeichen \\
(Zusammenhänge zwischen Sachverhalten & (Zusammenhänge zwischen Sachverhalten (Begriffe, \\
(Begriffe, Konzepte usw.), die mehr oder weniger & Konzepte usw.), die mehr oder weniger mehrdeutig \\
mehrdeutig im Text oder Textkomplex und mehr & im Text oder Textkomplex und mehr oder weniger \\
oder weniger differenziert und strukturiert reprä- & differenziert und strukturiert repräsentiert werden, \\
sentiert werden, sind zu identifizieren, dekodieren & sind zu identifizieren, dekodieren und zu deuten. \\
und zu deuten. Diese Zusammenhänge sind folglich & Diese Zusammenhänge sind folglich dem Text oder \\
dem Text oder Textkomplex inhärent und bedürfen & Textkomplex inhärent und bedürfen nicht zwingend \\
nicht zwingend externen Faktenwissens, jedoch & externen Faktenwissens, jedoch eine mehr oder \\
eine mehr oder weniger tiefe Auseinandersetzung & weniger tiefe Auseinandersetzung mit dem Text oder \\
mit dem Text oder Textkomplex als Ganzes) & Textkomplex als Ganzes) \\
\hline
\end{tabular}

lokale Kohärenz zwischen Lokalzeichen und praktischem Wissen

(Relation zwischen Einzelinformationen eines Sachverhalts aus kategorialen Tabellen/weiteren nichtkontinuierlichen Texten und Textkomplexen und einem knowing how zu Informationen und Sachverhalten)

lokale Kohärenz zwischen Lokalzeichen und Faktenwissen

(Relation zwischen Einzelinformationen eines Sachverhalts aus kategorialen Tabellen/weiteren nichtkontinuierlichen Texten und Textkomplexen und einem knowing that $\mathrm{zu}$ Informationen und Sachverhalten)

globale Kohärenz zwischen (eher) Globalzeichen und praktischem Wissen

Interferenz zwischen Sachverhalten und hinter Einzelinformationen stehenden Sachverhalten aus kategorialen Tabellen/weiteren nichtkontinuierlichen Texten und Textkomplexen und einem knowing how zu Informationen und Sachverhalten)

globale Kohärenz zwischen (eher) Globalzeichen und Faktenwissen

Interferenz zwischen Sachverhalten und hinter Einzelinformationen stehenden Sachverhalten aus kategorialen Tabellen/weiteren nichtkontinuierlichen Texten und Textkomplexen und einem knowing that zu Informationen und Sachverhalten)

Reflexion und/oder Bewertung: lokale/globale Kohärenz und Faktenwissen

(Reflexion und Bewertung von Informationen eines mehr oder weniger umfangreich textuell repräsentierten Sachverhalts bis hin zu Zusammenhängen zwischen mehr oder weniger umfangreich textuell repräsentierten Sachverhalten unter Rückgriff auf mehr oder weniger spezifisches externes Faktenwissen)
Reflexion und/oder Bewertung: lokale/globale Kohärenz und Faktenwissen/praktisches Wissen (unter Zuhilfenahme von Darstellungsstrategien und Superstrukturen und aufbauend auf Interferenzen zwischen Einzelinformationen zu Sachverhalten, übergeordneten Sachverhalten und hinter Einzelinformationen stehenden Sachverhalten aus kategorialen Tabellen/weiterer nichtkontinuierlicher Texte und Textkomplexe und knowing how/knowing zu Handlungen) 
Drittens können sich jene routinalisierten lokalen kulturellen Codes in den beiden gegenübergestellten sozialen Kontexten nicht decken. Dieses Ergebnis trägt jedoch die Position in sich, dass es diese Relevanz- und Sinnoptionen mit Texten/Textkomplexen in sozialen Praxen der Schule etwa nicht gäbe, sondern vielmehr sind sie nicht Teil dessen, was eine funktionale Grundbildung ausmacht. Eine skillful performance in textuellen Situationen entlang der Merkhilfe, Organisationshilfe, Kontrolle oder Weitergabe finden sich mehrfach im schulischen Alltag, sei es das Zeugnis, der Hausaufgabeneintrag, der Arbeitsplan oder die Lernzielvereinbarung. Bei diesen multikodalen Texten und Textkomplexen handelt es sich jedoch nicht dezidiert um Sach- und Informationstexte, welche im Sinne der Bildungsstandards oder des thematisierten Standard-Mehrebenenmodells zur Erklärung der Verarbeitung vor, während und im Zuge des Lesens einer Behaltensleistung oder Einstellungsänderung dienen. Funktionale Grundbildung im schulischen Alltag der Sekundarstufe I wird nicht mittels ihres Lesens festgemacht - es handelt sich nicht um ein Kriterium von vermittelbar/nicht-vermittelbar bzw. besser/schlechter des (inklusiven!) Bildungssystems (vgl. Kapitel 2.2.3). Die Schüler/innen erfahren entlang dieser Texte zur Merkhilfe, Organisationshilfe, Kontrolle oder Weitergabe keine Rückmeldung, inwiefern sie weniger oder ausgeprägter kompetent, lesen' können, respektive, das Lesen für die eine Gesellschaft (vgl. Kapitel 3.4.3) beherrschen.

Demgegenüber sind die textuellen Anforderungen on-the-job von Einfach(st)erwerbstätigkeiten nicht durch die Relevanz- und Sinnoption entlang der Einstellungsänderung geprägt. Hinsichtlich des pädagogischen Ziels der Einstellungsänderung eignen sich weder diese literalen Praxen dieser beruflichen Handlungssituationen noch ihre routinalisiert eingebundenen Texte und Textkomplexe auf den ersten Blick. Die erziehungswissenschaftliche Debatte um Projektionsarten in Weltkarten (Köller/Jucker-Kupper 2009) bis hin zu Fernsehsendungen mit Karten (z. B. „Mit offenen Karten“) unterstreichen jedoch eindeutig, dass sich für diese pädagogische Intention primär ikonische Texte und Textkomplexe grundsätzlich eignen können, respektive, der routinalisierte lokale kulturelle Code entlang der Einstellungsänderung denkbar ist. Die definite Auswahl von infiniten Informationen eines Sachverhalts in Diagrammen bis kategorialen Tabellen stellen einen weiteren möglichen Kandidaten für pädagogische intentionale Settings dar. Hierbei gilt jedoch an die Limitierung durch die Bandbreite der Sachverhalte und Relationen zu erinnern, die mit primär ikonischen Zeichen überhaupt repräsentiert werden können: In Kapitel 3.2 wurden bereits auf die Möglichkeiten der textuell repräsentierten Sachverhalte hingewiesen, die durch das Spektrum von kategorialen, terminologischen oder Verlaufsstrukturen auf der Ebene von Lokalzeichen und von zuvorderst Referenz, Relevanz, Widerspruch, Bekräftigung und Abfolge auf der Ebene von eher Globalzeichen eingegrenzt wurde. Diese finden sich als Teil des knowledge about how content area knowledge is represented bzw. knowledge about how document knowledge is represented wieder, welche analog Kapitel 3.3.3.3 über die starken Darstellungs- und Steuerungs-Kodes Spezifika bspw. einzelner Fachsprachen je Kontext erfassen. Ein weiteres Beispiel sind die Superstrukturen im Spiegel der ökonomischen, rechtlichen, politischen oder organisatorischen Herkunft der Texte.

Die Ergebnisse der Teilstudie zeigen somit zuvorderst auf, dass unter dem Voraussetzungscharakter von Bildung für (berufliche) Teilhabe an Einfach(st)arbeitsplätzen die schulische Förderung des Lesens in der berufsorientierenden Sekundarstufe I zwei fundamentale Erweiterungen bedarf:

- Eine skillful performance des Lesens außerhalb der Schule zeigt sich nicht zuvorderst entlang der Behaltensleistung und Einstellungsänderung. Den Voraussetzungs- und Auslösungscharakter von Bildung für Teilhabe mitzudenken, heißt konsequenterweise den Lernenden auch 
Settings zu eröffnen, welche der kompletten Bandbreite der sozialen Eingebundenheit von Texten entspricht. Es gilt die Vielfalt des situativ-kontextuellen Lesenkönnens erfahrbar zu machen und als task/goal model akzeptierend zu berücksichtigen. Neben den routinalisierten lokalen kulturellen Codes entlang der Behaltensleistung (Lernvorlage) und Einstellungsänderung müssen die Pendants entlang des Textgebrauchs, der Merkhilfe, der Organisationshilfe, der Weitergabe und der Kontrolle zu einem Teil dessen werden, was Lesekompetenzen insbesondere in berufsvorbereitenden Bildungsprogrammen ausmacht.

- Ziel müsste, zweitens, sein, dass die Lernenden die hierbei aktualisierten (hypothetischen) Lesekompetenzen entlang der Bandbreite an aufgedeckten routinalisierten textuellen Gegenständen erweitern können. Diese Forderung schließt u. a. die Identifikation primär ikonischer Lokal-bis Globalzeichen in multikodalen Texten und Textkomplexen entlang jeweils starker Darstellungs- und Steuerungscodes (=source model) oder im Zuge von Superstrukturen mit ein. Die initiierte oder begleitende dynamische Verarbeitungsmodellierung des Repräsentierten via ein content model verlangt aus den Annahmen des Arbeitsmodells zur Verarbeitung jener multikodalen Texte und Textkomplexe sowohl auf der kognitiven, personalen als auch sozialen Ebene entsprechende Erweiterungen. Diese umfassen insbesondere das aktualisierte verkörperlichte, implizite Wissen about how content area knowledge and document knowledge is represented in texts bis hin of the task to be completed und of the response to be output (vgl. Kapitel 3.3.3). 



\section{Primitive Kulturtechnik: Kulturell vernachlässigbar oder im ordentlichen Lesen ohnehin umfasst?}

\subsection{Design der quantitativen Teilstudien: Ergänzungen}

Aus der theoretischen Debatte manifestierte sich für den Erkenntnisgegenstand die markierte Problemstellung (siehe Kapitel 3.4.1) in einem Nicht-Mitdenken von primär ikonischen Zeichensystemen der mulitikodalen Texte und Textkomplexe in schulischen Leseprogrammen. Die Konkretisierung, ob diese Texte als per se „einfach" bzw. "primitiv" eingestuft und daher in institutionellen Bildungsprozessen nicht vernachlässigt werden könnten, wurde daher innerhalb der übergeordnet methodischen Debatte (siehe Kapitel 3.5) an zentrale Position gerückt: Die Intention ist von dem Ansatz geleitet, mittels eines probabilistischen Kausalitätsparadigmas zu untersuchen, ob es dieselben oder im Gegensatz, spezifische dahinterliegende Verarbeitungsprozesse sind, die nicht ohnehin durch die funktionalen Lesecompetencies bereits ausreichend erklärt werden. Die Problematik der Geltungsreichweite im Spiegel der konzeptionellen Validität unter dem Stichwort schwache Kausalität sowie der sozialen Gebundenheit von Kompetenzen wurden bereits ausführlich debattiert und sollen an dieser Stelle nicht nochmals wiederholt werden.

\subsubsection{Referenzstudien}

Es liegen zu den Lesecompetencies symbolischer (a) bzw. multikodaler Texte und Textkomplexe (b) von Jugendlichen des sonderpädagogischen Schwerpunkts Lernen nur bedingt Referenzstudien vor.

(a) Mit der KESS-7-F-Studie wurden die Lesecompetencies von Jugendlichen der 7. Klassenstufe an Hamburger Schulen mit dem sonderpädagogischen Schwerpunkt Lernen mittels des Instrumentariums KESS-4 untersucht. Dieses Instrumentarium richtete sich ursprünglich an Schüler/innen der 4. Klassenstufe der Primarschule (Wocken/Gröhlich 2009, 133f.) und betrachtet entlang veröffentlichter Beispielaufgaben konzeptionell ausschließlich Lesecompetencies auf Satz- und Textebene kontinuierlicher Texte (Bos/Pietsch 2004, 8ff.). Die KESS-7-Studie kann folglich nur m. E. als Referenzstudie für das Lesen kontinuierlicher Texte eingeschätzt werden. Dies gilt sowohl für berichtete Performanzverteilung der Siebtklässler/innen sowie für den Vergleich zu den Viertklässler/innen:

Tab. 29: Lesecompetencies kontinuierlicher Texte der KESS-7-F-Studie

\begin{tabular}{lllccc}
\hline Bildungsprogramm & Gruppenvariable & $\mathbf{n}$ & gültiger Anteil & M & SD \\
\hline Förderschulen & männlich & 345 & 58,8 & 439 & 76,5 \\
7. Klasse & weiblich & 242 & 41,2 & 440 & 81,0 \\
\hline & Gesamt & 587 & 100,0 & 440 & 78,3 \\
\hline
\end{tabular}

(Quelle: Wocken/Gröhlich 2009)

(b) Die Erhebung von Performanzen im Spiegel von Lesecompetencies sowohl kontinuierlicher als auch nichtkontinuierlicher Texte lag im Interesse sowohl einer Zusatzstudie zu PISA 2012 (Müller et al. 2017) als auch zur Berliner Erhebung arbeitsrelevanter Basiskom- 
petenzen (BELLA; Lehmann/Hoffmann 2009). Während sich die Zielgruppe der PISAZusatzstudie aus Jugendlichen des sonderpädagogischen Schwerpunkts Lernen sowie des Schwerpunkts Emotionale und soziale Entwicklung, betrachtete BELLA ausschließlich Schülerinnen und Schüler mit anerkanntem Förderbedarf Lernen.

Die PISA-Zusatzstudie folgt den konzeptionellen Grundannahmen und nutzt die Messinstrumente der internationalen Bildungsmonitoringstudie mit leichten organisatorischen, aber nicht inhaltlichen Modifikationen (Müller et al. 2017, 177f.). Die Performanzwerte der Stichprobe umfassen daher das Lesen sowohl kontinuierlicher als auch nichtkontinuierlicher Texte oder von multikodalen Texten primär symbolischer und primär ikonischer Kodalität (Kapitel 2.2.1). Diese Studie kann zur Bearbeitung der Forschungsfrage jedoch nur bedingt Auskunft geben, da zum einen die eingesetzten Texte ausschließlich von der in funktionalen Bildungssettings geprägten Behaltensleistung geprägt sind. Zum anderen handelt es sich bei den, neben kontinuierlichen Texten eingesetzten, nichtkontinuierlichen Texten jeweils um Repräsentationen, die nicht primär ikonischer Art sind. Die nichtkontinuierlichen Texte sind gerahmt durch in Fließtexten dargelegten Informationen sowie Fragen und gleichen schulischen Sachtexten. Darüber hinaus werden, wie in Kapitel 3.1 umfassend dargelegt, mit diesem Test Performanzen funktionaler Lesecompetencies entgegen der Leseforschung verengt als ein rein kognitives Konstrukt des Verarbeitens von Repräsentiertem beim Lesen erfasst. Diese Performanzen werden, vergleichbar der PISA-Studie selbst, in Veröffentlichungen nicht differenziert für die Leseleistung entlang kontinuierlicher vs. nichtkontinuierlicher Texte dargelegt, geschweige diskutiert. Durch die genannten Einschränkungen empfiehlt sich die PISA-Zusatzstudie nicht als Referenzstudie für das methodische Design. Die Performanzverteilung kann unter dem dargelegten Vorbehalt für die Einschätzung der eigenen Forschungsbefunde zurate gezogen werden: Der Mittelwert von 332 Punkten unter den Jugendlichen mit anerkanntem sonderpädagogischem Förderbedarf liegt einerseits - statistisch bedeutsam - niedriger im Vergleich zu den Schüler/ innen anderer Bildungsprogramme (vgl. Tabelle 30 und Tabelle 31).

Tab. 30: Stichprobenmerkmale der referentiellen PISA-Zusatzstudie

\begin{tabular}{llcc}
\hline Bildungsgang & Geschlecht & Häufigkeit & gültiger Anteil \\
\hline Förderschule & männlich & 410 & 59,4 \\
& weiblich & 281 & 40,6 \\
& Gesamt & 691 & \\
\hline
\end{tabular}

(Quelle: Müller et al. 2017, 181)

Tab. 31: Lesecompetencies der PISA-Zusatzstudie

\begin{tabular}{lcccccc}
\hline $\begin{array}{l}\text { Bildungs- } \\
\text { programm }\end{array}$ & $\begin{array}{c}\text { Anteil an } \\
\text { Stichprobe }\end{array}$ & $\mathbf{M}$ & $(\mathrm{SE})$ & SD & $(\mathrm{SE})$ & $\mathbf{d} \boldsymbol{\Delta}^{*}$ \\
\hline Förderschule & $3 \%$ & 332 & $(7.8)$ & 87 & $(5.0)$ & \\
Hauptschule & $11 \%$ & 417 & $(8.3)$ & 69 & $(3.0)$ & 4.08 \\
\hline
\end{tabular}

(Quelle: ebd., 184ff.)

Anmerkung:

Reliabilität: Plausible Values $=0.88$

*Effektstärken der Differenz zwischen Förderschule und den übrigen Bildungsprogrammen 
Andererseits muss hervorgehoben werden, dass insbesondere unter den Jugendlichen mit anerkanntem sonderpädagogischem Förderbedarf eine höhere Streuungsbandbreite der Lesecompetencies festgestellt werden muss. Dieser Befund spiegelt sich in der qualitativen Zuordnung der PISA-Scores in Stufen wider (vgl. Abbildung 43). Die 15\% dieser Jugendlichen erreichen die Lesecompetencies-Stufen II und höher und performten entlang der Testaufgaben demzufolge besser als die Achtklässler/innen der anderen Bildungsprogramme auf der Stufe I und darunter.

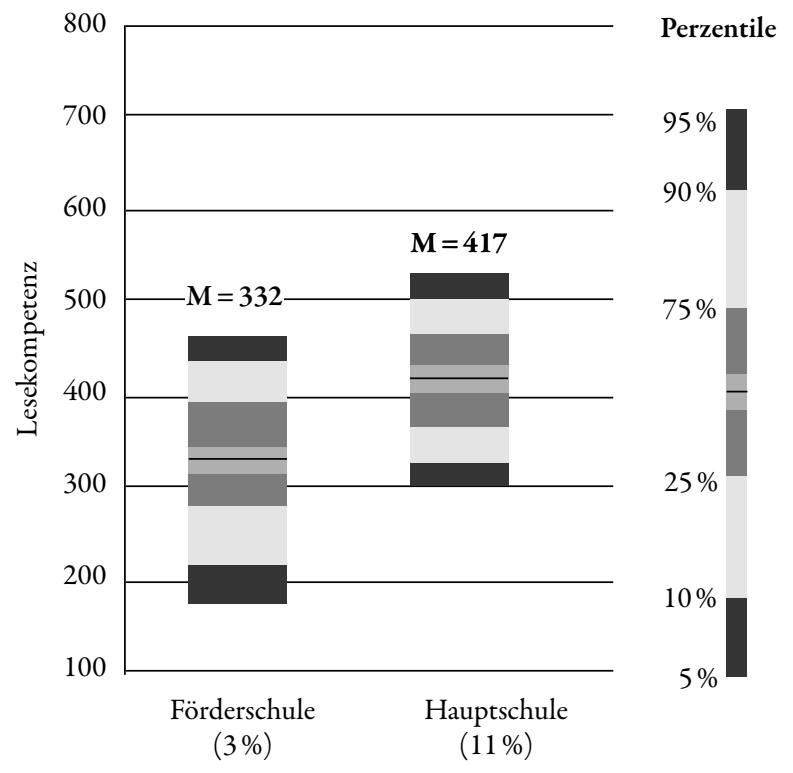

Abb. 43: Lesecompetencies-Stufen der PISA-Zusatzstudie (Quelle: Müller et al. 2017, 187; Auszug)

Die BELLA-Studie berücksichtigt im Leistungsbereich „Leseverständnis“ das Lesen kontinuierlicher und im Leistungsbereich „Informationsentnahme aus Tabellen, Graphiken und Karten“ das Lesen nichtkontinuierlicher (Sach-)Texte (Lehmann/Hoffmann 2009, 40ff.). Die Testsammlung bestand für beide Leistungsbereiche aus schriftsprachlich ausformulierten Fragen zu einerseits zwei Fließtexten und andererseits zwei Sachtexten. Die nachfolgenden Abbildungen $44 \& 45$ verdeutlichen, dass die Testitems das Lesen von multikodalen Texten charakterisieren, welche primär symbolischer und sekundär ikonischer Kodalität zu zumeist metrischen Sachverhalten und deren Relationen zuzuordnen sind (Kapitel 2.2.1). Oder in anderen Worten, das Leseverständnis dieser Texte in der BELLA-Studie berücksichtigt nicht das Lesen von multikodalen Texten und Textkomplexen.

Die Aufgaben erinnern zudem stark an schulische Sachaufgaben mit einer Verengung auf die routinalisierten textuellen Relevanz- und Sinnoptionen mit Tabellen, Graphiken und Karten, die typisch für die schulische Bildung sind. Unter diesem Vorbehalt sind die Ergebnisse in der Tabelle $33 \mathrm{zu}$ interpretieren, welche analog zu der PISA-Zusatzstudie auf dichotome Rasch-Modellierungen beruhen. Die Stichprobe umfasst 4.142 Schüler/innen mit anerkanntem sonderpädagogischen Förderbedarf Lernen, unterrichtet teils an separativen oder schulisch-inklusiven Bildungsprogrammen und teils an berufsqualifizierenden Schulen. 


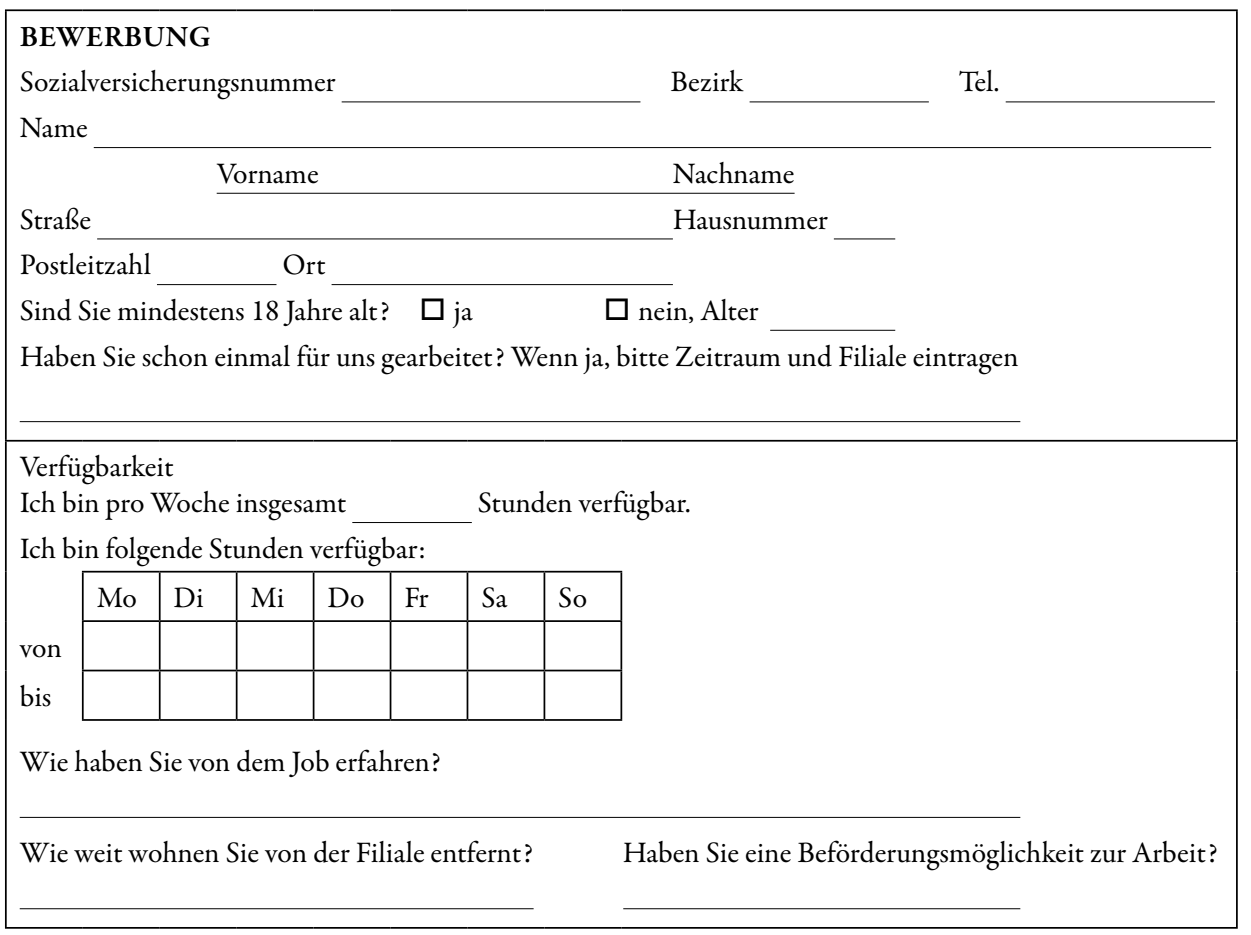

Abb. 44: Beispielaufgabe aus der BELLA-Studie zum Leseverständnis bei Sachtexten (Quelle: Ahrbeck et al. 2009, 41)

Tab. 32: Stichprobenmerkmale der referentiellen BELLA-Studie

\begin{tabular}{lllll}
\hline Bildungsgang & Geschlecht & Klassenstufe & Häufigkeit & gültiger Anteil \\
\hline Schwerpunkt Lernen & männlich & L 7-10 & & \\
$\begin{array}{l}\text { an Förderschulen } \\
\text { Lernen (L) }\end{array}$ & weiblich & L 7-10 & \\
\cline { 2 - 5 } & Gesamt & L 7-10 & 3065 \\
& Gesamt & L 7 & 714 \\
& Gesamt & L 8 & 854 & \\
& Gesamt & L 9 & 818 \\
\hline Schwerpunkt & Gesamt & L 10 & \\
Lernen an Schulen & weiblich & G 7-10 & \\
mit gemeinsamen & Gesamt & G 7-10 & \\
Unterricht (G) & Gesamt & G 7 & 608 \\
& Gesamt & G 8 & 210 \\
& Gesamt & G 9 & 118 \\
& Gesamt & G10 & \\
\hline
\end{tabular}

(Quelle: Lehmann/Hoffmann 2009, 41) 
Tab. 33: Lesecompetencies der BELLA-Studie zu Tabellen, Graphiken und Karten

\begin{tabular}{lcclclc}
\hline Subgruppe & $\mathbf{M}$ & SD & Subgruppe & M & SD & d $\boldsymbol{\Delta}^{*}$ \\
\hline L 7 & 87.65 & 24.20 & G 7 & 90.83 & 20.82 & -0.15 \\
L 8 & 93.96 & 23.10 & G 8 & 98.28 & 24.01 & -0.19 \\
L 9 & 103.07 & 23.97 & G 9 & 102.61 & 23.38 & 0.02 \\
L 10 & 114.53 & 23.56 & G 10 & 107.61 & 23.27 & 0.17 \\
\hline Gesamtgruppe & 100 & 25.00 & Berufsqual. & 117.05 & 24.42 & 0.68 \\
\hline
\end{tabular}

(Quelle: Lehmann/Hoffmann 2009, 44)

Anmerkung: FöS Lernen = Förderschule Lernen; Berufsqual. $=$ berufsqualifizierende Schule

Cronbachs Alpha $=0.83$

${ }^{*}$ Effektstärken der Differenz zwischen den Subgruppen

Die Verteilung der Lesecompetencies zu Tabellen, Graphiken und Karten in der BELLAStudie zeigt einerseits auf, dass diese sukzessiv über die Schuljahre ansteigen. Dieser Trend erweist sich deskriptiv stärker unter den Schüler/innen mit sonderpädagogischem Förderbedarf Lernen in separativen schulischen Bildungsprogrammen: Bei etwa ähnlichem Streuungsverhältnis steigt der Mittelwert um 23,88 Scores im Vergleich zu 16,78 Scores in schulisch-inklusiven Bildungsprogrammen.

In den berichteten Ergebnissen fallen insbesondere die Unterschiede in den Lesecompetencies zu Tabellen, Graphiken und Karten zwischen den Jugendlichen an berufsqualifizierenden Angeboten im Vergleich zur Gesamtgruppe auf. Dieser Unterschied ist mit $d=0.68$ somit mit einem mittleren Effekt statistisch relevant. Es verwundert daher nicht, dass die 14,1\% der Jugendlichen, die die Aufgaben des höchsten Schwierigkeitsbereichs korrekt bearbeiten konnten, zuvorderst unter den Schüler/innen des 10. Schuljahrs in berufsqualifizierenden Bildungsprogrammen zu finden sind. Merkmale, die die Aufgaben der einzelnen Schwierigkeitsbereichen abdecken, konnten nicht aufgefunden werden. Die höchste Schwierigkeitsstufe 5 charakterisiert nach den veröffentlichten Aufgaben wahrscheinlich die textuelle Anforderung, dass für den Teil der ikonischen Texte Relationen aus Sachverhalten erschlossen werden müssen, die aus einer Fülle von Sachverhalten bzw. Distraktoren ausgewählt werden müssen. Die Sachverhalte und ihre Relationen sind alle textuell repräsentiert. Die Autoren berichten andererseits v. a. von keinem statistischen Effekt durch das Geschlecht $(d=0.09)$.

Tab. 34: Schwierigkeitsbereiche der referentiellen BELLA-Studie zu Tabellen, Graphiken und Karten

\begin{tabular}{crc}
\hline Schwierigkeitsbereich & \multicolumn{1}{l}{ Scores } & Verteilung gesamt \\
\hline 1 & $0-68,43$ & 8,3 \\
2 & $68,44-94,47$ & 30,5 \\
3 & $94,48-107,74$ & 26,0 \\
4 & $107,75-$ & 21,1 \\
& 127,99 & \\
5 & über 127,99 & 14,1 \\
\hline
\end{tabular}


Verteilung der

Schülerleistungen
Testaufgaben

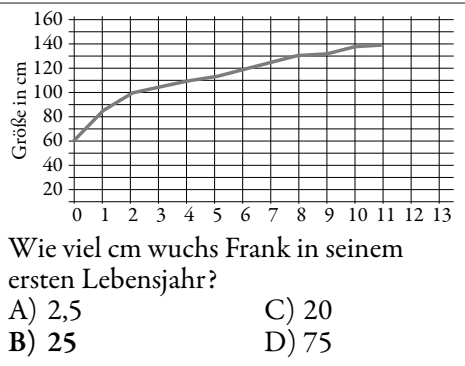

[Siehe Graphik der Aufgabe mit dem Skalenwert 128]

An welchem Geburtstag war Frank doppelt so groß wie bei seiner Geburt?

A) am 2. Geburtstag

B) am 4. Geburtstag

C) am 6. Geburtstag

D) am 8. Geburtstag

Fünf Kinder spielten Pfeilschießen. Je besser sie mit ihrem Pfeil in die Mitte der Zielscheibe trafen, desto mehr Punkte erhielten sie. Die Tabelle zeigt, wie viele Punkte jedes Kind in den ersten fünf Spielen erreicht hat.

\begin{tabular}{|l|r|c|r|r|r|c|}
\hline & 1 & 2 & 3 & 4 & 5 & Zs \\
\hline Peter & 100 & 60 & 75 & 100 & 100 & 435 \\
\hline Ina & 85 & 80 & 90 & 80 & 85 & 420 \\
\hline Rolf & 55 & 75 & 100 & 80 & 90 & 390 \\
\hline Sylvia & 80 & 85 & 90 & 95 & 100 & 450 \\
\hline Udo & 100 & 95 & 85 & 80 & 65 & 425 \\
\hline
\end{tabular}

In welchem Spiel erreichte jedes Kind 80 oder mehr Punkte?
A) im 5. Spiel
C) im 2. Spiel
B) im 3. Spiel
D) im 4. Spiel

Die Abbildung unten zeigt einen Ausschnitt aus einem Stadtplan. Darin sind die Namen der wichtigsten Gebäude eingetragen.

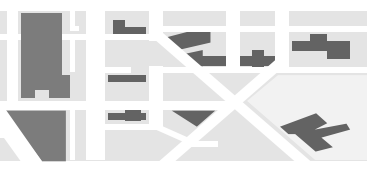

Welches Gebäude liegt am weitesten 1234567 Anzahl der Aufgaben von der Post entfernt?
A) das Krankenhaus
C) das Rathaus

B) der Bahnhof

D) das Kaufhaus Informationsentnahme

Gesamtstichprobe $(\mathrm{N}=4.142)$

Abb. 45: Schwierigkeitsbereiche und Aufgabenbeispiele der BELLA-Studie zu Tabellen, Graphiken und Karten (Quelle: ebd.) 


\subsubsection{Methodische Schlussfolgerungen aus den Referenzstudien}

Sowohl die PISA-Zusatzstudie als auch die BELLA-Studie manifestieren die enttarnte Setzung, dass das Lesen multikodaler Texte und Textkomplexe mit primär ikonischer Kodalität in schulischen Settings nicht explizit berücksichtigt werden muss. Insbesondere multikodale Textkomplexe primär ikonischer Kodalität finden sich nicht in den Aufgabensets anderer Studien zum Lesen der Zielgruppe. Nichtsdestotrotz vertreten diese Studien die Position, dass sie für das Lesen als Voraussetzung für Teilhabe in der Gesellschaft im Singular Auskunft geben. Vor dem Hintergrund der aufgedeckten textuellen Anforderungen an den untersuchten Einfach(st)erwerbstätigkeiten zuvorderst entlang multikodaler Texte und Textkomplexe mit primär ikonischer Kodalität verhärtet sich die dechiffrierte These, dass diese zu primitiv sind, da sie bereits schon immer durch die anerkannten funktionalen Lesecompetencies berücksichtigt sind (vgl. Kapitel 3.4.3 \& Kapitel 5).

Es resultierte begründet das Erkenntnisinteresse, die dechiffrierte Setzung wissenschaftlich kontrolliert nachzugehen, analog zu den Referenzstudien bearbeitet via eine standardisierte Erhebung mittels Testaufgaben mit anschließender multivariater Analyse über eine dichotome Rasch-Modellierung. Die Analyse der Performanzen entlang dieser Testaufgaben sollte die These der a priori eingeschätzten schwachen Kausalität zwischen den Lesecompetencies entlang von Texten des primär symbolischen zu denen des primär ikonischen Codes befragen. Das Auswertungsdesign wurde zuvorderst nach der Strategie konzipiert, zu prüfen, inwiefern sich die primär symbolischen Lesecompetencies als Prädiktor für die primär ikonischen Lesecompetencies eignen. Die Werte der interferenzstatistischen Regression werden als Indiz dafür interpretiert, dass die Lesecompetencies entlang eindeutig primär ikonischer Texte bis Textkomplexe bereits durch die Lesecompetencies entlang primär symbolisch erfasst werden. Für die explorative Erhebung und Analyse wird folglich theoriebasiert keine Unabhängigkeit zwischen den Lesecompetencies kontinuierliche und den Lesecompetencies nichtkontinuierlicher Texte und Textkomplexe prognostiziert.

In einem ersten Schritt galt es, die theoretischen Annahmen in ein angemessenes Instrumentarium für die Lesecompetencies kategorialer ikonischer Texte und Textkomplexe zu überführen. Es waren Aufgaben zu entwickeln, die - wie in Kapitel 6.4.3 noch darzulegen ist - die die Bandbreite an aufgedeckten routinalisiert eingebundenen multikodalen Texte abdecken, die an den untersuchten Einfach(st)arbeitsplätzen entlang der routinalisiert lokale kulturelle Codes eine skillful performance erst ausmachen (vgl. Kapitel 5.3). Die eingebundenen kategorialen Tabellen, Graphiken, Karten usw. werden nicht ohne symbolische Codes auskommen, jedoch nicht durch Fließtexte gedoppelt bis gerahmt. Innerhalb der Auseinandersetzung mit den starken Codes hinter Tabellen bis Diagramme wurde herausgearbeitet, dass symbolische Zeichen in diesen ikonischen Repräsentationen semiotisch eingebettet sind. Sie unterliegen jedoch textstrukturell den ikonischen Textstrukturen und lassen sich in der großen Mehrheit auf einzelne Wörter bis Satzphrasen beschränken (vgl. Kapitel 3.2).

Im besten Fall werden die Aufgaben im Zuge der Erhebung einen Datensatz erzeugen, mit dem via ein probabilistisches Modell zumindest statistisch akzeptabel abbildend auf die Lesecompetencies geschlossen werden kann, die in der Testsituation von den Probanden aktualisiert wurden. Primat gegenüber der Optimierung statistischer Kennwerte liegt daher in der Kontrolle der literalen situativen Einbettung der im Grunde simulierten literalen events. Die präferierte multivariate Analysemethode dieses Datensatzes ist die der dichotomen Rasch-Modellierung. Sie hat sich unlängst in diversen kognitiven Schulleistungsvergleichstests wie z. B. der PISA-Studie und so auch in der Referenzstudie der PISA-Zusatzstudie bewährt (Müller et al. 2017, 182ff.). 
Ihr entscheidender Vorteil ist es, dass die Schwierigkeitsgrade der Items nicht a priori festgelegt in die Codierregeln einfließen. Stattdessen erlaubt eine Rasch-Modellierung, dass die Performanzen der Stichprobe in ein Verhältnis zu den Schwierigkeitsgraden der Items gesetzt werden können. Oder in anderen Worten, Rasch-Modellierungen suchen zum einen Items bis hin zu Itemgruppen, die ähnlich häufig falsch bzw. richtig bearbeitet wurden, um im Anschluss für jene Items und Itemgruppen Schwierigkeitsgrade zu bestimmen. Items, die nur wenige Befragte korrekt bearbeiten, erhalten folglich einen höheren Testwert als Items, die nahezu von allen korrekt gelöst wurden (Moosbrugger 2008).

Es wird folglich zu zeigen sein, ob gar ein probabilistisches Modell spezifische Lesecompetencies entlang von Texten und Textkomplexen der primär ikonischen Kodalität empfehlen. In zweiter Reihe kann erst befragt werden, ob die Teilnehmenden bei z. B. bestimmten Lokal- bis Globalzeichen den routinalisiert lokalen kulturellen Codes entsprechend probabilistisch häufiger skillful performen, ergo, welche Lokal- bis Globalzeichen für bestimmte Schüler/innen gegenüber anderen Schüler/innen einfacher und welche schwieriger zu identifizieren und dekodieren sind.

\subsubsection{Material}

Das Untersuchungsdesign der Teilstudie sah für die Erhebung eine standardisierte schriftliche Befragung durchgeführt als Gruppenbefragung vor (Jankisz/Moosbrugger 2008; Raithel 2008), bei der die nachfolgenden Materialien Einsatz fanden: Fragebogen personenbezogener Daten, Diagramm zur Generierung eines Codes zur Pseudo-Anonymisierung und Testaufgaben multikodaler Textualität primär ikonischer Kodalität sowie primär symbolischer Kodalität (vgl. Tabelle 35). Ersterer Fragebogen sah vor, einige personenbezogene Daten zu erheben, die der Analyse der Zusammensetzung der Stichprobe dienen. In der späteren Analyse soll ein Stichprobenvergleich zu den Referenzstudien ermöglicht werden. Empfehlungen der Gestaltung von Fragebogen für die Zielgruppe entsprechend (Gaupp et al. 2006) wurde eine layouttechnische Klarheit sowie eine weitgehende Reduktion von insbesondere komplexen kontinuierlichen Erklärtexten und von Antwortmöglichkeiten angestrebt. Anstelle weiterer Antwortmöglichkeiten wurde ein freies Antwortfeld eingepflegt. Diese Empfehlungen prägten gleichermaßen die Gestaltung des Diagramms für die Generierung eines Pseudo-Kodes. Vor dem Hintergrund, dass die Testaufgaben über mehrere Tage hinweg bearbeitet werden konnten, wurde kein Pseudo-Kode je Teilnehmer/in vorgegeben, sondern von diesen aus einer Kombination ihrer personenbezogenen Daten selbst erzeugt. Der Vorteil gegenüber fest vorgegebenen PseudoKodes ist, dass diese auch bei Verlust wieder von den Teilnehmer/innen selbst unmittelbar rekonstruiert werden können.

Im Weiteren wird der Fokus auf der Darlegung der Entwicklung der Testaufgaben multikodaler Texte und Textkomplexe primär ikonischer Kodalität liegen, einschließlich der Durchführungsanweisung und dem Illustrationsmaterial. Im nachfolgenden Unterkapitel wird zuvor stark komprimiert auf die Testaufgaben für kontinuierliche Texte und Textkomplexe eingegangen. Für diese Testaufgaben konnte ein geeignetes bewährtes Verfahren gefunden werden, sodass keine eigenen Items entwickelt werden mussten.

\subsubsection{Testaufgaben zu multikodalen Texten primär symbolischer Kodalität}

Mit dem Ziel ökonomisch zu untersuchen, welche Fließtext-Lesecompetencies die Zielgruppe innerhalb der literalen Praxen der Schule hat, wurde nach einem theoretisch fundierten, praktisch erprobten und standardisierten Diagnoseinstrument Ausschau gehalten. Während es für die ersten Schuljahre bereits eine Vielzahl entsprechender Testverfahren gab, liegt seit 2012 mit dem im Hogrefe-Verlag erschienen Verfahren Lesen 8-9 ein Instrument vor, das neben techni- 
schen Lesefertigkeiten symbolischer Zeichen die Kohärenzbildung in Fließtexten umfasst (Bäuerlein et al. 2012, 11). Die technischen Lesefertigkeiten werden innerhalb des Verfahrens als eine grundlegende Voraussetzung für das sinnentnehmende Lesen von Fließtexten verstanden. Erhoben werden sie als Fertigkeiten, Grapheme mit einer angemessenen Geschwindigkeit und Genauigkeit in Phoneme übersetzen zu können, ohne dabei eine große Anstrengung aufwenden zu müssen (ebd., 12). Die Kohärenzbildung dagegen gilt als aktiver Rekonstruktionsprozess des Sinngehaltes geschriebener Wörter, Sätze, Absätze und Texte der Lesenden unter Einbeziehung ihres Weltwissens. Dabei können verschiedene Ebenen des Textverständnisses bzw. Formen der Textrepräsentation unterschieden werden. Auf Satzphrasenebene müssen lokale Kohärenzen gebildet werden, wohingegen bei komplexen Sätzen, Absätzen bis hin zur Gesamttextebene die Bildung globaler Kohärenzen erforderlich ist (ebd., 13ff.). Das Verfahren kann mit einer angegebenen reinen Bearbeitungszeit von unter 40 Minuten ökonomisch als Gruppentests bei Schüler/innen der Jahrgangsstufen 8 und 9 eingesetzt werden.

Die dargelegten Maßnahmen bis Kennwerte zur Einhaltung der Gütekriterien sprach für den Einsatz insbesondere des Untertests „Textverständnis“ zur Analyse der Lesecompetencies entlang der globalen Kohärenzbildung bei Fließtexten (ebd., 21ff.):

- Durchführungs-, Auswertungs- und Interpretationsobjektivität durch u.a. einen Durchführungsleitfaden, durch wörtlich vorgegebene und einzuhaltende Instruktionen, durch festgelegte Bearbeitungszeiten je Aufgabe bzw. durch die Auswertung mittels Normstichprobenergebnissen ( 945 Schüler/innen aus sieben Bundesländern);

- interne Reliabilität von Cronbachs Alpha =.87;

- Retest-Reliabilität von $r=.84$;

- inhaltliche Validität durch Einbindung von Texten unterschiedlicher Genres und von Verständnisfragen unterschiedlicher Schwierigkeitsgrade;

- Konstruktvalidität: Subtest korreliert bedeutsam mit anderen Leseverständnistests $(r=.59$; $r=.57)$;

- Kriteriumsvalidität: Absicherung durch Außenkriterien wie die Deutschnote und Einschätzung durch Lehrkräfte des Faches Deutsch.

Der Untertest zur Erfassung des Textverständnisses umfasst zwei Texte. Den Schüler/innen wird neben einem 706 Wörtern langen expositorischen Text ein 897 Wörter langer narrativer Text vorgelegt, um anschließend jeweils 19 Verständnisfragen zu bearbeiten. Es werden jeweils fünf Antwortalternativen vorgegeben, darunter je eine richtige. 21 der gestellten Fragen erfordern die Bildung lokaler Kohärenzen und 17 Fragen die Bildung globaler Kohärenzen (ebd., 18).

\subsubsection{Testaufgaben zu multikodalen Texten primär ikonischer Kodalität - von theoriebasierten Selektions- bis Durchführungskriterien}

Bei der Entwicklung der multikodalen Testaufgaben mit primär ikonischer Kodalität wurde entlang dem favorisierten Lesekompetenzansatz und Arbeitsmodell der Zeichenverarbeitung begründet, Abstand von einer vollständigen Aufösung von generalisierbaren Dispositionen im Lesenden zu nehmen. Gleiches gilt für den Ansatz einer mentalen Modellierung ausschließlich explizit-konzeptioneller kognitiver Verarbeitungsprozesse, die entlang abzulehnender textstrukturell inhärenter und somit für jegliche Lesesituationen generalisierbare hierarchische Komplexitätsgrade erschlossen werden könnten. Die Selektion der Texte und Textkomplexe in den Testunterlagen war demgemäß nicht primär davon geprägt, alle Sets von starken Codes der einzelnen multikodalen Texte in den Blick zu nehmen. Oder in anderen Worten, die semiotisch abgeleiteten textstrukturellen Komplexitätsgrade der jeweiligen inhärenten Relationen 
wie z. B. entlang der Hierachie der Listenstruktur bei Tabellen sensu Mosenthal und Kirsch (1990, 636ff.) wurden lediglich als sekundäres Kriterium für die Aufnahme von Texten in der standardisierten Erhebung herangezogen. Die Listenstrukturen der single lists, der combinded lists, der intersected lists und der nested lists wurde bei der Auswahl der Testaufgaben dahingehend zurate gezogen, um sicherzustellen, dass die komplette Bandbreite berücksichtigt wird (vgl. Tabelle 36). Eine umfassend auf Optimierung textstruktureller Merkmale ausgerichtete Selektion von Items war folglich theoriebasiert begründet und nicht angestrebt.

STRUKTUREN: Seite 1

Person-Kode:
Textcode:

\begin{tabular}{|c|c|c|c|}
\hline \multicolumn{4}{|c|}{ routinalisierte lokale kulturelle Codes } \\
\hline \multirow{7}{*}{ 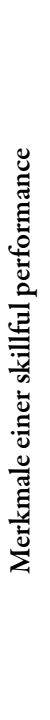 } & & ja & nein \\
\hline & $\begin{array}{l}\text { Textgebrauch (eine skillful performance des Lesens von Texten/Textkomplexen } \\
\text { macht sich an dem Erlernen fest, wie routiniert (z. B. Arbeitsplatz) eingesetzt } \\
\text { Texte und Textkomplexe bearbeitet werden) }\end{array}$ & & \\
\hline & $\begin{array}{l}\text { Lernvorlage (eine skillful performance des Lesens von Texten/Textkomplexen } \\
\text { macht sich an der Behaltensleistung routiniert (z. B. Arbeitsplatz) relevanten } \\
\text { Faktenwissens fest) }\end{array}$ & & \\
\hline & $\begin{array}{l}\text { Merkhilfe (eine skillful performance des Lesens von Texten/Textkomplexen } \\
\text { macht sich unmittelbar an der selbstständigen Ausführung von ansonsten } \\
\text { häufig routinierten Aufgaben mit Zeitdruck fest) }\end{array}$ & & \\
\hline & $\begin{array}{l}\text { Organisationshilfe (eine skillful performance des Lesens von Texten/Text- } \\
\text { komplexen macht sich an der selbstständigen Ausführung von ansonsten häufig } \\
\text { routinierten Aufgaben ohne Zeitdruck fest) }\end{array}$ & & \\
\hline & $\begin{array}{l}\text { Kontrolle (eine skillful performance des Lesens von Texten/Textkomplexen } \\
\text { macht sich an der ökonomisierten (z. B.betriebsinternen) Kontrolle von Status- } \\
\text { oder Verlaufswerten fest) }\end{array}$ & & \\
\hline & $\begin{array}{l}\text { Weitergabe (eine skillful performance des Lesens von Texten/Textkomplexen } \\
\text { macht sich an der ökonomisierten (z. B. betriebsinternen) Weitergabe von } \\
\text { Status- oder Verlaufswerten fest) }\end{array}$ & & \\
\hline
\end{tabular}

\begin{tabular}{|c|c|c|c|}
\hline \multicolumn{4}{|c|}{ routinalisierte multikodale Texte und Textkomplexe } \\
\hline \multirow{7}{*}{ 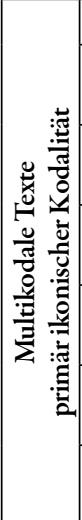 } & & ja & nein \\
\hline & $\begin{array}{l}\text { Schaubilder/graphische Darstellungen (bildl. Darstellung von quant. Daten/ } \\
\text { Bsp.: Diagramme, Graphen) }\end{array}$ & & \\
\hline & $\begin{array}{l}\text { Tabellen (Spalten- und Zeilendarstellung von quant. \& qualit. Daten/ } \\
\text { Bsp.: Stundenplan, Aufstellungen) }\end{array}$ & & \\
\hline & $\begin{array}{l}\text { Diagramme (bildliche Darstellung von qualit. Daten/ } \\
\text { Bsp.: Flussdiagramm, Streudiagramm) }\end{array}$ & & \\
\hline & $\begin{array}{l}\text { Karten (Darst. geographischer, sozialer oder physischer Beziehung/ } \\
\text { Bsp.: Landkarten, Fluchtpläne, Netzwerke) }\end{array}$ & & \\
\hline & $\begin{array}{l}\text { darstellende Bilder \& Fotos (Bsp.: realitätsnahe/-getreue Abbildungen, } \\
\text { Illustrationen) }\end{array}$ & & \\
\hline & $\begin{array}{l}\text { Ergänzung: Diskontinuierlicher Text ist ein Hypertext? } \\
\text { (Texte, die in unterschiedlichen Abfolgen gelesen werden können.) }\end{array}$ & $\square$ ja & $\square$ nein \\
\hline
\end{tabular}




\begin{tabular}{|c|c|c|c|}
\hline \multicolumn{4}{|c|}{ routinalisierte multikodale Texte und Textkomplexe } \\
\hline \multirow{8}{*}{ 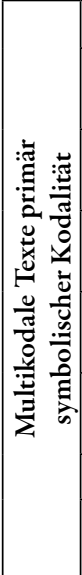 } & & ja & nein \\
\hline & $\begin{array}{l}\text { Beschreibung (physisch-räumliche Eigenschaften/Bsp.: Produktionsbeschrei- } \\
\text { bungen) }\end{array}$ & & \\
\hline & $\begin{array}{l}\text { Erzählung (zeitliche Eigenschaften/Bsp.: Märchen, Belletristik, Unfallbericht, } \\
\text { Protokolle) }\end{array}$ & & \\
\hline & $\begin{array}{l}\text { Darlegung (Darstellung von Konzepten \& Konstrukten/Bsp.: Lexikon-, Lehr- } \\
\text { buchartikel, Analysen) }\end{array}$ & & \\
\hline & $\begin{array}{l}\text { Argumentation (Beziehungen, Überzeugungsabsicht/Bsp.: Gutachten, } \\
\text { Gerichtsurteil, Gegenüberstellung, Kommentar) }\end{array}$ & & \\
\hline & $\begin{array}{l}\text { Anweisung (Verfahren, Regeln, Vorschriften, Gesetze; Bsp.: Garantiebeschr., } \\
\text { Kochrezept, Störungsbehebung) }\end{array}$ & & \\
\hline & Dokumente (Standardisierungen für Archivierung/Bsp.: abstracts) & & \\
\hline & $\begin{array}{l}\text { Ergänzung: Kontinuierlicher Text ist ein Hypertext? } \\
\text { (Texte, die in unterschiedlichen Abfolgen gelesen werden können.) }\end{array}$ & $\square_{\mathrm{ja}}$ & $\square$ nein \\
\hline
\end{tabular}

\begin{tabular}{|c|c|c|c|}
\hline \multicolumn{4}{|c|}{ Textgattung nichtkontinuierlicher Texte } \\
\hline & & $\mathrm{ja}$ & nein \\
\hline \multirow{5}{*}{ 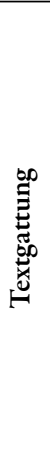 } & $\begin{array}{l}\text { Formular (formatierte, teils kodierte Texte zur Datenerhebung/Bsp.: Steuerer- } \\
\text { klärung, behördliche Anträge, Personalbogen, statistische Fragebögen) }\end{array}$ & & \\
\hline & $\begin{array}{l}\text { Informationsblatt (formatierte Weitergabe von Daten/Bsp.: Fahrpläne, Preislis- } \\
\text { ten, Kataloge, TV-Programm, Speisekarten, Kochrezepte) }\end{array}$ & & \\
\hline & $\begin{array}{l}\text { Aufruf/Anzeige (Texte, die auf Überzeugung, Aufmerksamkeit oder Hand- } \\
\text { lungen abzielen/Bsp.: Prospekte, Anzeigen, Flyer, Wahlplakate, Einladungen, } \\
\text { Aufrufe, Warnungen, Bekanntmachungen) }\end{array}$ & & \\
\hline & $\begin{array}{l}\text { Gutschein (Nachweis des Anspruchs auf bestimmte käufliche Leistungen/Bsp.: } \\
\text { Fahrschein, Rechnung, Kassenbeleg, Quittungen, Eintrittskarte) }\end{array}$ & & \\
\hline & $\begin{array}{l}\text { Bescheinigung (schriftliche Bestätigung für Verträge, Vereinbarungen oder } \\
\text { erbrachte Leistungen durch eine autorisierte Stelle/Bsp.: Schulzeugnisse, Dip- } \\
\text { lome, Verträge, Garantien) }\end{array}$ & & \\
\hline
\end{tabular}

Abb. 46: Primäres Selektionskriterium bei der Auswahl der Testaufgaben: routinalisierte lokale kulturelle Codes \& textuelle Gegenstände

Als primäres Selektionskriterium wurde stattdessen, wie bereits in der übergeordneten methodischen Debatte benannt und begründet (vgl. Kapitel 3.5 \& 6.4.2), die routinalisierten lokalen kulturellen Codes ausgewählt, die aus den untersuchten sozialen Praxen an Einfach(st)erwerbstätigkeiten hervorgingen (vgl. Kapitel 5.3). Diese situativ-kontextuelle Einbindung der multikodalen Texte mit primär ikonischer Kodalität erlaubt sich erst dem anzunähern, was eine skillful performance in den literalen Praxen ausmacht (vgl. Abbildung 46; vgl. Tabelle 35). Unter diesen Merkmalen routinalisierter textueller Relevanz- und Sinnoptionen mit diesen Texten für eine skillfulperformance waren aus dem Forschungsinteresse heraus insbesondere die entlang der Merkhilfe, der Organisationshilfe, der Kontrolle und der Weitergabe von Interesse. Sowohl die Pendants entlang der Lernvorlage als auch der Textgebrauch charakterisieren eine große Nähe zu der literalen Praktik in der Schule mit multikodalen Texten und Textkomplexen, die hier empirisch befragt wer- 
den sollen (vgl. Tabelle 27). Das in jener Auswahl routinalisierter textueller Relevanz- und Sinnoptionen von den Probanden aktualisierte knowing how beim Gebrauch und knowing that bei der Anwendung von ikonischen Texten und Textkomplexen bilden die Grundlage für die weitere Analyse. Ziel der Analyse ist unter Rückgriff auf dem (de)komponierten Arbeitsmodell die aktualisierten explizit-kognitiven bis implizit-embodied Verarbeitung von ikonischen Zeichen.

Im Folgenden wird die entlang beider Selektionskriterien kontrollierte Auswahl an multikodalen Testaufgaben mit primär ikonischer Kodalität, auch nicht exemplarisch, in z. B. ihrer semiotischen Struktur begründet vorgestellt. Argument ist, dass die ausgewählten Testaufgaben auf den textuellen Fundstücken der untersuchten Einfach(st)erbwerbstätigkeiten nahezu basieren, welche im Laufe dieser Niederschrift bereits größtenteils zur Illustration und Erklärung vorgestellt wurden. Zur Übersicht finden sich Seitenverweise zu den relevanten textuellen Fundstücken in den Tabelle 35 \& Tabelle 36 eingepflegt. Die dahinterliegenden Items werden innerhalb der Debatte um die Codierregeln in Kapitel 6.1.3.3 transparent gemacht. Aus diesen Dokumentationen geht hervor, dass die ausgewählten Testaufgaben die angestrebte Bandbreite routinalisierter lokaler kultureller Codes an den untersuchten Einfach(st)erwerbstätigkeiten abdecken: Während die Texte „Notfallverhalten“, „Serviceorganisation“ und „Lagerdokumentation“ jeweils einem Merkmal zugeordnet werden konnten, die eine skillful performance markieren. Die Textkomplexe „Wohngeldantrag“, „Montagedokumentation“ und „Autoverleih“ konnten jeweils zwei dieser Merkmale zugeordnet werden (vgl. Tabelle 35).

Tab. 35: Verteilung der Testaufgaben entlang des primären Selektionskriterium routinalisierte lokale kulturelle Codes

\begin{tabular}{|c|c|c|c|c|c|c|c|}
\hline \multirow[b]{2}{*}{$\begin{array}{l}\text { multikodale } \\
\text { Testaufgabe }\end{array}$} & \multirow[b]{2}{*}{$\begin{array}{l}\text { Text/ } \\
\text { Textkomplex }\end{array}$} & \multirow[b]{2}{*}{$\frac{\tilde{E}}{\tilde{E}} \cdot \overrightarrow{\tilde{\omega}}$} & \multicolumn{5}{|c|}{ Textgebrauch } \\
\hline & & & 志总 & 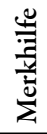 & 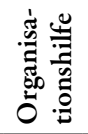 & 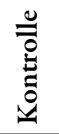 & 离 \\
\hline Wohngeldantrag & $\begin{array}{l}\text { Antragsformular; Tabelle \& } \\
\text { Dokumente (Mietvertrag) }\end{array}$ & & & & & $\mathrm{x}$ & $\mathrm{x}$ \\
\hline Notfallverhalten & Flussdiagramm & $(102)$ & & $\mathrm{x}$ & & & \\
\hline Serviceorganisation & Raumplan & & & & $\mathrm{x}$ & & \\
\hline Lagerdokumentation & Tabelle (Lieferschein) & $(105)$ & & & & $\mathrm{x}$ & \\
\hline $\begin{array}{l}\text { Montage- } \\
\text { dokumentation }\end{array}$ & $\begin{array}{l}\text { Tabellen (Empfangsschein; } \\
\text { Fehlerdokumentation; Fehlerliste) }\end{array}$ & $(97 ; 99)$ & & & & $\mathrm{x}$ & $\mathrm{x}$ \\
\hline Autoverleih & $\begin{array}{l}\text { Tabellen \& graphische Darstel- } \\
\text { lungen (Fahrzeugprotokolle) }\end{array}$ & (75) & & & $\mathrm{x}$ & $\mathrm{x}$ & \\
\hline
\end{tabular}




\begin{tabular}{|c|c|c|c|}
\hline \multicolumn{4}{|c|}{ Listenstruktur } \\
\hline & & & zutreffend \\
\hline \multirow{21}{*}{ 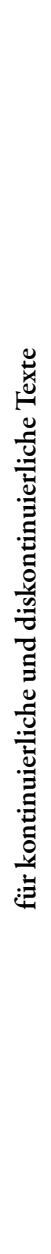 } & \multirow{5}{*}{$\begin{array}{l}\text { Die gesuchte } \\
\text { Information } \\
\text { ist ein/e ... }\end{array}$} & Person, Tier, Ort, Objekt. & $\square \rightarrow 1-5$ \\
\hline & & Betrag, Zeitangabe, Merkmal, Handlung, Position. & $\square \rightarrow 1-5$ \\
\hline & & Art und Weise, Ziel, Zweck, Bedingung, prädikatives Adjektiv. & $\square \rightarrow 2-5$ \\
\hline & & Grund, Ergebnis, Ursache, Beweis, Ähnlichkeit, Muster. & $\square \rightarrow 3-5$ \\
\hline & & Entsprechung, Differenz, Motiv. & $\square \rightarrow 5$ \\
\hline & \multirow{4}{*}{$\begin{array}{l}\text { Die gesuchte } \\
\text { Information } \\
\text { lässt sich } \\
\text { finden ... }\end{array}$} & $\begin{array}{l}\text { als wortwörtliche bzw. synonyme Entsprechung bzw. gar nicht, da es } \\
\text { eine persönliche Angabe ist (locating). }\end{array}$ & $\square \rightarrow 1-5$ \\
\hline & & $\begin{array}{l}\text { als Entsprechung nach dem Erkennen einer Bedingung oder Neudar- } \\
\text { stellung in einem anderen Informationstypus (cycling). }\end{array}$ & $\square \rightarrow 2-5$ \\
\hline & & $\begin{array}{l}\text { mit dem Einsatz von Spezialwissen oder mittels Erkennen einer über- } \\
\text { einstimmenden Beziehung (integrating). }\end{array}$ & $\square \rightarrow 3-5$ \\
\hline & & $\begin{array}{l}\text { nach dem Erkennen einer komplexen textbasierten Überstimmung, } \\
\text { z. B. Gegenüberstellung, Kategorisierung (generating). }\end{array}$ & $\square \rightarrow 5$ \\
\hline & \multirow{3}{*}{$\begin{array}{l}\text { Die gesuchte } \\
\text { Information } \\
\text { ist verteilt ... }\end{array}$} & innerhalb eines Absatzes. & $\square \rightarrow 1-5$ \\
\hline & & innerhalb mehrerer Absätze eines Dokuments. & $\square \rightarrow 2-5$ \\
\hline & & über mehrere Dokumente. & $\square \rightarrow 3-5$ \\
\hline & \multirow{5}{*}{$\begin{array}{l}\text { Die gesuchte } \\
\text { Information } \\
\text { ist mehr } \\
\text { oder weniger } \\
\text { triftig, da ... }\end{array}$} & keine ähnlichen, überschneidenden Informationen im Dokument. & $\square \rightarrow 1-5$ \\
\hline & & $\begin{array}{l}\text { synonyme, bzw. eindeutig ersichtliche, überschneidende Informatio- } \\
\text { nen, jedoch in einem anderen Abschnitt. }\end{array}$ & $\square \rightarrow 1-5$ \\
\hline & & $\begin{array}{l}\text { plausible, überschneidende Informationen in demselben Abschnitt; } \\
\text { stehen jedoch in einem anderen Zusammenhang. }\end{array}$ & $\square \rightarrow 2-5$ \\
\hline & & plausible, überschneidende Informationen in demselben Abschnitt. & $\square \rightarrow 4-5$ \\
\hline & & $\begin{array}{l}\text { sehr plausible, stark überschneidende Informationen in demselben } \\
\text { Abschnitt oder durch den Kontext. }\end{array}$ & $\square \rightarrow 5$ \\
\hline & \multirow{4}{*}{$\begin{array}{l}\text { Die gesuchte } \\
\text { Information } \\
\text { ist ... }\end{array}$} & kurz (kurzer Abschnitt) und übersichtlich gegliedert. & $\square \rightarrow 1-5$ \\
\hline & & $\begin{array}{l}\text { mittelmäßig lang (mehrere Abschnitte bis drei Seiten) und übersicht- } \\
\text { lich gegliedert. }\end{array}$ & $\square \rightarrow 2-5$ \\
\hline & & lang (mehr als drei Seiten) und übersichtlich gegliedert. & $\square \rightarrow 3-5$ \\
\hline & & lang (mehr als drei Seiten) und komplex. & $\square \rightarrow 3-5$ \\
\hline
\end{tabular}




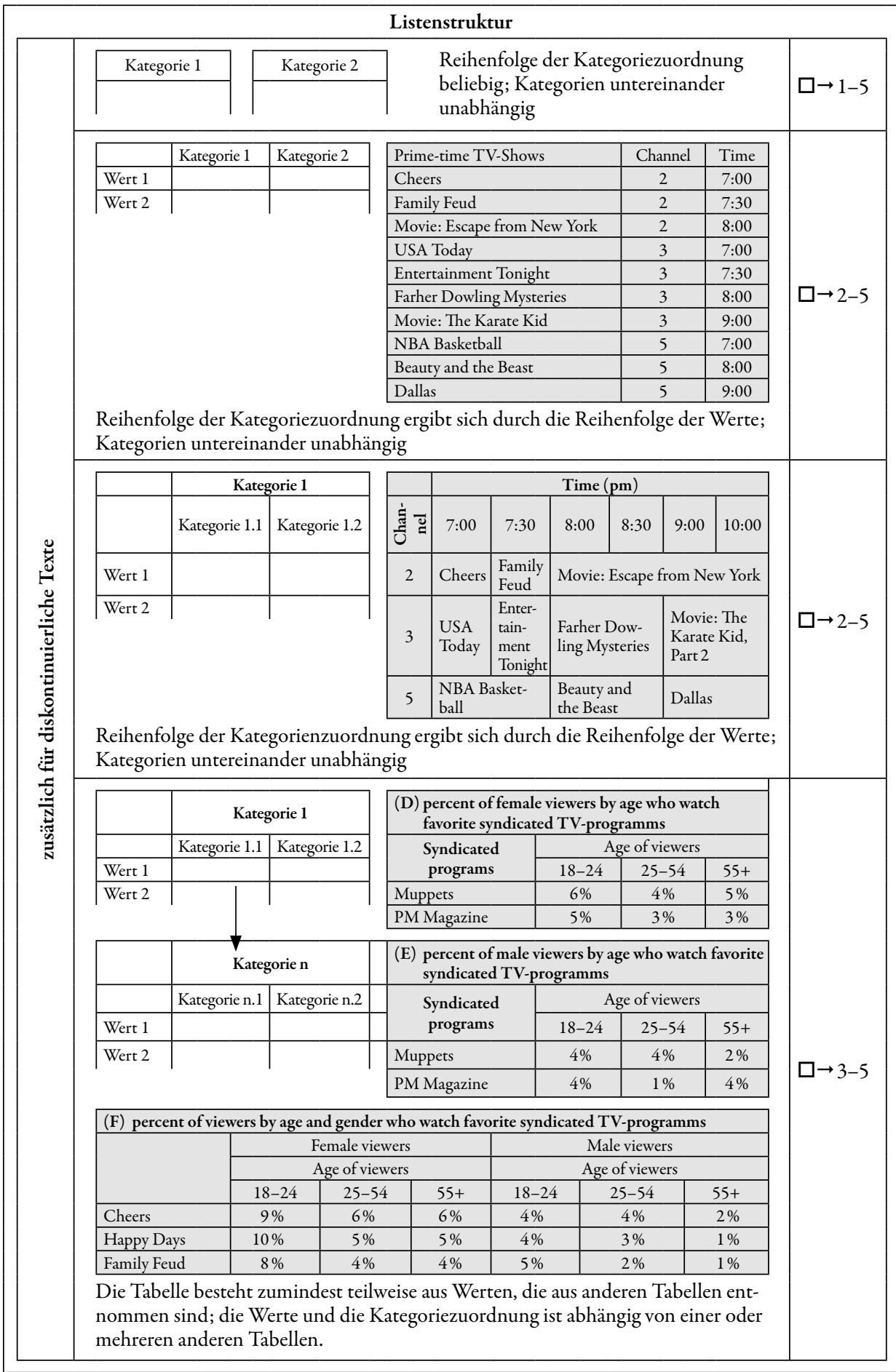

Abb. 47: Sekundäres Selektionskriterium bei der Auswahl der Testaufgaben: Listenstruktur 
Zur Wahrung des Datenschutzes wurden diese textuellen Fundstücke für die standardisierte Befragung imitiert sowie sämtliche Hinweise zur Herkunft eliminiert. Analog wurde die routinalisierten textuellen Relevanz- und Sinnoptionen mit diesen Texten übernommen, obgleich verkürzt bis simplifiziert. Bei dieser situativ-kontextuellen Adaption wurde Wert daraufgelegt, dass notwendige Einweisungen in die Lesesituation ohne jeglichen Fließtext auskommen; entsprechend den literalen events an den untersuchten Einfach(st)erwerbstätigkeiten. Anstelle der Einführung in die Testaufgaben mit je einem kontinuierlichen Text wurden verbale Einweisungen adaptiert. Die Lehrkräfte, die die Testsituationen durchführten, erhielten für diesen Zweck Durchführungsanweisungen, welche neben den Materialien, Vorbereitungen für die Durchführung auch Satzbausteine enthielten, mit welchen die Situation umschrieben werden konnte, in welche die Texte bzw. Textkomplexe in der Testsituation simuliert eingebunden sind. Das Verstehen dieser Situation war Ziel der Einführung und eben nicht die Texte als solche. Ziel dieser Maßnahmen war es, die Bedingungen on-the-job nicht zu „verschulen“, denn, wie in Kapitel 3.2.6 dargelegt, erhalten die Mitarbeitenden keine kontinuierlichen Einweisungstexte.

Aufgrund der Relevanz jener situativ-kontextuellen Einbettung wurde eigens im vorausgehenden Lehr- und Forschungsprojekt eine Explorationsstudie durchgeführt, welche eine Optimierung der verbalen Einweisungen zum Kern hatte. Jugendliche wurden befragt, wie sie die Situation und den Auftrag entlang der Testaufgaben verstehen. Als Ergebnis wurde für die multikodale Textaufgabe "Autoverleih“ eigens ein Video produziert und mehrere Adaptionen der sprachlichen Einweisung vorgenommen. Jedoch war auch in der Erhebung vorgesehen, dass die Nachvollziehbarkeit der Situation gesichert werden muss, weswegen Rückfragen durch die Probanden erlaubt waren.

Tab. 36: Verteilung der Testaufgaben entlang des sekundären Selektionskriterium Listenstruktur

\begin{tabular}{|c|c|c|c|c|c|c|}
\hline $\begin{array}{l}\text { multikodale } \\
\text { Testaufgabe }\end{array}$ & $\begin{array}{l}\text { Text/ } \\
\text { Textkomplex }\end{array}$ & $\frac{\frac{\vec{n}}{\bar{d}}}{\frac{\vec{d}}{\tilde{a}}}$ & 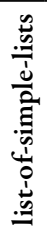 & 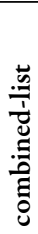 & 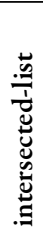 & 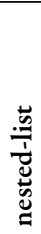 \\
\hline Wohngeldantrag & Antragsformular; Tabelle \& Dokumente (Mietvertrag) & & & & & $\mathrm{x}$ \\
\hline Notfallverhalten & Flussdiagramm & & & $\mathrm{x}$ & & \\
\hline Serviceorganisation & Raumplan & & & & $\mathrm{x}$ & \\
\hline Lagerdokumentation & Tabelle (Lieferschein) & & & & $\mathrm{x}$ & \\
\hline Montagedokumentation & Tabellen (Empfangsschein; Fehlerdokumentation; Fehlerliste) & & & & & $\mathrm{x}$ \\
\hline Autoverleih & Tabellen \& graphische Darstellungen (Fahrzeugprotokolle) & & & & & $\mathrm{x}$ \\
\hline
\end{tabular}

*Anmerkungen (vgl. Kapitel 3.4.2):

1 „Informationen ermitteln“: Informationen eines Sachverhalts, die mehr oder weniger tief in den Text oder Textkomplex eingebettet sind und mehr oder weniger klar differenzierbar innerhalb des Textes oder Textkomplexes lokalisierbar sind, sind zu identifizieren und zu dekodieren. Diese Informationen sind folglich dem Text oder Textkomplex inhärent und bedürfen nicht zwingend weder externen Faktenwissens noch einer tiefen Auseinandersetzung mit dem Text (= im Sinne der Herstellung „lokaler Kohärenz“).

2 „Textbezogenes Interpretieren“: Zusammenhänge zwischen Sachverhalten (Begriffe, Konzepte usw.), die mehr oder weniger mehrdeutig im Text oder Textkomplex und mehr oder weniger differenziert und strukturiert repräsentiert werden, sind zu identifizieren, dekodieren und zu deuten. Diese Zusammenhänge sind folglich dem Text oder Textkomplex inhärent und bedürfen nicht zwingend externen Faktenwissens, jedoch einer mehr oder weniger tiefen Auseinandersetzung mit dem Text oder Textkomplex als Ganzes (= im Sinne der Herstellung „globaler Kohärenz").

3 „Reflexion und Bewertung“ von Informationen eines mehr oder weniger umfangreich textuell repräsentierten Sachverhalts oder von Zusammenhängen zwischen mehr oder weniger umfangreich textuell repräsentierten Sachverhalten unter Rückgriff auf mehr oder weniger spezifisches externes Faktenwissen (= im Sinne der Herstellung „lokaler“ und "globaler Kohärenz") 


\subsubsection{Codierregeln zur Auswertung der Testaufgaben multikodaler Texte und Textkomplexe primär ikonischer Kodalität}

In der nachfolgenden Tabelle 37 ist die dichotome Einschätzung der als richtig bzw. als falsch bewerteten Angaben entlang der einzelnen standardisierten Befragungen zusammengefasst. Da zuvorderst die Verarbeitung der starken Codes im Spiegel der routinalisierten textuellen Relevanz- und Sinnoptionen beurteilt werden sollte, begründet sich, dass Rechtschreibfehler, Zahlendreher oder einzelne Auslassungen nicht als falsch interpretiert werden. Die Fehlerkategorien lassen nicht vermuten, dass die semiotische Struktur hinter den Tabellen bis Diagrammen nicht verstanden worden wären. Wurden dagegen die für eine skillfulperformance zu verarbeiteten Entitäten und Relationen zwischen Entitäten nicht markiert bzw. notiert, wurde dies dichotom als Fehler kategorisiert.

Tab. 37: Codierregeln zur standardisierten Befragung „Wohngeldantrag“

\begin{tabular}{|c|c|c|c|}
\hline Item & Beschreibung & $\begin{array}{l}\text { Richtig } \\
\text { (Beispiele für Ausnahmen) }\end{array}$ & $\begin{array}{l}\text { Falsch } \\
\text { (Beispiele aus den Daten) }\end{array}$ \\
\hline W_Art_1 & Antragsart & „Erstantrag“ & \\
\hline W_FNam_2 & Familienname & realistische Eintragung & (Mustermann) \\
\hline W_Gnam_3 & Geburtsname & $\begin{array}{l}\text { nicht ausgefüllt; anderer oder gleicher } \\
\text { Nachname }\end{array}$ & $\begin{array}{l}\text { ein Vorname } \\
\text { (Mustermann, Thomas) }\end{array}$ \\
\hline W_VNam_4 & Vorname & realistische Eintragung & (Thomas) \\
\hline W_GebD_5 & Geburtsdatum & Bsp. (0)4.(0)4.(19)94; $1992<\mathrm{Jahr}<2000$ & $\begin{array}{l}\text { unrealistische Eintragung } \\
(45.04 .1994 ; 04.13 .1994)\end{array}$ \\
\hline W_GebO_6 & Geburtsort & $\begin{array}{l}\text { falls D: Stadt; } \\
\text { falls Ausland: Stadt und/oder Land }\end{array}$ & unrealistische Eintragung \\
\hline W_Gesch_7 & Geschlecht & entsprechend dem Vornamen & unrealistische Eintragung \\
\hline W_Str_8 & Straße & realistische Eintragung & $\begin{array}{l}\text { Adresse des Vermieters; } \\
\text { unrealistische Eintragung }\end{array}$ \\
\hline W_HNr_9 & Hausnummer & realistische Eintragung & $\begin{array}{l}\text { Adresse des Vermieters; } \\
\text { unrealistische Eintragung }\end{array}$ \\
\hline W_PLZ_10 & Postleitzahl & realistische Eintragung & $\begin{array}{l}\text { Adresse des Vermieters; } \\
\text { unrealistische Eintragung }\end{array}$ \\
\hline W_Ort_11 & Ort & realistische Eintragung & $\begin{array}{l}\text { Adresse des Vermieters; } \\
\text { unrealistische Eintragung }\end{array}$ \\
\hline W_AW_12 & alte Wohnung & $\begin{array}{l}\text { nicht ausgefüllt, } \\
\text { andere oder vorherige Anschrift }\end{array}$ & $\begin{array}{l}\text { Adresse des Vermieters; } \\
\text { unrealistische Eintragung }\end{array}$ \\
\hline W_Fams_13 & Familienstand & angekreuzt: „ledig“, „verheiratet“ & unrealistische Eintragung \\
\hline W_PVer_14 & $\begin{array}{l}\text { Persönliche } \\
\text { Verhältnisse }\end{array}$ & $\begin{array}{l}\text { angekreuzt: „Auszubildender“, „arbeitslos“, } \\
\text { „Sonstiges“ }\end{array}$ & unrealistische Eintragung \\
\hline W_KTO_15 & Kontonummer & 5-10 Zahlen & unrealistische Eintragung \\
\hline W_BLZ_16 & Bankleitzahl & 6-10 Zahlen & unrealistische Eintragung \\
\hline W_Inst_17 & Kreditinstitut & $\begin{array}{l}\text { realistische Eintragung: Bank, Post, Visa, } \\
\text { Finanz... }\end{array}$ & unrealistische Eintragung \\
\hline W_BVer_18 & $\begin{array}{l}\text { Bank- } \\
\text { verbindung }\end{array}$ & \multicolumn{2}{|c|}{$\begin{array}{l}\text { Fall 1: „x“ bei „Antragsteller“ + Eintragung Ort unrealistische Eintragung } \\
\text { Fall 2: „x" bei „Antragsteller“ + Eintragung } \\
\text { Straße (Musterstraße, Sonnenstraße) } \\
\text { Fall 3: „x" bei „Antragsteller“ + Adresse }\end{array}$} \\
\hline
\end{tabular}




\begin{tabular}{llll}
\hline Item & Beschreibung & $\begin{array}{l}\text { Richtig } \\
\text { (Beispiele für Ausnahmen) }\end{array}$ & $\begin{array}{l}\text { Falsch } \\
\text { (Beispiele aus den Daten) }\end{array}$ \\
\hline W_WB_19 & $\begin{array}{l}\text { Wohngeld- } \\
\text { berechtigter }\end{array}$ & angekreuzt „Hauptmieter“, „Untermieter“ & unrealistische Eintragung \\
\hline W_VerNam_20 & $\begin{array}{l}\text { Name } \\
\text { Vermieter }\end{array}$ & Mustermann & unrealistische Eintragung \\
\hline W_VorVer_21 & $\begin{array}{l}\text { Vorname } \\
\text { Vermieter }\end{array}$ & Thomas & unrealistische Eintragung \\
\hline W_VerStr_22 & $\begin{array}{l}\text { Straße Ver- } \\
\text { mieter }\end{array}$ & Sonnenstraße & unrealistische Eintragung \\
\hline W_HNrVer_23 & $\begin{array}{l}\text { Hausnummer } \\
\text { Vermieter }\end{array}$ & 26 & unrealistische Eintragung \\
\hline W_PLZVer_24 & $\begin{array}{l}\text { Postleitzahl } \\
\text { Vermieter }\end{array}$ & 34117 & unrealistische Eintragung \\
\hline W_OrtVer_25 & Ort Vermieter & Kassel & unrealistische Eintragung \\
\hline W_EDat_26 & Einzugsdatum & 04.04 .2012 (andere Form möglich) & unrealistische Eintragung \\
\hline W_WFL_27 & Wohnfläche & 40 & unrealistische Eintragung \\
\hline W_MIET_28 & Mietegesamt & 330 & unrealistische Eintragung \\
\hline W_ZBeg_29 & Zahlungsbeginn & 04.04 .2012 (andere Form möglich) & unrealistische Eintragung \\
\hline
\end{tabular}

Tab. 38: Codierregeln zur standardisierten Befragung „Notfallverhalten“

\begin{tabular}{|c|c|c|c|}
\hline Item & Beschreibung & $\begin{array}{l}\text { Richtig } \\
\text { (Beispiele für Ausnahmen) }\end{array}$ & $\begin{array}{l}\text { Falsch } \\
\text { (Beispiele aus den Daten) }\end{array}$ \\
\hline N_awer_30 & Antwort zu Person & realistische Eintragung & unrealistische Eintragung \\
\hline N_awas_31 & Antwort zu Labor & realistische Eintragung & unrealistische Eintragung \\
\hline N_awo_32 & Anwort zu Adresse & realistische Eintragung & unrealistische Eintragung \\
\hline N_fwer_33 & Frage zu Person & realistische Eintragung & unrealistische Eintragung \\
\hline N_fwas_34 & Frage zu Labor & realistische Eintragung & unrealistische Eintragung \\
\hline N_fwo_35 & Frage zu Adresse & realistische Eintragung & unrealistische Eintragung \\
\hline N_Mark_36 & $\begin{array}{l}\text { Markierungen im } \\
\text { Plan }\end{array}$ & $\begin{array}{l}\text { Markierung } 2 \text { aus 3: } \\
\text { 1. Notfall Sachen, Brand, Explosion } \\
\text { 2. Feuerm. betät. und }[\ldots] \\
\text { 3. Zentrale Alarmierung }[\ldots] \\
\text { plus } \\
\text { Nicht-Markierung: } \\
\text { 1. Notfall Personen } \\
\text { 2. Notarzt }\end{array}$ & unrealistische Eintragung \\
\hline N_Prinzip_37 & $\begin{array}{l}\text { Verständnis des } \\
\text { Notfallplans }\end{array}$ & $\begin{array}{l}\text { Voraussetzungen: } \\
\text { 1. Feuer ist markiert oder es gibt mindes- } \\
\text { tens eine Verbindung zu oder von Feuer } \\
\text { 2. Es gibt mindestens zwei Markierungen. } \\
\text { 3. Es ist maximal die Hälfte markiert/ver- } \\
\text { bunden (Es zählen nun auch die vorher } \\
\text { nicht zu beachtenden Felder.) } \\
\text { 4. Es gibt keine Unterbrechungen (Der } \\
\text { Startpunkt muss jedoch nicht bei } \\
\text { Notfall oder Personen liegen). }\end{array}$ & $\begin{array}{l}\text { Bei Nicht-Erfüllung } \\
\text { der Voraussetzungen }\end{array}$ \\
\hline
\end{tabular}


Tab. 39: Codierregeln zur standardisierten Befragung „Lagerdokumentation“

\begin{tabular}{llll}
\hline Item & Beschreibung & $\begin{array}{l}\text { Richtig } \\
\text { (Beispiele für Ausnahmen) }\end{array}$ & $\begin{array}{l}\text { Falsch } \\
\text { (Beispiele aus den Daten) }\end{array}$ \\
\hline L_SÄGE_38 & Handkreissäge & $\begin{array}{l}\text { oben oder unten markiert: „568...KF } \\
\text { und/oder Akku-Handkreissäge ELEC“ }\end{array}$ & andere Markierung \\
\hline L_ZB_39 & Zubehör & $\begin{array}{l}\text { oben oder unten markiert: } \\
\text { „2x Akku Ladegerät Koffer“ }\end{array}$ & andere Markierung \\
\hline L_SNR_40 & Seriennummer & $\begin{array}{l}\text { oben oder unten markiert: } \\
\text { „(Serien Nr) 98567“ }\end{array}$ & andere Markierung \\
\hline L_Versand_41 & Versandart & $\begin{array}{l}\text { oben oder unten markiert: } \\
\text { „Versandart 2 und/oder Abholung } \\
\text { Electron-Shop“ }\end{array}$ & andere Markierung \\
& Abholung & $\begin{array}{l}\text { oben markiert: } \\
\text { „Neugerät mit Verschrotten“ }\end{array}$ & andere Markierung \\
\hline L_RepStat_42 & Reparaturstatus & $\begin{array}{l}\text { oben markiert: „Neugerät als } \\
\text { kostenpflichtige Ersatzlieferung“ }\end{array}$ & andere Markierung \\
\hline L_NGAKE_43 & Ersatzgerät & &
\end{tabular}

Tab. 40: Codierregeln zur standardisierten Befragung „Autoprotokoll“

\begin{tabular}{|c|c|c|c|}
\hline Item & Beschreibung & $\begin{array}{l}\text { Richtig } \\
\text { (Beispiele für Ausnahmen) }\end{array}$ & $\begin{array}{l}\text { Falsch } \\
\text { (Beispiele aus den Daten) }\end{array}$ \\
\hline A_Protokoll_44 & Auswahl Protokoll & Auswahl Protokol Golf-Klasse & Protokoll Passat-Klasse \\
\hline A_Tank_45 & Tankanzeige voll & Markierung bei etwa $1 / 1$; Abweichung $3 \mathrm{~mm}$ & Abweichung $>3 \mathrm{~mm}$ von $1 / 1$ \\
\hline A_Kennz_46 & Kennzeichen & $\begin{array}{l}\text { KA TE } 1985 \\
\text { (KA 1985; TE KA 1985; KA TE } 1895 ; \text {...) }\end{array}$ & anderer Eintrag \\
\hline A_CoDat_47 & CO-Datum & freigelassen & Eintrag \\
\hline A_CoKm_48 & CO-Kilometer & $161518(\mathrm{~km})$ (bis zu 2 Zahleninversionen) & $>3$ Zahleninversionen \\
\hline A_CiDat_49 & CI-Datum & freigelassen & Eintrag \\
\hline A_CiKm_50 & CI-Kilometer & freigelassen & Eintrag \\
\hline A_Spiegel_51 & Außenspiegel & Markierung eingezeichnet & Markierung fehlt \\
\hline A_Reifen_52 & Reifen & Markierung eingezeichnet & Markierung fehlt \\
\hline A_Wischer_53 & Scheibenwischer & Markierung eingezeichnet & Markierung fehlt \\
\hline A_Bremse_54 & Bremsen & Markierung eingezeichnet & Markierung fehlt \\
\hline A_Licht_55 & Licht & Markierung eingezeichnet & Markierung fehlt \\
\hline A_Instr_56 & Instrumente & Markierung eingezeichnet & Markierung fehlt \\
\hline A_Horn_57 & Signalhorn & Markierung fehlt & Markierung eingezeichnet \\
\hline A_BWerk_58 & Bordwerkzeug & Markierung eingezeichnet & Markierung fehlt \\
\hline A_Verband_59 & Verbandkasten & Markierung eingezeichnet & Markierung fehlt \\
\hline A_LadeB_60 & $\begin{array}{l}\text { Funktion } \\
\text { Ladebordwand }\end{array}$ & Markierung fehlt & Markierung eingezeichnet \\
\hline A_ERad_61 & Ersatzrad & Markierung eingezeichnet & Markierung fehlt \\
\hline A_LadeR_62 & Laderaumabdeckung & Markierung eingezeichnet & Markierung fehlt \\
\hline A_KFahr_63 & Kratzer Fahrertür & $\begin{array}{l}\text { strichhafte Markierung bei Fahrertür } \\
\text { (Schraffierung) }\end{array}$ & $\begin{array}{l}\text { Markierung fehlt; } \\
\text { Markierung der } \\
\text { Beifahrertüre }\end{array}$ \\
\hline
\end{tabular}




\begin{tabular}{llll}
\hline Item & Beschreibung & $\begin{array}{l}\text { Richtig } \\
\text { (Beispiele für Ausnahmen) }\end{array}$ & $\begin{array}{l}\text { Falsch } \\
\text { (Beispiele aus den Daten) }\end{array}$ \\
\hline A_KKoff_64 & Kratzer Kofferraum & $\begin{array}{l}\text { strichhafte Markierung bei Kofferraum (o.r.) } \\
\text { (Schraffierung) }\end{array}$ & Markierung fehlt \\
A_KStoss_65 & Kratzer Stoßstange & $\begin{array}{l}\text { strichhafte Markierung bei Stoßstange (o.r.) } \\
\text { (Schraffierung) } \\
\text { realisitische Unterschrift }\end{array}$ & Markierung fehlt \\
A_UVerm_66 & $\begin{array}{l}\text { Unterschrift } \\
\text { Vermieter }\end{array}$ & freigelassen & Keine Unterschrift \\
A_UMiet_67 & $\begin{array}{l}\text { Unterschrift } \\
\text { Mieter }\end{array}$ & & Unterschrift eingetragen \\
\hline
\end{tabular}

Tab. 41: Codierregeln zur standardisierten Befragung „Serviceorganisation“

\begin{tabular}{|c|c|c|c|}
\hline Item & Beschreibung & $\begin{array}{l}\text { Richtig } \\
\text { (Beispiele für Ausnahmen) }\end{array}$ & $\begin{array}{l}\text { Falsch } \\
\text { (Beispiele aus den Daten) }\end{array}$ \\
\hline S_AZ_68 & Arbeitszeit erkannt & Zeile Tanja; Spalte 7.7. à 9-15 & anderer Eintrag \\
\hline \multirow[t]{2}{*}{ S_T_69 } & $\begin{array}{l}\text { Einzudeckende } \\
\text { Tische }\end{array}$ & $\begin{array}{l}\text { Tische } 16,15,121,12,11,111,101,10, \\
4,3,2,1 \text { in Plan markiert und 23, 231, 22, } \\
221,21 \text { nicht markiert }\end{array}$ & $\begin{array}{l}\text { Falls: Tische aus mind. } 2 \\
\text { Tischreihen }(1-4,10-11 \text {, } \\
12-16) \text { fehlen }\end{array}$ \\
\hline & & $\begin{array}{l}\text { ( } 2 \text { der } 3 \text { Tischsets vollständig und } 1 \\
\text { Tischset unvollständig markiert) }\end{array}$ & $\begin{array}{l}\text { Falls: mind. } 2 \text { falsche } \\
\text { Tische markiert }\end{array}$ \\
\hline S_AZG_70 & $\begin{array}{l}\text { Anzahl der } \\
\text { Gedecke }\end{array}$ & $\begin{array}{l}64 \\
\text { ( } 48,68 \text { oder entsprechend der falschen } \\
\text { Markierung; Folgefehler) }\end{array}$ & $\begin{array}{l}\text { Rechnung ohne Bezug } \\
\text { z. B. bei Folgefehler }\end{array}$ \\
\hline
\end{tabular}

Tab. 42: Codierregeln zur standardisierten Befragung „Montagedokumentation“

\begin{tabular}{|c|c|c|c|}
\hline Item & Beschreibung & $\begin{array}{l}\text { Richtig } \\
\text { (Beispiele für Ausnahmen) }\end{array}$ & $\begin{array}{l}\text { Falsch } \\
\text { (Beispiele aus den Daten) }\end{array}$ \\
\hline M_Tür71 & $\begin{array}{l}\text { Schranktür } \\
\text { markieren }\end{array}$ & $\begin{array}{l}\text { „Holz-Winkeltür... 62842... Lack polarweiß“ } \\
\text { („Holz-Winkeltür... 62359“) }\end{array}$ & andere Markierung \\
\hline M_Licht72 & $\begin{array}{l}\text { Lichtleiste } \\
\text { markieren }\end{array}$ & 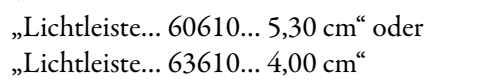 & andere Markierung \\
\hline M_TTür73 & $\begin{array}{l}\text { Teilnummer } \\
\text { Schranktür }\end{array}$ & $\begin{array}{l}\text { „141 Türen Folie/Lack“ } \\
(„ 142 . . . ”, 143 . . . ”, 144 \ldots . . \text { ) }\end{array}$ & andere Markierung \\
\hline M_FTür_74 & $\begin{array}{l}\text { Fehlernummer } \\
\text { Schranktür }\end{array}$ & $\begin{array}{l}\text { „21 Kratzer/Druckstellen/ } \\
\text { Abplatzungen“ oder „31 Oberfläche } \\
\text { verzogen/gerissen“ }\end{array}$ & andere Markierung \\
\hline M_TLicht_75 & $\begin{array}{l}\text { Teilnummer } \\
\text { Lichtleiste }\end{array}$ & $\begin{array}{l}\text { „240 Beleuchtung“(„038 Zubehör“ oder } \\
\text { „220 kompl. Elemente“) }\end{array}$ & andere Markierung \\
\hline M_Flicht_76 & $\begin{array}{l}\text { Fehlernummer } \\
\text { Lichtleiste }\end{array}$ & „52 Fehlteil“ & andere Markierung \\
\hline $\begin{array}{l}\text { M_Eint- } \\
\text { Tür_77 }\end{array}$ & $\begin{array}{l}\text { Eintragung } \\
\text { Türnummer }\end{array}$ & $\begin{array}{l}141-21-62842 \\
\text { (Folgefehler berücksichten; eine der drei } \\
\text { Positionen fehlt) }\end{array}$ & andere Markierung \\
\hline $\begin{array}{l}\text { M_Eint- } \\
\text { Lic_78 }\end{array}$ & $\begin{array}{l}\text { Eintragung } \\
\text { Lichtnummer }\end{array}$ & $\begin{array}{l}240-52-60610 / 63610 \text { (Folgefehler be- } \\
\text { rücksichtigen; eine der drei Positionen fehlt) }\end{array}$ & andere Markierung \\
\hline
\end{tabular}




\subsection{Durchführung und Stichprobe}

Die Teilstudie konnte aufgrund der zur Verfügung stehenden Ressourcen kein mehrschichtiges Verfahren zur Ziehung der Stichprobe auf Ebene der Schulen als auch auf der Ebene der Jugendlichen vorweisen. An deren Stelle tritt, wie bei vergleichbaren Untersuchungen, ein Bemühen, die gewünschten Teilnahmequoten zu erzielen. Es ist daher nicht auszuschließen, dass zum einen insbesondere die Schulen auf unsere zuerst telefonische und alsdann schriftliche Anfrage mit einem Beteiligungssignal reagierten, die der Thematik positiv gegenüberstehen. Zum anderen kann dies auch für die Jugendlichen bzw. ihre Eltern gelten, welche nach einer schriftlichen Information einer Teilnahme zustimmen mussten. Insbesondere die Zustimmung der Eltern ist ein Faktor, welcher in Explorationsstudien, wie der vorliegenden, mit Anforderungen von Zufallsstichproben kaum in Einklang zu bringen sind. Die Kontrolle der Zusammensetzung der Stichprobe kann daher nur über die Annäherung von sozialstrukturellen Merkmalen erfolgen.

Im Zuge der Auseinandersetzung mit Referenzstudien zum Lesen von nichtkontinuierlichen Texten, war auffällig, dass Jugendliche in berufsqualifizierenden Angeboten die besten Performanzen mit diesen Texten zeigten (vgl. Kapitel 6.4.1). Vor diesem Hintergrund war angestrebt, bei der explorativen Studie zu den Lesecompetencies von multikodalen Texten und Textkomplexen in achten Klassen auch sogenannte Praxisklassen anzufragen. In diesen Klassen erfüllen die Jugendlichen an drei Tagen ihre Schulpflicht im Unterricht an Schulen und an zwei Tagen im Langzeitpraktikum in Betrieben. Die Begleitung des Langzeitpraktikums wird in der Regel durch Sozialpädagogen flankiert. Die Jugendlichen an den Praxisklassen werden sich durch die institutionell implementierte Nähe zur Berufswelt und beruflichen Bildung von der sonstigen Stichprobe unterscheiden: Neben Schüler/innen mit anerkanntem sonderpädagogischem Förderbedarf Lernen sollten sich diese aus Jugendlichen im Bildungsgang des Ersten allgemeinbildenden Schulabschlusses (EAB) bzw. der Hauptschule zusammensetzen. Letztere Teilgruppe wurde vor dem Hintergrund mitbedacht, um die Ergebnisse mit den Referenzstudien durch Vergleiche prüfen zu können.

Es wurden für die Datenerhebung 27 Schulen angeschrieben, unter denen letztlich 13 Förderschulen und 6 Berufsschulen sich für eine Beteiligung entschieden. An den teilnehmenden Schulen konnte eine Gesamterhebung in den achten Klassen erzielt werden. Insgesamt nahmen an den Erhebungen 226 Jugendlichen teil, darunter 37 Schüler/innen des Bildungsgangs mit Ziel Förderschulabschluss Lernen. Mit 136 teilnehmenden Jugendlichen des Bildungsgangs mit Ziel EAB/ Hauptschulabschluss lag das Verhältnis bei etwa der 11\%:3\% der referentiellen PISA-Zusatzstudie. Das durchschnittliche Alter beträgt 14 Jahre und 10 Monate. Auch die Verteilung nach Geschlecht und Migrationshintergrund unter den befragten Jugendlichen des Bildungsgangs sonderpädagogischer Förderbedarf Lernen deckt sich nahezu mit der Stichprobe der referentiellen PISA-Zusatzstudie. Die gültigen Anteile differenzieren mit maximal 2,3\% kaum (vgl. Tabelle 44), sodass zusammenfassend von einer kontrollierten Stichprobe ausgegangen werden kann. Eine Repräsentativität der Stichprobe der Teilstudie Lesecompetencies wird nicht postuliert.

Tab. 43: Verteilung der teilnehmenden Jugendlichen auf Bildungsgänge

\begin{tabular}{llcc}
\hline Bildungsgang & Praxisklasse & Häufigkeit & gültiger Anteil \\
\hline Ziel Erster allgemeinbildender Schulabschluss (EAB) & nein & 136 & 70,8 \\
Ziel Förderschulabschluss Lernen (SPF L) & ja & 29 & 12,8 \\
& nein & 37 & 16,4 \\
& Gesamt & 66 & 29,2 \\
\hline Gesamt & & 226 & 100,0 \\
\hline
\end{tabular}


Tab. 44: Vergleich einzelner Stichprobenmerkmale im Bildungsgang des sonderpädagogischen Schwerpunkts Lernen zur referentiellen PISA-Zusatzstudie

\begin{tabular}{llcc}
\hline Merkmal & & $\begin{array}{c}\text { güliger Anteil: } \\
\text { Teilstudie }\end{array}$ & $\begin{array}{c}\text { gültiger Anteil: } \\
\text { Referenzstudie }\end{array}$ \\
\hline Geschlecht & männlich & 58,1 & 59,4 \\
& weiblich & 41,9 & 40,6 \\
\hline Migrationshintergrund & Gesamt & 33,3 & 31,0 \\
\hline
\end{tabular}

\subsection{Modellprüfung der standardisierten Erhebung von Lesecompetencies multikodaler Texte und Textkomplexe}

\subsubsection{Statistische Modellprüfung}

Eine Auskunft über die Messvalidität des Konstrukts Lesecompetencies multikodaler Texte und Textkomplexe kann mit Methoden der Klassischen Testtheorie (KTT) nicht erzielt werden. Hierzu besteht Einigkeit in den Referenzstudien, die stattdessen eine probabilistische Modellierung der latenten Variablen, beruhend auf der Item-Response-Theorie (IRT), empfehlen. In der vorliegenden Studie wird analog durch ein Latent-Trait-Modell von direkt beobachtbaren manifesten Items ausgegangen, die auf Merkmalsausprägungen einer nicht unmittelbar beobachtbaren dahinterliegenden Fähigkeit verweisen. Entgegen der Klassischen Testtheorie wird die Itemschwierigkeit infolge nicht a priori vorgegeben, sondern aus der Performanz der Stichprobe in der Explorationsstudie erst analysiert, indem die Lösungswahrscheinlichkeit einer manifesten Variablen mit den latenten der Probanden probabilistisch in Bezug gesetzt wird. Über Modelltests wird die Konstruktvalidität des Messmodells feststellbar; hier das multidimensionale Rasch-Modell (Moosbrugger 2008, 216ff.). Dieses Rasch-Modell für dichotome Antworten wurde entlang der in Kapitel 6.1.3.3 beschriebenen Codierregeln für die bearbeiteten Testaufgaben zu Lesecompetencies multikodaler Texte primär ikonischer Kodalität angewendet und verweist auf folgende Charakteristika: Jede Person hat eine individuelle latente Merkmalsausprägung $\vartheta_{v}$ und jedes Item der Testaufgaben eine Schwierigkeit $\sigma_{i}$, wobei Personen- und Itemparameter auf denselben, mehrdimensionalen Skalen dargestellt werden. Entscheidend für die Lösungswahrscheinlichkeit ist die Differenz zwischen der individuellen Merkmalsausprägung $\vartheta_{v}$ und der Schwierigkeit $\sigma_{i}$ des jeweiligen Items $i$.

Die Messgenauigkeit eines Tests wird mit dem Gütekriterium der Reliabilität beschrieben. Im Kontext der IRT werden zu ihrer Schätzung die geschätzte Varianz des zu messenden Merkmals und die Varianz der Expected a Posteriori-Schätzer (EAP) der individuellen Merkmalsausprägungen verwendet (oft mit dem Kürzel EAP/PV-Reliabilität bezeichnet; vgl. Rost, 2004). Die EAP/PV-Reliabilität bezieht sich auf die Messgenauigkeit von Items, die ein eindimensionales Merkmal erfassen. Wenn ein mehrdimensionales Konstrukt erfasst wird, wie es bei Kompetenztests in der Regel der Fall ist, sollte entsprechend separat für jede Dimension die Reliabilität betrachtet werden. (Terzer et al. 2013, 69)

Zur Analyse der Modellgültigkeit bei mehrdimensionalen Modellen wird empfohlen, für die ausgewählten dichotomen Rasch-Modellen die kumulierte Wahrscheinlichkeit mittels Informationsindizes zu ermitteln und mit alternativen Modellen zu vergleichen (ebd., 69f.). Über diese Indizes ist es möglich, Vergleiche zur Wahrscheinlichkeit anzustellen, mit der das Anwortmuster unter Annahme dieses Modells mit - hier - drei Dimensionen zu einem 
Modell mit zwei Dimensionen auftritt. Die Informationsindizes sagen darüber hinaus nichts über die Modellgüte per se aus. Nebst diesen statistischen Maßgaben an die Angemessenheit zur Prüfung von unterschiedlichen mehrdimensionalen Modellen wurde das Prinzip der Einfachheit (Rost 2004, 330f.) mit seinem Ziel der statistischen Optimierung der Anzahl an Parameter je Modell $\left(n_{P}\right)$ nur unter der Vorgabe verfolgt, dass eine möglichst umfassende Erfassung der situativ kontextuellen Einbindung der eingesetzten Texte und Textkomplexe im Zentrum steht. Die Reduktion auf möglichst statistisch aussagekräftige bzw. eindeutige Items war theoriebasiert durch die Anlage der Arbeit nicht angestrebt. Oder in anderen Worten, es sollten so wenig Items wie möglich und zugleich nur so viele Items wie nötig eliminiert werden; bspw., wenn die interne Konsistenz der Items statistisch bedenklich gefährdet war. Es wurde das Kriterium von heterogenen, mehrdimensionalen Tests bzw. von Evaluationsstudien von $r_{i t}>0,2$ angesetzt (Hirsig 1993).

Das eingesetzte Programm Acer ConQuest (Adams et al. 2015) bestimmt für jedes der zu vergleichenden Modelle die Personen- und Itemparameter so, dass diese Wahrscheinlichkeit Logarithmus der Likelihood $-D=-\log L$ maximal wird. Aus dem Programmoutput von „final Deviance " $=-2 \log L$ zusammen mit der Parameteranzahl des jeweiligen Modells $n_{P}$ wurden die gängigen Informationsindizes AIC (Akaikes Information Criterium $=2\left(n_{P}+D\right)$ ) und BIC (Bayes Information Criterium $=n_{P} \log N+2 D$ ) berechnet (siehe Tabelle 45). Hier ist zu ergänzen, dass mit 10 Items im 3-dimensionalen und mit 12 Items im 2-dimensionalen Modell eine nahezu identische Anzahl aus dem ursprünglichen Itempool gestrichen werden mussten, da die Cut-Off-Werte für weighted MNSQ Infit mit $0.8 £ 4.2$ und T£4.96 nicht erfüllt wurden. Mit graphischen Modelltests wurde abschließend getrennt für beide Dimensionen eine Stichprobenunabhängigkeit entlang des Merkmals Geschlecht kontrolliert.

Tab. 45: Vergleich der globalen Modellgüte

\begin{tabular}{lcccc}
\hline Bildungsgang & $\mathbf{n}_{\mathbf{p}}$ & AIC & BIC & c2-Test \\
\hline 2-dimensionales Modell & 63 & 11577 & 11802 & \multirow{2}{*}{ 3 } \\
3-dimensionales Modell & 68 & 11641 & 11880 & \\
\hline
\end{tabular}

Tab. 46: Modellvergleich durch Fit-Statistik-Kennwerten

\begin{tabular}{lccccc}
\hline Modell & Skala & Varianz & $\begin{array}{l}\text { EAP/PV- } \\
\text { Reliabilität }\end{array}$ & $\begin{array}{l}\text { latente } \\
\text { Korrelation }\end{array}$ & KMO \\
\hline 2-dimensionales Modell & Dim 1 & .976 & .802 & .655 & \\
& Dim 2 & 4.170 & .811 & & \\
\hline 3-dimensionales Modell & Dim 1 & 0.829 & .673 & .606 & .723 \\
& Dim 2 & 4.326 & .881 & & .730 \\
& Dim 3 & 4.720 & .827 & .552 & .793 \\
\hline
\end{tabular}

Neben einer tendenziellen, aber nicht absoluten Präferenz des Modells mit kleineren Informationsindizes wird in der Methodendebatte keine jeweilige Mindest-Differenz für die endgültige Selektion vorgegeben (ebd., 341). An deren Stelle tritt ein $c^{2}$-Signifikanztest als Prüfstatistik $\left(c^{2}=-2\left(\log L_{2 \mathrm{Dim}}-\log L_{3 \mathrm{Dim}}\right)\right)$. Dies ist möglich, da für das 2-dimensionale Rasch-Modell - wie noch zu zeigen ist - Modellgültigkeit angenommen werden kann und zudem das 2-dimensi- 
onale Rasch-Modell durch Parameterrestriktion aus dem 3-dimensionalen hervorgeht (ebd., 332). Der $c^{2}$-Test verdeutlicht, dass das 2-dimensionale Modell die Daten nicht signifikant angemessener abbildet als das komplexere 3-dimensionale Modell.

„Die MNSQ-Werte geben an, wie gut die Daten zum theoretischen Messmodell passen. Eine ideale Modellanpassung indizieren Items mit dem Wert 4. Fitwerte, die deutlich größer als 1 sind, weisen auf eine schlechte Anpassung, Werte deutlich kleiner als 1 auf eine „zu gute“ Anpassung, d.h. auf eine überdurchschnittlich hohe Trennschärfe hin. Die Fitwerte sollten zwischen 0.5 und 4.5 liegen [...], in PISA-2000 gelten Fit- Indizes zwischen 0.8 und 4.2 als messmethodisch akzeptabel“ (Lipowsky et al. 2006, 15).

Neben der globalen Modellgüte sprechen abschließend auch die Item-Fit- und Skalenwerte statistisch nicht für die Präferenz des komplexeren 3-dimensionalen Modells (vgl. Tabelle 46 bis Tabelle 48). Aufgrund fehlender zwingender statistischer Argumente wurde abschließend für die Auswahl die EAP/PV-Reliabilitätswerte der Referenzstudien herangezogen. Das 2-dimensionale Modell entspricht durchgängig nahezu dem Niveau von .88 der referentiellen Zusatzauswertung zu PISA 2012 (Müller et al. 2017, 184) und von .83 der BELLA-Studie (Lehmann/ Hoffmann 2009, 40). Das 2-dimensionale Rasch-Modell bildet den Datensatz in Summe vergleichend angemessener $\mathrm{ab}$ und wird für die weitere Analyse herangezogen. Es bildet die Grundlage u.a. für die spätere Berechnung der Weighted-Likelihood-Estimates im Sinne des besten Punktschätzer der dahinterliegenden Fähigkeit der Probanden (Rost 2004, 316). Die zugehörigen Items einschl. Schwierigkeitparameter $\sigma_{i}$ werden in den nachfolgenden Tabellen je Dimension zusammengefasst.

Tab. 47: Modelfit der Dimension 1 der Lesecompetencies multikodaler Texte und Textkomplexe

\begin{tabular}{lccccc}
\hline Item & $\sigma_{\mathrm{i}}$ & SE & $\begin{array}{c}\text { weighted } \\
\text { MNSQ }\end{array}$ & $\begin{array}{c}\text { weighted } \\
\text { T }\end{array}$ & $\mathbf{r}_{\text {it }}$ \\
\hline W_FNam_2 & -4.098 & .004 & .92 & -.9 & .5 \\
W_Gnam_3 &. .511 & .009 & .91 & -4.4 & .5 \\
W_VNam_4 & -4.098 & .008 & .90 & -4.2 & .5 \\
W_GebD_5 & -4.031 & .000 & .89 & -4.3 & .5 \\
W_GebO_6 & -.901 & .004 & .99 & -.0 & .4 \\
W_Gesch_7 & -3.020 & .005 & .99 & .1 & .4 \\
W_Str_8 & -.149 & .000 & .90 & -4.8 & .5 \\
W_HNr_9 & -.040 & .003 & .91 & -4.7 & .5 \\
W_PLZ_10 & -.397 & .007 & 4.03 & .4 & .3 \\
W_Ort_11 & -.454 & .010 & 4.02 & .3 & .3 \\
W_AW_12 & -4.424 & .005 & 4.04 & .4 & .4 \\
W_Fams_13 & -.258 & .009 & 4.07 & 4.2 & .3 \\
W_KTO_15 & .788 & .006 & 4.10 & 4.4 & .2 \\
W_Inst_17 & 4.505 & .000 & 4.09 & .9 & .2 \\
W_WFL_27 & 2.682 & .005 & 4.06 & .3 & .2 \\
W_MIET_28 & 2.761 & .004 & 4.04 & .2 & .2 \\
L_SÄGE_38 & .036 & .010 & 4.07 & 4.5 & .4 \\
L_SNR_40 & -4.493 & .005 & 4.13 & 4.4 & .3 \\
L_RepStat_42 & 4.424 & .001 & 4.08 & 4.0 & .3 \\
A_Protokoll_44 & -0.997 & .004 & 4.05 & .5 & .3 \\
A_Tank_45 & 2.157 & .005 & .106 & .4 & .2 \\
A_Kennz_46 & -4.511 & .003 & .95 &. .4 & .4 \\
& & & & &
\end{tabular}




\begin{tabular}{lccccc}
\hline Item & $\sigma_{\mathrm{i}}$ & SE & $\begin{array}{c}\text { weighted } \\
\text { MNSQ }\end{array}$ & $\begin{array}{c}\text { weighted } \\
\text { T }\end{array}$ & $\mathbf{r}_{\text {it }}$ \\
\hline A_Spiegel_51 & -2.444 & .012 & .89 & -.6 & .5 \\
A_Wischer_53 & -2.093 & .000 & .90 & -.7 & .5 \\
A_Verband_59 & -2.041 & .004 & .88 & -.8 & .5 \\
A_KFahr_63 & .123 & .013 & 4.08 & 4.6 & .4 \\
A_KKoff_64 & .542 & .005 & 4.00 & .0 & .4 \\
\hline A_KStoss_65 & .517 & .004 & 4.06 & 4.1 & .3 \\
M_Licht_72 & 2.092 & .000 & 4.05 & .4 & .3 \\
M_TTür773 & .864 & .004 & .93 & -4.1 & .4 \\
M_FTür_74 & .247 & .000 & .96 & -.7 & .3 \\
M_TLicht_75 & 4.234 & .000 & .94 & -.8 & .4 \\
M_Flicht_76 & 4.182 & .007 & .88 & -4.6 & .4 \\
M_EintTür77 & 2.916 & .003 & .97 & -.1 & .3 \\
M_EintLicht_78 & 3.677 & .009 & .91 & -.2 & .3 \\
\hline
\end{tabular}

Tab. 48: Modelfit der Dimension 2 der Lesecompetencies multikodaler Texte und Textkomplexe

\begin{tabular}{|c|c|c|c|c|c|}
\hline Item & $\sigma_{\mathrm{i}}$ & SE & $\begin{array}{c}\text { weighted } \\
\text { MNSQ }\end{array}$ & $\begin{array}{c}\text { weighted } \\
\mathrm{T} \\
\end{array}$ & $\mathbf{r}_{\mathrm{it}}$ \\
\hline W_PVer_14 & .794 & .004 & 4.05 & .8 & .2 \\
\hline W_BVer_18 & 2.232 & .003 & 4.04 & .3 & .2 \\
\hline W_WB_19 & 4.617 & .009 & .96 & -.4 & .3 \\
\hline W_VerNam_20 & 2.289 & .006 & .76 & -4.6 & .4 \\
\hline W_VorVer_21 & 2.411 & .000 & .78 & -4.3 & .3 \\
\hline W_VerStr_22 & 2.475 & .002 & .8 & -4.2 & .3 \\
\hline W_HNrVer_23 & 2.543 & .000 & .79 & -4.1 & .3 \\
\hline W_PLZVer_24 & 2.613 & .000 & .82 & -.9 & .3 \\
\hline W_OrtVer_25 & 2.475 & .004 & .82 & -4.1 & .3 \\
\hline N_fwer_33 & -.169 & .004 & 4.08 & 4.5 & .4 \\
\hline N_fwas_34 & -.825 & .005 & 4.11 & 4.5 & .3 \\
\hline N_fwo_35 & -4.347 & .005 & 4.08 & .8 & .3 \\
\hline A_CoDat_47 & -4.422 & .006 & 4.17 & 4.6 & .2 \\
\hline A_CiDat_49 & -2.227 & .007 & .99 & -.0 & .4 \\
\hline A_CiKm_50 & -2.521 & .005 & .94 & -.3 & .4 \\
\hline A_Reifen_52 & -4.457 & .003 & 4.01 & .1 & .3 \\
\hline A_Bremse_54 & .860 & .004 & 4.04 & .6 & .2 \\
\hline A_Licht_55 & -2.227 & .004 & .90 & -.6 & .5 \\
\hline A_Instr_56 & -.031 & .006 & 4.08 & 4.5 & .3 \\
\hline A_Horn_57 & 2.509 & .003 & .96 & -.2 & .2 \\
\hline A_BWerk_58 & .092 & .000 & 4.05 & .9 & .3 \\
\hline A_ERad_61 & -4.761 & .013 & .99 & -.0 & .4 \\
\hline A_LadeR_62 & -.750 & .012 & .98 & -.3 & .4 \\
\hline A_UMiet_67 & -2.175 & .007 & 4.06 & .4 & .3 \\
\hline S_T_69 & 4.257 & .005 & 4.15 & 4.8 & .3 \\
\hline
\end{tabular}




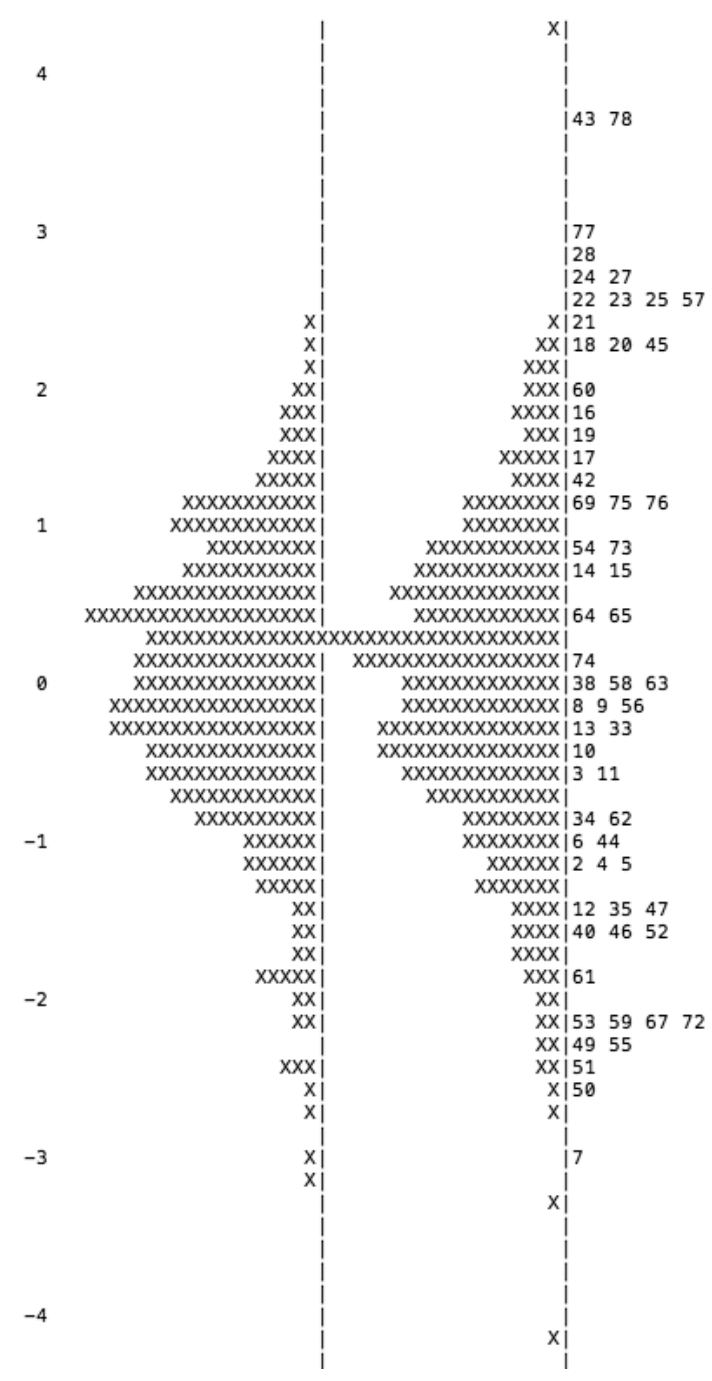

Abb. 48: Schwierigkeitsbereiche

\subsubsection{Inhaltliche Modellprüfung}

Die einzelnen Items der statistisch abgeleiteten zwei Dimensionen von Lesecompetencies multikodaler Texte und Textkomplexe primär ikonischer Kodalität wurden abschließend semiotisch analysiert. Als Grundlage der Analyse dienen die theoriebasierten Schwierigkeitsmerkmale, die bereits in der Auswertung zu den routinalisierten lokalen kulturellen Codes Anwendung fanden (vgl. Tabelle 28) und aus den semiotischen Schwierigkeitsmerkmalen nichtkontinuierlicher Texte der OECD abgeleitet wurden (vgl. Kapitel 3.4.2). Als Ergebnis verhärtet sich, dass die Items der 4. Dimension in Abgrenzung zur 2. Dimension Folgendes gemeinsam haben (vgl. Tabelle 49): 
- Informationen ermitteln durch Herstellung von lokaler Kohärenz (1);

- lokale Kohärenz zwischen Lokalzeichen und praktischem Wissen (1a);

- globale Kohärenz zwischen eher Lokalzeichen und praktischem Wissen (2a);

- Lokalzeichen eines multikodalen Textes primär ikonischer Kodalität (eine kategoriale Tabelle, eine graphische Darstellung/ein Piktogramm, eine Karte/ein Raumplan) (2a);

- Reflexion und Bewertung der Relation von Sachverhalten aus einem Text (3a);

- keine lokale Kohärenz zwischen Lokalzeichen und Faktenwissen (1b);

- keine globale Kohärenz zwischen eher Globalzeichen und Faktenwissen (2b);

- keine Reflexion und Bewertung der Relationen von Sachverhalten aus Textkomplexen (3b).

Während die 4. Dimension folglich Lesecompetencies multikodaler Texte primär ikonischer Kodalität mit einer Tendenz einer ansteigenden Komplexität von lokaler Kohärenz über globale Kohärenz und Reflexion/Bewertung abbilden, so trifft dies für die 2. Dimension für Lesecompetencies multikodaler Textkomplexe primär ikonischer Kodalität.

Tab. 49: Qualitative Kategorisierung der Items zu Lesecompetencies von multikodalen Texten vs. Textkomplexen

\begin{tabular}{l}
\hline Gegenstand - Einfach(st)erwerbstätigkeiten \\
\hline 1 lokale Kohärenz zwischen Lokalzeichen \\
(Informationen eines Sachverhalts, die mehr oder weni- \\
ger tief in den Text oder Textkomplex eingebettet sind \\
und mehr oder weniger klar differenzierbar innerhalb \\
des Textes oder Textkomplexes lokalisierbar sind, sind zu \\
identifizieren und zu dekodieren. Diese Informationen \\
sind folglich dem Text oder Textkomplex inhärent und \\
bedürfen nicht zwingend weder externen Faktenwissens \\
noch einer tiefen Auseinandersetzung mit dem Text)
\end{tabular}

1a lokale Kohärenz zwischen Lokalzeichen und praktischem Wissen

(Relation zwischen Einzelinformationen eines Sachverhalts aus kategorialen Tabellen/weiteren nichtkontinuierlichen Texten und Textkomplexen und einem knowing how zu Informationen und Sachverhalten)

Dimension 1: Aufgabenschwierigkeit $\sigma_{i}>-0.5$ Exemplarisch sind die Items im Wohngeldantrag, die nach dem Namen bis hin zum Geschlecht fragen. Für die Bearbeitung der Items ist lokale Kohärenz zwischen den Lokalzeichen des Formulars mit dem praktischen Wissen von Name bis Geschlecht herzustellen; somit beschränkt auf das Formular.

1b lokale Kohärenz zwischen Lokalzeichen und Faktenwissen

(Relation zwischen Einzelinformationen eines Sachverhalts aus kategorialen Tabellen/weiteren nichtkontinuierlichen Texten und Textkomplexen und einem knowing that zu Informationen und Sachverhalten)

Dimension 2: Aufgabenschwierigkeit $\sigma_{i}>-0.8$ Exemplarisch sind die Items im Fahrzeugprotokoll, die dem km-Stand vor und nach der Fahrzeugmiete fragen. Für die Bearbeitung der Items ist Kohärenz zwischen den Lokalzeichen des Formulars einschl. dem mündlich vorgegebenen Fachwissen herzustellen; somit verteilt auf einen Textkomplex.

\footnotetext{
2 globale Kohärenz zwischen (eher) Globalzeichen (Zusammenhänge zwischen Sachverhalten (Begriffe, Konzepte usw.), die mehr oder weniger mehrdeutig im Text oder Textkomplex und mehr oder weniger differenziert und strukturiert repräsentiert werden, sind zu identifizieren, dekodieren und zu deuten. Diese Zusammenhänge sind folglich dem Text oder Textkomplex inhärent und bedürfen nicht zwingend externen Faktenwissens, jedoch einer mehr oder weniger tiefen Auseinandersetzung mit dem Text oder Textkomplex als Ganzes)
} 


\begin{tabular}{|c|c|}
\hline igkeiten & \\
\hline $\begin{array}{l}\text { 2a globale Kohärenz zwischen (eher) Globalzeichen } \\
\text { und praktischem Wissen } \\
\text { Interferenz zwischen Sachverhalten und hinter } \\
\text { Einzelinformationen stehenden Sachverhalten aus } \\
\text { kategorialen Tabellen/weiteren nichtkontinuierlichen } \\
\text { Texten und Textkomplexen und einem knowing how } \\
\text { zu Informationen und Sachverhalten) }\end{array}$ & $\begin{array}{l}\text { Dimension 1: Aufgabenschwierigkeit }-.5>\sigma_{i}<4.1 \\
\text { Exemplarisch sind die Items im Wohngeldantrag, } \\
\text { die nach den eigenen Kontodaten fragen. Für die } \\
\text { Bearbeitung des Items ist Kohärenz zwischen dem } \\
\text { Sachverhalt und praktischem Wissen herzustellen; } \\
\text { somit beschränkt auf das Formular. }\end{array}$ \\
\hline $\begin{array}{l}\text { 2b globale Kohärenz zwischen (eher) Globalzeichen } \\
\text { und Faktenwissen } \\
\text { Interferenz zwischen Sachverhalten und hinter } \\
\text { Einzelinformationen stehenden Sachverhalten aus } \\
\text { kategorialen Tabellen/weiteren nichtkontinuierlichen } \\
\text { Texten und Textkomplexen und einem knowing that } \\
\text { zu Informationen und Sachverhalten) }\end{array}$ & $\begin{array}{l}\text { Dimension 2: Aufgabenschwierigkeit }-.8>\sigma_{i}<4.2 \\
\text { Exemplarisch die Items im Fahrzeugprotokoll, } \\
\text { die nach Gegenständen mit Fachbegriffen fragen, } \\
\text { die nicht mündlich vorgegeben wurden. Für } \\
\text { die Bearbeitung der Items ist globale Kohärenz } \\
\text { zwischen den Lokalzeichen des Formulars einschl. } \\
\text { Fachwissen mit den graphischen Darstellungen zu } \\
\text { Funktionsweise dieser Teile nach der Miete; somit } \\
\text { verteilt auf einen Textkomplex. }\end{array}$ \\
\hline
\end{tabular}

3 Reflexion und/oder Bewertung: lokale/globale Kohärenz und Faktenwissen/praktisches Wissen (unter Zuhilfenahme von Darstellungsstrategien und Superstrukturen und aufbauend auf Interferenzen zwischen Einzelinformationen zu Sachverhalten, übergeordneten Sachverhalten und hinter Einzelinformationen stehenden Sachverhalten aus kategorialen Tabellen/ weiterer nichtkontinuierlicher Texte und Textkomplexe und knowing how/knowing zu Handlungen)

3a beschränkt auf Texte Dimension 1: Aufgabenschwierigkeit $\sigma_{i}>4.1$ Exemplarisch ist das Item zum Lieferschein, in dem der Status „verschrottet“ bearbeitet werden muss. Für die Bearbeitung des Items ist eine Bewertung des Sachverhalts zum Sachverhalt herzustellen; somit beschränkt auf das Forumular.

3b beschränkt auf Textkomplexe

Dimension 2: Aufgabenschwierigkeit $\sigma_{i}>4.2$

Exemplarisch die Items im Wohngeldantrag, die nach Daten zum Vermieter fragen. Für die Bearbeitung der Items ist eine Bewertung des Sachverhalts zwischen dem Formular und dem Mietvertrag einschl. seiner Lokalzeichen zu den Daten des Vermieters herzustellen; somit verteilt auf einen Textkomplex.

\subsubsection{Deskriptive und vergleichend kriteriale Modellprüfung}

In den nachfolgenden Unterkapiteln werden zum einen die ermittelten Lesecompetencies multikodaler und nichtkontinuierlicher Texte/Textkomplexe deskriptiv zusammengefasst. Zum anderen werden diese Ergebnisse zu den ermittelten Lesecompetencies jeweils mit den Referenzstudien deskriptiv zur Kontrolle der Kriteriums- und Konstruktvalidität gegenübergestellt. Es wird gezeigt werden, dass letztlich von ähnlich statistisch bedeutsamen Verteilungen jener ermittelten Lesecompetencies entlang der analysierten Stichprobengruppen ausgegangen werden kann. 


\subsubsection{Deskriptive und vergleichend kriteriale Modellprüfung der Lesecompetencies multikodaler Texte und Textkomplexe}

Die Testaufgaben entlang von primär kategorialen Tabellen, graphischen Darstellungen bis Karten wurden zur Absicherung der Auswertungsobjektivität mittels der vorgestellten Codierregeln (siehe Kapitel 6.1.3.3) dichotom als richtig oder falsch bewertet. Die deskriptive Auswertung der Anteile an korrekten Antworten kann den nachfolgenden Tabellen 50 und 51 je Item getrennt für die Jugendlichen mit Bildungsziel Förderschulabschluss und mit Bildungsziel Hauptschulabschluss bzw. Erster allgemeinbildender Bildungsabschluss (EAB) entnommen werden. Entsprechend der qualitativen Skalierung in den beiden Dimensionen von Lesecompetencies multikodaler Textualität fielen den Jugendlichen die Aufgaben am einfachsten, in denen sie praktisches Wissen wie das eigene Geschlecht innerhalb eines Textes lokal bearbeiten mussten. In beiden Probandengruppen performen hier teils über $90 \%$ diese Anforderung skillful. Im Gegensatz dazu fielen den Probanden Aufgaben am schwierigsten, die Bewertung und Reflexion von repräsentierten Sachverhalten insbesondere in Textkomplexen. Manche dieser Items haben nur 2,7\% bis $15 \%$ korrekt bearbeitet. Deskriptiv auffällig ist zuvorderst, dass $27 \mathrm{der} 63$ Items und somit nahezu jede zweite Aufgabe nur von der Hälfte der Jugendlichen richtig bearbeitet werden konnte.

Die Vergleiche zwischen den Untergruppen zeigt bereits auf, dass sich die Schwierigkeitsverteilung deskriptiv vergleichbar verhält. Es muss jedoch festgehalten werden, dass es Jugendlichen eines Bildungsgangs deskriptiv auffällig einfacher fiel, einzelne Texte bzw. Textkomplexe korrekt zu bearbeiten. Die Jugendlichen des Bildungsgangs mit Ziel EAB lösten z. B. viele Items des Wohngeldantrags bis zu $20 \%$ häufiger richtig. Im Gegenzug fiel den Jugendlichen des Bildungsgangs mit Ziel Hauptschulabschluss die Bearbeitung des Lieferscheins in der Lagerorganisation im Trend deutlich einfacher.

Tab. 50: Anteil korrekter Antworten nach Bildungsgang innerhalb der Dimension 1

\begin{tabular}{lcclcc}
\hline Item & \multicolumn{2}{c}{ \% of total } & Item & \multicolumn{2}{c}{ \% of total } \\
\cline { 2 - 3 } & FöA & EAB/HS & & FöA & EAB/HS \\
& 81,1 & 70,6 & A_Protokoll_44 & 75,7 & 63,6 \\
W_FNam_2 & 78,4 & 57,4 & A_Tank_45 & & \\
W_Gnam_3 & 83,8 & 69,9 & A_Kennz_46 & 75,7 & 89,3 \\
W_VNam_4 & 83,8 & 68,4 & A_Spiegel_51 & 90.9 & 93,2 \\
W_GebD_5 & 83,8 & 65,4 & A_Wischer_53 & 87,9 & 91,3 \\
W_GebO_6 & 97,3 & 92,6 & A_Verband_59 & 84,8 & 90,3 \\
W_Gesch_7 & 67,6 & 47,8 & A_KFahr_63 & 43,9 & 55,3 \\
W_Str_8 & 67,6 & 47,8 & A_KKoff_64 & 50,0 & 51,5 \\
W_HNr_9 & 67,6 & 57,4 & A_KStoss_65 & 40,9 & 54,4 \\
W_PLZ_10 & 64,9 & 59,6 & M_Licht72 & 81,8 & 86,8 \\
W_Ort_11 & 91,9 & 79,2 & M_TTür73 & 50,0 & 42,7 \\
W_AW_12 & 54,1 & 57,4 & M_FTür_74 & 43,9 & 57,3 \\
W_Fams_13 & 35,1 & 34,6 & M_TLicht_75 & 47,0 & 23,6 \\
W_KTO_15 & 10,8 & 25,0 & M_EintTür77 & 30,3 & 34,6 \\
W_Inst_17 & 2,7 & 9,6 & M_EintLicht_78 & 19,7 & 26,5 \\
W_WFL_27 & 50.0 & 62,5 & & & \\
L_SÄGE_38 & 63,6 & 82,4 & & & \\
L_SNR_40 & 25,7 & 37,5 & & & \\
L_RepStat_42 & & & & &
\end{tabular}


Tab. 51: Anteil korrekter Antworten nach Bildungsgang innerhalb der Dimension 2

\begin{tabular}{|c|c|c|c|c|c|}
\hline \multirow[t]{2}{*}{ Item } & \multicolumn{2}{|c|}{$\%$ of total } & \multirow[t]{2}{*}{ Item } & \multicolumn{2}{|c|}{$\%$ of total } \\
\hline & $\begin{array}{c}\text { FöA } \\
\text { Gesamt }\end{array}$ & $\mathrm{EAB} / \mathrm{HS}$ & & $\begin{array}{c}\text { FöA } \\
\text { Gesamt }\end{array}$ & $\mathrm{EAB} / \mathrm{HS}$ \\
\hline W_PVer_14 & 48,6 & 30,9 & A_CoDat_47 & & \\
\hline W_BVer_18 & 19.8 & 16,8 & A_CiDat_49 & & \\
\hline W_WB_19 & 13,5 & 22,8 & A_CiKm_50 & 92,4 & 88,3 \\
\hline W_VerNam_20 & 2,7 & 15,4 & A_Reifen_52 & 84,8 & 74,8 \\
\hline W_VorVer_21 & 2,7 & 14,0 & A_Bremse_54 & 39,4 & 40,1 \\
\hline W_VerStr_22 & 2,7 & 13,2 & A_Licht_55 & 87,9 & 90,3 \\
\hline W_HNrVer_23 & 2,7 & 12,5 & A_Instr_56 & 48,5 & 55,3 \\
\hline W_PLZVer_24 & 2,7 & 12,5 & A_Horn_57 & 25,8 & 44,7 \\
\hline W_OrtVer_25 & 2,7 & 13,2 & A_BWerk_58 & 54,4 & 43,7 \\
\hline N_fwas_34 & 64,9 & 59,1 & A_ERad_61 & 83,3 & 88,3 \\
\hline \multirow[t]{3}{*}{ N_fwo_35 } & 64,9 & 66,7 & A_LadeR_62 & 63,6 & 71,8 \\
\hline & & & A_UMiet_67 & 78,8 & 92,2 \\
\hline & & & S_T_69 & 20,7 & 22,1 \\
\hline
\end{tabular}

Aus der methodischen Debatte ging hervor, dass die Personenfähigkeiten $\vartheta_{v}$ mit dem Programm ConQuest (Adams et al. 2015) über Weighted-Likelihood-Estimates bestimmt werden. Weighted-Likelihood-Estimates (WLE) gelten als die besten Punktschätzer der dahinterliegenden Fähigkeit der Probanden (Rost 2004, 316). Die Mittelwerte jener Personenfähigkeiten $\vartheta_{v}$ werden einerseits z-skaliert und andererseits T-skaliert in der nachfolgenden Tabelle zusammengefasst. Es werden die Werte in beiden Skalierungen berichtet, um eine einfachere Vergleichbarkeit mit den Ergebnissen der Lesecompetencies herstellen zu können, die im angewandten Testverfahren Lesen 8-9 in T-Werten ausgegeben werden.

Die Verteilung der analysierten Lesecompetencies in Texten und Textkomplexen zwischen den Jugendlichen des Bildungsgangs mit dem Ziel Förderschulabschluss Lernen an Förderschulen liegen mit $d=0.37$ bzw. 0.20 einerseits erwartungsgemäß statistisch relevant niedriger als bei den Gleichaltrigen an den berufsorientierenden Praxisklasse. Ein Ergebnis der BELLA-Studie war, dass Schüler/innen berufsqualifizierender Angebote beim Verarbeiten von ikonischen Zeichen statistisch bedeutsam mit einem mittleren Effekt besser performten als die Gesamtgruppe aus Förderschüler/innen mit anerkanntem Förderbedarf Lernen (vgl. Kapitel 6.3.3.1; Tabelle 52). Andererseits bestätigt sich gleichermaßen ein statistisch relevanter Unterschied zwischen den Lesecompetencies multikodaler Texte und Textkomplexe der Jugendlichen des Bildungsgangs mit dem Ziel Förderschulabschluss Lernen und der Jugendlichen mit dem Bildungsziel EAB wie er in der PISA-Zusatzstudie festgestellt wurde. Ein direkter Vergleich kann jedoch bekanntlich nicht gezogen werden, da in dieser Referenzstudie einerseits Lesecompetencies entlang von sowohl Fließ- als auch nichtkontinuierlichen Texten und andererseits von einem breiteren Spektrum an Bildungsprogrammen betrachtet wurden, die in der Hierarchie bis in die gymnasiale Sekundarstufe I reichen (vgl. Kapitel 6.3.3.1; vgl. Abbildung 49). Es kann als stimmig interpretiert werden, dass die Performanzen der Jugendlichen des Bildungsgangs mit Ziel Förderschulabschluss Lernen bzw. EAB in der referentiellen PISA-Zusatzstudie deutlich geringer ausfallen. Dies ist ein Ergebnis der Stichprobenwahl in der vorliegenden Erhebung von Lesecompetencies primär ikonischer Kodalität. 
Tab. 52: Verteilung der Lesecompetencies multikodaler Texte und Textkomplexe - Mittelwerte

\begin{tabular}{llcrrrc}
\hline Bildungsgang & Dimension & $\overline{\mathbf{x}}(\mathbf{z})$ & $\mathrm{SD}(\mathrm{z})$ & $\overline{\mathbf{x}}\left(\mathrm{T}^{\mathrm{a}}\right)$ & $\mathrm{SD}\left(\mathrm{T}^{\mathrm{a}}\right)$ & $\mathrm{d} \Delta^{*}$ \\
\hline FöS Praxisklasse & 1 Texte & -.045 & 4.000 & 50,45 & 10,00 & 0.37 \\
& 2 Textkomplexe & .008 & .752 & 50,09 & 7,52 & 0.20 \\
\hline FöS & 1 Texte & -.501 & 4.762 & 44,99 & 17,62 & \\
& 2 Textkomplexe & -.248 & 4.543 & 47,52 & 15,43 & \\
\hline EAB & 1 Texte & .022 & 4.056 & 50,22 & 10,56 & 0.42 \\
& 2Textkomplexe & .016 & 4.331 & 50,16 & 13,32 & 0.19 \\
\hline Gesamt & 1 Texte & -.042 & 4.169 & 49,58 & 11,68 & \\
& 2 Textkomplexe & -.019 & 4.283 & 49,81 & 12,83 & \\
\hline
\end{tabular}

Anmerkung: ${ }^{\mathrm{a}} \mathrm{T}=10 \mathrm{z}+50$

${ }^{*}$ Effektstärken der Differenz zu den Förderschulen Lernen

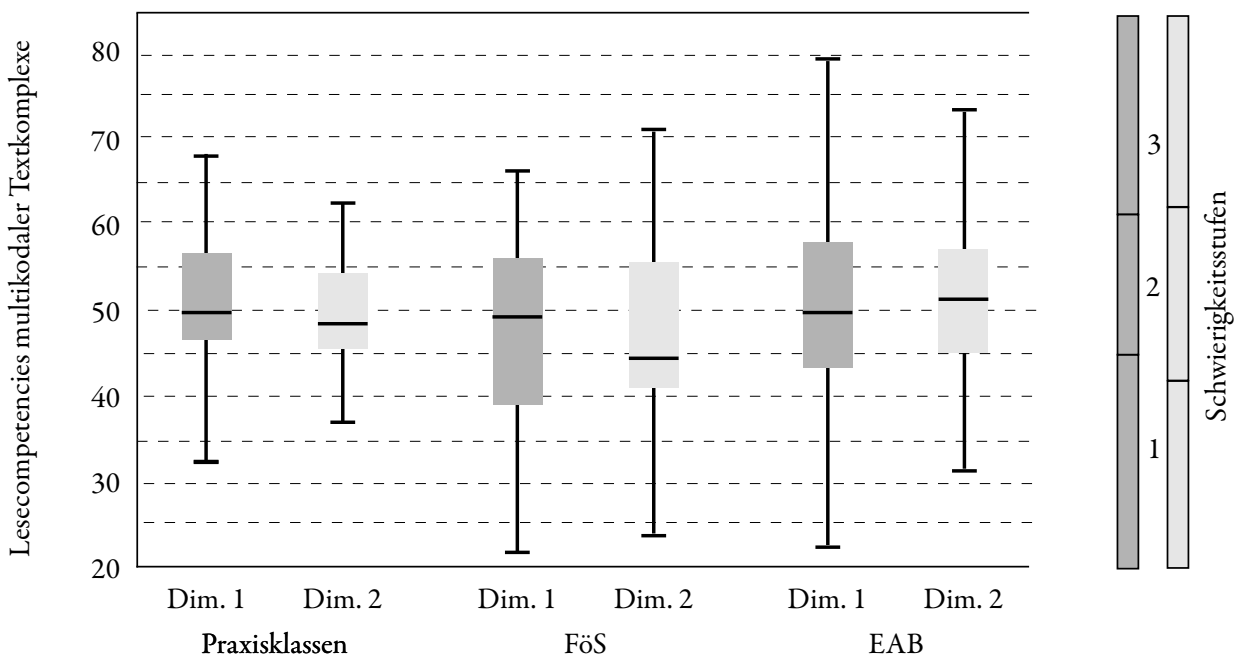

Abb. 49: Verteilung der Lesecompetencies multikodaler Texte und Textkomplexe - Quartile und Median

Eine weitere Analogie zu den benannten Referenzstudien verdeutlicht sich in der auffällig höheren Streuung insbesondere der erhobenen WLE-Personenfähigkeiten der Schüler/innen an den Förderschulen Lernen innerhalb der ersten Standardabweichung. Das heißt es muss auch für die Lesecompetencies multikodaler Texte und Textkomplexe festgehalten werden, dass einerseits kein geringer Anteil unter diesen Jugendlichen das durchschnittliche Leistungsniveau der Stichprobengruppe EAB erreicht. Andererseits trifft für ca. $45 \%$ unter den Schüler/innen an den Förderschulen Lernen zu, dass sie nur die Aufgaben des einfachsten Schwierigkeitsniveaus korrekt bearbeiten haben (vgl. Abbildung 49).

\subsubsection{Deskriptive und vergleichend kriteriale Auswertung der Lesecompetencies von Fließtexten}

Die Jugendlichen des Bildungsgangs mit Ziel Förderschulabschluss Lernen und EAB haben neben den Testaufgaben entlang primär ikonischer Texte und Textkomplexe zusätzlich den Test Lesen 8-9 bearbeitet. Den Jugendlichen an Praxisklassen wurde dieses standardisierte Verfahren 
aus einerseits „Basalen Lesekompetenzen“ auf der Wort- und Satzphrasenebene und andererseits dem „Textverständnis“ auf Satz- bis Textebene nicht vorgelegt. Die Performanzen beider Stichprobengruppen (vgl. Tabelle 53) zeigen erwartungsgemäß für die Dimension „Textverständnis“ eine statistisch bedeutsame Verteilung auf $(\mathrm{d}=0.87)$. In der referentiellen PISA-Zusatzstudie lag die statistische Signifikanz zwischen den Lesecompetencies bei Fließtexten der Probanden an den Förderschulen Lernen gegenüber dem Bildungsgang Lernen mit $\mathrm{d}=4.08$ vergleichbar im Bereich, der nach Cohen als starker Effekt bezeichnet werden kann. Eine vergleichsweise größere Streuung der Performanzen unter den Schüler/innen an den Förderschulen Lernen fällt wiederum für das „Textverständnis“ in beiden Testverfahren auf. Die Testkonstruktion von Lesen 7-9 sieht Items der „Basalen Lesekompetenzen“ mit ihrem Fokus der Verarbeitung auf Wort- bis Satzphrasenebene vor (vgl. Kapitel 6.1.3.1), die innerhalb der Rahmenkonzeption der PISA-Zusatzstudie explizit nicht wiederzufinden sind (vgl. Kapitel 6.4.1).

Tab. 53: Verteilung der Lesecompetencies kontinuierlicher Texte - Mittelwerte

\begin{tabular}{llccc}
\hline Bildungsgang & Dimension & $\overline{\mathbf{x}}(\mathrm{T})$ & $\mathrm{SD}(\mathrm{T})$ & $\mathrm{d} \boldsymbol{\Delta}^{*}$ \\
\hline FöS Praxisklasse & Gesamtskala & - & - & - \\
& 1 „Basale Lesekompetenzen“ & - & - & - \\
& 2 „Textverständnis“ & - & - & - \\
\hline FöS & Gesamtskala & 32.06 & 13.53 & \\
& 1 „Basale Lesekompetenzen“ & 37.12 & 7.00 & \\
& 2 „Textverständnis“ & 36.32 & 14.58 & \\
\hline EAB & Gesamtskala & 54.21 & 9.44 & 4.69 \\
& 1 „Basale Lesekompetenzen“ & 54.43 & 8.85 & 3.36 \\
& 2 „Textverständnis“ & 47.02 & 10.42 & 0.87 \\
\hline Gesamt & Gesamtskala & 43.19 & 14.73 & \\
& 1 „Basale Lesekompetenzen“ & 47.18 & 14.79 & \\
& 2 „Textverständnis“ & 42.54 & 13.35 & \\
\hline
\end{tabular}

Anmerkung: ${ }^{\mathrm{T}} \mathrm{T}=10 \mathrm{z}+50$

*Effektstärken der Differenz zu den Förderschulen Lernen

Jene Prüfkriterien aus der Gegenüberstellung mit der PISA-Zusatzstudie verdeutlichen nicht zuletzt, dass die Schüler/innen an den Förderschulen Lernen eindeutig unterdurchschnittliche Lesecompetencies der globalen Kohärenzbildung bei Fließtexten nachweisen können - eingebettet in eine Lesesituation mit der routinalisierten Sinn- und Relevanzoption entlang der Behaltensleistung. Insbesondere die „Basalen Lesekompetenzen“ fallen mit Blick auf die Performanzen der ca. 68,4\% unter diesen Jugendlichen innerhalb der ersten Standardabweichung besorgniserregend niedrig auf - und dies deutlicher im Kontrast zu den Lesecompetencies des „Textverständnisses“.

\subsection{Interferenzstatistische Auswertung}

In den zurückliegenden Kapiteln wurden Maßnahmen bzw. Merkmale zur Kontrolle der Durchführungs-, Auswertungs- und Interpretationsobjektivität bis hin zur internen und externen Modellgüte (Bäuerlein et al. 2012, 11ff.) der explorativen Teilstudie zu den Lesecompetencies multikodaler Texte und Textkomplexe primär ikonischer Kodalität und zu den Lesecompeten- 
cies entlang von Fließtexten zusammengetragen. Die dahinterliegenden Testaufgaben wurden vor dem Hintergrund des Erkenntnisinteresses entwickelt bzw. übernommen und angewandt, um explorativ zu prüfen, ob einerseits von einem überzufälligen Zusammenhang zwischen den erhobenen Lesecompetencies ausgegangen werden kann; d. h. zwischen den Lesecompetencies entlang (a) primär ikonisch vs. entlang (b) symbolisch repräsentierter Texte/Textkomplexe situativ eingebettet in den routinalisierten lokalen kulturellen Codes entlang der (b) Behaltensleistung vs. (a) Organisationshilfe, Merkhilfe, Kontrolle und Weitergabe. Andererseits akzentuiert die zweite Forschungsfrage der explorativen Teilstudie die Prüfung der prädikativen Aussagekraft erster Kategorie (a) für zweitere Kategorie (b) von Lesecompetencies.

Die nachfolgenden interferenzstatistischen Analysen beanspruchen hierbei keine Generalisierbarkeit. Einschränkungen wurden bereits umfassend im Spiegel der kognitiven Verarbeitung diskutiert. Das zugrundeliegende Arbeitsmodell der Verarbeitung von semiotischen Zeichen positioniert eindeutig diverse Einflussfaktoren für eine skillful performance entlang eines Textes. Es wurde von dem Mainstream-Ansatz einer mentalen Modellierung ausschließlich explizit-konzeptioneller kognitiver Verarbeitungsprozesse genommen, die entlang abzulehnender textstrukturell inhärenter und somit für jegliche Lesesituation generalisierbarer hierarchischer Komplexitätsgrade erschlossen werden könnten. Vor dem Hintergrund der Stichprobenabhängigkeit im zugrundeliegenden Rasch-Modell zur Analyse der Lesecompetencies multikodaler Texte und Textkomplexe primär ikonischer Kodalität muss hervorgehoben werden, dass die Ergebnisse insbesondere nicht auf Jugendliche anderer Bildungsprogramme übertragen werden können. Trotz Kontrolle der tatsächlichen Stichprobe nach einigen Personenmerkmalen kann keine Zufallstichprobe beansprucht werden - dies kann bei explorativen Studien nicht erwartet werden. Vorab muss geschlussfolgert werden, dass die Ergebnisse durch entsprechende Folgestudien geprüft werden müssen.

\subsubsection{Statistischer Zusammenhang zwischen den Dimensionen primär ikonischen multikodalen Lesens und symbolischen Lesens}

Es konnte - wie zu erwarten war - nachgewiesen werden, dass die jeweils beiden Dimensionen von Lesecompetencies positiv miteinander stark (Cohen 1988) korrelieren. Dieser wechselseitige Zusammenhang ist zwischen den Dimensionen der Lesecompetencies des symbolischen Lesens statistisch leicht stärker als bei den Lesecompetencies multikodaler Texte und Textkomplexe, aber vergleichbar bedeutsam. Die Korrelationswerte innerhalb der Dimensionen liegen jedoch unterhalb des Cut-Off-Werts des Korrelationskoeffizienten $r<0,7$ bzw. $r>-0,7$ bei der Prüfung der linearen Unabhängigkeit. Es liegt folglich keine Multikollinearität zwischen den jeweilig beiden Dimensionen je Kodalität von Lesecompetencies vor; dies ist zugleich eine Voraussetzung für die nachfolgende regressionsanalytische Auswertung.

Demgegenüber zeigt sich - was zu befragen war - ein statistisch weniger bedeutsamer, mittlerer Zusammenhang nach Cohen (ebd.) nur zwischen den Lesecompetencies multikodaler Texte primär ikonischer Kodalität und den Lesecompetencies entlang von Fließtexten, einhergehend mit den routinalisierten Sinn- und Relevanzoptionen der jeweiligen Lesesituation. Dieser Befund leitet einerseits über zur Analyse jener aufgedeckten notwendigen, aber nicht hinreichenden Bedingung eines kausalen Zusammenhangs und lässt daher andererseits die Schlussfolgerung zu, dass die Lesecompetencies multikodaler Textkomplexe in der untersuchten Stichprobe und in ihrer situativ-kontextuellen Einbettung im Sinne der Organisationshilfe, der Merkhilfe, der Kontrolle und der Weitergabe nicht durch die Lesecompetencies symbolischen Lesens erklärt werden kann. 
Tab. 54: Korrelation zwischen Dimensionen von Lesecompetencies symbolischer Texte quer zu multikodaler Texte

\begin{tabular}{llcccc}
\hline Lesecompetencies & Dimension & ikonTexte & ikonTkomp & „BasLk“ & „Textver“ \\
\hline ikonTexte & Pearson r & & $.491^{* *}$ & .136 & $.338^{*}$ \\
& $\mathrm{p}$ (2-seitig) & & .000 & .380 & .025 \\
\hline \multirow{2}{*}{ kkonTkomp } & Pearson r & & & -.059 & .108 \\
& $\mathrm{p}$ (2-seitig) & & .706 & .486 \\
\hline „BasLk“ & Pearson r & & & $.537^{* *}$ \\
& $\mathrm{p}$ (2-seitig) & & & .000 \\
\hline „Textver“ & Pearson r & & & \\
& $\mathrm{p}$ (2-seitig) & & & & \\
\hline
\end{tabular}

Anmerkungen: ${ }^{* *} p<0.01 ;{ }^{*} p<0.05$

ikonTexte $=$ Lesecompetencies multikodaler Texte primär ikonischer Kodalität eingebettet entlang der routinalisierten lokalen kulturellen Codes der Organisationshilfe, der Merkhilfe, der Kontrolle und der Weitergabe

ikonTkomp = Lesecompetencies multikodaler Textkomplexe primär ikonischer Kodalität eingebettet entlang der routinalisierten lokalen kulturellen Codes der Organisationshilfe, der Merkhilfe, der Kontrolle und der Weitergabe

„BasL"= Lesecompetencies auf der Ebene der lokalen Kohärenzbildung symbolischer Zeichen entlang der routinalisierten lokalen kulturellen Codes der Behaltensleistung

"Textverständnis" $=$ Lesecompetencies auf der Ebene der lokalen Kohärenzbildung symbolischer Zeichen entlang der routinalisierten lokalen kulturellen Codes der Behaltensleistung

\subsubsection{Kausaler Zusammenhang zwischen den Dimensionen primär ikonischen multikodalen Lesens und symbolischen Lesens}

Es wurde festgestellt, dass die Lesecompetencies multikodaler Texte (ikonTexte) sowohl mit den Lesecompetencies multikodaler Textkomplexe (ikonTkomp) stark korrelieren, als auch mit einem mittleren Effekt mit den Lesecompetencies auf der Ebene der globalen Kohärenzbildung entlang von Fließtexten („Textver“) und dies situativ-kontextuell eingebettet in Lesesituationen der entsprechenden routinalisierten Sinn- und Relevanzoptionen. Mit dem Ziel zu untersuchen, ob und mit welcher statistischen Aussagekraft die Lesecompetencies bei Fließtexten, eingebettet in die typische literale Praktik der Sekundarstufe I, die Performanz entlang multikodaler Texte - aber nicht Textkomplexe - determiniert werden, wurde eine einfache lineare Regressionsanalyse berechnet. Die Regressionsanalyse ergibt (vgl. Tabelle 55), dass der untersuchte Prädiktor Lesecompetencies bei Fließtexten statistisch signifikant die Performanzen entlang von multikodalen Texten in der Stichprobe voraussagen $(p<.05)$. Der Prädiktor kann etwa $9 \%$ der Gesamtvarianz aufklären. Ein entsprechend korrigierter multipler Determinationskoeffizient im Bereich von $.13>R^{2}>.02$ kann, jedoch nach Cohen (1988) nur eine geringe Varianzaufklärung tatsächlich zugesprochen werden.

Tab. 55: Lineare Regressionsanalyse zur Vorhersage der Lesecompetencies multikodaler Texte primär ikonischer Kodalität

\begin{tabular}{llccc}
\hline Einflussvariable & & unstandardisiert & standardisiert & Standardfehler \\
\hline Konstante & $\mathrm{t}$ & 44.645 & & \\
„Textver“ & $\mathrm{B}$ & $.220^{*}$ & $.338^{*}$ & $.094^{*}$ \\
\hline & $\mathrm{R}^{2}$ & .115 & & \\
& korr. $\mathrm{R}^{2}$ & .093 & & \\
& $\mathrm{~F}(\mathrm{df}=43)$ & 5.432 & & \\
& & & & \\
\hline
\end{tabular}

Anmerkungen: * $\mathrm{p}<0.05$

Durbin-Watson-Statistik $=2.295$ 
Neben der bereits dokumentierten Prüfung einer nicht vorliegenden Multikollinearität des Messmodells, kann entlang der Durbin-Watson-Statistik zudem von einer Unabhängigkeit der Residuen ausgegangen werden (vgl. Tabelle 55). Graphische Prüfungen lassen des Weiteren eine Homoskedastizität und Normalverteilung der Residuen postulieren.

\subsection{Ergebnis der Teilstudie}

(Quant_I) Sind die Anforderungen einer Handlungsfähigkeit entlang der an Einfach(st)arbeitsplätzen aufgefundenen ikonischen Textualität zu vernachlässigen, da die funktionalen Lesecompetencies im Sinne eines sozialen Identifikationskriteriums bereits eine Teilhabewahrscheinlichkeit in auch Einfach (st)arbeit ausreichend determinieren?

Die literalen Praxen des Lesens der Schule, welche das Bewertungsprogramm charakteriseren, sind durch kontinuierliche bis multikodale Texte primär symbolischer Kodalität, eingebettet in Lesesituationen entlang der Behaltensleistung und der Einstellungsänderung. Demgegenüber finden, für das Bewertungsprogramm entscheidend, multikodale Texte primär ikonischer Kodalität quer zu den routinalisierten lokalen kulturellen Codes der Organisationshilfe, der Merkhilfe, der Kontrolle und der Weitergabe keine Berücksichtigung. Typische schulische Sachtexte entsprechen weder der primären Kodalität noch den genannten Sinn- und Relevanzoptionen der Lesesituationen. Dieser Befund wurde vor dem Hintergrund der Voraussetzungen von Teilhabe an Einfach(st)arbeitsplätzen nur insofern als besorgniserregend interpretiert, dass die schulisch relevanten Lesecompetencies die Arbeitsplatz relevanten Lesecompetencies im besten Fall bereits umfassend abdecken.

Ergebnis der explorativen Teilstudie aus standardisierten Erhebungen jener beiden Lesecompetencies ist jedoch, dass das für das schulische Bewertungsprogramm relevante Lesen der Behaltensleistungnur eingeschränkt die textuellen Anforderungen an den untersuchten Einfach(st)arbeitsplätzen tatsächlich abdecken: Während eine skillful performance entlang dem Lesen von Textkomplexen aus einer Kombination aus kategorialen Tabellen, graphischen Darstellungen, Diagrammen und Karten in keinem statistisch bedeutsamen kausalen Zusammenhang steht, gilt dies durchaus für das Pendant des Lesens einer einzelnen kategorialen Tabelle, einer einzelnen graphischen Darstellung, eines kategorialen Diagramms und von Karten. Es muss jedoch hervorgehoben werden, dass höhere Performanzwerte bei der globalen Kohärenzbildung innerhalb der Stichprobe nur gering statistisch bedeutsam bessere Performanzwerte bei primär ikonischen Texten - und nicht Textkomplexen - erklären können. Dieses zentrale Ergebnis lässt sich vor dem Hintergrund der dargelegten Forschungslage zur Verarbeitung beider semiotischer Zeichen nachvollziehbar einordnen. Die Kohärenzbildung von primär ikonischen Texten setzt sich einerseits aus zu verarbeitenden Globalzeichen unterer Ordnung (vgl. Kapitel 3.3.1) und aus Lokalzeichen auseinander, oder in anderen Worten, aus Relationen von Sachverhalten, die einer kategorialen Tabelle, einer graphischen Darstellung, einem kategorialen Diagramm oder einer Karten bereits inhärent sind. Insbesondere die Lokalzeichen setzen sich repräsentional zwar primär aus, wie dargelegt, Sets starker ikonischer Darstellungs-Kodes zusammen, jedoch nicht selten sekundär aus symbolischen Steuerungs-Kodes. Beispielsweise sind Tabellenfelder explizit symbolisch gekennzeichnet. Es müssen beim Lesen folglich ähnlich wie bei der globalen Kohärenzbildung entlang von Fließtexten teils Relationen aus Sachverhalten (re)konstruiert bzw. aktualisiert werden. Obgleich die Repräsentation jener Relationen sich semiotisch unterscheiden, ist deren Verarbeitung - hier gemeint im Sinne des dekonstruierten Arbeitsmodell der Verarbeitung - jeweils Kern der Kohärenzbildung. Die Verarbeitung von Globalzeichen höherer Ordnung, welche bei multikodalen Textkomplexen den Kern der Kohärenzbildung markieren, unterliegt demgegenüber Anforderungen in der Modellierung, die nicht mehr viel mit denen beim Lesen von Fließtexten gemein haben. 


\section{Empirischer Blick auf das praxeologische „Wie“ des Lesens multikodaler Texte}

In Zusammenarbeit mit Judith Adam

\subsection{Forschungsfrage}

(Qual_II) Teilhabe an sozialen Praxen mit nichtkontinuierlichen Texten und Textkomplexen an Einfach(st)arbeitsplätzen eröffnet Lernprozesse auch explizit-konzeptionellen Wissens über semiotische Strukturen? (Können diese Unterschiede oder Gemeinsamkeiten mittels des theoretisch abgeleiteten Makromodells abgebildet werden? Respektive, zeigen sich eindeutig abzugrenzende Präferenzen oder eher wechselseitig sich beeinflussende implizit-embodied oder explizit-konzeptionelle kogntivite Verarbeitungsprozesse bei den Teilnehmenden?)

Aus den theoretischen Vorarbeiten ging das Forschungsinteresse hervor, sich insbesondere den implizit-embodied Verarbeitungsprozessen anzunähern, welche im dekonstruierten Arbeitsmodell zum Lesen multikodaler Texte und Textkomplexe in ein wechselseitiges Verhältnis zu den explizit-konzeptionellen Pendants gestellt wurden.

Aus der multivariaten Analyse der Lesecompetencies multikodaler Texte und Textkomplexe ging im Trend hervor, dass Schüler/innen mit anerkanntem sonderpädagogischem Bildungs-, Beratungs- und Unterstützungsbedarf im Schwerpunkt Lernen an berufsqualifizierenden Angeboten bzw. Praxisklassen statistisch bedeutsam besser performten als ihre Altersgenossen an Förderschulen Lernen. Vor diesem Hintergrund bot sich an, abschließend nicht ,nur' diesen Unterschied in den Lesecompetencies der Jugendlichen zu erklären, sondern auch - soweit mit den geringen finanziellen und personellen Ressourcen möglich - zu verstehen. Jenes Verstehen akzentuiert folglich die Befragung, inwiefern eher implizit-embodied und explizit-konzeptionelle Verarbeitungen bei Probanden mit hohen bzw. niedrigen Performanzwerten angenommen werden können.

\subsection{Design der Erhebung}

Es war folglich auf der Basis des theoretisch dekonstruierten Arbeitsmodells der Verarbeitung von multikodalen Texten und Textkomplexen (vgl. Kapitel 3.3.3.4) ein Forschungssetting zu entwickeln, um einzelne Indizien beider Verarbeitungsprozesse aufzudecken. Aus der bisherigen Darlegung bietet sich an, Spuren innerhalb einer victorious experience während der Bearbeitung multikodaler Text bzw. Textkomplexe aufzudecken - im Sinne einer rekontextualisierten Schematisierung, Habituation und Automation innerhalb konkret körpergebunden erfahrener Verarbeitung. Im Sinne eines sensibilisierenden Konzepts bieten sich u. a. folgende Indizien an: - Verbalisierung der Bearbeitung orientiert sich an körpergebundenen räumlich-zeitlich-sukzessiven Handlungsabläufen und -rahmenbedingungen entlang bspw. von Methaphern bis parasprachlichen Gesten eines

- „mehr/weniger“: z. B. verkörperlichte quasi-gustatorische Erfahrungen;

- „vorher/nachher“: z. B. verkörperlichte quasi-akustische Erfahrungen;

- „auf/ab“: z. B. verkörperlichte quasi-visuelle Erfahrungen;

- „hinter/vor“" z. B. verkörperlichte quasi-olfaktorische Erfahrungen;

- „zusammengehörend/nicht-zusammengehörend“: z. B. verkörperlicht erfahrene Bewegungen mit bspw. den Fingern horizontal und vertikal zu einem Punkt (quasi-haptisch/taktil); 
- Verbalisierung der Bearbeitung orientiert sich nicht an statischen Zuständen, zu denen zu memorierende Codes, Preise oder (Fach-)Begriffe im Zentrum der Erklärung stehen;

- Verbalisierung der Bearbeitung orientiert sich am knowledge about how content area knowledge is represented, und somit zu typischen Darstellungsstragien zum Sachverhalt oder der dahinterliegenden Merkmale und Attribute;

- Verbalisierung der Bearbeitung orientieren sich am knowledge about how document knowledge is represented und somit zum verkörperlichten ,Wissen' über Autorenschaft des Textes bzw. Textkomplexes, einschließlich der jeweiligen Verantwortungszuständigkeit, der jeweiligen Addressaten sowie der jeweiligen Intention;

- keine Verbalisierung der Textsorte, des Textformats, der Textsorte oder spezifischer Superstrukturen;

- keine Verbalisierung typischer Darstellungsstragien generell in ökonomischen, rechtlichen, politischen, organisatorischen usw. Kontexten;

- Verbalisierungen der Bearbeitung orientieren sich an verkörperlichten Handlungsabläufen zur internal representation of the task to be completed und of the response to be output mittels zuvorderst von Hinweisen zu Handlungsroutinen.

Die explikative Annäherung an dieses überwiegend implizit situativ aktualisierte Wissen, diese Werte, diese Einstellungen sowie an die sozialen Beziehungen, die eine Lesesituation sozial markieren, können über die deduktiven Methoden und Techniken des quantitativen Paradigmas gegenwärtig methodologisch schlüssig nicht operationalisiert werden. Diese Explikationen zielen dagegen auf Erklärungen begrenzter Reichweite, die jene raum-zeitlichen Bedingungen jener zuvorderst in der sozialen Realität implizit aktualisierten Werte, Einstellung und sozialen Beziehungen gegenstandsbezogen verankern und zu einem gewissen Grad verallgemeinern (Lamnek 2005, 117ff.): Umfassender Art basieren diese eher auf dem knowing how der teilnehmenden Personen, deren Regel- und/oder Sinnhaftigkeit dem Forschenden eher verborgen als zugänglich ist, dies umso mehr ,je tiefer diese in ihrer habitualisierten, routinemäßigen Handlungspraxis verankert sind“ (Bohnsack 2001, 336f. zit. nach ebd., 225).

Vor diesem Hintergrund wird das Erhebungsverfahren des Lauten Denkens zur Rekonstruktion von Erfahrungen und Wissen in Verarbeitungsprozessen empfohlen, die zu mentalen Modellierungen führen. Nach Konrad $(2010,481)$ bietet es ,am ehesten die Möglichkeit in Forschungsprogrammen handlungssteuernde Kognitionen zu beleuchten“, die dem qualitativen Paradigma und dem Ansatz folgen, in denen Kognition sozio-kulturell eingebettet verortet wird. Innerhalb der Debatte um die Methode zeichnet sich eine Präferenz von (teil-)strukturierten Settings ab, die keine Erfüllung der klassischen Gütekriterien, aber deren Kontrolle zum Kern haben. Neben gängigen kontrollierenden Maßnahmen wie z. B. der Multiperspektivität oder Theoriegeleitetheit (Scherer/Opitz 2010, 31ff.) können die interessierenden Lern- und Problemlösesequenzen in einem gewissen Grad strukturiert werden, der das Prinzip der Offenheit noch gewährleistet. Die Planung des Erhebungssettings sollte folglich berücksichtigen, dass die Probanden auch die Möglichkeit bekommen, ihre Entscheidungen während der Auseinandersetzung mit multikodalen Texten und Textkomplexen unmittelbar und frei benennen und kommentieren zu können. Diese Situationen sollen den Probanden möglichst nicht den Eindruck vermitteln, dass sie kontrolliert werden und nicht mit Zwang ihr Handeln augenblicklich oder unmittelbar an die Introspektion anschließend verbalisieren müssen (Konrad 2010, 478ff.; Yom et al. 2007, 637f.). Teilstrukturierte Settings ergänzen daher keine prompts oder verbale Verstärker, sprich Fragen während der Bear- 
beitung, sondern von medial unterstützten, sogenannte retroperspective protocols. Konkret heißt dies, dass die Probanden während der Bearbeitung einerseits zur freien Kommentierung (=concurrent protocols) des Vorgehens motiviert werden, während andererseits die Bearbeitung filmisch festgehalten wird. Die unmittelbar anschließende Sichtung des Videos gibt Impulse zur Retrospektion (=retrospective protocols). Diese Form wird insbesondere für Personen empfohlen, die ihre verbalen Fähigkeiten weniger eloquent einschätzen und nur zu wenigen Äußerungen während der Introspektion neigen (Konrad 2010, 481f.; Yom et al. 2007, 646f.).

Die favorisierte Methodenwahl lag in einer teilstandardisierten Einzelfallerhebung des Lauten Denkens zur Exploration möglicher impliziter, wie auch expliziter Verarbeitungsprozesse in mehrseitigen (komplexeren) Texten primär ikonischer Kodalität. Eine Einzelfallstudie ausgewählter Idealtypen erlaubte es am ehesten zu erschließen, ob es neben allen bestehenden Vorabannahmen einer skillful performance entlang von Texten, sich die Möglichkeit „herstellen“ lässt, „über individuelle Prozesse und Verläufe“ (Bortz/Döring 2006, 323) die Forschungsfrage der Teilstudie zu beantworten, respektive, Indikatoren dafür gegeben sind, wo, wie und auf welche Art und Weise die Probanden zu „Lösungen“ bzw. zu einer entsprechenden Verarbeitungstiefe gelangen. Es handelt sich hierbei um eine intensive Erforschung von kontrollierten Idealtypen. Die Kriterien werden innerhalb der Durchführung benannt (vgl. 7.3.1). An dieser Stelle soll lediglich darauf hingewiesen werden, dass die Idealtypen sozusagen, einmal den „Normalfall“ (keine berufsqualifizierenden Erfahrungen), den „Ausnahmefall“ (berufsqualifzierende, aber nur unmittelbare Erfahrungen im entsprechenden Berufsfeld) und den „Extremfall“ (deutliche und kontinuierliche Erfahrungen im entsprechenden Berufsfeld) abbilden.

\subsubsection{Teilstrukturierte Situation der Interspektion}

Als Setting wurde eine Situation über ein Rollenspiel simuliert, in der ein möglichst authentischer multikodaler Textkomplex primär ikonischer Kodalität zu bearbeiten war. Gleichermaßen zentral war die Simulation der Kriterien, die eine skillful performance in der Situation erst ausmacht, sprich entsprechend der routinalisierten Relevanz- und Sinnoptionen, die an den Einfach(st)arbeitsplätzen exploriert werden konnten. Oder in anderen Worten, es wurde ein möglichst authentischer Arbeitsauftrag nachempfunden, welcher ausschließlich mündlich eingeführt wurde. Der multikodale Textkomplex war nicht durch einen Fließtext begleitet, geschweige eingeführt oder als Repräsentationsdublette ergänzt.

Neben der Auswahl eines multikodalen Textkomplexes wurden Vorkehrungen einer Videographie der Simulation getroffen. Mit dem Ziel, dass sich die Probanden nicht dauerhaft kontrolliert fühlen und eine Retrospektion der Bearbeitungsprozesse initiiert werden kann, wurden primär die Texte per Video festgehalten. Die Videographie diente auf dieser Weise nicht nur als Input für die Retrospektion, sondern als Quelle für die spätere Transkription des laut Gesagten und laut Gedachten. Ergänzend wurden von den jeweilig Testleitenden Gedächtnisprotokolle zur gesamten Phase der videographierten Datenerhebung geführt. Die Methode „des lauten Denkens" wurde den teilnehmenden Schüler/innen als eine Möglichkeit, während des Arbeitsprozesses die eigenen Gedanken zu verbalisieren, angeboten, jedoch nicht explizit verlangt. Bei den vorgeschalteten Vorstudien zur Methodenentwicklung konnten die obig benannten Problematiken des „lauten Denkens“ bestätigt werden. Unter der Zielgruppe ist von Probanden auszugehen, die sich durch prompts unter Druck oder Kontrolle gesetzt fühlen und letztlich mit Irritationen reagieren würden. Die Erinnerung an die Introspektion beeinflusste sichtlich die Verarbeitung der multikodalen Textkomplexe. 
Im Rollenspiel schlüpfte der/die jeweilige Proband/in vorstrukturiert in die Rolle eines/einer Arbeitnehmers/in und der jeweilige Testleitende in die Rolle des Vorgesetzten, der dem Mitarbeitenden einen für ihn/sie neuen Arbeitsauftrag erteilt.

Ein Rollenspiel hat immer einen Als-ob-Charakter. Er wird durch eine Instruktion eingeleitet [...] und besteht aus einer Handlungs- oder Verhaltenssequenz, die einem Publikum präsentiert wird (vgl.: Sader, 1986, 1995). [...] Im Rollenspiel kann sich der Versuchsleiter in der Beobachterrolle befinden, oder selbst ,mitspielen' (ebd., 322).

Der jeweilig Testleitende wurde für seine/ihre Aufgabe der systematischen Beobachtung während des Rollenspiels entlang der Kriterien des sensibilisierenden Konzepts vorbereitet. Wichtig war sicherzustellen, dass er/sie an der Situation nur in der vorgesehenen Rolle aktiv beobachtend teilnimmt.

\subsubsection{Teilstrukturierte Situation der Retrospektion}

Ausgehend von verschiedenen Vorüberlegungen werden die Rollenspiele videographiert, um sie unmittelbar im Anschluss als Input für ein ex-post erhobenes Laut-Denken-Protokoll zur Verarbeitung des Textkomplexes heranzuziehen. Nicht weiter in der Debatte zum Lauten Denken behandelt, kann dieser Teil der Erhebung als ein problemzentriertes Interview interpretiert werden. Diese Interviewmethode wird gleichermaßen als „teilstandardisierte Methode klassifiziert“ (Reinders 2012, 101), das auf „Leitfragen aus einer Kombination von offenen, erzählgenerierenden Fragen und eher strukturierten Fragen“ (ebd.) beruht. Die Kriterien des sensibilisierenden Konzepts eingebettet, erschließt sich methodisch begründet die Möglichkeit, kontrolliert einzelne beobachtbare und/oder vermutete verkörperlichte vs. statische Verarbeitungsprozesse zu explizieren. Die Grundausrichtung aus deduktivem und induktivem Vorgehen empfiehlt, die gewonnenen Informationen und Einsichten während den Beobachtungen zur Modifikation und Präzisierung der Fragestellung heranzuziehen und ggf. die Fragestellung im Interview situativ anzupassen. Die drei Grundpositionen des problemzentrierten Interviews sensu Witzel (2000) konkretisieren die Erhebung:

- „Problemzentrierung“: Wie bearbeiten die Schüler/innen die textuellen Anforderungen, welche Strategien und welches Vorwissen können sie nutzen?

- „Gegenstandsorientierung“: Ändert sich die Verarbeitung einer/s Schüler/s bei semiotisch vermeintlich unterschiedlich komplexen Repräsentationen?

- „Prozessorientierung“: Möglichkeit zur ggf. notwendigen Modifikation der Aufgabenstellung, des Settings, des Interviews, der Forschungsfrage, etc.

Die Entwicklung des Leitfadens erfolgte zur Vorstudie und wurde für die explorative Einzelfallstudie weiterentwickelt. Anhand der mit ihnen durchgeführten Probedurchläufe konnten die Leitfragen zum problemzentrierten Interview erarbeitet und optimiert werden. Der Aufbau des Interviews folgt der Gliederung nach Lamnek (2010, 335f.) für ein problemzentriertes Interview: In der ersten Phase des Interviews liegt der Schwerpunkt auf den allgemeinen Befindlichkeiten der Schüler/innen und der Möglichkeit erste eigene Überlegungen zur Bearbeitung des Textkomplexes kundzutun. In der zweiten Phase gilt es, sofern die Schüler/innen nicht beide Phasen aus sich heraus bereits verknüpfen, erste Überlegungen zur interessierenden Verarbeitung zu äußern. Die dritte Phase nutzt sowohl die Möglichkeit des Zurückspiegelns, der Verständnisfrage wie auch der Konfrontation. An dieser Stelle sollten und konnten die datenerhebenden Testleitenden, je nach Beobachtung selbstständig eigene Schwerpunkte anhand des 
Leitfadens setzen. „In der (vor)letzten Phase sind nun Ad Hoc-Fragen möglich. Hier kann [und soll] der Interviewer direkte Fragen zu Themenbereichen stellen, die der Befragte bislang noch nicht von sich aus genannt hatte" (ebd.).

\subsubsection{Gedächtnisprotokoll}

Im Anschluss an die Interspektion und Retrospektion/problemzentrierte Interviews war durch die Testleitende ein Gedächtnisprotokoll (ebd., 557f.) zeitnah zu erstellen, wenn möglich nicht später als „Mit der Distanz der Nacht [, denn] vielleicht [ist] sogar [mit ihr] gewährleistet, dass die wesentlicheren Tatsachen notiert werden“" (ebd., 559). Neben der zeitlichen Vorgabe wurde der Inhalt entlang nachfolgender 5 Punkte via einer Vorlage vorstrukturiert (vgl. ebd., 562ff): Als erstes sollte der persönliche Eindruck über den/die Probanden/in festgehalten werden, ergänzt durch entsprechende Angaben, die durch den/die Probanden/in kommuniziert wurden. Zweitens wurde die faktische Situation der Datenerhebung dokumentiert, z. B. alle technischen Details wie Zeitpunkt und Dauer. Der dritte Aspekt galt der sozialen Situation der Probanden. Trat ein bestimmtes Verhalten zutage, traten soziale Störungen auf etc., so wurde auch dies notiert. Daraufhin galt es im vierten Aspekt der Dokumentation darzulegen, ob (bedeutsame) Auslassungen aufgefallen sind, so zum Beispiel, dass die Teilnehmenden ihre Arbeit verweigerten, verbunden mit dem Schritt, mögliche erklärende Vermutungen zu äußern. In derselben Weise galt es mit dem fünften Aspekt umzugehen, bezogen auf (bedeutsame) soziale Abweichungen, wie bspw. starke Gefühlsäußerungen der Teilnehmenden. Die Gedächtnisprotokollvorlage galt folglich der systematischen Dokumentation der Wahrnehmung. Dabei wurde der chronologische Aspekt bei besonderen Vorkommnissen bewusst außen vor gelassen, dennoch stand es den Testleitenden frei, Zeitmarkierungen später im Abgleich mit dem Video in ihrem Protokoll hinzuzufügen. Um eine Trennung zwischen Sachverhalt und Interpretation, zumindest in Ansätzen, zu gewährleisten, galt es in bewusster Absicht eine mögliche Interpretation bzw. Vermutung zu einem auftretenden Sachverhalt zu skizzieren.

\subsubsection{Auswertungsdesign}

Die Auswertung erfolgte in mehreren Schritten. Im Anschluss an die Festlegung des Materials galt es die Entstehungssituation zu analysieren. Zum Retrospektion-Interview wurde basierend auf einfachen Regeln ein Transkript erzeugt, ergänzt um Verweise zu den referentiellen Passagen im videographierten Rollenspiel. Das Angebot zur Introspektion wurden von keinem Probanden genutzt, sodass Verbalisierungen nicht im Transkript ergänzt werden mussten. Die Sichtung der Daten, d.h. sowohl der transkribierten Interviews, der Videoaufzeichnungen als auch der Gedächtnisprotokolle, erfolgte entlang der zuvor im Prozess aufbereiteten Kategorien des sensibilisierenden Konzepts. Es galt im Gestus der strukturierenden qualitativen Inhaltsanalyse zu prüfen (Mayring 2010), welche der deduktiven Kategorien mit welchem Abstraktionsgrad das Material wiedergibt. Es wurden gleichsam die Retrospektion-Videos dahingehend gesichtet, ob Facetten von literalen Praktiken im Umgang mit den vorgelegten Texten zu erkennen waren und wenn ja, ob die Schüler/innen die Funktion der Texte erkannten, wie auch eine kontextuelle Einordnung durch sie wahrzunehmen war und daraus sich ein mögliches „Nutzen“ erkennen ließ. Die daraus resultierenden Möglichkeitsräume der Codierung führten zu einer ersten Präzisierung der Forschungsfragen. Diese waren Grundlage für die ausführliche und schriftliche Aufarbeitung des Datenmaterials, welches daran anknüpfend in kompaktere Sequenzen gegliedert wurde, um in den Diskurs gestellt zu werden. Die Fragen, welche sich im Zuge der ersten Analyseschritte ergaben, lauten: 
- Wie lässt es sich feststellen, welche semiotischen Lokal- bis Globalzeichen verarbeitet wurden?

- Werden die routinalisierten lokalen kulturellen Codes erkannt, die eine skillful performance entlang des Textes ausmachen?

- Liegt ein Nutzen von eher statischen (expliziten Konzepten - knowing that) oder eher dynamischen (Tendenz zu knowing how - handelnden Konzepten) Verben/Satzphrasen vor? Gibt es entsprechende Indikatoren in den videographierten Interspektionen oder Retrospektionen?

Die weiteren Schritte der Analyse des Materials hatten die Befragung der Daten entlang bestehender deduktiver Kategorien und deren Subsumption oder Streichung zum Ziel. Gleichermaßen galt es die seither nicht berücksichtigten Kategorien nach dem Prinzip der Offenheit induktive herzuleiten. Diese Arbeitsphase konkretisiert sich an Reduktionen durch Paraphasierung des Datenmaterials je Proband mit anschließender Generalisierung und umfasste die Anwendung des hergeleiteten Kategoriensystems, womit eine deduktive Überprüfung vorgenommen wurde, ob sowohl die Struktur von Lokalzeichen und Globalzeichen erkannt als auch genutzt wurden. Besonderes Augenmerk galt hier der Überprüfung, ob und in welcher Form Steuerungs-Kodes erkannt und genutzt wurden.

Die gesamte Auswertung erfolgt immer unter dem Vorbehalt, dass es auf den Videos eindeutige Indikatoren zu ermitteln gab, bzw. diese im Interview durch die Schüler/innen anschließend expliziert wurden, sei es durch eine Verbalisierung oder ein (erneutes) Darstellen der eigenen Vorgehensweise. Des Weiteren wurde versucht, kognitive Strategien zur Kohärenzerschließung zu erfassen, sofern sie beobachtbar waren. Hierzu zählten Organisations-, Elaborations- und Wiederholungsstrategien, aber auch generelle Strategien der Planung, Regulation und (Selbst-) überwachung. Teilweise sind diese beobachtbar, teilweise nicht.

\subsection{Durchführung}

\subsubsection{Feldzugang und Kriterien zur Datenerhebung}

Die Auswahl der Schüler/innen erfolgte zuvorderst entlang der Zielsetzung, Einflussfaktoren der Berufsqualifizierung abzubilden. Kurzum, das erste Kriterium zielte darauf ab, Schüler/ innen zu finden, die bedingt bis eindeutig berufsfeldspezifische Erfahrungen aus dem Bereich Gastronomie, Montage oder bspw. Maschinenbau (KFZ-Bereich) mitbrachten. Eine eindeutig berufsfeldspezifische Erfahrung setzte ein mehrmonatiges Praktikum voraus. Als Äquivalent wurden mehrjährige berufsfeldspezifische Arbeitserfahrungen z. B. in der Form von Gelegenheits- bis Ferienjobs interpretiert. Ebenso sollten die Schüler/innen einen anerkannten sonderpädagogischen Bildungsbedarf im Schwerpunkt Lernen haben. Vor diesem Hintergrund wurden Schüler/innen der Berufsorientierungsstufe einer Schule gezielt angefragt, um sie für eine freiwillige Teilnahme an der Studie zu gewinnen.

Es ist nicht auszuschließen, dass durch die Anfrage insbesondere Jugendliche gewonnen werden konnten, die entlang ihrer Arbeitshaltung und aufgebrachten Aufmerksamkeitsfokussierung und -spanne besonders offen waren, sich auf die Situation einzulassen. Hier ist im Besonderen der Schüler 3 zu nennen. Darüber hinaus fragten bspw. Schülerin 1 und 2 in einem „Pausengespräch“, wann es in die „zweite Runde“ ginge. Als mögliches Indiz für den wahrgenommenen Ernstcharakter kann der folgende Ausspruch einer Schülerin festgehalten werden: „War ich bes- 
ser als die anderen zwei, oder was?" Aufgrund der Art der Prosodie könnte es jedoch auch eine weniger ernst gemeinte Rückfrage gewesen sein.

Grundsätzlich ist positiv zu erwähnen, dass die Kooperation zwischen Testleitenden, Schulleitung und Klassenlehrern/innen von Offenheit, Interesse und Engagement geprägt war. Die zur Verfügung gestellten Ressourcen, wie bspw. ein heller, leicht abgelegener, aber dennoch gut zu erreichender Raum zur Datenerhebung, sei ein positiv zu erwähnender Aspekt. Zusätzlich ist noch zu sagen, dass es auch Angebote der Klassenlehrer/innen gab, welche sich freiwillig für Rücksprachen bzgl. der einzelnen Schüler/innen bereit erklärten und im Anschluss an ihre Unterrichtstätigkeit zur Verfügung standen.

\subsubsection{Stichprobe: Idealtypen berufsfeldspezifischer Orientierung}

Die Vorstellung der Schüler/innen erfolgt an dieser Stelle immer bezogen auf das Aufgabenformat "Service“ im Berufsfeld Gastronomie. Ergänzend wird angeführt wie „bekannt“ die Schüler/innen mit ihren Testleitenden waren, als auch die Dauer der Videoaufzeichnung der Interspektion und des anschließenden Interviews zur Retrospektion.

Schüler 1 ist männlich und im achten Schulbesuchsjahr einer Förderschule mit dem Schwerpunkt Lernen. Er durchlief u.a. ein einmonatiges Praktikum im Berufsfeld Gastronomie, da es nach eigenen Angaben sein Ziel ist, im Anschluss an die allgemeinbildende Schulzeit den Beruf des Kochs zu erlernen. Auch nach Beendigung seines Praktikums war es diesem Schüler möglich, weitere Erfahrungen im Bereich der Gastronomie zu sammeln. Er konnte in der Vergangenheit sowohl in seinem Praktikumsbetrieb meist an einem Tag in der Woche im Service, als auch in den Schulferien in einem weiteren Gastronomiebetrieb einer Nebentätigkeit in der Küche nachgehen. Die Durchführung mit diesem Schüler oblag der Testleitung 2. Die Testleitung und der Schüler kannten sich bis zum Tag der Aufnahme lediglich durch einen kurzen Erstkontakt beim Vorabbesuch der Schule. Der Bekanntheitsgrad zwischen einer Coderin und dem Schüler ist näher, da diese mit wenigen Unterrichtsstunden bereits an der Schule tätig war. Die Dauer des Videos zum Rollenspiel beträgt mit Einführung in die Aufgabe 4 Minuten und 50 Sekunden, das anschließende Interview beträgt 4 Minuten und 30 Sekunden. Zu den Lesecompetencies entlang von Fließtexten dieses Schülers ist anzumerken, dass er sowohl in Leseflüssigkeit als auch in globaler Kohärenzbildung attestierte Auffälligkeiten zeigt.

Schüler 2 ist weiblich und besucht das neunte Schulbesuchsjahr selbiger Förderschule Lernen, aber in einer anderen Klassengemeinschaft als Schüler 1 und 3. Sie hat bereits mehrmonatige Praktikazeiten absolviert, so u. a. in einer Bäckerei mit angeschlossenem Café sowie einer Hotelfrühstückslounge. Dadurch konnte sie bedingt in den Service im engeren Sinne hineinschnuppern. Nach eigenen Auskünften sei der Beruf der Bäckereifachverkäuferin nicht unbedingt ihr Traumberuf, denn „mit Mathe könnte es schwer werden“. Träumen würde sie eher „,on etwas mit Tieren“. Die Testleitung 1 ist mit der Schülerin durch die Arbeit im Unterricht an der Schule bekannt. Es ist v. a. diesem Bekanntheitsgrad zu verdanken, dass sich die Schülerin für die Teilnahme bereit erklärt hat. Die Dauer des Videos zur Bearbeitung der Aufgabe im Rollenspiel umfasst in ihrem Fall 5 Minuten und 22 Sekunden, die des anschließenden Interviews 2 Minuten und 9 Sekunden. Die Lesecompetencies der Schülerin sind nicht näher durch die Schüler/ in oder ihre Lehrerin beschrieben worden.

Schüler 3 ist männlich, im neunten Schulbesuchsjahr selbiger Förderschule und hat ebenfalls mehrere Monate an Praktika absolviert. Nach eigenen Angaben ist sein absoluter Traumberuf Pilot. Da ihm dies selbst unrealistisch erscheint, „,es eben nur ein Traum sei“, hält er für sich den Beruf des Landmaschinenmechanikers als ein erstrebenswertes Berufsziel. Den Beruf lernte er erstmals in 
seinem zweiten Praktikum in einer größeren Agrarservicefirma kennen. Nach eigenen Angaben gefalle ihm dort besonders die körperliche Arbeit, das Reparieren von Fahrzeugen und anderen Geräten im Bereich der Landwirtschaft. Außerdem ist man an diesen Arbeitsplätzen „nicht so zimperlich, ein harter Ton sei immer gegeben, aber gut“.'Zur Testleitung 3 steht er in einem völlig unbekannten Verhältnis. Die Dauer der Videoaufzeichnung zu seinem Rollenspiel beträgt insgesamt rund 15 Minuten. Er führt einen sehr nach außen orientierten, dialogischen Arbeitsstil und bindet seine Testleitung kontinuierlich in die Bearbeitung der Aufgaben mit ein. Ergänzend sei an dieser Stelle erwähnt, dass dieser Schüler unter erhöhten Druck bzw. Stress zumeist mit körperlichen Bewegungen reagierte, die mit einem ,Hibbeln' in den Beinen einsetzten und in ein ,Kippeln auf dem Stuhl übergingen. Teils und noch relativ oft endeten die Reaktionen in Formen von körperlich und verbal aggressivem Verhalten, meist gegen sich selbst, aber auch gegen Gegenstände. In der Videoaufzeichnung werden eindeutig Formen dieses Verhaltens unter Stress sichtbar.

\subsubsection{Induktive Datensichtung entlang der präzisierten Fragestellung}

Schüler 1: Eigentlich nur gucken wie ein erwachsener Mann, äh, eine erwachsene Frau arbeiten muss, was die, wie sie nachdenken muss, wann sie Arbeit hat und wann sie anfangen muss und wann sie auch Ende hat.

Im Rahmen der ersten Annäherung an das erhobene Datenmaterial von Schüler 1 fallen einige Aspekte besonders auf. Gleich zu Beginn erweckt es den Anschein, dass es dem Schüler von besonderer Wichtigkeit ist, seinen Arbeitsauftrag derart vollständig erfasst zu haben, wie er durch mündliche Einweisung vorgegbenen war. Im Dialog mit der Testleitung versichert er sich, welche Arbeitsschritte nachfolgend anstehen. Der folgende Auszug aus dem Dialog verdeutlicht, dass Irritaitonen nicht ausblieben:

Testleiter: Genau, von wann bis wann, richtig, das sind erst mal die zwei Aufgaben, genau, und du weißt auch was im Servicebereich, was das bedeutet, so im Gasthaus?

Schüler 1: Mmh.

Die Situation stellt sich bei Schüler 1 entlang der in der Videosequenz beobachtbaren Körpersprache derart dar,dass keinerlei Irritationen zu vermuten waren, sondern sie zeugt vielmehr von einer in diesem Bereich bereits gemachten Erfahrung und dadurch wohl auch vermittelten inneren Gelassenheit. Die nun folgende Arbeitsphase lässt einen nahezu routinierten Umgang mit der größeren Anzahl von Arbeitsblättern des vorgegebenen multikodalen Textkomplexes primär ikonischer Kodalität in ihrer ersten Interpretation zu. Es sind Phasen zu beobachten, in denen der Schüler offensichtlich die einzelnen repräsentierten Sachverhalte erfasst und anschließend mit System die Blätter bearbeitet. Während dieser gesamten Phase der Strukturierung ist zu beobachten, dass er beim Lesen die Lippen bewegt. Es sind jedoch keine Verbalisierungen zu vernehmen, die ggf. Auf- bzw. Rückschlüsse auf seine eigenen Gedanken zulassen. Auffallend ist weiterhin, dass er für eine handschriftlich verfasste Liste deutlich mehr Zeit zum Erlesen aufwendet als für eine Tabelle mit Arbeitszeiten. Beim Lesen lässt sich beobachten, dass er die starken Codes hinter der X- und Y-Achse mit Kopfbewegungen zum einen begleitet. Zum anderen positionierte der Schüler, jeweils auf der linken, wie auch der rechten Tabellenseite, in Höhe des gesuchten Namens seinen Daumen. Angekommen beim Raumplan dreht er diesen ,routiniert' in eine offensichtlich notwendige Leserichtung und sortiert anschließend To-do-Liste, Arbeitsplan und Raumplan in diese Reihenfolge. Die handschriftliche Checkliste wird leicht zur Seite geschoben, liegt jedoch noch im Lesebereich des Schülers. Es erfolgt nun beobachtbar die Herstellung von Verknüpfungen 
zwischen der To-do-Liste und dem Raumplan; ablesbar durch die Führung der Zeigefinger. Nach einem Zählvorgang wird eine Selbstüberprüfung durch den Schüler beobachtbar, bevor es zu Notizen und damit vermutlich auch verbundenen Rechenvorgängen kommt. Es ist eine erneute Überprüfung der Verbindung zwischen To-do-Liste, Raumplan, eigenen Notizen und handschriftlicher Liste zu sehen. Auf dem Notizzettel des Schülers ist am Ende der Bearbeitungszeit u. a. eine Arbeitszeit und eine Anzahl der zu bedienenden Tische zu erkennen. Er ist aus seiner Sicht zu einem akzeptablen Ergebnis gekommen. Im anschließenden Interview benennt er als erstes eine auftretende Unstimmigkeit bzgl. der notwendigen Information zu Arbeitsbeginn. Auffallend wird nun im Interview eine Diskrepanz zwischen dem zu sehenden Umgang mit dem Arbeitsplan und der ersten Selbstauskunft auf die rückversichernde Frage zum Verständnis der Situation:

Schüler 1: Eigentlich nicht, ich habe eigentlich nur geschaut, Kim, noch einmal.

Testleiter: Das habe ich gesehen.

Schüler 1: F irgendwas, dann habe ich, denke ich mal, hier hat sie frei.

Testleiter: Ja.

Schüler 1: Und dann hier am 7.7. hat sie Arbeit. Vom 5. bis zum 7. hat die Arbeit.

[Während dieser Gesprächsphase zeigt der Schüler jeweils mit seinem Stift durch Bewegung an, wo und wie er sich auf dem Zeitplan orientiert und in welcher Reihenfolge er zu seinen Informationen gelang.]

Schüler 2: Mein Gott [S blättert die Zettel durch], oh, [ein Zettel ist heruntergefallen, dreht, sortiert neu und stapelt wieder]. Ja, die ganzen Zettel zusammensuchen und gucken wo das alles ist.

Schüler 2 zeigt zu Beginn der Testsituation eine zurückhaltende Körpersprache. Eine wahrnehmbare Lockerung der Situation tritt während der Kommunikation mit der Testleitung ein:

Testleiter: Also, du wirst eine Fachkraft sein, im Servicebereich. Du musst eine Aufgabe erledigen, die entspricht jetzt, quasi, wenn du ins Praktikum gehst und du bekommst einen Auftrag. [TL schiebt der Schülerin den Arbeitsauftrag und alle dazugehörigen Formulare hin, sodass durch die Handlung der Aufforderungscharakter der Bearbeitung entsteht.]

Schüler 2: Mh, aber das Lesen nicht.

Testleiter: Ich kann das auch vorlesen.

Die Situation der Einführung ist entgegen der Teilstandardisierung durch ein wechselseitiges Lesen zwischen TL und Schüler 2 geprägt. Die Abweichung ist laut Gesprächsprotokoll begründet, um die Situation durch Zusicherung der Hilfestellung zu entspannen. Die Arbeitsphase ist im Weiteren gekennzeichnet durch eine ruhige Arbeitshaltung beim Sortieren und Strukturieren des multikodalen Textkomplexes. Das beobachtbare Verhalten zeigt, dass der Fokus stets bei der Seite liegt, die sie bearbeitet. Seiten, die nicht bearbeitet werden, deckt Schüler 2 ab oder dreht sie um. Nach Bearbeitung der jeweiligen Seite des Textkomplexes erfolgt in Selbstüberprüfung ein Abgleich mit der Anforderung in der simulierten Situation, was durch die erneute Sortierung der Arbeitsmaterialien als auch entlang der beobachtbaren Handführung erkennbar ist. Sowohl das Arbeiten mit dem Raumplan wie auch das Lesen des Arbeitsplans wirken hierbei gekonnt. Beim Raumplan und beim Arbeitsplan wird dies durch die zugleich harmonische und stringente Handführung deutlich. Durch die Zielgerichtetheit zu den jeweilig entscheidenden ikonischen Entitäten liegt auch hier die Vermutung nahe, dass ihr der Umgang mit den Sets starker Codes hinter Tabellen bekannt ist. Infolge der Rückfrage zum Bearbeitungsstand durch die Testleitung in der Rolle als Vorgesetzte beendet Schüler 2 ihre Arbeiten und nimmt die Möglichkeit einer Zeitverlängerung nicht in Anspruch. In der unmittelbar an das Rollenspiel 
anschließenden Retrospektion gibt Schüler 2 zum videographierten Durchblättern aller zur Verfügung gestellten Dokumente die Auskunft, dass die Situation für sie „komisch“ war.

Schüler 2: Ja, die ganzen Zettel zusammensuchen und gucken wo das alles ist.

Die Frage, ob Schüler 2 schon einmal mit vergleichbaren Dokumenten gearbeitet hat, wird verneint. Die rückversichernde Nachfrage durch die TL, ob sämtliche Seiten gänzlich neu gewesen seien, bleibt im Interview unbeantwortet. Konkrete Antworten, die Einblicke eröffnen, warum sie die Textkomplexe wie auf den videographierten Ausschnitten bearbeitet hat, bleiben mit einer Ausnahme auch im weiteren Gespräch aus. Auf die direkte Frage nach einer eingesetzten Strategie während der selbstständigen Prüfung entlang des Arbeitsplans gab sie zur Auskunft, das „Ja, weil da steht halt Kim“. Jedes weitere Explizieren zur Verarbeitung kommentiert sie mit einem Fokus auf ihre gezeigte Arbeitshaltung: „Ich bin dann dabeigeblieben."

Schüler 3: Mhh (ausatmend), ach irgendwie, ich weiß nicht so ganz, was ich machen soll? ... Also, ich habe es nicht verstanden und konnte es dann irgendwie nicht richtig bearbeiten.

Die Erarbeitung und Bearbeitung des Aufgabenformates „Service“ durch den Schüler 3 ist dadurch gekennzeichnet, dass er einen äußerst kommunikativen Arbeitsstil pflegt. Er versucht immer wieder die Testleitung in seine Arbeitsprozesse zu integrieren. Der Umgang mit den Arbeitsmaterialien wirkt dabei ohne erkennbare Struktur und decken sich mit seinen eigenen Verbalisierungen. Das Erkennen der einzelnen ikonischen Lokalzeichen innerhalb der einzelnen Textdokumente gelingt ihm nicht eigenständig. In den Sequenzen, in welchen die Testleitung in der Rolle des Vorgesetzten insbesondere die Anweisung gibt, mit welchem Dokument oder Sachverhalt explizit zu arbeiten ist, liest der Schüler anschließend den angewiesenen Ausschnitt des jeweiligen Textes und orientiert sich, durch seinen Kugelschreiber angezeigt, in der graphischen Entität auf den Dokumenten. Daher ist folgerichtig festzuhalten, dass während des gesamten Rollenspiels und der anschließenden Retrospektion eine selbstständige Erarbeitung nicht zu beobachten war. Durch den extrovertierten Arbeitsstil des Schülers unterstreichen Selbstauskünfte sowohl explizit sprachlich diese Interpretation zur Verarbeitung als auch embodied/verkörperlicht. Der beobachtbare Umgang mit den Arbeitsmaterialien verstärkt die Interpretation. Insbesondere die Sinn- und Relevanzoptionen des Textkomplexes von der prozessualen textuellen Merkhilfe bis hin zur prozessualen textuellen Organisationshilfe für die notwendigen Arbeitsprozesse scheinen ihm verschlossen zu bleiben:

Schüler 3: Das passt nicht zu mir.

Testleiter: Passt nicht zu dir, ok. Wieso nicht?

Schüler 3: Es passt nicht.

Testleiter: Ja, kannst du es begründen?

Schüler 3: $\ddot{\text { Ähm. }}$

Testleiter: Also, konntest du dich nicht reinversetzen, weil es ein Mädchenname war?

Schüler 3: Ja, nein, weil ich konnte mich einfach nicht, ich konnte mich einfach nicht reinversetzen.

Testleiter: Ok, konntest dich nicht reinversetzen. Als du dir die Aufgabenstellung dann noch einmal durchgelesen hast, hast du es dann noch mal besser verstanden? Weil ich habe dir die Aufgabe am Anfang ja vorgelesen.

Schüler 3: Mmh.

Testleiter: Ja, und nachdem ich sie dir dann nochmal vorgelesen habe, hast du sie dann besser verstanden?

Schüler 3: Nein. 
Testleiter: Also, du konntest dich nicht reinversetzen und hast es dann beim zweiten Mal auch nicht besser verstanden. Haben dir, weil du hast mich ja dann nach den Zetteln gefragt, haben die dir dann noch geholfen? Du hattest ja hier noch so ein paar Zettel.

Schüler 3: Ja, ich, nein, ich habe eigentlich nur da von Tisch eins bis vier [ $S$ zeigt auf die Lokalzeichen], da habe ich dann das da gemacht [ $S$ zeigt auf die Tische eins bis vier im Raumplan].

Testleiter: Ok, hast also nur das gelesen und hast du die anderen Zettel, hast du die gebraucht?

Schüler 3: Die hier eigentlich gar nicht [Arbeitspan und To-do-Liste]. Ich habe darauf, da habe ich etwas geschaut [S zeigt auf tabellarischen Zeitplan]. Aber hier hat es nicht viel geholfen, ja weil hier, da ist eins bis vier [S zeigt auf die Lokalzeichen], aber nicht, wie viele Gedecke ich dafür brauche.

\subsubsection{Analyse entlang der deduktiven Kategorien}

Das deduktiv vorliegende Kategoriensystem ermöglichte es, den Blick gezielter auf die explizit-kognitive bis implizit-embodied Verarbeitung bei bestimmten semiotischen Zeichen des Testkomplexes zu richten. Ergänzend konnte eine Art individuelle Authentizität bzgl. der routinalisierten lokalen kulturellen Codes analysiert werden, welche eine skillful performance in den simulierten Situationen erst kennzeichnen.

Im Kontext der Simulation im Service der Gastronomie gilt es nun zu überprüfen, ob die Idealtypen die vorliegenden Strukturen im Textkomplex aus nichtkontinuierlichen Texten, d.h. in graphischen Darstellungen, kategorialen Tabellen und Karten, zum einen erkennen und zum anderen nutzen konnten. Die Analyse jenes „Erkennens“ und „Nutzens“ der Struktur der einzelnen Lokalzeichen auf Textebene bis hin zu deren Verknüpfungen als Globalzeichen auf Textkomplexebene werden über die sprachlich explizierte Retrospektion quer zu den verkörperlichten Handlungen aus den videographierten Rollenspielen nachfolgend thematisiert.

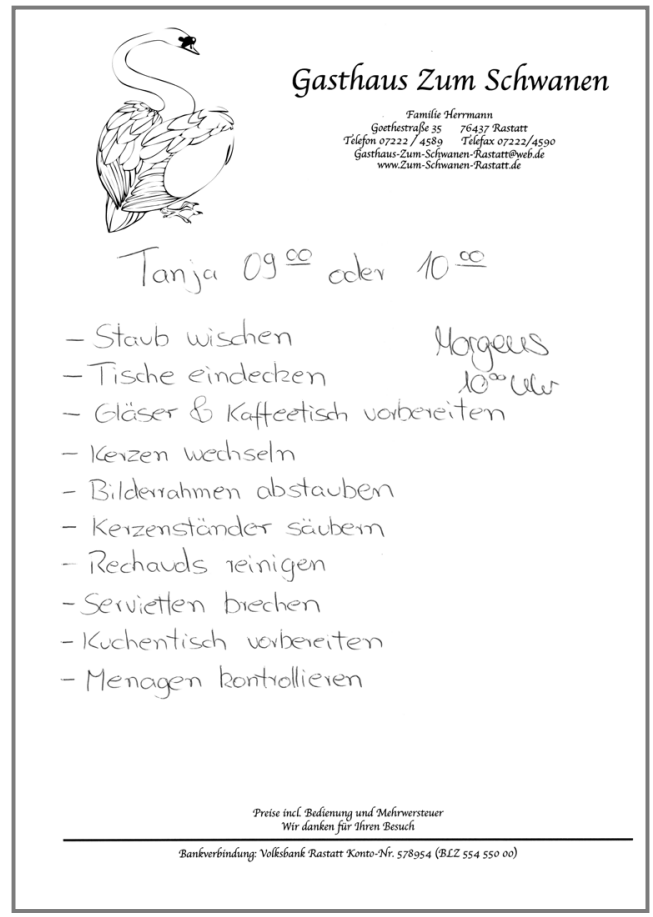

Abb. 50: Primär ikonischer Textkomplex im Service - To-do-Liste 


\section{Zeitplan (30.6. - 12.7.) \\ Sa So}

Sa So

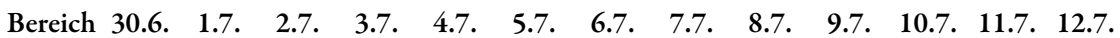

\begin{tabular}{|c|c|c|c|c|c|c|c|c|c|c|c|c|c|c|}
\hline & & & & & & & & & & & & & & \\
\hline Anne & $S$ & $\mathrm{f}$ & $\mathrm{f}$ & $\mathrm{f}$ & $17-1$ & $17-1$ & $9-15$ & $\mathrm{f}$ & $\mathrm{f}$ & $\mathrm{f}$ & $\mathrm{f}$ & $17-1$ & $17-1$ & $9-15$ \\
\hline Brigitte & $S$ & $\mathrm{f}$ & $\mathrm{f}$ & $\mathrm{f}$ & $9-15$ & -15 & -15 & 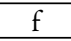 & $\mathrm{f}$ & $\mathrm{f}$ & -15 & $9-15$ & 15 & \\
\hline 7 & $\overline{\mathrm{K}}$ & -14 & -14 & -14 & 14 & $\mathrm{f}$ & $\mathrm{f}$ & -14 & -14 & -14 & -14 & $\mathrm{f}$ & 1 & -1 \\
\hline 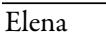 & & -1 & $f$ & $\mathrm{f}$ & 7 & $7-1$ & $7-1$ & $7-1$ & $f$ & S & $17-1$ & \begin{tabular}{|l|}
$17-1$ \\
\end{tabular} & $17-1$ & $\overline{7-1}$ \\
\hline & & $\mathrm{f}$ & & & $7-1$ & $7-1$ & $f$ & f & & & $f$ & $17-1$ & $17-1$ & $f$ \\
\hline & & -1 & $\mathrm{f}$ & $\mathrm{f}$ & $f$ & $f$ & $17-1$ & $17-1$ & & & & $\mathrm{f}$ & $7-1$ & $17-1$ \\
\hline & & $7-1$ & $7-1$ & $7-1$ & 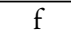 & f & $17-1$ & $17-1$ & $7-1$ & $17-1$ & $\mathrm{f}$ & & $17-1$ & $17-1$ \\
\hline & & $\mathrm{f}$ & $17-1$ & $17-1$ & $17-1$ & $17-1$ & $\mathrm{f}$ & $f$ & -1 & $17-1$ & $17-1$ & $17-1$ & $\mathrm{f}$ & $\mathrm{f}$ \\
\hline & B & -1 & $\mathrm{f}$ & $\mathrm{f}$ & $f$ & S & $\mathrm{f}$ & $17-1$ & $7-1$ & $\mathcal{f}$ & $\mathrm{f}$ & f & $\mathrm{f}$ & f \\
\hline & $\bar{K}$ & -14 & $5-14$ & $\mathrm{f}$ & 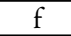 & $5-14$ & $5-14$ & $5-14$ & $5-14$ & & & $5-14$ & $5-14$ & $5-14$ \\
\hline lich & $\mathrm{K}$ & -23 & $\mathrm{f}$ & $14-23$ & $\mathrm{f}$ & $14-23$ & $f$ & $14-23$ & $\mathrm{f}$ & $14-23$ & $\mathrm{f}$ & $14-23$ & $\mathrm{f}$ & $14-$ \\
\hline & $S$ & $9-15$ & $9-15$ & $9-15$ & $\mathrm{f}$ & S & $\mathrm{f}$ & $9-15$ & $9-15$ & $9-15$ & $t$ & $f$ & & $9-1$ \\
\hline 15a & $\mathrm{K}$ & $14-23$ & $14-23$ & $14-23$ & $14-23$ & $\mathrm{f}$ & $\mathrm{f}$ & $14-23$ & $14-23$ & $14-23$ & $14-23$ & & $\mathrm{f}$ & $14-$ \\
\hline & $\mathrm{K}$ & $\mathrm{f}$ & $14-23$ & $\mathrm{f}$ & $14-23$ & $14-23$ & $4-23$ & $\mathrm{f}$ & $14-23$ & $\mathrm{f}$ & $14-23$ & $4-23$ & $14-23$ & $\mathrm{f}$ \\
\hline & $S$ & f & f & $\mathrm{f}$ & $9-15$ & $9-15$ & $9-15$ & $\mathrm{f}$ & $f$ & f & $9-15$ & $9-15$ & $9-15$ & $\mathrm{f}$ \\
\hline & B & $\mathrm{f}$ & $\mathrm{f}$ & $\mathrm{f}$ & $\mathrm{f}$ & $\mathrm{f}$ & $\mathrm{f}$ & $\mathrm{f}$ & $\mathrm{f}$ & & $\mathrm{f}$ & $\mathrm{f}$ & ST & f \\
\hline & $\mathrm{K}$ & 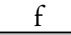 & $\mathrm{f}$ & -14 & $5-14$ & $5-14$ & $5-14$ & $\mathrm{f}$ & $\mathrm{f}$ & $5-14$ & $5-14$ & $5-14$ & $5-14$ & $f$ \\
\hline & $S$ & $9-15$ & $9-15$ & $9-15$ & $\mathrm{f}$ & $\mathrm{f}$ & $f$ & $9-15$ & $9-15$ & $9-15$ & $\mathrm{f}$ & $\mathrm{f}$ & $f$ & $9-15$ \\
\hline & B & $\mathrm{f}$ & $f$ & $\mathrm{f}$ & $17-1$ & $17-1$ & $\bar{f}$ & $\mathrm{f}$ & f & $\mathrm{f}$ & f & $17-1$ & $17-1$ & $6 f$ \\
\hline & B & f & $17-1$ & $17-1$ & $\mathrm{f}$ & $f$ & $\mathrm{f}$ & $\mathrm{f}$ & $\mathrm{f}$ & $17-1$ & $17-1$ & $\mathrm{f}$ & f & $f$ \\
\hline & $S$ & $\mathrm{f}$ & $17-1$ & $17-1$ & $17-1$ & -1 & $\mathrm{f}$ & $\mathrm{r}$ & $17-1$ & $17-1$ & $17-1$ & $17-1$ & $\mathrm{f}$ & $f$ \\
\hline$b e$ & B & $\mathrm{f}$ & f & $\mathrm{f}$ & $17-1$ & $17-1$ & $\mathrm{f}$ & $\mathrm{f}$ & $f$ & $\mathrm{f}$ & $\mathrm{f}$ & $17-1$ & $17-1$ & $f$ \\
\hline
\end{tabular}

\begin{tabular}{c|c|c|c|c|}
\hline 9-15 & \multicolumn{2}{|c|}{ Türseite \& Mitte \& Buffet } & \multicolumn{2}{c|}{ Stammtischseite \& Terrasse } \\
\hline $17-1$ & Türseite \& Mitte & Stammtischseite \& Buffet & Terrasse \\
\hline
\end{tabular}

Abb. 51: Primär ikonischer Textkomplex Service - Arbeitsplan

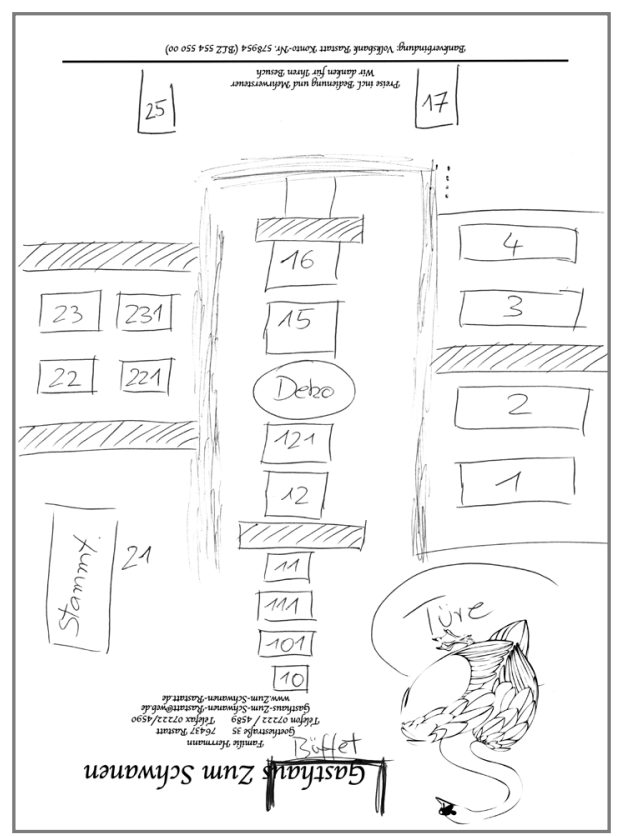

Abb. 52: Primär ikonischer Textkomplex im Service - Raumplan 


\subsubsection{Analyse des Arbeitsplans entlang der deduktiven Kategorien}

Für die Verarbeitung des Arbeitsplans entlang der Frage „Wann muss ich arbeiten?“ sind folgende Informationen zu generieren: Die Jugendlichen müssen initiierend oder begleitend durch Verarbeitung des routinalisierten lokalen kulturellen Code der Organisationshilfe (task/goal model) einerseits die einzelnen Lokalzeichen im Set starker Codes einer kategorialen nested-list aus zwei mit einem Schraffierungs-Steuerungs-Kode verknüpften Tabellen lokalisieren (source model) und verarbeiten (content model). Andererseits gilt es den gesuchten Sachverhalt in seiner Relation im Sinne einer Verlaufs-Struktur mit den Sachverhalten aus weiteren primär ikonischen Texten zu verknüpfen (siehe Tabelle 9):

- Schüler 1 orientiert sich zügig innerhalb der Tabelle. Anhand seiner beobachtbaren Kopfbewegung und Handführung verdeutlicht sich seine Verarbeitung des starken Codes hinter Tabellen über einerseits Spalten und andererseits Zeilen. Sein Zeigefinger markiert direkt das schraffierte Tabellenfeld in der „oberen“ Tabelle. Der zweite richtet sich auf das schraffierte Tabellenfeld in der „unteren Tabelle“. Im späteren Interview expliziert der Schüler dieses Vorgehen sowie weitere Informationen aus dem Arbeitsplan, die er wahrgenommen hat; und vermutlich indirekt als Ausschlusskriterium verwenden konnte. In der reinen Beobachtung ist das Ausschließen bestimmter Informationen nicht wahrzunehmen. Die Art seiner Beschreibung und das zügige Lesen lassen einen routinierten Umgang mit Tabelle als Teil von Textkomplexen interpretieren. Hier korrespondieren beobachtbare Handlungen mit Informationen zur Praxiserfahrung des Schülers und lassen bereits an dieser Stelle Interpretationen zu, welche Bedeutung das kontextuelle Lernen in Form von Praxiserfahrungen für Schüler/ innen haben könnte.

- Schüler 2 handelt mit dem Arbeitsplan durch „wandernde“ Finger. Sukzessiv werden auf der Y-Achse Informationen ausgeschlossen bis das treffende Feld unter dem Finger erscheint. Ab dieser Stelle begibt sich der Finger entlang der X-Achse der Tabelle auf die Suche. Er,,wandert“ bis zum gesuchten Sachverhalt der Uhrzeit im Arbeitsplan. Das Retrospektion-Interview selbst lässt keine Rückschlüsse zu, wie die Bewegungen der Finger auf dem Arbeitsplan zu verstehen sind. Die Kenntnis eines solchen Plans wird verneint. Dennoch bleibt die Vermutung bestehen, dass Schüler 2 die Sinn- und Relevanzoptionen der prozessualen textuellen Organisationshilfe nutzt, um skillful am Arbeitsplan zu performen. Offen bleibt in der Retrospektion auch, welche Überprüfungsstrategie Schüler 2 zugrunde gelegt hat, da sie nicht in der Lage dazu ist, zu explizieren.

Testleiter: Mit der Arbeitszeit? Weil du hast ja jetzt zuerst auf dieses Blatt geschaut [TL zeigt auf Aufgabenplan, der neben dem Arbeitsplan liegt.].

Schüler 2: Ja, weil da steht halt Kim.

Testleiter: Mh, und dann bist du noch mal zu dieser Tabelle übergegangen? Ähm, hast du ein Ergebnis erhalten, das irgendwie übereinstimmt? Oder bist du dann einfach dabeigeblieben?

Schüler 2: Ich bin dann dabeigeblieben.

Ob und auf welche Weise explizit-konzeptuelle Verarbeitungsstrategien bei Schüler 2 Anwendung fanden, ist durch die Person selbst nicht verbalisiert worden. Es bleibt im Bereich des Nicht-Explizierbaren auf Grundlage der Beobachtung, wie die Interaktion mit dem Arbeitsplan im Sinne einer prozessualen textuellen Organisationshilfe und des Arbeitsplans im Sinne einer Merkhilfe gestaltet. Zu Beginn der Arbeitsphase wird jener Arbeitsplan zur Seite gelegt, um später wieder herangezogen zu werden. Es bleibt ebenso unklar, warum die Fingerbewegungen - wie oben bereits dargelegt - an jeweils dem Tabellenfeld in Zeile und Spalte enden, die im Anschluss via Vereinigung der Finger verknüpft werden. Es ist somit deutlich zu erken- 
nen, dass X- und Y-Achse im Arbeitsplan verkörperlicht in der Zusammenführung aus Spalte und Zeile gelesen werden.

- Schüler 3 agiert mit dem Arbeitsplan gemeinsam mit der Testleitung. Er nimmt den Arbeitsplan erst zur Hand, nachdem er einen expliziten Hinweis der Testleitung erhalten hat. Das anschließende Agieren mit dem Arbeitsplan lässt Rückschlüsse auf eine Verarbeitung der Sets starker Codes hinter einer einzelnen intersected-list zu. Diese Schlussfolgerung zeigt sich entlang der Handhaltung und der Stiftführung - welche, so ist zu vermuten, die Lesetätigkeit unterstützt - sowie der gestellten Rückfragen vom „Mitarbeiter“ an den „Vorgesetzten“. Entlang seiner wiederholten Rückfragen wird demgegenüber ersichtlich, dass er weder die starken Codes hinter nested-lists noch die Sinn- und Relevanzoptionen des Arbeitsplans als Ganzes verarbeiten kann. Er formulierte wiederholt, dass er nicht genau verstehe, was er mit welchem Text des Textkomplexes machen soll. Die videographierte Sequenz zeigt deutlich, dass der Schüler seinen Kugelschreiber so über den Arbeitsplan führt, dass das Lokalisieren der notwendigen Einheiten auf Lokalzeichenebene offensichtlich gegeben ist, es jedoch zu keiner weiteren Verknüpfung kommt. Mit der durch die Testleitung angebotenen Anweisung zum Steuerungs-Kode der Schraffierung zwischen „oberer“ und „unterer“ Tabelle der nestedlist Arbeitsplan gelingt es dem Schüler wiederum, den Sachverhalt aus den repräsentierten Informationen auf der Ebene eines Lokalzeichens zu entnehmen.

\subsubsection{Analyse des Raumplans entlang der deduktiven Kategorien}

Die textuelle Anforderung einer skillful performance ist es zum einen, die topologischen Beziehungen in den graphischen Entitäten des Raumplans zu dechiffrieren, um zum anderen die kategorischen Relationen zwischen den graphischen Entitäten zu nutzen. Es gilt u.a., wesentliches von unwesentlichem Mobiliar zu unterscheiden, um herauszufinden, welche Tische einzudecken sind; so wie in der To-do-Liste vorgegeben. Anhand der deduktiven Kategorien wird deutlich, dass Schüler 1 sich unmittelbar im repräsentierten Raum orientieren kann und binnen kürzester Zeit die verarbeiteten Sachverhalte für den Bearbeitungsprozess nutzbar machen kann. Es wird insbesondere deutlich, dass die konkurrierenden Angaben keine Irritationen erzeugen, wenn es gilt, die zu deckenden Tische von anderem Mobiliar zu differenzieren. Ein Erkennen und Nutzen ist bei Schüler 1 mit hoher Wahrscheinlichkeit gegeben, denn es decken sich Handlungen und Verbalisierung der Verarbeitung. Seine Erfahrungen im Bereich der Gastronomie zeigen hier ein hohes Wissen bzgl. der notwendigen kulturellen Praxis und Routinen einer skillfulperformance. Für Schüler 2 gilt an dieser Stelle ebenfalls ein routiniertes Handeln zu bescheinigen, welches offenlegt, das Wesentliches von Unwesentlichem unterschieden werden konnte.

Deutliche Irritation durch konkurrierende Informationen gehen einher mit Schwierigkeiten mit der Lokalisierung und Nutzung der wesentlichen Informationen bei Schüler 3. Er ist, so lassen es die videographierten Handlungen vermuten, in der Lage, die Sets starker Codes hinter einem Raumplan zu erkennen. Wiederum liegt die Hauptschwierigkeit im Herausarbeiten der auf Globalzeichenebene repräsentierten Relation im Sinne von Verlaufsstrukturen zwischen den Sachverhalten im Raumplan mit den Sachverhalten in der To-do-Liste. Ihm scheint es nicht einsichtig, weder begleitend noch initiierend, dass jene Verarbeitung auf Globalzeicheneben durch den routinalisierten lokalen kulturellen Code entlang der Organisationshilfe sozio-kontextuell gerahmt ist. Die Sachverhalte hinter den Lokalzeichen jedes Textes bis Textkomplexes bleiben für sich isoliert. Während der Aufzeichnung verbalisiert er dies in folgender Gesprächssequenz mit der Testleitung: 
Testleiter: Gut, deine Aufgabe ist ja das Eindecken. Wie kannst du die Aufgabe lösen?

Schüler 3: Soll ich diese jetzt einzeichnen oder was? [S hat den Raumplan direkt vor sich liegen]

Testleiter: Du kannst es machen, wie du willst. Du sollst mir nur sagen, um die Tische einzudecken, was du brauchst.

Schüler 3: Eins bis vier? Acht Gedecke, da überall zwei dann ...

Testleiter: Die Frage ist ja, wie viel Gedecke benötigen du?

Schüler 3: Acht Stück, oder? Steht doch da.

Die Analysen zu Schüler 1 bis 3 verhärten bei Schüler 3 die Vermutung, dass sich ein „Wissen“ um die sozio-kontextuelle Sinn- und Relevanzoptionen entlang des Textkomplexes positiv auf die Verarbeitung auswirkt und unscharfe task-/goal-models deutliche Schwierigkeiten auftreten lassen.

\subsubsection{Analyse der To-do-Liste entlang der deduktiven Kategorien}

Die punktuelle textuelle Merkhilfe, welche eine skillfulperformance der To-do-Liste rahmt, wird von Schüler 1 im Retrospektion-Interview nicht explizit benannt. In der Videodokumentation ist zu sehen, dass er diese von oben nach unten sorgfältig liest. Sie wird anschließend für den restlichen Bearbeitungsprozess als nicht mehr von Bedeutung erkannt. An dieser Stelle liegt die Vermutung nahe, dass Schüler 1 von einer Checkliste aus seiner kulturellen Praxis heraus weiß, dass diese in routinierte Handlungen irrelevant wird:

Schüler 2 ist hingegen deutlich stärker mit der To-do-Liste beschäftigt. Es entsteht der Anschein, dass die Checkliste nicht in ihrer Funktion genutzt wird, sondern als eigenständige bzw. doppelte Informationsquelle zur Arbeitszeit. Es bleibt jedoch angesichts fehlender Hand- und Stiftführung sowie Explikation in der Retrospektion im Bereich der Spekulation, wie Schüler 2 sich mit der konkurrierenden Angabe einer Uhrzeit auseinandersetzt.

Alle weiteren Informationen der Checkliste sind für eine skillful performance entlang des Textkomplexes im Rollenspiel nicht von Relevanz und können somit, wie auch durch Schüler 2 erfolgt, sofort ausgeschlossen werden. Schüler 3 ist demgegenüber von der Checkliste verwirrt:

Schüler 3: Und, und dann soll ich jetzt Tische decken? [S hält Raumplan und die To-do-Liste in der Hand, den Raumplan dreht er leicht weg, sodass er für ihn und die TL nicht mehr lesbar ist]

Testleiter: Musst du schauen was du brauchst.

Schüler 3: Hier oder das. [zeigt erneut auf den Arbeitsplan und die To-do-Liste]

Der weitere Verarbeitungsverlauf ist bei Schüler 3 davon geprägt, dass er die Checkliste ganz zur Seite legt. Sie wird für ihn an keiner Stelle mehr sichtlich oder verbalisiert berücksichtigt - gleichermaßen die repräsentierten Sachverhalte, die in Relation mit Sachverhalten weiterer Texte des Textkomplexes stehen.

\subsubsection{Zusammenführung der drei Einzelfälle}

Aus der Analyse der drei Idealtypen von Berufsqualifizierung lässt sich vor dem Hintergrund der einleitend drei fokussierten Fragen zuvorderst festhalten, dass kein Proband explizit die eigene Verarbeitung begründet darlegen konnte. An dessen Stelle tritt vielmehr die Analyse der Erklärung auf der handelnden, darstellenden Ebene, ergänzt um Beschreibungen zum Wie der Verarbeitung. Die Zusammenführung der drei Einzelfälle erfolgt daher zuvorderst aus den Phasen der handelnden Verarbeitung. 
Tab. 56: Verengung der Verarbeitung auf Phasen der handelnden Verarbeitung

\begin{tabular}{|c|c|c|c|}
\hline In Bezug & Schüler 1 & Schüler 2 & Schüler 3 \\
\hline $\begin{array}{l}\text { Initiierender } \\
\text { Zugang zu den } \\
\text { Textkomplexen }\end{array}$ & $\begin{array}{l}\text { Zu Beginn der Bearbei- } \\
\text { tungszeit zeigt er ein } \\
\text {,schultypisches' Verhalten, } \\
\text { im Sinne des überprüfenden } \\
\text { Rückfragens, ob er den } \\
\text { Arbeitsauftrag auch korrekt } \\
\text { verstanden habe. Daran } \\
\text { anschließend setzt ein klar } \\
\text { organisierter und struktu- } \\
\text { rierter Verarbeitungsprozess } \\
\text { mit kurzen Sequenzen der } \\
\text { Selbstüberprüfung ein. Die } \\
\text { spätere Explizierung inner- } \\
\text { halb des Interviews belegt } \\
\text { Routinen. Die gesamte Ver- } \\
\text { arbeitung ist als selbstsicher } \\
\text { zu beschreiben. }\end{array}$ & $\begin{array}{l}\text { Wirkt zu Beginn stark ver- } \\
\text { unsichert, diese Unsicherheit } \\
\text { scheint im Laufe der Ein- } \\
\text { führungsphase schwächer } \\
\text { zu werden, besonders ab der } \\
\text { Stelle, in der sie „Wissen“ } \\
\text { explizieren und rückmelden } \\
\text { kann, dass beruflicher Alltag } \\
\text { „anders“ sei. Der Umgang } \\
\text { mit einer Fülle von Text- } \\
\text { material scheint erst einmal } \\
\text { Schwierigkeiten zu bereiten, } \\
\text { wird jedoch durch eigene } \\
\text { Organisationsstrategien (es } \\
\text { sind immer nur zwei Text- } \\
\text { seiten zu sehen, und nicht } \\
\text { zu Lesendes wird abgedeckt) } \\
\text { systematisiert und dadurch } \\
\text { in der Fülle minimiert. }\end{array}$ & $\begin{array}{l}\text { Zeigt über den gesamten } \\
\text { Bearbeitungszeitraum deut- } \\
\text { liche Anzeichen von Stress. } \\
\text { Wirkt sehr verunsichert und } \\
\text { ist scheinbar darauf bedacht } \\
\text { in ständiger Kommunikation } \\
\text { mit der Testleitung zu sein. } \\
\text { Seine Aussagen bewegen } \\
\text { sich in dem Spannungsfeld } \\
\text { zwischen: „Ich habe den } \\
\text { Arbeitsauftrag verstanden“ } \\
\text { und dem Gegenpol „Ich weiß } \\
\text { nicht was ich machen soll“. } \\
\text { Seine allgemeine Körperspra- } \\
\text { che spiegelt diese Unsicher- } \\
\text { heiten wider. Strategien der } \\
\text { Selbstorganisation, der Über- } \\
\text { prüfung oder das Einrichten } \\
\text { des Arbeitsplatzes scheinen } \\
\text { ihm nicht zu gelingen, bzw. } \\
\text { sind deutlich geprägt durch } \\
\text { einen kommunikativen Stil, } \\
\text { der es ihm ermöglicht, in } \\
\text { möglichst kleinen Schritten } \\
\text { zu den gewünschten Inhalten } \\
\text { zu gelangen. }\end{array}$ \\
\hline
\end{tabular}

Zum Erkennen Die Organisations- und und zur Nut- Strukturierungsprozesse des zung struktu- Schülers erfolgen scheinbar reller Einheiten nach einem bekannten Musund der damit ter. Die To-do-Liste wird im verbundenen Vergleich zu allen anderen Kategorien
Durch eigene Organisationsprozesse strukturiert dieser Schüler den Aufgabenkomplex und ist dadurch in der Lage, sowohl kleinere lokale Einheiten zu erfassen wie auch über mehrere Textseiten hinweggehende Kohärenzen zu nutzen. Besonders deutlich wird dies anhand der persönlichen Überprüfungsstrategie, sodass zwischen den einzelnen gewonnenen Informationen aus einer bestimmten Kategorie und dem ,im eigentlichen Arbeitssetting“ nicht so ausführlichen Arbeitsauftrag die Kohärenzen gebildet und genutzt werden können.
Im Diskurs mit der Testleitung gelingt es dem Schüler, bekannte Lesestrategien innerhalb einer Tabelle anzuwenden, er ist in der Lage, die entsprechenden Verbindungen zu ziehen, da er die notwendigen Steuerungscodes zum Lesen einer Tabelle verinnerlicht hat. Das eigenständige Erlesen im Kontext dieser komplexen Aufgabe und des unbekannten sozialen Settings lassen ihn in eine Überforderungssituation geraten, welche scheinbar einen Transfer verhindert. und Lesen solcher primä ikonischen Texte offen. 


\begin{tabular}{|c|c|c|c|}
\hline In Bezug & Schüler 1 & Schüler 2 & Schüler 3 \\
\hline $\begin{array}{l}\text { Zum Erkennen } \\
\text { und zur Nut- } \\
\text { zung struktu- } \\
\text { reller Einheiten } \\
\text { und der damit } \\
\text { verbundenen } \\
\text { Kategorien } \\
\text { (Fortsetzung) }\end{array}$ & $\begin{array}{l}\text { In ähnlicher Art und Weise, } \\
\text { jedoch nicht ganz so zügig, } \\
\text { erfolgt das Lesen des Ar- } \\
\text { beitsplans, samt zugehöriger } \\
\text { Legende. Hier werden die } \\
\text { Kohärenzbildungen über } \\
\text { Textgrenzen hinweg erkannt } \\
\text { und genutzt. }\end{array}$ & $\begin{array}{l}\text { Kohärenzen innerhalb der } \\
\text { zur Verfügung gestellten } \\
\text { Arbeitsmaterialien bleiben } \\
\text { offen bzw. ungenutzt, so } \\
\text { z.B. die Verknüpfung zwi- } \\
\text { schen punktuell textueller } \\
\text { Merkhilfe (Checkliste) und } \\
\text { prozessualer textueller Orga- } \\
\text { nisationshilfe (Arbeitsplan). }\end{array}$ & \\
\hline $\begin{array}{l}\text { Zu bekannter } \\
\text { kultureller/ } \\
\text { sozialer Praxis }\end{array}$ & $\begin{array}{l}\text { Bringt den höchsten Anteil } \\
\text { an Erfahrungen aus dem } \\
\text { Bereich dieser kulturellen/ } \\
\text { sozialen Praxis mit; es tritt } \\
\text { deutlich hervor, dass ihm } \\
\text { die Kontexte des Lesens } \\
\text { und die damit verbundene } \\
\text { Anwendung sehr vertraut } \\
\text { sind und ihm ein routinier- } \\
\text { tes Verhalten ermöglichen. } \\
\text { Er ist in der Lage, sein } \\
\text { Wissen zu explizieren und } \\
\text { begründet sein Handeln } \\
\text { mit dem bereits erworbenen } \\
\text { Praxiswissen. }\end{array}$ & $\begin{array}{l}\text { Bringt einen gemischten An- } \\
\text { teil an Erfahrungen aus dem } \\
\text { Bereich dieser kulturellen/ } \\
\text { sozialen Praxis ein; kann das } \\
\text { gezeigte Anwendungswis- } \\
\text { sen nicht explizieren; zeigt } \\
\text { jedoch deutlich auf, dass ein } \\
\text { Arbeitsauftrag im Kontext } \\
\text { des „Arbeitens“ bzw. eines } \\
\text { möglichen „Praktikums“ } \\
\text { in diesen Settings nicht } \\
\text { schriftlich, sondern münd- } \\
\text { lich kommuniziert wird. } \\
\text { Dies ermöglicht somit einen } \\
\text { deutlichen Hinweis auf ein } \\
\text { mögliches Praxiswissen im } \\
\text { Kontext von verschiedenen } \\
\text { Arbeitswelten. }\end{array}$ & $\begin{array}{l}\text { Bringt den geringsten Anteil } \\
\text { an Erfahrungen aus diesem } \\
\text { Bereich der kulturellen/ } \\
\text { sozialen Praxis ein; ist } \\
\text { deutlich mit der Bearbei- } \\
\text { tungssituation überfordert; } \\
\text { ist nicht in der Lage, bereits } \\
\text { erlernte Strategien, wie bspw. } \\
\text { das Lesen einer Tabelle, auf } \\
\text { die hier geforderte Aufgabe } \\
\text { selbstständig zu übertra- } \\
\text { gen und in den größeren } \\
\text { Kontext einzuordnen. Die } \\
\text { „fremde“ kulturelle/soziale } \\
\text { Praxis scheint Lösungswege } \\
\text { zu verhindern. }\end{array}$ \\
\hline $\begin{array}{l}\text { Zu den sich } \\
\text { öffnenden } \\
\text { Fragen und/ } \\
\text { oder möglichen } \\
\text { Vermutungen }\end{array}$ & $\begin{array}{l}\text { Das Wissen um die Bedeu- } \\
\text { tung von entsprechenden } \\
\text { Darstellungsformen, wie } \\
\text { auch das Wissen durch den } \\
\text { immer wiederkehrenden } \\
\text { persönlichen Praxisvollzug } \\
\text { scheinen bei diesem Schüler } \\
\text { zu einem routinierten, zü- } \\
\text { gigen Lesen und Bearbeiten } \\
\text { der gestellten Aufgabe zu } \\
\text { führen. Besonders auffal- } \\
\text { lend bleibt die Aussage: } \\
\text { „Eigentlich nur gucken wie } \\
\text { ein erwachsener Mann, } \\
\text { äh, 'ne erwachsene Frau } \\
\text { arbeiten muss, was die, wie } \\
\text { sie nachdenken muss, wann } \\
\text { sie Arbeit hat und wann sie } \\
\text { anfangen muss und wann sie } \\
\text { auch Ende hat." }\end{array}$ & $\begin{array}{l}\text { Unbeantwortet bleibt } \\
\text { aktuell die Frage im Raum } \\
\text { stehen, ob und wenn ja, } \\
\text { wie dieser Schüler die in } \\
\text { einem Praktikum gemachten } \\
\text { Erfahrungen, im erweiterten } \\
\text { Bereich der Gastronomie, in } \\
\text { sein Arbeiten integriert hat } \\
\text { und von daher Kohären- } \\
\text { zen zwischen Texten der } \\
\text { Merkhilfe (Checkliste) und } \\
\text { Texten der Organisationshil- } \\
\text { fe (Arbeitsplan) herstellen } \\
\text { konnte. Die Nichtexplizier- } \\
\text { barkeit durch den Schüler, } \\
\text { wie auch die nicht wirklich } \\
\text { an eindeutigen Indikatoren } \\
\text { festzumachende Beobach- } \\
\text { tung der Erstellung von } \\
\text { Verknüpfungen bleibt in } \\
\text { doppelter Hinsicht offen, }\end{array}$ & $\begin{array}{l}\text { An diesem Beispiel eröffnen } \\
\text { sich Fragen der generellen Le- } \\
\text { sefähigkeit und im Besonde- } \\
\text { ren der von nichtkontinuier- } \\
\text { lichen Texten in Verbindung } \\
\text { mit ikonischen Darstellun- } \\
\text { gen. Auf welche Art und } \\
\text { Weise werden Informationen } \\
\text { erfasst, verarbeitet und Ver- } \\
\text { knüpfungen zu bisherigem } \\
\text { Wissen hergestellt? }\end{array}$ \\
\hline
\end{tabular}




\begin{tabular}{|c|c|c|c|}
\hline In Bezug & Schüler 1 & Schüler 2 & Schüler 3 \\
\hline $\begin{array}{l}\text { Zu den sich } \\
\text { öffnenden } \\
\text { Fragen und/ } \\
\text { oder möglichen } \\
\text { Vermutungen } \\
\text { (Fortsetzung) }\end{array}$ & $\begin{array}{l}\text { Vielleicht liegt genau in } \\
\text { dieser Aussage der Schlüssel } \\
\text { zu möglichen Lesestrategien } \\
\text { und weiteren Formen der } \\
\text { Erkenntnis. Es erweckt den } \\
\text { Anschein, dass „schuli- } \\
\text { sches“ Wissen, „schulische“ } \\
\text { Lesekompetenz (bspw. von } \\
\text { kontinuierlichen Texten) } \\
\text { nicht unbedingt fördernd/ } \\
\text { förderlich für den Umgang } \\
\text { mit solchen Textkomplexen } \\
\text { aus dem beruflichen Kontext } \\
\text { sein könn(t)en. Die notwen- } \\
\text { digen Lesestrategien können } \\
\text { scheinbar primär in der Pra- } \\
\text { xis „erworben“ werden bzw. } \\
\text { wurden bereits bei diesem } \\
\text { Schüler „erworben“. }\end{array}$ & $\begin{array}{l}\text { zum einen da der Schüler } \\
\text { aufgrund des signalisier- } \\
\text { ten Endes der Zeit an } \\
\text { dieser Stelle sein Arbeiten } \\
\text { beendet, zum anderen, weil } \\
\text { dennoch im Arbeitsplan die } \\
\text { notwendigen Informationen } \\
\text { generiert wurden. Daran } \\
\text { schließt sich die Frage an, ob } \\
\text { ein höheres Maß an Routine } \\
\text { (an ein Wissen um bzw. ein } \\
\text { Wissen wie) an dieser Stelle } \\
\text { zu einem Weiterarbeiten } \\
\text { geführt hätte, wie auch, ob } \\
\text { die Nutzung der Checkliste, } \\
\text { damit in ihre eigentliche } \\
\text { Funktion wieder übergegan- } \\
\text { gen wäre und die Kohären- } \\
\text { zen durch ein routinierteres } \\
\text { Lesen leichter herzustellen } \\
\text { gewesen wären. }\end{array}$ & $\begin{array}{l}\text { Es ist eindeutig, dass Tabel- } \\
\text { len bereits von diesem Schü- } \\
\text { ler gelesen werden können, } \\
\text { das dahinterliegende System } \\
\text { bekannt ist, jedoch eine } \\
\text { Übertragung in komplexere } \\
\text { Kontexte offensichtlich } \\
\text { nicht gelingt, daran anknüp- } \\
\text { fend der Aspekt inwiefern } \\
\text { ein kulturelles/ein situatives } \\
\text { Verstehen und Kennen der } \\
\text { gewünschten sozialen Praxis } \\
\text { das Lesen und Verstehen } \\
\text { (Wissen um bzw. ein Wissen } \\
\text { wie) beeinflusst. }\end{array}$ \\
\hline
\end{tabular}

\subsection{Auswertung der Daten: Präzisierung der Forschungsfrage}

Wie lässt es sich feststellen, welche semiotischen Lokal- bis Globalzeichen verarbeitet wurden? Liegt ein Nutzen von eher statischen oder eher dynamischen Verben/Satzphrasen vor - während statische Verbalisierungen theoriebasiert auf explizit-konzeptionelle Verarbeitungsmodelle hinweisen, können dynamische Verbalisierungen als Indiz für implizit-embodied Verarbeitungsmodelle gelten? Gibt es entsprechende Indikatoren in den videographierten Interspektionen oder anschließenden Retrospektionen in Abhängigkeit der berufsfeldspezifischen Arbeitsplatzerfahrung?

Die Feststellung, wie die Jugendlichen die Texte verarbeitet haben, erwies sich als äußerst diffizil. Zum einen lassen sich ausschließlich durch die Beobachtung der Situation nur Vermutungen äußern, welche sich durch beobachtbare Handlungen erahnen lassen, wie z. B. durch einen Stift begleitetes Lesen bzw. andere körperliche Handlungen. Da keiner der Probanden die Interspektion anwendete, kann auf keine Verbalisierung zum eigenen Vorgehen in den videographierten Rollenspielen zurückgegriffen werden. Bereits bei den Vortests wurde deutlich, dass die Interspektion den Jugendlichen teilweise bzw. völlig unbekannt war. In den Gesprächsprotokollen wurden Rückmeldungen festgehalten, die auf die bekannte Problematik der Irritation bei der Verarbeitung verweist. Sie fühlten sich folglich nicht in der Lage parallel zu der anspruchsvollen Bearbeitung des Textkomplexes Auskunft über die Verarbeitung zu geben. Der Fokus lag für die Jugendlichen eher darin, zu einem Ergebnis zu kommen und sich bemüht zu zeigen.

Als Schlussfolgerung zu den Vorstudien wurde die videogestützte Retrospektion ergänzt. Diese Maßnahme im Erhebungsdesign hat dazu beigetragen, zumindest bei zwei Probanden vertiefte Einblicke in die Verarbeitung zu bekommen. In der Retrospektion konnte sowohl entlang von kongruenten Verbalisierungen gepaart mit (erneutem) Zeigen oder Verweisen auf die Handlungsschritte in einzelnen Sequenzen aufgedeckt werden, wie die Probanden zu ihrem Ergeb- 
nis entlang des Textkomplexes im Rollenspiel gekommen sind. Aufschlussreich sind zum einen zuvorderst die Sequenzen, die auf den ersten Blick eine Diskrepanz zwischen Verbalisierung und (videographierter) Handlung aufreißen. In der Regel wird in diesen Sequenzen mitgeteilt, man wisse nicht, wie man zu einer Lösung gekommen sei, jedoch wurde im Anschluss sukzessiv erklärt oder mit Handeln aufgezeigt, wie man vorgegangen ist. Auf den zweiten Blick verdeutlichen eben diese Sequenzen, dass die Jugendlichen implizit-konzeptionelle aber keine explizitkonzeptionellen Verarbeitungsmodelle in den Lesesituationen aktualisierten, geschweige denn über Fachbegriffe zum Prozess des Lesens verfügten. Zum anderen sind die Sequenzen von Interesse, in denen vage Indizien für explizit-konzeptionelle Verarbeitungsmodelle gegeben werden, welche sich jedoch ausschließlich auf das task-/goal-model beziehen. Kurzum es wird in diesen Sequenzen angedeutet, was eine skillful performance entlang der einzelnen Texte in der simulierten beruflichen Situation erst ausmacht. Es sind insbesondere die Jugendlichen mit berufsfeldoder gar arbeitsplatzspezifischen Erfahrungen, deren Verarbeitung auf routinalisierte lokale kulturelle Codes zurückgreift. Jenes knowing how zum task-/goal-model trägt in der Kontrastierung der drei Idealtypen dazu bei, sich im Textkomplex zu orientieren, d.h. die im primär ikonischen Textkomplex inhärent repräsentierte Relation von Sachverhalten aufzuschlüsseln. Ohne diese initiierende und begleitende Konstruktion des task-/goal-models erscheint eine wechselseitige Konsttrukion des source-models und weiter des content-models folglich erschwert. Was sich somit entlang der vorliegenden Beobachtungen festhalten lässt, ist, dass es den Probanden, welche über bereits bekannte Ideen in Form des Handelns verfügen, eher gelingt zu einer Lösung zu kommen, auch wenn ihnen die Gesamtstruktur des Textes nicht gleich zu Beginn als offensichtlich erscheint. Es sind die Jugendlichen, die auch in Momenten des vermeintlichen Stockens während des Arbeitsprozesses, erneut in den Arbeitsprozess einsteigen.

Als einen mehrfach zu beobachtender Zugang zur Verarbeitung des source-modells entpuppte sich das Ordnen der Arbeitsmaterialien. Die Ordnung erfolgt bereits während der Verarbeitung und verfolgt sichtlich die Strategie, möglichst wenige Quellen ausgebreitet vor sich zu haben. Deutlich wurde diese Tendenz insbesondere entlang der Texte des Textkomplexes, die einen auffällend höheren Anteil sekundär symbolischer Kodalität haben. Während der Leseprozesse verhärtet sich diese Tendenz, da weder Hände noch ein Stift jeweils zu den ,auf die Seite gelegten' entsprechenden Texten geführt werden. Vereinzelte Verbalisierungen in der Retrospektion stützen zudem diese Tendenz.

Dennoch bleibt die Frage offen, ob und inwiefern zwischen den explizit-konzeptionellen und implizit-embodied Verarbeitungsmodellen stets eindeutig differenziert werden kann; dies insbesondere vor dem Hintergrund des aufgedeckten Einflusses des task-/goal-sowie source models. Die Beschreibungen der Retrospektion quer zum videographierten Handeln bei der Verarbeitung lassen für die einzelnen analysierten Sequenzen quer über die Probanden hinweg zuvorderst die Relevanz jener Wechselbeziehung in den Mittelpunkt rücken. Es bestätigt sich damit jedoch die theoretische Positionierung innerhalb der Debatte um Lesekompetenz, dass von einem Ansatz Abstand zu nehmen ist, der von klar hierarchischen Schwierigkeitsstufen auf der Basis ausschließlich semiotischer Komplexitätsgrade ausgeht (vgl. Kapitel 3). Vielmehr erfährt die Rekonstruktion bzw. Aktualisierung der sozialen Bedingungen einer skillful performance mit dem Textkomplex primär ikonischer Kodalität an entscheidender Bedeutung, gepaart mit der Rekonstruktion der Relation der relevanten Sachverhalte über mehrere Texte hinweg. Jener Zusammenhang ist jedoch nicht derart zu interpretieren, dass task-/goal-sowie source model per se als explizit-konzeptionelles Wissen vorliegen müssen. Dies gilt u. a. für das knowledge about how content area knowledge is represented, knowledge about how document knowledge is represen- 
ted, representation of the task to be completed und of the response to be output. In der überwiegenden Anzahl der analysierten Sequenzen wird demgegenüber beschreibend erklärt, wie bspw. ein Textkomplex am simulierten Arbeitsplatz bzw. Berufsfeld zu erlesen ist.

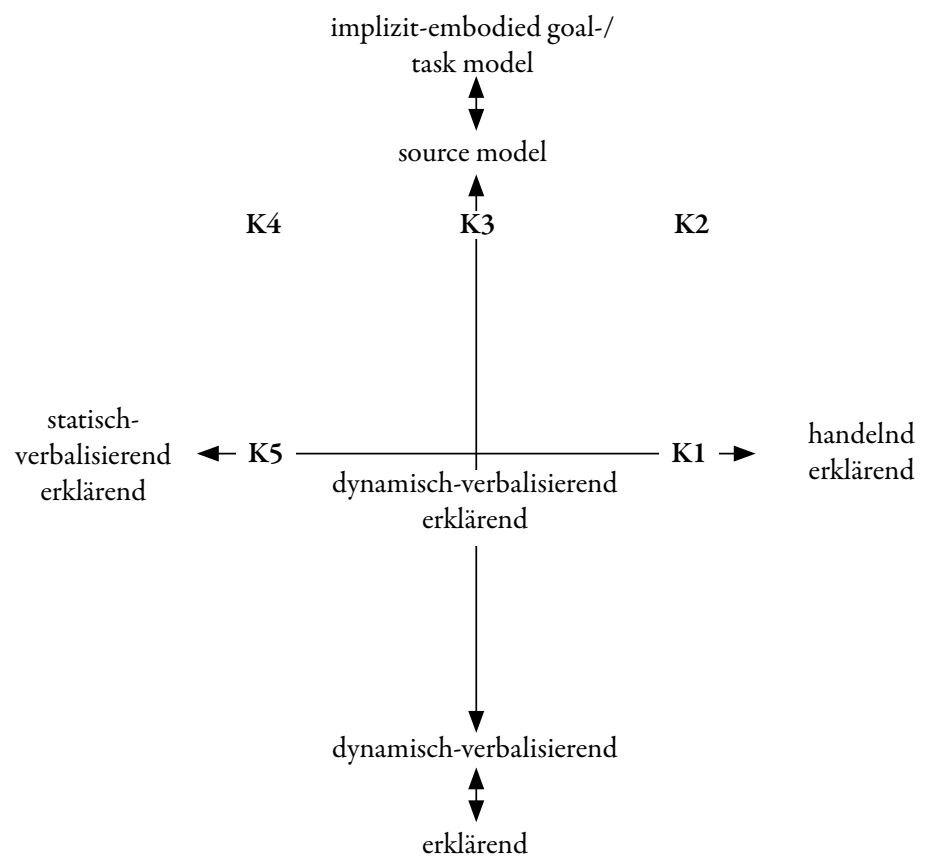

Abb. 53: Kategoriensystem zur Verarbeitung von primär ikonischen Textkomplexen

Tab. 57: Kategoriensystem zur Verarbeitung von primär ikonischen Textkomplexen

\begin{tabular}{|c|c|}
\hline Kategorie & Beschreibung \\
\hline $\begin{array}{l}\text { K1: Handlungsbasierte } \\
\text { Verarbeitung: „Ich } \\
\text { habe es verstanden ... }\end{array}$ & $\begin{array}{l}\text { Äußerungen sind mit dem beobachtbaren Handeln kongruent, begründen } \\
\text { jedoch kein Handeln. Die videographierte Sequenz zeigt einen routinierten } \\
\text { Umgang mit einzelnen Texten, jedoch nicht mit allen Texten und insbesondere } \\
\text { nicht deren Verknüpfung als Textkomplex. }\end{array}$ \\
\hline 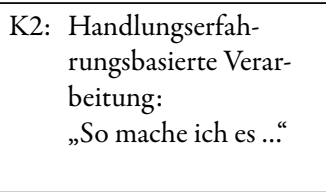 & $\begin{array}{l}\text { Äußerungen sind mit dem beobachtbaren Handeln kongruent, begründen } \\
\text { jedoch kein Handeln. Die videographierte Sequenz demonstriert einen } \\
\text { routinierten Umgang mit dem Textkomplex, d. h. eine gezielte Selektion der } \\
\text { Sachverhalte, die situativ-kontextuell in Relation gesetzt werden müssen. Das } \\
\text { Vorgehen kann nicht verbalisiert begründet werden. }\end{array}$ \\
\hline $\begin{array}{l}\text { K3: Texterfahrungsbasier- } \\
\text { te Verarbeitung: } \\
\text { "Ich mache es so, weil } \\
\text { man das so macht ..." }\end{array}$ & $\begin{array}{l}\text { Handeln und verbalisierte Begründung zeigen eine Kongruenz auf. Die } \\
\text { gegebene Begründung beruht auf dynamische Beschreibungen, wie Texte am } \\
\text { spezifischen Arbeitsplatz oder im Berufsfeld zu lesen sind. Dieses,Wissen` } \\
\text { hilft bei der Konstruktion der Relation aus Sachverhalten der einzelnen Texte. } \\
\text { Unbekannte fachspezifische Termini oder Texte können Verarbeitung dennoch } \\
\text { verlangsamen. }\end{array}$ \\
\hline
\end{tabular}




\begin{tabular}{|c|c|}
\hline Kategorie & Beschreibung \\
\hline $\begin{array}{l}\text { K4: Konzeptionelles } \\
\text { Handeln: } \\
\text { „Das ist so, weil dies } \\
\text { immer so gemacht } \\
\text { wird ... }\end{array}$ & $\begin{array}{l}\text { Handeln und verbalisierte Begründung zeigen eine Kongruenz auf. Wesentli- } \\
\text { che Informationen aus Dokumenten können entnommen werden und als sol- } \\
\text { che (wenn auch nicht zwingend als Fachbegriff und ggf. auch nur als schriftlich } \\
\text { Notiz) explizit benannt werden; und dies vor dem Hintergrund eines Wissens, } \\
\text { wie Texte am spezifischen Arbeitsplatz oder im Berufsfeld zu lesen sind. Dieses } \\
\text {,Wissen hilft bei der Konstruktion der Relation aus den explizit benannten } \\
\text { Sachverhalten zumindest eines einzelnen Textes. }\end{array}$ \\
\hline $\begin{array}{ll}\text { K5: } & \text { Konzeptionelles } \\
& \text { Handeln: } \\
& \text { „Das ist so erklären, } \\
& \text { dass ...” }\end{array}$ & $\begin{array}{l}\text { Wesentliche Informationen aus Dokumenten können entnommen werden } \\
\text { und als solche (wenn auch nicht zwingend als Fachbegriff) explizit benannt } \\
\text { werden. Die Zusammenhänge zwischen den Sachverhalten werden als solche } \\
\text { nicht explizit kommuniziert. Dies geschieht vor dem Hintergrund jedoch eines } \\
\text { formulierten Wissens zur Funktion der einzelnen Texte am Arbeitsplatz oder } \\
\text { im Berufsfeld, ohne dies mit Fachtermini benennen zu können. }\end{array}$ \\
\hline
\end{tabular}





\section{Teilhabe durch multikodales Lesen}

Ausgangspunkt der Studien, die zum Kern dieser Veröffentlichung wurden, war die Annäherung an das kompetente Lesen der Textualität an Einfach(st)erwerbstätigkeiten und damit die Befragung einer Handlungsfähigkeit entlang multikodaler Texte und Textkomplexe primär ikonischer Kodalität im Spannungsfeld von Bildung und Teilhabe. Nachfolgend werden die empirischen Ergebnisse in den Lesekompetenzansatz des Lesens als Set sozialer Praxen rückgebunden, um abschließend zentrale Konsequenzen für Bildung in Relation zu Teilhabe abzuleiten.

Insbesondere unter dem Aspekt einer gesellschaftlichen Teilhabe, die in unserer Informationsgesellschaft eng an kommunikative Kompetenzen gebunden ist, stellt der Umgang mit jenen multikodalen Texten und Textkomplexen sowohl eine Inhalts- als auch eine zentrale Zieldimension dar. Das Bilden von Relationen aus begrifflichen, topologischen oder Verlaufsstrukturen über Analogien und Metaphern sind wichtige schriftliche Kommunikationsformen primär nichtkontinuierlicher Texte und Textkomplexe an Arbeitsplätzen, welche, wie dargelegt, nicht durch Fließtexte ersetzt werden können. Diese Texte können das Behalten, das Organisieren, das Merken, die Weitergabe und die Kontrolle von relationalen Sachverhalten textuell ermöglichen. Gegenübersymbolischen weisen einige der aufgedeckten (starken) Darstellungs-Kodes eine größere Nähe zu verkörperlichten erfahrungsnahen Analogien und räumlichen Metaphern auf, welche eine Verarbeitung nahelegen, die in implizit-embodied Modellen konzipiert werden.

Die aufgedeckten historischen Spuren lassen zudem vermuten, dass die thematisierten Sets starker ikonischer Darstellungscodes eine Gültigkeit in mehreren international Schriftsystemen haben. Es müssten jedoch bspw. ethnographische Studien angestellt werden, dieser Schlussfolgerung nachzugehen.

Die Möglichkeiten eines alternativen Zeichensystems verdeutlicht sich zudem im Spiegel von Chancengleichheit, wie durch das Bundesgleichstellungsgesetz (BGG) geregelt. Das BGG zielt darauf, die Benachteiligung von Menschen mit Behinderung zu verhindern, garantiert die gleichberechtigte Teilhabe von Menschen mit Behinderung am Leben in der Gesellschaft und sichert eine selbstbestimmte Lebensführung. Das BGG legt in $\$ 7$ (1) ein Benachteiligungsverbot für Träger öffentlicher Gewalt fest. In $\$ 4$ des BGG ist die Barrierefreiheit, die jedem Menschen mit Behinderung zusteht, thematisiert. In $\$ 10$ BGG ist eine für die Zielgruppe angemessene Gestaltung von Bescheiden und Vordrucken vorgeschrieben. Das in einer Teilstudie befragte Formular zum Wohngeld widerspricht in seiner Gestaltung den „Regeln für leichte Sprache“ (MBMB, 2009). Zum Beispiel werden Fachwörter nicht erklärt bzw. es wird kein hypotaktischer Satzbau verwendet. Das mehrseitige Formular ist sehr textlastig und die Zeilenabstände sehr gering. Daneben könnte die stringente Gestaltung mittels ikonischen Darstellungs- und Steuerungs-Kodes die Verarbeitung jener öffentlichen multikodalen Texte deutlich erleichtern. Jene Formulare nicht bearbeiten zu können, kann sich unmittelbar auf die gesellschaftliche Teilhabe von sozialrechtlich benachteiligten Jugendlichen und jungen Erwachsenen auswirken, da diese nicht selten keinen Anspruch auf eine Assistenz haben.

\subsection{Theorie des multikodalen Lesens}

Der Mainstream-Ansatz von Lesekompetenz, das Lesen als Informationsentnahme/Kulturrechnik im Geltungskreis von handlungstheoretisch-kognitiven competencies, folgt, wie im 
Forschungsstand dargelegt, den Annahmen, dass diese als Abbild aus der Performanz in LeseAufgaben erschlossen werden kann. Dabei wird durch die Setzung, dass ,alle' Schüler/innen der 8. Klassen aus einer als gleich zu betrachtenden Schulsozialisation heraus jene Lese-Aufgaben bearbeiten, jegliche Relevanz des Kontexts als vernachlässigbar begründet, in welche diese eingebunden ist. Oder in anderen Worten, die Setzung postuliert, dass man aus der Performanz der ausgewählten Lese-Aufgaben unmittelbar auf die Rekonstruktionsleistungen für Texte jeglichen privaten, öffentlichen und beruflichen Kontext schließen kann (=Wissen). Da jene handlungstheoretisch-kognitiven competencies auch emotional-volitionale Facetten stets umfassen, folgt aus der sozialen Kontextfreiheit, dass mit einem Nicht-Wissen in der funktionalen Kompetenzeinschätzung monokausal ein Nicht-Wollen einhergeht.

Die theoretische Analyse zur historischen Ableitung von primär ikonischen Texten/Textkomplexen verdeutlichte demgegenüber erstens, dass der Kontext einer Lesesituation eindeutig von Zeit und Raum abhängig zu interpretieren ist. Die aufgedeckte statistisch schwach bedeutsame Abhängigkeit bis gar Unabhängigkeit der spezifischen Lesecompetencies von jenen primär ikonischen Texten/Textkomplexen unterstreicht zweitens die Kritik, dass nicht zwischen zu differenzierenden Lesecompetencies in Abhängigkeit der Kodalität der Texte unterschieden wird. Die explorative Studie zur verstehenden Analyse der Verarbeitung primär ikonischer Texte/ Textkomplexe verhärtet, drittens, die Relevanz des Kontextes zur Erklärung von Performanzunterschieden bei primär ikonischen Texten vs. Textkomplexen. Eine kontextfreie, sprich generalisierbare Lesekompetenz jeglicher Texte muss im Ergebnis als stark simplifiziert zurückgewiesen werden.

Die eigenen Teilstudien zum multikodalen Lesen primär ikonischer Texte und Textkomplexe verdeutlichen folglich, dass die Lesebegriffe des Lesens als Informationsentnahme wie auch Lesens als kulturelle Praxis inadäquat sind. Diese Ansätze haben gemeinsam, dass von letztlich explizierbaren universellen kognitiven Dispositionen ausgegangen wird, welche als Voraussetzung von Handeln das Lesen sämtlicher Texte bestimmen; und somit sämtlicher Texte unabhängig der Technik, des Mediums und der Kodalität des referentiellen Zeichensystems. Die mit den Befunden der vorliegenden Teilstudien nicht zu vereinenden Postulate einer sozialen Kontextfreiheit bzw. von universellen kognitiven Dispositionen tragen bereits immer in sich mit, dass das Lesen an den untersuchten Einfach(st)erwerbstätigkeiten keine anerkannte Berücksichtigung für Teilhabe und somit auch für Bildung erfahren sollte. In jenen Lesebegriffen wird folglich eine Perspektive in der Relation von Bildung und Teilhabe präferiert, in der Bildung ausschließlich als Voraussetzung von (beruflicher) Teilhabe betrachtet wird, jedoch (berufliche Teilhabe) nicht als Auslöser von Bildung (Walther 2012). Jene durch Bildung herzustellende Teilhabe eröffnet sich durch die Befähigung oder generell den Zugang zu lebenslangem Lernen in einer modernen Mediengesellschaft via Lesecompetencies für vermeintlich alle sozialen Lagen, für alle Generationen bis gar für jeden Arbeitsplatz ungeachtet des Qualifizierungsniveaus bzw. Prestiges.

Insbesondere hinter der Tradition des Lesens als kulturelle Praxis, aber auch hinter der des Lesens als kulturelle Praxen und des Lesens als Informationsverarbeitung/Kulturtechnik wird zudem eine Besonderheit in der Betrachtung schulischer Praxen deutlich, die auf das „Lernen“ über das „Leitmedium“ Text oder den Schriftspracherwerb routinalisiert ausgerichtet sind. Unter dieser Routiniertheit fallen bestimmte Kompositionen des Artefaktes Zeichensysteme: Hierbei handelt es sich fast ausschließlich um diverse kontinuierliche Texte und Textkomplexe. Tabellen, graphische Darstellungen, Diagramme und Karten sind insofern nur von Belang, wenn sie bspw. Sachtexten sekundär in die ansonsten primär symbolischen Fließtexte eingebunden sind. Diese 
formieren einerseits die textuellen Bausteine der Praktik des schulischen Lernens mit Texten (Behaltensleistung, Einstellungsänderung) im Spiegel eines Kompetenzstrukturmodells. Andererseits rahmen primär kontinuierliche Texte die Praktik des schulischen Schriftspracherwerbs im Spiegel eines Kompetenzentwicklungsmodells. Die Performanz soll alsdann Auskunft über die kognitiven Prozesse in einem Soll-Ist-Vergleich zu einem Normmodell geben, welche sämtliche fachdidaktisch hergeleitete hierarchieniedrigeren und -höheren kognitiven Prozesse bereits umfasst, oder, im zweiten Fall, entlang eines Normmodells von Entwicklungsstufen, welche den Erwerb dieser hierarchieniedrigeren und -höheren Prozesse thematisiert. Diese Besonderheit konstituiert sich aus der institutionellen Notwendigkeit des Primats einer zu explizierenden Bildung in der Relation von Bildung und Teilhabe, welche letztlich auch von ihrer Funktion eines sozialen Identifikationskriteriums nicht zu trennen ist: die soziale Markierung von mehr oder weniger, sowie von nicht textuell handlungsfähigen Schüler/innen.

In corpore, der Ansatz des Lesens als Set sozialer Praxen erweist sich als der theoretische Ansatz, der sich am ehesten als kohärent und konsistent zur Einordnung der empirischen Ergebnisse der vorliegenden Arbeit eignet. Dieser postuliert keine willkürliche Vielfalt von sozialen Anforderungen einer Handlungsfähigkeit mit Texten bis Textkomplexen. Stattdessen betont dieser Lesekompetenzansatz, eine Vielzahl von zugehörigen Lesesituationen (=events) einer literalen sozialen Praktik als Ausgangspunkt für die Analyse der Anforderungen an eine skillful performance entlang von Texten heranzuziehen. Hierbei bindet die literale soziale Praktik in den zugehörigen events einen nicht-willkürlichen, sondern routinalisiert eingebundenen Gebrauch von semiotischen Arte-fakten. Ein Auszug jener on-the-job explorierten routinalisierten lokalen kulturellen Codes ist in Tabelle 54 eingearbeitet. Die konzeptionelle Fixierung betont das Lesen in Lesesituationen, in denen das kompetente Lesen gerade nicht entlang der Behaltensleistung bestimmt ist. Oder in anderen Worten, die Teilhabe an einer sozialen Praktik mittels einem Text verlangt eine Aktualisierung dessen, was im jeweiligen Kontext sozial als kompetentes Lesen festgelegt ist. Das kompetente Lesen aktualisiert sich in einigen literalen Praxen entlang multikodaler Texte und Textkomplexe, die auf beiden Zeichensystemen beruhen. Eine sozial-literale Praktik, die ,Sinnwelt' miteinander vernetzter literal-sozialer events oder im Hier und Jetzt sozio-kontextuell identisch eingebetteter Lesesituationen, umfasst bzw. eröffnet sämtlich ,zum Einsatz kommendes Wissen'. Zu jenem impliziten knowing how zählt das ,zum Einsatz kommende Wissen', aus dem heraus u. a. konkrete Gegenstände bis abstrakte Entitäten wie semiotische Zeichen aus dem Repertoire der Artefakte eine Bedeutung erst routinalisiert erfahren.

Ohne an dieser Stelle die Grundlagen des praxeologischen Verständnisses zu repetieren, betont es das kollektive oder intersubjektive geteilte Bewusstsein auch über ,Literalität, Deutungen und Diskurse, d.h. Reflexionen über Literalität und deren Sinnhaftigkeit', welche bestimmten sozialen Konventionen und Einstellungen unterliegen können (vgl. Kapitel 3). Jene ,Sinnwelt', die bei den zugehörigen literal-sozialen events im Hier und Jetzt von kollektiven oder individuellen Akeuren via ,routinisierten, innovativen oder konflikthaften Interpretationsleistungen' mobilisiert wird, charakterisiert letztlich eine Betrachtung des Lesens als Prozess, bei dem über das Soziale auf das Handeln des Einzelnen entlang der ,anspruchsvollen Tätigkeit des Lesens' geschlossenen wird. Demgemäß sind die Abbildungen 54 und 55 von außen nach innen zu lesen. Dies verdeutlicht, dass die Analyse von Lesekompetenzen multikodaler Texte und Textkomplexe ausgehend von miteinander verschränkten sozial-literalen events möglich ist. Möglich ist dies im Sinne einer annähernden Explikation des von kollektiven und individuellen Akteuren in der Aktualisierung geteilten knowing how über die routinalisiert lokalen kulturellen Codes. Dieses knowing how wurde als „Wissen“ charakterisiert, was eine skillful performance im Hier und Jetzt überhaupt ausmacht. 


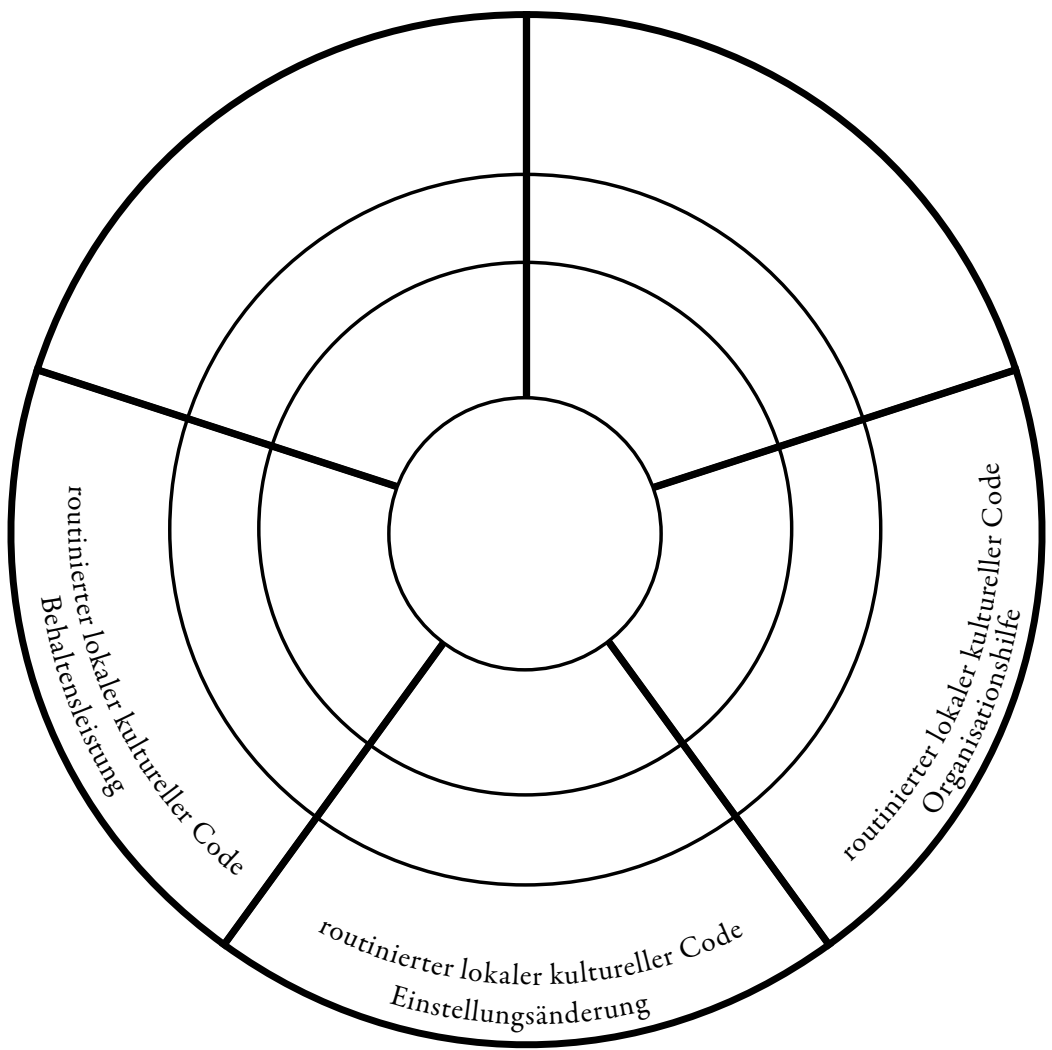

Abb. 54: Multikodales Lesen als Set sozial-literaler Praxen 1

Dies gilt gleichermaßen für soziale Praxen, die durch einen textuellen Input bestimmt/begleitet werden. Eine tatsächliche Explikation der jeweiligen ,Sinnwelt' der sozial-literalen Praktik kann auch über die Berücksichtigung mehrerer zugehöriger sozial-literaler events nicht die Intention sein. Die Forschungsergebnisse zur Verarbeitung multikodaler Texte und Textkomplexe betonen kohärent und konsistent, nicht nur die Relevanz der Aktualisierung jenes ,Wissens' um die routinalisierte Sinn- und Relevanzoptionen ausgehend vom sozialen Kontext der Lesesituation, sondern auch die Begrenzung, dieses ,Wissen' mit statischen Konzepten explizieren zu können. Deutlich wird dies insbesondere in der Debatte zu Makrotheorien der Kohärenzbildung: Wenn „eine Fülle von Einflußgrößen (sic!) berücksichtigt werden, die beim Textverstehen wirksam werden können" sind die Verarbeitungsprozesse infolge eines einhergehenden „großen Abstraktionsgrades einer direkten empirischen Überprüfung nicht zugänglich“" (Christmann/Groeben 2001, 171).

Die theoretischen Kongruenzen des Lesebegriffansatzes als Set sozialer Praxen mit einem funktional handlungsorientiert-situativen Kompetenzbegriff verhärten sich weiter (vgl. Basendowski 2013, 135ff.): Eine „skillful performance“ basiert auf einem „Handeln als Index für Wissen“, welches im Sinne von „,als-ob'-Klassifizierungen empirischer Beobachtungen“ sozio-kontextuell situativ rekonstruiert wird; und ausdrücklich nicht als Folge von Wissen im Sinne eines knowing that, respektive, nicht im Sinne von mentalen Entitäten im Subjekt als Quelle jeglicher Handlung. Zugleich begrenzt dieser Kompetenzbegriff den praxeologischen Lesebegriff: Er 
begrenzt ihn einerseits für die sozio-kulturell verketteten Situationen mit stark routinalisierten lokalen kulturellen Codes, bzw., mit vermeintlich „,explizierbaren“, ähnlichen symbolischen Orientierungssystemen. Andererseits begrenzt er ihn durch den Fokus auf demgemäß als-ob-klassifizierbare kognitive, emotional-motivationale und sozial-kommunikative Anteile, die entlang einer "skillful performance“ in einer Praktik beobachtbar sind. Ein nicht beliebiger Gebrauch der Zeichensysteme knüpft an eine routinalisierte Textualisierung an, samt deren praktizierten Funktion und praktizierten Relevanz. In das empirische Interesse rücken konsequenterweise zuvorderst die - hypothetisch-symbolischen - lokalen kulturellen Codes zur Beschreibung der textuellen Anforderungen in einer Praktik der Dokumentation eines Kleinbetrag-Kaufvertrags (Kassenbeleg), von geleisteten Arbeitszeiten (Arbeitsprotokoll) usw. in beruflichen Kontexten. Erst darauf aufbauend wäre ein Rückgriff auf einzelne Teilnehmende einer Praktik möglich. Der nicht beliebige Gebrauch der stark routinalisierten „Spielregeln“ des Artefaktes Zeichensysteme als lokale Kriterien einer „skillful performance“ eröffnet Zugang zu einer immer nur hypothetischen Konstruktion der sowohl kognitiven, emotional-motivationalen und sozial-kommunikativen Dispositionen eines/r Teilnehmers/in einer Praktik.

Jene skillful performance im Sonderfall sozial-literaler Praxen umfasst sodann auch ein ,Wissen über die Faktizität des Artefakts. Aus der praxeologischen Erkenntnistheorie entlehnt, verfügen die Artefakte, so auch semiotische Zeichen, per se jedoch keinen Eigenwert. Das angesprochene ,Wissen' bezieht sich schlussfolgernd nicht auf ein knowing how zum Gebrauch des routinalisiert eingebetteten literalen Artefakts. Aus der Analyse zu Makrotheorien der verstehenden Verarbeitung semiotischer Zeichen bietet sich durch die geforderte „semantische Leere“ dieses ,Wissen' über die Faktizität des semiotischen Artefakts folgender Kandidat an: die fundamentale Repräsentationsmächtigkeit mittels semiotischer Zeichen, welche auf der Ebene der Identifikation sowohl bei symbolischen als auch bei ikonischen Zeichen thematisiert wurde.

$\mathrm{Zu}$ der Ausdifferenzierung der fundamentalen Repräsentationsmächtigkeit für symbolische Schriftsysteme weiß man aus linguistischen Analysen (Dürscheid 2012, 67ff.), dass diese letztlich alle auf eine überschaubare Anzahl von arbiträren Repräsentationsanalogien zu gesprochenen Sprachen beruhen: Wenn die Schriftlinguistik zwischen logographischen und phonographischen Schrifttypen unterscheidet und zweitere weiter in alphabetisch-morphophonemische, alphabetisch-phonemische oder syllabische Subtypen ausdifferenziert werden können, so konkretisieren sich hierüber die einzelnen arbiträren Repräsentationsanalogien. Ein semiotisches Zeichen korrespondiert folglich stets mit einer, wenn auch unterschiedlichen Entität des Sprachsystems. Beispielsweise kann ein Zeichen für ein Phonem oder ein Set von Phonemen stehen, die zusammen einen Begriff bezeichnen oder ein/mehrere Phonem(e) als Entität eines Morphems wie im deutschen Schriftsystem. In anderen Schriftsystemen repräsentiert ein Graphem eine Sprachsilbe. Diese fundamentalen Repräsentationsanalogien symbolischer Zeichen stellen ein Ergebnis evolutionärer Entwicklungen dar und bieten letztlich Grundlage für die Ausdifferenzierung sämtlicher sozial-literaler Praxen. Angesichts dessen, dass spezifische Schriftsysteme evolutionär auf mehr oder weniger eigene arbiträre Repräsentationsanalogien beruhen, umfasst jenes, Wissen' ausschließlich die skizzierte fundamentale Repräsentationsmächtigkeit. Dieses daher im Weiteren als knowing how fundamentaler Repräsentationsanalogien bezeichnete ,Wissen', auf welches sozialliterale Praxen evolutionär beruhen, umfasst folglich bspw. nicht, dass ein bestimmtes symbolisches Zeichen für ein bestimmtes Wort, ein bestimmtes Phonem bzw. ein bestimmtes Phonem als Entität eines Morphems oder einer Silbe semiotisch in einem spezifischen Schriftsystem steht. Gleiches gilt für die Aktualisierung dieser Praxen via seiner sozial-literaler events, obgleich sie stets mindestens eines dieser fundamentalen Repräsentationsanalogien dezidiert mobilisieren. 


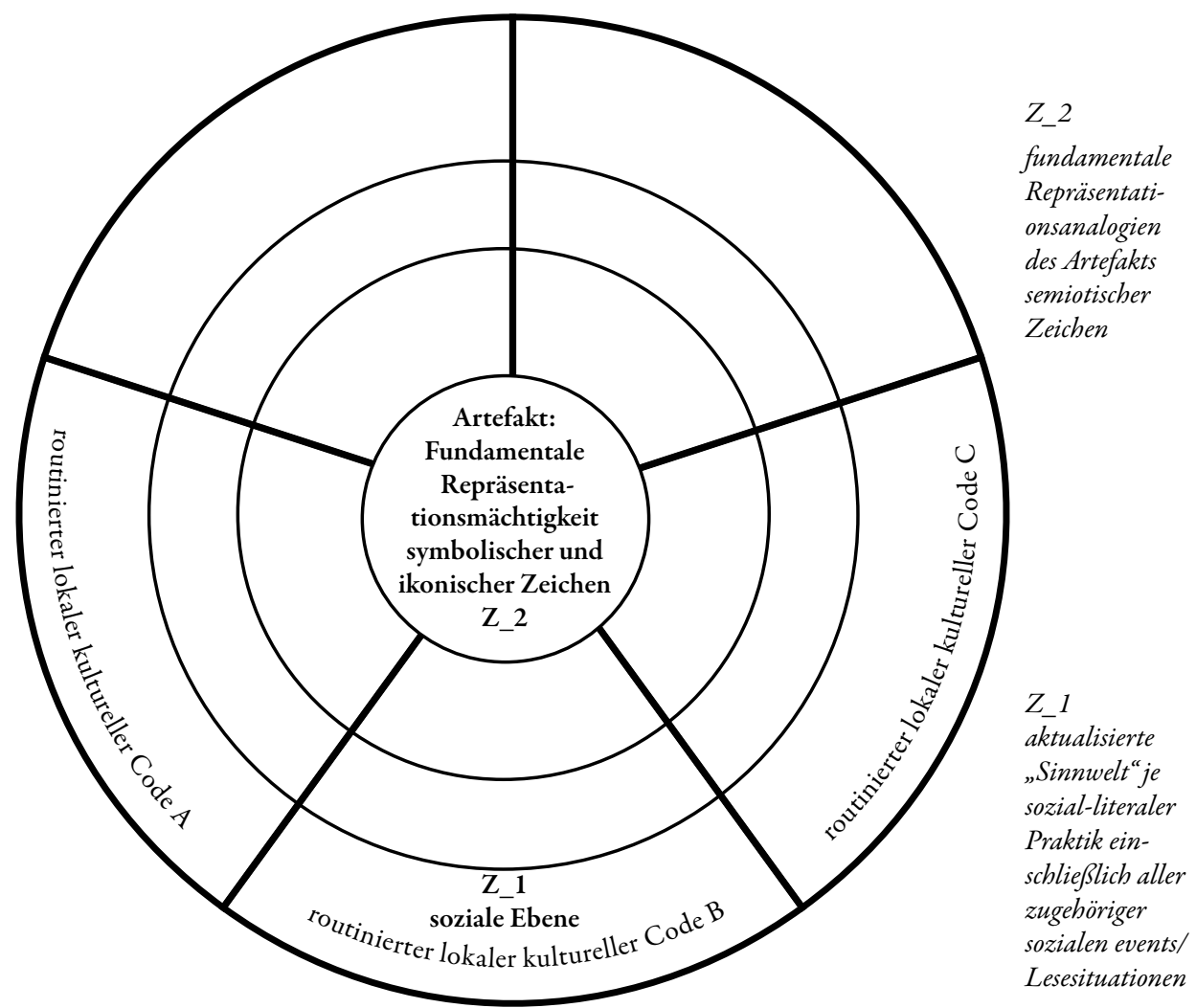

Abb. 55: Multikodales Lesen als Set sozial-literaler Praxen 2

Über diese einzelnen zugehörigen sozial-literalen events kann rückgeschlossen werden, welche ,Sinnwelt' und welches ,Wissen' semiotischer Repräsentationsmächtigkeit die Personen als Träger re-/konstruieren, wenn auch nur einen Auszug aus der, Sinnwelt' und dem, Wissen 'semiotischer Repräsentationsmächtigkeit, welche die sozial-literalen Praktik bspw. der Dokumentation eines Kleinbetrag-Kaufvertrags (Kassenbeleg) ausmachen. Über die dargelegten Makromodelle der verstehenden Verarbeitung von Texten kristallisiert sich die Vermutung heraus, dass sich jene Mobilisierung eines Auszugs um ,Wissen` semiotischer Repräsentationsmächtigkeit auf der Ebene der Identifikation von semiotischen Zeichen begründen lässt. Die Identifikation basiert, wie dargelegt, zumindest teils auf ein generalisierbares knowing that der Repräsentationsanalogie, ergo, dass z. B. ein Graphem für ein Phonem steht - oder, dass z. B. eine räumliche Anordnung zweier Ikone für eine Beziehung zwischen diesen beiden Ikonen steht, usw. Personen, die nicht über dieses knowing that semiotischer Repräsentationsmächtigkeit verfügen, vermögen nicht an events einer sozial-literalen Praktik, einer spezifischen ,Sinnwelt', tatsächlich teilzuhaben. Diese wertfreien, aber sinnlich wahrnehmbaren Anteile der Repräsentationsmächtigkeit sind Ergebnis evolutionärer Begrenzungen über Teilnahme an diversen sozial-literalen Praxen, für welche die Gestaltungsgesetze ein zentrales Beispiel darstellen.

Im Umkehrschluss verweist eine „semantisch leere“ Identifikation eines semiotischen Zeichens, wie sie bspw. auch im Sinne einer prä-attentiven Verarbeitung diskutiert wurde, auf explizier- 
bare kognitive Prozesse, welche eine Handlungsfähigkeit mit Texten als Voraussetzung beeinflussen: Sie umfasst aber, nur diejenigen Facetten von Repräsentationsanalogien unabhängig des Sozialen und Situativen, die auf Entitäten dieses einen spezifischen Zeichensystems und dem Bezeichneten zutreffen. Nur innerhalb dieser Bedingung gilt dieser Voraussetzungscharakter, ergo generalisierend für alle Texte der zugehörigen sozial-literalen events. Diese gehen folglich bereits immer von dem Spezialfall eines z.B. symbolischen und/oder ikonischen Zeichensystems aus und blenden konsequenterweise andere aus - ein Ausdruck der ,Sinnwelt', wofür die sozial-literale Praktik routinalisiert steht.

Für sozial-literale events der Praxen wie z.B. der Dokumentation eines Kleinbetrag-Kaufvertrags (Kassenbeleg) oder von geleisteten Arbeitszeiten (Arbeitsprotokoll) kommen, übertragen, insbesondere die arbiträren Repräsentationsanalogien infrage, welche hinter den zuvor thematisierten starken Darstellungs-Kodes von kategorial-tabellarischen Texten und Textkomplexen thematisiert wurden. Dem Ursprung dieser „semantisch leeren“ ikonisch-arbiträren Repräsentationsanalogien wurde eigens nachgegangen. Es wurde aufgezeigt, dass eine skillful performance der routinalisiert an Einfach(st)erwerbstätigkeiten angesprochenen sozial-literalen events nicht nur an dem praktischen Sinn der multikodalen Texte und Textkomplexe über routinalisierte lokale kulturelle Codes gebunden ist, sondern gleichermaßen auch an der Faktizität dieses spezifischen semiotischen Artefaktes (vgl. Abbildung 56).

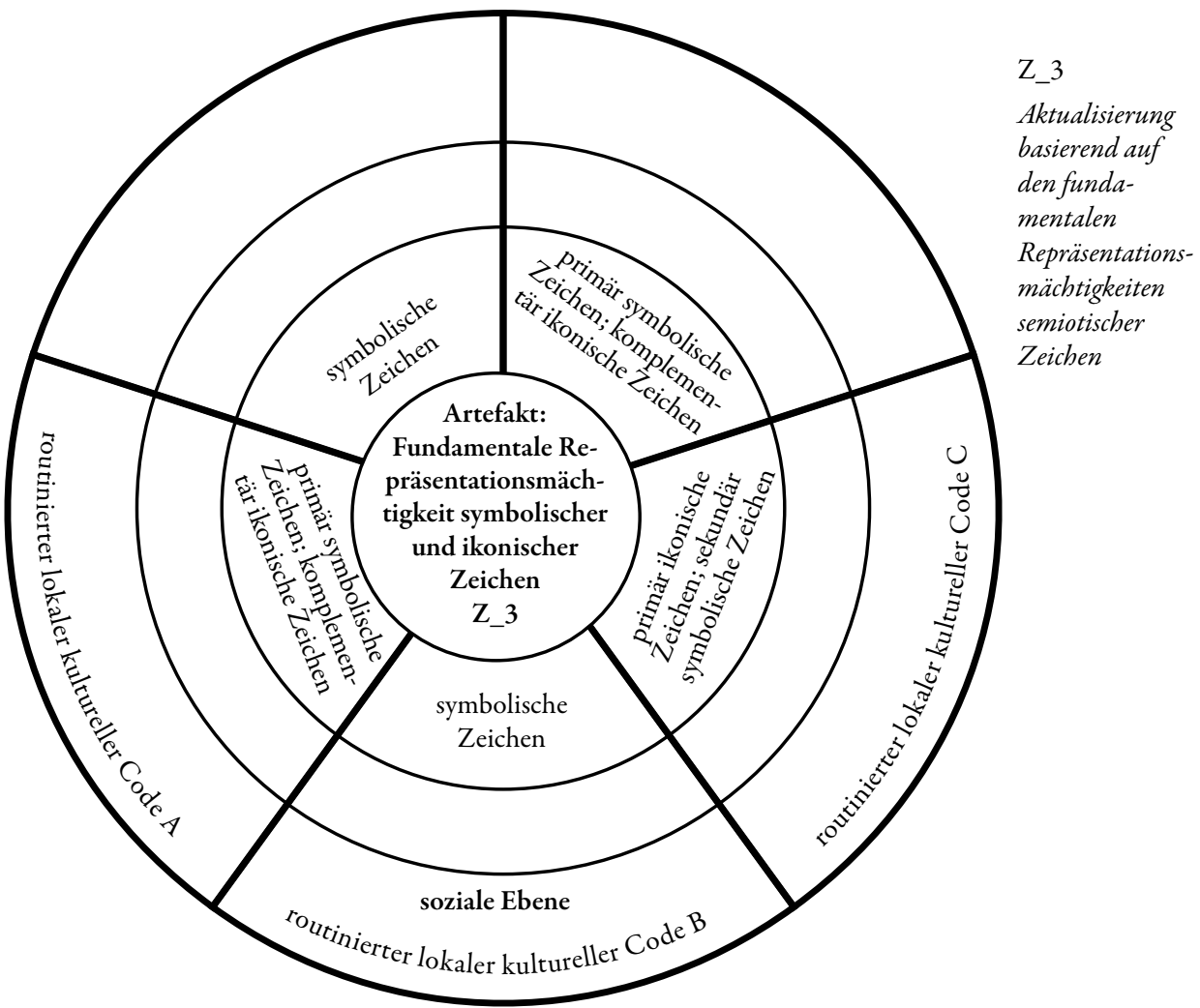

Abb. 56: Lesende in Lese-events einer sozial-literalen Praktik 3 
Die Handlungsfähigkeit mit Texten, respektive, eine verstehende Verarbeitung unter Berücksichtigung des Sozialen, Situativen und Verkörperlichten des einzelnen Lesenden lässt sich im Rückschluss über das aktualisierte,Wissen' erschließen, welche Personen als Träger sozial-literaler Praxen samt ihrer events offenbaren. Die Relevanz des routinalisiert ordentlichen Gebrauchs des semiotischen Artefakts innerhalb dieses Rückschlusses mitgedacht, bietet das theoretisch dekonstruierte Arbeitsmodell aus TRACE model und embodied theory der verstehenden Verarbeitung von Texten den folgenden theoretisch stringenten Erklärungsansatz, mit welchem sich die Forschungsergebnisse der vorliegenden Studie einordnen lassen: Sowohl das im Hier und Jetzt komplementär erzeugte source model und task/goal model als auch deren initiierende oder begleitetende verstehende Verarbeitung entlang eines multikodalen Texts bzw. Textkomplexes (content model) integrieren sich konzeptuell derart, dass sie über dynamische mentale Modellierungen zwischen den Polen konkret körpergebunden und abstrabiert körpergebunden erfahrenes ein ,Wissen' ansprechen, das wie gefordert, eindeutig auf knowing how verweist. „Von außen nach innen“ eröffnet dieser Rückschluss, erstens, einen Zugang zum „impliziten Sinn oder zu der Konzeption einer Art Idee zur bzw. internal representation of the task to be completed und of the response to be output. Jeglicher Rückschluss muss als annähernde Explikation der zugehörigen events aber letztlich nie explizierbaren sozial-literalen Praktik betrachtet werden, oder in anderen Worten, als einen Auszug der jedem event routinalisiert inneliegenden ,Sinnwelt'. „Von innen nach außen“ eröffnet sich, zweitens, ein Rückschluss auf den „impliziten Sinn des routinalisiert eingebundenen Gebrauchs der semiotischen Zeichen, respektive, einer Konzeption einer Art Idee about how content area and document knowledge is represented in texts. Sowohl von innen als auch von außen, ergo, sowohl ausgehend von den semiotischen Artekfakten bzw. Repräsentationsanalogien als auch von der ,Sinnwelt' der sozial-literalen Praktik routiniert beeinflusst, ergibt sich, drittens, ein Rückschluss auf deren victorious experience zum textuellen Input; und dies basierend auf der Voraussetzung, dass sie als semiotische Zeichen überhaupt erkannt wurden. Oder in anderen Worten, die angesprochene Aktualisierung des content models durch die Lesenden stellt eine Art Idee über das Bezeichnete der vorliegenden identifizierten semiotischen Zeichen dar. Da die victorious experience über Rekontextualisierung quasi-,räumlich-zeitlich' konkreter bis gar eher schematisierter, habitualisierter und automatisierter verköperlichter Erfahrungen sukzessiv aufgebaut wird, verhärtet sich über die Komplementarität von content, source und task/goal model, dass dieser Rückschluss nur vor dem Hintergrund der sozial-literalen Praxen vorgenommen werden kann, in welcher der/die untersuchte Leser/in seither routinalisierte Erfahrungen sammelte. Jene in der Person liegende Einflussfaktoren des Lesens prägen maßgeblich auch die verstehende Verarbeitung von Texten aus dem Arbeitsmodell zur Verarbeitung multikodaler Texte und Textkomplexe (vgl. Abbildung 57).

Ein jeweils vorliegender multikodaler Text oder Textkomplex legt aus jenem Arbeitsmodell des verarbeitenden Lesens folglich nahe, dass deren Entitäten nur einen textuellen Möglichkeitsraum während seiner sukzessiv verstehenden Verarbeitung durch eine/n Lesende/n erzeugen. Sie können ein Sampling von Texten bilden, mittels denen aus einem nicht beliebigen Gebrauch des Artefaktes im Spannungsfeld von lokalen kulturellen Codes alsdann nur hypothetisch auf die Handlungsfähigkeit von „allen“ Teilnehmenden kategorisch geschlossen werden kann; so gemäß dem Lesen als Set sozialer Praxen im Geltungskreis von handlungstheoretisch-situativen Kompetenzen.

Innerhalb jener Symbiose liegen in der interaktiven Komplementarität von task/goal model und content model zuvorderst emotional-motivationalen Einflussfaktoren des Lesens begründet. Hierbei handelt es sich jedoch um kein explizites Konstrukt von gar domänenunabhängiger Motivation, sondern vielmehr um ein impliziet verkörperlichtes Konstrukt in construal und integration auf Wort- und Satzphrasenebene (Stichwort: predictability) sowie auf Textebene 


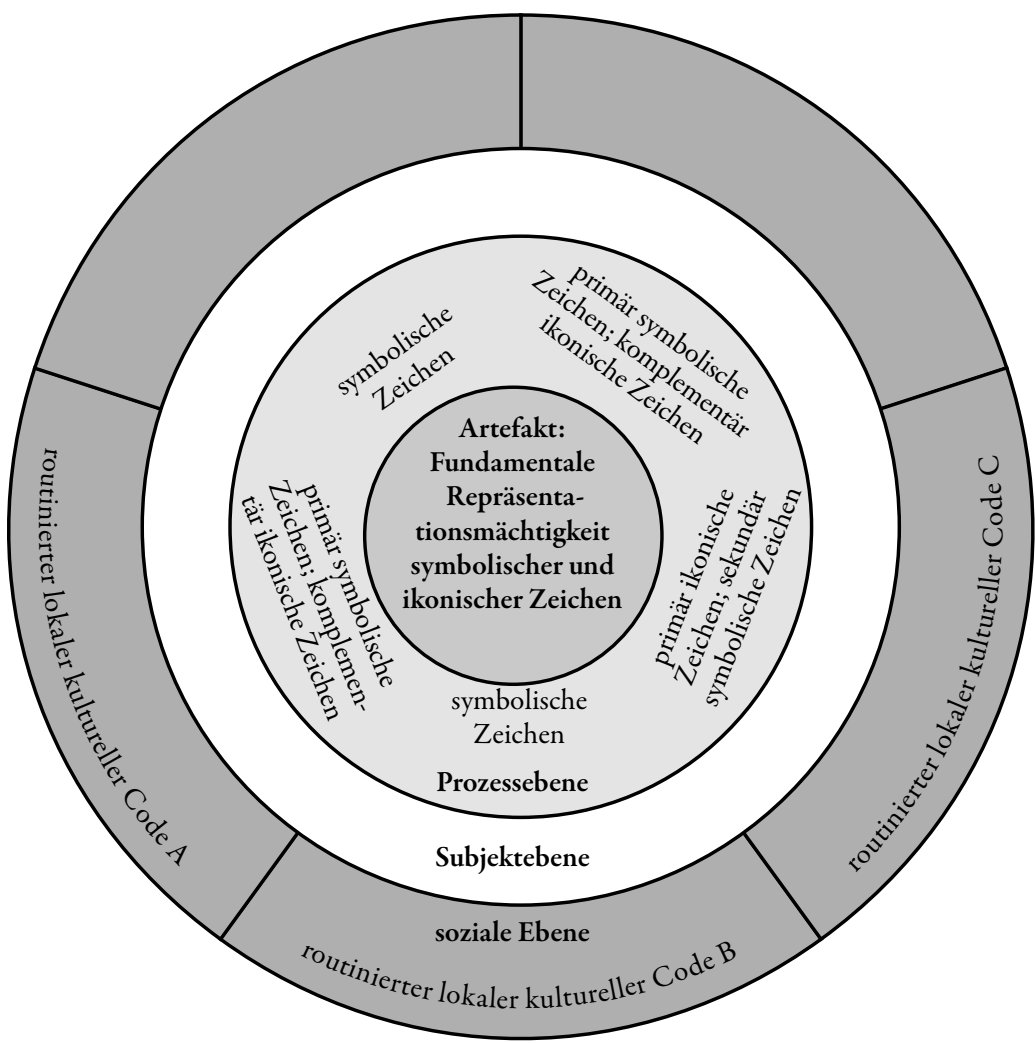

Aktualisiertes knowing how bis knowing that fundamentaler Repräsentationsanalogien

Aktualisiertes knowing how bis knowing that about how content area and document knowledge is represented in texts (source model)

Aktualisiertes knowing how bis knowing that about internal representation of the task to be completed und of the response to be output (task/goal model)

Abb. 57: Multikodales Lesen als Set sozial-literaler Praxen

(Stichwort: continuity assumption). Die Interaktion des task/goal models mit dem content model wurde in einer vorausgehenden oder begleitenden Evaluation via Subsumption, Verwerfung, Neufassung oder Modifikation erklärt. Jenes sozial, situativ und soziale Verständnis der emotional-motivationalen Ebene des Lesens zeigt sich bspw. daran, dass kurzzeitig zurückliegende oder besonders einprägsame Erfahrungen sich überlagern können. Während der verstehend verarbeitenden Interaktion mit einem multikodalen Text oder Textkomplex sei abschließend auf die Konzepte des verkörperlichten knowledge zu semantischen und syntaktischen Bedeutungsstrukturen hinzuweisen. Letztere basieren auf Schematisierung, Habitalisierung und Automatisierung des knowing how, welche innerhalb dynamischer mentaler Modelle über das eher abstrabiert körpergebunden erfahrene, Wissen' konzeptuell entsprochen wird. 


\subsection{Multikodales Lesen in der Relation von Bildung und Teilhabe}

Der Voraussetzungscharakter von Bildung für Teilhabe im Sinne von Walther (2012) wird in keinem der thematisierten Lesekompetenzansätze infrage gestellt. Jedoch unterscheidet sich die postulierte Geltungsreichweite jenes Voraussetzungscharakters eindrücklich an dem referentiellen Kulturkonzept (vgl. Tabelle 6). Den weitreichendsten Anspruch stellt der Ansatz des Lesens als Informationsverarbeitung/Kulturtechnik mit seiner postulierten universellen Gültigkeit für alle Mitglieder und jegliche Lesesituation der modernden Mediengesellschaft. Weniger beim Lesen als kulturelle Praxis, sondern vielmehr beim Lesen als kulturelle Praxen wird dieser universelle Geltungsanspruch nur noch für soziale Milieus mitgedacht, welche die Bildungssprache pflegen, sprich für die legitime und bedingt für die Mainstream-Literalitäten. Beim Lesen als Set sozialer Praxen ist dieser Geltungsanspruch am geringsten, da er sich nur noch auf die Personen bezieht, welche an einer textuell eingebundenen Situation teilhaben. Vor dem Hintergrund der aufgedeckten Mythen von Lesekompetenz zur Vorhersage von Teilhabe an Einfach(st)erwerbstätigkeiten verwundert nicht, dass lediglich jener Ansatz des Lesens als Set sozialer Praxen geeignet ist, kulturwissenschaftlich stringent die Relation von Bildung und Teilhabe in Arbeit tatsächlich mitzudenken. Die Forschungsergebnisse der vorliegenden Studien zum multikodalen Lesen von primär ikonischen Texten und Textkomplexen geben deutliche Indizien dafür, dass die weiteren Lesekompetenzansätze befragt werden müssen, ob sie den Voraussetzungscharakter für die tatsächliche Breite des Beschäftigungssystems abbilden können.

Problematischer sind die weiteren Lesekompetenzansätze jedoch mit Blick auf den Auslösungscharakter von Teilhabe für Bildung. Wird die Frage aufgeworfen, ob Teilhabe an Einfach(st) erwerbstätigkeiten - bspw. in der Form von berufsqualifizierenden Angeboten bis Arbeitserfahrungen - Bildungsprozesse auslösen können, bietet das enge Korsett des Lesens als Informationsverarbeitung/Kulturtechnik, des Lesens als kulturelle Praxis und des Lesens als kulturelle Praxen keine Konzepte oder Begriffe, um ausgelöste Bildungsprozesse für Lesekompetenzen überhaupt zu benennen. Die Bewertungsprogramme von Bildung sind, in corpore, dahingegen kritisch zu befragen, ob sie sich mit ihren Zertifikaten im Spiegel der Anforderung um Inklusion noch auf das Lesen alleinig von Fließtexten entlang der Sinn- und Relevanzoption der Behaltensleistung bzw. der Einstellungsänderung beschränken können. Sollen multikodale Texte und Textkomplexe primär ikonischer Kodalität Berücksichtigung finden, bedarf es der kritischen Befragung und Weiterentwicklung der Leitsemantiken. Die Antwort auf diese Frage ist untrennbar mit der Betrachtung verknüpft, ob die Teilhabe an Einfach(st)erwerbstätigkeiten überhaupt eine akzeptierte Form von Teilhabe im Lebensbereich Arbeit darstellt. Die vorliegende Arbeit folgt, wie begründet dargelegt, der Position, dass diese Tätigkeiten selbstsagend zu Teilhabe im Lebensbereich Arbeit beitragen (können) und daher in der Inklusionsdebatte mitzudenken sind.

Aus dem Lesen als kulturelle Praxen - es handelt sich nicht um den präferierten Ansatz - könnte man zur Illustration schlussfolgern, dass Bildungsprozesse dahingehend befragt werden müssten, ob sie nur einen engen Ausschnitt aus der Vielfalt des gesellschaftlichen Lesens tatsächlich fortführen müssen? Insbesondere mit Blick auf die Bewertungsgrundlage des kompetenten Lesens bedarf es einer Akzeptanz von Vielfalt unterschiedlicher kultureller Praxen entlang von Texten. Die geringe Akzeptanz der Basalen Literalität und die Ignoranz einer Literalität einfacher Arbeit gilt es auf der Grundlage dieser Arbeit zu erweitern, wenn Bildung als Voraussetzung sowie Auslöser von Teilhabe in der Bandbreite von Gesellschaften einschl. seiner Lebensbereiche mitgedacht wird (vgl. Grotlüschen 2010; 30ff.). 
Tab. 58: Literalität einfacher Arbeit eines Lesens als kulturelle Praxen

\begin{tabular}{|c|c|c|c|c|}
\hline Merkmale & $\begin{array}{l}\text { Legitime } \\
\text { Literalität }\end{array}$ & $\begin{array}{l}\text { Mainstream- } \\
\text { Literalität }\end{array}$ & $\begin{array}{l}\text { Basale } \\
\text { Literalität }\end{array}$ & $\begin{array}{l}\text { Literalität } \\
\text { einfacher Arbeit }\end{array}$ \\
\hline $\begin{array}{l}\text { Zeichen und } \\
\text { Umfang }\end{array}$ & Fließtexte & Fließtexte & $\begin{array}{l}\text { geringe Anzahl von } \\
\text { (symbolischen) } \\
\text { Zeichen kombiniert } \\
\text { mit Graphiken }\end{array}$ & $\begin{array}{l}\text { multikodale Texte } \\
\text { und Textkomplexe } \\
\text { primär ikonischer } \\
\text { Kodalität }\end{array}$ \\
\hline Themen & $\begin{array}{l}\text { kritisches Hinterfra- } \\
\text { gen von Berichten } \\
\text { etc.; Schriftgebrauch } \\
\text { für die Konservie- } \\
\text { rung; Qualifizierung }\end{array}$ & $\begin{array}{l}\text { Schriftgebrauch für } \\
\text { die Konservierung; } \\
\text { Qualifizierung }\end{array}$ & Alltagsbewältigung & $\begin{array}{l}\text { routinalisierte ein- } \\
\text { gebundene Texte am } \\
\text { Arbeitsplatz }\end{array}$ \\
\hline $\begin{array}{l}\text { kultureller } \\
\text { Code }\end{array}$ & $\begin{array}{l}\text { Kritik; Zitation; } \\
\text { Interessenlosigkeit; } \\
\text { Ironie; bestätigende } \\
\text { Gründe; soziale } \\
\text { Gründe }\end{array}$ & $\begin{array}{l}\text { Wissenserwerb; } \\
\text { Entspannung; } \\
\text { Soziale Gründe; } \\
\text { Bestätigende Gründe; } \\
\text { Instrumentelle } \\
\text { Gründe; Nahrichten- } \\
\text { bezogene Gründe }\end{array}$ & $\begin{array}{l}\text { Distinktion; Infor- } \\
\text { mationsentnahme; } \\
\text { nachrichtenbezogene } \\
\text { Gründe; instrumen- } \\
\text { telle Gründe }\end{array}$ & $\begin{array}{l}\text { Organisationshilfe, } \\
\text { Merkhilfe, Kont- } \\
\text { rolle, Weitergabe, } \\
\text { Behaltensleistung }\end{array}$ \\
\hline $\begin{array}{l}\text { Textuelle } \\
\text { Beispiele }\end{array}$ & $\begin{array}{l}\text { wissenschaftliche } \\
\text { Arbeiten; Poesie; } \\
\text { spezifische Fachzeit- } \\
\text { schriften; klassische } \\
\text { Komödien und } \\
\text { Dramen; Emails; } \\
\text { bzw. Briefe etc. }\end{array}$ & $\begin{array}{l}\text { Soziale Medien; um- } \\
\text { fassende Zeitungsbe- } \\
\text { richte; gebräuchliche } \\
\text { Fachzeitschriften; } \\
\text { Aufbauanleitungen; } \\
\text { Mietvertrag; Rech- } \\
\text { nungen; Romane; etc. }\end{array}$ & $\begin{array}{l}\text { kurze Zeitungsbe- } \\
\text { richte; Wetterbe- } \\
\text { richt; Todesanzeigen, } \\
\text { Wohnungsanzeigen; } \\
\text { Werbeprospekte; etc. }\end{array}$ & $\begin{array}{l}\text { Textkomplexe aus } \\
\text { Lieferscheinen, } \\
\text { Rechnungen und } \\
\text { Berichtsvorlagen; } \\
\text { Raumpläne; etc. }\end{array}$ \\
\hline Distinktion & $\begin{array}{l}\text { gegenüber basaler } \\
\text { und legitimer Lite- } \\
\text { ralität }\end{array}$ & $\begin{array}{l}\text { gegenüber Basaler } \\
\text { Literalität }\end{array}$ & $\begin{array}{l}\text { gegenüber Legitimer } \\
\text { und Mainstream- } \\
\text { Literalität }\end{array}$ & $\begin{array}{l}\text { gegenüber Legitimer } \\
\text { und Mainstream- } \\
\text { Literalität }\end{array}$ \\
\hline
\end{tabular}

Jene begründeten Ergänzungen des Lesens für eine auf Akzeptanz von sozialer Vielfalt bedachte Bildung in der Relation zu Teilhabe vorausgesetzt, lässt sich in einem zweiten Schritt eine zentrale Schlussfolgerung aus den vorliegenden Ergebnissen ziehen: Mit dieser Ergänzung geht nicht einher, dass einzelne soziale Milieus nur noch ihrer Literalität entsprechend gebildet werden sollen. Ergebnis ist vielmehr im Spiegel von formellen Bildungssettings in einem inklusiven Bildungssystem zu betonen, die Bandbreite des kompetenten Lesens mitzudenken und zu akzeptieren. Hierbei geht es insbesondere um die Bewusstwerdung, dass ein kompetentes Lesen mannigfaltig sein kann und vor diesem Hintergrund schulische Bewertungen vorzunehmen sind. Wenn folglich gefordert wird, dass Leseperformanzen von sozialrechtlich benachteiligten Jugendlichen, insbesondere unmittelbar vor Abschluss der obligatorischen Schulzeit, nicht mehr ausschließlich entlang einer Behaltensleistung und Einstellungsänderung mittels Fließtexten bemessen werden sollen, so kann es theoretisch stringent aus dem Lesen als Set sozialer Praxen im Umkehrschluss nur heißen, Lesekompetenzen anschlussfähig zu fördern: Unter Berücksichtigung potenzieller beruflicher Anschlusslösungen können relevante routinalisierte lokale kulturelle Codes einhergehend mit den entsprechenden multikodalen Texten und Textkomplexen ausgemacht werden. 
Ressourcen der Jugendlichen können konzeptionell stringent aufgedeckt werden, die in den gängigen funktionalen Lesekompetenzansätzen nicht mitbedacht werden.

Mit diesem Postulat geht somit letztlich die stärkere didaktische Fokussierung auf eine Verarbeitung mit multikodalen Texten und Textkomplexen primär ikonischer Kodalität einher. Die quantitative Teilstudie zeigte nicht zuletzt auf, dass gerade an allgemeinbildenden Bildungsgängen nur ca. 45 \% unter den Schüler/innen an den Förderschulen Lernen die Aufgaben des einfachsten Schwierigkeitsniveaus korrekt bearbeiten konnten (vgl. Abbildung 49) - die Zuordnung zum einfachsten Schwierigkeitsniveau wurde aus dem Lösungsverhalten der Stichprobe berechnet. Statistisch bedeutsam bessere Performanzen zeigten dagegen die Jugendlichen, die an Praxisklassen unterrichtet werden und somit institutionell geregelt regelmäßig in Arbeitsprozesse on-the-job auch von Einfach(st)erwerbstätigkeiten eingebunden sind. Dieser Befund leitet dazu über, dass die Schlussfolgerung nach einer stärkeren Berücksichtigung von multikodalen Texten und Textkomplexen primär ikonischer Kodalität nicht im tradierten Sinne begrenzt auf den Deutschunterricht erfolgen sollte. Die Performanzunterschiede lassen sich aus den vorliegenden Studien gerade durch ein knowing how der routinalisierten lokalen kulturellen Codes verstehen - und nicht erklären. Jenes innerhalb des Lesens als Set sozialer Praxen per se implizite und verkörperlichte Wissen kann vielmehr thematisiert und intentionaler Aspekt von Bildungsprozessen werden, wenn diese in Handlungen eingebunden sind. Es gilt Jugendliche an sozialen Praxen mit multikodalen Texten teilhaben zu lassen, in denen eine skillful performance entlang bspw. einer Merkhilfe, Organisationshilfe, Kontrolle und Weitergabe routinalisiert geprägt sind. Übertragen aus dem „Immersed Experiencer Framework" sind Erfahrungen zu sammeln und zu reflektieren im Sinne einer Rekontextualisierung quasi-,räumlich-zeitlicher' konkreter bis gar eher schematisierter, habitualisierter und automatisierter verkörperlichter Erfahrungen. Da unser Alltag eine Vielzahl an Begegnungen mit multikodalen Texten und Textkomplexen primär ikonischer Kodalität nahezu erzwingt, könnten Lesesituationen an jener Relevanz- und Sinnoptionen bereits früh ansetzen, indem sie bspw. in der Schule alle Arten von Formularen als Lernanlass und -gegenstand nutzen. In schulischen Handlungsfeldern finden sich Anlässe beim Anlegen von Ämterplänen in der Klasse, beim Führen von Klassenlisten oder bei der Organisation des Leihwesens der Schulbibliothek. Für die Auswahl der Textsorten heißt dies zwangsweise, dass multikodale Texte und Textkomplexe deutlich stärker in Unterricht einzubeziehen sind als dies bislang der Fall ist. Darunter sind einerseits insbesondere jene Tabellen, Formulare, Internetseiten usw. gemeint, die primär ikonisch und kategorial aufgebaut sind und eben nicht numerisch bzw. als Dopplung eines Fließtextes. Andererseits sind gezielt auch Kombinationen dieser primär ikonisch repräsentierten Texte gemeint, sprich multikodale Textkomplexe primär ikonischer Kodalität, die ohne Fließtext auskommen.

Insofern die Anbahnung von Lesekompetenzen eng gekoppelt wird mit der Vermittlung basaler schriftsprachlicher Kompetenzen sowie dem Verstehen der sozialen Funktionen von Schrift, dem funktionalen Ablauf des Lesens und der Kenntnis aller Buchstaben usw. (Rosebrock/Nix 2008, 15ff.), sind unter fachspezifischer und sachstruktureller Sicht Parallelen für eine zielgerichtete Förderung auch jener multikodalen Texte und Textkomplexe zu ziehen. Entlang einer vereinfachten Gegenüberstellung sollen abschließend aus einer didaktischen Perspektive einzelne Schlussfolgerungen aus den vorliegenden Befunden für die Verarbeitung von Zeichen auf der Prozessebene auszugsartig gezogen werden. Mit Abbildung 58 und Tabelle 59 wird verdeutlicht, dass die unterschiedlichen Sets starker Darstellungs-Kodes hinter kategorialen Tabellen, Diagrammen, graphischen Darstellungen und Karten nebst den Steuerungs-Kodes vergleichbar von Relevanz sind, wie die phonologische und morphologische Bewusstheit einschl. GraphemPhonem-Korrespondenz im deutschen symbolischen Schriftsystem. 


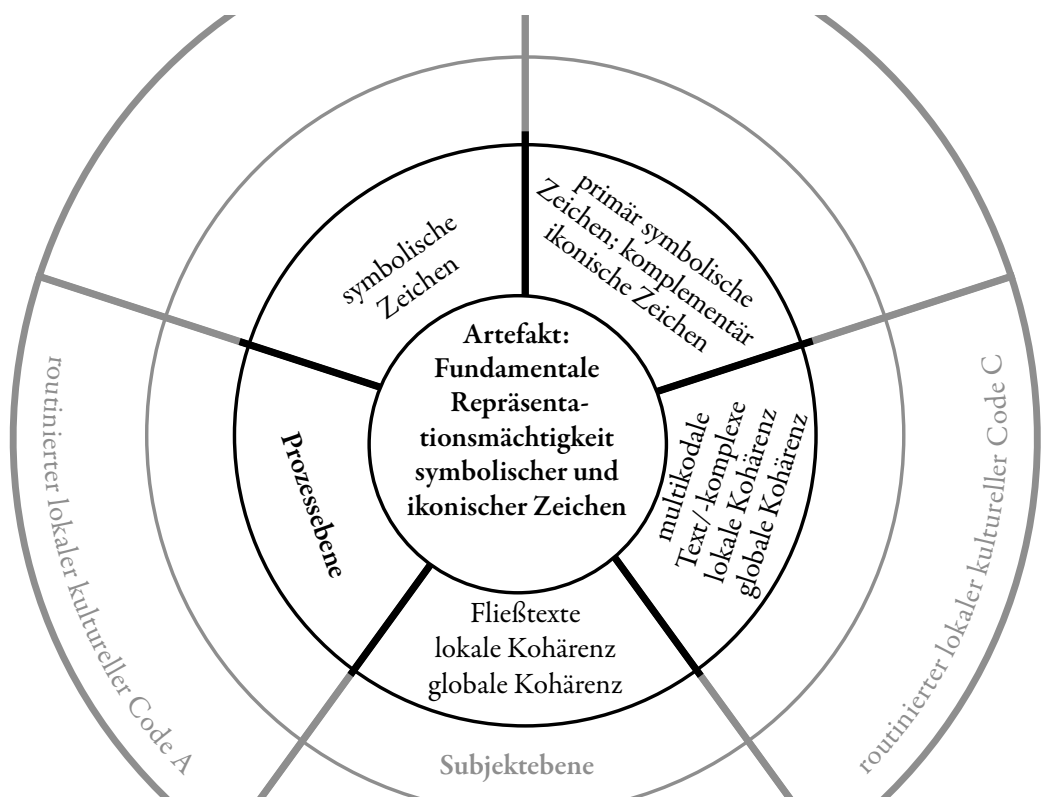

Abb. 58: Verarbeitung multikodaler Texte als Set sozial-literaler Praxen

Tab. 59: Auszug der Verarbeitungsprozesse multikodaler Texte und Textkomplexe

\begin{tabular}{lll}
\hline Verarbeitung & \multicolumn{1}{c}{ multikodale Texte } \\
\cline { 2 - 3 } $\begin{array}{l}\text { Repräsentations- } \\
\text { analogie }\end{array}$ & $\begin{array}{l}\text { Erkennen (auf den ersten Blick), Identifi- } \\
\text { zieren und Gruppieren graphischer Enti- } \\
\text { täten als semiotische Einheiten, sprich als } \\
\text { symbolische Zeichen, die im deutschen } \\
\text { Schriftsystem für Phoneme als Teil von } \\
\text { tifizieren und Gruppieren graphischer } \\
\text { Eontïäten und räumlicher Strukturen zwi- } \\
\text { schen graphischen Entitäten als ikonische } \\
\text { Elementar- oder Globalzeichen }\end{array}$ \\
\hline Lokale Kohärenz & $\begin{array}{l}\text { Worterkennung } \\
\text { (phonematisch/morphematisch) }\end{array}$ & $\begin{array}{l}\text { mentale Modellierungen, die es ermög- } \\
\text { lichen die graphischen Einheiten zu } \\
\text { analysieren und zu verstehen }\end{array}$ \\
& $\begin{array}{l}\text { Buchstabenkombinationen hemmen/ } \\
\text { aktivieren Zugriff auf lexikalisches }\end{array}$ & $\begin{array}{l}\text { 4-7 Entitäten können parallel verarbeitet } \\
\text { werden }\end{array}$ \\
& $\begin{array}{l}\text { Gedächtnis } \\
\text { Konstruktion von Wortbedeutungen und }\end{array}$ & $\begin{array}{l}\text { Aktivierung: goal/task-model; source } \\
\text { model (bspw. graphische Schemata, }\end{array}$ \\
& $\begin{array}{l}\text { Mikropropositionen (Prädikat-Argu- } \\
\text { ment-Aussagen) }\end{array}$ & Darstellungs-Kodes, Steuerungs-Kodes) \\
& $\begin{array}{l}\text { Aktivierung: goal/task-model (bspw. } \\
\text { intentional, exentional, wissensbasiert); } \\
\text { source model (bspw. textbasiert) }\end{array}$ \\
\hline
\end{tabular}




\begin{tabular}{lll}
\hline Verarbeitung & \multicolumn{1}{c}{ multikodale Texte } \\
\cline { 2 - 3 } Globale Kohärenz & \multicolumn{1}{c}{ Symbolische Zeichen } & \multicolumn{1}{c}{ Ikonische Zeichen } \\
& $\begin{array}{l}\text { Online-Verdichtung/Verknüpfung meh- } \\
\text { rerer Mikropropositionen zu Makropro- } \\
\text { positionen durch Auslassungen, Seligie- } \\
\text { ren, Generalisieren und Konstruieren }\end{array}$ & $\begin{array}{l}\text { Online-Verdichtung/Verknüpfung } \\
\text { mehrere Elementarzeichen zu/zum } \\
\text { Globalzeichen unterschiedlicher Hier- } \\
\text { archie durch Auslassungen, Seligieren, } \\
\text { Generalisieren und Konstruieren }\end{array}$ \\
& $\begin{array}{l}\text { Nutzen konventioneller Strukturen } \\
\text { bestimmter Texttypen (Superstrukturen) }\end{array}$ & $\begin{array}{l}\text { Nutzen konventioneller Darstellungs-/ } \\
\text { Steuerungs-Kodes bestimmter routinali- } \\
\text { siert eingebundener Texte }\end{array}$ \\
& $\begin{array}{l}\text { Analyse von rhetorischen und/oder } \\
\text { stilistischen Formen (metalinguistische } \\
\text { Betrachtung) }\end{array}$ & $\begin{array}{l}\text { Metanalyse von Sets starker Darstel- } \\
\text { lungs-Steuerungs-Kodes }\end{array}$ \\
Aktivierung: goal/task-model (bspw. & $\begin{array}{l}\text { Aktivierung: goal/task-model (bspw. } \\
\text { Interessen, Erwartungen); source model } \\
\text { (bspw. Superstrukturen) }\end{array}$ & $\begin{array}{l}\text { Interessen, Erwartungen); source model } \\
\text { (bspw. graphische Schemata, Darstel- } \\
\text { lungscodes, Steuerungscodes) }\end{array}$ \\
\hline
\end{tabular}

Vor dem Hintergrund, dass die Forschungsergebnisse der vorliegenden Gesamtstudie der implizit-embodied Verarbeitung jener multikodalen Texte primär ikonischer Kodalität Potenziale zuspricht, sind demzufolge verkörperlichte Erfahrungssequenzen zu ermöglichen, die die Interferenzen innerhalb nichtkontinuierlicher Texte und Textkomplexe herstellen können, welche zuvorderst auf terminologische, kategoriale und auf Verlaufsstrukturen eines repräsentierten Sachverhalts verweisen. Es gilt das Lesen dieser Texte über ein Hinein- und Herauszoomen oder visuelle Routinen zu verknüpfen, welche „quasi-zeitlich-räumlich“ auf ein „mehr/weniger“, „vorher/nachher“, „auf/ab“, „hinter/vor“ und „einschließend/ausschließend“ verweisen. Diese Settings sollen den Lesenden ermöglichen, implizit-embodied und nicht sprachlich explizit verkörperlicht erfahrungsnahe Analogie und räumlichen Metaphern herzustellen. Neben diesen verkörperlichten quasi-visuellen Erfahrungssequenzen sind gleichsam die weiteren quasi-sensorischen Pendants mitzudenken, so z. B., wenn quasihaptisch erfahrene Handlungsabfolgen mit den Händen rekontextualisiert werden. Vor dem Hintergrund dessen, dass sowohl für die Kohärenzherstellung der Relationen verkörperlicht erfahrungsnahe Analogien und räumliche Metapher für nichtkontinuierliche Texte und Textkomplexe von Relevanz sind, wurde abgeleitet, dass den entsprechenden Darstellungs-Kodes bereits sämtliche terminologische, kategoriale sowie Verlaufsstrukturen des repräsentierten Sachverhalts inhärent sind. Gleiches gilt für die externen Steuerungs-Kodes wie Pfeile, durch die die Richtung einer repräsentierten Kausalität und Effekte von Kausalitäten hervorgehoben werden können.

Für die Verarbeitung empfehlen sich entsprechende verkörperlichte erfahrungsnabe Analogien und räumliche Metapher begleitet durch parasprachlichen Gesten von "mehr/weniger“ über z. B. verkörperlichte quasi-gustatorische Erfahrungen, von „vorher/nachher“ über z. B. verkörperlichte quasi-akustische Erfahrungen, von „auf/ab“ über z. B. verkörperlichte quasivisuelle Erfahrungen, von „hinter/vor“ über z. B. verkörperlichte quasi-olfaktorischen Erfahrungen sowie von „zusammengehörend/nicht-zusammengehörend“ über z. B. verkörperlicht erfahrene Bewegungen mit bspw. den Fingern horizontal und vertikal zu einem Punkt. Die Unterstützung bei der Verarbeitung sollte sich nicht nur an den repräsentierten statischen Zuständen orientieren, z.B. an den in Sachverhalten repräsentierten Codes, Preisen oder 
(Fach-)Begriffen. Vielmehr wird für eine Orientierung am knowledge about how content area knowledge is represented plädiert und somit an typischen Repräsentationsstrategien aus Sets starker Darstellungs- und Steuerungs-Kodes zum Sachverhalt oder der dahinterliegenden Merkmale und Attribute in den jeweiligen sozialen Lesesituationen. Dies leitet über zur angestrebten Bewusstwerdung des zentralen knowledge about how document knowledge is represented und somit zum verkörperlichten ,Wissen' über Autorenschaft des Textes bzw. Textkomplexes, einschließlich der jeweiligen Intention aus der Aktualisierung der sozialen literalen Praxis. 



\section{Verzeichnisse}

\section{Literaturverzeichnis}

Adams, Raymont J./Wu, Margaret L./Wilson, Mark R. (2015). ACER ConQuest: Generalised Item Response Modelling Software, Camberwell: Australian Council for Educational Research

Adamzik, Kirsten (2011). in: Habscheid, Stephan (Hrsg.): Textsorten, Handlungsmuster, Oberflächen. Linguistische Typologien der Kommunikation, De Gruyter Lexikon. Berlin: De Gruyter, 367-386

Ahrbeck, Bernd/Lehmann, Rainer H./Fickler-Stang, Ulrike/Kretschmer, Annette/et al. (2009). ENEBS. Evaluationsstudie des Netzwerks Berliner Schülerfirmen, abrufbar unter: https://www.asig-berlin.de/ASIG_NEBS/downloads/ ENEBS_Webfassung.pdf (letzter Zugriff: 15.5.2013)

Autorengruppe Bildungsberichterstattung (Hrsg.) (2008). Bildung in Deutschland 2008: Ein indikatorengestützter Bericht mit einer Analyse zu Übergängen im Anschluss an den Sekundarbereich I, Bielefeld: Bertelsmann

Barton, David/Hamilton, Mary/Ivanič, Roz (Hrsg.) (2000). Situated Literacies. Reading and writing in context, London: Routledge

Basendowski, Sven (2013). Die soziale Frage an (mathematische) Grundbildung: Eine empirische Studie zu dem Wesen, der Funktion und der Relevanz mathematischer Kompetenzen in einfachen Erwerbstätigkeiten sowie Analysen für didaktische Implikationen/Sven Basendowski, Bad Heilbrunn: Klinkhardt, Julius

Basendowski, Sven (2014). Grundbildung - Ein Konzept für alle in einem inklusiven Bildungssystem? Erste Befunde und Schlussfolgerungen, in: Vierteljahresschrift für Heilpädagogik und ibre Nachbargebiete, Vol. 3, 191-204

Basendowski, Sven (2015). Unified Training System Versus Complementary Qualification Routes. How the Finnish Educational System Deals with Vulnerable Groups - from a German Point of View, in: Schroeder, Joachim (Hrsg.): Breaking Down Barriers from Education to Employment. The journey towards inclusion for vulnerable groups, Sofia: Investpress, 227-246

Bäuerlein, Kerstin/Lenhard, Wolfgang/Schneider, Wolfgang (2012). LESEN 8-9. Lesetestbatterie für die Klassenstufen 8-9. Verfahren zur Erfassung der basalen Lesekompetenz und des Textverständnisses, Göttingen: Hogrefe

Bellmann, Johannes/Priddat, Birger P. (2006). Pädagogische und ökonomische Lernwelten zwischen Differenzierung und Entdifferenzierung, in: Ehrenspeck, Yvonne/Lenzen, Dieter (Hrsg.): Beobachtungen des Erziehungssystems systemtheoretische Perspektiven, Wiesbaden: VS Verlag für Sozialwissenschaften, 153-177

Bertschi-Kaufmann, Andrea (2010). In Textwelten Erfahrungen sammeln. Medienbildung und kulturelle Partizipation, in: Bachmair, Ben (Hrsg.): Medienbildung in neuen Kulturräumen. Die deutschsprachige und britische Diskussion, Wiesbaden: VS Verlag für Sozialwissenschaften, 339-354

Bertschi-Kaufmann, Andrea (2004). Lese- und Schreibaktivitäten in multimedialen Umgebungen. Langzeitbeobachtungen, in: Bertschi-Kaufmann, Andrea/Kassis, Wassilis/Sieber, Peter (Hrsg.): Mediennutzung und Schriftlernen. Analysen und Ergebnisse zur literalen und medialen Sozialisation, Lesesozialisation und Medien. Weinheim: Juventa, 75-95

$B I B B$, Bundesinstitut für Berufsbildung (2013). Datenreport zum Berufsbildungsbericht 2013. Informationen und analysen zur Entwicklung der beruflichen Bildung, Bonn: BIBB, abrufbar unter: http://datenreport.bibb.de/ media2013/BIBB_Datenreport_2013.pdf(letzter Zugriff: 15.3.2019)

Bishop, Alan J. (1988). Mathematics Education in Its Cultural Context, in: Educational Studies in Mathematics, Vol. 19(2), 179-191

Blömeke, Sigrid (2003). Lehren und Lernen mit neuen Medien - Forschungsstand und Forschungsperspektiven, in: Unterrichtswissenschaft, Vol. 31(1), 57-82

Bortz, Jürgen/Döring, Nicola (2006). Forschungsmethoden und Evaluation für Human- und Sozialwissenschaftler, Berlin, Heidelberg: Springer-Verlag Berlin Heidelberg

Bos, Wilfried/Pietsch, Marcus (2004). Erste Ergebnisse aus KESS 4. Kurzbericht, abrufbar unter: http://www.erzwiss. uni-hamburg.de/kess/kurzbericht.pdf (letzter Zugriff: 18.9.2019)

Brandherm, Ruth (2007). Nicht so einfach! Situation und Perspektiven der einfachen Arbeit, in: WISO direkt, (Juni), 1-4

Brandt, Deborah (2009). Literacy and learning: reflections on writing, reading, and society, San Francisco: Jossey-Bass

Butterfield, Lee D./Borgen, William A./Amundson, Norman E./Maglio, Asa-Sophia T. (2005). Fifty years of the critical incident technique: 1954-2004 and beyond, in: Qualitative Research, Vol. 5(4), 475-497 
Campbell-Kelly, Martin/Croarken, Mary/Flood, Raymond/Robson, Eleanor (Hrsg.) (2003). The history of mathematical tables: from Sumer to spreadsheets, Oxford: Oxford University Press

Chasles, Michel (1839). Geschichte der Geometrie, hauptsächlich mit Bezug auf die neueren Methoden. Aus d. Franz. übertragen durch Sohncke, Ludwig Adolph, Halle: Gebauersche Buchhandlung

Christmann, Ursula/Groeben, Norbert (1999). Psychologie des Lesens, in: Franzmann, Bodo/Jäger, Georg/Löffler, Dietrich (Hrsg.): Handbuch Lesen, München: Saur, 145-223

Cohen, Jacob (1988). Statistical power analysis for the behaviorsal science, Hillsdale: Lawrence Erlbaum

Deutsches PISA-Konsortium (2001). PISA 2000: Basiskompetenzen von Schülerinnen und Schülern im internationalen Vergleich, Opladen: Leske + Budrich

DUK, Deutsche UNESCO-Kommission e.V. (2014). Inklusion: Leitlinien für die Bildungspolitik, Bonn: DUK, abrufbar unter: https://www.unesco.de/fileadmin/medien/Dokumente/Bildung/2014_Leitlinien_inklusive_Bildung.pdf

Dürscheid, Christa (2012). Einführung in die Schriftlinguistik, Göttingen: Vandenhoeck \& Ruprecht

Eco, Umberto (2009). The Infinity of Lists. An illustrated Essay; übersetzt von Alastair McEwen, New York: Rizzoli

Enggruber, Ruth/Euler, Dieter/Gidion, Gerd/Wilke, Jürgen (2003). Pfade für Jugendliche in Ausbildung und Betrieb. Gutachten zur Darstellung der Hintergründe der unzureichenden Ausbildungs- und Beschäftigungschancen von benachteiligten Jugendlichen in Baden-Württemberg sowie deren Verbesserungsmöglichkeiten für das Wirtschaftsministerium Baden-Württemberg, abrufbar unter: http://www.mfw.baden-wuerttemberg.de/fm7/1106/Jugendliche_Ausbildung.pdf(letzter Zugriff: 15.9.2011)

Flap, Henk/Boxman, Ed (2001). Getting started: The influence of social capital on the start of the occupational career, in: Lin, Nan/Cook, Karen S./Burt, Ronald S. (Hrsg.): Social Capital: Theory and Research, Sociology and economics, New York: Aldine de Gruyter, 159-182

Franklin, Benjamin (1916). Autobiography of Benjamin Franklin. Edited by Frank Woodworth Pine, Rathway: The Quinn \& Boden Co. Press, abrufbar unter: http://www.gutenberg.org/files/20203/20203-h/20203-h.htm\#IX (letzter Zugriff: 20.3.2014)

Fuchs, Peter (1997). Adressabilität als Grundbegriff der soziologischen Systemtheorie. non sunt multiplicanda entia praeter necessitatem, in: Soziale Systeme, Vol. 3(1), 57-79

Garbe, Christine (2009). Lesekompetenz, in: Garbe, Christine/Holle, Karl/Jesch, Tatjana (Hrsg.): Texte lesen: Lesekompetenz - Textverstehen - Lesedidaktik - Lesesozialisation, Paderborn: Schöningh, 13-38

Gaupp, Nora/Geier, Boris/Hupka-Brunner, Sandra (2012). Chancen bildungsbenachteiligter junger Erwachsener in der Schweiz und in Deutschland: Die (Nicht-Bewältigung) der zweiten Schwelle, in: Zeitschrift für Soziologie der Erziehung und Sozialisation, Vol. 32(3), 299-318

Gaupp, Nora/Kuhnke, Ralf/Schweigard, Eva (2006). Vergleich unterschiedlicher Erhebungsmethoden. Arbeitsbericht im Rahmen der Dokumentationsreihe: Methodische Erträge aus dem „DJI-Übergangspanel“, Arbeitspapier, München, Halle: DJI

Gläser, Jochen/Laudel, Grit (2008). Experteninterviews und qualitative Inhaltsanalyse. Als Instrumente rekonstruierender Untersuchungen, Wiesbaden: VS Verlag für Sozialwissenschaften

Grotlüschen, Anke (2011). Zur Aufösung von Mythen. Eine theoretische Verortung des Forschungsansatzes lea. - Literalitätsentwicklung von Arbeitskräften, in: Grotlüschen, Anke/Kretschmann, Rudolf, Rudolf/Quante-Brandt, Eva, Eva/Wolf, Karsten D. (Hrsg.): Literalitätsentwicklung von Arbeitskräften, Alphabetisierung und Grundbildung, Münster: Waxmann, 13-40

Haible, Ulrike Barbara (2011). Diskontinuierliche Texte. Der Umgang mit diskontinuierlichen Darstellungsformen holt die medialen Alltagserfahrungen in die Schule und fördert die Lesekompetenz, in: Lehren und lernen, Vol. 37(5), 4-7

Hake, Günter/Grünreich, Dietmar/Meng, Liqiu (2002). Kartographie. Visualisierung raum-zeitlicher Informationen, Berlin: De Gruyter

Hillmert, Steffen (2009). Soziale Inklusion und Exklusion: die Rolle von Bildung, in: Stichweh, Rudolf/Windolf, Paul (Hrsg.): Inklusion und Exklusion: Analysen zur Sozialstruktur und sozialen Ungleichheit, Wiesbaden: VS Verlag für Sozialwissenschaften, 85-100

Hirsig, René (1993). Methodische Grundlagen der Testpsychologie, Zürich: Psychologisches Institut der Universität Zürich

Horn, Klaus-Peter/Kemnitz, Heidemarie/Marotzki, Winfried/Sandfuchs, Uwe (Hrsg.) (2012). Klinkhardt Lexikon Erziehungswissenschaft. Band 2: Gruppenpuzzle - Pflegewissenschaft, in: Bad Heilbrunn: Klinkhardt, Julius

Horz, Holger (2009). Medien, in: Wild, Elke/Möller, Jens (Hrsg.): Pädagogische Psychologie, Heidelberg: Springer Medizin, 103-133 
Hradil, Stefan (2006). Die Sozialstruktur Deutschlands im internationalen Vergleich, Wiesbaden: VS Verlag für Sozialwissenschaften

Hupka-Brunner, Sandra/Meyer, Thomas/Stalder, Barbara E./Keller, Anita (2011). PISA-Kompetenzen und Übergangswege. Ergebnisse aus der Schweizer TREE-Studie, in: Krekel, Elisabeth M./Lex, Tilly (Hrsg.): Neue Jugend, neue Ausbildung?: Beiträge aus der Jugend- und Bildungsforschung, Bielefeld: Bertelsmann, 173-188

Hurrelmann, Bettina (2002). Leseleistung, Lesekompetenz. Folgerung aus PISA, mit einem Plädoyer für ein didaktisches Konzept des Lesens als kultureller Praxis, in: Praxis Deutsch, Vol. 176, 6-18

Hurrelmann, Bettina (2009a). Literalität und Bildung, in: Bertschi-Kaufmann, Andrea/Rosebrock, Cornelia (Hrsg.): Literalität Bildungsaufgabe und Forschungsfeld, Weinheim: Juventa, 21-42

Hurrelmann, Bettina (2010). Modelle und Merkmale der Lesekompetenz, in: Bertschi-Kaufmann, Andrea (Hrsg.): Lesekompetenz, Leseleistung, Leseförderung. Grundlagen, Modelle und Materialien, Lehren lernen, Zug: Klett und Balmer

Hurrelmann, Bettina (2009b). Prototypische Merkmale der Lesekompetenz, in: Groeben, Norbert/Hurrelmann, Bettina (Hrsg.): Lesekompetenz. Bedingungen, Dimensionen, Funktionen, Weinheim: Juventa, 275-286

Jankisz, Eva/Moosbrugger, Helfried (2008). Planung und Entwicklung von psychologischen Tests und Fragebogen, in: Moosbrugger, Helfried/Kelava, Augustin (Hrsg.): Testtheorie und Fragebogenkonstruktion,Springer-Lehrbuch, Berlin, Heidelberg: Springer-Verlag Berlin-Heidelberg, 27-72

Kamil, Michael L./Kim Chou, Helen (2009). Comprehension and Computer Technology: Rast Results, Current Knowledge and Future Promises, in: Israel, Susan. E./Duffy, Gerald G. (Hrsg.): Handbook of Research on Reading Comprehension, New York: Routledge, 289-304

Kelle, Udo (2008). Die Integration qualitativer und quantitativer Methoden in der empirischen Sozialforschung. Theoretische Grundlagen und methodologische Konzepte, Wiesbaden: VS Verlag für Sozialwissenschaften

Keller, Berndt/Schulz, Susanne/Seifert, Hartmut (2012). Entwicklungen und Strukturmerkmale der atypisch Beschäftigten in Deutschland bis 2010, Düsseldorf: Wirtschafts- und Sozialwissenschaftliches Institut der Hans-BöcklerStiftung

Kintsch, Walter (2012). Psychological Models of Reading Comprehension and Their Implications for Assessment, in: Sabatini, John P/Albro, Elizabeth/O'Reilly, Tenaha (Hrsg.): Measuring up: advances in how we assess reading ability, Lanham: Rowman \& Littlefield Education, 21-37

Kirsch, Irwin S./Mosenthal, Peter B. (1990). Understanding Documents. Understanding Forms, Part II, in: Journal of Reading, Vol. 33(8), 636-641

$K M K$, Ständige Konferenz der Kultusminister der Länder in der Bundesrepublik Deutschland (2005). Bildungsstandards im Fach Deutsch für den Hauptschulabschluss. Beschluss vom 15.10.2004, abrufbar unter: http://www.kmk. org/fileadmin/veroeffentlichungen_beschluesse/2004/2004_10_15-Bildungsstandards-Deutsch-Haupt.pdf (letzter Zugriff: 15.6.2019)

Kock, Klaus (2008). Auf Umwegen in den Beruf. Destandardisierte und prekäre Beschäftigung von Jugendlichen an der zweiten Schwelle - eine Auswertung empirischer Befunde, Dortmund: Sfs

Köhler, Christoph/Struck, Olaf/Bultemeier, Anja/Grotheer, Michael/et al. (2004). Beschäftigungsstabilität und betriebliche Beschäftigungssysteme in West- und Ostdeutschland. SFB 580: Gesellschaftliche Entwicklungen nach dem Systemumbruch, Jena: SFB 580

Köller, Christopher/Jucker-Kupper, Patrick (Hrsg.) (2009). Karten, Kartographie und Geschichte: von der Visualisierung der Macht zur Macht der Visualisierung = Cartes, cartographie et histoire, Zürich: Chronos-Verlag

Konrad, Klaus (2010). Lautes Denken, in: Mey, Günter/Mruck, Katja (Hrsg.): Handbuch Qualitative Forschung in der Psychologie, Wiesbaden: VS Verlag für Sozialwissenschaften, 476-490

Kretschmann, Rudolf (2011). Kompetenzverfahren „Leseverständnis“ (KLV), in: Grotlüschen, Anke/Kretschmann, Rudolf/Quante-Brandt, Eva/Wolf, Karsten D. (Hrsg.): Literalitätsentwicklung von Arbeitskräften, Alphabetisierung und Grundbildung, Münster: Waxmann, 41-57

Kurtz, Thomas (2006). Erziehung, Kommunikation, Person. Zur Stellung des Erziehungssystems in einem besonderen Quartett gesellschaftlicher Funktionen, in: Ehrenspeck, Yvonne/Lenzen, Dieter (Hrsg.): Beobachtungen des Erziehungssystems: Systemtheoretische Perspektiven, Wiesbaden: VS Verlag für Sozialwissenschaften, 113-131

Lamnek, Siegfried (2005). Qualitative Sozialforschung: Lehrbuch, Weinheim: Beltz, PVU

Lamnek, Siegfried (2010). Qualitative Sozialforschung: Lehrbuch, Weinheim: Beltz

Lehmann, Rainer/Hoffmann, Ellen (Hrsg.) (2009). BELLA: Berliner Erhebung arbeitsrelevanter Basiskompetenzen von Schülerinnen und Schülern mit Förderbedarf „Lernen“, Münster: Waxmann

Leopold, Claudia (2009). Lernstrategien und Textverstehen. Spontaner Einsatz und Förderung von Lernstrategien, Münster: Waxmann 
Linde, Andrea (2007). Alphabetisierung, Grundbildung oder Literalität?, in: Grotlüschen, Anke/Linde, Andrea (Hrsg.): Literalität, Grundbildung oder Lesekompetenz? Beiträge zu einer Theorie-Praxis-Diskussion, Münster: Waxmann, 90-99

Linde, Andrea (2008). Literalität und Lernen. Eine Studie über das Lesen- und Schreibenlernen im Erwachsenenalter, Münster: Waxmann

Link, Jürgen (1999). Versuch über den Normalismus. Wie Normalität produziert wird, Opladen: Westdt. Verlag

Lipowsky, Frank/Drollinger-Vetter, Barbara/Hartig, Johannes/Klieme, Eckhard (2006). Dokumentation der Erhebungsund Auswertungsinstrumente zur schweizerisch-deutschen Videostudie „Unterrichtsqualität, Lernverhalten und mathematisches Verständnis“ 2 Leistungstests, Frankfurt am Main: DIPF

Luhmann, Niklas (2002). Das Erziehungssystem der Gesellschaft. Hg. von Dieter Lenzen, Frankfurt am Main: Suhrkamp

Luhmann, Niklas (2004). Schriften zur Pädagogik, Frankfurt am Main: Suhrkamp

Luhmann, Niklas (1984). Soziale Systeme: Grundriss einer allgemeinen Theorie, Frankfurt am Main: Suhrkamp

Lubmann, Niklas (2009). Soziologische Aufklärung 3. Soziales System, Gesellschaft, Organisation, Wiesbaden: VS Verlag für Sozialwissenschaften

Mayring, Philipp (2010). Qualitative Inhaltsanalyse: Grundlagen und Techniken Dr. nach Typoskript, Weinheim: Beltz Meier, Brian P./Schnall, Simone/Schwarz, Norbert/Bargh, John A. (2012). Embodiment in Social Psychology, in: Topics in Cognitive Science, Vol. 4(4), 705-716

Mertens, Dieter (1985). Das Qualifikationsparadoxon. Bildung und Beschäftigung bei kritischer Arbeitsmarktperspektive, in: Zeitschrift für Pädagogik, Vol. 30, 439-455

Mey, Günter/Mruck, Katja (2011). Grounded Theory Reader, Wiesbaden: VS Verlag für Sozialwissenschafte

Moosbrugger, Helfried (2008). Item-Response-Theorie (IRT), in: Moosbrugger, Helfried/Kelava, Augustin (Hrsg.): Testtheorie und Fragebogenkonstruktion, Berlin, Heidelberg: Springer-Verlag Berlin-Heidelberg, 215-259

Mosenthal, Peter B./Kirsch, Irwin S. (1998). A New Measure for Assessing Document Complexity. The PMOSE/ IKIRSCH Document Readability Formula, in: Journal of Adolescent \& Adult Literacy, Vol. 41(8), 638-657

Müller, Katharina/Prenzel, Manfred/Sälzer, Christine/Mang, Julia/et al. (2017). Wie schneiden Schülerinnen und Schüler an Sonder- und Förderschulen bei PISA ab? Analysen aus der PISA 2012-Zusatzerhebung zu Jugendlichen mit sonderpädagogischem Förderbedarf, in: Unterrichtswissenschaft, Vol. 45(2), 175-192

Müller, Kirstin (2008). Schlüsselkompetenzen und beruflicher Verbleib, Bielefeld: Bertelsmann

Niederhaus, Constanze (2011). Informierende Bilder in Fachkundebüchern: berufsfeldabhängige Variation und didaktische Konsequenzen, in: bwp@ Spezial 5 - Hochschultage Berufliche Bildung 2011, Fachtagung 18, abrufbar unter: http://www.bwpat.de/ht2011/ft18/niederhaus_ft18-ht2014.pdf(letzter Zugriff: 3.5.2019)

OECD, Organisation for Economic Co-operation and Development (Hrsg.) (2010). PISA 2009 Ergebnisse, Bielefeld: Bertelsmann

OECD, Organisation for Economic Co-operation and Development (Hrsg.) (2002). Lesen kann die Welt verändern : Leistung und Engagement im Ländervergleich Ergebnisse PISA 2000, Paris: OECD

Peirce, Charles S. (1991). Naturordnung und Zeichenprozess. Schriften über Semiotik und Naturphilosophie. Hrsg. und eingeleitet von Helmut Pape. Übers. von Bertram Kienzle, Frankfurt am Main: Suhrkamp

Philipp, Maik (2011). Lesesozialisation in Kindheit und Jugend. Lesemotivation, Leseverhalten und Lesekompetenz in Familie, Schule und Peer-Beziehungen, Stuttgart: Kohlhammer

Pieper, Irene/Rosebrock, Cornelia/Wirthwein, Heike/Volz, Steffen (2004). Lesesozialisation in schriftfernen Lebenswelten: Lektüre und Mediengebrauch von HauptschülerInnen, Weinheim: Juventa

Plake, Klaus (2010). Schule als Konstrukt der Öffentlichkeit. Bilder - Strategien - Wirklichkeiten, Wiesbaden: VS, Verlag für Sozialwissenschaften

Projektgruppe VERA (2005). VERA 2005 Deutsch. Didaktische Erläuterungen - Lesen, abrufbar unter: http://veraweb.uni-landau.de/verapub/fileadmindownloads/VERA_Deutsch_didaktische_Erlaeuterungen_2005.pdf (letzter Zugriff: 15.6.2019)

Raithel, Jürgen (2008). Die Befragung als dominantes Datenerhebungsverfahren, in: Raithel, Jürgen (Hrsg.): Quantitative Forschung: Ein Praxiskurs, Wiesbaden: VS Verlag für Sozialwissenschaften, 65-82

Reckwitz, Andreas (2003). Grundelemente einer Theorie sozialer Praktiken. Eine sozialtheoretische Perspektive, in: Zeitschrift für Soziologie, Vol. 32(4), 282-301

Reckwitz, Andreas (2005). Kulturelle Differenzen aus praxeologischer Perspektive. Kulturelle Globalisierung jenseits von Modernisierungstheorie und Kulturessentialismus, in: Srubar, Ilja/Renn, Joachim/Wenzel, Ulrich (Hrsg.): Kulturen vergleichen: Sozial- und kulturwissenschaftliche Grundlagen und Kontroversen, Wiesbaden: VS Verlag für Sozialwissenschaften, 92-111 
Reckwitz, Andreas (2010). Unscharfe Grenzen. Perspektiven der Kultursoziologie, Bielefeld: Transcript

Reinders, Heinz (2012). Qualitative Interviews mit Jugendlichen führen. Ein Leitfaden, München: Oldenbourg

Robson, Eleanor (2003). Tables and tabular formatting in Sumer, Babylonia, and Assyria, 2500 bce-50 ce, in: Campbell-Kelly, Martin/Croarken, Mary/Flood, Raymond/Robson, Eleanor (Hrsg.): The history of mathematical tables: from Sumer to spreadsheets, Oxford: Oxford University Press, 19-47

Roelcke, Thorsten (2001). Fachsprachen im Alltag. Probleme und Perspektiven der Kommunikation zwischen Experten und Laien, in: Lehr, Andrea/Kammerer, Matthias/Konerding, Klaus-Pter/Storrer, Angelika/et al. (Hrsg.): Sprache im Alltag. Beiträge zu neuen Perspektiven in der Linguistik; Herbert Ernst Wiegand zum 65. Geburtstag gewidmet, Berlin: De Gruyter, 219-232

Römer, Christine/Matzke, Brigitte (2005). Lexikologie des Deutschen: eine Einführung, Tübingen: Narr

Rosebrock, Cornelia/Nix, Daniel (2008). Grundlagen der Lesedidaktik und der systematischen schulischen Leseförderung, Baltmannsweiler: Schneider Verlag Hohengehren

Rost, Jürgen (2004). Lehrbuch Testtheorie - Testkonstruktion, Bern: Huber

Rouet, Jean-François (2006). The Skills of Document Use: From Text Comprehension to Web-Based Learning, Mahwah: Erlbaum

Scherer, Petra/Opitz, Elisabeth Moser (2010). Fördern im Mathematikunterricht der Primarstufe, Heidelberg: Spektrum Akademischer Verlag

Scherf, Daniel (2013). Leseförderung aus Lehrersicht, Wiesbaden: Springer Fachmedien

Schmidt, Claudia (2010). Lesen und neue Medien, in: Lutjeharms, Madeline/Schmidt, Claudia (Hrsg.): Lesekompetenz in Erst-, Zweit- und Fremdsprache, Gießener Beiträge zur Fremdsprachendidaktik. Tübingen: Narr, 27-38

Schneider-Wiejowski, Karina/Kellermeier-Rehbein, Birte/Haselhuber, Jakob (Hrsg.) (2013). Vielfalt, Variation und Stellung der deutschen Sprache, Berlin: De Gruyter

Schnotz, Wolfgang (1994). Wissenserwerb mit logischen Bildern, in: Weidenmann, Bernd (Hrsg.): Wissenserwerb mit Bildern: Instruktionale Bilder in Printmedien, Film/Video und Computerprogrammen, Bern, H. Huber, 95-147

Schroeder, Joachim (2011). Elaborierte Codes: Semiotische Überlegungen zur Textualität in einfacharbeitsplätzen, in: Bindl, Anne-Kristin/Schroeder, Joachim/Thielen, Marc (Hrsg.): Arbeitsrealitäten und Lernbedarfe wenig qualifizierter Menschen, Bad Heilbrunn: Klinkhardt, Julius, 209-236

Schroeder, Joachim (2012). Schulen für schwierige Lebenslagen. Studien zu einem Sozialatlas der Bildung, Münster: Waxmann

Seufert, Tina (2003). Wissenserwerb mit multiplen Repräsentationen. Wirksamkeit von Kohärenzbildungshilfen, Berlin: Logos-Verlag

Sloane, Peter F.E. (2007). Bildungsstandards in der beruflichen Bildung. Wirkungssteuerung beruflicher Bildung, Paderborn: Eusl-Verl.-Ges.

Stamm, Margrit (2012). Schulabbrecher in unserem Bildungssystem, Wiesbaden: VS Verlag für Sozialwissenschaften

Stichweh, Rudolf (2013). Inklusion und Exklusion in der Weltgesellschaft - am Beispiel der Schule und des Erziehungssystems, in: Zeitschrift für Inklusion; Nr. 1 (2013)

Stichweh, Rudolf (2009). Leitgesichtspunkte einer Soziologie der Inklusion und Exklusion, in: Stichweh, Rudolf/Windolf, Paul (Hrsg.): Inklusion und Exklusion: Analysen zur Sozialstruktur und sozialen Ungleichheit, Wiesbaden: VS Verlag für Sozialwissenschaften, 29-44

Stitt-Gohdes, Wanda L./Lambrecht, Judith J./Redmann, Donna H. (2000). The Critical-Incident Technique in Job Behavior Research, in: Journal of Vocational Education Research, Vol. 25(1), 59-84

Tenorth, Heinz-Elmar (2010). Arbeit an der Theorie. Kritik, Analyse, Konstruktion, in: Friebertshäuser, Barbara/Langer, Antje/Prengel, Annedore (Hrsg.): Handbuch qualitative Forschungsmethoden in der Erziehungswissenschaft, Weinheim: Juventa, 89-100

Terzer, Eva/Hartig, Johannes/Upmeier zu Belzen, Annette (2013). Systematische Konstruktion eines Tests zu Modellkompetenz im Biologieunterricht unter Berücksichtigung von Gütekriterien, in: Zeitschrift für Didaktik der Naturwissenschafte, Vol. 19, 51-76

Thimus, Albert, Freiherr von (1868). Die harmonikale Symbolik des Alterthums. Erste Abtheilung Köln: Verlag der M. DuMont-Schauberg'schen Buchhandlung

Tobies, Renate/Tournès, Dominique (2011). Mini-Workshop. History of Numerical and Graphical Tables. Report No. 12/2014. Mathematisches Forschungsinstitut Oberwolfach, abrufbar unter: http://www.ems-ph.org/doi/10.4171/ OWR/2011/12 (letzter Zugriff: 12.10.2019) 
Tomlin, Alison/Baker, Dave/Street, Brian (2002). Home and school numeracy practices: Where are the borders and overlaps?, in: Valero, Paola/Skovsmose, Ole (Hrsg.): Proceedings of the 3rd International MES Conference, Copenhagen: Centre for Research in Learning Mathematics, 1-10

Ullrich, Mark/Schnotz, Wolfgang/Horz, Holger/McElvany, Nele/et al. (2012). Kognitionspsychologische Aspekte eines Kompetenzmodells zur Bild-Text-Integration, in: Psychologische Rundschau, Vol. 63(1), 11-17

Urban, Michael (2009). Form, System und Psyche: zur Funktion von psychischem System und struktureller Kopplung in der Systemtheorie, Wiesbaden: VS Verlag für Sozialwissenschaften

Vanderstraeten, Ralf (2006). Die Unwahrscheinlichkeit der pädagogischen Kommunikation, in: Ehrenspeck, Yvonne/ Lenzen, Dieter (Hrsg.): Beobachtungen des Erziehungssystems: Systemtheoretische Perspektiven, Wiesbaden: VS Verlag für Sozialwissenschaften, 95-112

Volz, Steffen (2005). Literaturerwerb im Bildungskeller. Befunde zur literarischen Sozialisation und zu literarischen Kompetenzen bildungsferner Jugendlicher, Heidelberg: unveröffentl. Diss., abrufbar unter: http://archiv.ub.uniheidelberg.de/volltextserver/volltexte/2006/6215/pdf/Bildungskeller4.pdf (letzter Zugriff: 16.5.2018)

Walter, Sybille/Walther, Andreas (2007). „Context matters“. Anforderungen, Risiken und Spielräume im deutschen Übergangssystem, in: Stauber, Barbara/Pohl, Axel/Walther, Andreas (Hrsg.): Subjektorientierte Übergangsforschung. Rekonstruktion und Unterstützung biografischer Übergänge junger Erwachsener, Weinheim: Juventa, 65-96

Walther, Andreas (2014). Kompetenz und Partizipation. Dilemmata der Jugendhilfe in der Wissensgesellschaft, in: Faas, Stefan/Bauer, Petra/Treptow, Rainer (Hrsg.): Kompetenz, Performanz, Soziale Teilhabe. Sozialpädagogische Perspektiven auf ein bildungstheoretisches Konstrukt, Wiesbaden: Springer VS, 155-170

Weidenmann, Bernd (1994). Informierende Bilder, in: Weidenmann, Bernd (Hrsg.): Wissenserwerb mit Bildern: Instruktionale Bilder in Printmedien, Film/Video und Computerprogrammen, Bern, Huber, 9-58

Weinbach, Christine (2004).... und gemeinsam zeugen sie geistige Kinder: Erotische Phantasien um Niklas Luhmann und Pierre Bourdieu, in: Nassehi, Armin/Nollmann, Gerd (Hrsg.): Bourdieu und Luhmann. Ein Theorievergleich, Frankfurt am Main: Suhrkamp, 57-84

Weinert, Franz E. (2001). Vergleichende Leistungsmessung in Schulen - eine umstrittene Selbstverständlichkeit, in: Weinert, Franz E. (Hrsg.): Leistungsmessungen in Schulen, Weinheim: Beltz, 17-31

Weinkopf, Claudia/Jaehrling, Karen (2005). Stellenbesetzungsprobleme bei „Einfacharbeitsplätzen bzw. Niedriglohnjobs“, in: Hoffmann, Thomas (Hrsg.): Einfache Arbeit für gering Qualifizierte: Material und Handlungshilfen, Eschborn: RKW-Verlag, 16-17

Werlich, Egon (1975). Typologie der Texte. Entwurf eines textlinguisten Modells zur Grundlegung einer Textgrammatik, Heidelberg: Quelle \& Meyer

Windolf, Paul (2009). Einleitung: Inklusion und soziale Ungleichheit, in: Stichweh, Rudolf/Windolf, Paul (Hrsg.): Inklusion und Exklusion: Analysen zur Sozialstruktur und sozialen Ungleichheit, Wiesbaden: VS Verlag für Sozialwissenschaften, 11-27

Witzel, Andreas (2000). Das problemzentrierte Interview, in: Forum: Qualitative Social Research, Vol. 1(1), Art. 22

Wocken, Hans/Gröhlich, Carola (2009). Kompetenzen von Schülerinnen und Schülern an Hamburger Förderschulen, in: Bos, Wilfried/Bonsen, Martin/Gröhlich, Carola (Hrsg.): KESS 7. Kompetenzen und Einstellungen von Schülerinnen und Schülern an Hamburger Schulen zu Beginn der Jahrgangsstufe 7, HANSE - Hamburger Schriften zur Qualität im Bildungswesen, Münster: Waxmann, 133-142

Yom, Miriam/Wilhelm, Thorsten H./Gauert, Stefanie (2007). Protokolle lauten Denkens und Site Covering, in: Buber, Renate/Holzmüller, Hartmut H. (Hrsg.): Qualitative Marktforschung, Wiesbaden: Gabler, 635-652

Zwaan, Rolf A. (2004). The Immersed Experiencer. Toward an Embodied Theory of Language Comprehension, in: Ross, Brian H. (Hrsg.): The psychology of learning and motivation. Advances in research and theory, New York: Elsevier Science, 35-62 


\section{Tabellenverzeichnis}

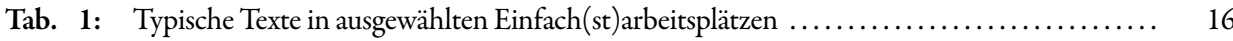

Tab. 2: Niedriglohnbeschäftigung nach Geschlecht und höchstem Ausbildungsabschluss ........ 21

Tab. 3: Typische vs. atypische Beschäftigungsverhältnisse nach Qualifikation im zeitlichen Vergleich ....

Tab. 4: Kontrastierung von Kompetenzbegriffen entlang ihrer handlungstheoretischen Annahmen ...................................................... 48

Tab. 5: Hierarchisches Modell der kognitiven Anforderungen von Texten „Basaler Literalität“ .... 56

Tab. 6: Gegenüberstellung der vier Begriffe des Lesens nach Kulturkonzepten (a) . ..............

Tab. 7: Gegenüberstellung textueller Beispiele und Strukturen ikonischer Zeichen ...............

Tab. 8: Semiotische Aspekte des Zusammenhangs zwischen Repräsention und repräsentiertem Sachverhalt bei graphischen Darstellungen, Karten und Diagrammen .............. 71

Tab. 9: Semiotische Aspekte des Zusammenhangs zwischen Repräsentation und repräsentiertem Sachverhalt bei Tabellen ............................................ 75

Tab. 10: Set starker Codes hinter Diagrammen (qualitativ/qualitativ; qualitativ/quantitativ) .......

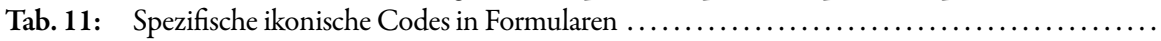

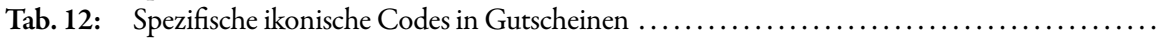

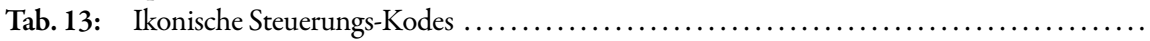

Tab. 14: Hierarchie-niedrige Prozessebene: interaktive Identifikation von Lokalzeichen ...........

Tab. 15: Hierarchie-niedrige Prozessebene: interaktive Verarbeitung von Lokalzeichen .............

Tab. 16: Prozessebene: interaktive Verarbeitung von Globalzeichen $\ldots \ldots \ldots \ldots \ldots \ldots \ldots \ldots \ldots . . . \ldots \ldots$

Tab. 17: Routinalisierte lokale kulturelle Codes in sozialen Lesepraxen der Sekundarstufe I .........

Tab. 18: Routinalisierte lokale kulturelle Codes in sozialen Lesepraxen der Primarstufe und außerhalb von Lesecompetencies .................................... 122

Tab. 19: Niedriglohnbeschäftigung nach Geschlecht und höchstem Ausbildungsabschluss ....... 156

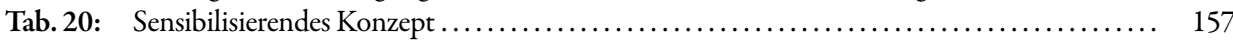

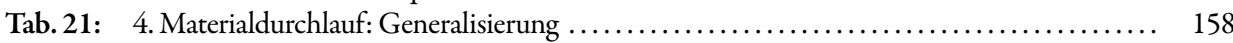

Tab. 22: Erster Materialdurchlauf mit Bezug auf kontinuierliche Texte $\ldots \ldots \ldots \ldots \ldots \ldots \ldots \ldots \ldots \ldots$

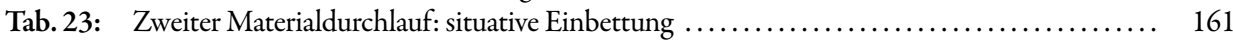

Tab. 24: Zweiter Materialdurchlauf: situative Einbettung und textueller Gegenstand ............ 161

Tab. 25: Zweiter Materialdurchlauf: einige Kategorien situativer Einbettungen im Produktions-/Logistik- und Dienstleistungssektor .........................

Tab. 26: Situativ-textuelle routinalisierte lokale kulturelle Codes zur Annäherung an eine skillful performance mit nichtkontinuierlichen Texten und Textkomplexen .............

Tab.27: Gegenüberstellung der routinalisierten lokalen kulturellen Codes in und von Texten in sozialen Praxen an der obligatorischen Schule und an den untersuchten Einfach(st)arbeitsplätzen

Tab. 28: Gegenüberstellung von textuellen Gegenständen des Lesens einer skillful performance entlang sozialer Praxen der obligatorischen Schulbildung (mit Referenz der funktionalen Grundbildungscompentencies) und an Einfach(st)arbeitsplätzen ..........

Tab. 29: Lesecompetencies kontinuierlicher Texte der KESS-7-F-Studie $\ldots \ldots \ldots \ldots \ldots \ldots \ldots \ldots \ldots$

Tab. 30: Stichprobenmerkmale der referentiellen PISA-Zusatzstudie $\ldots \ldots \ldots \ldots \ldots \ldots \ldots \ldots \ldots$

Tab. 31: Lesecompetencies der PISA-Zusatzstudie .................................

Tab. 32: Stichprobenmerkmale der referentiellen BELLA-Studie $\ldots \ldots \ldots \ldots \ldots \ldots \ldots \ldots \ldots \ldots \ldots$

Tab. 33: Lesecompetencies der BELLA-Studie zu Tabellen, Graphiken und Karten ...............

Tab. 34: Schwierigkeitsbereiche der referentiellen BELLA-Studie zu Tabellen, Graphiken

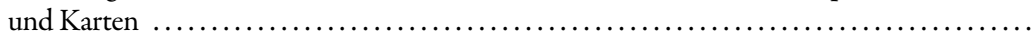

Tab. 35: Verteilung der Testaufgaben entlang des primären Selektionskriterium routinalisierte lokale kulturelle Codes ...............................................

Tab. 36: Verteilung der Testaufgaben entlang des sekundären Selektionskriterium Listenstruktur 
Tab. 37: Codierregeln zur standardisierten Befragung „Wohngeldantrag“ “................. 190

Tab. 38: Codierregeln zur standardisierten Befragung „Notfallverhalten“ $\ldots \ldots \ldots \ldots \ldots \ldots \ldots \ldots . \ldots 1$

Tab. 39: Codierregeln zur standardisierten Befragung „Lagerdokumentation“ ............... 192

Tab.40: Codierregeln zur standardisierten Befragung „Autoprotokoll“ $\ldots \ldots \ldots \ldots \ldots \ldots \ldots \ldots . \ldots 2$

Tab. 41: Codierregeln zur standardisierten Befragung „Serviceorganisation“ ................. 193

Tab. 42: Codierregeln zur standardisierten Befragung „Montagedokumentation“ “............ 193

Tab. 43: Verteilung der teilnehmenden Jugendlichen auf Bildungsgänge $\ldots \ldots \ldots \ldots \ldots \ldots \ldots \ldots \ldots \ldots$

Tab. 44: Vergleich einzelner Stichprobenmerkmale im Bildungsgang des sonderpädagogischen

Schwerpunkts Lernen zur referentiellen PISA-Zusatzstudie ...................... 195

Tab. 45: Vergleich der globalen Modelgüte ....................................... 196

Tab. 46: Modellvergleich durch Fit-Statistik-Kennwerten .......................... 196

Tab.47: Modelfit der Dimension 1 der Lesecompetencies multikodaler Texte und Textkomplexe .............................................. 197

Tab. 48: Modelfit der Dimension 2 der Lesecompetencies multikodaler Texte und Textkomplexe

Tab.49: Qualitative Kategorisierung der Items zu Leseompetencies von multikodalen Texten vs. Textkomplexen ............................................ 200

Tab. 50: Anteil korrekter Antworten nach Bildungsgang innerhalb der Dimension $1 \ldots \ldots \ldots \ldots \ldots .202$

Tab. 51: Anteil korrekter Antworten nach Bildungsgang innerhalb der Dimension 2 ........... 203

Tab. 52: Verteilung der Lesecompetencies multikodaler Texte und Textkomplexe - Mittelwerte ... 204

Tab. 53: Verteilung der Lesecompetencies kontinuierlicher Texte - Mittelwerte ............. 205

Tab. 54: Korrelation zwischen Dimensionen von Lesecompetencies symbolischer Texte quer

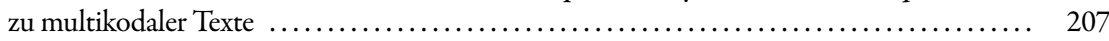

Tab. 55: Lineare Regressionsanalyse zur Vorhersage der Lesecompetencies multikodaler Texte primär ikonischer Kodalität ........................................ 207

Tab. 56: Verengung der Verarbeitung auf Phasen der handelnden Verarbeitung ................. 224

Tab. 57: Kategoriensystem zur Verarbeitung von primär ikonischen Textkomplexen ............. 228

Tab. 58: Literalität einfacher Arbeit eines Lesens als kulturelle Praxen $\ldots \ldots \ldots \ldots \ldots \ldots \ldots \ldots \ldots . \ldots \ldots \ldots$

Tab. 59: Auszug der Verarbeitungsprozesse multikodaler Texte und Textkomplexe $\ldots \ldots \ldots \ldots \ldots .243$ 


\section{Abbildungsverzeichnis}

Abb. 1: Deskripitiv-kategoriale Gliederung nichtkontinuierlicher Texte (Quelle: Haible 2011) . 13

Abb. 2: Abbildungen von Hasen mit zunehmender Realitätsnähe (Quelle: Horz 2009, 110) ... 14

Abb. 3: Beschreibungsschema entlang von Mitgliedsbedingungen an die Komplimentärrollen

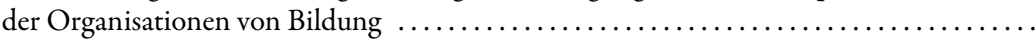

Abb. 4: Inklusive und inkludierend exklusive Mitgliedsbedingungen der Organisationen

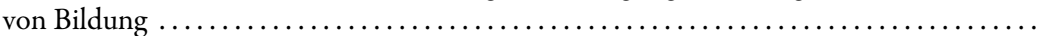

Abb. 5: Exkludierend inklusive und exklusive Mitgliedsbedingungen der Organisationen

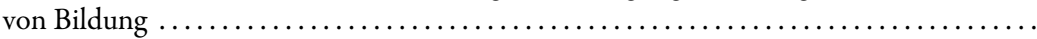

Abb. 6: Beschreibungsschema entlang von Mitgliedsbedingungen an die Leistungsrollen der

Abb. 7: Mitgliedsbedingungen an die Komplementärrollen der Organisationen von Bildung (1)

Abb. 8: Mitgliedsbedingungen an die Komplementärrollen der Organisationen von Bildung (2)

Abb. 9: Determinierung des textuellen Lesens im Spannungsfeld von Kompetenz/en ...........

Abb. 10: Mehrebenenmodell des Lesens als Beispiel des Lesebegriffs als kulturelle Praxis

(Quelle: Rosebrock/Nix 2008)

Abb. 12: Bautagebuch in der Gartenpflege (Quelle: Imitation GRAWIRA) $\ldots \ldots \ldots \ldots \ldots \ldots \ldots$

Abb. 13: Starke Codes in einem Strukturdiagramm (4. Ebene) $\ldots \ldots \ldots \ldots \ldots \ldots \ldots \ldots \ldots \ldots$

Abb. 14: Starke Codes in einem Strukturdiagramm (2. Ebene) $\ldots \ldots \ldots \ldots \ldots \ldots \ldots \ldots \ldots \ldots \ldots$

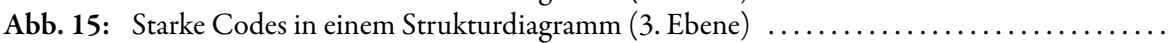

Abb. 16: Starker Kode hinter einer (senkrechten) simple-list bzw. list-of-simple-lists (4. Ebene)

(Quelle: adaptiert nach Mosenthal/Kirsch 1998)

Abb. 17: Starker Kode hinter einer (senkrechten) combined-list (2. Ebene)

Abb. 18: Starker Kode hinter einer (senkrechten) intersected-list (3. Ebene)

(Quelle: adaptiert nach Mosenthal/Kirsch 1998) ...................... 8

Abb. 19: Arbeitsplan in der Gastronomie (Quelle: Basendowski 2013; Imitation) . . . . . . . . 81

Abb. 20: Starker Kode hinter einer (senkrechten) nested-list (4. Ebene) $\ldots \ldots \ldots \ldots \ldots \ldots \ldots \ldots . \ldots 2$

Abb. 21: Kassenbon und Variation seiner tabellarischen Felder ....................... 85

Abb. 22: Beispiel einer Rechnung nach DIN 676 und DIN $5008 \ldots \ldots \ldots \ldots \ldots \ldots \ldots \ldots \ldots \ldots$

Abb. 23: Empfangsschein in der Möbelmontage (Quelle: Imitation; GRAWIRA) . . . . ...... 89

Abb. 24: Serviceanforderung in der Möbelmontage (Quelle: Imitation; GRAWIRA) ........ 91

Abb. 25: Schulungsmappe zu den Formularen in der Möbelmontage

(Quelle: Kopie; GRAWIRA) . . . . . . . . . . . . . 92

Abb. 26: Aushang Brandverhalten in der Produktion (Quelle: Imitation; Studie Basendowski) ... 93

Abb. 27: Lieferschein in der Lagerlogistik (Quelle: Imitation; Studie Basendowski) . ......... 97

Abb. 28: Functional webs im Immersed Experiencer Framework (Quelle: ebd., 42) . ......... 113

Abb. 29: Beispiele textstrukturell syntaktischer Lokalzeichen-Lokalzeichen-Relationen ........ 124

Abb. 30: Beispiele textstrukturell syntaktischer Globalzeichen-Globalzeichen-Interferenzen . . . . 125

Abb. 31: Beispiele textstrukturell semantischer Lokalzeichen-Lokalzeichen-Relationen ........ 125

Abb. 32: Beispiele textstrukturell semantischer Globalzeichen-Globalzeichen-Interferenzen . . . 126

Abb. 33: Arbeitsmodell der attentiven (implizit-embodied bis explizit-konzeptionellen)

Verarbeitung von primär ikonisch repräsentierten Texten und Textkomplexen ........ 128

Abb. 34: Struktur der Vernetzung (unten) der einzelnen Forschungsfragen (oben) . . ......... 134

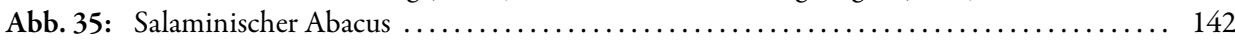

Abb. 36: Multiplikationstafel/Abacus-Tabelle aus dem europäischen Mittelalter ........... 143

Abb. 37: Erste bekannte gedruckte Fassung einer Multiplikationstafel . . . . . . . . . . . . . 145

Abb. 38: Bürokratische Archivierung einer Anzahl von Schafen und Ziegen

(Quelle: Robson 2003, 24) ................................ 147

Abb. 39: To-do-Liste von Benjamin Franklin (Quelle: Franklin 1916) . . . . . . . . . . . 148 
Abb. 40: Historische Fahrpläne im Eisenbahnpersonenverkehr (1839) (Quelle: 1839, http://www. scienceandsociety.co.uk/results.asp?image $=10562991) \ldots \ldots \ldots \ldots \ldots \ldots \ldots \ldots$

Abb. 41: Historische Fahrpläne im Eisenbahnpersonenverkehr (1841) (Quelle: 1841, http://leicester. contentdm.oclc.org/cdm/ref/collection/p15407coll9/id/1264) ............. 150

Abb. 42: Checkliste Frühstücksdienst (Quelle: Imitation; Studie Basendowski)

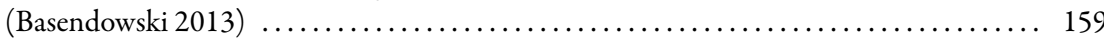

Abb. 43: Lesecompetencies-Stufen der PISA-Zusatzstudie

(Quelle: Müller et al. 2017, 187; Auszug) ......................... 177

Abb. 44: Beispielaufgabe aus der BELLA-Studie zum Leseverständnis bei Sachtexten

(Quelle: Ahrbeck et al. 2009, 41) .............................. 178

Abb. 45: Schwierigkeitsbereiche und Aufgabenbeispiele der BELLA-Studie zu Tabellen,

Graphiken und Karten (Quelle: ebd.) . ........................... 180

Abb. 46: Primäres Selektionskriterium bei der Auswahl der Testaufgaben: routinalisierte

lokale kulturelle Codes \& textuelle Gegenstände ....................... 185

Abb. 47: Sekundäres Selektionskriterium bei der Auswahl der Testaufgaben: Listenstruktur ...... 188

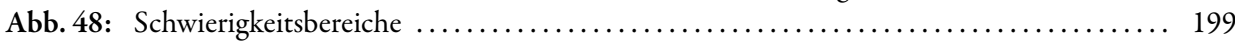

Abb. 49: Verteilung der Lesecompetencies multikodaler Texte und Textkomplexe -

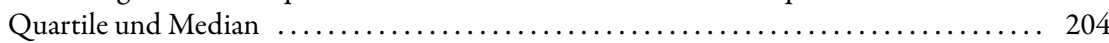

Abb. 50: Primär ikonischer Textkomplex Service - To-do-Liste ...................... 219

Abb. 51: Primär ikonischer Textkomplex Service - Arbeitsplan ..................... 220

Abb. 52: Primär ikonischer Textkomplex Service - Raumplan ..................... 220

Abb. 53: Kategoriensystem zur Verarbeitung von primär ikonischen Textkomplexen .......... 228

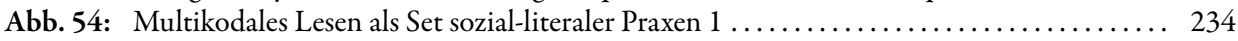

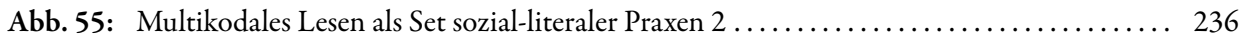

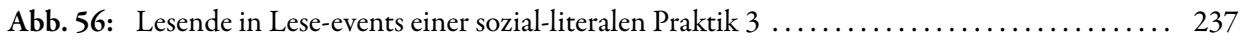

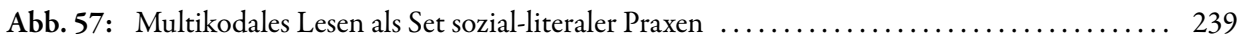

Abb. 58: Verarbeitung multikodaler Texte als Set sozial-literaler Praxen $\ldots \ldots \ldots \ldots \ldots \ldots \ldots \ldots 243$ 


\section{Anhang}

\section{Anhang 1: Beispiel Retrospektion}

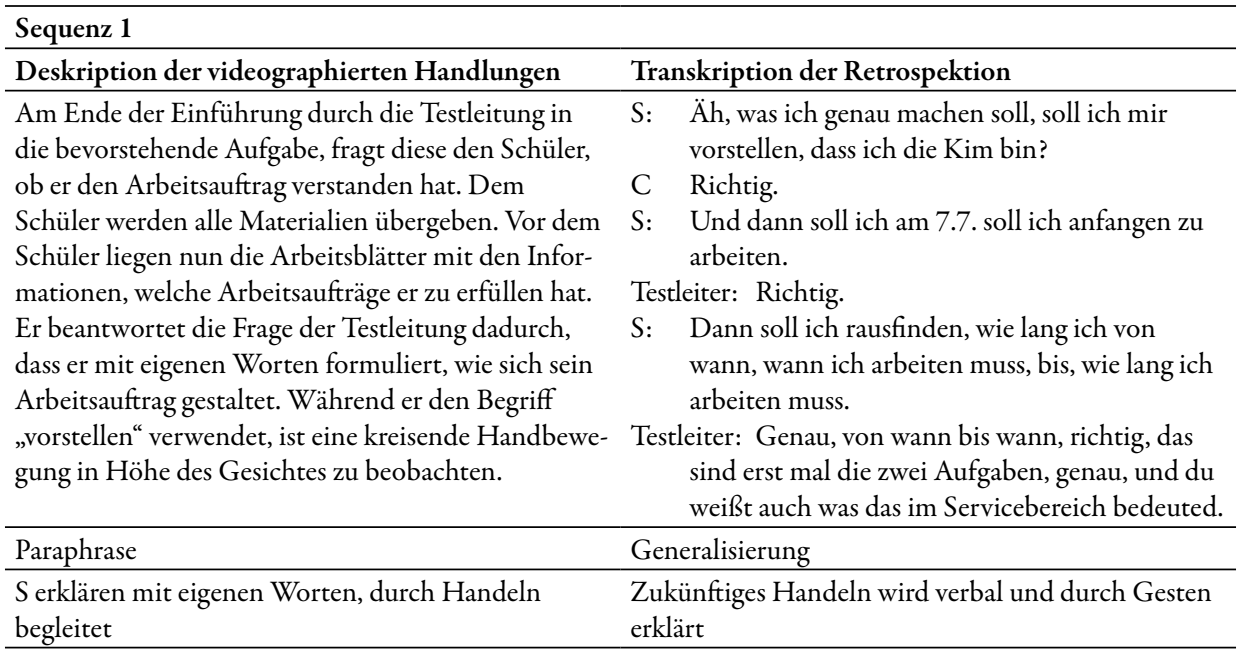

\begin{tabular}{|c|c|}
\hline Sequenz 2 & \\
\hline Deskription der videographierten Handlungen & Transkription der Retrospektion \\
\hline $\begin{array}{l}\text { Die Testleitung wiederholt, welche Aufgaben im } \\
\text { Bereich des Service eines Gasthauses zu erledigen } \\
\text { sind und stellt dabei nochmals die Arbeitsblätter vor. } \\
\text { Der Schüler nickt währenddessen kontinuierlich mit } \\
\text { seinem Kopf. }\end{array}$ & $\begin{array}{l}\text { Es liegt keine parallele Verbalisierung zu dieser } \\
\text { Sequenz vor. }\end{array}$ \\
\hline Paraphrase & Generalisierung \\
\hline Testleiter erklärt. & Verbale Testeinführung und Materialvorstellung \\
\hline \multicolumn{2}{|l|}{ Sequenz 3} \\
\hline Deskription der videographierten Handlungen & Transkription der Retrospektion \\
\hline $\begin{array}{l}\text { Die Testleitung verbalisiert den Beginn der Arbeits- } \\
\text { phase. Der Schüler liest das Aufgabenblatt durch, auf } \\
\text { welchem der Arbeitsauftrag steht. Während des Le- } \\
\text { sens bewegt er die Lippen; es sind keine akustischen } \\
\text { Signale zu vernehmen. Der Schüler sitzt leicht im } \\
\text { Oberkörper gebeugt in Richtung der Arbeitsblätter } \\
\text { und des Tisches. }\end{array}$ & $\begin{array}{l}\text { Es liegt keine parallele Verbalisierung zu dieser } \\
\text { Sequenz vor. }\end{array}$ \\
\hline Paraphrase & Generalisierung \\
\hline S liest, beobachtbare Lippenbewegung & $\begin{array}{l}\text { Lesen (Informationsentnahme) durch Lippenbewe- } \\
\text { gung }\end{array}$ \\
\hline
\end{tabular}




\begin{tabular}{|c|c|}
\hline \multicolumn{2}{|l|}{ Sequenz 4} \\
\hline ibung der beobachtbaren Handlung & $\begin{array}{l}\text { Transkription der Retroperspektiven verbalen } \\
\text { Äußerungen }\end{array}$ \\
\hline $\begin{array}{l}\text { Nachdem der Schüler das Lesen der Aufgaben- } \\
\text { stellung beendet hat, greift er zielgerichtet zum } \\
\text { Arbeitsplan. Beim Lesen des Planes bewegt er ebenso } \\
\text { die Lippen, ohne dass ein Laut zu vernehmen ist. Der } \\
\text { Daumen seiner linken Hand ruht etwa in Höhe des } \\
\text { Namens [12.4.10], welchen es zu identifizieren gilt. } \\
\text { Was mit seiner rechten Hand geschieht, ist zu diesem } \\
\text { Zeitpunkt nicht beobachtbar. Der Schüler bewegt } \\
\text { den Kopf von links nach rechts. An dieser Stelle wird } \\
\text { nun sichtbar, dass der Schüler auch den Daumen } \\
\text { der rechten Hand in Höhe des zu identifizierenden } \\
\text { Namens hält. Der Arbeitsplan wird nun vom Schüler } \\
\text { zur Seite gelegt. }\end{array}$ & $\begin{array}{l}\text { Testleiter: Mh, super, gut und wie war das hier mit } \\
\text { dem Zeitplan. Das wäre ja die erste Aufgabe } \\
\text { eigentlich. Hast du das jetzt verstanden, was die } \\
\text { Kim eigentlich jetzt am 7.7. machen muss? } \\
\text { S: } \quad \text { Eigentlich nein, ich habe da, ich habe eigentlich } \\
\text { nur geguckt, nach Kim, noch mal. } \\
\text { Testleiter: Mh, das hab ich gesehen. } \\
\text { S: F irgendwas, dann hab ich, denk mal hier hat sie } \\
\text { frei. } \\
\text { Testleiter: Ja. } \\
\text { S: Und dann hier am 7.7. hat sie Arbeit von } \\
\text { fünften bis zum siebten hat die Arbeit [S zeigt } \\
\text { während dieser Gesprächsphase jeweils mit } \\
\text { seinem Stift genau an, wo und wie er sich auf } \\
\text { dem Zeitplan bewegt(e)]. }\end{array}$ \\
\hline Paraphrase & Generalisierung \\
\hline $\begin{array}{l}\text { S liest, beobachtbare Lippen- und Kopfbewegung, } \\
\text { Hand/Finger ruht auf Höhe der Informationsent- } \\
\text { nahme }\end{array}$ & $\begin{array}{l}\text { Lesen (Informationsentnahme) durch Lippenbewe- } \\
\text { gung, Handverortung [und anschließende verbale } \\
\text { Explikation] }\end{array}$ \\
\hline
\end{tabular}

\begin{tabular}{|c|c|}
\hline \multicolumn{2}{|l|}{ Sequenz 5} \\
\hline Deskription der videographierten Handlungen & Transkription der Retrospektion \\
\hline $\begin{array}{l}\text { Es liegt der Raumplan oben auf in der linken Hand } \\
\text { des Schülers. Dieser Plan wird nach knapp einer Se- } \\
\text { kunde um } 180 \text { Grad gedreht, sodass die handschriftli- } \\
\text { chen Informationen zu lesen sind, Piktogramme [15] } \\
\text { und sonstige Informationen [16], wie Bankverbin- } \\
\text { dung [17] etc. stehen nun auf dem Kopf. Der Schüler } \\
\text { betrachtet den Raumplan, es ist keine Augen- oder } \\
\text { Handbewegung zu beobachten, seine Lippen zeigen } \\
\text { minimale Bewegungen an. Der Raumplan wird er- } \\
\text { neut um } 180 \text { Grad gedreht, für ca. } 2 \text { bis } 3 \text { Sekunden } \\
\text { angesehen und zusammen mit dem Arbeitsplan und } \\
\text { der Aufgabenstellung gestapelt, die drei Blätter ver- } \\
\text { bleiben in der linken Hand des Schülers, etwas links } \\
\text { oberhalb der Mitte, ihre Reihenfolge ist: zu oberst } \\
\text { die Aufgabenstellung, dann der Arbeitsplan und zu } \\
\text { unterst der Raumplan. }\end{array}$ & $\begin{array}{l}\text { Testleiter: Ok, und was hat besonders gut geklappt? } \\
\text { Bei den Aufgaben? Wo hast du dich jetzt sicher } \\
\text { gefühlt und hast dir gedacht, oh, das hab ich } \\
\text { jetzt aber echt gut verstanden? } \\
\text { S: Da wo, wo ich die Tische eins bis vier zu de- } \\
\text { cken, zu decken waren. Das hab ich kapiert. }\end{array}$ \\
\hline Paraphrase & Generalisierung \\
\hline $\begin{array}{l}\text { S liest, ohne beobachtbare Lippen- und Kopfbewe- } \\
\text { gung }\end{array}$ & $\begin{array}{l}\text { Lesen (Informationsentnahme) ohne Lippen-, Kopf- } \\
\text { und Handbewegung [anschließende verbale Explika- } \\
\text { tion] }\end{array}$ \\
\hline
\end{tabular}




\begin{tabular}{|c|c|}
\hline Sequenz 6 & \\
\hline Deskription der videographierten Handlungen & Transkription der Retrospektion \\
\hline $\begin{array}{l}\text { Mit der rechten Hand hält der Schüler die Check- } \\
\text { liste fest, welche in etwa mittig vor ihm liegt. Die } \\
\text { Checkliste wird nun durch den Schüler gelesen, } \\
\text { hierbei ist wieder eine deutliche Lippenbewegung zu } \\
\text { beobachten (allerdings ohne akustisch wahrnehm- } \\
\text { bare Laute), dabei bewegt sich der Kopf minimal in } \\
\text { Leserichtung mit. Nach Beendigung dieses Lesens } \\
\text { fä̈hert der Schüler einmal kurz die Arbeitsblätter } \\
\text { in seiner linken Hand auf und stößt sie dann unter } \\
\text { zur Hilfenahme seiner rechten Hand oberhalb der } \\
\text { Checkliste auf dem Tisch zu einem „sauberen und } \\
\text { ordentlichen“ Stapel zusammen. Diesen legt er nun } \\
\text { mittig leicht links etwas oberhalb ab, direkt vor sich } \\
\text { hat der Schüler die Checkliste liegen und greift mit } \\
\text { der rechten Hand nach einem Stift, mit der linken } \\
\text { Hand zieht er das leere Blatt heran und verschiebt } \\
\text { leicht die Checkliste. }\end{array}$ & $\begin{array}{l}\text { S: Denn bevor sie anfangen muss, ist neun oder } \\
\text { zehn Uhr, habe ich auch verstanden, } \\
\text { Testleiter: Mh, } \\
\text { S: Äh, plus die Erwachsene müsste acht Stunden } \\
\text { arbeiten, eigentlich, das müsst ich dazu rech- } \\
\text { nen, eigentlich. }\end{array}$ \\
\hline Paraphrase & Generalisierung \\
\hline S liest, beobachtbare Lippen- und Kopfbewegung & $\begin{array}{l}\text { Lesen (Informationsentnahme) durch Lippenbewe- } \\
\text { gung, [und anschließende verbale Explikation der } \\
\text { Diskrepanz der Arbeitszeiten] }\end{array}$ \\
\hline
\end{tabular}

\begin{tabular}{|c|c|}
\hline Sequenz 7 & \\
\hline Deskription der videographierten Handlungen & Transkription der Retrospektion \\
\hline $\begin{array}{l}\text { Der Schüler beginnt nun auf das weiße Blatt zu sch- } \\
\text { reiben. Sein Kopf bewegt sich von links nach rechts. } \\
\text { Anschließend führt er in einem kurzen Abstand den } \\
\text { Stift über die Checkliste, er verharrt minimal in der } \\
\text { Höhe der Arbeitszeitinformationen [22]. }\end{array}$ & $\begin{array}{l}\text { Testleiter: Mh, eh, eh, genau, super und ja, wie bist } \\
\text { du denn jetzt vorgegangen? Du hast erst die } \\
\text { Aufgabe gelesen, was du machen sollst, und } \\
\text { dann, was hast du dann gemacht? } \\
\text { S: } \quad \begin{array}{l}\text { Dann hab ich mir ein paar Stichpunkte aufge- } \\
\text { schrieben. }\end{array}\end{array}$ \\
\hline Paraphrase & Generalisierung \\
\hline $\begin{array}{l}\text { S liest, beobachtbare Lippen-, Kopf- und Handbewe- } \\
\text { gung, Stift ruht auf Höhe der Informationsentnahme }\end{array}$ & $\begin{array}{l}\text { Lesen (Informationsentnahme) mit Lippen-, } \\
\text { Kopf- und Handbewegung, [anschließende verbale } \\
\text { Explikation des Handelns] }\end{array}$ \\
\hline
\end{tabular}

\begin{tabular}{|c|c|}
\hline Sequenz 8 & \\
\hline Deskription der videographierten Handlungen & Transkription der Retrospektion \\
\hline $\begin{array}{l}\text { Daran anschließend greift er zur Aufgabenstellung, } \\
\text { der Schüler scheint etwas zu lesen (es ist keine Beob- } \\
\text { achtung des Kopfes, oder der Hände möglich), dann } \\
\text { behält er die Aufgabenstellung in der linken Hand } \\
\text { und zieht mit der Rechten den Raumplan näher zu } \\
\text { sich heran. }\end{array}$ & $\begin{array}{l}\text { Es liegt keine parallele Verbalisierung zu dieser } \\
\text { Sequenz vor. }\end{array}$ \\
\hline Paraphrase & Generalisierung \\
\hline $\begin{array}{l}\text { S verbindet Textdokumente, durch Handlung; } \\
\text { [Lesen nicht zu beobachten und belegbar durch } \\
\text { bisherige Indikatoren (Lippenbewegung etc.)] }\end{array}$ & Verbindung wird durch Handeln hergestellt/sichtbar \\
\hline
\end{tabular}




\begin{tabular}{|c|c|}
\hline & \\
\hline Deskription der videographierten Handlungen & Transkription der Retrospektion \\
\hline $\begin{array}{l}\text { Der Raumplan liegt nun auf der Checkliste, welcher } \\
\text { sofort um } 180 \text { Grad gedreht wird. Mit dem Kugel- } \\
\text { schreiber in der rechten Hand erfolgt eine abzäh- } \\
\text { lende Bewegung über die Tische des Raumplans } \\
\text { [17.2, 17.4] nun wechselt er in den mittleren Teil des } \\
\text { Raumplans, der Stift wird erst zum unteren Ende des } \\
\text { Blattes bewegt, wird direkt an das obere Blattende } \\
\text { geführt und es beginnt eine abzählende Bewegung } \\
\text { [18.8, 18.6, 18.4, 18.2] fließend ist der Übergang zu } \\
\text { den Tischen [19.3] und Stammtisch [19.1]. Nach } \\
\text { Beendigung dieses Zählvorgangs erfolgt ein erneutes } \\
\text { Abzählen mittels des Kugelschreibers deutlich ange- } \\
\text { zeigt durch die Kennzeichnungen } 17.2,17.4,18.2 \text {, } \\
18.4,18.6, \text { (ohne } 18.8 \text { und } 19,1 \text { (Stammtisch)) und } \\
\text { 19.3 dann, 18.8. Nun notiert sich der Schüler etwas } \\
\text { auf seinem weißen Blatt. }\end{array}$ & $\begin{array}{l}\text { S: Äh, das sind achtzehn Tische. Habe ich so, habe } \\
\text { ich mir dann, habe ich dann mal durchgezählt. } \\
\text { S: Ich habe gedacht, wenn man Koch werden will, } \\
\text { weil ich Koch werden will, } \\
\text { Testleiter: Äh, } \\
\text { S: muss man ja auch rechnen, wie viel du einneh- } \\
\text { men musst und welche Tische gedeckt werden: } \\
\text { Wann, wann, wann, wenn jetzt ne Klasse } \\
\text { kommt mit fünfzehn Mann, welche Tische } \\
\text { gedeckt werden, wie viel, wie viel Essen man } \\
\text { portioniert und dann hab ich mir gedacht, ist } \\
\text { eigentlich gar nicht so schlimm. } \\
\text { Testleiter: Mh, hast du dafür die Finger genommen? } \\
\text { Nein, du hast dann durchgezählt, genau super. } \\
\text { Das habe ich gesehen. So, wenn du jetzt zurück- } \\
\text { gehen würdest in deine Klasse, ja, was würdest } \\
\text { du deinen Mitschülern sagen, was du eigentlich } \\
\text { jetzt hier machen musstest? So mal in deinen } \\
\text { eigenen Worten. } \\
\text { Testleiter: Mh, mh } \\
\text { S: Der Plan, Tisch für vier decken, mit Geschirr } \\
\text { und so. }\end{array}$ \\
\hline Paraphrase & Generalisierung \\
\hline $\begin{array}{l}\text { S liest, beobachtbare Lippen-, Kopf- und Handbewe- } \\
\text { gung, Stift wird zur Informationsentnahme sichtbar } \\
\text { über das Dokument geführt (Prozess beobachtbar, } \\
\text { expliziert im Interview, wirkt sehr routiniert) }\end{array}$ & $\begin{array}{l}\text { S erklärt sein Tun verbal und durch (erneutes) } \\
\text { Handeln. }\end{array}$ \\
\hline
\end{tabular}




\begin{tabular}{|c|c|}
\hline Sequenz 10 & \\
\hline Deskription der videographierten Handlungen & Transkription der Retrospektion \\
\hline $\begin{array}{l}\text { Es wird der Raumplan auf die Checkliste gelegt, der } \\
\text { Schüler nimmt nun in beide Hände die Aufgaben- } \\
\text { stellung und beginnt zu lesen (es sind minimale } \\
\text { Lippenbewegungen zu erkennen). Es erfolgt nun ein } \\
\text { Abgleich zwischen Aufgabenblatt und Raumplan, } \\
\text { der Stift wird kurz über die Tischreihen geführt, } \\
\text { und es erfolgt ein erneutes notierten auf dem weisen } \\
\text { Blatt. \{Vermutung: hier scheint die integrierte Rech- } \\
\text { nung notiert und ausgerechnet zu werden\} }\end{array}$ & $\begin{array}{l}\text { S: Dann habe ich mir ein paar Stichpunkte aufge- } \\
\text { schrieben. Um neun kann die Arbeit anfangen. } \\
\text { Testleiter: ehä }\end{array}$ \\
\hline Paraphrase & Generalisierung \\
\hline $\begin{array}{l}\text { S liest beobachtbare Lippenbewegung, S verbindet } \\
\text { Textdokumente mittels beobachtbarer Stiftführung/ } \\
\text { Handlung }\end{array}$ & $\begin{array}{l}\text { Verbindung durch Handlung herstellen, Handeln } \\
\text { explizierbar }\end{array}$ \\
\hline
\end{tabular}

\begin{tabular}{ll}
\hline Sequenz 11 & \\
\hline Deskription der videographierten Handlungen & Transkription der Retrospektion \\
\hline $\begin{array}{l}\text { Nun wird der Raumplan in die linke Hand gegeben, } \\
\text { wo sich bereits das Arbeitsblatt mit der Aufgaben- }\end{array}$ & Es liegt keine parallele Verbalisierung zu dieser \\
stellung befindet und auf diesen gelegt. & Sequenz vor. \\
\hline Paraphrase & Generalisierung \\
\hline S organisiert Arbeitsplatz, handelnd & Arbeitsplatzorganisation \\
\hline
\end{tabular}

\begin{tabular}{|c|c|}
\hline \multicolumn{2}{|l|}{ Sequenz 12} \\
\hline Deskription der videographierten Handlungen & Transkription der Retrospektion \\
\hline $\begin{array}{l}\text { Mit dem Kugelschreiber in der rechten Hand be- } \\
\text { ginnt der Schüler die Checkliste, welche nun wieder } \\
\text { gut sichtbar mittig vor ihm liegt, zu lesen. Sowohl } \\
\text { der Kugelschreiber wie auch der Kopf des Schülers } \\
\text { wandern von links nach rechts über das Blatt, die } \\
\text { Lippen des Schülers bewegen sich. }\end{array}$ & $\begin{array}{l}\text { Es liegt keine parallele Verbalisierung zu dieser } \\
\text { Sequenz vor. }\end{array}$ \\
\hline Paraphrase & Generalisierung \\
\hline $\begin{array}{l}\text { S liest, beobachtbare Lippen-, Kopf- und Handbewe- } \\
\text { gung mittels Stifts. }\end{array}$ & $\begin{array}{l}\text { Lesen (Informationsentnahme) durch Lippenbewe- } \\
\text { gung, Handverortung }\end{array}$ \\
\hline \multicolumn{2}{|l|}{ Sequenz 13} \\
\hline Deskription der videographierten Handlungen & Transkription der Retrospektion \\
\hline $\begin{array}{l}\text { Der Schüler dreht den Kopf in Richtung der Ka- } \\
\text { mera, in dem Moment, als die Testleitung ihm eine } \\
\text { Frage stellt. }\end{array}$ & $\begin{array}{l}\text { Testleiter: So, die Zeit ist jetzt um. Brauchst du noch } \\
\text { ein bisschen Zeit, oder möchtest du das jetzt been- } \\
\text { den? } \\
\text { S: } \quad \text { Können wir beenden. }\end{array}$ \\
\hline Paraphrase & Generalisierung \\
\hline S fokussiert Aufmerksamkeit auf TL & \\
\hline
\end{tabular}





Für erwachsene ehemalige „PISA-Risikoschüler“ und -schülerinnen bestätigen Forschungsstudien wiederkehrend die Feststellung, dass diese Personengruppe nicht über die funktionalen Lesekompetenzen - „vor dem Komma“ - verfügt und daher linear-kausal höchstwahrscheinlich nicht in sämtlichen Lebensbereichen teilhaben kann. Erlauben jedoch die psychometrischen Tests tatsächlich eine Aussage über die Inklusion in Arbeit im Beschäftigungssystem der tätigkeitsbezogenen qualifizierten Arbeitsplätze?

Kann die Relation von Bildung und Teilhabe (in Arbeit) lediglich über den Voraussetzungscharakter von Bildung erschlossen werden? Dieser übergeordneten Perspektive folgend, wird das Lesen kulturwissenschaftlich verortet und werden die dahinterliegenden Verarbeitungsmodelle von arbiträren Zeichen erweitert, um textuellen Anforderungen on-the-job überhaupt ressourcenorientiert empirisch nachgehen zu können. Abschließende empirische Studien beschäftigen sich mit der Annäherung, ob „PISA-Risikoschüler“ und -schülerinnen über die Lesekompetenz multikodaler Texte und Textkomplexe (aus zuvorderst kategorialen Tabellen, Grafiken etc. und einzelnen Wörtern bis kurzen Absätzen) verfügen, mit denen sie sich in einer Auswahl von Arbeitsplätzen konfrontiert sehen.

Der Autor

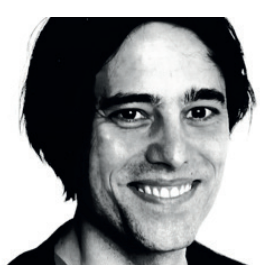

Sven Basendowski, Dr. phil., ist Juniorprofessor für Pädagogik im Förderschwerpunkt Lernen am Institut für Sonderpädagogische Entwicklungsförderung und Rehabilitation an der Universität Rostock. Seine Arbeit-/Interessenschwerpunkte sind kulturtechnische und berufsorientierende Bildungsprozesse unter prekären Bedingungen, netzwerkanalytische Betrachtungen von sozialer Benachteiligung, Strukturen der Übergangsregimes Schule/Arbeit im Spiegel sozialer Benachteiligung.

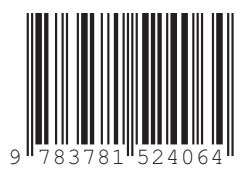

